University of Nebraska - Lincoln

DigitalCommons@University of Nebraska - Lincoln

Faculty Papers and Publications in Animal

Science

Animal Science Department

8-2013

\title{
Genome-wide prediction of age at puberty and reproductive longevity in sows
}

\author{
Julie Kathleen Tart \\ University of Nebraska-Lincoln, tart.julie@gmail.com \\ Rodger K. Johnson \\ University of Nebraska-Lincoln, rjohnson5@unl.edu \\ Justin W. Bundy \\ University of Nebraska-Lincoln, jbundy2@unl.edu \\ N. N. Ferdinand \\ University of Nebraska-Lincoln
}

A. M. McKnite

University of Nebraska-Lincoln

See next page for additional authors

Follow this and additional works at: https://digitalcommons.unl.edu/animalscifacpub

Part of the Genetics and Genomics Commons, and the Meat Science Commons

Tart, Julie Kathleen; Johnson, Rodger K.; Bundy, Justin W.; Ferdinand, N. N.; McKnite, A. M.; Wood, Jennifer R.; Miller, Phillip S.; Rothschild, M. F.; Spangler, Matthew L.; Garrick, Dorian J.; Kachman, Stephen D.; and Ciobanu, Daniel C., "Genome-wide prediction of age at puberty and reproductive longevity in sows" (2013). Faculty Papers and Publications in Animal Science. 803.

https://digitalcommons.unl.edu/animalscifacpub/803

This Article is brought to you for free and open access by the Animal Science Department at DigitalCommons@University of Nebraska - Lincoln. It has been accepted for inclusion in Faculty Papers and Publications in Animal Science by an authorized administrator of DigitalCommons@University of Nebraska Lincoln. 


\section{Authors}

Julie Kathleen Tart, Rodger K. Johnson, Justin W. Bundy, N. N. Ferdinand, A. M. McKnite, Jennifer R.

Wood, Phillip S. Miller, M. F. Rothschild, Matthew L. Spangler, Dorian J. Garrick, Stephen D. Kachman, and

Daniel C. Ciobanu 


\title{
Genome-wide prediction of age at puberty and reproductive longevity in sows
}

\author{
J. K. Tart, ${ }^{1}$ R. K. Johnson, ${ }^{1}$ J. W. Bundy, ${ }^{1}$ N. N. Ferdinand, ${ }^{1}$ A. M. McKnite, ${ }^{1}$ J. R. Wood,${ }^{1}$ \\ P. S. Miller, ${ }^{1}$ M. F. Rothschild, ${ }^{2}$ M. L. Spangler, ${ }^{1}$ D. J. Garrick, ${ }^{2}$ S. D. Kachman, ${ }^{3}$ \& D. C. Ciobanu ${ }^{1}$ \\ 1. Animal Science Department, University of Nebraska-Lincoln, Lincoln, NE, USA \\ 2. Department of Animal Science and Center for Integrated Animal Genomics, lowa State University, Ames, IA, USA \\ 3. Department of Statistics, University of Nebraska-Lincoln, Lincoln, NE, USA
}

Corresponding author - D. C. Ciobanu, Animal Science Department, University of Nebraska-Lincoln, Lincoln, PO Box 830908, NE 68583-0908, USA; email dciobanu2@unl.edu

\begin{abstract}
Traditional selection for sow reproductive longevity is ineffective due to low heritability and late expression of the trait. Incorporation of DNA markers into selection programs is potentially a more practical approach for improving sow lifetime productivity. Using a resource population of crossbred gilts, we explored pleiotropic sources of variation that influence age at puberty and reproductive longevity. Of the traits recorded before breeding, only age at puberty significantly affected the probability that females would produce a first parity litter. The genetic variance explained by 1-Mb windows of the sow genome, compared across traits, uncovered regions that influence both age at puberty and lifetime number of parities. Allelic variants of SNPs located on SSC5 (27-28 Mb), SSC8 (36-37 Mb) and SSC12 (1.2-2 Mb) exhibited additive effects and were associated with both early expression of puberty and a greater than average number of lifetime parities. Combined analysis of these SNPs showed that an increase in the number of favorable alleles had positive impact on reproductive longevity, increasing number of parities by up to 1.36 . The region located on SSC5 harbors non-synonymous alleles in the arginine vasopressin receptor 1A (AVPR1A) gene, a G-protein-coupled receptor associated with social and reproductive behaviors in voles and humans and a candidate for the observed effects. This region is characterized by high levels of linkage disequilibrium in different lines and could be exploited in marker-assisted selection programs across populations to increase sow reproductive longevity.
\end{abstract}

Keywords: DNA, genetics, genome-wide association, marker, pig, reproduction

\section{Introduction}

Through improved management, effective selection for litter size and use of crossbreeding systems, reproductive efficiency has been substantially improved in most of today's commercial sows. Despite this progress, a significant proportion of sows are unable to produce three or more parities, which is required to cover development and maintenance costs of breeding females (Stalder et al., 2003). Reproductive failure is currently the most frequent reason for early culling of sows (Mote et al. 2009) and represents a major economic and welfare problem for swine producers.

Reproductive longevity is a composite trait with different components that are repeatedly expressed throughout the life of the sow and is dependent on the physiological potential of females to resume ovar- ian cyclicity, rebreed and farrow following successful parities. Major components of reproductive longevity include age at puberty, ovulation rate, age at first service, conception rate, number of piglets born alive and weaned, wean-to-service interval, weight loss during lactation and number of litters generated per lifetime. Most of these traits are associated with reproductive functions and have significant environmental variation. Age at puberty is moderately heritable and the earliest phenotypic indicator of reproductive longevity (Serenius \& Stalder 2006; Young et al. 2008). Gilts that express first estrus early in life, mate and farrow at a younger age have improved reproductive longevity (e.g. Stalder 2004; Serenius \& Stalder 2006; Patterson et al. 2010; Knauer et al. 2011).

Traditional selection for age at puberty requires daily estrus detection in the presence of a boar, beginning at 
approximately 140 days of age (Miller et al. 2011). Collecting age-at-puberty data is therefore tedious and labor intensive and not practiced in most commercial or seedstock herds. Selection could be greatly simplified with the availability of molecular genetic predictors to identify and select individuals with propensity for early onset of estrus. Analyses of the genetic sources of variation in sow age at puberty have been based primarily on genome scans to detect quantitative trait loci (QTL) (Rohrer et al. 1999; Cassady et al. 2001; Holl et al. 2004; Yang et al. 2008) followed by a positional candidate gene approach (Nonneman et al. 2006; Kuehn et al. 2009). However, practical use of those results in swine breeding programs is limited primarily due to the lack of power from the structure of family-based QTL studies, lack of segregation of the QTL in commercial populations and the use of lowdensity and unevenly spaced marker panels. The introduction of massive parallel DNA sequencing and multiplex genotyping approaches has generated the resources and tools necessary to screen large populations for thousands of polymorphisms uniformly covering the entire genome. These developments have improved the capacity to dissect and identify genetic sources of phenotypic variation in complex traits.

The objectives of this study were to explore the genetic sources of variation in age at puberty and reproductive longevity expressed in the University of Nebraska-Lincoln (UNL) resource population. This resource population combines genetic information from multiple commercial lines that vary in an array of reproductive and developmental traits (Petry \& Johnson 2004; Johnson et al. 2008; Miller et al. 2011). By employing high-density genotyping and genome-wide association studies (GWAS), the specific objectives of this research were to (i) determine the proportion of additive genetic variation explained by the Porcine SNP60K Bead Array for age at puberty, reproductive longevity and other reproductive traits; (ii) identify major chromosomal regions and genes that explain differences in age at puberty; and (iii) explore common sources of genetic variation that influence both age at puberty and reproductive longevity.

\section{Materials and methods}

\section{Resource population}

The UNL resource population was developed to determine the influence of genetic factors and energy inputs on sow development, reproductive performance and longevity. The dams of the project gilts were commercial Large White $\times$ Landrace crossbreds $(\mathrm{LW} \times \mathrm{LR})$ or Nebraska Index Line (NIL) (Petry \& Johnson 2004) sows. The NIL had been selected for increased litter size for 29 generations and included within litter selection for increased growth and decreased backfat during the last 12 generations. The NIL dams used in this research were from generation 24 through 27. Both $L W \times L R$ and NIL dams were inseminated with semen from two unrelated LR-based industry lines (LR1 and LR2) to produce families of half-sib litters (Miller et al. 2011). Project gilts were produced in seven batches using single boar semen with a number of boars represented in each batch. The LR1 sire line was used in the first four (1-4) and LR2 was used in the last three batches (5-7). From 275 litters sired by 56 sires, 852 gilts contributed to this study after being randomly selected at approximately 56 days of age and subsequently exhibiting puberty before 240 days of age.

\section{Nutrition}

Gilts received the same diet and management from birth to 123 days of age. From 123 days of age until they were moved to the breeding barn at 225-240 days of age, gilts were allowed either ad libitum access to feed (A) or were placed on a restricted feeding regimen (R), receiving a daily allotment of feed that was $75 \%$ of the energy consumed by gilts on the A regimen (Miller et al. 2011). The $\mathrm{R}$ diet was formulated similarly to the A diet except that it was fortified to maintain similar intakes of amino acids, minerals and vitamins. During the breeding period and thereafter, all experimental animals received the same diet.

\section{Phenotypes}

This research was approved by the University of Nebraska Institutional Animal Care and Use Committee. Detection of age at puberty was initiated at 140 days of age and continued until approximately 240 days of age. The majority of the gilts (91\%) expressed estrus in this interval. Gilts were moved once daily from their pen to an adjacent room and exposed to a mature boar for $15 \mathrm{~min}$. This process continued until gilts were moved to the breeding barn or until all gilts within a pen had been observed in estrus at least twice. Gilts were maintained through four parities unless they were culled or died. Culling was performed only for failure to conceive or farrow a litter, or for structural problems. Total number born of fully formed pigs (TNB), number born alive (NBA), number mummified and number stillborn were recorded through the fourth parity.

\section{DNA isolation and genotyping}

The DNA was isolated from tail or ear tissues using the DNeasy or Puregene blood and tissue kits (Qiagen). The DNA quantity and quality were assessed by the NanoDrop Spectrophotometer (Thermo Scientific) and agarose gel electrophoresis. All 852 gilts were genotyped using the Porcine SNP60K BeadArray (Illumina) that includes 62183 SNP assays. The majority of the SNPs $(88.2 \%)$ were mapped on the build 10.2 reference assembly of the porcine genome. Illumina data analy- 
sis software was used to assign quality scores (gencall) for each genotype. A minimum gencall genotype quality score of 0.40 and a sample and SNP call rate of 0.80 were used as cutoff thresholds for removing low-quality genotypes. As a result, 57761 SNPs representing 822 genotyped samples were used in the genome-wide association analyses. Selective genotyping of the AVPR1A G256D SNPs using a subset of 78 samples of the UNL resource population was performed using KASPar technology (KBioscience).

\section{Statistical analyses}

Restricted maximum likelihood (REML) estimates of variance components and heritability were obtained from jmp (SAS Institute, Inc.) using a sire model. Variables were assumed to be normally distributed and analyzed with a linear mixed model including fixed effects of dam line, batch, gilt development, diet nested within batch and including random effects of sire and litter. Heritability was estimated as four times the sire variance divided by the phenotypic variance. Traits recorded before breeding (birth weight, weaning weight, age at puberty, 230-day weight, 230-day backfat thickness and 230-day longissimus muscle area) were assessed for their effect on the probability that females would produce a first parity litter by fitting these traits as covariates in generalized linear mixed models.

The proportion of genetic variance for age at puberty, litter size traits, reproductive longevity and productivity was estimated from high-density SNP genotypes using the Bayes B model (Kizilkaya et al. 2010) implemented via the gensel software package (Fernando \& Garrick 2008). The analyses were performed with $\Pi$ value set equal to 0.995 , assuming prior probability of 0.005 SNPs having a nonzero effect for the traits of interest. Previous research has shown that faster convergence is reached when only a small subset of markers is assumed to have a nonzero effect in each iteration (Onteru et al. 2012). Fixed effects included line, batch and diet. Markov chain Monte Carlo included 41000 iterations with the first 1000 samples being discarded as burn-in. Effects sampled from Bayes B at every 40th iteration were used to derive the posterior distribution for the genetic variance explained by every $1-\mathrm{Mb}$ genome window. The variance explained by a window was calculated as the variance across animals of the genomic values obtained from sampled SNP effects within the window, expressed as a proportion of the genetic variance obtained using sampled SNP effects from the whole genome. These values were obtained repeatedly for each window to derive the posterior distributions for inference. Under the null hypothesis that a 1-Mb fragment was not associated with a QTL, we estimated the probability of each window having an effect greater than 0. Alternatively, under the assumption the trait was infinitesimal and every window accounted for the same fraction of the genomic variation, we estimated the prob- ability of each window explaining a variance greater than the average proportion of variance. Potential common sources of phenotypic variation for different traits were evaluated by (i) pairwise correlation of genomic prediction values, (ii) comparing the variance explained by $1-\mathrm{Mb}$ regions across traits following Bayesian analysis and (iii) single-marker association analyses across the traits for major SNP contributors to phenotypic variation.

Associations between single markers that represent major QTL areas for age at puberty and lifetime number of parities were tested using a linear mixed model fitted by jmp that included marker genotype and replicate as fixed effects and sire, litter and diet as random effects. A similar model was used to evaluate the effect of the number of favorable alleles across three additive SNPs (ALGA0064320, ALGA0106255 and BGIS0007637) on age at puberty and lifetime number of parities; due to low frequency $(n=2)$, the individuals that carried six favorable alleles across the three SNPs were excluded from the analysis.

\section{Gene ontology}

Windows of $1 \mathrm{Mb}$ that were associated with the largest fraction of genetic variation were extended by $1 \mathrm{Mb}$ in both directions for functional characterization and to search for positional candidate genes using the Sus scrofa build 10.2 assembly. Human orthologs of swine positional candidate genes were obtained using the Ensembl Genes 67 database and the biomart data-mining tool: http://uswest.ensembl.org/biomart/martview/ 9883b5f936490aef948937cd5de40e1b . Functional annotation of the human orthologs, enrichment of gene ontology terms (GO, BP, FAT) and pathway analysis were performed using david: http://david.abcc.ncifcrf.gov/ .

\section{cDNA sequencing and SNP discovery}

Hypothalamic region, pituitary, ovarian cortex and granulosa cells were dissected from a random sample of 10 pre-pubertal gilts (with ages of 161-165 days). The gilts were the offspring of eight dams that expressed first estrus early ( $<154$ days of age), midrange (160170 days of age) and late (> 191 days of age). The pubertal status of the gilts was based on ovarian morphology and progesterone profile (Coat-A-Count Progesterone Kit, Diagnostic Products Corporation). The RNA was isolated from each tissue using TRIzol (Life Technologies). RNA pools were set up for each of the tissues with equal RNA contributions from the experimental animals. First-strand cDNA synthesis (GE Healthcare) of the RNA pools was followed by PCR of the cDNA samples (GoTaq Flexi DNA polymerase; Promega) for six positional candidate genes (AVPR1A, BAIAP2, CRTC1, OR2G3, PAPPA and PRKAA2) located in five QTL regions reported in this study (Table S1). Presence or absence of expression was evaluated by agarose electrophoresis. PCR products were treated with 
ExoSAP-IT (USB Corporation) and sequenced using dye terminators on an ABI PRISM 3130 Genetic Analyzer (Applied Biosystems). sequencher software (Gene Codes) was used to align the sequences and to identify polymorphisms.

Haplotype analyses and linkage disequilibrium estimates of five major BeadArray SNPs (ALGA0031465: 27356329 bp; DIAS0004594: 24499250 bp; BGIS0007637, 27499925 bp; ASGA0025214: 27633346 bp; and ALGA0031474: $27819353 \mathrm{bp}$ ) from a window located on SSC5 were performed by haploview 4.2 (Barrett et al. 2005) using the UNL resource population and a set of Duroc $\times$ Hampshire crossbreds $(\mathrm{D} \times \mathrm{H}, n=87)$.

\section{Results and discussion}

Variation in age at puberty is defined by multiple loci that overlap previously reported candidate genes and QTL

Heritability estimates of sow reproductive traits in the UNL resource population were low to moderate. Age at puberty was most heritable (0.38), followed by NBA $(0.18)$ and TNB (0.16) at first parity. Heritabilities for reproductive longevity (expressed as number of parities generated during a sow's lifetime) and lifetime productivity (expressed as lifetime TNB and NBA) were 0.04 and 0.01 respectively.

Combined SNP effects explained 26\% of phenotypic differences in age at puberty (Table 1). The contribution of SNP variation to the phenotypic variance of the lifetime number of parities was $19 \%$, whereas the contribution to litter size traits (TNB and NBA) at first parity was very limited $(5 \%)$. Genomic prediction values were obtained for each gilt using estimated SNP effects

Table 1. Posterior means of variance components of sow reproductive traits based on 57761 SNP effects estimated by genomewide association studies. The average genotyping call rate of the samples used in the analyses varied from 80.2 to $93.6 \%$ with a mean of $93.0 \%$. The average of the SNP call rate was $98.7 \%$ and varied among SNPs with 207 SNPs generating genotypes for all samples and 37878 SNPs generating genotypes for at least $99.0 \%$ of the samples. A large proportion of informative SNPs were characterized by minor allelic frequency of at least 0.10 (77.6\%). The fraction of monomorphic SNPs was $4 \%$

\begin{tabular}{|c|c|c|c|c|c|}
\hline Trait $^{1}$ & $n$ & $\begin{array}{l}\text { Genetic } \\
\text { variance }\end{array}$ & $\begin{array}{l}\text { Residual } \\
\text { variance }\end{array}$ & $\begin{array}{l}\text { Total } \\
\text { variance }\end{array}$ & $\begin{array}{l}\text { Proportion of } \\
\text { phenotypic } \\
\text { variance } \\
\text { explained } \\
\text { by SNPS }\end{array}$ \\
\hline AP & 822 & 90.37 & 253.12 & 343.47 & 0.26 \\
\hline TNB-LT & 550 & 3.41 & 301.83 & 305.24 & 0.01 \\
\hline NBA-LT & 547 & 5.75 & 261.70 & 267.46 & 0.02 \\
\hline NP & 739 & 0.23 & 1 & 1.23 & 0.19 \\
\hline TNB_P1 & 622 & 0.44 & 8.48 & 8.92 & 0.05 \\
\hline NBA_P1 & 620 & 0.42 & 8.60 & 9.01 & 0.05 \\
\hline
\end{tabular}

1. AP, age at puberty; TNB-LT, lifetime total number born; NBA-LT, lifetime number born alive; NP, number of lifetime parities; TNB, total number born; and NBA, number born alive at parity 1 (P1).
(Fernando \& Garrick 2008). Substantial differences between sires' average genome-wide predictions of their daughters' age at puberty existed, providing potential opportunities for selection (Figure S1).

A large proportion of the SNPs (77.6\%) used in the analyses were characterized by minor allelic frequency of at least 0.10 . The relatively large proportion of SNPs that displayed substantial allelic variation was expected because all of the SNPs present in the Porcine SNP60K BeadArray have been previously validated in common swine breeds (Ramos et al. 2009). Regions such as those located on SSC1 (231.5-243.4 Mb), SSC8 (59.4-61.3 Mb) and SSC15 $(90.4-93.5 \mathrm{Mb})$ were characterized almost exclusively by SNPs with very low minor allele frequencies $(q<0.1 ; 14-125 \mathrm{SNPs} /$ region). These SNPs were less abundant in an unrelated paternal dataset of $\mathrm{D} \times \mathrm{H}$ crossbreds. The region located on SSC1 displayed important differences in allelic frequencies and in the number of fixed and less polymorphic SNPs $(q<0.10)$ between UNL and $\mathrm{D} \times \mathrm{H}$ datasets. This low polymorphic profile observed for this region in the UNL population resource represents a potential signature of artificial selection for traits emphasized in maternal lines.

Analysis of the genetic variance explained by each $1-\mathrm{Mb}$ window of the swine genome, based on posterior distributions of the SNP effects, uncovered major regions associated with puberty onset, reproductive longevity and litter size traits. The top $1 \%$ of the 2593 windows of $1 \mathrm{Mb}$ combined explained $11 \%$ of additive genetic variation and included multiple regions from SSC1 (31-32, 94.2-94.9, 287-288, 94.1-94.9 Mb), SSC3 (16-16.9, 71.1-72 Mb), SSC6 (115.1-116, 144$145 \mathrm{Mb})$, SSC8 (36-37, 37-38 Mb), SSC9 (21.1-22, 139$140 \mathrm{Mb})$, SSC12 (1.2-2, 2.1-3, 11-11.9 Mb), SSC13 (117$117.9,142.1-142.9 \mathrm{Mb})$ and SSC14 (19-20, 28-29, 66-67, 68-69 Mb) and unique regions from SSC2 (87-88 Mb), SSC4 (7-8 Mb), SSC5 (30-30.9 Mb), SSC7 (39.1-40 Mb) and SSC18 (60-60.9 Mb) (Table S2, Figure 1). Most of these regions are characterized by clusters of SNPs associated with the largest effects on age at puberty onset (top $0.05 \%$ ) (Table S3). The region located at the proximal end of SSC4 (7-8 Mb) explained the largest amount of additive genetic variation $(1.22 \%)$, having a model frequency of $36 \%$, although the variation explained by this window was not significantly greater than the average explained by $1-\mathrm{Mb}$ windows $(P<0.32)$ (Table S2).

Several of the detected regions for age at puberty overlap previously reported candidate genes and QTL. The windows at the proximal end of SSC12 (1-2, 2.1$3,11-11.9 \mathrm{Mb}$ ) are adjacent to a QTL for age at puberty in a cross between NIL, selected for increased ovulation rate and embryonic survival, and a control line subjected to random mating (Holl et al. 2004). NIL is one of the ancestral contributors to the UNL resource population, and overlapping results from Holl et al. (2004) were expected for any real QTL. Compared with other commercial lines, the NIL line has higher prolificacy, greater 


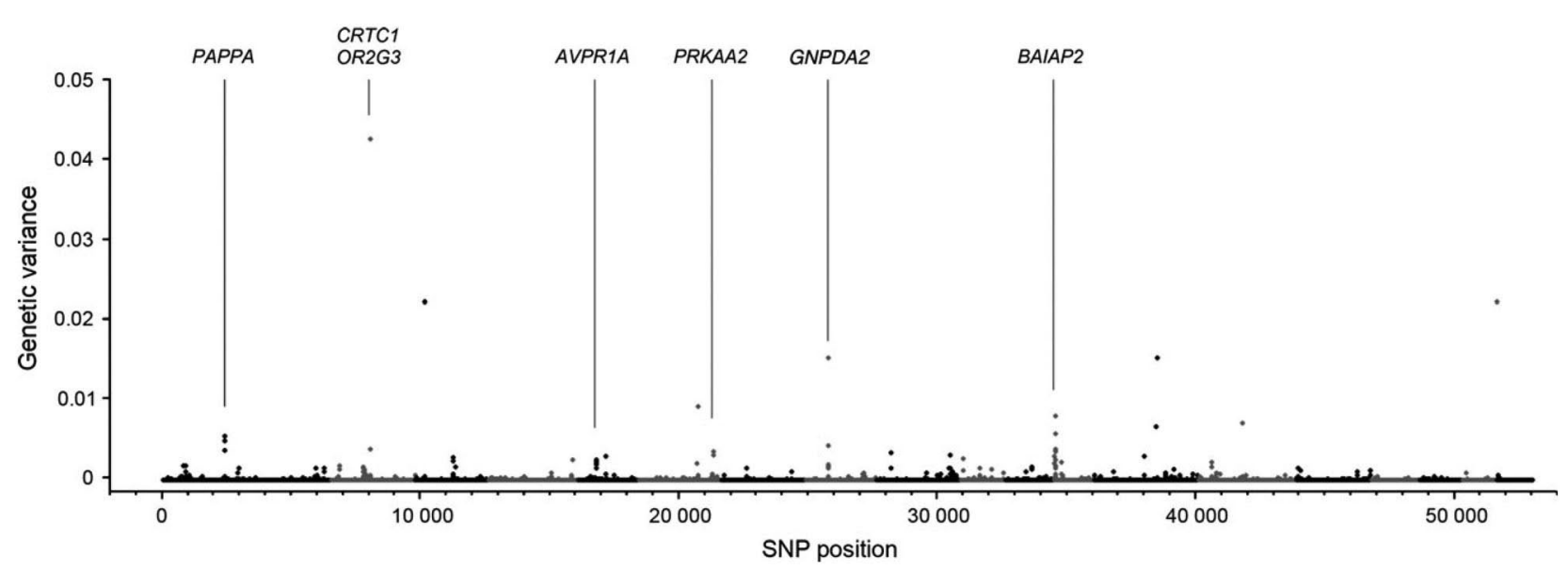

Figure 1. Genome-wide association analysis between 57761 SNPs and age at puberty. Each dot represents the proportion of genetic variance explained by five consecutive SNPs. The $x$-axis represents the location of the SNPs in the swine genome. The $y$ axis represents the contribution of that marker to the genetic variance. Alternate shadings represent autosomes, from SSC1 to 18 , followed by chromosome $\mathrm{X}$.

reproductive longevity and earlier expression of puberty (Moeller et al. 2004). The NIL is a parental line of GPK347, one of the six commercial lines used by National Pork Producers Council in a comparative study of reproductive longevity and of traits associated with this complex trait (Serenius et al. 2006; Knauer et al., 2010). GPK347 sows expressed estrus earlier and had the lowest risk of being culled due to reproductive failure, especially at first parity, of all lines. GPK347 also was one of the lines with the slowest growth and smallest longissimus muscle area. Potential sources of variation located in the region on SSC12 include genes involved in the generation of precursor metabolites and energy $(G A A, 2.3 \mathrm{Mb})$ or cellular response to hormonal stimuli (BAIAP2, $1.5 \mathrm{Mb}$ ).

The window associated with age at puberty on SSC6 (144-145 Mb) overlaps a QTL mapped by Bidanel et al. (2008) in a three-generation cross between Meishan and Large White. Candidate genes in this region include genes involved in regulation of fatty acid metabolic (PRKAA2, $143.5 \mathrm{Mb})$ and catabolic $(C P T 2,146.7 \mathrm{Mb})$ processes and reproductive (DMRTB1, $146.5 \mathrm{Mb})$ and vasculature (PPAP2B, $143.7 \mathrm{Mb})$ development.

CREB-regulated transcription coactivator 1 (CRTC1, $58.8 \mathrm{Mb}$ ) is a candidate gene located in the expanded area of the QTL mapped on SSC2 (58.4-61 Mb) whose expression is modulated by leptin, a hormone known to influence age at puberty (reviewed in Kaplowitz 2008). Inactive CRTC1 (Crtc1-\%) is associated with hyperphagia, obesity and infertility in mice, with females displaying low levels of circulating luteinizing hormone (Altarejos et al. 2008). Overexpression of CRTC1 in hypothalamic cells increases expression of kisspeptin, which activates secretion of gonadotrophin-releasing hormone (Elks \& Ong 2011) and contributes to the onset of puberty (Li et al. 2008). A recent meta-analysis of GWAS uncovered an intronic SNP in CRTC1 associated with age at menarche in humans (Elks \& Ong 2011).
Top-ranking windows, such as those located on SSC1 (31-32 Mb) and SSC12 (11-11.9 Mb), overlapped or were adjacent to loci associated with age at puberty reported by Nonneman et al. (2011). Another potential QTL mapped on SSC10 $(70-70.9 \mathrm{Mb})$ is located next to AKR1C2 $(71.8 \mathrm{Mb})$, a member of a family of genes known to be involved in obesity, polycystic ovary syndrome and delayed age at menarche in humans (Rittner et al. 1997; Blouin et al. 2005; Qin et al. 2006). A non-synonymous SNP located in AKR1C2 was associated with age at puberty in a swine composite population that included Meishan, Landrace and Large White (Nonneman et al. 2006).

Functional characterization of major GWAS-derived QTL regions uncovered candidate genes for age at puberty

The top 1\% 1-Mb windows with the largest contribution to genetic variance were subjected to functional annotation, enrichment and pathway analyses based on human orthologs due to limited annotation of the swine transcriptome. Significant enrichments were observed for genes involved in regulation of small GTPase-mediated signal transduction $(P<0.01$; e.g., DOK1, DAB1), positive regulation of vasoconstriction $(P<0.05$, e.g., AVPR1A, PTGS2), RAS protein signal transduction $(P<0.05$, e.g., DHCR24), etc. Additional processes enriched at the suggestive level $(P<0.10)$ that could potentially influence age at puberty and reproductive longevity included genes involved in the regulation of the lipid biosynthetic process (e.g., FASN, PRKAA2), the prostaglandin biosynthetic process (e.g., PLA2G4A, AVPR1A), eye photoreceptor cell development and differentiation (e.g., ALMS1), etc. Pathways enriched $(P<0.10)$ in genes located in these regions include $A B C$ transporters (e.g., $A B C A 5$ ), endocytosis (e.g., $R A B 4 A$ ) and insulin signaling pathways (e.g., RPTOR, HK2). Individual candidate genes of interest in top-ranked windows include 
AVPR1A involved in reproductive behavior, RPTOR in cellular response to nutrient levels, CRTC1 in regulation of transcription, PRKAA2 in energy metabolism, RAB3A in neurotransmitter secretion, BAIAP2 in neuronal growth, PAPPA in female pregnancy and OR2G3 in detection of chemical stimulus.

Positional candidate genes from the GWAS-derived QTL areas are polymorphic and expressed in tissues from the hypothalamic-pituitary-ovarian axis

Puberty is the process of reaching reproductive maturity and is characterized by activation of the hypothalamic-pituitary-ovarian (HPO) axis, which triggers the ovulation of an oocyte competent for fertilization and embryonic development (Senger 2005). A positional candidate approach combined with gene expression profiling in tissues from the HPO axis and resequencing were employed to uncover functional genes and polymorphisms responsible for variation in age at puberty. Presence or absence of the expression of six positional candidate genes located in five GWAS-derived QTL regions, and involved in biological processes described above that could impact expression of first estrus and reproductive longevity (AVPR1A, BAIAP2, CRTC1, OR2G3, PAPPA and PRKAA2), was evaluated in the hypothalamus, pituitary and ovarian cortex of pre-pubertal gilts (161-165 days of age). The candidate genes are involved in sexual and social behavior, such as AVPR1A (Walum et al. 2008; Gobrogge et al. 2009); energy metabolism (Kemp et al. 2003) and porcine oocyte maturation (Mayes et al. 2007), such as PRKAA2; onset of puberty, such as CRTC1 (Li et al. 2008); follicular maturation (Monget et al. 2003), placental development (Sahraravand et al. 2011), pregnancy (Vandenberghe et al. 2011) and growth (Rehage et al. 2007), such as PAPPA; neuritis growth (Bockmann et al. 2002) and neuronal disorders (Ribases et al. 2009), such as BAIAP2; and initiate a neuronal response triggered by odorant molecules such as OR2G3 (Malnic et al. 2004). AVPR1A and OR2G3 were the only genes expressed in the hypothalamic region. Expression of OR2G3, AVPR1A, BAIAP2, CRTC1, PAPPA and PRKAA2 was detected in granulosa cells and the ovarian cortex. With the exception of PAPPA, a similar presence also was observed in the pituitary. Although potential transient expression triggered by an environmental stimulus could be easily missed, this approach provides opportunities to explore transcript and sequence variation in the UNL population. Partial cDNA sequencing of these genes uncovered SNPs that trigger amino acid substitutions in AVPR1A (ss518151313 and ss518151314) and BAIAP2 (ss518151315; synonymous SNPs in AVPR1A (ss518151323), OR2G3 (ss518151308, ss518151309 and ss518151310) and PAPPA (ss518151311 and ss518151312); and one SNP located in the 3' UTR of the PRKAA2 (ss518151316).
Genetic relationship between age at puberty and reproductive longevity

Fertility is substantially influenced by a range of components expressed during different developmental stages. Some of these components might also influence other traits recorded before breeding. We estimated the effects of traits recorded before breeding on the probability that females would produce a first parity litter. Of the traits evaluated (birth weight, weaning weight, age at puberty, 230-day weight, 230-day backfat thickness and 230-day longissimus muscle area), only age at puberty was a significant determinant $(P=0.002)$. Regardless of genetic line or the gilt developmental regimen, the likelihood of a parity 1 litter decreased as age at puberty increased (Figure 2). A moderate relationship based on the SNP effects, estimated by Bayes B, also was detected between age at puberty and reproductive longevity, here expressed as the number of parities produced by a sow during lifetime $(r=-0.45, P<0.0001)$. Similar trends were observed between age at puberty and other measures of lifetime productivity, such as lifetime TNB $(-0.25, P<0.0001)$ and NBA $(-0.28, P<0.0001)$. Gilts that express puberty early in life have increased rebreeding success and produce more litters and piglets during their lifetime.

We hypothesized that common sources of variation and overlapping genetic networks influence both the variability of age at puberty and the components of reproductive longevity. Exploring the genetic variance explained by each $1-\mathrm{Mb}$ window across traits revealed that four of the top $1 \%(n=26)$ windows that influence age at puberty (SSC12, 1-2, 2-3 Mb; SSC5, 30$30.9 \mathrm{Mb}$; SSC18, 60-60.9 Mb) ranked high (top $2 \%$ ) for lifetime number of parities (Table S2 and S4). The windows located at the proximal end of SSC1 $(1-2,2-3 \mathrm{Mb})$ also ranked high for lifetime productivity traits such as

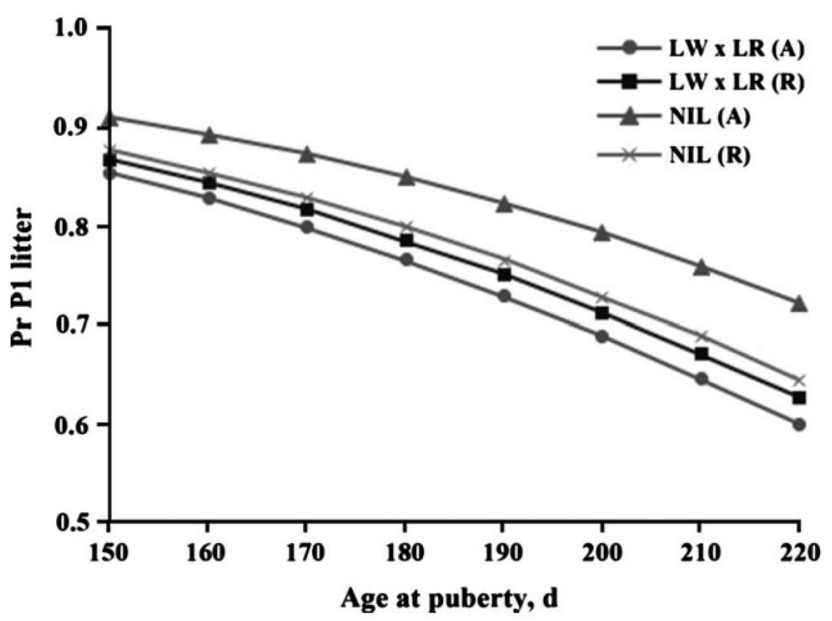

Figure 2. Effect of increased age at puberty on the probability of generating a parity 1 litter. Regardless of genetic line (NIL or $L W \times L R)$ or the gilt developmental regimen $(A=$ ad libitum, $R=$ energy restricted), the likelihood of a parity 1 litter decreased as age at puberty increased in the four batches of the study. 
lifetime TNB and NBA. These pleiotropic regions are potential sources of the moderate correlations between genomic predictions of age at puberty and reproductive longevity. In contrast, only one of the top 1\% windows for age at puberty ranked high for litter size traits (TNB and NBA) at parity 1.

The SNPs associated with the largest age at puberty effect in each of the top $1 \%(n=26) 1$-Mb GWAS-derived QTL regions described above were evaluated in single-marker association analysis to investigate the presence of common genetic sources of variation with reproductive longevity. Most of these SNPs $(92 \%)$ explained significant differences in variation in age at puberty that varied between homozygote genotypes from 3.4 (ALGA0106255, SSC $8,36.5 \mathrm{Mb}, P<0.05$ ) to 11.1 days of age (ASGA0105637, SSC2, 87.2 Mb, $P<0.01)$. In addition to explaining differences in age at puberty, BGIS0007637 (SSC5, $30.7 \mathrm{Mb}, P<0.01)$ and ASGA0003702 (SSC1, $94.3 \mathrm{Mb}, P<0.05)$ also explained important variation in the lifetime number of parities $(P<0.05)$ (Table 2). Suggestive trends $(P<0.15)$ that captured the negative relationships between age at puberty and number of lifetime parities were observed for four SNPs located on SSC8, SSC12 and SSC13. Given that both traits are dependent on the function of the hypothalamic-pituitary-gonadal axis, we anticipated that the variation of both traits would be influenced by the same loci. Additive properties of the SNPs were evaluated by integrating three SNPs exhibiting additive $(P<0.10)$ and similar effects that varied from 0.18 parities for ALGA0064320 (SSC12) and ALGA0106255 (SSC8) to 0.26 for BGIS0007637 (SSC5). An increase in the number of favorable alleles across these SNPs was associated with an early age at puberty and a positive impact on the number of lifetime parities $(P<0.001$, Table 3$)$. The individuals that carried five favorable alleles across the three loci generated 1.36 more parities than did individuals where the favorable alleles were absent $(P<0.05)$. The distribution of the allelic combinations underlines opportunities for genetic improvement by increasing the frequency of favorable alleles.

The window located on SSC5 (27-28 Mb) represents one of the pleiotropic regions that partially explains the negative relationship between age at puberty and reproductive longevity. BGIS0007637 is a non-synonymous SNP (G31E) located in AVPR1A, one of the candidate genes located in this window. AVPR1A is a G-proteincoupled receptor expressed in many tissues including several regions of the brain such as the hypothalamic arcuate nucleus (Caldwell et al. 2008). Recent studies associated AVPR1A with social and reproductive behaviors in voles and humans (Walum et al. 2008; Gobrogge et al. 2009). The AA genotype of BGIS0007637 was associated with a 5.8-day later expression of first estrus compared with genotype GG $(P<0.05)$ and a 3.6-day later expression compared with genotype AG $(P<0.09)$. In contrast, the GG genotype was associated with 0.53 more lifetime parities than AA $(P<0.01)$ and 0.33 more parities than CT $(P<0.08)$ genotypes.

Re-sequencing AVPR1A uncovered two novel nonsynonymous SNPs (G256D and K377Q) in addition to BGIS0007637. The nature of the residue substitutions generated by the AVPR1A alleles has the potential to change the expression of first estrus and to impact reproductive longevity. BGIS0007637 (G31E) is located in the extracellular $\mathrm{NH}_{2}$-terminus and is characterized by a change from a small residue $(\mathrm{G})$ to an acidic residue (E). The $\mathrm{NH}_{2}$-terminus of AVPR1A has an important role in agonist binding and intracellular signaling; induced mutations at the conserved Arg46 and Glu54 sites affected arginine vasopressin affinity and disrupted signaling (Hawtin et al. 2000, 2005). The SNP G256D (SSC5: $27417070 \mathrm{bp}$ ) is located in the third intracellular loop of AVPR1A and is characterized by a substitution, from a small residue $(G)$ characterized by flexibility to an acidic residue (D). The SNP K377Q (SSC5: $27413729 \mathrm{bp}$ ) is located at the $\mathrm{C}$ terminus, known to have an important role in coordinating protein interactions with AVPR1A (Thibonnier et al. 2001). Alignment of AVPR1A protein orthologs using clustalw2 (Larkin et al. 2007) showed the K377 variant is well conserved across species, being present in human, dog, mouse, rat and swine, whereas the Q377 variant is present only in swine. Similarly, the presence of an acidic residue at position 31 of AVPR1A is conserved across species: glutamic acid (E31) is present in human, mouse, rat, swine and vole; aspartic acid (D31) is present in cow, dog and sheep; whereas the glycine (G31) is only present in swine.

Genotyping a random subset of samples $(n=78)$ using the G256D SNP followed by haplotype analysis showed that in the UNL resource population, this novel AVPR1A polymorphism is in complete linkage disequilibrium (LD) with five SNPs from the Porcine SNP60K BeadChip. Analysis of the AVPR1A region in additional breeds showed that this block is characterized by high LD in multiple populations. The first four SNPs of the block (ALGA0031465, DIAS0004594, BGIS0007637 and ASGA0025214) that also includes the AVPR1A gene are in complete LD in commercial crossbreds ( $\mathrm{LW} \times \mathrm{LR}$ ) and pure $(\mathrm{LW})$ lines from a different maternal resource population $(n=820$, Onteru et al. 2012). Similar results were obtained in a UNL paternal dataset of $\mathrm{D} \times \mathrm{H}$ crossbreds $(n=87)$; the first three SNPs of the block, which include the AVPR1A gene, were characterized by high levels of $\operatorname{LD}\left(\mathrm{r}^{2}>0.93\right)$.

The frequency of the favorable haplotype (0.49) in our resource population indicates potential opportunities for increasing its frequency and thus improving reproductive longevity in commercial maternal lines by marker-assisted selection. Mote et al. (2006) reported that an improvement from three to four parities produced by a sow would increase the sow net present 
Table 2. Single-marker association results between the genotypes of the SNPs that represent each of the 10 GWAS-derived QTL regions and age at puberty (AP) and number of lifetime parities (NP) in the UNL resource population

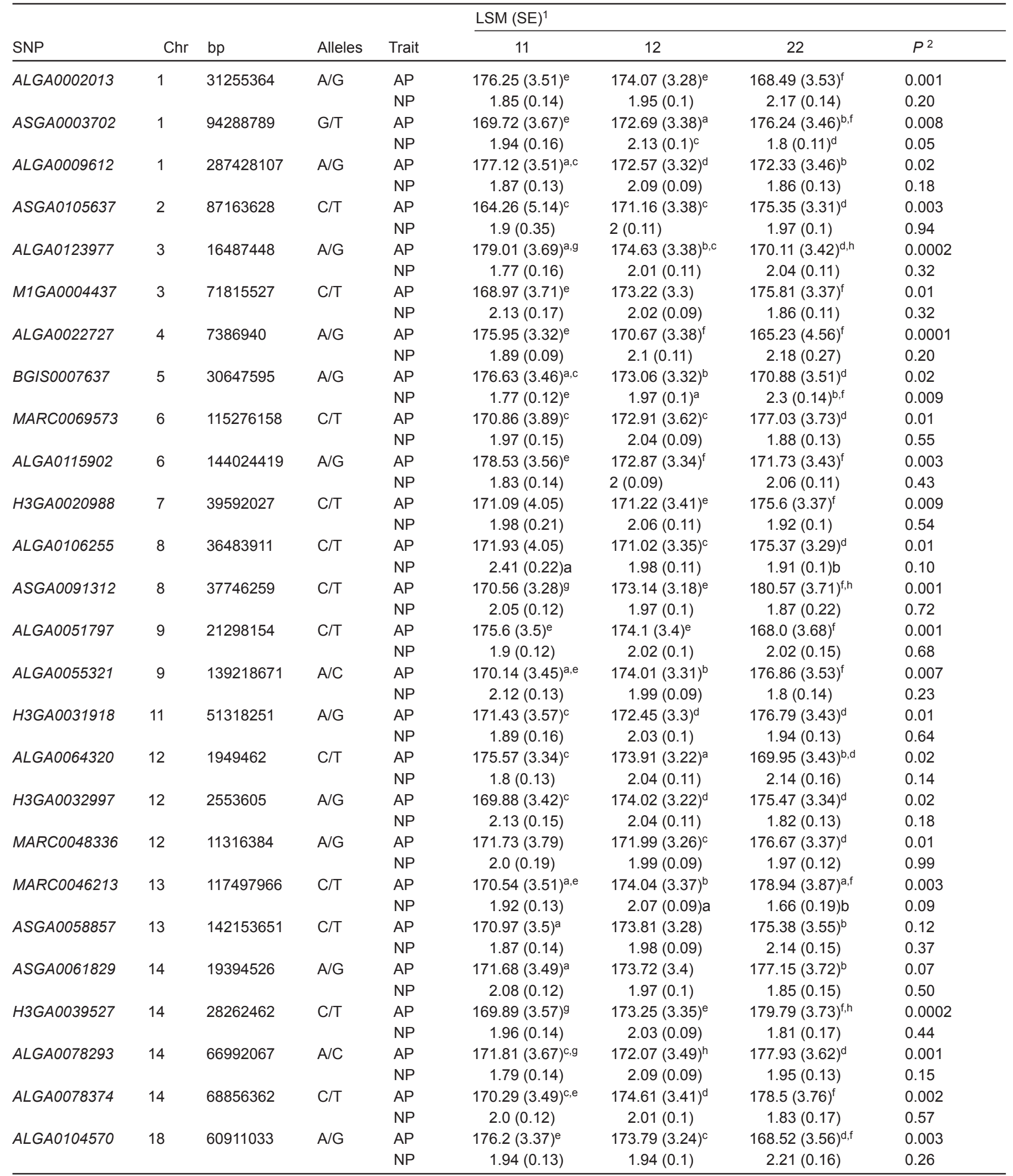

1. Least square means (LSM) estimates with different superscript differ: a, b, $P<0.1$; c, d, $P<0.05 ; \mathrm{e}, \mathrm{f}, P<0.01 ; \mathrm{g}, \mathrm{h}, P<0.001$. The alleles $(1$ and 2) are designated based on the alphabetical order of SNPs variants (A, C, G and T).

2. $P$-value for overall test of the effect of genotypes. The number of animals per each genotyping class varied: $n=50-479(11), 324-422,(12)$ and 38-416 (22) for AP, and $n=63-423$ (11), 296-390 (12) and 38-321 (22) for NP. 
Table 3. The combined effect of the favorable alleles from SNPs ALGA0064320 (SSC12), ALGA0106255 (SSC8) and $B G / S 0007637$ (SSC5) on age at puberty (AP) and number of lifetime parities (NP) in the UNL resource population

\begin{tabular}{lcrl}
\hline Trait & $\begin{array}{l}\text { Number of } \\
\text { favorable alleles }\end{array}$ & $n$ & LSM $(\mathrm{SE})^{1}$ \\
\hline AP & 0 & 35 & $180.42(4.34)^{\mathrm{c}, \mathrm{d}}$ \\
& 1 & 140 & $178.44(3.33)^{\mathrm{e}, \mathrm{f}}$ \\
& 2 & 254 & $175.2(3.15)^{\mathrm{a}, \mathrm{b}}$ \\
& 3 & 247 & $169.94(3.17)^{\mathrm{a}, \mathrm{c}, \mathrm{f}}$ \\
& 4 & 109 & $168.57(3.46)^{\mathrm{b}, \mathrm{d}, \mathrm{e}}$ \\
$\mathrm{NP}$ & 5 & 29 & $173.74(4.58)$ \\
& 6 & 2 & - \\
& 0 & 140 & $1.42(0.28)^{\mathrm{b}}$ \\
& 1 & 254 & $1.93(0.16)$ \\
& 2 & 247 & $1.73(0.13)^{\mathrm{a}, \mathrm{c}}$ \\
& 3 & 109 & $2.18(0.13)^{\mathrm{c}}$ \\
& 4 & 29 & $2.25(0.18)$ \\
& 5 & 2 & - \\
\hline
\end{tabular}

1. Least square means (LSM) estimates with the same superscript differ: a, b, c, d, $P<0.05$; e, $P<0.01 ; \mathrm{f}, P<0.001$.

value by $\$ 77.38$ in farrow-to-finish operations. Using these estimates and assuming the frequency of the haplotypes are in Hardy-Weinberg equilibrium, we can estimate that an increase in the frequency of the favorable haplotype from 0.49 to 0.90 would generate an increase in the sow net present value by $\$ 17.64$. Although the effect of this locus can vary from population to population, we expect that sows with the favorable genotype will farrow 5.1 more pigs and 4.3 more pigs sold per litter during their lifetime compared with the alternate homozygote haplotype if we consider that an average number of pigs born alive and number of pigs sold per litter are 10.2 and 8.5 respectively. This change could have a potentially important impact given that an improvement of just 0.10 in the average number of parities produced by a sow would increase profit in the USA alone by approximately $\$ 15$ million per year (Mote et al. 2006).

\section{Conclusions}

Using a population that includes genetics from several commercial maternal lines, we explored and identified two significant and three suggestive pleiotropic sources of variation of age at puberty and reproductive longevity. One of these sources is represented by a region located on SSC5 that harbors the AVPR1A gene and carries allelic variants associated with early expression of puberty and a greater number of lifetime parities. The favorable homozygote genotype of one of the SNPs located in AVPR1A (BGIS0007637) is responsible for an almost 6-day earlier expression of first estrus and a half a parity more during lifetime than the alternate homozygote. As the alleles and frequencies present in one population are not necessarily present in others, this study provides evidence that this region is informative in different resource populations as well. Combined analysis of SNPs that exhibited additive properties (including BGIS0007637) showed that an increase in the number of favorable alleles had positive impact on reproductive longevity, increasing the lifetime number of parities to 1.36. Three of the four genetic lines that contributed to the UNL resource population have an important contribution to the maternal genetics of the U.S. pig industry as well as having a worldwide presence. Although it is unclear whether genetic variants of $A V P R 1 A$ represent the functional determinants of the variation in age at puberty and reproductive longevity, the presence of a relatively large DNA block in LD, conserved in different lines, could represent a potential molecular resource that could be applied to improve age at puberty and reproductive longevity across populations without knowledge of the causal gene or mutation.

Acknowledgments - This work was supported by the National Pork Board, Nebraska Pork Producers Association, U.S. Pig Genome Coordinator, University of Nebraska Agricultural Research Division, Iowa State University and Hatch funds. We thank Matthew Anderson, Jeffrey Perkins, Donald McClure, Thomas McGargill, Roman Moreno, William Pohlmeier, John Kammermann and Brett White for their technical assistance. We thank John Bastiaansen for suggestions and comments, Martien Groenen for providing updated SNP locations on Build 10.2 and Suneel Onteru for providing LD data of the AVPR1A region from his recent research (Onteru et al. 2012).

\section{References}

Altarejos J. Y., Goebel N., Conkright M. D., Inoue H., Xie J., Arias C. M., Sawchenko P. E. \& Montminy M. (2008) The Creb1 coactivator Crtc1 is required for energy balance and fertility. Nature Medicine 14, 1112-17.

Barrett J. C., Fry B., Maller J. \& Daly M. J. (2005) HAPLOVIEW: Analysis and visualization of LD and haplotype maps. Bioinformatics 21, 263-5.

Bidanel J. P., Rosendo A., Iannuccelli N., Riquet J., Gilbert H., Caritez J. C., Billon Y., Amigues Y., Prunier A. \& Milan D. (2008) Detection of quantitative trait loci for teat number and female reproductive traits in Meishan x Large White F2 pigs. Animal 2, 813-20.

Blouin K., Blanchette S., Richard C., Dupont P., Luu-The V. \& Tchernof A. (2005) Expression and activity of steroid aldoketoreductases $1 \mathrm{C}$ in omental adipose tissue are positive correlates of adiposity in women. American Journal of Physiology. Endocrinology and Metabolism 288, E398-404.

Bockmann J., Kreutz M. R., Gundelfinger E. D. \& Bockers T. M. (2002) ProSAP/Shank postsynaptic density proteins interact with insulin receptor tyrosine kinase substrate IRSp53. Journal of Neurochemistry 83, 1013-17.

Caldwell H. K., Lee H. J., Macbeth A. H. \& Young W. S. 3rd (2008) Vasopressin: Behavioral roles of an "original" neuropeptide. Progress in Neurobiology 84, 1-24. 
Cassady J. P., Johnson R. K., Pomp D., Rohrer G. A., Van Vleck L. D., Spiegel E. K. \& Gilson K. M. (2001) Identification of quantitative trait loci affecting reproduction in pigs. Journal of Animal Science 79, 623-33.

Elks C. E. \& Ong K. K. (2011) Whole genome associated studies for age at menarche. Briefings in Functional Genomics 10, 91-7.

Fernando R. L. \& Garrick D. J. (2008) Gensel User Manual for a Portfolio of Genomic Selection Related Analyses. Animal Breeding and Genetics, Iowa State University, Ames.

Gobrogge K. L., Liu Y., Young L. J. \& Wang Z. (2009) Anterior hypothalamic vasopressin regulates pair-bonding and druginduced aggression in a monogamous rodent. Proceedings of the National Academy of Sciences of the United States of America 106, 19144-9.

Hawtin S. R., Wesley V. J., Parslow R. A., Patel S. \& Wheatley M. (2000) Critical role of a subdomain of the N-terminus of the V1a vasopressin receptor for binding agonists but not antagonists; functional rescue by the oxytocin receptor $\mathrm{N}$ terminus. Biochemistry 39, 13524-33.

Hawtin S. R., Wesley V. J., Simms J., Argent C. C., Latif K., \& Wheatley M. (2005) The N-terminal juxtamembrane segment of the V1a vasopressin receptor provides two independent epitopes required for high-affinity agonist binding and signaling. Molecular Endocrinology 19, 2871-81.

Holl J. W., Cassady J. P., Pomp D. \& Johnson R. K. (2004) A genome scan for quantitative trait loci and imprinted regions affecting reproduction in pigs. Journal of Animal Science 82, 3421-9.

Kaplowitz P. B. (2008) Link between body fat and the timing of puberty. Pediatrics 121 (Suppl 3), S208-17.

Kemp B. E., Stapleton D., Campbell D. J. et al. (2003) AMP-activated protein kinase, super metabolic regulator. Biochemical Society Transactions 31, 162-8.

Kizilkaya K., Fernando R. L. \& Garrick D. J. (2010) Genomic prediction of simulated multibreed and purebred performance using observed fifty thousand single nucleotide polymorphism genotypes. Journal of Animal Science 88, 544-51.

Knauer M. T., Cassady J. P., Newcom D. W. \& See M. T. (2011) Phenotypic and genetic correlations between gilt estrus, puberty, growth, composition, and structural conformation traits with first-litter reproductive measures. Journal of Animal Science 89, 935-42.

Knauer M., Stalder K. J., Serenius T. et al. (2010) Factors associated with sow stayability in 6 genotypes. Journal of Animal Science 88, 3486-92.

Kuehn L. A., Nonneman D. J., Klindt J. M. \& Wise T. H. (2009) Genetic relationships of body composition, serum leptin, and age at puberty in gilts. Journal of Animal Science 87, 477-83.

Larkin M. A., Blackshields G., Brown N. P. et al. (2007) CLUSTALW and CLUSTALX version 2. 0. Bioinformatics 23, 2947-8.

Li S., Ren J., Yang G., Guo Y. \& Huang L. (2008) Characterization of the porcine kisspeptins receptor gene and evaluation as candidate for timing of puberty in sows. Journal of Animal Breeding and Genetics 21, 9-227.

Malnic B., Godfrey P. A. \& Buck L. B. (2004) The human olfactory receptor gene family. Proceedings of the National Academy of Sciences of the United States of America 101, 2584-9.
Mayes M. A., Laforest M. F., Guillemette C., Gilchrist R. B. \& Richard F. J. (2007) Adenosine 5'-monophosphate kinaseactivated protein kinase (PRKA) activators delay meiotic resumption in porcine oocytes. Biology of reproduction 76, 589-97.

Miller P. S., Moreno R. \& Johnson R. K. (2011) Effects of restricting energy during the gilt developmental period on growth and reproduction of lines differing in lean growth rate: Responses in feed intake, growth, and age at puberty. Journal of Animal Science 89, 342-54.

Moeller S. J., Goodwin R. N., Johnson R. K., Mabry J. W., Baas T. J. \& Robison O. W. (2004) The National Pork Producers Council Maternal Line National Genetic Evaluation Program: A comparison of six maternal genetic lines for female productivity measures over four parities. Journal of Animal Science 82, 41-53.

Monget P., Mazerbourg S., Delpuech T., Maurel M. C., Maniere S., Zapf J., Lalmanach G., Oxvig C. \& Overgaard M. T. (2003) Pregnancy-associated plasma protein-A is involved in insulin-like growth factor binding protein-2 (IGFBP-2) proteolytic degradation in bovine and porcine preovulatory follicles: Identification of cleavage site and characterization of IGFBP-2 degradation. Biology of reproduction 68, 77-86.

Mote B. E., Stalder K. J. \& Rothschild M. F. (2006) Candidate Genes Associated with Sow Longevity. National Swine Improvement Federation. Nashville, TN.

Mote B. E., Koehler K. J., Mabry J. W., Stalder K. J. \& Rothschild M. F. (2009) Identification of genetic markers for productive life in commercial sows. Journal of Animal Science 87, 2187-95

Nonneman D. J., Wise T. H., Ford J. J., Kuehn L. A. \& Rohrer G. A. (2006) Characterization of the aldo-keto reductase 1C gene cluster on pig chromosome 10: Possible associations with reproductive traits. BMC Veterinary Research 2,28 .

Nonneman D. J., Rohrer G. A., Rempel L. A., Wiedmann R. T. \& Vallet J. L. (2011) Genome-wide associations for age at puberty in a Duroc-Landrace-Yorkshire swine population; Proceedings of the Plant and Animal Genome Conference XIX, January 15-19, 2011, San Diego, CA. pp. P609.

Onteru S. K., Fan B., Du Z. Q., Garrick D. J., Stalder K. J. \& Rothschild M. F. (2012) A whole-genome association study for pig reproductive traits. Animal Genetics 43, 18-26.

Patterson J. L., Beltranema E. \& Foxcroft G. R. (2010) The effect of gilt age at first estrus and breeding on third estrus on sow body weight changes and long-term, reproductive performance. Journal of Animal Science 88: 2500-13.

Petry D. B. \& Johnson R. K. (2004) Responses to 19 generations of litter size selection in the Nebraska Index line. I. Reproductive responses estimated in pure line and crossbred litters. Journal of Animal Science 82, 1000-6.

Qin K., Ehrmann D. A., Cox N., Refetoff S. \& Rosenfield R. L. (2006) Identification of a functional polymorphism of the human type 5 17beta-hydroxysteroid dehydrogenase gene associated with polycystic ovary syndrome. Journal of Clinical Endocrinology and Metabolism 91, 270-6.

Ramos A. M., Crooijmans R. P., Affara N. A. et al. (2009) Design of a high density SNP genotyping assay in the pig using SNPs identified and characterized by next generation sequencing technology. PLoS ONE 4, e6524. 
Rehage M., Mohan S., Wergedal J. E., Bonafede B., Tran K., Hou D., Phang D., Kumar A. \& Qin X. (2007) Transgenic overexpression of pregnancy-associated plasma protein-A increases the somatic growth and skeletal muscle mass in mice. Endocrinology 148, 6176-85.

Ribases M., Bosch R., Hervas A. et al. (2009) Case-control study of six genes asymmetrically expressed in the two cerebral hemispheres: association of BAIAP2 with attention-deficit/ hyperactivity disorder. Biological psychiatry 66, 926-34.

Rittner H. L., Lee P. D., Blum W. F., Doerr H. G., Steiss J., Kreuder J., Rascher W., Kiess W. (1997) Developmental patterns of serum 3 alpha-androstanediol glucuronide. Journal of Endocrinological Investigation 20, 245-50.

Rohrer G. A., Ford J. J., Wise T. H., Vallet J. L. \& Christenson R. K. (1999) Identification of quantitative trait loci affecting female reproductive traits in a multigeneration Meishan-White composite swine population. Journal of Animal Science 77, 1385-91.

Sahraravand M., Jarvela I. Y., Laitinen P., Tekay A. H. \& Ryynanen M. (2011) The secretion of PAPP-A, ADAM12, and PP13 correlates with the size of the placenta for the first month of pregnancy. Placenta 32, 999-1003.

Senger P. (2005) Puberty. In: P. Senger, ed., Pathways to Pregnancy and Parturition. Current Conceptions, Inc. Pullman, WA, 128-42.

Serenius T. \& Stalder K. J. (2006) Selection for sow longevity. Journal of Animal Science 84(Suppl), E166-71.

Serenius T., Stalder K. J., Baas T. J., Mabry J. W., Goodwin R. N., Johnson R. K., Robison O. W., Tokach M. \& Miller R. K. (2006) National Pork Producers Council Maternal Line National Genetic Evaluation Program: a comparison of sow longevity and trait associations with sow longevity. Journal of Animal Science 84, 2590-5.
Stalder K. J., Lacy R. C., Cross T. L. \& Conatser G. E. (2003) Financial impact of average parity of culled females in a breed-to-wean swine operation using replacement gilt net present value analysis. Journal of Swine Health and Production 11, 69-74.

Stalder K. J. (2004) Sow longevity scrutinized. National Hog Farmer, Primedia Magazines and Media Inc., Overland Park, KS, 49, 26- 30.

Thibonnier M., Plesnicher C. L., Berrada K. \& Berti-Mattera L. (2001) Role of the human V1 vasopressin receptor $\mathrm{COOH}$ terminus in internalization and mitogenic signal transduction. American Journal of Physiology. Endocrinology and Metabolism 281, E81-92.

Vandenberghe G., Mensink I., Twisk J. W., Blankenstein M. A., Heijboer A. C. \& van Vugt J. M. (2011) First trimester screening for intra-uterine growth restriction and early-onset pre-eclampsia. Prenatal Diagnosis 31, 955-61.

Walum H., Westberg L., Henningsson S. et al. (2008) Genetic variation in the vasopressin receptor $1 a$ gene (AVPR1A) associates with pair-bonding behavior in humans. Proceedings of the National Academy of Sciences of the United States of America 105, 14153-6.

Yang G., Ren J., Li S., Mao H., Guo Y., Zou Z., Ren D., Ma J. \& Huang L. (2008) Genome-wide identification of QTL for age at puberty in gilts using a large intercross F2 population between White Duroc 9 Erhualian. Genetics, selection, evolution: GSE 40, 529-39.

Young M. G., Tokach M. D., Aherne F. X., Dritz S. S., Goodband R. D. et al. (2008) Effect of space allowance during rearing and selection criteria on performance of gilts over three parities in a commercial swine production system. Journal of Animal Science 86, 3181-93.

\section{Supporting information}

\section{Additional supporting information follows:}

Figure S1. Sire's genomic breeding values estimated as the average of their daughter's genomic predicted values for age at puberty.

Table S1. PCR primers for CDNA sequencing of the positional candidate genes.

Table S2. Genetic variance of age at puberty explained by 1-Mb windows.

Table S3. Regions with major clusters of SNPs associated with the largest effects on puberty onset.

Table S4. Genetic variance of lifetime number of parities explained by 1-Mb windows. 
Figure S1. Sire's genomic breeding values estimated as the average of their daughter' genomic predicted values for age at puberty.

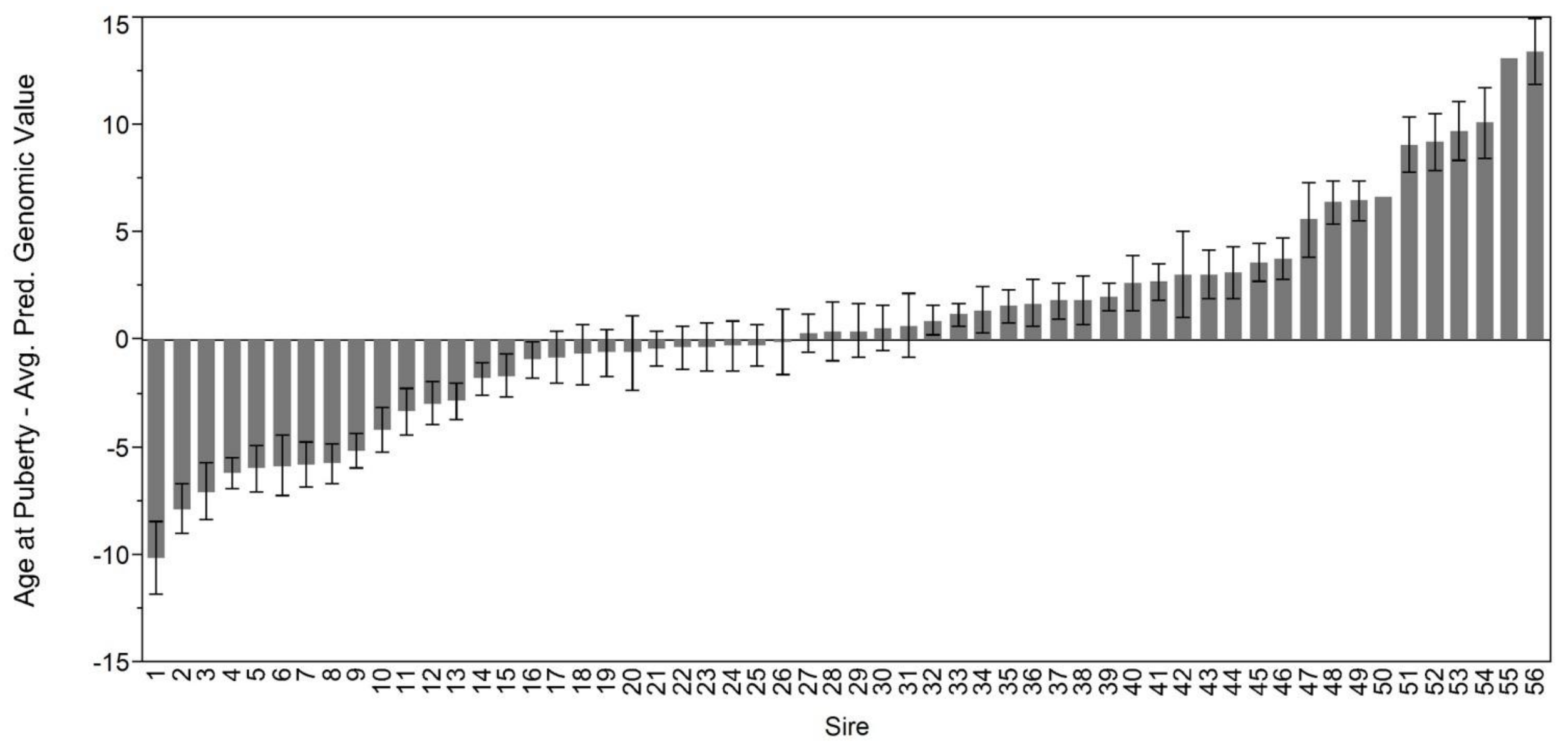


Table S1. PCR primers for cDNA sequencing of the positional candidate genes.

\begin{tabular}{c|l|l}
\hline $\begin{array}{c}\text { Gene } \\
\text { symbol }\end{array}$ & \multicolumn{1}{|c}{ Forward primer } & \multicolumn{1}{c}{ Reverse primer } \\
\hline AVPRIA & $\begin{array}{l}\text { AGATGCCAATACCAGTCG } \\
\text { TGTTTGCATCCGCTGAAGA } \\
\text { ATGGTCGAGGTGAGCAATGT }\end{array}$ & $\begin{array}{l}\text { TGACATTGCTCACCTCGACCAT } \\
\text { TCAGCCAAGGAGCTGAAAAGTC } \\
\text { TTCGTGTTTGGCAGCATGG }\end{array}$ \\
\hline BAIAP2 & $\begin{array}{l}\text { AGAGCGAGAAGACCAAGATG } \\
\text { CATCGATGCCATCAGCAACA }\end{array}$ & $\begin{array}{l}\text { AGGCAGGTGGTAACGAAACA } \\
\text { GTGTAGGAGAAGGGAAACCA }\end{array}$ \\
\hline \multirow{2}{*}{ ORTC1 $2 G 3$} & $\begin{array}{l}\text { TGCCCAACGTGAACCAGATT } \\
\text { AGCAGGTGTCACCCACTCTCT }\end{array}$ & $\begin{array}{l}\text { TGAGTAAAGAAGGCGTAGGGCAAC } \\
\text { CCAGCGTGCTGGTCAGTTCTT }\end{array}$ \\
\hline ACCTCTTGACCCTTGTGGGAAA & $\begin{array}{l}\text { CTCTATTTCAGCGGGAGA } \\
\text { GAGGCCTTCAGGCATTACAACA }\end{array}$ & $\begin{array}{l}\text { CGATCTTGCTGATGTCGCAGTT } \\
\text { TCTGGACACCAGGTCCAGGTAACA } \\
\text { CCCGTCACCATCATGGAAAT } \\
\text { TGCTTGTCCATTTGCCTTCC } \\
\text { TTTGACAACTCCTGGTCCTTCC }\end{array}$ \\
& $\begin{array}{l}\text { CGAAGGTGAAGAATGCGATGA } \\
\text { CGGGATGACGAACTGATCAAGA }\end{array}$ & $\begin{array}{l}\text { AGCGTAAAGCCTGCCGAAAT } \\
\text { TTCAAAGGTATTGAGTGTCCTCTGGA } \\
\text { TCACACACTTCTTTCACAGCCTCA }\end{array}$ \\
\hline
\end{tabular}


Table S2. Genetic variance of age at puberty explained by $1 \mathrm{Mb}$ windows

\begin{tabular}{|c|c|c|c|c|c|c|c|c|c|c|c|c|c|}
\hline Window & start & end & \#SNPs & $\% \operatorname{Var}$ & Cum\%Var & $p>0$ & $\mathrm{p}>$ Average & map & pos0 & map & posn & chr & $\mathrm{Mb}$ \\
\hline 2593 & 30809 & 14634 & 4799 & 8.66 & 0 & 1 & 1 & 21 & 0 & 21 & 0 & 21 & 0 \\
\hline 1641 & 29706 & 49340 & 16 & 0.98 & 2.2 & 0.347347 & 0.327327 & 12 & 1169606 & 12 & 1998701 & 12 & 1 \\
\hline 1208 & 32044 & 24165 & 19 & 0.62 & 3.52 & 0.229229 & 0.205205 & 8 & 37029609 & 8 & 37983458 & 8 & 37 \\
\hline 1642 & 43103 & 30214 & 25 & 0.53 & 4.05 & 0.249249 & 0.228228 & 12 & 2065310 & 12 & 2979580 & 12 & 2 \\
\hline 494 & 45465 & 19141 & 19 & 0.53 & 4.57 & 0.235235 & 0.202202 & 3 & 16029880 & 3 & 16920601 & 3 & 16 \\
\hline 94 & 45969 & 723 & 15 & 0.48 & 5.05 & 0.193193 & 0.178178 & 1 & 94180981 & 1 & 94935963 & 1 & 94 \\
\hline 1991 & 39038 & 59753 & 32 & 0.42 & 6.33 & 0.249249 & 0.196196 & 14 & 68005023 & 14 & 68982424 & 14 & 68 \\
\hline 2452 & 44846 & 14947 & 12 & 0.37 & 6.7 & 0.16016 & 0.138138 & 18 & 60063061 & 18 & 60911033 & 18 & 60 \\
\hline 1076 & 46993 & 23505 & 34 & 0.36 & 7.06 & 0.264264 & 0.235235 & 7 & 39051601 & 7 & 39985401 & 7 & 39 \\
\hline 1989 & 11090 & 11108 & 30 & 0.35 & 7.41 & 0.228228 & 0.203203 & 14 & 66049762 & 14 & 66992067 & 14 & 66 \\
\hline 1023 & 17625 & 16282 & 23 & 0.34 & 7.75 & 0.186186 & 0.153153 & 6 & 144024419 & 6 & 144977414 & 6 & 144 \\
\hline 1942 & 10729 & 10757 & 27 & 0.33 & 8.08 & 0.208208 & 0.171171 & 14 & 19012273 & 14 & 19994178 & 14 & 19 \\
\hline 1603 & 8898 & 58640 & 30 & 0.27 & 9.53 & 0.219219 & 0.178178 & 11 & 51024403 & 11 & 51986201 & 11 & 51 \\
\hline 1207 & 30711 & 31235 & 19 & 0.26 & 9.79 & 0.17017 & 0.14014 & 8 & 36098692 & 8 & 36972953 & 8 & 36 \\
\hline 1341 & 24536 & 61555 & 28 & 0.25 & 10.05 & 0.213213 & 0.168168 & 9 & 21032490 & 9 & 21990942 & 9 & 21 \\
\hline 31 & 15632 & 54565 & 32 & 0.25 & 10.3 & 0.217217 & 0.174174 & 1 & 31008523 & 1 & 31952459 & 1 & 31 \\
\hline 549 & 18957 & 3074 & 24 & 0.25 & 10.55 & 0.202202 & 0.166166 & 3 & 71100014 & 3 & 71946686 & 3 & 71 \\
\hline 1846 & 57493 & 60468 & 16 & 0.24 & 10.79 & 0.165165 & 0.136136 & 13 & 142131038 & 13 & 142902305 & 13 & 142 \\
\hline 1951 & 10819 & 49467 & 37 & 0.24 & 11.03 & 0.235235 & 0.178178 & 14 & 28021727 & 14 & 28972892 & 14 & 28 \\
\hline 1973 & 55273 & 43758 & 31 & 0.21 & 11.25 & 0.196196 & 0.161161 & 14 & 50012997 & 14 & 50992818 & 14 & 50 \\
\hline 993 & 5508 & 37793 & 22 & 0.2 & 11.45 & 0.192192 & 0.155155 & 6 & 114005432 & 6 & 114926648 & 6 & 114 \\
\hline 91 & 19926 & 36128 & 28 & 0.2 & 11.65 & 0.19019 & 0.162162 & 1 & 91015459 & 1 & 91983106 & 1 & 91 \\
\hline 756 & 34031 & 35791 & 35 & 0.2 & 11.84 & 0.245245 & 0.194194 & 4 & 133019096 & 4 & 133997198 & 4 & 133 \\
\hline 301 & 40386 & 40397 & 31 & 0.19 & 12.04 & 0.208208 & 0.17017 & 1 & 301003643 & 1 & 301983854 & 1 & 301 \\
\hline 727 & 41212 & 22251 & 27 & 0.19 & 12.23 & 0.184184 & 0.151151 & 4 & 104027961 & 4 & 104999962 & 4 & 104 \\
\hline
\end{tabular}




\begin{tabular}{|c|c|c|c|c|c|c|c|c|c|c|c|c|c|}
\hline 1206 & 17252 & 33461 & 26 & 0.18 & 13.16 & 0.166166 & 0.124124 & 8 & 35031484 & 8 & 35944997 & 8 & 35 \\
\hline 1458 & 8103 & 24932 & 34 & 0.18 & 13.33 & 0.235235 & 0.181181 & 9 & 138014373 & 9 & 138986678 & 9 & 138 \\
\hline 286 & 40341 & 20558 & 25 & 0.17 & 13.51 & 0.182182 & 0.154154 & 1 & 286007661 & 1 & 286998337 & 1 & 286 \\
\hline 1865 & 26437 & 43503 & 24 & 0.17 & 13.68 & 0.148148 & 0.123123 & 13 & 161008278 & 13 & 161962013 & 13 & 161 \\
\hline 819 & 29636 & 52487 & 19 & 0.17 & 13.85 & 0.133133 & 0.108108 & 5 & 52019205 & 5 & 52937921 & 5 & 52 \\
\hline 608 & 3358 & 21626 & 18 & 0.17 & 14.01 & 0.156156 & 0.133133 & 3 & 130036775 & 3 & 130877498 & 3 & 130 \\
\hline 1544 & 8576 & 53828 & 33 & 0.16 & 14.18 & 0.199199 & 0.163163 & 10 & 70020888 & 10 & 70904101 & 10 & 70 \\
\hline 1867 & 15397 & 57476 & 16 & 0.16 & 14.34 & 0.151151 & 0.124124 & 13 & 163002035 & 13 & 163963155 & 13 & 163 \\
\hline 376 & 20912 & 54049 & 17 & 0.16 & 14.5 & 0.106106 & 0.083083 & 2 & 60022633 & 2 & 60953615 & 2 & 60 \\
\hline 1731 & 36190 & 26033 & 35 & 0.16 & 14.66 & 0.208208 & 0.159159 & 13 & 27003930 & 13 & 27982732 & 13 & 27 \\
\hline 1788 & 61695 & 10019 & 15 & 0.16 & 14.82 & 0.112112 & 0.091091 & 13 & 84062043 & 13 & 84751325 & 13 & 84 \\
\hline 36 & 39862 & 308 & 38 & 0.15 & 14.97 & 0.193193 & 0.146146 & 1 & 36021536 & 1 & 36976294 & 1 & 36 \\
\hline 1972 & 47627 & 47631 & 22 & 0.15 & 15.12 & 0.159159 & 0.124124 & 14 & 49115640 & 14 & 49990314 & 14 & 49 \\
\hline 1654 & 61557 & 49358 & 30 & 0.15 & 15.27 & 0.189189 & 0.14014 & 12 & 14013189 & 12 & 14984702 & 12 & 14 \\
\hline 1115 & 38008 & 38015 & 26 & 0.15 & 15.42 & 0.2002 & 0.159159 & 7 & 78031358 & 7 & 78971281 & 7 & 78 \\
\hline 2084 & 11840 & 19362 & 27 & 0.15 & 15.57 & 0.162162 & 0.133133 & 15 & 7009701 & 15 & 7998827 & 15 & 7 \\
\hline 2098 & 44097 & 11960 & 27 & 0.15 & 15.71 & 0.18018 & 0.139139 & 15 & 21094100 & 15 & 21993474 & 15 & 21 \\
\hline 758 & 41310 & 34359 & 29 & 0.15 & 15.86 & 0.148148 & 0.117117 & 4 & 135010909 & 4 & 135982856 & 4 & 135 \\
\hline 56 & 60375 & 16290 & 28 & 0.15 & 16.01 & 0.178178 & 0.142142 & 1 & 56004411 & 1 & 56836525 & 1 & 56 \\
\hline 1295 & 7326 & 7332 & 21 & 0.14 & 16.15 & 0.136136 & 0.112112 & 8 & 124059371 & 8 & 124932778 & 8 & 124 \\
\hline 1640 & 45525 & 50520 & 21 & 0.14 & 16.29 & 0.155155 & 0.12012 & 12 & 79502 & 12 & 980500 & 12 & 0 \\
\hline 2230 & 53489 & 32220 & 11 & 0.14 & 16.43 & 0.124124 & 0.097097 & 15 & 153014360 & 15 & 153829568 & 15 & 153 \\
\hline 1981 & 51624 & 47641 & 30 & 0.13 & 16.57 & 0.169169 & 0.132132 & 14 & 58002810 & 14 & 58984594 & 14 & 58 \\
\hline 374 & 57638 & 2321 & 9 & 0.13 & 16.7 & 0.077077 & 0.063063 & 2 & 58377827 & 2 & 58871412 & 2 & 58 \\
\hline 685 & 32042 & 35889 & 30 & 0.13 & 16.84 & 0.173173 & 0.142142 & 4 & 62027221 & 4 & 62973622 & 4 & 62 \\
\hline 2039 & 39144 & 52595 & 23 & 0.13 & 16.97 & 0.159159 & 0.125125 & 14 & 116309545 & 14 & 116970199 & 14 & 116 \\
\hline 1316 & 29295 & 19170 & 47 & 0.13 & 17.1 & 0.221221 & 0.167167 & 8 & 145036187 & 8 & 145994240 & 8 & 145 \\
\hline 992 & 29558 & 16483 & 20 & 0.13 & 17.23 & 0.139139 & 0.117117 & 6 & 113074700 & 6 & 113818056 & 6 & 113 \\
\hline 1457 & 8091 & 8104 & 34 & 0.13 & 17.37 & 0.19019 & 0.141141 & 9 & 137007027 & 9 & 137947776 & 9 & 137 \\
\hline 381 & 2307 & 37117 & 15 & 0.13 & 17.5 & 0.118118 & 0.087087 & 2 & 66048253 & 2 & 66776495 & 2 & 66 \\
\hline 1402 & 7849 & 24787 & 21 & 0.13 & 17.63 & 0.136136 & 0.107107 & 9 & 82004163 & 9 & 82930808 & 9 & 82 \\
\hline 375 & 53440 & 20913 & 10 & 0.13 & 17.76 & 0.114114 & 0.095095 & 2 & 59243470 & 2 & 59957369 & 2 & 59 \\
\hline 1022 & 41725 & 23200 & 24 & 0.13 & 17.88 & 0.153153 & 0.111111 & 6 & 143021550 & 6 & 143976085 & 6 & 143 \\
\hline 1634 & 34637 & 9166 & 28 & 0.13 & 18.01 & 0.175175 & 0.138138 & 11 & 82019810 & 11 & 82972974 & 11 & 82 \\
\hline 22 & 19578 & 19127 & 26 & 0.12 & 18.13 & 0.154154 & 0.114114 & 1 & 22042965 & 1 & 22993058 & 1 & 22 \\
\hline 328 & 2029 & 35259 & 38 & 0.12 & 18.26 & 0.189189 & 0.153153 & 2 & 12047540 & 2 & 12999985 & 2 & 12 \\
\hline 2061 & 34382 & 27445 & 33 & 0.12 & 18.38 & 0.181181 & 0.141141 & 14 & 138031007 & 14 & 138971702 & 14 & 138 \\
\hline 1702 & 55843 & 33509 & 34 & 0.12 & 18.5 & 0.167167 & 0.125125 & 12 & 62026594 & 12 & 62962634 & 12 & 62 \\
\hline 1506 & 8380 & 42798 & 26 & 0.12 & 18.62 & 0.181181 & 0.133133 & 10 & 32008178 & 10 & 32971037 & 10 & 32 \\
\hline
\end{tabular}




\begin{tabular}{|c|c|c|c|c|c|c|c|c|c|c|c|c|}
\hline 2211 & 36231 & 12532 & 22 & 0.12 & 18.74 & 0.154154 & 0.12012 & 15 & 134006803 & 15 & 134994861 & 15 \\
\hline 674 & 41090 & 3831 & 23 & 0.12 & 18.86 & 0.151151 & 0.117117 & 4 & 51036347 & 4 & 51937058 & 4 \\
\hline 1149 & 6589 & 57088 & 30 & 0.12 & 18.98 & 0.164164 & 0.116116 & 7 & 113020823 & 7 & 113908824 & 7 \\
\hline 1298 & 58793 & 7341 & 17 & 0.12 & 19.09 & 0.128128 & 0.1001 & 8 & 127052406 & 8 & 127976364 & 8 \\
\hline 1467 & 56527 & 42719 & 35 & 0.12 & 19.21 & 0.199199 & 0.157157 & 9 & 147006399 & 9 & 147992536 & 9 \\
\hline 1436 & 58155 & 34813 & 27 & 0.12 & 19.33 & 0.178178 & 0.132132 & 9 & 116099220 & 9 & 116927941 & 9 \\
\hline 798 & 4764 & 41437 & 19 & 0.12 & 19.44 & 0.112112 & 0.084084 & 5 & 31161895 & 5 & 31980027 & 5 \\
\hline 965 & 32376 & 23056 & 23 & 0.11 & 19.56 & 0.169169 & 0.125125 & 6 & 86017603 & 6 & 86940350 & 6 \\
\hline 1263 & 7178 & 42323 & 34 & 0.11 & 19.67 & 0.183183 & 0.141141 & 8 & 92067978 & 8 & 92963940 & 8 \\
\hline 2456 & 14641 & 32232 & 31 & 0.11 & 19.78 & 0.174174 & 0.143143 & 19 & 3024537 & 19 & 3979293 & 19 \\
\hline 2040 & 11517 & 43949 & 27 & 0.11 & 19.89 & 0.146146 & 0.117117 & 14 & 117011153 & 14 & 117886869 & 14 \\
\hline 2223 & 49601 & 44295 & 26 & 0.11 & 20 & 0.164164 & 0.132132 & 15 & 146016611 & 15 & 146927575 & 15 \\
\hline 1997 & 39060 & 39065 & 16 & 0.11 & 20.11 & 0.115115 & 0.088088 & 14 & 74155370 & 14 & 74990114 & 14 \\
\hline 1504 & 25138 & 17677 & 25 & 0.11 & 20.22 & 0.136136 & 0.114114 & 10 & 30012240 & 10 & 30978174 & 10 \\
\hline 2078 & 27597 & 27585 & 24 & 0.11 & 20.33 & 0.164164 & 0.131131 & 15 & 1015164 & 15 & 1975870 & 15 \\
\hline 433 & 40646 & 2557 & 27 & 0.11 & 20.44 & 0.152152 & 0.122122 & 2 & 118010721 & 2 & 118934685 & 2 \\
\hline 25 & 52428 & 36511 & 19 & 0.11 & 20.55 & 0.138138 & 0.104104 & 1 & 25010377 & 1 & 25927201 & 1 \\
\hline 629 & 3436 & 21719 & 31 & 0.11 & 20.65 & 0.171171 & 0.138138 & 4 & 6007874 & 4 & 6977891 & 4 \\
\hline 1974 & 10991 & 10997 & 21 & 0.11 & 20.76 & 0.117117 & 0.09009 & 14 & 51006694 & 14 & 51937785 & 14 \\
\hline 7 & 18327 & 39767 & 28 & 0.11 & 20.87 & 0.153153 & 0.12012 & 1 & 7149806 & 1 & 7983109 & 1 \\
\hline 2012 & 11281 & 11289 & 20 & 0.1 & 20.97 & 0.14014 & 0.103103 & 14 & 89013097 & 14 & 89670530 & 14 \\
\hline 996 & 23113 & 14773 & 17 & 0.1 & 21.07 & 0.136136 & 0.103103 & 6 & 117172816 & 6 & 117926852 & 6 \\
\hline 448 & 21120 & 16166 & 31 & 0.1 & 21.18 & 0.155155 & 0.118118 & 2 & 133019436 & 2 & 133937811 & 2 \\
\hline 673 & 16349 & 3816 & 14 & 0.1 & 21.28 & 0.113113 & 0.094094 & 4 & 50110206 & 4 & 50997149 & 4 \\
\hline 1132 & 54761 & 6452 & 28 & 0.1 & 21.38 & 0.169169 & 0.129129 & 7 & 96000929 & 7 & 96970994 & 7 \\
\hline 1466 & 8157 & 31453 & 27 & 0.1 & 21.48 & 0.172172 & 0.129129 & 9 & 146021041 & 9 & 146997147 & 9 \\
\hline 1021 & 57735 & 5649 & 28 & 0.1 & 21.58 & 0.135135 & 0.101101 & 6 & 142036921 & 6 & 142989178 & 6 \\
\hline 288 & 20567 & 20576 & 32 & 0.1 & 21.68 & 0.173173 & 0.129129 & 1 & 288024268 & 1 & 288885054 & 1 \\
\hline 121 & 46021 & 54553 & 15 & 0.1 & 21.78 & 0.082082 & 0.053053 & 1 & 121128221 & 1 & 121999146 & 1 \\
\hline 1993 & 11130 & 39052 & 25 & 0.1 & 21.88 & 0.138138 & 0.097097 & 14 & 70019958 & 14 & 70993366 & 14 \\
\hline 329 & 45775 & 40461 & 10 & 0.1 & 21.98 & 0.074074 & 0.063063 & 2 & 13005151 & 2 & 13902607 & 2 \\
\hline 1633 & 38630 & 25654 & 34 & 0.1 & 22.08 & 0.184184 & 0.133133 & 11 & 81016745 & 11 & 81997858 & 11 \\
\hline 2333 & 13268 & 28437 & 31 & 0.1 & 22.17 & 0.171171 & 0.125125 & 17 & 11026407 & 17 & 11952380 & 17 \\
\hline 1586 & 8822 & 47348 & 14 & 0.1 & 22.27 & 0.1001 & 0.071071 & 11 & 34013189 & 11 & 34890371 & 11 \\
\hline 866 & 5160 & 5146 & 35 & 0.1 & 22.37 & 0.186186 & 0.127127 & 5 & 99010975 & 5 & 99972762 & 5 \\
\hline 481 & 37250 & 33526 & 27 & 0.1 & 22.47 & 0.156156 & 0.104104 & 3 & 3029529 & 3 & 3936783 & 3 \\
\hline 1646 & 48323 & 29730 & 39 & 0.1 & 22.56 & 0.187187 & 0.127127 & 12 & 6120038 & 12 & 6998897 & 12 \\
\hline 1624 & 43040 & 9077 & 26 & 0.1 & 22.66 & 0.145145 & 0.109109 & 11 & 72031850 & 11 & 72966366 & 11 \\
\hline 285 & 18644 & 40340 & 27 & 0.1 & 22.75 & 0.162162 & 0.109109 & 1 & 285026326 & 1 & 285985500 & 1 \\
\hline
\end{tabular}




\begin{tabular}{|c|c|c|c|c|c|c|c|c|c|c|c|c|c|}
\hline 1618 & 9013 & 9025 & 32 & 0.09 & 22.85 & 0.176176 & 0.131131 & 11 & 66014418 & 11 & 66991408 & 11 & 66 \\
\hline 1857 & 15094 & 51557 & 16 & 0.09 & 22.94 & 0.133133 & 0.092092 & 13 & 153245989 & 13 & 153940892 & 13 & \\
\hline 447 & 21116 & 2642 & 20 & 0.09 & 23.04 & 0.133133 & 0.106106 & 2 & 132012813 & 2 & 132997331 & 2 & . \\
\hline 1883 & 10378 & 56500 & 14 & 0.09 & 23.13 & 0.078078 & 0.068068 & 13 & 179045118 & 13 & 179990010 & 13 & \\
\hline 1317 & 16299 & 15805 & 33 & 0.09 & 23.22 & 0.154154 & 0.107107 & 8 & 146042078 & 8 & 146958932 & 8 & 1 \\
\hline 1955 & 51375 & 43700 & 17 & 0.09 & 23.32 & 0.109109 & 0.089089 & 14 & 32000259 & 14 & 32974206 & 14 & \\
\hline 635 & 21769 & 3500 & 37 & 0.09 & 23.41 & 0.193193 & 0.137137 & 4 & 12003384 & 4 & 12980361 & 4 & \\
\hline 1517 & 54523 & 57266 & 22 & 0.09 & 23.5 & 0.117117 & 0.089089 & 10 & 43015513 & 10 & 43858335 & 10 & \\
\hline 1650 & 25726 & 53234 & 23 & 0.09 & 23.6 & 0.137137 & 0.112112 & 12 & 10017988 & 12 & 10938363 & 12 & \\
\hline 1943 & 10758 & 26750 & 27 & 0.09 & 23.69 & 0.151151 & 0.111111 & 14 & 20034189 & 14 & 20912572 & 14 & \\
\hline 1086 & 6073 & 49020 & 33 & 0.09 & 23.78 & 0.178178 & 0.125125 & 7 & 49013454 & 7 & 49982901 & 7 & \\
\hline 373 & 59106 & 20917 & 11 & 0.09 & 23.87 & 0.086086 & 0.062062 & 2 & 57011989 & 2 & 57924527 & 2 & \\
\hline 550 & 54572 & 15140 & 17 & 0.09 & 23.97 & 0.094094 & 0.078078 & 3 & 72009115 & 3 & 72838665 & 3 & \\
\hline 1861 & 10288 & 26421 & 17 & 0.09 & 24.06 & 0.098098 & 0.07007 & 13 & 157024403 & 13 & 157993717 & 13 & 157 \\
\hline 1716 & 45087 & 9550 & 38 & 0.09 & 24.15 & 0.181181 & 0.133133 & 13 & 12004442 & 13 & 12986957 & 13 & \\
\hline 1992 & 27075 & 39049 & 28 & 0.09 & 24.24 & 0.136136 & 0.098098 & 14 & 69015482 & 14 & 69980983 & 14 & \\
\hline 1493 & 30638 & 45259 & 31 & 0.09 & 24.33 & 0.161161 & 0.119119 & 10 & 19081508 & 10 & 19975279 & 10 & \\
\hline 28 & 215 & 221 & 27 & 0.09 & 24.42 & 0.159159 & 0.112112 & 1 & 28050709 & 1 & 28899322 & 1 & \\
\hline 639 & 3531 & 16086 & 38 & 0.09 & 24.51 & 0.189189 & 0.131131 & 4 & 16001400 & 4 & 16995545 & 4 & \\
\hline 122 & 52120 & 40042 & 20 & 0.09 & 24.6 & 0.113113 & 0.084084 & 1 & 122019338 & 1 & 122922363 & 1 & 12 \\
\hline 1190 & 32735 & 24097 & 38 & 0.09 & 24.68 & 0.198198 & 0.145145 & 8 & 19014542 & 8 & 19969058 & 8 & \\
\hline 2054 & 11610 & 27398 & 29 & 0.09 & 24.77 & 0.151151 & 0.12012 & 14 & 131033758 & 14 & 131991212 & 14 & 13 \\
\hline 1652 & 25739 & 38637 & 25 & 0.09 & 24.86 & 0.14014 & 0.101101 & 12 & 12040820 & 12 & 12957003 & 12 & \\
\hline 2082 & 27562 & 11838 & 13 & 0.09 & 24.95 & 0.084084 & 0.07007 & 15 & 5034616 & 15 & 5936184 & 15 & \\
\hline 1455 & 24905 & 8084 & 30 & 0.09 & 25.04 & 0.164164 & 0.129129 & 9 & 135034104 & 9 & 135978071 & 9 & 13 \\
\hline 1141 & 23847 & 23858 & 27 & 0.09 & 25.12 & 0.134134 & 0.097097 & 7 & 105022346 & 7 & 105980155 & 7 & 10 \\
\hline 93 & 50671 & 19939 & 23 & 0.09 & 25.21 & 0.134134 & 0.097097 & 1 & 93019315 & 1 & 93750500 & 1 & \\
\hline 1571 & 32103 & 31549 & 18 & 0.09 & 25.29 & 0.121121 & 0.089089 & 11 & 19306043 & 11 & 19998464 & 11 & \\
\hline 1697 & 14997 & 9441 & 32 & 0.09 & 25.38 & 0.17017 & 0.128128 & 12 & 57002739 & 12 & 57950908 & 12 & \\
\hline 527 & 21348 & 53475 & 32 & 0.09 & 25.47 & 0.173173 & 0.114114 & 3 & 49011623 & 3 & 49877554 & 3 & \\
\hline 1619 & 25566 & 9037 & 28 & 0.09 & 25.55 & 0.171171 & 0.131131 & 11 & 67039090 & 11 & 67975596 & 11 & \\
\hline 401 & 2345 & 20942 & 20 & 0.09 & 25.64 & 0.134134 & 0.098098 & 2 & 86090869 & 2 & 86970329 & 2 & \\
\hline 1456 & 24911 & 8092 & 25 & 0.08 & 25.72 & 0.149149 & 0.115115 & 9 & 136006502 & 9 & 136977536 & 9 & \\
\hline 1110 & 23689 & 47077 & 21 & 0.08 & 25.81 & 0.129129 & 0.093093 & 7 & 73011207 & 7 & 73938361 & 7 & \\
\hline 1958 & 59895 & 10883 & 27 & 0.08 & 25.89 & 0.142142 & 0.104104 & 14 & 35036848 & 14 & 35973121 & 14 & \\
\hline 2299 & 13022 & 44435 & 24 & 0.08 & 25.98 & 0.138138 & 0.112112 & 16 & 64085517 & 16 & 64990189 & 16 & \\
\hline 2313 & 44466 & 44470 & 28 & 0.08 & 26.06 & 0.149149 & 0.116116 & 16 & 78001888 & 16 & 78984075 & 16 & \\
\hline 653 & 3655 & 3663 & 24 & 0.08 & 26.14 & 0.124124 & 0.098098 & 4 & 30026623 & 4 & 30990527 & 4 & \\
\hline 1792 & 10033 & 26259 & 31 & 0.08 & 26.23 & 0.174174 & 0.121121 & 13 & 88018134 & 13 & 88989523 & 13 & \\
\hline
\end{tabular}




\begin{tabular}{|c|c|c|c|c|c|c|c|c|c|c|c|c|c|}
\hline 1969 & 36069 & 54351 & 33 & 0.08 & 26.31 & 0.169169 & 0.109109 & 14 & 46043439 & 14 & 46983442 & 14 & 46 \\
\hline 1862 & 58565 & 10297 & 21 & 0.08 & 26.4 & 0.118118 & 0.093093 & 13 & 158099578 & 13 & 158992883 & 13 & 158 \\
\hline 1189 & 24087 & 58423 & 33 & 0.08 & 26.48 & 0.162162 & 0.111111 & 8 & 18001094 & 8 & 18974551 & 8 & 18 \\
\hline 2016 & 43880 & 51099 & 29 & 0.08 & 26.56 & 0.146146 & 0.116116 & 14 & 93096651 & 14 & 93968851 & 14 & 93 \\
\hline 269 & 20483 & 46359 & 21 & 0.08 & 26.64 & 0.12012 & 0.094094 & 1 & 269008819 & 1 & 269972373 & 1 & 269 \\
\hline 1301 & 49143 & 30100 & 27 & 0.08 & 26.73 & 0.133133 & 0.094094 & 8 & 130012979 & 8 & 130997013 & 8 & 130 \\
\hline 953 & 23029 & 16694 & 41 & 0.08 & 26.81 & 0.195195 & 0.142142 & 6 & 74002918 & 6 & 74982947 & 6 & 74 \\
\hline 1995 & 27092 & 43822 & 29 & 0.08 & 26.89 & 0.149149 & 0.104104 & 14 & 72010321 & 14 & 72950668 & 14 & 72 \\
\hline 897 & 49937 & 5274 & 39 & 0.08 & 26.97 & 0.178178 & 0.128128 & 6 & 18019907 & 6 & 18982722 & 6 & 18 \\
\hline 2399 & 52077 & 15161 & 29 & 0.08 & 27.05 & 0.167167 & 0.123123 & 18 & 7052901 & 18 & 7985651 & 18 & 7 \\
\hline 960 & 45681 & 48918 & 31 & 0.08 & 27.14 & 0.175175 & 0.122122 & 6 & 81021081 & 6 & 81987568 & 6 & 81 \\
\hline 1041 & 5722 & 5729 & 29 & 0.08 & 27.22 & 0.152152 & 0.111111 & 7 & 4033259 & 7 & 4988931 & 7 & 4 \\
\hline 1929 & 26641 & 61516 & 22 & 0.08 & 27.3 & 0.133133 & 0.101101 & 14 & 6036903 & 14 & 6992243 & 14 & 6 \\
\hline 52 & 45898 & 19734 & 24 & 0.08 & 27.38 & 0.145145 & 0.116116 & 1 & 52062069 & 1 & 52999008 & 1 & 52 \\
\hline 961 & 5399 & 33393 & 25 & 0.08 & 27.46 & 0.12012 & 0.096096 & 6 & 82014983 & 6 & 82929466 & 6 & 82 \\
\hline 1884 & 10380 & 10384 & 13 & 0.08 & 27.54 & 0.1001 & 0.078078 & 13 & 180057818 & 13 & 180923495 & 13 & 180 \\
\hline 2348 & 28504 & 56461 & 19 & 0.08 & 27.62 & 0.124124 & 0.086086 & 17 & 26152267 & 17 & 26991897 & 17 & 26 \\
\hline 1998 & 27107 & 11179 & 30 & 0.08 & 27.7 & 0.156156 & 0.106106 & 14 & 75048108 & 14 & 75991444 & 14 & 75 \\
\hline 2079 & 11871 & 53733 & 32 & 0.08 & 27.78 & 0.15015 & 0.109109 & 15 & 2009887 & 15 & 2990508 & 15 & 2 \\
\hline 914 & 52509 & 29711 & 18 & 0.08 & 27.86 & 0.108108 & 0.086086 & 6 & 35011158 & 6 & 35967061 & 6 & 35 \\
\hline 30 & 19613 & 31537 & 35 & 0.08 & 27.94 & 0.185185 & 0.133133 & 1 & 30017551 & 1 & 30978281 & 1 & 30 \\
\hline 1331 & 7522 & 7528 & 32 & 0.08 & 28.02 & 0.149149 & 0.118118 & 9 & 11012156 & 9 & 11997728 & 9 & 11 \\
\hline 1980 & 43776 & 11033 & 21 & 0.08 & 28.09 & 0.126126 & 0.098098 & 14 & 57113686 & 14 & 57982523 & 14 & 57 \\
\hline 1658 & 50249 & 54233 & 30 & 0.08 & 28.17 & 0.145145 & 0.11011 & 12 & 18147178 & 12 & 18890652 & 12 & 18 \\
\hline 554 & 37294 & 15024 & 20 & 0.08 & 28.25 & 0.122122 & 0.088088 & 3 & 76044809 & 3 & 76994672 & 3 & 76 \\
\hline 1529 & 31097 & 25246 & 17 & 0.08 & 28.33 & 0.107107 & 0.076076 & 10 & 55063135 & 10 & 55999312 & 10 & 55 \\
\hline 1705 & 43234 & 25916 & 23 & 0.08 & 28.41 & 0.123123 & 0.095095 & 13 & 1022508 & 13 & 1982864 & 13 & 1 \\
\hline 2037 & 34461 & 27313 & 23 & 0.08 & 28.49 & 0.122122 & 0.097097 & 14 & 114007859 & 14 & 114987900 & 14 & 114 \\
\hline 2208 & 16001 & 58144 & 35 & 0.08 & 28.56 & 0.16016 & 0.105105 & 15 & 131030369 & 15 & 131996123 & 15 & 131 \\
\hline 2369 & 13525 & 56669 & 22 & 0.08 & 28.64 & 0.12012 & 0.077077 & 17 & 47086750 & 17 & 47981889 & 17 & 47 \\
\hline 2 & 19451 & 39756 & 29 & 0.08 & 28.72 & 0.173173 & 0.13013 & 1 & 2010349 & 1 & 2995480 & 1 & 2 \\
\hline 2457 & 54441 & 44855 & 26 & 0.08 & 28.8 & 0.149149 & 0.106106 & 19 & 4006006 & 19 & 4977485 & 19 & 4 \\
\hline 1154 & 6642 & 42124 & 34 & 0.08 & 28.87 & 0.161161 & 0.117117 & 7 & 118001966 & 7 & 118979653 & 7 & 118 \\
\hline 1910 & 17196 & 15151 & 23 & 0.08 & 28.95 & 0.114114 & 0.085085 & 13 & 206171736 & 13 & 206956346 & 13 & 206 \\
\hline 983 & 31756 & 23097 & 24 & 0.08 & 29.03 & 0.13013 & 0.092092 & 6 & 104100988 & 6 & 104981251 & 6 & 104 \\
\hline 1330 & 57942 & 35967 & 35 & 0.08 & 29.1 & 0.169169 & 0.13013 & 9 & 10050483 & 9 & 10972428 & 9 & 10 \\
\hline 1020 & 5635 & 57523 & 32 & 0.08 & 29.18 & 0.169169 & 0.122122 & 6 & 141025042 & 6 & 141988774 & 6 & 141 \\
\hline 1655 & 29638 & 9253 & 32 & 0.08 & 29.26 & 0.16016 & 0.11011 & 12 & 15000817 & 12 & 15974791 & 12 & 15 \\
\hline 1556 & 54532 & 8624 & 25 & 0.08 & 29.33 & 0.13013 & 0.109109 & 11 & 4003595 & 11 & 4989840 & 11 & 4 \\
\hline
\end{tabular}




\begin{tabular}{|c|c|c|c|c|c|c|c|c|c|c|c|c|c|}
\hline 1928 & 47556 & 26639 & 25 & 0.08 & 29.41 & 0.141141 & 0.106106 & 14 & 5009281 & 14 & 5995892 & 14 & 5 \\
\hline 2013 & 27176 & 11298 & 27 & 0.08 & 29.48 & 0.151151 & 0.116116 & 14 & 90035986 & 14 & 90968956 & 14 & 90 \\
\hline 2310 & 28331 & 54335 & 37 & 0.08 & 29.56 & 0.164164 & 0.109109 & 16 & 75024639 & 16 & 75983297 & 16 & 75 \\
\hline 1699 & 52072 & 14562 & 30 & 0.08 & 29.64 & 0.156156 & 0.109109 & 12 & 59032217 & 12 & 59982065 & 12 & 59 \\
\hline 1297 & 54817 & 7338 & 18 & 0.08 & 29.71 & 0.115115 & 0.091091 & 8 & 126024923 & 8 & 126952312 & 8 & 126 \\
\hline 1074 & 23489 & 5987 & 36 & 0.08 & 29.79 & 0.169169 & 0.125125 & 7 & 37026669 & 7 & 37967748 & 7 & 37 \\
\hline 1988 & 27052 & 43804 & 21 & 0.07 & 29.86 & 0.112112 & 0.093093 & 14 & 65015603 & 14 & 65969593 & 14 & 65 \\
\hline 2241 & 59592 & 28090 & 36 & 0.07 & 29.94 & 0.184184 & 0.126126 & 16 & 6013914 & 16 & 6989080 & 16 & 6 \\
\hline 1843 & 26371 & 54558 & 16 & 0.07 & 30.01 & 0.079079 & 0.057057 & 13 & 139142736 & 13 & 139998680 & 13 & 139 \\
\hline 1889 & 10399 & 43531 & 26 & 0.07 & 30.09 & 0.12012 & 0.077077 & 13 & 185067136 & 13 & 185935406 & 13 & 185 \\
\hline 1324 & 24449 & 24456 & 27 & 0.07 & 30.16 & 0.127127 & 0.092092 & 9 & 4014490 & 9 & 4990218 & 9 & 4 \\
\hline 1948 & 26773 & 26784 & 24 & 0.07 & 30.23 & 0.132132 & 0.086086 & 14 & 25077228 & 14 & 25976982 & 14 & 25 \\
\hline 769 & 41347 & 53366 & 29 & 0.07 & 30.31 & 0.157157 & 0.113113 & 5 & 2021416 & 5 & 2975876 & 5 & 2 \\
\hline 906 & 22965 & 29298 & 16 & 0.07 & 30.38 & 0.097097 & 0.07007 & 6 & 27402328 & 6 & 27995521 & 6 & 27 \\
\hline 425 & 37172 & 37174 & 22 & 0.07 & 30.46 & 0.125125 & 0.087087 & 2 & 110005387 & 2 & 110955851 & 2 & 110 \\
\hline 100 & 19961 & 766 & 23 & 0.07 & 30.53 & 0.131131 & 0.101101 & 1 & 100021690 & 1 & 100995739 & 1 & 100 \\
\hline 436 & 21064 & 21074 & 36 & 0.07 & 30.6 & 0.182182 & 0.119119 & 2 & 121032595 & 2 & 121991931 & 2 & 121 \\
\hline 504 & 61170 & 18828 & 35 & 0.07 & 30.68 & 0.176176 & 0.128128 & 3 & 26002460 & 3 & 26978899 & 3 & 26 \\
\hline 1093 & 60723 & 57604 & 39 & 0.07 & 30.75 & 0.171171 & 0.121121 & 7 & 56025148 & 7 & 56971231 & 7 & 56 \\
\hline 55 & 439 & 19744 & 28 & 0.07 & 30.82 & 0.16016 & 0.113113 & 1 & 55012053 & 1 & 55965347 & 1 & 55 \\
\hline 1635 & 25663 & 60061 & 26 & 0.07 & 30.9 & 0.145145 & 0.099099 & 11 & 83011472 & 11 & 83976808 & 11 & 83 \\
\hline 1187 & 38098 & 31881 & 29 & 0.07 & 30.97 & 0.134134 & 0.095095 & 8 & 16014162 & 8 & 16983112 & 8 & 16 \\
\hline 908 & 61575 & 5328 & 27 & 0.07 & 31.04 & 0.15015 & 0.094094 & 6 & 29076106 & 6 & 29958963 & 6 & 29 \\
\hline 737 & 4304 & 4323 & 33 & 0.07 & 31.11 & 0.163163 & 0.114114 & 4 & 114013243 & 4 & 114969990 & 4 & 114 \\
\hline 2359 & 44589 & 44602 & 28 & 0.07 & 31.18 & 0.123123 & 0.099099 & 17 & 37018486 & 17 & 37982038 & 17 & 37 \\
\hline 1952 & 52661 & 43688 & 32 & 0.07 & 31.26 & 0.178178 & 0.127127 & 14 & 29004529 & 14 & 29985434 & 14 & 29 \\
\hline 326 & 2014 & 2017 & 30 & 0.07 & 31.33 & 0.15015 & 0.1001 & 2 & 10028746 & 2 & 10999714 & 2 & 10 \\
\hline 1617 & 9002 & 25562 & 18 & 0.07 & 31.4 & 0.11011 & 0.076076 & 11 & 65022245 & 11 & 65973607 & 11 & 65 \\
\hline 736 & 4291 & 4305 & 37 & 0.07 & 31.47 & 0.195195 & 0.121121 & 4 & 113016911 & 4 & 113982378 & 4 & 113 \\
\hline 962 & 53460 & 59865 & 19 & 0.07 & 31.54 & 0.111111 & 0.075075 & 6 & 83038514 & 6 & 83954643 & 6 & 83 \\
\hline 1342 & 7582 & 18715 & 33 & 0.07 & 31.61 & 0.168168 & 0.121121 & 9 & 22008802 & 9 & 22985623 & 9 & 22 \\
\hline 1976 & 11004 & 26978 & 22 & 0.07 & 31.68 & 0.126126 & 0.092092 & 14 & 53021844 & 14 & 53902642 & 14 & 53 \\
\hline 526 & 40799 & 15457 & 19 & 0.07 & 31.75 & 0.12012 & 0.076076 & 3 & 48022855 & 3 & 48924957 & 3 & 48 \\
\hline 997 & 5522 & 57839 & 18 & 0.07 & 31.82 & 0.111111 & 0.081081 & 6 & 118009798 & 6 & 118954409 & 6 & 118 \\
\hline 265 & 51666 & 20455 & 22 & 0.07 & 31.89 & 0.121121 & 0.083083 & 1 & 265041087 & 1 & 265964227 & 1 & 265 \\
\hline 2253 & 39400 & 28125 & 35 & 0.07 & 31.96 & 0.167167 & 0.118118 & 16 & 18027175 & 16 & 18998881 & 16 & 18 \\
\hline 2238 & 28072 & 28078 & 30 & 0.07 & 32.03 & 0.134134 & 0.096096 & 16 & 3010789 & 16 & 3964902 & 16 & 3 \\
\hline 1116 & 6302 & 6311 & 21 & 0.07 & 32.1 & 0.124124 & 0.085085 & 7 & 79025097 & 7 & 79997832 & 7 & 79 \\
\hline 1844 & 57629 & 52216 & 16 & 0.07 & 32.17 & 0.097097 & 0.074074 & 13 & 140032848 & 13 & 140851043 & 13 & 140 \\
\hline
\end{tabular}




\begin{tabular}{|c|c|c|c|c|c|c|c|c|c|c|c|c|c|}
\hline 1264 & 33341 & 56846 & 36 & 0.07 & 32.24 & 0.183183 & 0.135135 & 8 & 93062852 & 8 & 93956344 & 8 & 93 \\
\hline 422 & 31987 & 21011 & 26 & 0.07 & 32.31 & 0.147147 & 0.107107 & 2 & 107011848 & 2 & 107975061 & 2 & 107 \\
\hline 407 & 2388 & 32193 & 23 & 0.07 & 32.38 & 0.125125 & 0.092092 & 2 & 92017036 & 2 & 92965042 & 2 & 92 \\
\hline 1385 & 14587 & 24724 & 34 & 0.07 & 32.44 & 0.131131 & 0.1001 & 9 & 65022738 & 9 & 65959223 & 9 & 65 \\
\hline 684 & 3886 & 35992 & 22 & 0.07 & 32.51 & 0.103103 & 0.078078 & 4 & 61038550 & 4 & 61946905 & 4 & 61 \\
\hline 1182 & 32605 & 6807 & 39 & 0.07 & 32.58 & 0.195195 & 0.129129 & 8 & 11003327 & 8 & 11977623 & 8 & 11 \\
\hline 718 & 46728 & 56180 & 34 & 0.07 & 32.64 & 0.166166 & 0.118118 & 4 & 95000157 & 4 & 95988078 & 4 & 95 \\
\hline 2374 & 33960 & 28654 & 24 & 0.07 & 32.71 & 0.119119 & 0.085085 & 17 & 52023322 & 17 & 52970123 & 17 & 52 \\
\hline 834 & 51848 & 4950 & 32 & 0.07 & 32.78 & 0.177177 & 0.137137 & 5 & 67063213 & 5 & 67994769 & 5 & 67 \\
\hline 2303 & 45432 & 28301 & 22 & 0.07 & 32.85 & 0.131131 & 0.089089 & 16 & 68067101 & 16 & 68845016 & 16 & 68 \\
\hline 2007 & 11245 & 43857 & 24 & 0.07 & 32.91 & 0.099099 & 0.076076 & 14 & 84042439 & 14 & 84917246 & 14 & 84 \\
\hline 1979 & 39017 & 57764 & 14 & 0.07 & 32.98 & 0.087087 & 0.065065 & 14 & 56002473 & 14 & 56894114 & 14 & 56 \\
\hline 2219 & 15908 & 12594 & 30 & 0.07 & 33.04 & 0.147147 & 0.111111 & 15 & 142018466 & 15 & 142992475 & 15 & 142 \\
\hline 986 & 52629 & 32537 & 15 & 0.07 & 33.11 & 0.081081 & 0.062062 & 6 & 107035144 & 6 & 107975607 & 6 & 107 \\
\hline 1636 & 49330 & 25681 & 24 & 0.07 & 33.18 & 0.138138 & 0.101101 & 11 & 84167373 & 11 & 84977439 & 11 & 84 \\
\hline 118 & 866 & 40032 & 15 & 0.07 & 33.24 & 0.085085 & 0.053053 & 1 & 118042320 & 1 & 118992060 & 1 & 118 \\
\hline 33 & 19636 & 36543 & 35 & 0.07 & 33.31 & 0.176176 & 0.114114 & 1 & 33008187 & 1 & 33957887 & 1 & 33 \\
\hline 61 & 39921 & 497 & 25 & 0.07 & 33.37 & 0.114114 & 0.084084 & 1 & 61002086 & 1 & 61949887 & 1 & 61 \\
\hline 838 & 58110 & 41523 & 29 & 0.07 & 33.44 & 0.151151 & 0.11011 & 5 & 71056490 & 5 & 71996403 & 5 & 71 \\
\hline 1916 & 26576 & 57961 & 30 & 0.07 & 33.5 & 0.162162 & 0.121121 & 13 & 212011618 & 13 & 212967360 & 13 & 212 \\
\hline 24 & 50659 & 197 & 33 & 0.07 & 33.57 & 0.167167 & 0.11011 & 1 & 24006028 & 1 & 24953606 & 1 & 24 \\
\hline 1780 & 29911 & 60459 & 18 & 0.07 & 33.63 & 0.105105 & 0.076076 & 13 & 76026446 & 13 & 76870123 & 13 & 76 \\
\hline 1777 & 9963 & 56515 & 29 & 0.07 & 33.7 & 0.138138 & 0.099099 & 13 & 73023057 & 13 & 73966542 & 13 & 73 \\
\hline 2205 & 39348 & 12494 & 20 & 0.06 & 33.76 & 0.107107 & 0.083083 & 15 & 128002384 & 15 & 128894663 & 15 & 128 \\
\hline 2261 & 51282 & 53898 & 30 & 0.06 & 33.83 & 0.113113 & 0.078078 & 16 & 26019525 & 16 & 26898740 & 16 & 26 \\
\hline 1441 & 7997 & 8010 & 31 & 0.06 & 33.89 & 0.141141 & 0.091091 & 9 & 121014874 & 9 & 121983147 & 9 & 121 \\
\hline 474 & 17340 & 17340 & 1 & 0.06 & 33.96 & 0.025025 & 0.022022 & 2 & 159250446 & 2 & 159250446 & 2 & 159 \\
\hline 1949 & 26783 & 10812 & 22 & 0.06 & 34.02 & 0.133133 & 0.098098 & 14 & 26020243 & 14 & 26951421 & 14 & 26 \\
\hline 1940 & 38987 & 43657 & 25 & 0.06 & 34.09 & 0.122122 & 0.092092 & 14 & 17079159 & 14 & 17981100 & 14 & 17 \\
\hline 645 & 53331 & 3610 & 28 & 0.06 & 34.15 & 0.127127 & 0.082082 & 4 & 22012168 & 4 & 22978568 & 4 & 22 \\
\hline 1486 & 25070 & 25077 & 29 & 0.06 & 34.21 & 0.163163 & 0.104104 & 10 & 12003897 & 10 & 12991741 & 10 & 12 \\
\hline 600 & 35543 & 3322 & 33 & 0.06 & 34.28 & 0.179179 & 0.126126 & 3 & 122042998 & 3 & 122976843 & 3 & 122 \\
\hline 1978 & 26984 & 17883 & 15 & 0.06 & 34.34 & 0.088088 & 0.066066 & 14 & 55003669 & 14 & 55935436 & 14 & 55 \\
\hline 2305 & 44442 & 13063 & 27 & 0.06 & 34.4 & 0.154154 & 0.101101 & 16 & 70031425 & 16 & 70989867 & 16 & 70 \\
\hline 1291 & 53845 & 7318 & 25 & 0.06 & 34.47 & 0.124124 & 0.093093 & 8 & 120028517 & 8 & 120996107 & 8 & 120 \\
\hline 1864 & 26433 & 26436 & 20 & 0.06 & 34.53 & 0.085085 & 0.059059 & 13 & 160014224 & 13 & 160894746 & 13 & 160 \\
\hline 267 & 1671 & 1683 & 34 & 0.06 & 34.59 & 0.163163 & 0.107107 & 1 & 267026000 & 1 & 267994055 & 1 & 267 \\
\hline 229 & 40225 & 40227 & 27 & 0.06 & 34.66 & 0.144144 & 0.099099 & 1 & 229000633 & 1 & 229999129 & 1 & 229 \\
\hline 627 & 48680 & 3424 & 40 & 0.06 & 34.72 & 0.164164 & 0.109109 & 4 & 4001700 & 4 & 4957104 & 4 & 4 \\
\hline
\end{tabular}




\begin{tabular}{|c|c|c|c|c|c|c|c|c|c|c|c|c|c|}
\hline 1131 & 42052 & 6439 & 33 & 0.06 & 34.78 & 0.159159 & 0.112112 & 7 & 95010323 & 7 & 95983910 & 7 & 95 \\
\hline 2050 & 27380 & 47783 & 19 & 0.06 & 34.85 & 0.115115 & 0.092092 & 14 & 127001828 & 14 & 127981685 & 14 & 127 \\
\hline 1050 & 23338 & 5803 & 25 & 0.06 & 34.91 & 0.132132 & 0.087087 & 7 & 13023831 & 7 & 13906473 & 7 & 13 \\
\hline 1744 & 9757 & 30598 & 20 & 0.06 & 34.97 & 0.13013 & 0.081081 & 13 & 40050308 & 13 & 40905892 & 13 & 40 \\
\hline 426 & 60980 & 37178 & 27 & 0.06 & 35.03 & 0.141141 & 0.105105 & 2 & 111019898 & 2 & 111947482 & 2 & 111 \\
\hline 1089 & 60256 & 36441 & 35 & 0.06 & 35.1 & 0.15015 & 0.104104 & 7 & 52038431 & 7 & 52978945 & 7 & 52 \\
\hline 1535 & 8530 & 58456 & 37 & 0.06 & 35.16 & 0.172172 & 0.127127 & 10 & 61014593 & 10 & 61965891 & 10 & 61 \\
\hline 1667 & 9300 & 25792 & 32 & 0.06 & 35.22 & 0.157157 & 0.119119 & 12 & 27013251 & 12 & 27972588 & 12 & 27 \\
\hline 864 & 35055 & 33233 & 22 & 0.06 & 35.28 & 0.139139 & 0.088088 & 5 & 97022279 & 5 & 97980633 & 5 & 97 \\
\hline 2032 & 49505 & 11462 & 30 & 0.06 & 35.34 & 0.142142 & 0.103103 & 14 & 109016085 & 14 & 109988613 & 14 & 109 \\
\hline 2067 & 54405 & 52164 & 30 & 0.06 & 35.41 & 0.131131 & 0.081081 & 14 & 144001271 & 14 & 144927544 & 14 & 144 \\
\hline 90 & 45959 & 697 & 19 & 0.06 & 35.47 & 0.087087 & 0.061061 & 1 & 90085632 & 1 & 90991697 & 1 & 90 \\
\hline 872 & 41582 & 22893 & 32 & 0.06 & 35.53 & 0.156156 & 0.119119 & 5 & 105059227 & 5 & 105954521 & 5 & 105 \\
\hline 2068 & 58897 & 44040 & 27 & 0.06 & 35.59 & 0.124124 & 0.098098 & 14 & 145001826 & 14 & 145945750 & 14 & 145 \\
\hline 423 & 40633 & 55037 & 20 & 0.06 & 35.65 & 0.122122 & 0.096096 & 2 & 108005632 & 2 & 108954634 & 2 & 108 \\
\hline 26 & 205 & 53569 & 24 & 0.06 & 35.71 & 0.116116 & 0.084084 & 1 & 26091832 & 1 & 26995287 & 1 & 26 \\
\hline 2036 & 11487 & 11494 & 27 & 0.06 & 35.77 & 0.126126 & 0.098098 & 14 & 113018677 & 14 & 113973779 & 14 & 113 \\
\hline 2329 & 58631 & 18713 & 20 & 0.06 & 35.84 & 0.088088 & 0.068068 & 17 & 7027710 & 17 & 7923768 & 17 & 7 \\
\hline 697 & 35695 & 46681 & 25 & 0.06 & 35.9 & 0.135135 & 0.095095 & 4 & 74095578 & 4 & 74999752 & 4 & 74 \\
\hline 53 & 419 & 36621 & 25 & 0.06 & 35.96 & 0.126126 & 0.099099 & 1 & 53023914 & 1 & 53978051 & 1 & 53 \\
\hline 722 & 22214 & 22217 & 22 & 0.06 & 36.02 & 0.122122 & 0.094094 & 4 & 99012920 & 4 & 99808905 & 4 & 99 \\
\hline 2330 & 44499 & 28423 & 27 & 0.06 & 36.08 & 0.132132 & 0.094094 & 17 & 8048013 & 17 & 8987674 & 17 & 8 \\
\hline 2058 & 47805 & 11667 & 37 & 0.06 & 36.14 & 0.166166 & 0.104104 & 14 & 135016019 & 14 & 135991043 & 14 & 135 \\
\hline 1970 & 47623 & 57316 & 31 & 0.06 & 36.2 & 0.131131 & 0.1001 & 14 & 47010896 & 14 & 47976511 & 14 & 47 \\
\hline 2066 & 33925 & 47829 & 26 & 0.06 & 36.26 & 0.125125 & 0.09009 & 14 & 143000381 & 14 & 143979213 & 14 & 143 \\
\hline 2352 & 61285 & 50865 & 27 & 0.06 & 36.32 & 0.117117 & 0.084084 & 17 & 30009989 & 17 & 30994140 & 17 & 30 \\
\hline 1628 & 9093 & 43057 & 29 & 0.06 & 36.38 & 0.131131 & 0.088088 & 11 & 76044951 & 11 & 76999888 & 11 & 76 \\
\hline 1368 & 7693 & 7706 & 35 & 0.06 & 36.44 & 0.16016 & 0.117117 & 9 & 48020021 & 9 & 48972341 & 9 & 48 \\
\hline 1081 & 23527 & 23532 & 23 & 0.06 & 36.5 & 0.119119 & 0.075075 & 7 & 44021990 & 7 & 44974706 & 7 & 44 \\
\hline 775 & 48823 & 4626 & 31 & 0.06 & 36.56 & 0.151151 & 0.104104 & 5 & 8031569 & 5 & 8937125 & 5 & 8 \\
\hline 1133 & 23805 & 59484 & 27 & 0.06 & 36.62 & 0.122122 & 0.097097 & 7 & 97036575 & 7 & 97981809 & 7 & 97 \\
\hline 1762 & 9869 & 9874 & 28 & 0.06 & 36.68 & 0.146146 & 0.103103 & 13 & 58006732 & 13 & 58977959 & 13 & 58 \\
\hline 779 & 41393 & 22547 & 33 & 0.06 & 36.74 & 0.152152 & 0.101101 & 5 & 12002670 & 5 & 12992563 & 5 & 12 \\
\hline 2227 & 31461 & 31910 & 39 & 0.06 & 36.8 & 0.181181 & 0.119119 & 15 & 150020921 & 15 & 150963456 & 15 & 150 \\
\hline 1692 & 9416 & 43212 & 30 & 0.06 & 36.86 & 0.142142 & 0.096096 & 12 & 52006043 & 12 & 52989009 & 12 & 52 \\
\hline 889 & 15933 & 57273 & 34 & 0.06 & 36.92 & 0.148148 & 0.088088 & 6 & 10012272 & 6 & 10950015 & 6 & 10 \\
\hline 1018 & 59933 & 50769 & 24 & 0.06 & 36.97 & 0.117117 & 0.083083 & 6 & 139045500 & 6 & 139965340 & 6 & 139 \\
\hline 2353 & 13407 & 13417 & 31 & 0.06 & 37.03 & 0.147147 & 0.099099 & 17 & 31034493 & 17 & 31972942 & 17 & 31 \\
\hline 456 & 2711 & 31847 & 48 & 0.06 & 37.09 & 0.198198 & 0.118118 & 2 & 141030437 & 2 & 141983114 & 2 & 141 \\
\hline
\end{tabular}




\begin{tabular}{|c|c|c|c|c|c|c|c|c|c|c|c|c|c|}
\hline 2198 & 44240 & 47953 & 22 & 0.06 & 37.15 & 0.134134 & 0.083083 & 15 & 121000357 & 15 & 121778781 & 15 & 121 \\
\hline 2355 & 13423 & 49666 & 31 & 0.06 & 37.21 & 0.153153 & 0.115115 & 17 & 33009145 & 17 & 33993354 & 17 & 33 \\
\hline 1663 & 52557 & 55255 & 23 & 0.06 & 37.27 & 0.129129 & 0.081081 & 12 & 23012589 & 12 & 23998144 & 12 & 23 \\
\hline 2463 & 55031 & 60055 & 30 & 0.06 & 37.32 & 0.163163 & 0.113113 & 19 & 10005053 & 19 & 10911377 & 19 & 10 \\
\hline 644 & 3583 & 37400 & 38 & 0.06 & 37.38 & 0.152152 & 0.101101 & 4 & 21086107 & 4 & 21990523 & 4 & 21 \\
\hline 1714 & 43249 & 17565 & 16 & 0.06 & 37.44 & 0.099099 & 0.059059 & 13 & 10009490 & 13 & 10819124 & 13 & 10 \\
\hline 2387 & 61707 & 13664 & 29 & 0.06 & 37.5 & 0.156156 & 0.1001 & 17 & 65109674 & 17 & 65995368 & 17 & 65 \\
\hline 162 & 45571 & 51307 & 28 & 0.06 & 37.56 & 0.123123 & 0.076076 & 1 & 162006116 & 1 & 162976215 & 1 & 162 \\
\hline 1710 & 9490 & 9504 & 28 & 0.06 & 37.62 & 0.124124 & 0.079079 & 13 & 6000732 & 13 & 6978113 & 13 & 6 \\
\hline 2280 & 39448 & 28227 & 16 & 0.06 & 37.67 & 0.12012 & 0.093093 & 16 & 45027412 & 16 & 45829750 & 16 & 45 \\
\hline 1682 & 16358 & 50592 & 45 & 0.06 & 37.73 & 0.204204 & 0.13013 & 12 & 42035618 & 12 & 42984312 & 12 & 42 \\
\hline 2341 & 13304 & 44529 & 30 & 0.06 & 37.79 & 0.152152 & 0.099099 & 17 & 19005250 & 17 & 19978814 & 17 & 19 \\
\hline 116 & 40026 & 34229 & 20 & 0.06 & 37.85 & 0.091091 & 0.069069 & 1 & 116031370 & 1 & 116992830 & 1 & 116 \\
\hline 34 & 275 & 39857 & 39 & 0.06 & 37.9 & 0.161161 & 0.106106 & 1 & 34001467 & 1 & 34993536 & 1 & 34 \\
\hline 2088 & 39213 & 60898 & 14 & 0.06 & 37.96 & 0.084084 & 0.057057 & 15 & 11001978 & 15 & 11904651 & 15 & 11 \\
\hline 1029 & 15413 & 39742 & 24 & 0.06 & 38.02 & 0.107107 & 0.074074 & 6 & 150101096 & 6 & 150848334 & 6 & 150 \\
\hline 1439 & 55989 & 31056 & 15 & 0.06 & 38.08 & 0.096096 & 0.068068 & 9 & 119023630 & 9 & 119766208 & 9 & 119 \\
\hline 1490 & 8313 & 8318 & 26 & 0.06 & 38.13 & 0.146146 & 0.1001 & 10 & 16004340 & 10 & 16993029 & 10 & 16 \\
\hline 1625 & 14740 & 43042 & 38 & 0.06 & 38.19 & 0.163163 & 0.114114 & 11 & 73002067 & 11 & 73978500 & 11 & 73 \\
\hline 2381 & 28691 & 28701 & 27 & 0.06 & 38.25 & 0.14014 & 0.099099 & 17 & 59004882 & 17 & 59993508 & 17 & 59 \\
\hline 1915 & 60573 & 10541 & 30 & 0.06 & 38.31 & 0.143143 & 0.096096 & 13 & 211000701 & 13 & 211989807 & 13 & 211 \\
\hline 1505 & 51971 & 42795 & 21 & 0.06 & 38.36 & 0.116116 & 0.094094 & 10 & 31124017 & 10 & 31983675 & 10 & 31 \\
\hline 92 & 35188 & 710 & 24 & 0.06 & 38.42 & 0.132132 & 0.086086 & 1 & 92004937 & 1 & 92967007 & 1 & 92 \\
\hline 808 & 61891 & 37601 & 18 & 0.06 & 38.48 & 0.093093 & 0.073073 & 5 & 41151243 & 5 & 41831002 & 5 & 41 \\
\hline 987 & 60585 & 5492 & 19 & 0.06 & 38.53 & 0.097097 & 0.073073 & 6 & 108119233 & 6 & 108934597 & 6 & 108 \\
\hline 656 & 52078 & 3692 & 31 & 0.06 & 38.59 & 0.14014 & 0.08008 & 4 & 33016884 & 4 & 33955625 & 4 & 33 \\
\hline 1487 & 8294 & 34771 & 37 & 0.06 & 38.64 & 0.164164 & 0.113113 & 10 & 13042978 & 10 & 13958065 & 10 & 13 \\
\hline 1202 & 6931 & 37353 & 27 & 0.06 & 38.7 & 0.14014 & 0.1001 & 8 & 31034477 & 8 & 31972646 & 8 & 31 \\
\hline 1700 & 14464 & 14590 & 48 & 0.06 & 38.76 & 0.192192 & 0.138138 & 12 & 60002492 & 12 & 60982548 & 12 & 60 \\
\hline 612 & 15104 & 21645 & 27 & 0.06 & 38.81 & 0.14014 & 0.094094 & 3 & 134008198 & 3 & 134990636 & 3 & 134 \\
\hline 837 & 57141 & 22776 & 22 & 0.06 & 38.87 & 0.123123 & 0.09009 & 5 & 70001568 & 5 & 70984258 & 5 & 70 \\
\hline 1435 & 39645 & 7974 & 16 & 0.06 & 38.92 & 0.077077 & 0.047047 & 9 & 115076491 & 9 & 115974419 & 9 & 115 \\
\hline 1707 & 49399 & 16338 & 31 & 0.06 & 38.98 & 0.135135 & 0.099099 & 13 & 3072331 & 13 & 3982402 & 13 & 3 \\
\hline 470 & 33029 & 32699 & 39 & 0.06 & 39.04 & 0.187187 & 0.128128 & 2 & 155011592 & 2 & 155939920 & 2 & 155 \\
\hline 2080 & 14524 & 27558 & 27 & 0.06 & 39.09 & 0.139139 & 0.091091 & 15 & 3017242 & 15 & 3985728 & 15 & 3 \\
\hline 2086 & 27574 & 11858 & 23 & 0.06 & 39.15 & 0.125125 & 0.096096 & 15 & 9026506 & 15 & 9997960 & 15 & 9 \\
\hline 357 & 2202 & 2225 & 25 & 0.06 & 39.2 & 0.146146 & 0.101101 & 2 & 41011761 & 2 & 41998239 & 2 & 41 \\
\hline 683 & 3866 & 3882 & 18 & 0.06 & 39.26 & 0.09009 & 0.062062 & 4 & 60030169 & 4 & 60899193 & 4 & 60 \\
\hline 2373 & 16275 & 32952 & 32 & 0.06 & 39.31 & 0.161161 & 0.111111 & 17 & 51005799 & 17 & 51974554 & 17 & 51 \\
\hline
\end{tabular}




\begin{tabular}{|c|c|c|c|c|c|c|c|c|c|c|c|c|c|}
\hline 377 & 36445 & 2314 & 5 & 0.06 & 39.37 & 0.043043 & 0.037037 & 2 & 61161245 & 2 & 61822605 & 2 & 61 \\
\hline 2038 & 11499 & 18041 & 18 & 0.06 & 39.42 & 0.098098 & 0.081081 & 14 & 115013554 & 14 & 115960354 & 14 & 115 \\
\hline 1787 & 53061 & 31248 & 28 & 0.06 & 39.48 & 0.122122 & 0.087087 & 13 & 83003977 & 13 & 83965641 & 13 & 83 \\
\hline 1765 & 9894 & 30145 & 23 & 0.05 & 39.53 & 0.108108 & 0.087087 & 13 & 61014675 & 13 & 61982576 & 13 & 61 \\
\hline 963 & 29681 & 14395 & 11 & 0.05 & 39.59 & 0.062062 & 0.049049 & 6 & 84010187 & 6 & 84927021 & 6 & 84 \\
\hline 266 & 1657 & 1670 & 30 & 0.05 & 39.64 & 0.116116 & 0.084084 & 1 & 266007391 & 1 & 266974660 & 1 & 266 \\
\hline 1143 & 16438 & 18349 & 22 & 0.05 & 39.7 & 0.109109 & 0.067067 & 7 & 107177261 & 7 & 107995062 & 7 & 107 \\
\hline 2244 & 28095 & 44324 & 19 & 0.05 & 39.75 & 0.1001 & 0.069069 & 16 & 9060044 & 16 & 9999843 & 16 & 9 \\
\hline 1732 & 38724 & 9683 & 27 & 0.05 & 39.81 & 0.127127 & 0.095095 & 13 & 28078702 & 13 & 28974982 & 13 & 28 \\
\hline 1860 & 32613 & 26418 & 16 & 0.05 & 39.86 & 0.086086 & 0.062062 & 13 & 156202355 & 13 & 156983040 & 13 & 156 \\
\hline 2331 & 32511 & 58474 & 25 & 0.05 & 39.92 & 0.138138 & 0.096096 & 17 & 9014186 & 17 & 9992606 & 17 & 9 \\
\hline 2414 & 28844 & 39544 & 26 & 0.05 & 39.97 & 0.115115 & 0.076076 & 18 & 22043702 & 18 & 22991003 & 18 & 22 \\
\hline 744 & 22346 & 41275 & 34 & 0.05 & 40.03 & 0.16016 & 0.103103 & 4 & 121010319 & 4 & 121989854 & 4 & 121 \\
\hline 2308 & 28320 & 49641 & 27 & 0.05 & 40.08 & 0.139139 & 0.093093 & 16 & 73039198 & 16 & 73996806 & 16 & 73 \\
\hline 634 & 56887 & 52761 & 30 & 0.05 & 40.14 & 0.139139 & 0.097097 & 4 & 11002675 & 4 & 11982774 & 4 & 11 \\
\hline 415 & 40621 & 40624 & 18 & 0.05 & 40.19 & 0.09009 & 0.056056 & 2 & 100248260 & 2 & 100951727 & 2 & 100 \\
\hline 1888 & 34357 & 60318 & 16 & 0.05 & 40.24 & 0.08008 & 0.062062 & 13 & 184018627 & 13 & 184921505 & 13 & 184 \\
\hline 1396 & 24759 & 7829 & 19 & 0.05 & 40.3 & 0.092092 & 0.07007 & 9 & 76041058 & 9 & 76981023 & 9 & 76 \\
\hline 1822 & 30134 & 26325 & 15 & 0.05 & 40.35 & 0.093093 & 0.072072 & 13 & 118012221 & 13 & 118998431 & 13 & 118 \\
\hline 896 & 17827 & 36070 & 34 & 0.05 & 40.41 & 0.165165 & 0.114114 & 6 & 17189599 & 6 & 17980080 & 6 & 17 \\
\hline 729 & 22255 & 50937 & 29 & 0.05 & 40.46 & 0.142142 & 0.108108 & 4 & 106018138 & 4 & 106907287 & 4 & 106 \\
\hline 1453 & 51398 & 8072 & 22 & 0.05 & 40.51 & 0.118118 & 0.082082 & 9 & 133007769 & 9 & 133969269 & 9 & 133 \\
\hline 1166 & 23998 & 42180 & 25 & 0.05 & 40.57 & 0.12012 & 0.093093 & 7 & 130031038 & 7 & 130982643 & 7 & 130 \\
\hline 1760 & 47450 & 9861 & 26 & 0.05 & 40.62 & 0.114114 & 0.084084 & 13 & 56018886 & 13 & 56933573 & 13 & 56 \\
\hline 1653 & 25745 & 45545 & 33 & 0.05 & 40.67 & 0.132132 & 0.091091 & 12 & 13007194 & 12 & 13901807 & 12 & 13 \\
\hline 1183 & 14727 & 38092 & 24 & 0.05 & 40.73 & 0.123123 & 0.083083 & 8 & 12144490 & 8 & 12984077 & 8 & 12 \\
\hline 2401 & 28788 & 44733 & 21 & 0.05 & 40.78 & 0.099099 & 0.073073 & 18 & 9010445 & 18 & 9979869 & 18 & 9 \\
\hline 1091 & 6132 & 45578 & 37 & 0.05 & 40.83 & 0.143143 & 0.097097 & 7 & 54010631 & 7 & 54997566 & 7 & 54 \\
\hline 101 & 767 & 45985 & 26 & 0.05 & 40.89 & 0.101101 & 0.069069 & 1 & 101018800 & 1 & 101977893 & 1 & 101 \\
\hline 2027 & 27255 & 11416 & 30 & 0.05 & 40.94 & 0.133133 & 0.09009 & 14 & 104029253 & 14 & 104954378 & 14 & 104 \\
\hline 2221 & 50825 & 39364 & 35 & 0.05 & 40.99 & 0.156156 & 0.096096 & 15 & 144002309 & 15 & 144989420 & 15 & 144 \\
\hline 1509 & 17080 & 54705 & 14 & 0.05 & 41.05 & 0.095095 & 0.068068 & 10 & 35068814 & 10 & 35912867 & 10 & 35 \\
\hline 1738 & 18534 & 26069 & 26 & 0.05 & 41.1 & 0.095095 & 0.074074 & 13 & 34112306 & 13 & 34993798 & 13 & 34 \\
\hline 1019 & 5627 & 5634 & 29 & 0.05 & 41.15 & 0.134134 & 0.103103 & 6 & 140009596 & 6 & 140829602 & 6 & 140 \\
\hline 1887 & 38920 & 10393 & 13 & 0.05 & 41.21 & 0.092092 & 0.07007 & 13 & 183007199 & 13 & 183927662 & 13 & 183 \\
\hline 279 & 37018 & 58710 & 34 & 0.05 & 41.26 & 0.151151 & 0.087087 & 1 & 279014971 & 1 & 279972181 & 1 & 279 \\
\hline 1659 & 25772 & 53295 & 25 & 0.05 & 41.31 & 0.129129 & 0.095095 & 12 & 19195079 & 12 & 19884388 & 12 & 19 \\
\hline 528 & 55209 & 34822 & 21 & 0.05 & 41.36 & 0.116116 & 0.078078 & 3 & 50118060 & 3 & 50856818 & 3 & 50 \\
\hline 1629 & 25626 & 25633 & 36 & 0.05 & 41.42 & 0.165165 & 0.1001 & 11 & 77015017 & 11 & 77964900 & 11 & 77 \\
\hline
\end{tabular}




\begin{tabular}{|c|c|c|c|c|c|c|c|c|c|c|c|c|c|}
\hline 2001 & 11332 & 27119 & 30 & 0.05 & 41.47 & 0.131131 & 0.097097 & 14 & 78011535 & 14 & 78995471 & 14 & 78 \\
\hline 1114 & 6289 & 38007 & 10 & 0.05 & 41.52 & 0.059059 & 0.048048 & 7 & 77009237 & 7 & 77973037 & 7 & 77 \\
\hline 416 & 17209 & 58078 & 20 & 0.05 & 41.57 & 0.126126 & 0.089089 & 2 & 101065109 & 2 & 101995816 & 2 & 101 \\
\hline 2139 & 30906 & 12175 & 26 & 0.05 & 41.63 & 0.117117 & 0.072072 & 15 & 62033445 & 15 & 62990144 & 15 & 62 \\
\hline 1620 & 9035 & 25582 & 34 & 0.05 & 41.68 & 0.152152 & 0.103103 & 11 & 68089906 & 11 & 68982930 & 11 & 68 \\
\hline 907 & 32847 & 60920 & 17 & 0.05 & 41.73 & 0.095095 & 0.063063 & 6 & 28054235 & 6 & 28694089 & 6 & 28 \\
\hline 2053 & 61369 & 27393 & 24 & 0.05 & 41.78 & 0.122122 & 0.082082 & 14 & 130079124 & 14 & 130962219 & 14 & 130 \\
\hline 1069 & 5937 & 41868 & 30 & 0.05 & 41.84 & 0.132132 & 0.096096 & 7 & 32016844 & 7 & 32997273 & 7 & 32 \\
\hline 449 & 34248 & 31355 & 24 & 0.05 & 41.89 & 0.118118 & 0.083083 & 2 & 134056711 & 2 & 134992155 & 2 & 134 \\
\hline 2262 & 39411 & 49620 & 25 & 0.05 & 41.94 & 0.114114 & 0.082082 & 16 & 27017719 & 16 & 27972337 & 16 & 27 \\
\hline 839 & 22778 & 4985 & 22 & 0.05 & 41.99 & 0.108108 & 0.071071 & 5 & 72009882 & 5 & 72971559 & 5 & 72 \\
\hline 1420 & 45102 & 31415 & 34 & 0.05 & 42.04 & 0.158158 & 0.104104 & 9 & 100019532 & 9 & 100985297 & 9 & 100 \\
\hline 1975 & 47635 & 36432 & 20 & 0.05 & 42.1 & 0.116116 & 0.077077 & 14 & 52003103 & 14 & 52994112 & 14 & 52 \\
\hline 4 & 20 & 48373 & 30 & 0.05 & 42.15 & 0.13013 & 0.089089 & 1 & 4022389 & 1 & 4979417 & 1 & 4 \\
\hline 11 & 45674 & 39779 & 30 & 0.05 & 42.2 & 0.151151 & 0.105105 & 1 & 11012998 & 1 & 11945736 & 1 & 11 \\
\hline 2266 & 12822 & 12829 & 23 & 0.05 & 42.25 & 0.091091 & 0.061061 & 16 & 31016837 & 16 & 31983325 & 16 & 31 \\
\hline 1186 & 56317 & 24076 & 32 & 0.05 & 42.3 & 0.153153 & 0.103103 & 8 & 15012662 & 8 & 15992070 & 8 & 15 \\
\hline 633 & 3477 & 37374 & 27 & 0.05 & 42.35 & 0.117117 & 0.069069 & 4 & 10016061 & 4 & 10835685 & 4 & 10 \\
\hline 406 & 37135 & 40611 & 22 & 0.05 & 42.4 & 0.107107 & 0.084084 & 2 & 91003962 & 2 & 91992594 & 2 & 91 \\
\hline 947 & 59771 & 23021 & 35 & 0.05 & 42.46 & 0.135135 & 0.085085 & 6 & 68066489 & 6 & 68993837 & 6 & 68 \\
\hline 489 & 52392 & 2817 & 36 & 0.05 & 42.51 & 0.163163 & 0.104104 & 3 & 11054114 & 3 & 11960567 & 3 & 11 \\
\hline 490 & 62076 & 51843 & 27 & 0.05 & 42.56 & 0.156156 & 0.105105 & 3 & 12001168 & 3 & 12944285 & 3 & 12 \\
\hline 1543 & 25307 & 49263 & 44 & 0.05 & 42.61 & 0.184184 & 0.109109 & 10 & 69008968 & 10 & 69985989 & 10 & 69 \\
\hline 655 & 3670 & 37429 & 19 & 0.05 & 42.66 & 0.097097 & 0.073073 & 4 & 32034432 & 4 & 32960684 & 4 & 32 \\
\hline 998 & 35291 & 15499 & 34 & 0.05 & 42.71 & 0.153153 & 0.087087 & 6 & 119079398 & 6 & 119994720 & 6 & 119 \\
\hline 1113 & 6285 & 38001 & 9 & 0.05 & 42.76 & 0.064064 & 0.049049 & 7 & 76111756 & 7 & 76907606 & 7 & 76 \\
\hline 1173 & 6764 & 16676 & 23 & 0.05 & 42.81 & 0.103103 & 0.075075 & 8 & 2023121 & 8 & 2902485 & 8 & 2 \\
\hline 1718 & 14782 & 9567 & 38 & 0.05 & 42.86 & 0.177177 & 0.114114 & 13 & 14054487 & 13 & 14975251 & 13 & 14 \\
\hline 865 & 33611 & 22870 & 21 & 0.05 & 42.91 & 0.109109 & 0.079079 & 5 & 98025183 & 5 & 98986619 & 5 & 98 \\
\hline 120 & 20034 & 52208 & 25 & 0.05 & 42.96 & 0.12012 & 0.085085 & 1 & 120136077 & 1 & 120994029 & 1 & 120 \\
\hline 1730 & 43283 & 54454 & 34 & 0.05 & 43.01 & 0.163163 & 0.083083 & 13 & 26001164 & 13 & 26988390 & 13 & 26 \\
\hline 1111 & 37986 & 37989 & 17 & 0.05 & 43.06 & 0.096096 & 0.07007 & 7 & 74030057 & 7 & 74980796 & 7 & 74 \\
\hline 2129 & 12115 & 12118 & 26 & 0.05 & 43.11 & 0.126126 & 0.087087 & 15 & 52006633 & 15 & 52955798 & 15 & 52 \\
\hline 2091 & 27608 & 11902 & 17 & 0.05 & 43.16 & 0.102102 & 0.072072 & 15 & 14019896 & 15 & 14940640 & 15 & 14 \\
\hline 358 & 2224 & 2235 & 36 & 0.05 & 43.21 & 0.173173 & 0.11011 & 2 & 42026841 & 2 & 42989311 & 2 & 42 \\
\hline 1109 & 37972 & 47075 & 16 & 0.05 & 43.26 & 0.073073 & 0.054054 & 7 & 72026564 & 7 & 72991035 & 7 & 72 \\
\hline 1078 & 6014 & 6023 & 28 & 0.05 & 43.31 & 0.144144 & 0.098098 & 7 & 41004531 & 7 & 41984352 & 7 & 41 \\
\hline 1684 & 43190 & 9384 & 21 & 0.05 & 43.36 & 0.1001 & 0.076076 & 12 & 44080140 & 12 & 44971304 & 12 & 44 \\
\hline 1125 & 6369 & 47120 & 34 & 0.05 & 43.41 & 0.147147 & 0.098098 & 7 & 89014128 & 7 & 89999425 & 7 & 89 \\
\hline
\end{tabular}




\begin{tabular}{|c|c|c|c|c|c|c|c|c|c|c|c|c|c|}
\hline 1476 & 25011 & 8213 & 31 & 0.05 & 43.46 & 0.15015 & 0.104104 & 10 & 2015307 & 10 & 2870481 & 10 & 2 \\
\hline 785 & 51000 & 22588 & 33 & 0.05 & 43.51 & 0.142142 & 0.108108 & 5 & 18012619 & 5 & 18992892 & 5 & 18 \\
\hline 1937 & 57122 & 26708 & 29 & 0.05 & 43.56 & 0.138138 & 0.101101 & 14 & 14005194 & 14 & 14998570 & 14 & 14 \\
\hline 1386 & 24725 & 54763 & 34 & 0.05 & 43.61 & 0.141141 & 0.09009 & 9 & 66001271 & 9 & 66941538 & 9 & 66 \\
\hline 1478 & 25018 & 25027 & 27 & 0.05 & 43.66 & 0.14014 & 0.101101 & 10 & 4010500 & 10 & 4939665 & 10 & 4 \\
\hline 1723 & 25991 & 34226 & 29 & 0.05 & 43.71 & 0.147147 & 0.09009 & 13 & 19066947 & 13 & 19932514 & 13 & 19 \\
\hline 123 & 46027 & 40044 & 13 & 0.05 & 43.76 & 0.082082 & 0.062062 & 1 & 123220315 & 1 & 123968294 & 1 & 123 \\
\hline 795 & 4753 & 4757 & 16 & 0.05 & 43.81 & 0.083083 & 0.063063 & 5 & 28124798 & 5 & 28898534 & 5 & 28 \\
\hline 1369 & 24652 & 33877 & 29 & 0.05 & 43.86 & 0.129129 & 0.092092 & 9 & 49030981 & 9 & 49997890 & 9 & 49 \\
\hline 1320 & 51548 & 50018 & 36 & 0.05 & 43.91 & 0.159159 & 0.117117 & 9 & 21708 & 9 & 974310 & 9 & 0 \\
\hline 1244 & 58287 & 7095 & 20 & 0.05 & 43.96 & 0.086086 & 0.067067 & 8 & 73036634 & 8 & 73926862 & 8 & 73 \\
\hline 1156 & 57055 & 6668 & 28 & 0.05 & 44.01 & 0.141141 & 0.114114 & 7 & 120016852 & 7 & 120936305 & 7 & 120 \\
\hline 1920 & 17160 & 29629 & 15 & 0.05 & 44.06 & 0.083083 & 0.052052 & 13 & 216046101 & 13 & 216721489 & 13 & 216 \\
\hline 1866 & 43502 & 58439 & 13 & 0.05 & 44.11 & 0.083083 & 0.065065 & 13 & 162011185 & 13 & 162889030 & 13 & 162 \\
\hline 1909 & 16239 & 45642 & 22 & 0.05 & 44.16 & 0.113113 & 0.09009 & 13 & 205022285 & 13 & 205872598 & 13 & 205 \\
\hline 929 & 45764 & 45793 & 21 & 0.05 & 44.21 & 0.104104 & 0.064064 & 6 & 50006716 & 6 & 50922233 & 6 & 50 \\
\hline 1811 & 53091 & 52772 & 22 & 0.05 & 44.25 & 0.086086 & 0.06006 & 13 & 107023094 & 13 & 107980182 & 13 & 107 \\
\hline 1994 & 27085 & 47659 & 22 & 0.05 & 44.3 & 0.113113 & 0.078078 & 14 & 71155797 & 14 & 71975247 & 14 & 71 \\
\hline 1348 & 32410 & 59968 & 43 & 0.05 & 44.35 & 0.177177 & 0.119119 & 9 & 28035750 & 9 & 28998609 & 9 & 28 \\
\hline 949 & 18988 & 41654 & 34 & 0.05 & 44.4 & 0.135135 & 0.093093 & 6 & 70023840 & 6 & 70996798 & 6 & 70 \\
\hline 2085 & 15676 & 27573 & 21 & 0.05 & 44.45 & 0.117117 & 0.087087 & 15 & 8023920 & 15 & 8907355 & 15 & 8 \\
\hline 662 & 21934 & 3741 & 23 & 0.05 & 44.5 & 0.102102 & 0.068068 & 4 & 39025906 & 4 & 39998067 & 4 & 39 \\
\hline 525 & 59494 & 2950 & 16 & 0.05 & 44.55 & 0.077077 & 0.065065 & 3 & 47040078 & 3 & 47979489 & 3 & 47 \\
\hline 1462 & 45541 & 42701 & 28 & 0.05 & 44.6 & 0.132132 & 0.089089 & 9 & 142004595 & 9 & 142950929 & 9 & 142 \\
\hline 2249 & 12721 & 30312 & 18 & 0.05 & 44.65 & 0.093093 & 0.068068 & 16 & 14013912 & 16 & 14823848 & 16 & 14 \\
\hline 432 & 2541 & 2549 & 27 & 0.05 & 44.69 & 0.108108 & 0.08008 & 2 & 117038311 & 2 & 117962577 & 2 & 117 \\
\hline 134 & 20070 & 46053 & 12 & 0.05 & 44.74 & 0.075075 & 0.049049 & 1 & 134037038 & 1 & 134796719 & 1 & 134 \\
\hline 1594 & 14540 & 30172 & 13 & 0.05 & 44.79 & 0.072072 & 0.052052 & 11 & 42043634 & 11 & 42953643 & 11 & 42 \\
\hline 400 & 37125 & 40589 & 30 & 0.05 & 44.84 & 0.124124 & 0.087087 & 2 & 85036318 & 2 & 85956326 & 2 & 85 \\
\hline 2402 & 44734 & 56864 & 24 & 0.05 & 44.89 & 0.109109 & 0.086086 & 18 & 10016629 & 18 & 10868283 & 18 & 10 \\
\hline 1365 & 42507 & 49821 & 34 & 0.05 & 44.94 & 0.134134 & 0.093093 & 9 & 45011963 & 9 & 45974573 & 9 & 45 \\
\hline 1005 & 23147 & 58753 & 27 & 0.05 & 44.98 & 0.117117 & 0.082082 & 6 & 126023550 & 6 & 126991726 & 6 & 126 \\
\hline 1188 & 29688 & 15068 & 25 & 0.05 & 45.03 & 0.133133 & 0.098098 & 8 & 17019937 & 8 & 17992352 & 8 & 17 \\
\hline 959 & 34180 & 19311 & 31 & 0.05 & 45.08 & 0.128128 & 0.089089 & 6 & 80008558 & 6 & 80979535 & 6 & 80 \\
\hline 1454 & 8073 & 42675 & 14 & 0.05 & 45.13 & 0.076076 & 0.062062 & 9 & 134004914 & 9 & 134966767 & 9 & 134 \\
\hline 1460 & 38393 & 32548 & 24 & 0.05 & 45.17 & 0.108108 & 0.075075 & 9 & 140003794 & 9 & 140971189 & 9 & 140 \\
\hline 408 & 15455 & 37141 & 28 & 0.05 & 45.22 & 0.108108 & 0.076076 & 2 & 93152911 & 2 & 93992622 & 2 & 93 \\
\hline 1438 & 34121 & 7990 & 25 & 0.05 & 45.27 & 0.109109 & 0.079079 & 9 & 118021130 & 9 & 118989834 & 9 & 118 \\
\hline 465 & 17098 & 31420 & 40 & 0.05 & 45.32 & 0.158158 & 0.107107 & 2 & 150000460 & 2 & 150931547 & 2 & 150 \\
\hline
\end{tabular}




\begin{tabular}{|c|c|c|c|c|c|c|c|c|c|c|c|c|}
\hline 35 & 39858 & 54559 & 27 & 0.05 & 45.37 & 0.137137 & 0.096096 & 1 & 35046144 & 1 & 35987086 & 1 \\
\hline 2248 & 12717 & 39394 & 13 & 0.05 & 45.41 & 0.089089 & 0.066066 & 16 & 13007287 & 16 & 13970080 & 16 \\
\hline 2166 & 12298 & 39291 & 26 & 0.05 & 45.46 & 0.142142 & 0.086086 & 15 & 89097103 & 15 & 89953222 & 15 \\
\hline 2087 & 39210 & 27582 & 23 & 0.05 & 45.51 & 0.104104 & 0.078078 & 15 & 10038766 & 15 & 10975125 & 15 \\
\hline 1871 & 26454 & 16773 & 18 & 0.05 & 45.55 & 0.098098 & 0.067067 & 13 & 167022760 & 13 & 167997645 & 13 \\
\hline 32 & 36536 & 19635 & 36 & 0.05 & 45.6 & 0.167167 & 0.121121 & 1 & 32021937 & 1 & 32981735 & 1 \\
\hline 968 & 35273 & 58618 & 20 & 0.05 & 45.65 & 0.107107 & 0.076076 & 6 & 89123167 & 6 & 89986075 & 6 \\
\hline 1134 & 6461 & 56328 & 36 & 0.05 & 45.7 & 0.156156 & 0.113113 & 7 & 98003978 & 7 & 98998276 & 7 \\
\hline 2375 & 57872 & 13567 & 26 & 0.05 & 45.74 & 0.12012 & 0.074074 & 17 & 53166870 & 17 & 53996435 & 17 \\
\hline 1310 & 38197 & 39652 & 36 & 0.05 & 45.79 & 0.141141 & 0.094094 & 8 & 139013496 & 8 & 139970751 & 8 \\
\hline 17 & 53439 & 146 & 42 & 0.05 & 45.84 & 0.175175 & 0.104104 & 1 & 17004757 & 1 & 17991862 & 1 \\
\hline 360 & 20868 & 2246 & 38 & 0.05 & 45.89 & 0.144144 & 0.096096 & 2 & 44033531 & 2 & 44984555 & 2 \\
\hline 1914 & 47545 & 10531 & 26 & 0.05 & 45.93 & 0.128128 & 0.093093 & 13 & 210026312 & 13 & 210866603 & 13 \\
\hline 1632 & 25641 & 9142 & 31 & 0.05 & 45.98 & 0.136136 & 0.092092 & 11 & 80007010 & 11 & 80996526 & 11 \\
\hline 467 & 2756 & 2765 & 26 & 0.05 & 46.03 & 0.133133 & 0.091091 & 2 & 152016160 & 2 & 152972957 & 2 \\
\hline 1776 & 56340 & 9962 & 21 & 0.05 & 46.07 & 0.089089 & 0.065065 & 13 & 72033973 & 13 & 72975884 & 13 \\
\hline 344 & 19353 & 48275 & 39 & 0.05 & 46.12 & 0.166166 & 0.109109 & 2 & 28013337 & 2 & 28977392 & 2 \\
\hline 1566 & 58185 & 8686 & 21 & 0.05 & 46.17 & 0.115115 & 0.071071 & 11 & 14013915 & 11 & 14978831 & 11 \\
\hline 2458 & 14037 & 59417 & 36 & 0.05 & 46.22 & 0.162162 & 0.108108 & 19 & 5061093 & 19 & 5997290 & 19 \\
\hline 2433 & 28934 & 36288 & 23 & 0.05 & 46.26 & 0.102102 & 0.069069 & 18 & 41063892 & 18 & 41968802 & 18 \\
\hline 2389 & 44718 & 28759 & 31 & 0.05 & 46.31 & 0.146146 & 0.097097 & 17 & 67014254 & 17 & 67991230 & 17 \\
\hline 434 & 40657 & 2564 & 22 & 0.05 & 46.36 & 0.112112 & 0.075075 & 2 & 119044783 & 2 & 119970583 & 2 \\
\hline 870 & 5174 & 14894 & 24 & 0.05 & 46.4 & 0.119119 & 0.085085 & 5 & 103013980 & 5 & 103936081 & 5 \\
\hline 2454 & 29637 & 44851 & 26 & 0.05 & 46.45 & 0.132132 & 0.088088 & 19 & 1141455 & 19 & 1968265 & 19 \\
\hline 2108 & 34926 & 19281 & 31 & 0.05 & 46.5 & 0.134134 & 0.096096 & 15 & 31033635 & 15 & 31995248 & 15 \\
\hline 883 & 17685 & 33718 & 37 & 0.05 & 46.54 & 0.17017 & 0.101101 & 6 & 4060304 & 6 & 4871179 & 6 \\
\hline 12 & 88 & 36478 & 29 & 0.05 & 46.59 & 0.134134 & 0.084084 & 1 & 12024877 & 1 & 12946299 & 1 \\
\hline 1489 & 36031 & 42771 & 26 & 0.05 & 46.63 & 0.115115 & 0.079079 & 10 & 15042704 & 10 & 15992149 & 10 \\
\hline 424 & 35153 & 21019 & 21 & 0.05 & 46.68 & 0.11011 & 0.081081 & 2 & 109012528 & 2 & 109934488 & 2 \\
\hline 1209 & 38133 & 6984 & 19 & 0.05 & 46.73 & 0.1001 & 0.072072 & 8 & 38293338 & 8 & 38972975 & 8 \\
\hline 2077 & 31605 & 11886 & 23 & 0.05 & 46.77 & 0.122122 & 0.082082 & 15 & 4535 & 15 & 993283 & 15 \\
\hline 1042 & 41768 & 5734 & 31 & 0.05 & 46.82 & 0.121121 & 0.084084 & 7 & 5020698 & 7 & 5975746 & 7 \\
\hline 244 & 1531 & 58547 & 12 & 0.05 & 46.87 & 0.066066 & 0.049049 & 1 & 244008725 & 1 & 244996464 & 1 \\
\hline 399 & 20929 & 37124 & 19 & 0.05 & 46.91 & 0.095095 & 0.068068 & 2 & 84026553 & 2 & 84987807 & 2 \\
\hline 969 & 49991 & 16685 & 17 & 0.05 & 46.96 & 0.103103 & 0.078078 & 6 & 90124294 & 6 & 90920481 & 6 \\
\hline 1315 & 16475 & 16051 & 29 & 0.05 & 47 & 0.13013 & 0.086086 & 8 & 144034145 & 8 & 144999383 & 8 \\
\hline 1246 & 52937 & 7109 & 26 & 0.05 & 47.05 & 0.113113 & 0.084084 & 8 & 75063893 & 8 & 75981660 & 8 \\
\hline 581 & 21499 & 55369 & 17 & 0.05 & 47.1 & 0.084084 & 0.066066 & 3 & 103029683 & 3 & 103973714 & 3 \\
\hline 1314 & 7432 & 34330 & 34 & 0.05 & 47.14 & 0.159159 & 0.104104 & 8 & 143011607 & 8 & 143998135 & 8 \\
\hline
\end{tabular}




\begin{tabular}{|c|c|c|c|c|c|c|c|c|c|c|c|c|c|}
\hline 1898 & 15621 & 38950 & 20 & 0.05 & 47.19 & 0.111111 & 0.077077 & 13 & 194099336 & 13 & 194986995 & 13 & 194 \\
\hline 1626 & 43043 & 43047 & 33 & 0.05 & 47.23 & 0.131131 & 0.089089 & 11 & 74019999 & 11 & 74978970 & 11 & 74 \\
\hline 1212 & 45666 & 6997 & 25 & 0.05 & 47.28 & 0.125125 & 0.082082 & 8 & 41047757 & 8 & 41970562 & 8 & 41 \\
\hline 1174 & 31570 & 24030 & 26 & 0.05 & 47.32 & 0.141141 & 0.113113 & 8 & 3027824 & 8 & 3982853 & 8 & 3 \\
\hline 1057 & 5848 & 41835 & 37 & 0.05 & 47.37 & 0.173173 & 0.113113 & 7 & 20072507 & 7 & 20985785 & 7 & 20 \\
\hline 771 & 48814 & 58654 & 24 & 0.05 & 47.42 & 0.107107 & 0.071071 & 5 & 4092291 & 5 & 4916323 & 5 & 4 \\
\hline 1142 & 6524 & 6538 & 29 & 0.05 & 47.46 & 0.132132 & 0.083083 & 7 & 106002380 & 7 & 106937807 & 7 & 106 \\
\hline 282 & 20526 & 52816 & 32 & 0.05 & 47.51 & 0.156156 & 0.097097 & 1 & 282001963 & 1 & 282998551 & 1 & 282 \\
\hline 2362 & 13473 & 44612 & 20 & 0.05 & 47.55 & 0.11011 & 0.081081 & 17 & 40021954 & 17 & 40996438 & 17 & 40 \\
\hline 1255 & 33058 & 7148 & 22 & 0.05 & 47.6 & 0.095095 & 0.06006 & 8 & 84029728 & 8 & 84978671 & 8 & 84 \\
\hline 498 & 31622 & 21277 & 32 & 0.05 & 47.65 & 0.137137 & 0.079079 & 3 & 20058173 & 3 & 20983778 & 3 & 20 \\
\hline 2259 & 30054 & 44360 & 28 & 0.05 & 47.69 & 0.135135 & 0.093093 & 16 & 24096772 & 16 & 24940106 & 16 & 24 \\
\hline 757 & 41307 & 58188 & 32 & 0.05 & 47.74 & 0.134134 & 0.088088 & 4 & 134029125 & 4 & 134976773 & 4 & 134 \\
\hline 1892 & 33188 & 38939 & 26 & 0.05 & 47.78 & 0.113113 & 0.081081 & 13 & 188095940 & 13 & 188953021 & 13 & 188 \\
\hline 1644 & 43107 & 9196 & 20 & 0.05 & 47.83 & 0.103103 & 0.077077 & 12 & 4010569 & 12 & 4992763 & 12 & 4 \\
\hline 105 & 50887 & 806 & 31 & 0.05 & 47.87 & 0.124124 & 0.08008 & 1 & 105018683 & 1 & 105985626 & 1 & 105 \\
\hline 557 & 30375 & 40849 & 20 & 0.05 & 47.92 & 0.109109 & 0.078078 & 3 & 79053106 & 3 & 79981209 & 3 & 79 \\
\hline 617 & 58200 & 9423 & 21 & 0.05 & 47.96 & 0.102102 & 0.072072 & 3 & 139055473 & 3 & 139956551 & 3 & 139 \\
\hline 706 & 46700 & 22141 & 24 & 0.05 & 48.01 & 0.106106 & 0.073073 & 4 & 83025851 & 4 & 83846382 & 4 & 83 \\
\hline 2265 & 12813 & 16826 & 23 & 0.05 & 48.05 & 0.119119 & 0.078078 & 16 & 30081210 & 16 & 30976457 & 16 & 30 \\
\hline 898 & 22948 & 5282 & 22 & 0.05 & 48.1 & 0.106106 & 0.081081 & 6 & 19002312 & 6 & 19952347 & 6 & 19 \\
\hline 1924 & 43599 & 55048 & 23 & 0.05 & 48.14 & 0.113113 & 0.069069 & 14 & 1000181 & 14 & 1929126 & 14 & 1 \\
\hline 2064 & 39191 & 27474 & 38 & 0.05 & 48.19 & 0.177177 & 0.111111 & 14 & 141004641 & 14 & 141996996 & 14 & 141 \\
\hline 975 & 32278 & 34060 & 12 & 0.05 & 48.23 & 0.066066 & 0.045045 & 6 & 96057833 & 6 & 96764659 & 6 & 96 \\
\hline 6 & 41 & 15913 & 32 & 0.05 & 48.28 & 0.137137 & 0.1001 & 1 & 6010365 & 1 & 6986608 & 1 & 6 \\
\hline 2000 & 43829 & 11331 & 17 & 0.05 & 48.32 & 0.096096 & 0.072072 & 14 & 77013888 & 14 & 77974347 & 14 & 77 \\
\hline 1919 & 55230 & 30948 & 27 & 0.04 & 48.37 & 0.121121 & 0.077077 & 13 & 215012282 & 13 & 215936425 & 13 & 215 \\
\hline 778 & 4642 & 46790 & 22 & 0.04 & 48.41 & 0.107107 & 0.067067 & 5 & 11029721 & 5 & 11981380 & 5 & 11 \\
\hline 2591 & 49812 & 19344 & 14 & 0.04 & 48.46 & 0.074074 & 0.061061 & 19 & 143013614 & 19 & 143838682 & 19 & 143 \\
\hline 469 & 35662 & 33975 & 39 & 0.04 & 48.5 & 0.158158 & 0.104104 & 2 & 154040051 & 2 & 154980475 & 2 & 154 \\
\hline 1153 & 6633 & 47173 & 30 & 0.04 & 48.55 & 0.138138 & 0.099099 & 7 & 117036639 & 7 & 117950905 & 7 & 117 \\
\hline 1761 & 38756 & 34634 & 16 & 0.04 & 48.59 & 0.089089 & 0.075075 & 13 & 57191330 & 13 & 57921952 & 13 & 57 \\
\hline 1148 & 6579 & 6588 & 21 & 0.04 & 48.64 & 0.103103 & 0.078078 & 7 & 112053041 & 7 & 112991344 & 7 & 112 \\
\hline 66 & 19814 & 53423 & 26 & 0.04 & 48.68 & 0.111111 & 0.073073 & 1 & 66015914 & 1 & 66820206 & 1 & 66 \\
\hline 700 & 61827 & 22102 & 29 & 0.04 & 48.73 & 0.129129 & 0.093093 & 4 & 77034601 & 4 & 77999066 & 4 & 77 \\
\hline 1614 & 49307 & 53735 & 16 & 0.04 & 48.77 & 0.084084 & 0.057057 & 11 & 62019748 & 11 & 62976117 & 11 & 62 \\
\hline 455 & 2702 & 21167 & 33 & 0.04 & 48.82 & 0.162162 & 0.11011 & 2 & 140002186 & 2 & 140996142 & 2 & 140 \\
\hline 2351 & 13393 & 39515 & 26 & 0.04 & 48.86 & 0.123123 & 0.086086 & 17 & 29081895 & 17 & 29964161 & 17 & 29 \\
\hline 1999 & 11180 & 39077 & 30 & 0.04 & 48.9 & 0.128128 & 0.083083 & 14 & 76005264 & 14 & 76979840 & 14 & 76 \\
\hline
\end{tabular}




\begin{tabular}{|c|c|c|c|c|c|c|c|c|c|c|c|c|c|}
\hline 632 & 21742 & 3476 & 27 & 0.04 & 48.95 & 0.132132 & 0.074074 & 4 & 9005118 & 4 & 9946393 & 4 & 9 \\
\hline 1165 & 42173 & 6739 & 25 & 0.04 & 48.99 & 0.119119 & 0.085085 & 7 & 129019539 & 7 & 129994916 & 7 & 129 \\
\hline 1967 & 47616 & 26906 & 27 & 0.04 & 49.04 & 0.142142 & 0.082082 & 14 & 44011983 & 14 & 44977107 & 14 & 44 \\
\hline 2492 & 48158 & 49782 & 30 & 0.04 & 49.08 & 0.125125 & 0.078078 & 19 & 39062455 & 19 & 39994318 & 19 & 39 \\
\hline 1964 & 26879 & 26888 & 26 & 0.04 & 49.13 & 0.112112 & 0.078078 & 14 & 41000376 & 14 & 41993819 & 14 & 41 \\
\hline 2445 & 49749 & 44830 & 25 & 0.04 & 49.17 & 0.116116 & 0.078078 & 18 & 53009145 & 18 & 53889747 & 18 & 53 \\
\hline 318 & 45597 & 40436 & 23 & 0.04 & 49.21 & 0.108108 & 0.068068 & 2 & 2010989 & 2 & 2992926 & 2 & 2 \\
\hline 1572 & 35913 & 25432 & 34 & 0.04 & 49.26 & 0.118118 & 0.077077 & 11 & 20016772 & 11 & 20989421 & 11 & 20 \\
\hline 1536 & 59203 & 29373 & 31 & 0.04 & 49.3 & 0.155155 & 0.107107 & 10 & 62022807 & 10 & 62985219 & 10 & 62 \\
\hline 1957 & 47600 & 26837 & 26 & 0.04 & 49.34 & 0.138138 & 0.089089 & 14 & 34028866 & 14 & 34991538 & 14 & 34 \\
\hline 29 & 224 & 227 & 31 & 0.04 & 49.39 & 0.15015 & 0.091091 & 1 & 29026999 & 1 & 29949287 & 1 & 29 \\
\hline 1527 & 29405 & 34983 & 15 & 0.04 & 49.43 & 0.085085 & 0.063063 & 10 & 53037792 & 10 & 53939074 & 10 & 53 \\
\hline 1530 & 25247 & 42843 & 31 & 0.04 & 49.48 & 0.115115 & 0.084084 & 10 & 56024865 & 10 & 56998330 & 10 & 56 \\
\hline 746 & 41276 & 4409 & 42 & 0.04 & 49.52 & 0.139139 & 0.093093 & 4 & 123001093 & 4 & 123983260 & 4 & 123 \\
\hline 1070 & 23457 & 5953 & 35 & 0.04 & 49.56 & 0.144144 & 0.098098 & 7 & 33017627 & 7 & 33991469 & 7 & 33 \\
\hline 483 & 52620 & 48594 & 33 & 0.04 & 49.61 & 0.151151 & 0.106106 & 3 & 5004645 & 3 & 5897010 & 3 & 5 \\
\hline 322 & 20721 & 20726 & 37 & 0.04 & 49.65 & 0.133133 & 0.092092 & 2 & 6018125 & 2 & 6998911 & 2 & 6 \\
\hline 2419 & 13823 & 13835 & 31 & 0.04 & 49.69 & 0.144144 & 0.101101 & 18 & 27001689 & 18 & 27981092 & 18 & 27 \\
\hline 657 & 21900 & 37432 & 31 & 0.04 & 49.74 & 0.115115 & 0.073073 & 4 & 34074856 & 4 & 34965838 & 4 & 34 \\
\hline 1568 & 25413 & 53591 & 29 & 0.04 & 49.78 & 0.142142 & 0.096096 & 11 & 16046333 & 11 & 16996424 & 11 & 16 \\
\hline 701 & 4016 & 22109 & 30 & 0.04 & 49.82 & 0.135135 & 0.087087 & 4 & 78027085 & 4 & 78946182 & 4 & 78 \\
\hline 1075 & 23496 & 55765 & 24 & 0.04 & 49.87 & 0.113113 & 0.078078 & 7 & 38006859 & 7 & 38984501 & 7 & 38 \\
\hline 1210 & 6985 & 6988 & 23 & 0.04 & 49.91 & 0.124124 & 0.078078 & 8 & 39014921 & 8 & 39851468 & 8 & 39 \\
\hline 863 & 58755 & 5129 & 18 & 0.04 & 49.95 & 0.095095 & 0.062062 & 5 & 96024130 & 5 & 96953817 & 5 & 96 \\
\hline 325 & 2007 & 2015 & 31 & 0.04 & 50 & 0.141141 & 0.092092 & 2 & 9098547 & 2 & 9987663 & 2 & 9 \\
\hline 894 & 22936 & 53721 & 31 & 0.04 & 50.04 & 0.147147 & 0.096096 & 6 & 15000888 & 6 & 15985029 & 6 & 15 \\
\hline 1672 & 35905 & 25804 & 30 & 0.04 & 50.08 & 0.134134 & 0.085085 & 12 & 32009788 & 12 & 32777362 & 12 & 32 \\
\hline 1510 & 45136 & 8399 & 22 & 0.04 & 50.13 & 0.121121 & 0.075075 & 10 & 36057791 & 10 & 36991693 & 10 & 36 \\
\hline 513 & 2924 & 2927 & 22 & 0.04 & 50.17 & 0.105105 & 0.069069 & 3 & 35024144 & 3 & 35978570 & 3 & 35 \\
\hline 342 & 2103 & 2108 & 29 & 0.04 & 50.21 & 0.143143 & 0.101101 & 2 & 26004268 & 2 & 26978443 & 2 & 26 \\
\hline 999 & 5533 & 5542 & 25 & 0.04 & 50.26 & 0.105105 & 0.07007 & 6 & 120008587 & 6 & 120983888 & 6 & 120 \\
\hline 2438 & 55793 & 13941 & 24 & 0.04 & 50.3 & 0.104104 & 0.077077 & 18 & 46190367 & 18 & 46983072 & 18 & 46 \\
\hline 666 & 21950 & 21956 & 26 & 0.04 & 50.34 & 0.125125 & 0.088088 & 4 & 43005049 & 4 & 43983124 & 4 & 43 \\
\hline 1932 & 43625 & 10642 & 22 & 0.04 & 50.38 & 0.098098 & 0.071071 & 14 & 9010230 & 14 & 9915945 & 14 & 9 \\
\hline 740 & 46759 & 17226 & 31 & 0.04 & 50.43 & 0.137137 & 0.095095 & 4 & 117005641 & 4 & 117950885 & 4 & 117 \\
\hline 1090 & 37937 & 52186 & 35 & 0.04 & 50.47 & 0.146146 & 0.103103 & 7 & 53018942 & 7 & 53981796 & 7 & 53 \\
\hline 2386 & 55455 & 49703 & 39 & 0.04 & 50.51 & 0.155155 & 0.09009 & 17 & 64009869 & 17 & 64997324 & 17 & 64 \\
\hline 1494 & 8338 & 50400 & 24 & 0.04 & 50.56 & 0.1001 & 0.068068 & 10 & 20017052 & 10 & 20952466 & 10 & 20 \\
\hline 787 & 51925 & 4711 & 26 & 0.04 & 50.6 & 0.116116 & 0.083083 & 5 & 20035192 & 5 & 20954929 & 5 & 20 \\
\hline
\end{tabular}




\begin{tabular}{|c|c|c|c|c|c|c|c|c|c|c|c|c|}
\hline 552 & 52286 & 36373 & 23 & 0.04 & 50.64 & 0.095095 & 0.063063 & 3 & 74026878 & 3 & 74957221 & 3 \\
\hline 970 & 5433 & 15678 & 29 & 0.04 & 50.68 & 0.132132 & 0.094094 & 6 & 91031831 & 6 & 91946493 & 6 \\
\hline 822 & 37643 & 22718 & 20 & 0.04 & 50.73 & 0.122122 & 0.068068 & 5 & 55008930 & 5 & 55999452 & 5 \\
\hline 1690 & 9402 & 25876 & 23 & 0.04 & 50.77 & 0.113113 & 0.067067 & 12 & 50043147 & 12 & 50907615 & 12 \\
\hline 2260 & 18827 & 12787 & 23 & 0.04 & 50.81 & 0.103103 & 0.06006 & 16 & 25038049 & 16 & 25999742 & 16 \\
\hline 505 & 29633 & 21295 & 28 & 0.04 & 50.85 & 0.135135 & 0.098098 & 3 & 27041644 & 3 & 27988181 & 3 \\
\hline 966 & 61750 & 36045 & 16 & 0.04 & 50.9 & 0.081081 & 0.055055 & 6 & 87000361 & 6 & 87968836 & 6 \\
\hline 2097 & 60291 & 11949 & 20 & 0.04 & 50.94 & 0.097097 & 0.064064 & 15 & 20011263 & 15 & 20934106 & 15 \\
\hline 1933 & 10647 & 10663 & 30 & 0.04 & 50.98 & 0.126126 & 0.093093 & 14 & 10012490 & 14 & 10994010 & 14 \\
\hline 762 & 4547 & 22462 & 25 & 0.04 & 51.02 & 0.105105 & 0.082082 & 4 & 139015407 & 4 & 139989597 & 4 \\
\hline 2356 & 13429 & 44578 & 27 & 0.04 & 51.07 & 0.119119 & 0.079079 & 17 & 34032332 & 17 & 34975107 & 17 \\
\hline 604 & 3336 & 3341 & 27 & 0.04 & 51.11 & 0.126126 & 0.085085 & 3 & 126010993 & 3 & 126989393 & 3 \\
\hline 117 & 36762 & 48422 & 22 & 0.04 & 51.15 & 0.129129 & 0.093093 & 1 & 117018825 & 1 & 117930744 & 1 \\
\hline 252 & 40262 & 35883 & 26 & 0.04 & 51.19 & 0.129129 & 0.071071 & 1 & 252002865 & 1 & 252997864 & 1 \\
\hline 2400 & 34151 & 34727 & 32 & 0.04 & 51.23 & 0.133133 & 0.092092 & 18 & 8002708 & 18 & 8998286 & 18 \\
\hline 909 & 5329 & 22985 & 27 & 0.04 & 51.28 & 0.121121 & 0.082082 & 6 & 30009402 & 6 & 30942669 & 6 \\
\hline 1996 & 11156 & 39059 & 23 & 0.04 & 51.32 & 0.11011 & 0.065065 & 14 & 73021315 & 14 & 73984104 & 14 \\
\hline 1112 & 47078 & 47082 & 17 & 0.04 & 51.36 & 0.094094 & 0.067067 & 7 & 75033437 & 7 & 75893840 & 7 \\
\hline 78 & 621 & 18214 & 20 & 0.04 & 51.4 & 0.096096 & 0.061061 & 1 & 78021030 & 1 & 78960119 & 1 \\
\hline 1511 & 51024 & 59520 & 16 & 0.04 & 51.45 & 0.099099 & 0.072072 & 10 & 37039997 & 10 & 37984810 & 10 \\
\hline 135 & 55868 & 946 & 7 & 0.04 & 51.49 & 0.05005 & 0.045045 & 1 & 135320152 & 1 & 135930910 & 1 \\
\hline 875 & 5220 & 22906 & 22 & 0.04 & 51.53 & 0.107107 & 0.074074 & 5 & 108020226 & 5 & 108968821 & 5 \\
\hline 38 & 36560 & 322 & 32 & 0.04 & 51.57 & 0.131131 & 0.076076 & 1 & 38044337 & 1 & 38953492 & 1 \\
\hline 1247 & 50137 & 7117 & 32 & 0.04 & 51.61 & 0.163163 & 0.092092 & 8 & 76109453 & 8 & 76977941 & 8 \\
\hline 2435 & 28947 & 48120 & 24 & 0.04 & 51.66 & 0.104104 & 0.072072 & 18 & 43012691 & 18 & 43976955 & 18 \\
\hline 2444 & 28984 & 28988 & 28 & 0.04 & 51.7 & 0.124124 & 0.083083 & 18 & 52001599 & 18 & 52977587 & 18 \\
\hline 582 & 3203 & 3213 & 23 & 0.04 & 51.74 & 0.121121 & 0.082082 & 3 & 104064335 & 3 & 104974525 & 3 \\
\hline 720 & 22195 & 59950 & 29 & 0.04 & 51.78 & 0.137137 & 0.088088 & 4 & 97012116 & 4 & 97993218 & 4 \\
\hline 306 & 1952 & 48501 & 26 & 0.04 & 51.82 & 0.128128 & 0.073073 & 1 & 306010138 & 1 & 306907352 & 1 \\
\hline 1600 & 8877 & 59105 & 26 & 0.04 & 51.86 & 0.11011 & 0.073073 & 11 & 48014416 & 11 & 48937000 & 11 \\
\hline 2267 & 39421 & 12836 & 32 & 0.04 & 51.91 & 0.132132 & 0.088088 & 16 & 32024005 & 16 & 32979912 & 16 \\
\hline 1983 & 11044 & 11054 & 29 & 0.04 & 51.95 & 0.135135 & 0.097097 & 14 & 60106278 & 14 & 60939220 & 14 \\
\hline 1719 & 43256 & 25981 & 26 & 0.04 & 51.99 & 0.129129 & 0.081081 & 13 & 15058867 & 13 & 15976193 & 13 \\
\hline 339 & 46425 & 40489 & 21 & 0.04 & 52.03 & 0.108108 & 0.084084 & 2 & 23018675 & 2 & 23936325 & 2 \\
\hline 1701 & 31005 & 45198 & 31 & 0.04 & 52.07 & 0.125125 & 0.088088 & 12 & 61042519 & 12 & 61949547 & 12 \\
\hline 391 & 53479 & 54937 & 7 & 0.04 & 52.12 & 0.057057 & 0.036036 & 2 & 76037122 & 2 & 76667109 & 2 \\
\hline 1106 & 42008 & 47066 & 24 & 0.04 & 52.16 & 0.131131 & 0.083083 & 7 & 69000948 & 7 & 69913988 & 7 \\
\hline 1772 & 52171 & 9941 & 18 & 0.04 & 52.2 & 0.109109 & 0.073073 & 13 & 68100531 & 13 & 68968779 & 13 \\
\hline 2022 & 43899 & 11376 & 18 & 0.04 & 52.24 & 0.083083 & 0.058058 & 14 & 99111537 & 14 & 99978645 & 14 \\
\hline
\end{tabular}




\begin{tabular}{|c|c|c|c|c|c|c|c|c|c|c|c|c|c|}
\hline 1382 & 38268 & 24708 & 27 & 0.04 & 52.28 & 0.138138 & 0.099099 & 9 & 62083176 & 9 & 62996659 & 9 & 62 \\
\hline 1162 & 23970 & 49082 & 23 & 0.04 & 52.32 & 0.112112 & 0.075075 & 7 & 126014404 & 7 & 126852902 & 7 & 126 \\
\hline 751 & 4435 & 62066 & 30 & 0.04 & 52.36 & 0.143143 & 0.085085 & 4 & 128001137 & 4 & 128996039 & 4 & 128 \\
\hline 1085 & 6062 & 32577 & 27 & 0.04 & 52.4 & 0.127127 & 0.09009 & 7 & 48105743 & 7 & 48953568 & 7 & 48 \\
\hline 1092 & 56408 & 51854 & 36 & 0.04 & 52.45 & 0.151151 & 0.109109 & 7 & 55030156 & 7 & 55985056 & 7 & 55 \\
\hline 803 & 61241 & 22675 & 26 & 0.04 & 52.49 & 0.131131 & 0.085085 & 5 & 36115758 & 5 & 36973186 & 5 & 36 \\
\hline 659 & 3717 & 54368 & 24 & 0.04 & 52.53 & 0.099099 & 0.07007 & 4 & 36005051 & 4 & 36930500 & 4 & 36 \\
\hline 781 & 58093 & 4675 & 31 & 0.04 & 52.57 & 0.147147 & 0.101101 & 5 & 14008737 & 5 & 14972422 & 5 & 14 \\
\hline 726 & 46738 & 35076 & 28 & 0.04 & 52.61 & 0.137137 & 0.087087 & 4 & 103067357 & 4 & 103960092 & 4 & 103 \\
\hline 446 & 32191 & 2639 & 12 & 0.04 & 52.65 & 0.073073 & 0.06006 & 2 & 131005861 & 2 & 131999506 & 2 & 131 \\
\hline 443 & 54670 & 46468 & 25 & 0.04 & 52.69 & 0.12012 & 0.072072 & 2 & 128229377 & 2 & 128982107 & 2 & 128 \\
\hline 2099 & 27644 & 11969 & 28 & 0.04 & 52.73 & 0.121121 & 0.079079 & 15 & 22028463 & 15 & 22961387 & 15 & 22 \\
\hline 2072 & 11794 & 27527 & 32 & 0.04 & 52.77 & 0.128128 & 0.087087 & 14 & 149002010 & 14 & 149990362 & 14 & 149 \\
\hline 514 & 40785 & 45456 & 24 & 0.04 & 52.81 & 0.098098 & 0.071071 & 3 & 36026450 & 3 & 36894931 & 3 & 36 \\
\hline 1570 & 8708 & 47337 & 17 & 0.04 & 52.86 & 0.094094 & 0.063063 & 11 & 18175690 & 11 & 18868868 & 11 & 18 \\
\hline 2127 & 32696 & 27721 & 19 & 0.04 & 52.9 & 0.099099 & 0.064064 & 15 & 50037970 & 15 & 50967710 & 15 & 50 \\
\hline 1577 & 8768 & 34020 & 35 & 0.04 & 52.94 & 0.147147 & 0.098098 & 11 & 25001589 & 11 & 25970861 & 11 & 25 \\
\hline 1305 & 53922 & 33229 & 25 & 0.04 & 52.98 & 0.123123 & 0.081081 & 8 & 134024195 & 8 & 134960890 & 8 & 134 \\
\hline 1926 & 26615 & 10597 & 19 & 0.04 & 53.02 & 0.097097 & 0.067067 & 14 & 3034421 & 14 & 3966701 & 14 & 3 \\
\hline 723 & 22219 & 22229 & 26 & 0.04 & 53.06 & 0.123123 & 0.087087 & 4 & 100212108 & 4 & 100985004 & 4 & 100 \\
\hline 124 & 895 & 36766 & 20 & 0.04 & 53.1 & 0.092092 & 0.064064 & 1 & 124014542 & 1 & 124987731 & 1 & 124 \\
\hline 1616 & 8997 & 25559 & 17 & 0.04 & 53.14 & 0.091091 & 0.062062 & 11 & 64018740 & 11 & 64995107 & 11 & 64 \\
\hline 2298 & 13017 & 34982 & 15 & 0.04 & 53.18 & 0.081081 & 0.058058 & 16 & 63072207 & 16 & 63944017 & 16 & 63 \\
\hline 1733 & 9684 & 9690 & 33 & 0.04 & 53.22 & 0.141141 & 0.079079 & 13 & 29002222 & 13 & 29980015 & 13 & 29 \\
\hline 64 & 19796 & 45919 & 24 & 0.04 & 53.26 & 0.11011 & 0.079079 & 1 & 64018444 & 1 & 64959341 & 1 & 64 \\
\hline 2342 & 13318 & 28472 & 25 & 0.04 & 53.3 & 0.121121 & 0.068068 & 17 & 20006406 & 17 & 20971854 & 17 & 20 \\
\hline 1968 & 10944 & 26913 & 20 & 0.04 & 53.34 & 0.093093 & 0.069069 & 14 & 45068764 & 14 & 45950397 & 14 & 45 \\
\hline 1793 & 26260 & 38806 & 27 & 0.04 & 53.38 & 0.126126 & 0.078078 & 13 & 89014753 & 13 & 89982824 & 13 & 89 \\
\hline 763 & 37531 & 22469 & 25 & 0.04 & 53.42 & 0.127127 & 0.089089 & 4 & 140011062 & 4 & 140899838 & 4 & 140 \\
\hline 764 & 41334 & 4564 & 27 & 0.04 & 53.46 & 0.123123 & 0.08008 & 4 & 141117946 & 4 & 141966625 & 4 & 141 \\
\hline 529 & 17458 & 56912 & 26 & 0.04 & 53.5 & 0.129129 & 0.086086 & 3 & 51053503 & 3 & 51939345 & 3 & 51 \\
\hline 1927 & 10598 & 43606 & 21 & 0.04 & 53.54 & 0.098098 & 0.067067 & 14 & 4117715 & 14 & 4999819 & 14 & 4 \\
\hline 2069 & 44041 & 62004 & 35 & 0.04 & 53.58 & 0.142142 & 0.093093 & 14 & 146002787 & 14 & 146982251 & 14 & 146 \\
\hline 1686 & 14877 & 54189 & 25 & 0.04 & 53.62 & 0.123123 & 0.076076 & 12 & 46023473 & 12 & 46979274 & 12 & 46 \\
\hline 76 & 45935 & 36682 & 26 & 0.04 & 53.66 & 0.135135 & 0.081081 & 1 & 76030269 & 1 & 76975200 & 1 & 76 \\
\hline 334 & 2047 & 40469 & 40 & 0.04 & 53.7 & 0.154154 & 0.096096 & 2 & 18091227 & 2 & 18926886 & 2 & 18 \\
\hline 458 & 14862 & 29926 & 33 & 0.04 & 53.74 & 0.134134 & 0.094094 & 2 & 143053245 & 2 & 143991472 & 2 & 143 \\
\hline 640 & 17151 & 34932 & 34 & 0.04 & 53.78 & 0.141141 & 0.102102 & 4 & 17033119 & 4 & 17975684 & 4 & 17 \\
\hline 1054 & 23365 & 37883 & 30 & 0.04 & 53.82 & 0.127127 & 0.074074 & 7 & 17027287 & 7 & 17994605 & 7 & 17 \\
\hline
\end{tabular}




\begin{tabular}{|c|c|c|c|c|c|c|c|c|c|c|c|c|c|}
\hline 132 & 20064 & 40064 & 16 & 0.04 & 53.86 & 0.055055 & 0.045045 & 1 & 132029570 & 1 & 132999230 & 1 & 132 \\
\hline 284 & 55247 & 19250 & 28 & 0.04 & 53.9 & 0.102102 & 0.064064 & 1 & 284045369 & 1 & 284998024 & 1 & 284 \\
\hline 2264 & 45160 & 44369 & 22 & 0.04 & 53.94 & 0.096096 & 0.059059 & 16 & 29044133 & 16 & 29952113 & 16 & \\
\hline 2439 & 15130 & 30144 & 37 & 0.04 & 53.98 & 0.167167 & 0.091091 & 18 & 47041773 & 18 & 47988831 & 18 & \\
\hline 2270 & 31841 & 28189 & 31 & 0.04 & 54.02 & 0.12012 & 0.071071 & 16 & 35014537 & 16 & 35997155 & 16 & \\
\hline 1472 & 51756 & 8181 & 31 & 0.04 & 54.06 & 0.148148 & 0.099099 & 9 & 152109779 & 9 & 152989337 & 9 & \\
\hline 2347 & 13361 & 61623 & 26 & 0.04 & 54.1 & 0.115115 & 0.075075 & 17 & 25073964 & 17 & 25894376 & 17 & \\
\hline 1332 & 49169 & 35842 & 29 & 0.04 & 54.14 & 0.132132 & 0.086086 & 9 & 12012638 & 9 & 12980703 & 9 & \\
\hline 1126 & 47121 & 47126 & 26 & 0.04 & 54.18 & 0.127127 & 0.086086 & 7 & 90011443 & 7 & 90978100 & 7 & \\
\hline 611 & 30377 & 50036 & 30 & 0.04 & 54.22 & 0.126126 & 0.088088 & 3 & 133094186 & 3 & 133992535 & 3 & 13 \\
\hline 780 & 22548 & 55397 & 24 & 0.04 & 54.26 & 0.135135 & 0.091091 & 5 & 13000041 & 5 & 13990580 & 5 & \\
\hline 2174 & 12357 & 47922 & 11 & 0.04 & 54.3 & 0.066066 & 0.049049 & 15 & 97254108 & 15 & 97940788 & 15 & \\
\hline 2334 & 48019 & 52200 & 23 & 0.04 & 54.34 & 0.096096 & 0.072072 & 17 & 12059087 & 17 & 12967074 & 17 & \\
\hline 1541 & 8566 & 51447 & 20 & 0.04 & 54.38 & 0.094094 & 0.056056 & 10 & 67023689 & 10 & 67993259 & 10 & \\
\hline 96 & 729 & 54275 & 24 & 0.04 & 54.42 & 0.1001 & 0.067067 & 1 & 96015876 & 1 & 96971456 & 1 & \\
\hline 939 & 18379 & 14676 & 29 & 0.04 & 54.46 & 0.118118 & 0.083083 & 6 & 60051563 & 6 & 60993805 & 6 & \\
\hline 493 & 40755 & 18076 & 26 & 0.04 & 54.5 & 0.131131 & 0.088088 & 3 & 15010975 & 3 & 15925307 & 3 & \\
\hline 967 & 23061 & 60617 & 28 & 0.04 & 54.54 & 0.133133 & 0.078078 & 6 & 88011983 & 6 & 88964845 & 6 & 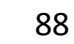 \\
\hline 1555 & 61046 & 25349 & 25 & 0.04 & 54.58 & 0.106106 & 0.064064 & 11 & 3251630 & 11 & 3991836 & 11 & \\
\hline 784 & 56684 & 48832 & 30 & 0.04 & 54.62 & 0.139139 & 0.091091 & 5 & 17055970 & 5 & 17949392 & 5 & 1 \\
\hline 2318 & 52112 & 45828 & 32 & 0.04 & 54.65 & 0.135135 & 0.094094 & 16 & 83023456 & 16 & 83958725 & 16 & 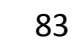 \\
\hline 413 & 46449 & 2427 & 20 & 0.04 & 54.69 & 0.093093 & 0.063063 & 2 & 98002439 & 2 & 98997793 & 2 & 98 \\
\hline 2283 & 12940 & 28241 & 25 & 0.04 & 54.73 & 0.128128 & 0.079079 & 16 & 48020257 & 16 & 48994238 & 16 & 40 \\
\hline 321 & 20706 & 48521 & 30 & 0.04 & 54.77 & 0.135135 & 0.093093 & 2 & 5004637 & 2 & 5984348 & 2 & \\
\hline 1340 & 17589 & 42468 & 30 & 0.04 & 54.81 & 0.122122 & 0.084084 & 9 & 20002748 & 9 & 20979753 & 9 & 20 \\
\hline 450 & 32857 & 29616 & 33 & 0.04 & 54.85 & 0.126126 & 0.087087 & 2 & 135007186 & 2 & 135935412 & 2 & 135 \\
\hline 1350 & 42484 & 54468 & 26 & 0.04 & 54.89 & 0.118118 & 0.078078 & 9 & 30061785 & 9 & 30795185 & 9 & 30 \\
\hline 1313 & 34735 & 50899 & 31 & 0.04 & 54.93 & 0.14014 & 0.087087 & 8 & 142003177 & 8 & 142950987 & 8 & 142 \\
\hline 1631 & 9121 & 59190 & 26 & 0.04 & 54.97 & 0.135135 & 0.086086 & 11 & 79016735 & 11 & 79920806 & 11 & $T$ \\
\hline 1483 & 25047 & 8270 & 27 & 0.04 & 55.01 & 0.126126 & 0.074074 & 10 & 9003663 & 10 & 9970331 & 10 & \\
\hline 643 & 21835 & 3579 & 30 & 0.04 & 55.05 & 0.144144 & 0.094094 & 4 & 20010437 & 4 & 20985135 & 4 & 2 \\
\hline 459 & 16843 & 31979 & 39 & 0.04 & 55.09 & 0.163163 & 0.1001 & 2 & 144000942 & 2 & 144958040 & 2 & 14 \\
\hline 2206 & 14907 & 61833 & 19 & 0.04 & 55.12 & 0.086086 & 0.06006 & 15 & 129031744 & 15 & 129798767 & 15 & $12 S$ \\
\hline 1934 & 26673 & 10675 & 37 & 0.04 & 55.16 & 0.145145 & 0.092092 & 14 & 11022529 & 14 & 11989764 & 14 & 1 \\
\hline 2002 & 55239 & 55906 & 29 & 0.04 & 55.2 & 0.13013 & 0.078078 & 14 & 79023018 & 14 & 79985244 & 14 & 7 \\
\hline 291 & 1845 & 1856 & 27 & 0.04 & 55.24 & 0.111111 & 0.059059 & 1 & 291021694 & 1 & 291903522 & 1 & 29 \\
\hline 1245 & 7096 & 56258 & 23 & 0.04 & 55.28 & 0.087087 & 0.057057 & 8 & 74017045 & 8 & 74976900 & 8 & \\
\hline 1390 & 51173 & 58953 & 25 & 0.04 & 55.32 & 0.106106 & 0.065065 & 9 & 70035504 & 9 & 70936501 & 9 & \\
\hline 1096 & 6190 & 6195 & 23 & 0.04 & 55.36 & 0.086086 & 0.057057 & 7 & 59039109 & 7 & 59986169 & 7 & \\
\hline
\end{tabular}




\begin{tabular}{|c|c|c|c|c|c|c|c|c|c|c|c|c|c|}
\hline 991 & 56220 & 18151 & 21 & 0.04 & 55.4 & 0.098098 & 0.067067 & 6 & 112091485 & 6 & 112990093 & 6 & 112 \\
\hline 2093 & 36390 & 45309 & 25 & 0.04 & 55.44 & 0.12012 & 0.082082 & 15 & 16070249 & 15 & 16953219 & 15 & \\
\hline 65 & 50270 & 36650 & 33 & 0.04 & 55.47 & 0.14014 & 0.087087 & 1 & 65008123 & 1 & 65994430 & 1 & \\
\hline 955 & 17656 & 5390 & 27 & 0.04 & 55.51 & 0.105105 & 0.076076 & 6 & 76056354 & 6 & 76948482 & 6 & \\
\hline 281 & 1765 & 30435 & 33 & 0.04 & 55.55 & 0.131131 & 0.079079 & 1 & 281095974 & 1 & 281969962 & 1 & \\
\hline 836 & 22766 & 16572 & 35 & 0.04 & 55.59 & 0.137137 & 0.094094 & 5 & 69012014 & 5 & 69897370 & 5 & \\
\hline 1936 & 10687 & 38982 & 27 & 0.04 & 55.63 & 0.137137 & 0.085085 & 14 & 13030843 & 14 & 13982953 & 14 & \\
\hline 753 & 4458 & 4471 & 36 & 0.04 & 55.67 & 0.155155 & 0.106106 & 4 & 130003887 & 4 & 130976284 & 4 & \\
\hline 1077 & 62009 & 41903 & 33 & 0.04 & 55.71 & 0.139139 & 0.077077 & 7 & 40007647 & 7 & 40992238 & 7 & \\
\hline 142 & 46073 & 20096 & 20 & 0.04 & 55.74 & 0.088088 & 0.059059 & 1 & 142041071 & 1 & 142992212 & 1 & 1 \\
\hline 371 & 16909 & 58969 & 7 & 0.04 & 55.78 & 0.036036 & 0.029029 & 2 & 55200111 & 2 & 55894230 & 2 & \\
\hline 63 & 19788 & 34371 & 29 & 0.04 & 55.82 & 0.129129 & 0.084084 & 1 & 63025137 & 1 & 63991546 & 1 & \\
\hline 1647 & 58634 & 33422 & 30 & 0.04 & 55.86 & 0.129129 & 0.086086 & 12 & 7009507 & 12 & 7976640 & 12 & \\
\hline 491 & 31278 & 2825 & 21 & 0.04 & 55.9 & 0.1001 & 0.062062 & 3 & 13040547 & 3 & 13922837 & 3 & \\
\hline 544 & 30156 & 21408 & 28 & 0.04 & 55.93 & 0.112112 & 0.083083 & 3 & 66040600 & 3 & 66993596 & 3 & \\
\hline 1880 & 43523 & 57865 & 15 & 0.04 & 55.97 & 0.089089 & 0.07007 & 13 & 176029424 & 13 & 176997245 & 13 & 17 \\
\hline 1094 & 6171 & 53464 & 34 & 0.04 & 56.01 & 0.14014 & 0.098098 & 7 & 57012925 & 7 & 57971318 & 7 & \\
\hline 1334 & 24505 & 16564 & 28 & 0.04 & 56.05 & 0.113113 & 0.068068 & 9 & 14007105 & 9 & 14991770 & 9 & \\
\hline 777 & 22527 & 22535 & 26 & 0.04 & 56.09 & 0.111111 & 0.077077 & 5 & 10080308 & 5 & 10947872 & 5 & \\
\hline 954 & 33596 & 30815 & 31 & 0.04 & 56.13 & 0.14014 & 0.089089 & 6 & 75138271 & 6 & 75952956 & 6 & - \\
\hline 1498 & 8595 & 25116 & 21 & 0.04 & 56.16 & 0.107107 & 0.074074 & 10 & 24011021 & 10 & 24984526 & 10 & \\
\hline 1775 & 38786 & 26194 & 22 & 0.04 & 56.2 & 0.101101 & 0.082082 & 13 & 71007063 & 13 & 71998014 & 13 & 1 \\
\hline 1941 & 10745 & 10730 & 25 & 0.04 & 56.24 & 0.13013 & 0.086086 & 14 & 18052581 & 14 & 18990098 & 14 & 18 \\
\hline 619 & 3396 & 57721 & 21 & 0.04 & 56.28 & 0.115115 & 0.081081 & 3 & 141006420 & 3 & 141885076 & 3 & 141 \\
\hline 806 & 31940 & 46816 & 20 & 0.04 & 56.32 & 0.089089 & 0.059059 & 5 & 39028335 & 5 & 39916475 & 5 & 39 \\
\hline 2023 & 59709 & 31236 & 24 & 0.04 & 56.35 & 0.113113 & 0.074074 & 14 & 100004352 & 14 & 100971708 & 14 & 10 \\
\hline 1923 & 43597 & 49442 & 19 & 0.04 & 56.39 & 0.085085 & 0.05005 & 14 & 167845 & 14 & 957393 & 14 & \\
\hline 2396 & 32079 & 31473 & 30 & 0.04 & 56.43 & 0.128128 & 0.088088 & 18 & 4013414 & 18 & 4997045 & 18 & \\
\hline 1025 & 34853 & 5672 & 21 & 0.04 & 56.47 & 0.1001 & 0.067067 & 6 & 146248545 & 6 & 146923286 & 6 & 14 \\
\hline 2212 & 27983 & 12553 & 34 & 0.04 & 56.5 & 0.132132 & 0.093093 & 15 & 135059821 & 15 & 135999617 & 15 & 13 \\
\hline 609 & 17394 & 55812 & 20 & 0.04 & 56.54 & 0.101101 & 0.052052 & 3 & 131057295 & 3 & 131906132 & 3 & 13 \\
\hline 876 & 5211 & 22917 & 20 & 0.04 & 56.58 & 0.114114 & 0.083083 & 5 & 109014722 & 5 & 109990527 & 5 & 10 \\
\hline 1786 & 9996 & 43399 & 29 & 0.04 & 56.62 & 0.146146 & 0.09009 & 13 & 82003709 & 13 & 82988910 & 13 & 8 \\
\hline 2325 & 13203 & 13209 & 18 & 0.04 & 56.66 & 0.092092 & 0.07007 & 17 & 3129112 & 17 & 3989769 & 17 & \\
\hline 62 & 56204 & 508 & 29 & 0.04 & 56.69 & 0.118118 & 0.075075 & 1 & 62058511 & 1 & 62989866 & 1 & 6 \\
\hline 804 & 61199 & 46814 & 21 & 0.04 & 56.73 & 0.095095 & 0.058058 & 5 & 37025932 & 5 & 37974847 & 5 & \\
\hline 2063 & 44017 & 47824 & 40 & 0.04 & 56.77 & 0.166166 & 0.103103 & 14 & 140004840 & 14 & 140944805 & 14 & 14 \\
\hline 1609 & 43009 & 38598 & 16 & 0.04 & 56.81 & 0.087087 & 0.06006 & 11 & 57041894 & 11 & 57992782 & 11 & \\
\hline 1526 & 25241 & 29360 & 29 & 0.04 & 56.84 & 0.122122 & 0.09009 & 10 & 52025204 & 10 & 52991516 & 10 & \\
\hline
\end{tabular}




\begin{tabular}{|c|c|c|c|c|c|c|c|c|c|c|c|c|c|}
\hline 1052 & 37873 & 58620 & 27 & 0.04 & 56.88 & 0.131131 & 0.085085 & 7 & 15057420 & 7 & 15995030 & 7 & 15 \\
\hline 1084 & 23542 & 23550 & 21 & 0.04 & 56.92 & 0.111111 & 0.075075 & 7 & 47037298 & 7 & 47934829 & 7 & 47 \\
\hline 136 & 947 & 950 & 16 & 0.04 & 56.96 & 0.084084 & 0.061061 & 1 & 136015038 & 1 & 136891518 & 1 & 136 \\
\hline 1431 & 54155 & 7956 & 14 & 0.04 & 57 & 0.081081 & 0.054054 & 9 & 111000802 & 9 & 111952227 & 9 & 111 \\
\hline 109 & 54801 & 36750 & 17 & 0.04 & 57.03 & 0.081081 & 0.056056 & 1 & 109003582 & 1 & 109912929 & 1 & 109 \\
\hline 1984 & 27028 & 11061 & 27 & 0.04 & 57.07 & 0.104104 & 0.071071 & 14 & 61003207 & 14 & 61937913 & 14 & 61 \\
\hline 1327 & 7496 & 57828 & 37 & 0.04 & 57.11 & 0.14014 & 0.089089 & 9 & 7010644 & 9 & 7987695 & 9 & 7 \\
\hline 1540 & 14859 & 25303 & 26 & 0.04 & 57.15 & 0.12012 & 0.085085 & 10 & 66020622 & 10 & 66971701 & 10 & 66 \\
\hline 829 & 60143 & 33985 & 36 & 0.04 & 57.18 & 0.151151 & 0.088088 & 5 & 62000754 & 5 & 62972049 & 5 & 62 \\
\hline 670 & 37445 & 46612 & 24 & 0.04 & 57.22 & 0.105105 & 0.072072 & 4 & 47043904 & 4 & 47963187 & 4 & 47 \\
\hline 2256 & 60138 & 14578 & 28 & 0.04 & 57.26 & 0.104104 & 0.074074 & 16 & 21011283 & 16 & 21993561 & 16 & 21 \\
\hline 2183 & 61219 & 27857 & 17 & 0.04 & 57.29 & 0.074074 & 0.054054 & 15 & 106045290 & 15 & 106977147 & 15 & 106 \\
\hline 2250 & 33334 & 45602 & 19 & 0.04 & 57.33 & 0.1001 & 0.062062 & 16 & 15022443 & 16 & 15992155 & 16 & 15 \\
\hline 1176 & 38085 & 33119 & 28 & 0.04 & 57.37 & 0.127127 & 0.088088 & 8 & 5043216 & 8 & 5984514 & 8 & 5 \\
\hline 536 & 21376 & 21383 & 27 & 0.04 & 57.41 & 0.11011 & 0.078078 & 3 & 58013180 & 3 & 58999300 & 3 & 58 \\
\hline 730 & 52513 & 35537 & 31 & 0.04 & 57.44 & 0.12012 & 0.079079 & 4 & 107002176 & 4 & 107959349 & 4 & 107 \\
\hline 944 & 48911 & 5374 & 26 & 0.04 & 57.48 & 0.11011 & 0.069069 & 6 & 65081101 & 6 & 65940489 & 6 & 65 \\
\hline 2379 & 28680 & 28687 & 33 & 0.04 & 57.52 & 0.136136 & 0.092092 & 17 & 57039720 & 17 & 57942798 & 17 & 57 \\
\hline 835 & 22760 & 48846 & 29 & 0.04 & 57.56 & 0.139139 & 0.095095 & 5 & 68011832 & 5 & 68998629 & 5 & 68 \\
\hline 472 & 18404 & 2779 & 37 & 0.04 & 57.59 & 0.163163 & 0.108108 & 2 & 157004735 & 2 & 157957344 & 2 & 157 \\
\hline 702 & 22110 & 46690 & 31 & 0.04 & 57.63 & 0.14014 & 0.089089 & 4 & 79008114 & 4 & 79794714 & 4 & 79 \\
\hline 1698 & 43220 & 33169 & 26 & 0.04 & 57.67 & 0.103103 & 0.069069 & 12 & 58042164 & 12 & 58992046 & 12 & 58 \\
\hline 1087 & 6091 & 53052 & 32 & 0.04 & 57.7 & 0.133133 & 0.084084 & 7 & 50028824 & 7 & 50991091 & 7 & 50 \\
\hline 1674 & 32709 & 43177 & 41 & 0.04 & 57.74 & 0.163163 & 0.113113 & 12 & 34002369 & 12 & 34995000 & 12 & 34 \\
\hline 618 & 30981 & 61276 & 16 & 0.04 & 57.78 & 0.083083 & 0.054054 & 3 & 140070086 & 3 & 140798715 & 3 & 140 \\
\hline 364 & 2269 & 53385 & 17 & 0.04 & 57.82 & 0.081081 & 0.061061 & 2 & 48125078 & 2 & 48927541 & 2 & 48 \\
\hline 1763 & 17218 & 26152 & 39 & 0.04 & 57.85 & 0.144144 & 0.1001 & 13 & 59015422 & 13 & 59941517 & 13 & 59 \\
\hline 1071 & 5954 & 46984 & 30 & 0.04 & 57.89 & 0.1001 & 0.079079 & 7 & 34026230 & 7 & 34978383 & 7 & 34 \\
\hline 2257 & 12758 & 12770 & 24 & 0.04 & 57.93 & 0.108108 & 0.074074 & 16 & 22095735 & 16 & 22991610 & 16 & 22 \\
\hline 902 & 33237 & 41617 & 26 & 0.04 & 57.96 & 0.11011 & 0.071071 & 6 & 23005830 & 6 & 23918938 & 6 & 23 \\
\hline 2113 & 44127 & 59289 & 19 & 0.04 & 58 & 0.107107 & 0.067067 & 15 & 36011179 & 15 & 36905893 & 15 & 36 \\
\hline 1325 & 42421 & 38220 & 12 & 0.04 & 58.04 & 0.064064 & 0.05005 & 9 & 5226276 & 9 & 5988229 & 9 & 5 \\
\hline 598 & 3310 & 33552 & 18 & 0.04 & 58.07 & 0.103103 & 0.07007 & 3 & 120040818 & 3 & 120863426 & 3 & 120 \\
\hline 1034 & 48936 & 30300 & 22 & 0.04 & 58.11 & 0.095095 & 0.065065 & 6 & 155139641 & 6 & 155985335 & 6 & 155 \\
\hline 1203 & 32565 & 6945 & 25 & 0.04 & 58.15 & 0.105105 & 0.068068 & 8 & 32014830 & 8 & 32984498 & 8 & 32 \\
\hline 2384 & 49695 & 39535 & 27 & 0.04 & 58.18 & 0.128128 & 0.069069 & 17 & 62012263 & 17 & 62979104 & 17 & 62 \\
\hline 1004 & 16509 & 5563 & 33 & 0.04 & 58.22 & 0.154154 & 0.091091 & 6 & 125030939 & 6 & 125996452 & 6 & 125 \\
\hline 652 & 37422 & 21881 & 21 & 0.04 & 58.26 & 0.082082 & 0.049049 & 4 & 29042837 & 4 & 29908735 & 4 & 29 \\
\hline 1068 & 5924 & 23446 & 36 & 0.04 & 58.29 & 0.132132 & 0.084084 & 7 & 31011932 & 7 & 31989509 & 7 & 31 \\
\hline
\end{tabular}




\begin{tabular}{|c|c|c|c|c|c|c|c|c|c|c|c|c|}
\hline 752 & 4447 & 4457 & 27 & 0.04 & 58.33 & 0.12012 & 0.074074 & 4 & 129016956 & 4 & 129991207 & 4 \\
\hline 2271 & 28190 & 53481 & 28 & 0.04 & 58.37 & 0.101101 & 0.07007 & 16 & 36026675 & 16 & 36933143 & 16 \\
\hline 686 & 22021 & 55977 & 19 & 0.04 & 58.4 & 0.1001 & 0.072072 & 4 & 63054580 & 4 & 63988841 & 4 \\
\hline 926 & 17213 & 30893 & 11 & 0.04 & 58.44 & 0.059059 & 0.044044 & 6 & 47104925 & 6 & 47852634 & 6 \\
\hline 1935 & 26683 & 10686 & 32 & 0.04 & 58.48 & 0.132132 & 0.083083 & 14 & 12010383 & 14 & 12997847 & 14 \\
\hline 2003 & 27127 & 11220 & 31 & 0.04 & 58.51 & 0.112112 & 0.07007 & 14 & 80010535 & 14 & 80984032 & 14 \\
\hline 2228 & 49606 & 28051 & 34 & 0.04 & 58.55 & 0.142142 & 0.092092 & 15 & 151027575 & 15 & 151968517 & 15 \\
\hline 745 & 37490 & 22359 & 24 & 0.04 & 58.59 & 0.119119 & 0.082082 & 4 & 122078780 & 4 & 122988956 & 4 \\
\hline 884 & 30000 & 37752 & 30 & 0.04 & 58.62 & 0.135135 & 0.086086 & 6 & 5041803 & 6 & 5951885 & 6 \\
\hline 725 & 29900 & 56117 & 27 & 0.04 & 58.66 & 0.131131 & 0.084084 & 4 & 102103941 & 4 & 102928877 & 4 \\
\hline 2020 & 27213 & 50842 & 32 & 0.04 & 58.7 & 0.133133 & 0.082082 & 14 & 97018128 & 14 & 97994997 & 14 \\
\hline 2106 & 45643 & 39240 & 29 & 0.04 & 58.73 & 0.125125 & 0.066066 & 15 & 29029368 & 15 & 29947470 & 15 \\
\hline 980 & 35612 & 37781 & 24 & 0.04 & 58.77 & 0.099099 & 0.073073 & 6 & 101036334 & 6 & 101986509 & 6 \\
\hline 1363 & 15377 & 38261 & 34 & 0.04 & 58.81 & 0.143143 & 0.097097 & 9 & 43064592 & 9 & 43992201 & 9 \\
\hline 130 & 46045 & 929 & 22 & 0.04 & 58.84 & 0.088088 & 0.067067 & 1 & 130035771 & 1 & 130848369 & 1 \\
\hline 1016 & 30067 & 35315 & 26 & 0.04 & 58.88 & 0.122122 & 0.083083 & 6 & 137044171 & 6 & 137985648 & 6 \\
\hline 1164 & 23981 & 23992 & 30 & 0.04 & 58.91 & 0.12012 & 0.076076 & 7 & 128019403 & 7 & 128999444 & 7 \\
\hline 414 & 54043 & 58908 & 11 & 0.04 & 58.95 & 0.067067 & 0.044044 & 2 & 99022293 & 2 & 99964100 & 2 \\
\hline 2383 & 56176 & 13628 & 37 & 0.04 & 58.99 & 0.151151 & 0.09009 & 17 & 61012026 & 17 & 61978820 & 17 \\
\hline 1947 & 10785 & 58020 & 23 & 0.04 & 59.02 & 0.099099 & 0.067067 & 14 & 24039632 & 14 & 24906177 & 14 \\
\hline 380 & 32083 & 48550 & 13 & 0.04 & 59.06 & 0.071071 & 0.053053 & 2 & 65057680 & 2 & 65994755 & 2 \\
\hline 1013 & 5599 & 45249 & 20 & 0.04 & 59.09 & 0.073073 & 0.051051 & 6 & 134051144 & 6 & 134996322 & 6 \\
\hline 974 & 60765 & 55766 & 24 & 0.04 & 59.13 & 0.136136 & 0.081081 & 6 & 95085460 & 6 & 95959657 & 6 \\
\hline 719 & 22191 & 35230 & 26 & 0.04 & 59.17 & 0.105105 & 0.063063 & 4 & 96048342 & 4 & 96996047 & 4 \\
\hline 1518 & 25193 & 31354 & 20 & 0.04 & 59.2 & 0.09009 & 0.066066 & 10 & 44144334 & 10 & 44971089 & 10 \\
\hline 1539 & 19076 & 17601 & 29 & 0.04 & 59.24 & 0.128128 & 0.083083 & 10 & 65022553 & 10 & 65996645 & 10 \\
\hline 1049 & 23328 & 23337 & 27 & 0.04 & 59.27 & 0.123123 & 0.074074 & 7 & 12019377 & 7 & 12984109 & 7 \\
\hline 1666 & 55925 & 43162 & 27 & 0.04 & 59.31 & 0.11011 & 0.076076 & 12 & 26022727 & 12 & 26969612 & 12 \\
\hline 9 & 19480 & 75 & 31 & 0.04 & 59.35 & 0.143143 & 0.089089 & 1 & 9007321 & 1 & 9984842 & 1 \\
\hline 1180 & 24046 & 42205 & 23 & 0.04 & 59.38 & 0.09009 & 0.064064 & 8 & 9026929 & 8 & 9891321 & 8 \\
\hline 1987 & 11074 & 27051 & 30 & 0.04 & 59.42 & 0.12012 & 0.071071 & 14 & 64014394 & 14 & 64974040 & 14 \\
\hline 1574 & 8741 & 8745 & 22 & 0.04 & 59.45 & 0.116116 & 0.071071 & 11 & 22013261 & 11 & 22990483 & 11 \\
\hline 1139 & 6512 & 49056 & 21 & 0.04 & 59.49 & 0.113113 & 0.083083 & 7 & 103001352 & 7 & 103999954 & 7 \\
\hline 2019 & 47711 & 11349 & 24 & 0.04 & 59.52 & 0.108108 & 0.076076 & 14 & 96103451 & 14 & 96986147 & 14 \\
\hline 336 & 30990 & 2066 & 26 & 0.04 & 59.56 & 0.116116 & 0.085085 & 2 & 20008168 & 2 & 20975241 & 2 \\
\hline 916 & 45445 & 18381 & 31 & 0.04 & 59.6 & 0.12012 & 0.08008 & 6 & 37040339 & 6 & 37983742 & 6 \\
\hline 1734 & 9691 & 43291 & 23 & 0.04 & 59.63 & 0.115115 & 0.083083 & 13 & 30023501 & 13 & 30990361 & 13 \\
\hline 2021 & 11363 & 43897 & 26 & 0.04 & 59.67 & 0.107107 & 0.073073 & 14 & 98020117 & 14 & 98941715 & 14 \\
\hline 492 & 37254 & 55645 & 38 & 0.04 & 59.7 & 0.173173 & 0.094094 & 3 & 14007265 & 3 & 14998247 & 3 \\
\hline
\end{tabular}




\begin{tabular}{|c|c|c|c|c|c|c|c|c|c|c|c|c|c|}
\hline 1108 & 49040 & 6266 & 21 & 0.04 & 59.74 & 0.069069 & 0.049049 & 7 & 71050477 & 7 & 71974687 & 7 & 71 \\
\hline 658 & 21908 & 3716 & 29 & 0.04 & 59.77 & 0.131131 & 0.088088 & 4 & 35008828 & 4 & 35986572 & 4 & 35 \\
\hline 1533 & 60045 & 32115 & 30 & 0.04 & 59.81 & 0.142142 & 0.092092 & 10 & 59082877 & 10 & 59954227 & 10 & 59 \\
\hline 821 & 18149 & 46840 & 11 & 0.04 & 59.85 & 0.069069 & 0.051051 & 5 & 54100199 & 5 & 54967272 & 5 & 54 \\
\hline 57 & 53965 & 39908 & 29 & 0.04 & 59.88 & 0.133133 & 0.088088 & 1 & 57012775 & 1 & 57981015 & 1 & 57 \\
\hline 60 & 36637 & 19773 & 28 & 0.04 & 59.92 & 0.119119 & 0.084084 & 1 & 60022622 & 1 & 60987629 & 1 & 60 \\
\hline 2236 & 28064 & 16889 & 21 & 0.04 & 59.95 & 0.107107 & 0.081081 & 16 & 1016806 & 16 & 1883120 & 16 & 1 \\
\hline 1294 & 34692 & 7327 & 12 & 0.04 & 59.99 & 0.082082 & 0.062062 & 8 & 123027874 & 8 & 123996880 & 8 & 123 \\
\hline 1602 & 25511 & 8897 & 20 & 0.04 & 60.02 & 0.09009 & 0.058058 & 11 & 50012027 & 11 & 50973313 & 11 & 50 \\
\hline 1073 & 41880 & 5974 & 19 & 0.04 & 60.06 & 0.073073 & 0.055055 & 7 & 36004578 & 7 & 36991143 & 7 & 36 \\
\hline 2255 & 44339 & 32998 & 34 & 0.04 & 60.09 & 0.144144 & 0.084084 & 16 & 20018423 & 16 & 20985329 & 16 & 20 \\
\hline 3 & 50179 & 24 & 34 & 0.04 & 60.13 & 0.132132 & 0.076076 & 1 & 3008501 & 1 & 3977158 & 1 & 3 \\
\hline 1507 & 38445 & 25162 & 18 & 0.04 & 60.16 & 0.085085 & 0.057057 & 10 & 33060083 & 10 & 33996942 & 10 & 33 \\
\hline 292 & 18920 & 37049 & 22 & 0.04 & 60.2 & 0.111111 & 0.08008 & 1 & 292036476 & 1 & 292998740 & 1 & 92 \\
\hline 703 & 4041 & 4048 & 16 & 0.04 & 60.23 & 0.07007 & 0.054054 & 4 & 80111898 & 4 & 80963726 & 4 & 80 \\
\hline 1170 & 24015 & 15870 & 27 & 0.04 & 60.27 & 0.138138 & 0.088088 & 7 & 134005327 & 7 & 134721771 & 7 & 134 \\
\hline 1059 & 41841 & 41843 & 20 & 0.04 & 60.3 & 0.088088 & 0.061061 & 7 & 22026124 & 7 & 22987329 & 7 & 22 \\
\hline 546 & 3054 & 21418 & 21 & 0.04 & 60.34 & 0.079079 & 0.058058 & 3 & 68009516 & 3 & 68984089 & 3 & 68 \\
\hline 1488 & 15962 & 31220 & 23 & 0.04 & 60.37 & 0.108108 & 0.068068 & 10 & 14031320 & 10 & 14993971 & 10 & 14 \\
\hline 1879 & 26465 & 43522 & 11 & 0.04 & 60.41 & 0.059059 & 0.048048 & 13 & 175005291 & 13 & 175985883 & 13 & 175 \\
\hline 137 & 20075 & 51457 & 29 & 0.04 & 60.44 & 0.12012 & 0.069069 & 1 & 137035325 & 1 & 137998037 & 1 & 137 \\
\hline 2309 & 13096 & 13106 & 32 & 0.04 & 60.48 & 0.14014 & 0.091091 & 16 & 74053569 & 16 & 74982852 & 16 & 74 \\
\hline 146 & 999 & 1007 & 23 & 0.04 & 60.51 & 0.111111 & 0.074074 & 1 & 146032525 & 1 & 146988836 & 1 & 146 \\
\hline 1779 & 26203 & 51448 & 24 & 0.03 & 60.55 & 0.101101 & 0.073073 & 13 & 75066887 & 13 & 75849184 & 13 & 75 \\
\hline 440 & 17984 & 2606 & 32 & 0.03 & 60.58 & 0.123123 & 0.086086 & 2 & 125009891 & 2 & 125989308 & 2 & 125 \\
\hline 593 & 40910 & 57175 & 24 & 0.03 & 60.62 & 0.094094 & 0.064064 & 3 & 115009623 & 3 & 115996607 & 3 & 115 \\
\hline 163 & 20181 & 1098 & 24 & 0.03 & 60.65 & 0.086086 & 0.057057 & 1 & 163013923 & 1 & 163977023 & 1 & 163 \\
\hline 1492 & 25101 & 57818 & 28 & 0.03 & 60.69 & 0.114114 & 0.078078 & 10 & 18001886 & 10 & 18973140 & 10 & 18 \\
\hline 1845 & 43467 & 51393 & 21 & 0.03 & 60.72 & 0.094094 & 0.063063 & 13 & 141032836 & 13 & 141902985 & 13 & 141 \\
\hline 1882 & 51545 & 39670 & 15 & 0.03 & 60.76 & 0.072072 & 0.049049 & 13 & 178024707 & 13 & 178860766 & 13 & 178 \\
\hline 1155 & 51515 & 34475 & 27 & 0.03 & 60.79 & 0.115115 & 0.079079 & 7 & 119018157 & 7 & 119962301 & 7 & 119 \\
\hline 1177 & 34275 & 60870 & 26 & 0.03 & 60.83 & 0.111111 & 0.086086 & 8 & 6009251 & 8 & 6999002 & 8 & 6 \\
\hline 732 & 50660 & 22279 & 31 & 0.03 & 60.86 & 0.113113 & 0.072072 & 4 & 109015657 & 4 & 109969089 & 4 & 09 \\
\hline 1322 & 16747 & 31404 & 28 & 0.03 & 60.9 & 0.111111 & 0.075075 & 9 & 2305353 & 9 & 2978499 & 9 & 2 \\
\hline 1966 & 26893 & 26901 & 26 & 0.03 & 60.93 & 0.115115 & 0.077077 & 14 & 43015985 & 14 & 43990421 & 14 & 43 \\
\hline 1593 & 38556 & 25494 & 17 & 0.03 & 60.97 & 0.096096 & 0.075075 & 11 & 41062108 & 11 & 41970836 & 11 & 41 \\
\hline 696 & 37458 & 46678 & 25 & 0.03 & 61 & 0.109109 & 0.071071 & 4 & 73017408 & 4 & 73907009 & 4 & 73 \\
\hline 1309 & 54482 & 7411 & 26 & 0.03 & 61.04 & 0.108108 & 0.068068 & 8 & 138036642 & 8 & 138999549 & 8 & 138 \\
\hline 2286 & 30153 & 48339 & 11 & 0.03 & 61.07 & 0.059059 & 0.047047 & 16 & 51117642 & 16 & 51990539 & 16 & 51 \\
\hline
\end{tabular}




\begin{tabular}{|c|c|c|c|c|c|c|c|c|c|c|c|c|c|}
\hline 268 & 60483 & 1693 & 31 & 0.03 & 61.11 & 0.132132 & 0.081081 & 1 & 268014122 & 1 & 268986440 & 1 & 268 \\
\hline 1567 & 61641 & 8693 & 21 & 0.03 & 61.14 & 0.103103 & 0.067067 & 11 & 15065868 & 11 & 15982103 & 11 & 15 \\
\hline 1859 & 18749 & 10284 & 20 & 0.03 & 61.18 & 0.087087 & 0.06006 & 13 & 155052948 & 13 & 155870725 & 13 & 155 \\
\hline 1953 & 10843 & 10849 & 26 & 0.03 & 61.21 & 0.112112 & 0.081081 & 14 & 30116923 & 14 & 30970606 & 14 & 30 \\
\hline 1107 & 23672 & 6263 & 28 & 0.03 & 61.25 & 0.107107 & 0.072072 & 7 & 70000093 & 7 & 70975451 & 7 & 70 \\
\hline 861 & 5106 & 5116 & 29 & 0.03 & 61.28 & 0.143143 & 0.081081 & 5 & 94018164 & 5 & 94958858 & 5 & 94 \\
\hline 694 & 61238 & 46673 & 23 & 0.03 & 61.32 & 0.09009 & 0.066066 & 4 & 71081956 & 4 & 71941276 & 4 & 71 \\
\hline 1123 & 6350 & 52211 & 28 & 0.03 & 61.35 & 0.12012 & 0.092092 & 7 & 87029852 & 7 & 87977236 & 7 & 87 \\
\hline 431 & 2535 & 21047 & 19 & 0.03 & 61.38 & 0.086086 & 0.072072 & 2 & 116053045 & 2 & 116939471 & 2 & 116 \\
\hline 2215 & 27964 & 60269 & 21 & 0.03 & 61.42 & 0.097097 & 0.07007 & 15 & 138046832 & 15 & 138987291 & 15 & 138 \\
\hline 2213 & 12552 & 44258 & 29 & 0.03 & 61.45 & 0.105105 & 0.072072 & 15 & 136021602 & 15 & 136981095 & 15 & 136 \\
\hline 2237 & 55021 & 44306 & 16 & 0.03 & 61.49 & 0.064064 & 0.041041 & 16 & 2142400 & 16 & 2996993 & 16 & 2 \\
\hline 1950 & 47583 & 57648 & 19 & 0.03 & 61.52 & 0.085085 & 0.062062 & 14 & 27119203 & 14 & 27942025 & 14 & 27 \\
\hline 842 & 54214 & 22797 & 25 & 0.03 & 61.56 & 0.095095 & 0.068068 & 5 & 75044596 & 5 & 75969214 & 5 & 75 \\
\hline 49 & 19716 & 392 & 31 & 0.03 & 61.59 & 0.143143 & 0.088088 & 1 & 49026306 & 1 & 49988544 & 1 & 49 \\
\hline 2197 & 39316 & 12447 & 24 & 0.03 & 61.63 & 0.127127 & 0.082082 & 15 & 120015248 & 15 & 120942551 & 15 & 120 \\
\hline 1262 & 38164 & 49136 & 20 & 0.03 & 61.66 & 0.086086 & 0.063063 & 8 & 91033467 & 8 & 91991327 & 8 & 91 \\
\hline 920 & 18166 & 50247 & 19 & 0.03 & 61.69 & 0.101101 & 0.065065 & 6 & 41004930 & 6 & 41983038 & 6 & 41 \\
\hline 1067 & 37906 & 5925 & 37 & 0.03 & 61.73 & 0.137137 & 0.098098 & 7 & 30036530 & 7 & 30978428 & 7 & 30 \\
\hline 366 & 32305 & 31301 & 24 & 0.03 & 61.76 & 0.114114 & 0.076076 & 2 & 50036228 & 2 & 50999217 & 2 & 50 \\
\hline 1366 & 49178 & 50954 & 20 & 0.03 & 61.8 & 0.102102 & 0.064064 & 9 & 46041782 & 9 & 46991931 & 9 & 46 \\
\hline 58 & 36632 & 39914 & 34 & 0.03 & 61.83 & 0.141141 & 0.094094 & 1 & 58006061 & 1 & 58990256 & 1 & 58 \\
\hline 2041 & 27326 & 11539 & 26 & 0.03 & 61.87 & 0.11011 & 0.081081 & 14 & 118017826 & 14 & 118988272 & 14 & 118 \\
\hline 1349 & 16910 & 42483 & 22 & 0.03 & 61.9 & 0.089089 & 0.063063 & 9 & 29002522 & 9 & 29982654 & 9 & 29 \\
\hline 147 & 48429 & 40089 & 21 & 0.03 & 61.93 & 0.105105 & 0.082082 & 1 & 147019363 & 1 & 147989923 & 1 & 147 \\
\hline 1575 & 47339 & 25448 & 30 & 0.03 & 61.97 & 0.112112 & 0.074074 & 11 & 23031513 & 11 & 23991998 & 11 & 23 \\
\hline 1444 & 38377 & 47289 & 27 & 0.03 & 62 & 0.125125 & 0.063063 & 9 & 124079367 & 9 & 124959430 & 9 & 124 \\
\hline 671 & 3806 & 51466 & 16 & 0.03 & 62.04 & 0.059059 & 0.035035 & 4 & 48069819 & 4 & 48936417 & 4 & 48 \\
\hline 453 & 55412 & 46472 & 30 & 0.03 & 62.07 & 0.11011 & 0.077077 & 2 & 138000735 & 2 & 138911144 & 2 & 138 \\
\hline 48 & 374 & 19715 & 22 & 0.03 & 62.1 & 0.09009 & 0.065065 & 1 & 48013733 & 1 & 48925115 & 1 & 48 \\
\hline 368 & 2287 & 57780 & 10 & 0.03 & 62.14 & 0.064064 & 0.042042 & 2 & 52013286 & 2 & 52674728 & 2 & 52 \\
\hline 2246 & 44326 & 52133 & 21 & 0.03 & 62.17 & 0.089089 & 0.072072 & 16 & 11013570 & 16 & 11999198 & 16 & 11 \\
\hline 488 & 31616 & 31091 & 27 & 0.03 & 62.21 & 0.118118 & 0.085085 & 3 & 10004418 & 3 & 10973478 & 3 & 10 \\
\hline 1147 & 6569 & 47162 & 24 & 0.03 & 62.24 & 0.115115 & 0.07007 & 7 & 111019262 & 7 & 111972610 & 7 & 111 \\
\hline 874 & 5208 & 41588 & 29 & 0.03 & 62.27 & 0.113113 & 0.077077 & 5 & 107029477 & 5 & 107978337 & 5 & 107 \\
\hline 107 & 19994 & 19999 & 31 & 0.03 & 62.31 & 0.119119 & 0.08008 & 1 & 107013020 & 1 & 107964728 & 1 & 107 \\
\hline 1440 & 18433 & 7996 & 20 & 0.03 & 62.34 & 0.087087 & 0.059059 & 9 & 120039871 & 9 & 120972491 & 9 & 120 \\
\hline 735 & 22288 & 53568 & 28 & 0.03 & 62.38 & 0.139139 & 0.076076 & 4 & 112014396 & 4 & 112988683 & 4 & 112 \\
\hline 892 & 45319 & 18258 & 27 & 0.03 & 62.41 & 0.132132 & 0.083083 & 6 & 13006700 & 6 & 13977172 & 6 & 13 \\
\hline
\end{tabular}




\begin{tabular}{|c|c|c|c|c|c|c|c|c|c|c|c|c|}
\hline 1954 & 47592 & 10856 & 31 & 0.03 & 62.44 & 0.135135 & 0.092092 & 14 & 31036695 & 14 & 31983579 & 14 \\
\hline 442 & 53661 & 18498 & 28 & 0.03 & 62.48 & 0.129129 & 0.083083 & 2 & 127056884 & 2 & 127964422 & 2 \\
\hline 792 & 50436 & 4736 & 27 & 0.03 & 62.51 & 0.121121 & 0.079079 & 5 & 25027517 & 5 & 25995235 & 5 \\
\hline 1485 & 50926 & 25069 & 31 & 0.03 & 62.54 & 0.142142 & 0.092092 & 10 & 11045137 & 10 & 11986190 & 10 \\
\hline 1693 & 9419 & 32021 & 25 & 0.03 & 62.58 & 0.093093 & 0.066066 & 12 & 53007660 & 12 & 53999623 & 12 \\
\hline 796 & 31262 & 22633 & 11 & 0.03 & 62.61 & 0.065065 & 0.053053 & 5 & 29078553 & 5 & 29952075 & 5 \\
\hline 2284 & 28242 & 28245 & 20 & 0.03 & 62.64 & 0.107107 & 0.075075 & 16 & 49050041 & 16 & 49868072 & 16 \\
\hline 1648 & 25713 & 25719 & 40 & 0.03 & 62.68 & 0.148148 & 0.098098 & 12 & 8013844 & 12 & 8987734 & 12 \\
\hline 1695 & 54085 & 35831 & 20 & 0.03 & 62.71 & 0.094094 & 0.075075 & 12 & 55002053 & 12 & 55962023 & 12 \\
\hline 721 & 46731 & 22211 & 29 & 0.03 & 62.74 & 0.147147 & 0.088088 & 4 & 98016950 & 4 & 98986789 & 4 \\
\hline 2354 & 60959 & 44566 & 25 & 0.03 & 62.78 & 0.122122 & 0.079079 & 17 & 32009043 & 17 & 32986542 & 17 \\
\hline 420 & 17253 & 61777 & 17 & 0.03 & 62.81 & 0.082082 & 0.052052 & 2 & 105029085 & 2 & 105980680 & 2 \\
\hline 468 & 21211 & 33815 & 32 & 0.03 & 62.84 & 0.139139 & 0.077077 & 2 & 153002555 & 2 & 153940974 & 2 \\
\hline 2344 & 13336 & 54404 & 27 & 0.03 & 62.88 & 0.104104 & 0.069069 & 17 & 22009494 & 17 & 22937340 & 17 \\
\hline 1676 & 25826 & 31535 & 27 & 0.03 & 62.91 & 0.119119 & 0.069069 & 12 & 36015100 & 12 & 36829763 & 12 \\
\hline 10 & 58158 & 61852 & 32 & 0.03 & 62.94 & 0.135135 & 0.084084 & 1 & 10010333 & 1 & 10949247 & 1 \\
\hline 126 & 46036 & 36768 & 17 & 0.03 & 62.98 & 0.062062 & 0.048048 & 1 & 126271024 & 1 & 126961319 & 1 \\
\hline 597 & 21576 & 3312 & 36 & 0.03 & 63.01 & 0.143143 & 0.083083 & 3 & 119006428 & 3 & 119979845 & 3 \\
\hline 2209 & 27936 & 56680 & 26 & 0.03 & 63.04 & 0.115115 & 0.082082 & 15 & 132116033 & 15 & 132949028 & 15 \\
\hline 1724 & 32249 & 59457 & 27 & 0.03 & 63.08 & 0.126126 & 0.078078 & 13 & 20004436 & 13 & 20991970 & 13 \\
\hline 54 & 432 & 45902 & 18 & 0.03 & 63.11 & 0.091091 & 0.06006 & 1 & 54001826 & 1 & 54991599 & 1 \\
\hline 201 & 36874 & 20295 & 31 & 0.03 & 63.14 & 0.099099 & 0.062062 & 1 & 201005516 & 1 & 201988814 & 1 \\
\hline 1151 & 6606 & 6617 & 30 & 0.03 & 63.18 & 0.116116 & 0.075075 & 7 & 115014212 & 7 & 115998395 & 7 \\
\hline 2437 & 54052 & 18288 & 22 & 0.03 & 63.21 & 0.097097 & 0.061061 & 18 & 45142069 & 18 & 45949396 & 18 \\
\hline 717 & 4144 & 4156 & 28 & 0.03 & 63.24 & 0.115115 & 0.066066 & 4 & 94003968 & 4 & 94978683 & 4 \\
\hline 486 & 55141 & 60330 & 11 & 0.03 & 63.28 & 0.067067 & 0.035035 & 3 & 8021716 & 3 & 8941384 & 3 \\
\hline 343 & 54505 & 60363 & 39 & 0.03 & 63.31 & 0.156156 & 0.081081 & 2 & 27137409 & 2 & 27978087 & 2 \\
\hline 2367 & 44625 & 44633 & 27 & 0.03 & 63.34 & 0.12012 & 0.079079 & 17 & 45002413 & 17 & 45985972 & 17 \\
\hline 699 & 37460 & 46684 & 27 & 0.03 & 63.38 & 0.125125 & 0.088088 & 4 & 76004484 & 4 & 76985054 & 4 \\
\hline 2390 & 28760 & 15313 & 26 & 0.03 & 63.41 & 0.114114 & 0.067067 & 17 & 68031429 & 17 & 68990915 & 17 \\
\hline 1901 & 30701 & 51285 & 15 & 0.03 & 63.44 & 0.083083 & 0.053053 & 13 & 197079707 & 13 & 197960155 & 13 \\
\hline 1911 & 33808 & 49428 & 23 & 0.03 & 63.47 & 0.087087 & 0.066066 & 13 & 207108562 & 13 & 207969771 & 13 \\
\hline 359 & 48542 & 2241 & 33 & 0.03 & 63.51 & 0.154154 & 0.097097 & 2 & 43009789 & 2 & 43985424 & 2 \\
\hline 946 & 34221 & 45175 & 32 & 0.03 & 63.54 & 0.13013 & 0.082082 & 6 & 67024466 & 6 & 67975744 & 6 \\
\hline 2059 & 27422 & 11676 & 30 & 0.03 & 63.57 & 0.115115 & 0.07007 & 14 & 136018609 & 14 & 136939847 & 14 \\
\hline 603 & 15834 & 40935 & 23 & 0.03 & 63.61 & 0.094094 & 0.06006 & 3 & 125018914 & 3 & 125979839 & 3 \\
\hline 687 & 3910 & 3919 & 27 & 0.03 & 63.64 & 0.12012 & 0.07007 & 4 & 64137468 & 4 & 64983207 & 4 \\
\hline 760 & 60100 & 4539 & 34 & 0.03 & 63.67 & 0.146146 & 0.092092 & 4 & 137017974 & 4 & 137950544 & 4 \\
\hline 1279 & 47235 & 34695 & 22 & 0.03 & 63.7 & 0.086086 & 0.055055 & 8 & 108068948 & 8 & 108982801 & 8 \\
\hline
\end{tabular}




\begin{tabular}{|c|c|c|c|c|c|c|c|c|c|c|c|c|c|}
\hline 1323 & 16236 & 62053 & 18 & 0.03 & 63.74 & 0.084084 & 0.059059 & 9 & 3016833 & 9 & 3993696 & 9 & 3 \\
\hline 365 & 37111 & 30623 & 20 & 0.03 & 63.77 & 0.094094 & 0.067067 & 2 & 49185691 & 2 & 49903368 & 2 & 49 \\
\hline 411 & 56707 & 20980 & 18 & 0.03 & 63.8 & 0.08008 & 0.055055 & 2 & 96053472 & 2 & 96992420 & 2 & 96 \\
\hline 1977 & 55282 & 57093 & 15 & 0.03 & 63.83 & 0.066066 & 0.044044 & 14 & 54006581 & 14 & 54867498 & 14 & 54 \\
\hline 1930 & 10620 & 36194 & 25 & 0.03 & 63.87 & 0.114114 & 0.078078 & 14 & 7028801 & 14 & 7976149 & 14 & 7 \\
\hline 1254 & 7134 & 7138 & 17 & 0.03 & 63.9 & 0.088088 & 0.054054 & 8 & 83018908 & 8 & 83968789 & 8 & 83 \\
\hline 309 & 56395 & 1963 & 25 & 0.03 & 63.93 & 0.111111 & 0.059059 & 1 & 309037070 & 1 & 309932123 & 1 & 309 \\
\hline 957 & 17263 & 56288 & 21 & 0.03 & 63.96 & 0.084084 & 0.059059 & 6 & 78048683 & 6 & 78938720 & 6 & 78 \\
\hline 74 & 36678 & 19858 & 25 & 0.03 & 64 & 0.121121 & 0.084084 & 1 & 74019662 & 1 & 74974844 & 1 & 74 \\
\hline 1778 & 16584 & 32736 & 22 & 0.03 & 64.03 & 0.087087 & 0.069069 & 13 & 74047780 & 13 & 74874236 & 13 & 74 \\
\hline 1064 & 23420 & 23424 & 29 & 0.03 & 64.06 & 0.142142 & 0.093093 & 7 & 27019705 & 7 & 27999311 & 7 & 27 \\
\hline 901 & 41616 & 34796 & 15 & 0.03 & 64.09 & 0.067067 & 0.052052 & 6 & 22021386 & 6 & 22868204 & 6 & 22 \\
\hline 1580 & 42962 & 8784 & 22 & 0.03 & 64.12 & 0.097097 & 0.068068 & 11 & 28019183 & 11 & 28992353 & 11 & 28 \\
\hline 895 & 17873 & 15478 & 22 & 0.03 & 64.16 & 0.115115 & 0.079079 & 6 & 16013020 & 6 & 16859988 & 6 & 16 \\
\hline 558 & 3105 & 21456 & 19 & 0.03 & 64.19 & 0.076076 & 0.055055 & 3 & 80053531 & 3 & 80978886 & 3 & 80 \\
\hline 1907 & 26535 & 26540 & 28 & 0.03 & 64.22 & 0.127127 & 0.078078 & 13 & 203080799 & 13 & 203989627 & 13 & 203 \\
\hline 2363 & 13478 & 13488 & 35 & 0.03 & 64.25 & 0.155155 & 0.09009 & 17 & 41034762 & 17 & 41957670 & 17 & 41 \\
\hline 438 & 21082 & 32175 & 23 & 0.03 & 64.29 & 0.107107 & 0.072072 & 2 & 123010574 & 2 & 123982380 & 2 & 123 \\
\hline 2497 & 44927 & 30069 & 14 & 0.03 & 64.32 & 0.08008 & 0.061061 & 19 & 44092699 & 19 & 44988606 & 19 & 44 \\
\hline 2407 & 28818 & 13743 & 19 & 0.03 & 64.35 & 0.094094 & 0.061061 & 18 & 15079181 & 18 & 15991827 & 18 & 15 \\
\hline 1046 & 37854 & 5772 & 30 & 0.03 & 64.38 & 0.127127 & 0.078078 & 7 & 9003631 & 7 & 9976814 & 7 & 9 \\
\hline 251 & 60174 & 1566 & 21 & 0.03 & 64.41 & 0.081081 & 0.044044 & 1 & 251044409 & 1 & 251957006 & 1 & 251 \\
\hline 2105 & 27663 & 59905 & 21 & 0.03 & 64.45 & 0.09009 & 0.067067 & 15 & 28020404 & 15 & 28991507 & 15 & 28 \\
\hline 1299 & 39715 & 33939 & 21 & 0.03 & 64.48 & 0.106106 & 0.069069 & 8 & 128039415 & 8 & 128966268 & 8 & 128 \\
\hline 1352 & 51795 & 33612 & 27 & 0.03 & 64.51 & 0.112112 & 0.071071 & 9 & 32073965 & 9 & 32928892 & 9 & 32 \\
\hline 1128 & 38033 & 23780 & 34 & 0.03 & 64.54 & 0.13013 & 0.086086 & 7 & 92008578 & 7 & 92964028 & 7 & 92 \\
\hline 1196 & 18440 & 24113 & 9 & 0.03 & 64.57 & 0.056056 & 0.037037 & 8 & 25020120 & 8 & 25948033 & 8 & 25 \\
\hline 698 & 22082 & 48720 & 20 & 0.03 & 64.61 & 0.094094 & 0.058058 & 4 & 75016304 & 4 & 75991872 & 4 & 75 \\
\hline 1367 & 19193 & 24642 & 29 & 0.03 & 64.64 & 0.104104 & 0.072072 & 9 & 47035782 & 9 & 47994172 & 9 & 47 \\
\hline 356 & 2192 & 48540 & 23 & 0.03 & 64.67 & 0.108108 & 0.071071 & 2 & 40072508 & 2 & 40993644 & 2 & 40 \\
\hline 1346 & 52819 & 30658 & 32 & 0.03 & 64.7 & 0.111111 & 0.069069 & 9 & 26005437 & 9 & 26987809 & 9 & 26 \\
\hline 41 & 334 & 36576 & 25 & 0.03 & 64.73 & 0.111111 & 0.069069 & 1 & 41017553 & 1 & 41894961 & 1 & 41 \\
\hline 348 & 20826 & 2148 & 31 & 0.03 & 64.76 & 0.127127 & 0.092092 & 2 & 32027800 & 2 & 32993535 & 2 & 32 \\
\hline 1329 & 42430 & 24481 & 26 & 0.03 & 64.8 & 0.102102 & 0.071071 & 9 & 9019463 & 9 & 9995702 & 9 & 9 \\
\hline 2404 & 13720 & 35278 & 28 & 0.03 & 64.83 & 0.109109 & 0.072072 & 18 & 12050532 & 18 & 12981450 & 18 & 12 \\
\hline 759 & 55823 & 22436 & 23 & 0.03 & 64.86 & 0.108108 & 0.07007 & 4 & 136004128 & 4 & 136979735 & 4 & 136 \\
\hline 2242 & 49614 & 39381 & 11 & 0.03 & 64.89 & 0.07007 & 0.048048 & 16 & 7013478 & 16 & 7634155 & 16 & 7 \\
\hline 2273 & 12883 & 12890 & 27 & 0.03 & 64.92 & 0.123123 & 0.088088 & 16 & 38004706 & 16 & 38989787 & 16 & 38 \\
\hline 1729 & 16980 & 32836 & 32 & 0.03 & 64.95 & 0.125125 & 0.082082 & 13 & 25073183 & 13 & 25929578 & 13 & 25 \\
\hline
\end{tabular}




\begin{tabular}{|c|c|c|c|c|c|c|c|c|c|c|c|c|c|}
\hline 1863 & 52197 & 26432 & 20 & 0.03 & 64.99 & 0.079079 & 0.057057 & 13 & 159004143 & 13 & 159985503 & 13 & 159 \\
\hline 860 & 19256 & 5107 & 26 & 0.03 & 65.02 & 0.108108 & 0.062062 & 5 & 93029076 & 5 & 93996098 & 5 & 93 \\
\hline 421 & 17823 & 55280 & 18 & 0.03 & 65.05 & 0.066066 & 0.047047 & 2 & 106206301 & 2 & 106982661 & 2 & 106 \\
\hline 2172 & 14575 & 48272 & 19 & 0.03 & 65.08 & 0.07007 & 0.046046 & 15 & 95042255 & 15 & 95919710 & 15 & 95 \\
\hline 1503 & 25131 & 8374 & 17 & 0.03 & 65.11 & 0.084084 & 0.051051 & 10 & 29045919 & 10 & 29991683 & 10 & 29 \\
\hline 2018 & 11327 & 51323 & 19 & 0.03 & 65.14 & 0.093093 & 0.066066 & 14 & 95195496 & 14 & 95961878 & 14 & 95 \\
\hline 2055 & 27399 & 27405 & 36 & 0.03 & 65.17 & 0.125125 & 0.073073 & 14 & 132022319 & 14 & 132972206 & 14 & 132 \\
\hline 1053 & 5817 & 37878 & 28 & 0.03 & 65.2 & 0.123123 & 0.091091 & 7 & 16062680 & 7 & 16974151 & 7 & 16 \\
\hline 507 & 15353 & 15494 & 29 & 0.03 & 65.24 & 0.121121 & 0.072072 & 3 & 29024154 & 3 & 29894909 & 3 & 29 \\
\hline 8 & 19474 & 19481 & 32 & 0.03 & 65.27 & 0.148148 & 0.083083 & 1 & 8004890 & 1 & 8988180 & 1 & 8 \\
\hline 1358 & 7645 & 7660 & 35 & 0.03 & 65.3 & 0.127127 & 0.083083 & 9 & 38006578 & 9 & 38993114 & 9 & 38 \\
\hline 1669 & 34952 & 17691 & 9 & 0.03 & 65.33 & 0.064064 & 0.047047 & 12 & 29022063 & 12 & 29700187 & 12 & 29 \\
\hline 1694 & 53957 & 9421 & 13 & 0.03 & 65.36 & 0.073073 & 0.054054 & 12 & 54003633 & 12 & 54837708 & 12 & 54 \\
\hline 2449 & 29007 & 14015 & 21 & 0.03 & 65.39 & 0.08008 & 0.054054 & 18 & 57033078 & 18 & 57957917 & 18 & 57 \\
\hline 278 & 1735 & 46383 & 25 & 0.03 & 65.42 & 0.107107 & 0.07007 & 1 & 278256541 & 1 & 278989901 & 1 & 278 \\
\hline 2030 & 11431 & 11442 & 28 & 0.03 & 65.45 & 0.123123 & 0.082082 & 14 & 107033485 & 14 & 107993512 & 14 & 107 \\
\hline 950 & 14987 & 45195 & 35 & 0.03 & 65.49 & 0.121121 & 0.077077 & 6 & 71001575 & 6 & 71995060 & 6 & 71 \\
\hline 2188 & 12409 & 35070 & 20 & 0.03 & 65.52 & 0.09009 & 0.056056 & 15 & 111088143 & 15 & 111917903 & 15 & 111 \\
\hline 973 & 18094 & 41675 & 22 & 0.03 & 65.55 & 0.1001 & 0.067067 & 6 & 94015473 & 6 & 94838066 & 6 & 94 \\
\hline 2278 & 12918 & 53338 & 8 & 0.03 & 65.58 & 0.053053 & 0.04004 & 16 & 43093614 & 16 & 43762162 & 16 & 43 \\
\hline 801 & 17044 & 4790 & 36 & 0.03 & 65.61 & 0.139139 & 0.087087 & 5 & 34011426 & 5 & 34945860 & 5 & 34 \\
\hline 1736 & 9705 & 26055 & 30 & 0.03 & 65.64 & 0.112112 & 0.075075 & 13 & 32068194 & 13 & 32997777 & 13 & 32 \\
\hline 69 & 549 & 553 & 22 & 0.03 & 65.67 & 0.115115 & 0.07007 & 1 & 69039001 & 1 & 69968051 & 1 & 69 \\
\hline 1347 & 7606 & 33018 & 27 & 0.03 & 65.7 & 0.113113 & 0.074074 & 9 & 27001512 & 9 & 27995647 & 9 & 27 \\
\hline 1894 & 38943 & 57167 & 24 & 0.03 & 65.73 & 0.123123 & 0.071071 & 13 & 190029235 & 13 & 190969594 & 13 & 190 \\
\hline 1795 & 35327 & 38815 & 28 & 0.03 & 65.76 & 0.103103 & 0.071071 & 13 & 91031788 & 13 & 91993044 & 13 & 91 \\
\hline 2057 & 59279 & 11648 & 27 & 0.03 & 65.8 & 0.127127 & 0.078078 & 14 & 134026947 & 14 & 134988252 & 14 & 134 \\
\hline 2306 & 28315 & 31773 & 25 & 0.03 & 65.83 & 0.115115 & 0.062062 & 16 & 71043012 & 16 & 71984043 & 16 & 71 \\
\hline 913 & 40575 & 14993 & 18 & 0.03 & 65.86 & 0.075075 & 0.05005 & 6 & 34031908 & 6 & 34960452 & 6 & 34 \\
\hline 661 & 3731 & 3735 & 20 & 0.03 & 65.89 & 0.074074 & 0.049049 & 4 & 38015849 & 4 & 38985579 & 4 & 38 \\
\hline 537 & 21384 & 21386 & 18 & 0.03 & 65.92 & 0.092092 & 0.058058 & 3 & 59012808 & 3 & 59995247 & 3 & 59 \\
\hline 755 & 4485 & 17260 & 33 & 0.03 & 65.95 & 0.133133 & 0.091091 & 4 & 132039333 & 4 & 132970877 & 4 & 132 \\
\hline 2442 & 44820 & 35514 & 27 & 0.03 & 65.98 & 0.109109 & 0.081081 & 18 & 50016394 & 18 & 50984974 & 18 & 50 \\
\hline 418 & 34648 & 46451 & 21 & 0.03 & 66.01 & 0.081081 & 0.06006 & 2 & 103008035 & 2 & 103803677 & 2 & 103 \\
\hline 807 & 51608 & 37599 & 12 & 0.03 & 66.04 & 0.074074 & 0.05005 & 5 & 40106617 & 5 & 40915894 & 5 & 40 \\
\hline 2210 & 27944 & 27951 & 29 & 0.03 & 66.07 & 0.098098 & 0.069069 & 15 & 133052815 & 15 & 133970166 & 15 & 133 \\
\hline 1152 & 51192 & 6632 & 34 & 0.03 & 66.1 & 0.145145 & 0.091091 & 7 & 116032422 & 7 & 116954912 & 7 & 116 \\
\hline 1903 & 36152 & 10488 & 27 & 0.03 & 66.13 & 0.11011 & 0.068068 & 13 & 199017905 & 13 & 199927172 & 13 & 199 \\
\hline 1836 & 51019 & 10186 & 24 & 0.03 & 66.17 & 0.106106 & 0.07007 & 13 & 132017643 & 13 & 132985654 & 13 & 132 \\
\hline
\end{tabular}




\begin{tabular}{|c|c|c|c|c|c|c|c|c|c|c|c|c|c|}
\hline 1146 & 23876 & 38050 & 22 & 0.03 & 66.2 & 0.101101 & 0.06006 & 7 & 110024659 & 7 & 110993314 & 7 & 110 \\
\hline 538 & 40825 & 3023 & 22 & 0.03 & 66.23 & 0.106106 & 0.075075 & 3 & 60015652 & 3 & 60998171 & 3 & 60 \\
\hline 1066 & 35927 & 5911 & 26 & 0.03 & 66.26 & 0.108108 & 0.073073 & 7 & 29040724 & 7 & 29987393 & 7 & 29 \\
\hline 738 & 4324 & 60684 & 25 & 0.03 & 66.29 & 0.083083 & 0.05005 & 4 & 115009742 & 4 & 115961449 & 4 & 115 \\
\hline 1728 & 9640 & 31794 & 33 & 0.03 & 66.32 & 0.127127 & 0.074074 & 13 & 24026709 & 13 & 24969288 & 13 & 24 \\
\hline 140 & 977 & 20087 & 18 & 0.03 & 66.35 & 0.076076 & 0.054054 & 1 & 140022043 & 1 & 140842490 & 1 & 140 \\
\hline 245 & 36368 & 46306 & 24 & 0.03 & 66.38 & 0.086086 & 0.045045 & 1 & 245008421 & 1 & 245981247 & 1 & 245 \\
\hline 990 & 57369 & 51975 & 16 & 0.03 & 66.41 & 0.091091 & 0.057057 & 6 & 111047373 & 6 & 111974448 & 6 & 111 \\
\hline 509 & 15333 & 51401 & 26 & 0.03 & 66.44 & 0.132132 & 0.08008 & 3 & 31012875 & 3 & 31931466 & 3 & 31 \\
\hline 628 & 21699 & 21709 & 29 & 0.03 & 66.47 & 0.128128 & 0.082082 & 4 & 5006687 & 4 & 5985544 & 4 & 5 \\
\hline 1205 & 60701 & 53391 & 29 & 0.03 & 66.5 & 0.128128 & 0.078078 & 8 & 34026040 & 8 & 34996041 & 8 & 34 \\
\hline 2192 & 12422 & 44235 & 15 & 0.03 & 66.53 & 0.076076 & 0.05005 & 15 & 115026418 & 15 & 115836301 & 15 & 115 \\
\hline 672 & 17290 & 46616 & 9 & 0.03 & 66.56 & 0.069069 & 0.045045 & 4 & 49093079 & 4 & 49917991 & 4 & 49 \\
\hline 1741 & 61426 & 43314 & 22 & 0.03 & 66.59 & 0.1001 & 0.073073 & 13 & 37122998 & 13 & 37987928 & 13 & 37 \\
\hline 2346 & 13351 & 28499 & 24 & 0.03 & 66.62 & 0.102102 & 0.069069 & 17 & 24013333 & 17 & 24856383 & 17 & 24 \\
\hline 900 & 5287 & 46901 & 27 & 0.03 & 66.65 & 0.133133 & 0.083083 & 6 & 21014463 & 6 & 21988030 & 6 & 21 \\
\hline 471 & 57006 & 61257 & 26 & 0.03 & 66.68 & 0.114114 & 0.076076 & 2 & 156071705 & 2 & 156982877 & 2 & 156 \\
\hline 2074 & 49549 & 56774 & 36 & 0.03 & 66.71 & 0.154154 & 0.087087 & 14 & 151015261 & 14 & 151993696 & 14 & 151 \\
\hline 2024 & 39109 & 47718 & 23 & 0.03 & 66.74 & 0.084084 & 0.053053 & 14 & 101124119 & 14 & 101995631 & 14 & 101 \\
\hline 800 & 4774 & 50667 & 20 & 0.03 & 66.77 & 0.089089 & 0.05005 & 5 & 33007354 & 5 & 33957565 & 5 & 33 \\
\hline 2573 & 29194 & 29203 & 31 & 0.03 & 66.8 & 0.116116 & 0.062062 & 19 & 125006304 & 19 & 125972086 & 19 & 125 \\
\hline 580 & 46511 & 40883 & 25 & 0.03 & 66.83 & 0.107107 & 0.067067 & 3 & 102090846 & 3 & 102994163 & 3 & 102 \\
\hline 2235 & 44393 & 12650 & 20 & 0.03 & 66.87 & 0.098098 & 0.069069 & 16 & 15148 & 16 & 973962 & 16 & 0 \\
\hline 439 & 15493 & 54534 & 31 & 0.03 & 66.9 & 0.132132 & 0.086086 & 2 & 124021778 & 2 & 124984584 & 2 & 124 \\
\hline 626 & 40968 & 59395 & 23 & 0.03 & 66.93 & 0.105105 & 0.063063 & 4 & 3004624 & 4 & 3984843 & 4 & 3 \\
\hline 367 & 49893 & 46435 & 23 & 0.03 & 66.96 & 0.095095 & 0.064064 & 2 & 51003535 & 2 & 51995031 & 2 & 51 \\
\hline 1311 & 30826 & 56282 & 22 & 0.03 & 66.99 & 0.116116 & 0.081081 & 8 & 140246531 & 8 & 140986050 & 8 & 140 \\
\hline 2182 & 12389 & 31116 & 10 & 0.03 & 67.02 & 0.053053 & 0.042042 & 15 & 105198145 & 15 & 105993430 & 15 & 105 \\
\hline 2048 & 39150 & 11581 & 19 & 0.03 & 67.05 & 0.092092 & 0.062062 & 14 & 125016745 & 14 & 125978735 & 14 & 125 \\
\hline 460 & 31325 & 35487 & 31 & 0.03 & 67.08 & 0.113113 & 0.068068 & 2 & 145012848 & 2 & 145963826 & 2 & 145 \\
\hline 2094 & 27624 & 56383 & 27 & 0.03 & 67.11 & 0.113113 & 0.061061 & 15 & 17030766 & 15 & 17976658 & 15 & 17 \\
\hline 1312 & 24406 & 7430 & 32 & 0.03 & 67.14 & 0.153153 & 0.093093 & 8 & 141003882 & 8 & 141980614 & 8 & 141 \\
\hline 1221 & 24189 & 7023 & 18 & 0.03 & 67.17 & 0.094094 & 0.061061 & 8 & 50029364 & 8 & 50863927 & 8 & 50 \\
\hline 1982 & 43779 & 51270 & 23 & 0.03 & 67.2 & 0.102102 & 0.072072 & 14 & 59004595 & 14 & 59986607 & 14 & 59 \\
\hline 741 & 22325 & 17379 & 28 & 0.03 & 67.23 & 0.113113 & 0.071071 & 4 & 118076498 & 4 & 118994554 & 4 & 118 \\
\hline 1931 & 43619 & 10635 & 24 & 0.03 & 67.26 & 0.091091 & 0.064064 & 14 & 8228429 & 14 & 8993832 & 14 & 8 \\
\hline 567 & 60904 & 3143 & 22 & 0.03 & 67.29 & 0.093093 & 0.062062 & 3 & 89138287 & 3 & 89999192 & 3 & 89 \\
\hline 2378 & 49689 & 28679 & 24 & 0.03 & 67.32 & 0.116116 & 0.074074 & 17 & 56020741 & 17 & 56981167 & 17 & 56 \\
\hline 952 & 29480 & 23030 & 23 & 0.03 & 67.35 & 0.105105 & 0.063063 & 6 & 73127988 & 6 & 73980831 & 6 & 73 \\
\hline
\end{tabular}




\begin{tabular}{|c|c|c|c|c|c|c|c|c|c|c|c|c|c|}
\hline 2302 & 59624 & 61393 & 13 & 0.03 & 67.38 & 0.061061 & 0.034034 & 16 & 67092208 & 16 & 67897442 & 16 & 67 \\
\hline 616 & 3390 & 54821 & 22 & 0.03 & 67.4 & 0.094094 & 0.06006 & 3 & 138053335 & 3 & 138992352 & 3 & 138 \\
\hline 2324 & 13194 & 13200 & 22 & 0.03 & 67.43 & 0.086086 & 0.054054 & 17 & 2011617 & 17 & 2991834 & 17 & 2 \\
\hline 1839 & 10202 & 10205 & 14 & 0.03 & 67.46 & 0.073073 & 0.053053 & 13 & 135034976 & 13 & 135995420 & 13 & 135 \\
\hline 1195 & 29447 & 16102 & 18 & 0.03 & 67.49 & 0.08008 & 0.057057 & 8 & 24033377 & 8 & 24901498 & 8 & 24 \\
\hline 692 & 22049 & 41123 & 26 & 0.03 & 67.52 & 0.104104 & 0.066066 & 4 & 69018203 & 4 & 69896058 & 4 & 69 \\
\hline 497 & 35761 & 2845 & 27 & 0.03 & 67.55 & 0.125125 & 0.07007 & 3 & 19077421 & 3 & 19951828 & 3 & 19 \\
\hline 405 & 2374 & 40607 & 27 & 0.03 & 67.58 & 0.121121 & 0.083083 & 2 & 90053305 & 2 & 90971450 & 2 & 90 \\
\hline 327 & 33139 & 15348 & 19 & 0.03 & 67.61 & 0.092092 & 0.063063 & 2 & 11066709 & 2 & 11888058 & 2 & 11 \\
\hline 2062 & 27447 & 11707 & 28 & 0.03 & 67.64 & 0.133133 & 0.082082 & 14 & 139040157 & 14 & 139971668 & 14 & 139 \\
\hline 691 & 34624 & 52522 & 22 & 0.03 & 67.67 & 0.101101 & 0.074074 & 4 & 68024939 & 4 & 68766745 & 4 & 68 \\
\hline 81 & 53680 & 52031 & 23 & 0.03 & 67.7 & 0.098098 & 0.057057 & 1 & 81036664 & 1 & 81998047 & 1 & 81 \\
\hline 2388 & 28744 & 49711 & 24 & 0.03 & 67.73 & 0.108108 & 0.075075 & 17 & 66020349 & 17 & 66966792 & 17 & 66 \\
\hline 1757 & 9835 & 59902 & 23 & 0.03 & 67.76 & 0.102102 & 0.07007 & 13 & 53002465 & 13 & 53914708 & 13 & 53 \\
\hline 809 & 37602 & 41457 & 15 & 0.03 & 67.79 & 0.054054 & 0.038038 & 5 & 42081631 & 5 & 42945018 & 5 & 42 \\
\hline 667 & 61805 & 3782 & 27 & 0.03 & 67.82 & 0.115115 & 0.077077 & 4 & 44003320 & 4 & 44997961 & 4 & 44 \\
\hline 2224 & 28039 & 12623 & 23 & 0.03 & 67.85 & 0.102102 & 0.056056 & 15 & 147013651 & 15 & 147944719 & 15 & 147 \\
\hline 14 & 51106 & 114 & 28 & 0.03 & 67.88 & 0.125125 & 0.083083 & 1 & 14038761 & 1 & 14874949 & 1 & 14 \\
\hline 1531 & 8506 & 8509 & 23 & 0.03 & 67.91 & 0.1001 & 0.053053 & 10 & 57044576 & 10 & 57997759 & 10 & 57 \\
\hline 867 & 35078 & 52364 & 21 & 0.03 & 67.94 & 0.109109 & 0.072072 & 5 & 100037065 & 5 & 100881229 & 5 & 100 \\
\hline 2090 & 27601 & 27609 & 20 & 0.03 & 67.97 & 0.087087 & 0.061061 & 15 & 13000324 & 15 & 13990176 & 15 & 13 \\
\hline 1938 & 26709 & 61669 & 30 & 0.03 & 68 & 0.128128 & 0.085085 & 14 & 15020070 & 14 & 15993135 & 14 & 15 \\
\hline 2358 & 28563 & 44588 & 30 & 0.03 & 68.03 & 0.128128 & 0.069069 & 17 & 36007878 & 17 & 36999204 & 17 & 36 \\
\hline 728 & 4233 & 4240 & 26 & 0.03 & 68.05 & 0.099099 & 0.067067 & 4 & 105017514 & 4 & 105998663 & 4 & 105 \\
\hline 2240 & 44315 & 14603 & 27 & 0.03 & 68.08 & 0.109109 & 0.066066 & 16 & 5008766 & 16 & 5923147 & 16 & 5 \\
\hline 1031 & 34144 & 23224 & 28 & 0.03 & 68.11 & 0.104104 & 0.068068 & 6 & 152208520 & 6 & 152976959 & 6 & 152 \\
\hline 2272 & 61969 & 28200 & 25 & 0.03 & 68.14 & 0.106106 & 0.068068 & 16 & 37039866 & 16 & 37966104 & 16 & 37 \\
\hline 1387 & 33254 & 51176 & 20 & 0.03 & 68.17 & 0.085085 & 0.066066 & 9 & 67063113 & 9 & 67814275 & 9 & 67 \\
\hline 2009 & 11258 & 35673 & 22 & 0.03 & 68.2 & 0.083083 & 0.056056 & 14 & 86073571 & 14 & 86979241 & 14 & 86 \\
\hline 1749 & 38737 & 9783 & 27 & 0.03 & 68.23 & 0.1001 & 0.055055 & 13 & 45053798 & 13 & 45985901 & 13 & 45 \\
\hline 1717 & 52035 & 17912 & 28 & 0.03 & 68.26 & 0.098098 & 0.072072 & 13 & 13026095 & 13 & 13971542 & 13 & 13 \\
\hline 2031 & 27273 & 11451 & 21 & 0.03 & 68.29 & 0.101101 & 0.068068 & 14 & 108016508 & 14 & 108992984 & 14 & 108 \\
\hline 412 & 40618 & 2422 & 18 & 0.03 & 68.32 & 0.086086 & 0.056056 & 2 & 97005674 & 2 & 97884804 & 2 & 97 \\
\hline 43 & 349 & 357 & 26 & 0.03 & 68.35 & 0.111111 & 0.071071 & 1 & 43045248 & 1 & 43986463 & 1 & 43 \\
\hline 1072 & 5961 & 23481 & 21 & 0.03 & 68.38 & 0.081081 & 0.053053 & 7 & 35002839 & 7 & 35973567 & 7 & 35 \\
\hline 2570 & 29188 & 31667 & 13 & 0.03 & 68.41 & 0.049049 & 0.034034 & 19 & 122081895 & 19 & 122863117 & 19 & 122 \\
\hline 2422 & 55319 & 13856 & 27 & 0.03 & 68.44 & 0.109109 & 0.073073 & 18 & 30091996 & 18 & 30963795 & 18 & 30 \\
\hline 305 & 48495 & 48498 & 42 & 0.03 & 68.47 & 0.165165 & 0.087087 & 1 & 305110723 & 1 & 305987689 & 1 & 305 \\
\hline 13 & 39783 & 19509 & 32 & 0.03 & 68.49 & 0.128128 & 0.08008 & 1 & 13005744 & 1 & 13972300 & 1 & 13 \\
\hline
\end{tabular}




\begin{tabular}{|c|c|c|c|c|c|c|c|c|c|c|c|c|c|}
\hline 2392 & 52114 & 45479 & 21 & 0.03 & 68.52 & 0.102102 & 0.057057 & 18 & 720 & 18 & 989459 & 18 & 0 \\
\hline 1664 & 25781 & 45651 & 32 & 0.03 & 68.55 & 0.129129 & 0.085085 & 12 & 24025660 & 12 & 24979525 & 12 & 24 \\
\hline 747 & 46767 & 4417 & 27 & 0.03 & 68.58 & 0.111111 & 0.069069 & 4 & 124043519 & 4 & 124982191 & 4 & 124 \\
\hline 156 & 20141 & 46108 & 29 & 0.03 & 68.61 & 0.117117 & 0.078078 & 1 & 156019595 & 1 & 156990269 & 1 & 156 \\
\hline 649 & 3626 & 61543 & 24 & 0.03 & 68.64 & 0.087087 & 0.061061 & 4 & 26012865 & 4 & 26994265 & 4 & 26 \\
\hline 1017 & 26600 & 46943 & 19 & 0.03 & 68.67 & 0.074074 & 0.051051 & 6 & 138009749 & 6 & 138921625 & 6 & 138 \\
\hline 989 & 60372 & 18638 & 21 & 0.03 & 68.7 & 0.102102 & 0.063063 & 6 & 110077183 & 6 & 110996934 & 6 & 110 \\
\hline 1137 & 42073 & 6502 & 29 & 0.03 & 68.73 & 0.112112 & 0.073073 & 7 & 101022085 & 7 & 101965379 & 7 & 101 \\
\hline 1524 & 8467 & 42833 & 23 & 0.03 & 68.76 & 0.1001 & 0.068068 & 10 & 50018944 & 10 & 50993659 & 10 & 50 \\
\hline 2440 & 13951 & 28968 & 29 & 0.03 & 68.78 & 0.121121 & 0.077077 & 18 & 48005643 & 18 & 48998765 & 18 & 48 \\
\hline 2405 & 28807 & 33208 & 29 & 0.03 & 68.81 & 0.139139 & 0.079079 & 18 & 13006832 & 18 & 13974542 & 18 & 13 \\
\hline 2029 & 39130 & 27265 & 20 & 0.03 & 68.84 & 0.094094 & 0.059059 & 14 & 106074877 & 14 & 106992825 & 14 & 106 \\
\hline 831 & 41491 & 37668 & 24 & 0.03 & 68.87 & 0.105105 & 0.071071 & 5 & 64000265 & 5 & 64971001 & 5 & 64 \\
\hline 843 & 22798 & 59361 & 15 & 0.03 & 68.9 & 0.082082 & 0.056056 & 5 & 76016635 & 5 & 76994567 & 5 & 76 \\
\hline 1102 & 23657 & 47057 & 24 & 0.03 & 68.93 & 0.091091 & 0.065065 & 7 & 65004224 & 7 & 65984458 & 7 & 65 \\
\hline 99 & 45981 & 760 & 22 & 0.03 & 68.96 & 0.093093 & 0.063063 & 1 & 99051689 & 1 & 99989884 & 1 & 99 \\
\hline 2312 & 56411 & 56478 & 29 & 0.03 & 68.99 & 0.117117 & 0.064064 & 16 & 77046740 & 16 & 77939638 & 16 & 77 \\
\hline 1869 & 33372 & 10325 & 13 & 0.03 & 69.01 & 0.059059 & 0.038038 & 13 & 165058078 & 13 & 165948194 & 13 & 165 \\
\hline 304 & 1935 & 54002 & 30 & 0.03 & 69.04 & 0.135135 & 0.07007 & 1 & 304042460 & 1 & 304994318 & 1 & 304 \\
\hline 648 & 37405 & 21866 & 27 & 0.03 & 69.07 & 0.124124 & 0.08008 & 4 & 25019533 & 4 & 25984211 & 4 & 25 \\
\hline 351 & 57275 & 37093 & 25 & 0.03 & 69.1 & 0.093093 & 0.063063 & 2 & 35054445 & 2 & 35962897 & 2 & 35 \\
\hline 435 & 56294 & 2570 & 17 & 0.03 & 69.13 & 0.077077 & 0.055055 & 2 & 120009260 & 2 & 120974703 & 2 & 120 \\
\hline 636 & 41007 & 3514 & 30 & 0.03 & 69.16 & 0.099099 & 0.067067 & 4 & 13008743 & 4 & 13903080 & 4 & 13 \\
\hline 466 & 19201 & 59062 & 29 & 0.03 & 69.19 & 0.118118 & 0.066066 & 2 & 151012311 & 2 & 151909224 & 2 & 151 \\
\hline 887 & 41597 & 52796 & 33 & 0.03 & 69.21 & 0.137137 & 0.081081 & 6 & 8019390 & 6 & 8966343 & 6 & 8 \\
\hline 2070 & 56792 & 11783 & 29 & 0.03 & 69.24 & 0.119119 & 0.079079 & 14 & 147003080 & 14 & 147903117 & 14 & 147 \\
\hline 1610 & 47386 & 8956 & 11 & 0.03 & 69.27 & 0.071071 & 0.05005 & 11 & 58007243 & 11 & 58988824 & 11 & 58 \\
\hline 1083 & 47007 & 23541 & 18 & 0.03 & 69.3 & 0.079079 & 0.057057 & 7 & 46295528 & 7 & 46996404 & 7 & 46 \\
\hline 750 & 4430 & 14793 & 21 & 0.03 & 69.33 & 0.097097 & 0.06006 & 4 & 127072671 & 4 & 127895687 & 4 & 127 \\
\hline 1437 & 7986 & 17888 & 14 & 0.03 & 69.36 & 0.069069 & 0.045045 & 9 & 117012701 & 9 & 117851340 & 9 & 117 \\
\hline 1055 & 5833 & 5843 & 33 & 0.03 & 69.38 & 0.115115 & 0.067067 & 7 & 18009952 & 7 & 18984509 & 7 & 18 \\
\hline 1939 & 31367 & 26720 & 31 & 0.03 & 69.41 & 0.131131 & 0.073073 & 14 & 16009830 & 14 & 16993535 & 14 & 16 \\
\hline 1200 & 6915 & 57731 & 27 & 0.03 & 69.44 & 0.115115 & 0.074074 & 8 & 29026480 & 8 & 29990918 & 8 & 29 \\
\hline 1058 & 5855 & 58462 & 29 & 0.03 & 69.47 & 0.134134 & 0.09009 & 7 & 21006494 & 7 & 21875537 & 7 & 21 \\
\hline 951 & 60140 & 17969 & 24 & 0.03 & 69.5 & 0.103103 & 0.065065 & 6 & 72055444 & 6 & 72949935 & 6 & 72 \\
\hline 1597 & 25501 & 38578 & 15 & 0.03 & 69.53 & 0.087087 & 0.063063 & 11 & 45049289 & 11 & 45992991 & 11 & 45 \\
\hline 578 & 21483 & 3187 & 26 & 0.03 & 69.55 & 0.101101 & 0.07007 & 3 & 100098050 & 3 & 100905327 & 3 & 100 \\
\hline 1127 & 6394 & 38034 & 27 & 0.03 & 69.58 & 0.109109 & 0.063063 & 7 & 91019546 & 7 & 91996584 & 7 & 91 \\
\hline 1671 & 55472 & 51029 & 17 & 0.03 & 69.61 & 0.08008 & 0.057057 & 12 & 31037865 & 12 & 31975933 & 12 & 31 \\
\hline
\end{tabular}




\begin{tabular}{|c|c|c|c|c|c|c|c|c|c|c|c|c|c|}
\hline 1691 & 9413 & 9414 & 17 & 0.03 & 69.64 & 0.083083 & 0.061061 & 12 & 51190108 & 12 & 51984256 & 12 & 51 \\
\hline 289 & 59996 & 40353 & 26 & 0.03 & 69.67 & 0.117117 & 0.067067 & 1 & 289048882 & 1 & 289983854 & 1 & 289 \\
\hline 1508 & 8391 & 25168 & 20 & 0.03 & 69.69 & 0.086086 & 0.06006 & 10 & 34168331 & 10 & 34945662 & 10 & 34 \\
\hline 1482 & 51292 & 25048 & 27 & 0.03 & 69.72 & 0.119119 & 0.076076 & 10 & 8003008 & 10 & 8965689 & 10 & 8 \\
\hline 1908 & 26541 & 16206 & 11 & 0.03 & 69.75 & 0.047047 & 0.04004 & 13 & 204016747 & 13 & 204969242 & 13 & 204 \\
\hline 1627 & 25614 & 36383 & 32 & 0.03 & 69.78 & 0.141141 & 0.087087 & 11 & 75009361 & 11 & 75996081 & 11 & 75 \\
\hline 185 & 1202 & 40165 & 32 & 0.03 & 69.81 & 0.138138 & 0.091091 & 1 & 185048329 & 1 & 185965822 & 1 & 185 \\
\hline 2451 & 56337 & 54740 & 15 & 0.03 & 69.83 & 0.083083 & 0.052052 & 18 & 59086075 & 18 & 59971337 & 18 & 59 \\
\hline 138 & 20078 & 40073 & 19 & 0.03 & 69.86 & 0.084084 & 0.064064 & 1 & 138050612 & 1 & 138927010 & 1 & 138 \\
\hline 768 & 29255 & 41348 & 26 & 0.03 & 69.89 & 0.087087 & 0.063063 & 5 & 1007763 & 5 & 1981830 & 5 & 1 \\
\hline 1337 & 15372 & 15154 & 29 & 0.03 & 69.92 & 0.107107 & 0.068068 & 9 & 17004309 & 9 & 17992016 & 9 & 17 \\
\hline 2128 & 60799 & 12113 & 22 & 0.03 & 69.95 & 0.096096 & 0.062062 & 15 & 51028020 & 15 & 51979193 & 15 & 51 \\
\hline 1681 & 51978 & 18544 & 27 & 0.03 & 69.97 & 0.106106 & 0.077077 & 12 & 41032283 & 12 & 41951866 & 12 & 41 \\
\hline 1522 & 25211 & 49249 & 33 & 0.03 & 70 & 0.129129 & 0.081081 & 10 & 48014763 & 10 & 48960017 & 10 & 48 \\
\hline 893 & 31738 & 5262 & 27 & 0.03 & 70.03 & 0.125125 & 0.071071 & 6 & 14016657 & 6 & 14947951 & 6 & 14 \\
\hline 2462 & 29042 & 14061 & 19 & 0.03 & 70.06 & 0.095095 & 0.062062 & 19 & 9077280 & 19 & 9970257 & 19 & 9 \\
\hline 80 & 58855 & 19881 & 25 & 0.03 & 70.09 & 0.121121 & 0.073073 & 1 & 80057576 & 1 & 80997487 & 1 & 80 \\
\hline 1157 & 23937 & 6673 & 18 & 0.03 & 70.11 & 0.078078 & 0.051051 & 7 & 121017867 & 7 & 121984372 & 7 & 121 \\
\hline 2448 & 28999 & 29008 & 24 & 0.03 & 70.14 & 0.122122 & 0.079079 & 18 & 56020136 & 18 & 56988923 & 18 & 56 \\
\hline 419 & 52106 & 36463 & 13 & 0.03 & 70.17 & 0.062062 & 0.047047 & 2 & 104256453 & 2 & 104830050 & 2 & 104 \\
\hline 1191 & 6858 & 24098 & 26 & 0.03 & 70.2 & 0.119119 & 0.075075 & 8 & 20003228 & 8 & 20948717 & 8 & 20 \\
\hline 2314 & 39478 & 28358 & 27 & 0.03 & 70.22 & 0.118118 & 0.068068 & 16 & 79012137 & 16 & 79991804 & 16 & 79 \\
\hline 1464 & 29448 & 8146 & 33 & 0.03 & 70.25 & 0.141141 & 0.072072 & 9 & 144006070 & 9 & 144965956 & 9 & 144 \\
\hline 613 & 40955 & 30011 & 32 & 0.03 & 70.28 & 0.14014 & 0.085085 & 3 & 135011301 & 3 & 135940918 & 3 & 135 \\
\hline 1426 & 38339 & 30177 & 17 & 0.03 & 70.31 & 0.073073 & 0.046046 & 9 & 106274400 & 9 & 106920454 & 9 & 106 \\
\hline 1560 & 47324 & 25377 & 28 & 0.03 & 70.34 & 0.115115 & 0.07007 & 11 & 8012239 & 11 & 8927330 & 11 & 8 \\
\hline 1318 & 15864 & 55548 & 25 & 0.03 & 70.36 & 0.108108 & 0.072072 & 8 & 147002408 & 8 & 147963935 & 8 & 147 \\
\hline 1755 & 9824 & 53467 & 20 & 0.03 & 70.39 & 0.083083 & 0.055055 & 13 & 51115180 & 13 & 51982923 & 13 & 51 \\
\hline 1740 & 43305 & 43310 & 28 & 0.03 & 70.42 & 0.113113 & 0.071071 & 13 & 36016140 & 13 & 36964009 & 13 & 36 \\
\hline 280 & 1752 & 40325 & 31 & 0.03 & 70.45 & 0.127127 & 0.072072 & 1 & 280005940 & 1 & 280937085 & 1 & 280 \\
\hline 820 & 4859 & 4862 & 13 & 0.03 & 70.47 & 0.057057 & 0.033033 & 5 & 53182979 & 5 & 53998123 & 5 & 53 \\
\hline 1805 & 52500 & 10098 & 17 & 0.03 & 70.5 & 0.078078 & 0.055055 & 13 & 101029096 & 13 & 101993885 & 13 & 101 \\
\hline 589 & 37330 & 3262 & 21 & 0.03 & 70.53 & 0.081081 & 0.063063 & 3 & 111033114 & 3 & 111986113 & 3 & 111 \\
\hline 1706 & 9470 & 43237 & 15 & 0.03 & 70.56 & 0.065065 & 0.043043 & 13 & 2049440 & 13 & 2947636 & 13 & 2 \\
\hline 1668 & 43163 & 14943 & 19 & 0.03 & 70.58 & 0.082082 & 0.056056 & 12 & 28071542 & 12 & 28779167 & 12 & 28 \\
\hline 1371 & 56630 & 14511 & 25 & 0.03 & 70.61 & 0.097097 & 0.074074 & 9 & 51113384 & 9 & 51965451 & 9 & 51 \\
\hline 1252 & 42309 & 16241 & 26 & 0.03 & 70.64 & 0.119119 & 0.066066 & 8 & 81017438 & 8 & 81998579 & 8 & 81 \\
\hline 437 & 40665 & 2591 & 27 & 0.03 & 70.67 & 0.115115 & 0.079079 & 2 & 122010013 & 2 & 122886290 & 2 & 122 \\
\hline 886 & 5242 & 5251 & 34 & 0.03 & 70.69 & 0.122122 & 0.081081 & 6 & 7000417 & 6 & 7943484 & 6 & 7 \\
\hline
\end{tabular}




\begin{tabular}{|c|c|c|c|c|c|c|c|c|c|c|c|c|c|}
\hline 1579 & 42964 & 54130 & 25 & 0.03 & 70.72 & 0.095095 & 0.062062 & 11 & 27007918 & 11 & 27954931 & 11 & 27 \\
\hline 1061 & 23410 & 56644 & 21 & 0.03 & 70.75 & 0.087087 & 0.066066 & 7 & 24017747 & 7 & 24848207 & 7 & 24 \\
\hline 1837 & 10187 & 34408 & 23 & 0.03 & 70.78 & 0.074074 & 0.054054 & 13 & 133032413 & 13 & 133979002 & 13 & 133 \\
\hline 1484 & 8271 & 42757 & 28 & 0.03 & 70.8 & 0.132132 & 0.072072 & 10 & 10002850 & 10 & 10995624 & 10 & 10 \\
\hline 575 & 32369 & 21475 & 19 & 0.03 & 70.83 & 0.091091 & 0.06006 & 3 & 97223093 & 3 & 97974345 & 3 & 97 \\
\hline 1 & 39749 & 17 & 23 & 0.03 & 70.86 & 0.096096 & 0.07007 & 1 & 1049272 & 1 & 1958514 & 1 & 1 \\
\hline 2490 & 53138 & 29090 & 16 & 0.03 & 70.89 & 0.058058 & 0.038038 & 19 & 37029211 & 19 & 37990102 & 19 & 37 \\
\hline 1538 & 8547 & 42859 & 33 & 0.03 & 70.91 & 0.112112 & 0.061061 & 10 & 64020297 & 10 & 64996997 & 10 & 64 \\
\hline 2184 & 59912 & 12400 & 14 & 0.03 & 70.94 & 0.06006 & 0.035035 & 15 & 107048443 & 15 & 107945927 & 15 & 107 \\
\hline 1615 & 38602 & 47398 & 13 & 0.03 & 70.97 & 0.062062 & 0.043043 & 11 & 63178157 & 11 & 63978325 & 11 & 63 \\
\hline 942 & 33525 & 53905 & 23 & 0.03 & 71 & 0.111111 & 0.072072 & 6 & 63053534 & 6 & 63986347 & 6 & 63 \\
\hline 2432 & 31687 & 44795 & 31 & 0.03 & 71.02 & 0.114114 & 0.074074 & 18 & 40067536 & 18 & 40992858 & 18 & 40 \\
\hline 1415 & 24811 & 53117 & 21 & 0.03 & 71.05 & 0.094094 & 0.063063 & 9 & 95008515 & 9 & 95973005 & 9 & 95 \\
\hline 1193 & 18779 & 59134 & 19 & 0.03 & 71.08 & 0.078078 & 0.053053 & 8 & 22007040 & 8 & 22904022 & 8 & 22 \\
\hline 1099 & 6208 & 41994 & 23 & 0.03 & 71.1 & 0.098098 & 0.06006 & 7 & 62010270 & 7 & 62954233 & 7 & 62 \\
\hline 1100 & 47042 & 41997 & 21 & 0.03 & 71.13 & 0.095095 & 0.057057 & 7 & 63015572 & 7 & 63961157 & 7 & 63 \\
\hline 445 & 52718 & 51230 & 14 & 0.03 & 71.16 & 0.074074 & 0.049049 & 2 & 130043084 & 2 & 130977850 & 2 & 130 \\
\hline 2317 & 44476 & 28376 & 39 & 0.03 & 71.19 & 0.139139 & 0.074074 & 16 & 82017160 & 16 & 82981442 & 16 & 82 \\
\hline 2300 & 44436 & 13031 & 17 & 0.03 & 71.21 & 0.089089 & 0.061061 & 16 & 65033887 & 16 & 65908026 & 16 & 65 \\
\hline 1033 & 23230 & 45268 & 28 & 0.03 & 71.24 & 0.096096 & 0.061061 & 6 & 154110204 & 6 & 154972467 & 6 & 154 \\
\hline 2052 & 11598 & 43981 & 19 & 0.03 & 71.27 & 0.081081 & 0.043043 & 14 & 129055323 & 14 & 129966461 & 14 & 129 \\
\hline 1986 & 39030 & 11073 & 20 & 0.03 & 71.29 & 0.07007 & 0.044044 & 14 & 63006966 & 14 & 63962796 & 14 & 63 \\
\hline 2049 & 11582 & 11590 & 20 & 0.03 & 71.32 & 0.1001 & 0.059059 & 14 & 126018137 & 14 & 126980313 & 14 & 126 \\
\hline 1105 & 6242 & 37965 & 21 & 0.03 & 71.35 & 0.078078 & 0.052052 & 7 & 68047313 & 7 & 68854659 & 7 & 68 \\
\hline 599 & 45286 & 16351 & 17 & 0.03 & 71.37 & 0.083083 & 0.059059 & 3 & 121035021 & 3 & 121972266 & 3 & 121 \\
\hline 1449 & 24889 & 24892 & 30 & 0.03 & 71.4 & 0.1001 & 0.08008 & 9 & 129005001 & 9 & 129988850 & 9 & 129 \\
\hline 1521 & 25204 & 25210 & 23 & 0.03 & 71.43 & 0.095095 & 0.06006 & 10 & 47025504 & 10 & 47958278 & 10 & 47 \\
\hline 1499 & 15273 & 25118 & 21 & 0.03 & 71.45 & 0.093093 & 0.065065 & 10 & 25028197 & 10 & 25912932 & 10 & 25 \\
\hline 1395 & 7807 & 7818 & 22 & 0.03 & 71.48 & 0.106106 & 0.071071 & 9 & 75067877 & 9 & 75973705 & 9 & 75 \\
\hline 2370 & 53621 & 28634 & 19 & 0.03 & 71.51 & 0.101101 & 0.05005 & 17 & 48108999 & 17 & 48985762 & 17 & 48 \\
\hline 83 & 53174 & 45952 & 23 & 0.03 & 71.53 & 0.096096 & 0.055055 & 1 & 83022518 & 1 & 83976962 & 1 & 83 \\
\hline 1261 & 24263 & 7167 & 19 & 0.03 & 71.56 & 0.083083 & 0.055055 & 8 & 90096395 & 8 & 90995810 & 8 & 90 \\
\hline 270 & 37003 & 59492 & 16 & 0.03 & 71.59 & 0.064064 & 0.045045 & 1 & 270102816 & 1 & 270881981 & 1 & 270 \\
\hline 1595 & 8852 & 54236 & 22 & 0.03 & 71.61 & 0.091091 & 0.061061 & 11 & 43085921 & 11 & 43951738 & 11 & 43 \\
\hline 2450 & 55697 & 29015 & 19 & 0.03 & 71.64 & 0.088088 & 0.049049 & 18 & 58006718 & 18 & 58958866 & 18 & 58 \\
\hline 427 & 2506 & 2509 & 16 & 0.03 & 71.67 & 0.085085 & 0.053053 & 2 & 112079833 & 2 & 112559732 & 2 & 112 \\
\hline 1742 & 38732 & 9749 & 24 & 0.03 & 71.69 & 0.103103 & 0.064064 & 13 & 38085096 & 13 & 38948602 & 13 & 38 \\
\hline 1257 & 32418 & 35245 & 17 & 0.03 & 71.72 & 0.072072 & 0.049049 & 8 & 86016419 & 8 & 86928240 & 8 & 86 \\
\hline 482 & 17466 & 16986 & 17 & 0.03 & 71.75 & 0.074074 & 0.043043 & 3 & 4032373 & 3 & 4980011 & 3 & 4 \\
\hline
\end{tabular}




\begin{tabular}{|c|c|c|c|c|c|c|c|c|c|c|c|c|c|}
\hline 335 & 15309 & 51754 & 25 & 0.03 & 71.77 & 0.106106 & 0.068068 & 2 & 19099121 & 2 & 19991449 & 2 & 19 \\
\hline 2220 & 55924 & 50445 & 24 & 0.03 & 71.8 & 0.101101 & 0.068068 & 15 & 143021121 & 15 & 143987209 & 15 & 143 \\
\hline 293 & 40360 & 20605 & 26 & 0.03 & 71.83 & 0.092092 & 0.056056 & 1 & 293020147 & 1 & 293991451 & 1 & 293 \\
\hline 1300 & 15673 & 17137 & 23 & 0.03 & 71.85 & 0.113113 & 0.068068 & 8 & 129127706 & 8 & 129878095 & 8 & 129 \\
\hline 1445 & 47290 & 24877 & 19 & 0.03 & 71.88 & 0.082082 & 0.057057 & 9 & 125100668 & 9 & 125928425 & 9 & 125 \\
\hline 1956 & 57718 & 26827 & 23 & 0.03 & 71.91 & 0.096096 & 0.062062 & 14 & 33040198 & 14 & 33959799 & 14 & 33 \\
\hline 2287 & 49627 & 28253 & 15 & 0.03 & 71.93 & 0.057057 & 0.042042 & 16 & 52012266 & 16 & 52903897 & 16 & 52 \\
\hline 2200 & 33559 & 12466 & 15 & 0.03 & 71.96 & 0.056056 & 0.037037 & 15 & 123052463 & 15 & 123902773 & 15 & 123 \\
\hline 1391 & 24746 & 45412 & 27 & 0.03 & 71.99 & 0.102102 & 0.06006 & 9 & 71170725 & 9 & 71989525 & 9 & 71 \\
\hline 1181 & 14756 & 14648 & 26 & 0.03 & 72.01 & 0.114114 & 0.078078 & 8 & 10036134 & 8 & 10993731 & 8 & 10 \\
\hline 395 & 54741 & 29454 & 12 & 0.03 & 72.04 & 0.053053 & 0.04004 & 2 & 80129606 & 2 & 80974584 & 2 & 80 \\
\hline 2297 & 44430 & 13014 & 16 & 0.03 & 72.07 & 0.082082 & 0.051051 & 16 & 62091147 & 16 & 62939945 & 16 & 62 \\
\hline 2214 & 12540 & 44255 & 23 & 0.03 & 72.09 & 0.102102 & 0.066066 & 15 & 137038624 & 15 & 137973386 & 15 & 137 \\
\hline 89 & 56625 & 51012 & 13 & 0.03 & 72.12 & 0.06006 & 0.041041 & 1 & 89210836 & 1 & 89896098 & 1 & 89 \\
\hline 2406 & 53817 & 61408 & 24 & 0.03 & 72.15 & 0.099099 & 0.061061 & 18 & 14053458 & 18 & 14934067 & 18 & 14 \\
\hline 1417 & 57174 & 38331 & 15 & 0.03 & 72.17 & 0.065065 & 0.048048 & 9 & 97051426 & 9 & 97912451 & 9 & 97 \\
\hline 1523 & 38463 & 25228 & 26 & 0.03 & 72.2 & 0.104104 & 0.071071 & 10 & 49042878 & 10 & 49993854 & 10 & 49 \\
\hline 1308 & 30257 & 14621 & 17 & 0.03 & 72.23 & 0.084084 & 0.058058 & 8 & 137006114 & 8 & 137855568 & 8 & 137 \\
\hline 1030 & 29748 & 58690 & 23 & 0.03 & 72.25 & 0.098098 & 0.069069 & 6 & 151053568 & 6 & 151865659 & 6 & 151 \\
\hline 159 & 20159 & 40116 & 28 & 0.03 & 72.28 & 0.112112 & 0.068068 & 1 & 159034424 & 1 & 159990673 & 1 & 159 \\
\hline 1035 & 61205 & 49847 & 27 & 0.03 & 72.3 & 0.105105 & 0.067067 & 6 & 156179469 & 6 & 156993286 & 6 & 156 \\
\hline 2092 & 54749 & 50716 & 23 & 0.03 & 72.33 & 0.097097 & 0.06006 & 15 & 15047103 & 15 & 15904702 & 15 & 15 \\
\hline 847 & 53969 & 16989 & 28 & 0.03 & 72.36 & 0.14014 & 0.076076 & 5 & 80009101 & 5 & 80967561 & 5 & 80 \\
\hline 1737 & 48353 & 43296 & 21 & 0.03 & 72.38 & 0.088088 & 0.067067 & 13 & 33023006 & 13 & 33967886 & 13 & 33 \\
\hline 979 & 31792 & 35888 & 21 & 0.03 & 72.41 & 0.091091 & 0.058058 & 6 & 100016231 & 6 & 100924422 & 6 & 100 \\
\hline 606 & 48654 & 21618 & 22 & 0.03 & 72.44 & 0.1001 & 0.063063 & 3 & 128135951 & 3 & 128988657 & 3 & 128 \\
\hline 545 & 54159 & 31836 & 26 & 0.03 & 72.46 & 0.088088 & 0.06006 & 3 & 67011470 & 3 & 67954066 & 3 & 67 \\
\hline 42 & 61252 & 348 & 20 & 0.03 & 72.49 & 0.082082 & 0.059059 & 1 & 42076935 & 1 & 42964863 & 1 & 42 \\
\hline 1592 & 59345 & 8847 & 13 & 0.03 & 72.51 & 0.066066 & 0.047047 & 11 & 40013884 & 11 & 40982195 & 11 & 40 \\
\hline 1338 & 15737 & 36164 & 16 & 0.03 & 72.54 & 0.078078 & 0.059059 & 9 & 18002888 & 9 & 18971973 & 9 & 18 \\
\hline 1785 & 43395 & 55189 & 20 & 0.03 & 72.57 & 0.082082 & 0.059059 & 13 & 81015331 & 13 & 81902962 & 13 & 81 \\
\hline 556 & 3098 & 30405 & 14 & 0.03 & 72.59 & 0.071071 & 0.05005 & 3 & 78029770 & 3 & 78944101 & 3 & 78 \\
\hline 2199 & 12455 & 45428 & 13 & 0.03 & 72.62 & 0.068068 & 0.053053 & 15 & 122166717 & 15 & 122998623 & 15 & 122 \\
\hline 1160 & 49073 & 14910 & 31 & 0.03 & 72.64 & 0.129129 & 0.072072 & 7 & 124018132 & 7 & 124941962 & 7 & 124 \\
\hline 2202 & 12471 & 12477 & 28 & 0.03 & 72.67 & 0.102102 & 0.057057 & 15 & 125026754 & 15 & 125988978 & 15 & 125 \\
\hline 1918 & 26587 & 26593 & 23 & 0.03 & 72.7 & 0.118118 & 0.069069 & 13 & 214005140 & 13 & 214973846 & 13 & 214 \\
\hline 179 & 1169 & 1177 & 24 & 0.03 & 72.72 & 0.097097 & 0.065065 & 1 & 179002367 & 1 & 179959509 & 1 & 179 \\
\hline 2217 & 49597 & 12580 & 33 & 0.03 & 72.75 & 0.133133 & 0.078078 & 15 & 140007776 & 15 & 140944699 & 15 & 140 \\
\hline 1789 & 10020 & 47465 & 12 & 0.03 & 72.77 & 0.065065 & 0.046046 & 13 & 85011406 & 13 & 85970514 & 13 & 85 \\
\hline
\end{tabular}




\begin{tabular}{|c|c|c|c|c|c|c|c|c|c|c|c|c|c|}
\hline 283 & 20534 & 40334 & 34 & 0.03 & 72.8 & 0.12012 & 0.066066 & 1 & 283010283 & 1 & 283959003 & 1 & 283 \\
\hline 1946 & 43663 & 43668 & 25 & 0.03 & 72.83 & 0.098098 & 0.063063 & 14 & 23027457 & 14 & 23992439 & 14 & \\
\hline 1563 & 59349 & 25394 & 25 & 0.03 & 72.85 & 0.117117 & 0.068068 & 11 & 11007618 & 11 & 11987246 & 11 & \\
\hline 1525 & 42834 & 8477 & 26 & 0.03 & 72.88 & 0.095095 & 0.062062 & 10 & 51028164 & 10 & 51975023 & 10 & \\
\hline 250 & 40257 & 46323 & 28 & 0.03 & 72.9 & 0.126126 & 0.035035 & 1 & 250018705 & 1 & 250959302 & 1 & \\
\hline 1271 & 24291 & 24294 & 16 & 0.03 & 72.93 & 0.076076 & 0.046046 & 8 & 100219507 & 8 & 100921095 & 8 & \\
\hline 660 & 52747 & 21926 & 25 & 0.03 & 72.95 & 0.092092 & 0.061061 & 4 & 37069150 & 4 & 37995302 & 4 & \\
\hline 2418 & 13810 & 28871 & 29 & 0.03 & 72.98 & 0.125125 & 0.072072 & 18 & 26004852 & 18 & 26977792 & 18 & \\
\hline 844 & 61670 & 37683 & 23 & 0.03 & 73.01 & 0.094094 & 0.067067 & 5 & 77016025 & 5 & 77994370 & 5 & \\
\hline 1339 & 33592 & 38230 & 26 & 0.03 & 73.03 & 0.109109 & 0.075075 & 9 & 19079143 & 9 & 19956110 & 9 & \\
\hline 2111 & 27681 & 12024 & 23 & 0.03 & 73.06 & 0.1001 & 0.067067 & 15 & 34128573 & 15 & 34973841 & 15 & \\
\hline 742 & 4366 & 22338 & 27 & 0.03 & 73.08 & 0.104104 & 0.07007 & 4 & 119022315 & 4 & 119974157 & 4 & \\
\hline 1713 & 51119 & 25952 & 26 & 0.03 & 73.11 & 0.114114 & 0.066066 & 13 & 9056247 & 13 & 9889880 & 13 & \\
\hline 177 & 1158 & 46156 & 18 & 0.03 & 73.13 & 0.07007 & 0.05005 & 1 & 177006345 & 1 & 177966091 & 1 & \\
\hline 2304 & 49638 & 28309 & 16 & 0.03 & 73.16 & 0.061061 & 0.046046 & 16 & 69042784 & 16 & 69938536 & 16 & \\
\hline 1039 & 52963 & 23259 & 27 & 0.03 & 73.19 & 0.097097 & 0.062062 & 7 & 2034057 & 7 & 2936612 & 7 & \\
\hline 1129 & 6407 & 23771 & 28 & 0.03 & 73.21 & 0.124124 & 0.074074 & 7 & 93005460 & 7 & 93885374 & 7 & J \\
\hline 1359 & 38257 & 14772 & 21 & 0.03 & 73.24 & 0.091091 & 0.066066 & 9 & 39034059 & 9 & 39882600 & 9 & 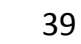 \\
\hline 1204 & 6944 & 6946 & 25 & 0.03 & 73.26 & 0.115115 & 0.082082 & 8 & 33033004 & 8 & 33985796 & 8 & \\
\hline 854 & 34683 & 31894 & 22 & 0.03 & 73.29 & 0.083083 & 0.057057 & 5 & 87048659 & 5 & 87950997 & 5 & 0 \\
\hline 1711 & 9505 & 50269 & 29 & 0.03 & 73.31 & 0.124124 & 0.072072 & 13 & 7091760 & 13 & 7981207 & 13 & \\
\hline 1660 & 18588 & 43151 & 15 & 0.03 & 73.34 & 0.075075 & 0.053053 & 12 & 20135020 & 12 & 20987983 & 12 & 20 \\
\hline 595 & 45806 & 3293 & 25 & 0.03 & 73.36 & 0.12012 & 0.074074 & 3 & 117022370 & 3 & 117988230 & 3 & 117 \\
\hline 2427 & 30201 & 30921 & 18 & 0.03 & 73.39 & 0.078078 & 0.052052 & 18 & 35243439 & 18 & 35993025 & 18 & 35 \\
\hline 559 & 21457 & 36248 & 16 & 0.03 & 73.41 & 0.074074 & 0.044044 & 3 & 81005314 & 3 & 81986256 & 3 & 81 \\
\hline 341 & 40493 & 2102 & 31 & 0.03 & 73.44 & 0.126126 & 0.083083 & 2 & 25056011 & 2 & 25977149 & 2 & 25 \\
\hline 337 & 40478 & 61121 & 25 & 0.03 & 73.46 & 0.11011 & 0.069069 & 2 & 21003130 & 2 & 21970774 & 2 & 2 \\
\hline 830 & 14362 & 4932 & 31 & 0.03 & 73.49 & 0.148148 & 0.086086 & 5 & 63039669 & 5 & 63996259 & 5 & 63 \\
\hline 539 & 36378 & 33523 & 21 & 0.03 & 73.51 & 0.068068 & 0.05005 & 3 & 61035420 & 3 & 61967071 & 3 & 6 \\
\hline 1824 & 26327 & 38849 & 13 & 0.03 & 73.54 & 0.05005 & 0.04004 & 13 & 120017132 & 13 & 120825463 & 13 & 120 \\
\hline 183 & 1189 & 1194 & 22 & 0.03 & 73.56 & 0.092092 & 0.052052 & 1 & 183094335 & 1 & 183971871 & 1 & 18 \\
\hline 2339 & 60615 & 48027 & 17 & 0.03 & 73.59 & 0.07007 & 0.041041 & 17 & 17004115 & 17 & 17972781 & 17 & 1 \\
\hline 2125 & 33919 & 47869 & 11 & 0.03 & 73.61 & 0.063063 & 0.04004 & 15 & 48336880 & 15 & 48970076 & 15 & 4 \\
\hline 1858 & 52073 & 58834 & 13 & 0.03 & 73.64 & 0.051051 & 0.029029 & 13 & 154021170 & 13 & 154899831 & 13 & 15 \\
\hline 1891 & 38928 & 10419 & 23 & 0.03 & 73.66 & 0.092092 & 0.064064 & 13 & 187024787 & 13 & 187968272 & 13 & 18 \\
\hline 495 & 19363 & 40764 & 16 & 0.03 & 73.69 & 0.083083 & 0.047047 & 3 & 17091063 & 3 & 17859317 & 3 & \\
\hline 1899 & 38951 & 30784 & 11 & 0.03 & 73.72 & 0.065065 & 0.051051 & 13 & 195007274 & 13 & 195871973 & 13 & 19 \\
\hline 1198 & 47191 & 42240 & 28 & 0.03 & 73.74 & 0.106106 & 0.066066 & 8 & 27009610 & 8 & 27992239 & 8 & \\
\hline 1383 & 24709 & 58649 & 21 & 0.03 & 73.77 & 0.094094 & 0.068068 & 9 & 63029187 & 9 & 63978822 & 9 & \\
\hline
\end{tabular}




\begin{tabular}{|c|c|c|c|c|c|c|c|c|c|c|c|c|c|}
\hline 2285 & 56469 & 53505 & 14 & 0.03 & 73.79 & 0.063063 & 0.037037 & 16 & 50384871 & 16 & 50977092 & 16 & 50 \\
\hline 1739 & 26070 & 62127 & 27 & 0.03 & 73.82 & 0.116116 & 0.064064 & 13 & 35018578 & 13 & 35985818 & 13 & 35 \\
\hline 2279 & 14366 & 28225 & 16 & 0.03 & 73.84 & 0.06006 & 0.044044 & 16 & 44062106 & 16 & 44919236 & 16 & 44 \\
\hline 2293 & 28264 & 28269 & 13 & 0.02 & 73.87 & 0.069069 & 0.045045 & 16 & 58031082 & 16 & 58593026 & 16 & 58 \\
\hline 1343 & 16035 & 61619 & 18 & 0.02 & 73.89 & 0.085085 & 0.05005 & 9 & 23018947 & 9 & 23888625 & 9 & 23 \\
\hline 47 & 18111 & 45887 & 21 & 0.02 & 73.92 & 0.109109 & 0.072072 & 1 & 47165930 & 1 & 47997868 & 1 & 47 \\
\hline 338 & 2077 & 40486 & 21 & 0.02 & 73.94 & 0.109109 & 0.068068 & 2 & 22230468 & 2 & 22996263 & 2 & 22 \\
\hline 2315 & 44471 & 57102 & 29 & 0.02 & 73.96 & 0.12012 & 0.075075 & 16 & 80020682 & 16 & 80946630 & 16 & 80 \\
\hline 754 & 4472 & 4484 & 27 & 0.02 & 73.99 & 0.102102 & 0.06006 & 4 & 131001920 & 4 & 131918945 & 4 & 131 \\
\hline 793 & 4741 & 57971 & 14 & 0.02 & 74.01 & 0.067067 & 0.043043 & 5 & 26081086 & 5 & 26929026 & 5 & 26 \\
\hline 2489 & 48295 & 30457 & 9 & 0.02 & 74.04 & 0.044044 & 0.025025 & 19 & 36313690 & 19 & 36933196 & 19 & 36 \\
\hline 1150 & 6597 & 38063 & 23 & 0.02 & 74.06 & 0.098098 & 0.06006 & 7 & 114081936 & 7 & 114977348 & 7 & 114 \\
\hline 1726 & 9628 & 9638 & 26 & 0.02 & 74.09 & 0.114114 & 0.06006 & 13 & 22030419 & 13 & 22892852 & 13 & 22 \\
\hline 452 & 2669 & 21148 & 31 & 0.02 & 74.11 & 0.134134 & 0.073073 & 2 & 137046877 & 2 & 137986855 & 2 & 137 \\
\hline 1097 & 6196 & 35881 & 28 & 0.02 & 74.14 & 0.096096 & 0.054054 & 7 & 60010451 & 7 & 60969455 & 7 & 60 \\
\hline 1394 & 25003 & 57148 & 19 & 0.02 & 74.16 & 0.099099 & 0.062062 & 9 & 74006034 & 9 & 74961114 & 9 & 74 \\
\hline 818 & 46830 & 46832 & 15 & 0.02 & 74.19 & 0.064064 & 0.045045 & 5 & 51014577 & 5 & 51824431 & 5 & 51 \\
\hline 2185 & 57606 & 34669 & 14 & 0.02 & 74.21 & 0.075075 & 0.04004 & 15 & 108002667 & 15 & 108889109 & 15 & 108 \\
\hline 688 & 22029 & 41111 & 19 & 0.02 & 74.24 & 0.081081 & 0.056056 & 4 & 65016011 & 4 & 65976827 & 4 & 65 \\
\hline 2081 & 11823 & 44077 & 30 & 0.02 & 74.26 & 0.129129 & 0.076076 & 15 & 4061992 & 15 & 4920014 & 15 & 4 \\
\hline 1283 & 18301 & 24327 & 24 & 0.02 & 74.29 & 0.1001 & 0.074074 & 8 & 112078084 & 8 & 112989184 & 8 & 112 \\
\hline 1194 & 35004 & 18179 & 24 & 0.02 & 74.31 & 0.101101 & 0.069069 & 8 & 23033602 & 8 & 23935848 & 8 & 23 \\
\hline 324 & 20738 & 52444 & 27 & 0.02 & 74.33 & 0.101101 & 0.068068 & 2 & 8022443 & 2 & 8997005 & 2 & 8 \\
\hline 891 & 22929 & 52834 & 20 & 0.02 & 74.36 & 0.1001 & 0.062062 & 6 & 12005914 & 6 & 12980787 & 6 & 12 \\
\hline 1015 & 45289 & 31146 & 21 & 0.02 & 74.38 & 0.086086 & 0.048048 & 6 & 136045327 & 6 & 136990113 & 6 & 136 \\
\hline 1468 & 42721 & 34292 & 28 & 0.02 & 74.41 & 0.13013 & 0.083083 & 9 & 148001849 & 9 & 148997867 & 9 & 148 \\
\hline 977 & 41681 & 56425 & 15 & 0.02 & 74.43 & 0.06006 & 0.05005 & 6 & 98001106 & 6 & 98924562 & 6 & 98 \\
\hline 247 & 45600 & 20396 & 29 & 0.02 & 74.46 & 0.1001 & 0.064064 & 1 & 247017119 & 1 & 247962534 & 1 & 247 \\
\hline 1573 & 35946 & 57717 & 23 & 0.02 & 74.48 & 0.083083 & 0.052052 & 11 & 21011462 & 11 & 21968682 & 11 & 21 \\
\hline 2268 & 28176 & 35962 & 28 & 0.02 & 74.51 & 0.11011 & 0.071071 & 16 & 33002382 & 16 & 33954882 & 16 & 33 \\
\hline 871 & 15672 & 22889 & 26 & 0.02 & 74.53 & 0.103103 & 0.063063 & 5 & 104000831 & 5 & 104987144 & 5 & 104 \\
\hline 577 & 35590 & 21481 & 16 & 0.02 & 74.56 & 0.082082 & 0.06006 & 3 & 99288792 & 3 & 99987765 & 3 & 99 \\
\hline 1355 & 24585 & 24591 & 22 & 0.02 & 74.58 & 0.086086 & 0.06006 & 9 & 35012606 & 9 & 35998370 & 9 & 35 \\
\hline 1661 & 38645 & 38646 & 21 & 0.02 & 74.6 & 0.101101 & 0.062062 & 12 & 21017764 & 12 & 21955485 & 12 & 21 \\
\hline 2394 & 19410 & 28772 & 25 & 0.02 & 74.63 & 0.098098 & 0.064064 & 18 & 2082165 & 18 & 2998461 & 18 & 2 \\
\hline 1764 & 9882 & 38767 & 30 & 0.02 & 74.65 & 0.116116 & 0.068068 & 13 & 60024211 & 13 & 60980258 & 13 & 60 \\
\hline 40 & 39878 & 61731 & 23 & 0.02 & 74.68 & 0.096096 & 0.062062 & 1 & 40017655 & 1 & 40974578 & 1 & 40 \\
\hline 2561 & 14262 & 34117 & 20 & 0.02 & 74.7 & 0.098098 & 0.058058 & 19 & 113078996 & 19 & 113962590 & 19 & 113 \\
\hline 2434 & 28937 & 48119 & 22 & 0.02 & 74.73 & 0.095095 & 0.062062 & 18 & 42030856 & 18 & 42938796 & 18 & 42 \\
\hline
\end{tabular}




\begin{tabular}{|c|c|c|c|c|c|c|c|c|c|c|c|c|c|}
\hline 501 & 2859 & 31107 & 27 & 0.02 & 74.75 & 0.117117 & 0.072072 & 3 & 23015607 & 3 & 23987557 & 3 & 23 \\
\hline 1519 & 29913 & 38459 & 16 & 0.02 & 74.77 & 0.083083 & 0.062062 & 10 & 45101309 & 10 & 45903094 & 10 & 45 \\
\hline 2159 & 12245 & 12246 & 7 & 0.02 & 74.8 & 0.049049 & 0.035035 & 15 & 82310352 & 15 & 82835779 & 15 & 82 \\
\hline 1945 & 54961 & 26760 & 24 & 0.02 & 74.82 & 0.097097 & 0.061061 & 14 & 22000499 & 14 & 22998419 & 14 & 22 \\
\hline 228 & 1433 & 1441 & 22 & 0.02 & 74.85 & 0.074074 & 0.038038 & 1 & 228038772 & 1 & 228976153 & 1 & 228 \\
\hline 2376 & 13568 & 28664 & 20 & 0.02 & 74.87 & 0.102102 & 0.048048 & 17 & 54025507 & 17 & 54986042 & 17 & 54 \\
\hline 515 & 58503 & 55385 & 21 & 0.02 & 74.9 & 0.08008 & 0.047047 & 3 & 37023012 & 3 & 37919636 & 3 & 37 \\
\hline 2385 & 13635 & 49697 & 24 & 0.02 & 74.92 & 0.1001 & 0.054054 & 17 & 63006246 & 17 & 63977352 & 17 & 63 \\
\hline 1657 & 9262 & 17231 & 24 & 0.02 & 74.94 & 0.098098 & 0.061061 & 12 & 17081939 & 12 & 17946048 & 12 & 17 \\
\hline 1735 & 38727 & 45082 & 24 & 0.02 & 74.97 & 0.11011 & 0.061061 & 13 & 31070091 & 13 & 31969896 & 13 & 31 \\
\hline 148 & 40088 & 1020 & 18 & 0.02 & 74.99 & 0.076076 & 0.047047 & 1 & 148011932 & 1 & 148999915 & 1 & 148 \\
\hline 21 & 59958 & 19577 & 21 & 0.02 & 75.02 & 0.108108 & 0.071071 & 1 & 21017894 & 1 & 21899292 & 1 & 21 \\
\hline 2060 & 44006 & 11688 & 26 & 0.02 & 75.04 & 0.095095 & 0.048048 & 14 & 137063119 & 14 & 137979007 & 14 & 137 \\
\hline 172 & 58719 & 17815 & 18 & 0.02 & 75.07 & 0.079079 & 0.059059 & 1 & 172013706 & 1 & 172960921 & 1 & 172 \\
\hline 873 & 22894 & 5207 & 22 & 0.02 & 75.09 & 0.094094 & 0.055055 & 5 & 106014977 & 5 & 106979109 & 5 & 106 \\
\hline 75 & 591 & 19861 & 26 & 0.02 & 75.11 & 0.101101 & 0.067067 & 1 & 75002664 & 1 & 75994076 & 1 & 75 \\
\hline 2073 & 49544 & 53633 & 25 & 0.02 & 75.14 & 0.103103 & 0.065065 & 14 & 150010047 & 14 & 150984209 & 14 & 150 \\
\hline 631 & 48689 & 3465 & 27 & 0.02 & 75.16 & 0.099099 & 0.066066 & 4 & 8074964 & 4 & 8963064 & 4 & 8 \\
\hline 541 & 35473 & 37273 & 27 & 0.02 & 75.19 & 0.109109 & 0.055055 & 3 & 63016890 & 3 & 63964964 & 3 & 63 \\
\hline 689 & 34682 & 46662 & 22 & 0.02 & 75.21 & 0.084084 & 0.051051 & 4 & 66002995 & 4 & 66988557 & 4 & 66 \\
\hline 858 & 46870 & 46875 & 32 & 0.02 & 75.24 & 0.136136 & 0.08008 & 5 & 91016023 & 5 & 91991652 & 5 & 91 \\
\hline 716 & 4133 & 22177 & 27 & 0.02 & 75.26 & 0.112112 & 0.072072 & 4 & 93043178 & 4 & 93979546 & 4 & 93 \\
\hline 2316 & 17367 & 13154 & 29 & 0.02 & 75.28 & 0.114114 & 0.081081 & 16 & 81058129 & 16 & 81997191 & 16 & 81 \\
\hline 2010 & 43864 & 61591 & 29 & 0.02 & 75.31 & 0.116116 & 0.074074 & 14 & 87034503 & 14 & 87975446 & 14 & 87 \\
\hline 1136 & 6481 & 42072 & 21 & 0.02 & 75.33 & 0.074074 & 0.052052 & 7 & 100038150 & 7 & 100997092 & 7 & 100 \\
\hline 155 & 40101 & 1050 & 25 & 0.02 & 75.36 & 0.113113 & 0.062062 & 1 & 155011670 & 1 & 155984596 & 1 & 155 \\
\hline 307 & 45528 & 60767 & 19 & 0.02 & 75.38 & 0.09009 & 0.062062 & 1 & 307006485 & 1 & 307934149 & 1 & 307 \\
\hline 607 & 21619 & 40940 & 24 & 0.02 & 75.4 & 0.1001 & 0.063063 & 3 & 129005942 & 3 & 129847043 & 3 & 129 \\
\hline 772 & 4597 & 22510 & 29 & 0.02 & 75.43 & 0.108108 & 0.067067 & 5 & 5011010 & 5 & 5995341 & 5 & 5 \\
\hline 15 & 115 & 124 & 33 & 0.02 & 75.45 & 0.113113 & 0.063063 & 1 & 15045014 & 1 & 15995234 & 1 & 15 \\
\hline 457 & 17525 & 2723 & 27 & 0.02 & 75.48 & 0.116116 & 0.072072 & 2 & 142053892 & 2 & 142924314 & 2 & 142 \\
\hline 1393 & 42732 & 42735 & 16 & 0.02 & 75.5 & 0.074074 & 0.049049 & 9 & 73040157 & 9 & 73942367 & 9 & 73 \\
\hline 184 & 20255 & 1200 & 22 & 0.02 & 75.52 & 0.11011 & 0.078078 & 1 & 184000929 & 1 & 184954399 & 1 & 184 \\
\hline 2051 & 47784 & 47788 & 15 & 0.02 & 75.55 & 0.061061 & 0.043043 & 14 & 128001688 & 14 & 128987436 & 14 & 128 \\
\hline 748 & 41286 & 22370 & 16 & 0.02 & 75.57 & 0.078078 & 0.051051 & 4 & 125054205 & 4 & 125948453 & 4 & 125 \\
\hline 1434 & 32716 & 17988 & 13 & 0.02 & 75.6 & 0.057057 & 0.037037 & 9 & 114021432 & 9 & 114916229 & 9 & 114 \\
\hline 302 & 46407 & 1927 & 32 & 0.02 & 75.62 & 0.157157 & 0.075075 & 1 & 302009919 & 1 & 302980607 & 1 & 302 \\
\hline 398 & 53826 & 37120 & 18 & 0.02 & 75.64 & 0.097097 & 0.055055 & 2 & 83080749 & 2 & 83985760 & 2 & 83 \\
\hline 733 & 22280 & 4274 & 20 & 0.02 & 75.67 & 0.098098 & 0.069069 & 4 & 110004343 & 4 & 110972254 & 4 & 110 \\
\hline
\end{tabular}




\begin{tabular}{|c|c|c|c|c|c|c|c|c|c|c|c|c|c|}
\hline 1649 & 9211 & 53205 & 28 & 0.02 & 75.69 & 0.09009 & 0.059059 & 12 & 9004984 & 12 & 9992535 & 12 & 9 \\
\hline 1362 & 8190 & 61588 & 15 & 0.02 & 75.72 & 0.084084 & 0.059059 & 9 & 42014140 & 9 & 42938387 & 9 & 42 \\
\hline 1643 & 18062 & 31826 & 17 & 0.02 & 75.74 & 0.075075 & 0.056056 & 12 & 3097741 & 12 & 3928785 & 12 & 3 \\
\hline 20 & 162 & 39815 & 27 & 0.02 & 75.76 & 0.106106 & 0.066066 & 1 & 20009618 & 1 & 20996770 & 1 & 20 \\
\hline 1269 & 53884 & 24286 & 8 & 0.02 & 75.79 & 0.036036 & 0.026026 & 8 & 98547289 & 8 & 98985483 & 8 & 98 \\
\hline 271 & 1703 & 20495 & 22 & 0.02 & 75.81 & 0.095095 & 0.063063 & 1 & 271028077 & 1 & 271984966 & 1 & 271 \\
\hline 540 & 29679 & 52501 & 28 & 0.02 & 75.83 & 0.123123 & 0.075075 & 3 & 62059336 & 3 & 62994493 & 3 & 62 \\
\hline 591 & 48642 & 21546 & 31 & 0.02 & 75.86 & 0.127127 & 0.067067 & 3 & 113053130 & 3 & 113993246 & 3 & 113 \\
\hline 1345 & 18378 & 18505 & 16 & 0.02 & 75.88 & 0.06006 & 0.04004 & 9 & 25082159 & 9 & 25971114 & 9 & 25 \\
\hline 310 & 20687 & 1973 & 16 & 0.02 & 75.91 & 0.077077 & 0.046046 & 1 & 310005846 & 1 & 310999663 & 1 & 310 \\
\hline 2014 & 43877 & 43878 & 20 & 0.02 & 75.93 & 0.083083 & 0.055055 & 14 & 91013066 & 14 & 91762659 & 14 & 91 \\
\hline 1665 & 29997 & 9297 & 24 & 0.02 & 75.95 & 0.093093 & 0.062062 & 12 & 25006232 & 12 & 25983929 & 12 & 25 \\
\hline 300 & 1904 & 40384 & 15 & 0.02 & 75.98 & 0.056056 & 0.036036 & 1 & 300316974 & 1 & 300929374 & 1 & 300 \\
\hline 1552 & 42880 & 56735 & 23 & 0.02 & 76 & 0.096096 & 0.05005 & 11 & 22488 & 11 & 939424 & 11 & 0 \\
\hline 1495 & 17792 & 49237 & 14 & 0.02 & 76.02 & 0.073073 & 0.056056 & 10 & 21021888 & 10 & 21954770 & 10 & 21 \\
\hline 1578 & 19431 & 42965 & 27 & 0.02 & 76.05 & 0.098098 & 0.058058 & 11 & 26022055 & 11 & 26971685 & 11 & 26 \\
\hline 1921 & 45363 & 43595 & 11 & 0.02 & 76.07 & 0.052052 & 0.036036 & 13 & 217027218 & 13 & 217743121 & 13 & 217 \\
\hline 2429 & 31656 & 13898 & 24 & 0.02 & 76.09 & 0.083083 & 0.059059 & 18 & 37000204 & 18 & 37926205 & 18 & 37 \\
\hline 175 & 20212 & 1152 & 12 & 0.02 & 76.12 & 0.066066 & 0.049049 & 1 & 175226043 & 1 & 175979464 & 1 & 175 \\
\hline 51 & 36604 & 36610 & 21 & 0.02 & 76.14 & 0.099099 & 0.069069 & 1 & 51037015 & 1 & 51998764 & 1 & 51 \\
\hline 799 & 22640 & 4773 & 17 & 0.02 & 76.17 & 0.075075 & 0.06006 & 5 & 32015964 & 5 & 32986570 & 5 & 32 \\
\hline 654 & 21887 & 41040 & 19 & 0.02 & 76.19 & 0.045045 & 0.03003 & 4 & 31003651 & 4 & 31982461 & 4 & 31 \\
\hline 2382 & 28702 & 13616 & 29 & 0.02 & 76.21 & 0.105105 & 0.064064 & 17 & 60017456 & 17 & 60992562 & 17 & 60 \\
\hline 1398 & 15099 & 38289 & 23 & 0.02 & 76.24 & 0.087087 & 0.058058 & 9 & 78070603 & 9 & 78963458 & 9 & 78 \\
\hline 2372 & 13537 & 28644 & 29 & 0.02 & 76.26 & 0.144144 & 0.088088 & 17 & 50025811 & 17 & 50988229 & 17 & 50 \\
\hline 917 & 30003 & 55336 & 19 & 0.02 & 76.28 & 0.064064 & 0.051051 & 6 & 38026430 & 6 & 38961317 & 6 & 38 \\
\hline 1708 & 33588 & 25928 & 20 & 0.02 & 76.31 & 0.08008 & 0.054054 & 13 & 4023767 & 13 & 4922900 & 13 & 4 \\
\hline 638 & 37381 & 3533 & 31 & 0.02 & 76.33 & 0.118118 & 0.052052 & 4 & 15016104 & 4 & 15977749 & 4 & 15 \\
\hline 2319 & 32088 & 45124 & 21 & 0.02 & 76.35 & 0.079079 & 0.05005 & 16 & 84005681 & 16 & 84890972 & 16 & 84 \\
\hline 1906 & 32040 & 47542 & 22 & 0.02 & 76.38 & 0.079079 & 0.056056 & 13 & 202031500 & 13 & 202882988 & 13 & 202 \\
\hline 1159 & 23948 & 6684 & 23 & 0.02 & 76.4 & 0.088088 & 0.054054 & 7 & 123132345 & 7 & 123997457 & 7 & 123 \\
\hline 1124 & 23731 & 23742 & 27 & 0.02 & 76.42 & 0.111111 & 0.072072 & 7 & 88077718 & 7 & 88970583 & 7 & 88 \\
\hline 263 & 53934 & 1646 & 17 & 0.02 & 76.45 & 0.063063 & 0.044044 & 1 & 263044178 & 1 & 263972707 & 1 & 263 \\
\hline 311 & 20690 & 46418 & 18 & 0.02 & 76.47 & 0.081081 & 0.047047 & 1 & 311027658 & 1 & 311995820 & 1 & 311 \\
\hline 1138 & 23834 & 38044 & 23 & 0.02 & 76.49 & 0.068068 & 0.039039 & 7 & 102013846 & 7 & 102949951 & 7 & 102 \\
\hline 1885 & 58248 & 52122 & 6 & 0.02 & 76.52 & 0.038038 & 0.028028 & 13 & 181014066 & 13 & 181995091 & 13 & 181 \\
\hline 512 & 51158 & 48617 & 23 & 0.02 & 76.54 & 0.093093 & 0.065065 & 3 & 34016624 & 3 & 34989088 & 3 & 34 \\
\hline 2225 & 12624 & 50103 & 16 & 0.02 & 76.56 & 0.065065 & 0.036036 & 15 & 148072735 & 15 & 148972866 & 15 & 148 \\
\hline 665 & 21944 & 41073 & 24 & 0.02 & 76.59 & 0.114114 & 0.073073 & 4 & 42037215 & 4 & 42984787 & 4 & 42 \\
\hline
\end{tabular}




\begin{tabular}{|c|c|c|c|c|c|c|c|c|c|c|c|c|c|}
\hline 1881 & 34925 & 60213 & 16 & 0.02 & 76.61 & 0.081081 & 0.051051 & 13 & 177033643 & 13 & 177999254 & 13 & 177 \\
\hline 2096 & 11933 & 11944 & 29 & 0.02 & 76.63 & 0.117117 & 0.076076 & 15 & 19076645 & 15 & 19989529 & 15 & 19 \\
\hline 323 & 54914 & 20737 & 20 & 0.02 & 76.66 & 0.089089 & 0.059059 & 2 & 7016156 & 2 & 7998883 & 2 & 7 \\
\hline 1537 & 56537 & 18536 & 27 & 0.02 & 76.68 & 0.102102 & 0.062062 & 10 & 63088598 & 10 & 63934312 & 10 & 63 \\
\hline 2226 & 12631 & 16591 & 25 & 0.02 & 76.7 & 0.097097 & 0.057057 & 15 & 149140529 & 15 & 149953164 & 15 & 149 \\
\hline 1433 & 24844 & 47287 & 13 & 0.02 & 76.73 & 0.048048 & 0.028028 & 9 & 113051200 & 9 & 113964106 & 9 & 113 \\
\hline 1161 & 49076 & 23969 & 31 & 0.02 & 76.75 & 0.097097 & 0.061061 & 7 & 125008742 & 7 & 125986792 & 7 & 125 \\
\hline 2428 & 36233 & 55359 & 21 & 0.02 & 76.77 & 0.1001 & 0.058058 & 18 & 36145263 & 18 & 36938024 & 18 & 36 \\
\hline 2117 & 14888 & 47863 & 26 & 0.02 & 76.8 & 0.093093 & 0.058058 & 15 & 40012527 & 15 & 40974908 & 15 & 40 \\
\hline 1854 & 10254 & 54605 & 14 & 0.02 & 76.82 & 0.07007 & 0.043043 & 13 & 150022400 & 13 & 150958697 & 13 & 150 \\
\hline 2311 & 28336 & 57447 & 25 & 0.02 & 76.84 & 0.09009 & 0.063063 & 16 & 76026438 & 16 & 76990076 & 16 & 76 \\
\hline 2349 & 13377 & 13386 & 21 & 0.02 & 76.87 & 0.073073 & 0.051051 & 17 & 27035671 & 17 & 27855668 & 17 & 27 \\
\hline 1621 & 25581 & 9054 & 22 & 0.02 & 76.89 & 0.09009 & 0.059059 & 11 & 69005136 & 11 & 69986366 & 11 & 69 \\
\hline 1870 & 26449 & 26455 & 12 & 0.02 & 76.91 & 0.059059 & 0.037037 & 13 & 166070864 & 13 & 166984099 & 13 & 166 \\
\hline 139 & 46064 & 55827 & 23 & 0.02 & 76.94 & 0.086086 & 0.055055 & 1 & 139217790 & 1 & 139996752 & 1 & 139 \\
\hline 1144 & 47158 & 51503 & 19 & 0.02 & 76.96 & 0.085085 & 0.05005 & 7 & 108071051 & 7 & 108972110 & 7 & 108 \\
\hline 1000 & 51607 & 51345 & 26 & 0.02 & 76.98 & 0.097097 & 0.067067 & 6 & 121120053 & 6 & 121994247 & 6 & 121 \\
\hline 1696 & 15978 & 49389 & 23 & 0.02 & 77 & 0.124124 & 0.081081 & 12 & 56000017 & 12 & 56868845 & 12 & 56 \\
\hline 1720 & 58329 & 9593 & 22 & 0.02 & 77.03 & 0.082082 & 0.049049 & 13 & 16082398 & 13 & 16946257 & 13 & 16 \\
\hline 409 & 2403 & 46447 & 13 & 0.02 & 77.05 & 0.062062 & 0.047047 & 2 & 94085488 & 2 & 94631894 & 2 & 94 \\
\hline 1550 & 58359 & 16921 & 13 & 0.02 & 77.07 & 0.058058 & 0.033033 & 10 & 76156036 & 10 & 76994582 & 10 & 76 \\
\hline 396 & 55142 & 40579 & 16 & 0.02 & 77.1 & 0.076076 & 0.049049 & 2 & 81034415 & 2 & 81845545 & 2 & 81 \\
\hline 2114 & 12032 & 45481 & 23 & 0.02 & 77.12 & 0.112112 & 0.072072 & 15 & 37073268 & 15 & 37957138 & 15 & 37 \\
\hline 2239 & 12664 & 12674 & 26 & 0.02 & 77.14 & 0.112112 & 0.072072 & 16 & 4016044 & 16 & 4964893 & 16 & 4 \\
\hline 1405 & 24792 & 24798 & 22 & 0.02 & 77.17 & 0.097097 & 0.063063 & 9 & 85001751 & 9 & 85988046 & 9 & 85 \\
\hline 761 & 22445 & 22454 & 25 & 0.02 & 77.19 & 0.078078 & 0.052052 & 4 & 138123252 & 4 & 138987333 & 4 & 138 \\
\hline 1558 & 8633 & 57537 & 24 & 0.02 & 77.21 & 0.114114 & 0.06006 & 11 & 6056322 & 11 & 6988731 & 11 & 6 \\
\hline 233 & 34453 & 50432 & 19 & 0.02 & 77.23 & 0.077077 & 0.049049 & 1 & 233021161 & 1 & 233975946 & 1 & 233 \\
\hline 2167 & 27823 & 12318 & 30 & 0.02 & 77.26 & 0.146146 & 0.068068 & 15 & 90020714 & 15 & 90992066 & 15 & 90 \\
\hline 882 & 59770 & 16401 & 24 & 0.02 & 77.28 & 0.085085 & 0.061061 & 6 & 3005254 & 6 & 3989070 & 6 & 3 \\
\hline 1335 & 19264 & 42460 & 25 & 0.02 & 77.3 & 0.105105 & 0.071071 & 9 & 15001911 & 9 & 15999539 & 9 & 15 \\
\hline 1960 & 43715 & 43721 & 32 & 0.02 & 77.33 & 0.116116 & 0.066066 & 14 & 37100789 & 14 & 37968250 & 14 & 37 \\
\hline 1275 & 7228 & 14453 & 13 & 0.02 & 77.35 & 0.051051 & 0.035035 & 8 & 104001629 & 8 & 104961048 & 8 & 104 \\
\hline 776 & 41383 & 22526 & 22 & 0.02 & 77.37 & 0.096096 & 0.062062 & 5 & 9096232 & 5 & 9942508 & 5 & 9 \\
\hline 27 & 36519 & 214 & 19 & 0.02 & 77.4 & 0.091091 & 0.049049 & 1 & 27020977 & 1 & 27899646 & 1 & 27 \\
\hline 1479 & 51717 & 8238 & 23 & 0.02 & 77.42 & 0.1001 & 0.067067 & 10 & 5008012 & 10 & 5973172 & 10 & 5 \\
\hline 2254 & 28126 & 12747 & 21 & 0.02 & 77.44 & 0.097097 & 0.065065 & 16 & 19022068 & 16 & 19997593 & 16 & 19 \\
\hline 379 & 14713 & 14713 & 1 & 0.02 & 77.46 & 0.017017 & 0.017017 & 2 & 64970665 & 2 & 64970665 & 2 & 64 \\
\hline 2160 & 54612 & 27790 & 21 & 0.02 & 77.49 & 0.079079 & 0.05005 & 15 & 83086792 & 15 & 83881219 & 15 & 83 \\
\hline
\end{tabular}




\begin{tabular}{|c|c|c|c|c|c|c|c|c|c|c|c|c|c|}
\hline 948 & 23020 & 45757 & 12 & 0.02 & 77.51 & 0.073073 & 0.051051 & 6 & 69006213 & 6 & 69930291 & 6 & 69 \\
\hline 2207 & 12503 & 14405 & 24 & 0.02 & 77.53 & 0.092092 & 0.057057 & 15 & 130001470 & 15 & 130968782 & 15 & 130 \\
\hline 1520 & 25201 & 8447 & 17 & 0.02 & 77.56 & 0.081081 & 0.058058 & 10 & 46084707 & 10 & 46983347 & 10 & \\
\hline 1044 & 37848 & 23298 & 28 & 0.02 & 77.58 & 0.114114 & 0.065065 & 7 & 7001964 & 7 & 7981170 & 7 & \\
\hline 487 & 59773 & 31966 & 23 & 0.02 & 77.6 & 0.096096 & 0.057057 & 3 & 9000666 & 3 & 9962466 & 3 & \\
\hline 535 & 21371 & 46483 & 27 & 0.02 & 77.62 & 0.099099 & 0.071071 & 3 & 57006572 & 3 & 57996439 & 3 & 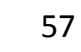 \\
\hline 169 & 20203 & 60501 & 12 & 0.02 & 77.65 & 0.063063 & 0.037037 & 1 & 169007648 & 1 & 169984967 & 1 & 169 \\
\hline 1098 & 47031 & 47040 & 14 & 0.02 & 77.67 & 0.069069 & 0.038038 & 7 & 61067623 & 7 & 61988185 & 7 & 61 \\
\hline 553 & 35325 & 61996 & 13 & 0.02 & 77.69 & 0.066066 & 0.046046 & 3 & 75020115 & 3 & 75980661 & 3 & \\
\hline 1673 & 9330 & 33165 & 22 & 0.02 & 77.71 & 0.09009 & 0.047047 & 12 & 33064079 & 12 & 33982636 & 12 & \\
\hline 2332 & 28445 & 49658 & 21 & 0.02 & 77.74 & 0.093093 & 0.056056 & 17 & 10039697 & 17 & 10939739 & 17 & \\
\hline 340 & 40490 & 2094 & 19 & 0.02 & 77.76 & 0.089089 & 0.057057 & 2 & 24003403 & 2 & 24998348 & 2 & \\
\hline 1373 & 47265 & 42547 & 21 & 0.02 & 77.78 & 0.083083 & 0.053053 & 9 & 53008122 & 9 & 53975532 & 9 & \\
\hline 650 & 51018 & 37416 & 9 & 0.02 & 77.81 & 0.037037 & 0.031031 & 4 & 27041022 & 4 & 27846293 & 4 & \\
\hline 362 & 37107 & 20887 & 18 & 0.02 & 77.83 & 0.079079 & 0.053053 & 2 & 46053572 & 2 & 46987103 & 2 & \\
\hline 584 & 46516 & 37321 & 20 & 0.02 & 77.85 & 0.075075 & 0.047047 & 3 & 106010885 & 3 & 106987656 & 3 & 106 \\
\hline 972 & 58007 & 56126 & 29 & 0.02 & 77.87 & 0.121121 & 0.069069 & 6 & 93012456 & 6 & 93942039 & 6 & \\
\hline 2110 & 12013 & 44120 & 23 & 0.02 & 77.9 & 0.102102 & 0.052052 & 15 & 33013541 & 15 & 33883655 & 15 & \\
\hline 2065 & 11741 & 11750 & 25 & 0.02 & 77.92 & 0.103103 & 0.065065 & 14 & 142014167 & 14 & 142951787 & 14 & \\
\hline 596 & 40918 & 35106 & 25 & 0.02 & 77.94 & 0.107107 & 0.069069 & 3 & 118132370 & 3 & 118993318 & 3 & \\
\hline 1040 & 23258 & 48953 & 22 & 0.02 & 77.96 & 0.091091 & 0.06006 & 7 & 3056769 & 7 & 3993079 & 7 & \\
\hline 1475 & 25009 & 42737 & 19 & 0.02 & 77.99 & 0.094094 & 0.065065 & 10 & 1045768 & 10 & 1994574 & 10 & \\
\hline 1296 & 7334 & 18435 & 16 & 0.02 & 78.01 & 0.086086 & 0.057057 & 8 & 125012854 & 8 & 125885539 & 8 & \\
\hline 695 & 32940 & 3965 & 16 & 0.02 & 78.03 & 0.08008 & 0.04004 & 4 & 72005343 & 4 & 72955795 & 4 & \\
\hline 45 & 19704 & 36591 & 29 & 0.02 & 78.05 & 0.11011 & 0.058058 & 1 & 45116274 & 1 & 45997932 & 1 & \\
\hline 506 & 40772 & 2885 & 26 & 0.02 & 78.08 & 0.112112 & 0.055055 & 3 & 28053055 & 3 & 28995377 & 3 & \\
\hline 485 & 33489 & 35827 & 10 & 0.02 & 78.1 & 0.041041 & 0.029029 & 3 & 7099006 & 3 & 7846008 & 3 & \\
\hline 1051 & 37863 & 5807 & 28 & 0.02 & 78.12 & 0.117117 & 0.068068 & 7 & 14059560 & 7 & 14901075 & 7 & \\
\hline 2395 & 49932 & 28778 & 26 & 0.02 & 78.14 & 0.1001 & 0.068068 & 18 & 3045578 & 18 & 3945470 & 18 & \\
\hline 77 & 29511 & 36684 & 23 & 0.02 & 78.17 & 0.091091 & 0.057057 & 1 & 77011485 & 1 & 77999034 & 1 & \\
\hline 1546 & 39669 & 45537 & 21 & 0.02 & 78.19 & 0.087087 & 0.055055 & 10 & 72248720 & 10 & 72941077 & 10 & \\
\hline 1606 & 47372 & 38593 & 22 & 0.02 & 78.21 & 0.101101 & 0.059059 & 11 & 54006230 & 11 & 54954986 & 11 & \\
\hline 444 & 2621 & 17641 & 25 & 0.02 & 78.23 & 0.11011 & 0.057057 & 2 & 129003155 & 2 & 129999370 & 2 & \\
\hline 352 & 60206 & 16792 & 22 & 0.02 & 78.26 & 0.105105 & 0.065065 & 2 & 36008750 & 2 & 36919501 & 2 & \\
\hline 1576 & 8755 & 8764 & 30 & 0.02 & 78.28 & 0.117117 & 0.065065 & 11 & 24007939 & 11 & 24974538 & 11 & \\
\hline 2269 & 12847 & 36370 & 22 & 0.02 & 78.3 & 0.075075 & 0.049049 & 16 & 34003949 & 16 & 34854206 & 16 & \\
\hline 905 & 5311 & 19392 & 25 & 0.02 & 78.32 & 0.094094 & 0.05005 & 6 & 26110849 & 6 & 26948992 & 6 & \\
\hline 2294 & 44419 & 44425 & 26 & 0.02 & 78.34 & 0.11011 & 0.063063 & 16 & 59138735 & 16 & 59988426 & 16 & \\
\hline 704 & 52553 & 41150 & 26 & 0.02 & 78.37 & 0.104104 & 0.057057 & 4 & 81026612 & 4 & 81984659 & 4 & \\
\hline
\end{tabular}




\begin{tabular}{|c|c|c|c|c|c|c|c|c|c|c|c|c|c|}
\hline 932 & 23002 & 5366 & 16 & 0.02 & 78.39 & 0.075075 & 0.044044 & 6 & 53001243 & 6 & 53966129 & 6 & 53 \\
\hline 226 & 1418 & 36922 & 19 & 0.02 & 78.41 & 0.071071 & 0.044044 & 1 & 226149776 & 1 & 226989901 & 1 & 226 \\
\hline 1406 & 24799 & 30566 & 15 & 0.02 & 78.43 & 0.08008 & 0.046046 & 9 & 86034248 & 9 & 86869637 & 9 & 86 \\
\hline 1630 & 25634 & 47412 & 29 & 0.02 & 78.46 & 0.111111 & 0.065065 & 11 & 78000180 & 11 & 78952414 & 11 & 78 \\
\hline 1877 & 43517 & 43519 & 14 & 0.02 & 78.48 & 0.069069 & 0.043043 & 13 & 173078781 & 13 & 173838695 & 13 & 173 \\
\hline 2443 & 33039 & 13976 & 31 & 0.02 & 78.5 & 0.124124 & 0.075075 & 18 & 51012467 & 18 & 51975074 & 18 & 51 \\
\hline 2130 & 52901 & 47879 & 24 & 0.02 & 78.52 & 0.086086 & 0.056056 & 15 & 53067599 & 15 & 53869001 & 15 & 53 \\
\hline 2229 & 55291 & 30146 & 18 & 0.02 & 78.54 & 0.079079 & 0.047047 & 15 & 152015494 & 15 & 152938240 & 15 & 152 \\
\hline 16 & 48394 & 18555 & 28 & 0.02 & 78.57 & 0.122122 & 0.075075 & 1 & 16038746 & 1 & 16870187 & 1 & 16 \\
\hline 2201 & 27903 & 34783 & 14 & 0.02 & 78.59 & 0.062062 & 0.046046 & 15 & 124030230 & 15 & 124792285 & 15 & 124 \\
\hline 1306 & 42379 & 31043 & 23 & 0.02 & 78.61 & 0.098098 & 0.064064 & 8 & 135031919 & 8 & 135960757 & 8 & 135 \\
\hline 2365 & 44622 & 55572 & 21 & 0.02 & 78.63 & 0.096096 & 0.063063 & 17 & 43013416 & 17 & 43931913 & 17 & 43 \\
\hline 548 & 32460 & 61294 & 15 & 0.02 & 78.66 & 0.061061 & 0.044044 & 3 & 70024504 & 3 & 70939407 & 3 & 70 \\
\hline 82 & 50124 & 652 & 23 & 0.02 & 78.68 & 0.085085 & 0.061061 & 1 & 82050327 & 1 & 82964894 & 1 & 82 \\
\hline 1481 & 25032 & 25038 & 24 & 0.02 & 78.7 & 0.104104 & 0.068068 & 10 & 7002238 & 10 & 7983066 & 10 & 7 \\
\hline 428 & 40638 & 2524 & 22 & 0.02 & 78.72 & 0.086086 & 0.058058 & 2 & 113003444 & 2 & 113985753 & 2 & 113 \\
\hline 1850 & 15050 & 43483 & 21 & 0.02 & 78.74 & 0.098098 & 0.059059 & 13 & 146120125 & 13 & 146920356 & 13 & 146 \\
\hline 956 & 50125 & 29287 & 18 & 0.02 & 78.77 & 0.085085 & 0.045045 & 6 & 77035698 & 6 & 77847902 & 6 & 77 \\
\hline 1722 & 59215 & 61918 & 25 & 0.02 & 78.79 & 0.111111 & 0.073073 & 13 & 18076357 & 13 & 18960253 & 13 & 18 \\
\hline 2133 & 44158 & 47882 & 14 & 0.02 & 78.81 & 0.055055 & 0.035035 & 15 & 56060543 & 15 & 56937444 & 15 & 56 \\
\hline 1344 & 24544 & 54125 & 25 & 0.02 & 78.83 & 0.095095 & 0.073073 & 9 & 24011516 & 9 & 24695732 & 9 & 24 \\
\hline 332 & 53436 & 46421 & 19 & 0.02 & 78.85 & 0.085085 & 0.054054 & 2 & 16030402 & 2 & 16770525 & 2 & 16 \\
\hline 647 & 21856 & 52159 & 16 & 0.02 & 78.88 & 0.072072 & 0.05005 & 4 & 24167536 & 4 & 24884374 & 4 & 24 \\
\hline 1598 & 8870 & 15264 & 22 & 0.02 & 78.9 & 0.087087 & 0.05005 & 11 & 46050226 & 11 & 46997995 & 11 & 46 \\
\hline 1581 & 8783 & 25476 & 14 & 0.02 & 78.92 & 0.074074 & 0.045045 & 11 & 29014105 & 11 & 29994790 & 11 & 29 \\
\hline 1443 & 18340 & 42650 & 17 & 0.02 & 78.94 & 0.086086 & 0.039039 & 9 & 123051900 & 9 & 123972496 & 9 & 123 \\
\hline 849 & 5041 & 5049 & 21 & 0.02 & 78.96 & 0.101101 & 0.051051 & 5 & 82045018 & 5 & 82988313 & 5 & 82 \\
\hline 715 & 37473 & 22171 & 24 & 0.02 & 78.99 & 0.1001 & 0.055055 & 4 & 92004679 & 4 & 92991564 & 4 & 92 \\
\hline 510 & 34006 & 17010 & 21 & 0.02 & 79.01 & 0.078078 & 0.048048 & 3 & 32058308 & 3 & 32920176 & 3 & 32 \\
\hline 511 & 30935 & 2918 & 18 & 0.02 & 79.03 & 0.083083 & 0.061061 & 3 & 33145490 & 3 & 33947247 & 3 & 33 \\
\hline 1036 & 50006 & 51808 & 23 & 0.02 & 79.05 & 0.1001 & 0.064064 & 6 & 157003423 & 6 & 157734339 & 6 & 157 \\
\hline 145 & 61688 & 40086 & 21 & 0.02 & 79.07 & 0.089089 & 0.058058 & 1 & 145008895 & 1 & 145991718 & 1 & 145 \\
\hline 2112 & 12025 & 27687 & 27 & 0.02 & 79.09 & 0.11011 & 0.071071 & 15 & 35010372 & 15 & 35973145 & 15 & 35 \\
\hline 2556 & 45258 & 14243 & 14 & 0.02 & 79.12 & 0.068068 & 0.048048 & 19 & 108206131 & 19 & 108915748 & 19 & 108 \\
\hline 1709 & 47431 & 25934 & 18 & 0.02 & 79.14 & 0.076076 & 0.051051 & 13 & 5013989 & 13 & 5963268 & 13 & 5 \\
\hline 833 & 14412 & 48843 & 26 & 0.02 & 79.16 & 0.098098 & 0.059059 & 5 & 66015525 & 5 & 66918435 & 5 & 66 \\
\hline 363 & 40549 & 2268 & 15 & 0.02 & 79.18 & 0.073073 & 0.043043 & 2 & 47055959 & 2 & 47984857 & 2 & 47 \\
\hline 923 & 51628 & 35328 & 11 & 0.02 & 79.2 & 0.054054 & 0.04004 & 6 & 44004031 & 6 & 44985575 & 6 & 44 \\
\hline 1374 & 7727 & 24675 & 21 & 0.02 & 79.22 & 0.088088 & 0.06006 & 9 & 54010624 & 9 & 54991166 & 9 & 54 \\
\hline
\end{tabular}




\begin{tabular}{|c|c|c|c|c|c|c|c|c|c|c|c|c|c|}
\hline 248 & 36945 & 40255 & 31 & 0.02 & 79.25 & 0.124124 & 0.059059 & 1 & 248065286 & 1 & 248988370 & 1 & 248 \\
\hline 2295 & 28275 & 56116 & 27 & 0.02 & 79.27 & 0.115115 & 0.079079 & 16 & 60082082 & 16 & 60988882 & 16 & 60 \\
\hline 543 & 55295 & 34223 & 14 & 0.02 & 79.29 & 0.053053 & 0.04004 & 3 & 65094505 & 3 & 65993379 & 3 & 65 \\
\hline 2033 & 43922 & 11470 & 23 & 0.02 & 79.31 & 0.081081 & 0.053053 & 14 & 110103413 & 14 & 110983342 & 14 & 110 \\
\hline 97 & 39983 & 39986 & 25 & 0.02 & 79.33 & 0.099099 & 0.059059 & 1 & 97039899 & 1 & 97978461 & 1 & 97 \\
\hline 1292 & 61470 & 16133 & 14 & 0.02 & 79.35 & 0.073073 & 0.051051 & 8 & 121026032 & 8 & 121909646 & 8 & 121 \\
\hline 330 & 30120 & 30120 & 1 & 0.02 & 79.38 & 0.018018 & 0.014014 & 2 & 14035728 & 2 & 14035728 & 2 & 14 \\
\hline 2222 & 12608 & 44292 & 27 & 0.02 & 79.4 & 0.101101 & 0.063063 & 15 & 145007171 & 15 & 145990809 & 15 & 145 \\
\hline 2076 & 47846 & 44070 & 13 & 0.02 & 79.42 & 0.054054 & 0.037037 & 14 & 153114220 & 14 & 153786761 & 14 & 153 \\
\hline 2277 & 12910 & 53804 & 15 & 0.02 & 79.44 & 0.073073 & 0.041041 & 16 & 42082484 & 16 & 42978682 & 16 & 42 \\
\hline 1375 & 24676 & 24680 & 23 & 0.02 & 79.46 & 0.091091 & 0.063063 & 9 & 55009244 & 9 & 55977696 & 9 & 55 \\
\hline 2461 & 14051 & 59871 & 18 & 0.02 & 79.48 & 0.066066 & 0.039039 & 19 & 8044093 & 19 & 8938817 & 19 & 8 \\
\hline 1608 & 25529 & 8944 & 27 & 0.02 & 79.5 & 0.1001 & 0.064064 & 11 & 56034371 & 11 & 56992066 & 11 & 56 \\
\hline 1140 & 23842 & 47156 & 21 & 0.02 & 79.53 & 0.086086 & 0.051051 & 7 & 104030356 & 7 & 104914125 & 7 & 104 \\
\hline 710 & 22147 & 22151 & 20 & 0.02 & 79.55 & 0.072072 & 0.049049 & 4 & 87007876 & 4 & 87889676 & 4 & 87 \\
\hline 1130 & 6399 & 47132 & 17 & 0.02 & 79.57 & 0.069069 & 0.046046 & 7 & 94045990 & 7 & 94980822 & 7 & 94 \\
\hline 1178 & 24041 & 17629 & 28 & 0.02 & 79.59 & 0.113113 & 0.071071 & 8 & 7050948 & 8 & 7964676 & 8 & 7 \\
\hline 782 & 37544 & 56372 & 21 & 0.02 & 79.61 & 0.087087 & 0.049049 & 5 & 15052496 & 5 & 15908465 & 5 & 15 \\
\hline 971 & 33304 & 58628 & 16 & 0.02 & 79.63 & 0.08008 & 0.055055 & 6 & 92269778 & 6 & 92978013 & 6 & 92 \\
\hline 1584 & 38540 & 47347 & 19 & 0.02 & 79.65 & 0.103103 & 0.061061 & 11 & 32013457 & 11 & 32913253 & 11 & 32 \\
\hline 625 & 54334 & 21676 & 24 & 0.02 & 79.68 & 0.092092 & 0.063063 & 4 & 2035615 & 4 & 2990838 & 4 & 2 \\
\hline 1409 & 33904 & 7887 & 6 & 0.02 & 79.7 & 0.036036 & 0.03003 & 9 & 89128614 & 9 & 89984248 & 9 & 89 \\
\hline 832 & 22748 & 17603 & 24 & 0.02 & 79.72 & 0.107107 & 0.063063 & 5 & 65003383 & 5 & 65950020 & 5 & 65 \\
\hline 1944 & 10762 & 26755 & 18 & 0.02 & 79.74 & 0.096096 & 0.058058 & 14 & 21017604 & 14 & 21963563 & 14 & 21 \\
\hline 1771 & 17550 & 43371 & 17 & 0.02 & 79.76 & 0.063063 & 0.033033 & 13 & 67066590 & 13 & 67934836 & 13 & 67 \\
\hline 585 & 16076 & 31446 & 21 & 0.02 & 79.78 & 0.083083 & 0.052052 & 3 & 107073046 & 3 & 107985092 & 3 & 107 \\
\hline 2328 & 13229 & 35077 & 17 & 0.02 & 79.8 & 0.056056 & 0.035035 & 17 & 6024213 & 17 & 6989362 & 17 & 6 \\
\hline 1491 & 8319 & 25102 & 24 & 0.02 & 79.82 & 0.084084 & 0.062062 & 10 & 17018794 & 10 & 17948352 & 10 & 17 \\
\hline 774 & 48820 & 4620 & 19 & 0.02 & 79.85 & 0.078078 & 0.051051 & 5 & 7013047 & 5 & 7992138 & 5 & 7 \\
\hline 129 & 34413 & 46044 & 15 & 0.02 & 79.87 & 0.066066 & 0.044044 & 1 & 129025180 & 1 & 129911267 & 1 & 129 \\
\hline 2322 & 28388 & 13180 & 20 & 0.02 & 79.89 & 0.067067 & 0.042042 & 17 & 15175 & 17 & 927919 & 17 & 0 \\
\hline 503 & 19654 & 31842 & 23 & 0.02 & 79.91 & 0.096096 & 0.062062 & 3 & 25018550 & 3 & 25990903 & 3 & 25 \\
\hline 2142 & 56423 & 12204 & 24 & 0.02 & 79.93 & 0.092092 & 0.062062 & 15 & 65014590 & 15 & 65866223 & 15 & 65 \\
\hline 2204 & 39346 & 27915 & 21 & 0.02 & 79.95 & 0.065065 & 0.037037 & 15 & 127201004 & 15 & 127982302 & 15 & 127 \\
\hline 87 & 36699 & 39967 & 21 & 0.02 & 79.97 & 0.077077 & 0.047047 & 1 & 87012148 & 1 & 87852879 & 1 & 87 \\
\hline 888 & 18733 & 29842 & 31 & 0.02 & 79.99 & 0.12012 & 0.055055 & 6 & 9149467 & 6 & 9957369 & 6 & 9 \\
\hline 1725 & 52499 & 9627 & 26 & 0.02 & 80.01 & 0.097097 & 0.061061 & 13 & 21009181 & 13 & 21999054 & 13 & 21 \\
\hline 642 & 37392 & 51299 & 35 & 0.02 & 80.04 & 0.105105 & 0.065065 & 4 & 19015552 & 4 & 19958367 & 4 & 19 \\
\hline 1201 & 24131 & 6930 & 32 & 0.02 & 80.06 & 0.129129 & 0.075075 & 8 & 30007914 & 8 & 30952610 & 8 & 30 \\
\hline
\end{tabular}




\begin{tabular}{|c|c|c|c|c|c|c|c|c|c|c|c|c|c|}
\hline 1469 & 31132 & 24979 & 20 & 0.02 & 80.08 & 0.094094 & 0.048048 & 9 & 149000274 & 9 & 149972040 & 9 & 149 \\
\hline 1985 & 11064 & 43795 & 20 & 0.02 & 80.1 & 0.08008 & 0.048048 & 14 & 62002777 & 14 & 62975744 & 14 & 62 \\
\hline 2122 & 12071 & 12075 & 16 & 0.02 & 80.12 & 0.067067 & 0.033033 & 15 & 45022930 & 15 & 45981536 & 15 & 45 \\
\hline 1307 & 15992 & 38195 & 21 & 0.02 & 80.14 & 0.083083 & 0.055055 & 8 & 136113975 & 8 & 136985884 & 8 & 136 \\
\hline 1551 & 29275 & 31887 & 17 & 0.02 & 80.16 & 0.076076 & 0.054054 & 10 & 77119998 & 10 & 77903646 & 10 & 77 \\
\hline 856 & 5080 & 5085 & 18 & 0.02 & 80.18 & 0.075075 & 0.052052 & 5 & 89017490 & 5 & 89879433 & 5 & 89 \\
\hline 622 & 52046 & 52339 & 7 & 0.02 & 80.2 & 0.048048 & 0.025025 & 3 & 144009549 & 3 & 144353522 & 3 & 144 \\
\hline 1534 & 53777 & 38465 & 20 & 0.02 & 80.22 & 0.08008 & 0.054054 & 10 & 60034333 & 10 & 60965150 & 10 & 60 \\
\hline 2498 & 29543 & 44930 & 25 & 0.02 & 80.25 & 0.084084 & 0.056056 & 19 & 45004996 & 19 & 45972443 & 19 & 45 \\
\hline 1721 & 25983 & 9603 & 32 & 0.02 & 80.27 & 0.111111 & 0.062062 & 13 & 17000021 & 13 & 17956613 & 13 & 17 \\
\hline 2046 & 53060 & 39149 & 23 & 0.02 & 80.29 & 0.092092 & 0.057057 & 14 & 123006309 & 14 & 123957615 & 14 & 123 \\
\hline 583 & 3214 & 21509 & 22 & 0.02 & 80.31 & 0.095095 & 0.057057 & 3 & 105002922 & 3 & 105933402 & 3 & 105 \\
\hline 1095 & 23626 & 23629 & 20 & 0.02 & 80.33 & 0.078078 & 0.055055 & 7 & 58003125 & 7 & 58927990 & 7 & 58 \\
\hline 693 & 22058 & 45095 & 23 & 0.02 & 80.35 & 0.077077 & 0.05005 & 4 & 70092569 & 4 & 70979785 & 4 & 70 \\
\hline 1545 & 18323 & 60676 & 13 & 0.02 & 80.37 & 0.062062 & 0.038038 & 10 & 71078073 & 10 & 71713257 & 10 & 71 \\
\hline 2011 & 47696 & 56621 & 27 & 0.02 & 80.39 & 0.103103 & 0.066066 & 14 & 88077735 & 14 & 88987258 & 14 & 88 \\
\hline 86 & 51445 & 19912 & 18 & 0.02 & 80.41 & 0.087087 & 0.053053 & 1 & 86014836 & 1 & 86991865 & 1 & 86 \\
\hline 1003 & 53717 & 23141 & 27 & 0.02 & 80.43 & 0.087087 & 0.063063 & 6 & 124059477 & 6 & 124954647 & 6 & 124 \\
\hline 1117 & 6312 & 6320 & 20 & 0.02 & 80.45 & 0.08008 & 0.047047 & 7 & 80027963 & 7 & 80985441 & 7 & 80 \\
\hline 104 & 54519 & 55902 & 22 & 0.02 & 80.47 & 0.079079 & 0.054054 & 1 & 104002361 & 1 & 104937362 & 1 & 104 \\
\hline 690 & 46663 & 22040 & 23 & 0.02 & 80.5 & 0.069069 & 0.041041 & 4 & 67015811 & 4 & 67992709 & 4 & 67 \\
\hline 403 & 40599 & 2362 & 26 & 0.02 & 80.52 & 0.092092 & 0.062062 & 2 & 88090537 & 2 & 88987913 & 2 & 88 \\
\hline 1803 & 30291 & 10087 & 22 & 0.02 & 80.54 & 0.087087 & 0.055055 & 13 & 99016394 & 13 & 99980492 & 13 & 99 \\
\hline 462 & 36434 & 36220 & 15 & 0.02 & 80.56 & 0.061061 & 0.041041 & 2 & 147103000 & 2 & 147952931 & 2 & 147 \\
\hline 350 & 2153 & 20840 & 18 & 0.02 & 80.58 & 0.072072 & 0.043043 & 2 & 34084545 & 2 & 34977540 & 2 & 34 \\
\hline 1965 & 26887 & 47615 & 19 & 0.02 & 80.6 & 0.069069 & 0.048048 & 14 & 42013177 & 14 & 42997445 & 14 & 42 \\
\hline 2478 & 55085 & 59759 & 13 & 0.02 & 80.62 & 0.071071 & 0.049049 & 19 & 25015600 & 19 & 25930877 & 19 & 25 \\
\hline 708 & 51990 & 21893 & 26 & 0.02 & 80.64 & 0.092092 & 0.058058 & 4 & 85011629 & 4 & 85824398 & 4 & 85 \\
\hline 2247 & 12709 & 28104 & 19 & 0.02 & 80.66 & 0.086086 & 0.055055 & 16 & 12073651 & 16 & 12976031 & 16 & 12 \\
\hline 1913 & 54867 & 26560 & 15 & 0.02 & 80.68 & 0.05005 & 0.032032 & 13 & 209095174 & 13 & 209954223 & 13 & 209 \\
\hline 2320 & 32464 & 49655 & 20 & 0.02 & 80.7 & 0.085085 & 0.057057 & 16 & 85011017 & 16 & 85843098 & 16 & 85 \\
\hline 1512 & 25179 & 8412 & 19 & 0.02 & 80.72 & 0.082082 & 0.052052 & 10 & 38009455 & 10 & 38986300 & 10 & 38 \\
\hline 223 & 36910 & 53243 & 11 & 0.02 & 80.74 & 0.042042 & 0.025025 & 1 & 223043310 & 1 & 223992839 & 1 & 223 \\
\hline 1688 & 38667 & 33231 & 16 & 0.02 & 80.77 & 0.065065 & 0.038038 & 12 & 48001470 & 12 & 48863354 & 12 & 48 \\
\hline 1243 & 57506 & 31821 & 14 & 0.02 & 80.79 & 0.054054 & 0.044044 & 8 & 72077897 & 8 & 72934282 & 8 & 72 \\
\hline 319 & 48515 & 20702 & 16 & 0.02 & 80.81 & 0.067067 & 0.044044 & 2 & 3024804 & 2 & 3972464 & 2 & 3 \\
\hline 508 & 2890 & 30765 & 29 & 0.02 & 80.83 & 0.138138 & 0.067067 & 3 & 30021862 & 3 & 30988651 & 3 & 30 \\
\hline 563 & 54568 & 3128 & 19 & 0.02 & 80.85 & 0.077077 & 0.046046 & 3 & 85125505 & 3 & 85886007 & 3 & 85 \\
\hline 531 & 2975 & 53227 & 17 & 0.02 & 80.87 & 0.073073 & 0.049049 & 3 & 53003881 & 3 & 53810019 & 3 & 53 \\
\hline
\end{tabular}




\begin{tabular}{|c|c|c|c|c|c|c|c|c|c|c|c|c|c|}
\hline 2323 & 57301 & 39484 & 23 & 0.02 & 80.89 & 0.09009 & 0.054054 & 17 & 1018025 & 17 & 1963091 & 17 & 1 \\
\hline 1781 & 32349 & 26217 & 15 & 0.02 & 80.91 & 0.077077 & 0.054054 & 13 & 77087193 & 13 & 77894260 & 13 & 77 \\
\hline 1397 & 34397 & 7834 & 21 & 0.02 & 80.93 & 0.087087 & 0.065065 & 9 & 77019488 & 9 & 77970228 & 9 & 77 \\
\hline 2245 & 44325 & 28100 & 10 & 0.02 & 80.95 & 0.054054 & 0.036036 & 16 & 10023136 & 16 & 10985374 & 16 & 10 \\
\hline 157 & 1061 & 61987 & 20 & 0.02 & 80.97 & 0.081081 & 0.05005 & 1 & 157003137 & 1 & 157966730 & 1 & 157 \\
\hline 614 & 60112 & 58635 & 22 & 0.02 & 80.99 & 0.087087 & 0.053053 & 3 & 136029014 & 3 & 136995452 & 3 & 136 \\
\hline 1886 & 60429 & 10389 & 6 & 0.02 & 81.01 & 0.036036 & 0.025025 & 13 & 182315832 & 13 & 182817467 & 13 & 182 \\
\hline 496 & 31870 & 50533 & 20 & 0.02 & 81.03 & 0.074074 & 0.037037 & 3 & 18042146 & 3 & 18985624 & 3 & 18 \\
\hline 1470 & 18814 & 24982 & 18 & 0.02 & 81.05 & 0.072072 & 0.044044 & 9 & 150097891 & 9 & 150980233 & 9 & 150 \\
\hline 1637 & 25682 & 9186 & 21 & 0.02 & 81.07 & 0.077077 & 0.053053 & 11 & 85016603 & 11 & 85932032 & 11 & 85 \\
\hline 1006 & 55733 & 17029 & 17 & 0.02 & 81.09 & 0.07007 & 0.048048 & 6 & 127003301 & 6 & 127961520 & 6 & 127 \\
\hline 542 & 3035 & 3039 & 14 & 0.02 & 81.11 & 0.059059 & 0.047047 & 3 & 64003964 & 3 & 64872620 & 3 & 64 \\
\hline 5 & 62015 & 40 & 18 & 0.02 & 81.13 & 0.068068 & 0.042042 & 1 & 5008585 & 1 & 5732081 & 1 & 5 \\
\hline 1354 & 55573 & 24584 & 23 & 0.02 & 81.15 & 0.103103 & 0.071071 & 9 & 34056153 & 9 & 34993225 & 9 & 34 \\
\hline 1797 & 30209 & 38825 & 15 & 0.02 & 81.17 & 0.06006 & 0.041041 & 13 & 93191917 & 13 & 93956431 & 13 & 93 \\
\hline 1904 & 29324 & 14927 & 17 & 0.02 & 81.19 & 0.067067 & 0.047047 & 13 & 200005691 & 13 & 200940291 & 13 & 200 \\
\hline 851 & 5055 & 29541 & 36 & 0.02 & 81.21 & 0.134134 & 0.059059 & 5 & 84005513 & 5 & 84932150 & 5 & 84 \\
\hline 1808 & 34971 & 31213 & 12 & 0.02 & 81.23 & 0.053053 & 0.039039 & 13 & 104031218 & 13 & 104807793 & 13 & 104 \\
\hline 480 & 32077 & 61938 & 16 & 0.02 & 81.25 & 0.06006 & 0.042042 & 3 & 2013852 & 3 & 2992936 & 3 & 2 \\
\hline 2028 & 39126 & 34756 & 23 & 0.02 & 81.27 & 0.107107 & 0.063063 & 14 & 105006631 & 14 & 105966472 & 14 & 105 \\
\hline 1179 & 14449 & 6790 & 16 & 0.02 & 81.29 & 0.066066 & 0.04004 & 8 & 8145247 & 8 & 8993808 & 8 & 8 \\
\hline 2034 & 43926 & 27299 & 30 & 0.02 & 81.31 & 0.099099 & 0.062062 & 14 & 111089602 & 14 & 111970799 & 14 & 111 \\
\hline 1288 & 7293 & 7306 & 30 & 0.02 & 81.33 & 0.092092 & 0.058058 & 8 & 117017825 & 8 & 117994361 & 8 & 117 \\
\hline 1392 & 60561 & 18765 & 22 & 0.02 & 81.35 & 0.089089 & 0.052052 & 9 & 72053046 & 9 & 72946896 & 9 & 72 \\
\hline 2460 & 14047 & 29036 & 20 & 0.02 & 81.37 & 0.081081 & 0.055055 & 19 & 7185187 & 19 & 7979887 & 19 & 7 \\
\hline 2371 & 28637 & 28639 & 17 & 0.02 & 81.39 & 0.09009 & 0.059059 & 17 & 49014076 & 17 & 49985893 & 17 & 49 \\
\hline 294 & 40365 & 19322 & 24 & 0.02 & 81.41 & 0.112112 & 0.066066 & 1 & 294012711 & 1 & 294890555 & 1 & 294 \\
\hline 1905 & 57209 & 10495 & 11 & 0.02 & 81.43 & 0.058058 & 0.035035 & 13 & 201035915 & 13 & 201929107 & 13 & 201 \\
\hline 1158 & 42143 & 49072 & 17 & 0.02 & 81.45 & 0.078078 & 0.05005 & 7 & 122018910 & 7 & 122910008 & 7 & 122 \\
\hline 1680 & 54822 & 25837 & 16 & 0.02 & 81.47 & 0.067067 & 0.049049 & 12 & 40019047 & 12 & 40544362 & 12 & 40 \\
\hline 1280 & 42341 & 7256 & 25 & 0.02 & 81.49 & 0.082082 & 0.047047 & 8 & 109015913 & 8 & 109852339 & 8 & 109 \\
\hline 1559 & 38486 & 42909 & 25 & 0.02 & 81.51 & 0.103103 & 0.071071 & 11 & 7000946 & 11 & 7976689 & 11 & 7 \\
\hline 1876 & 38897 & 59204 & 8 & 0.02 & 81.53 & 0.035035 & 0.028028 & 13 & 172047752 & 13 & 172744897 & 13 & 172 \\
\hline 1727 & 9639 & 29949 & 21 & 0.02 & 81.55 & 0.103103 & 0.056056 & 13 & 23032326 & 13 & 23944165 & 13 & 23 \\
\hline 1045 & 23299 & 23308 & 25 & 0.02 & 81.57 & 0.104104 & 0.067067 & 7 & 8003501 & 7 & 8967851 & 7 & 8 \\
\hline 1463 & 54512 & 17582 & 25 & 0.02 & 81.59 & 0.104104 & 0.056056 & 9 & 143029683 & 9 & 143890847 & 9 & 143 \\
\hline 1135 & 59132 & 42070 & 21 & 0.02 & 81.61 & 0.072072 & 0.036036 & 7 & 99021825 & 7 & 99992288 & 7 & 99 \\
\hline 1605 & 8909 & 47371 & 26 & 0.02 & 81.63 & 0.097097 & 0.064064 & 11 & 53000238 & 11 & 53920963 & 11 & 53 \\
\hline 143 & 54626 & 57690 & 16 & 0.02 & 81.65 & 0.07007 & 0.046046 & 1 & 143013233 & 1 & 143979942 & 1 & 143 \\
\hline
\end{tabular}




\begin{tabular}{|c|c|c|c|c|c|c|c|c|c|c|c|c|c|}
\hline 404 & 37134 & 2376 & 30 & 0.02 & 81.67 & 0.117117 & 0.068068 & 2 & 89007639 & 2 & 89995349 & 2 & 89 \\
\hline 2409 & 28829 & 13760 & 17 & 0.02 & 81.69 & 0.073073 & 0.05005 & 18 & 17013331 & 18 & 17876780 & 18 & 17 \\
\hline 1381 & 42570 & 24702 & 30 & 0.02 & 81.71 & 0.126126 & 0.065065 & 9 & 61003307 & 9 & 61986456 & 9 & 61 \\
\hline 1872 & 10335 & 43510 & 10 & 0.02 & 81.73 & 0.041041 & 0.031031 & 13 & 168097806 & 13 & 168856970 & 13 & 168 \\
\hline 824 & 52862 & 37653 & 21 & 0.02 & 81.75 & 0.077077 & 0.048048 & 5 & 57011829 & 5 & 57885800 & 5 & 57 \\
\hline 19 & 48399 & 19566 & 28 & 0.02 & 81.77 & 0.113113 & 0.064064 & 1 & 19017181 & 1 & 19992800 & 1 & 19 \\
\hline 347 & 56000 & 53156 & 25 & 0.02 & 81.79 & 0.099099 & 0.052052 & 2 & 31062461 & 2 & 31950516 & 2 & 31 \\
\hline 922 & 48276 & 14624 & 18 & 0.02 & 81.81 & 0.079079 & 0.043043 & 6 & 43076940 & 6 & 43969345 & 6 & 43 \\
\hline 299 & 20625 & 16654 & 16 & 0.02 & 81.83 & 0.084084 & 0.047047 & 1 & 299033446 & 1 & 299937327 & 1 & 299 \\
\hline 850 & 38165 & 61626 & 22 & 0.02 & 81.85 & 0.097097 & 0.059059 & 5 & 83011120 & 5 & 83918631 & 5 & 83 \\
\hline 1407 & 33069 & 29544 & 12 & 0.02 & 81.87 & 0.054054 & 0.04004 & 9 & 87009539 & 9 & 87977671 & 9 & 87 \\
\hline 1557 & 25354 & 38484 & 24 & 0.02 & 81.89 & 0.096096 & 0.061061 & 11 & 5016353 & 11 & 5987234 & 11 & 5 \\
\hline 164 & 46120 & 40123 & 26 & 0.02 & 81.91 & 0.095095 & 0.053053 & 1 & 164042900 & 1 & 164917958 & 1 & 164 \\
\hline 2410 & 17427 & 28834 & 15 & 0.02 & 81.93 & 0.072072 & 0.049049 & 18 & 18053144 & 18 & 18911413 & 18 & 18 \\
\hline 2586 & 45015 & 14343 & 20 & 0.02 & 81.95 & 0.092092 & 0.051051 & 19 & 138175988 & 19 & 138988497 & 19 & 138 \\
\hline 1874 & 26461 & 19317 & 15 & 0.02 & 81.97 & 0.067067 & 0.046046 & 13 & 170055214 & 13 & 170572438 & 13 & 170 \\
\hline 903 & 15916 & 54135 & 30 & 0.02 & 81.99 & 0.129129 & 0.053053 & 6 & 24005557 & 6 & 24928813 & 6 & 24 \\
\hline 724 & 41197 & 4211 & 22 & 0.02 & 82.01 & 0.094094 & 0.058058 & 4 & 101009461 & 4 & 101975122 & 4 & 101 \\
\hline 1791 & 36302 & 36334 & 18 & 0.02 & 82.03 & 0.056056 & 0.042042 & 13 & 87089543 & 13 & 87988310 & 13 & 87 \\
\hline 451 & 30625 & 59596 & 26 & 0.02 & 82.05 & 0.094094 & 0.055055 & 2 & 136043108 & 2 & 136947901 & 2 & 136 \\
\hline 2423 & 50754 & 61143 & 26 & 0.02 & 82.07 & 0.105105 & 0.061061 & 18 & 31024660 & 18 & 31995431 & 18 & 31 \\
\hline 1249 & 56689 & 24235 & 23 & 0.02 & 82.09 & 0.1001 & 0.06006 & 8 & 78021944 & 8 & 78996665 & 8 & 78 \\
\hline 1192 & 6861 & 34320 & 23 & 0.02 & 82.1 & 0.093093 & 0.053053 & 8 & 21000928 & 8 & 21987255 & 8 & 21 \\
\hline 1613 & 8974 & 8978 & 11 & 0.02 & 82.12 & 0.06006 & 0.036036 & 11 & 61033874 & 11 & 61996760 & 11 & 61 \\
\hline 2056 & 43991 & 11641 & 21 & 0.02 & 82.14 & 0.085085 & 0.049049 & 14 & 133005108 & 14 & 133783308 & 14 & 133 \\
\hline 2005 & 36169 & 47679 & 29 & 0.02 & 82.16 & 0.109109 & 0.035035 & 14 & 82017521 & 14 & 82965316 & 14 & 82 \\
\hline 1184 & 6812 & 38094 & 17 & 0.02 & 82.18 & 0.088088 & 0.048048 & 8 & 13111033 & 8 & 13951282 & 8 & 13 \\
\hline 855 & 45712 & 5079 & 15 & 0.02 & 82.2 & 0.077077 & 0.05005 & 5 & 88001856 & 5 & 88978805 & 5 & 88 \\
\hline 1677 & 52789 & 25830 & 18 & 0.02 & 82.22 & 0.074074 & 0.047047 & 12 & 37005248 & 12 & 37778827 & 12 & 37 \\
\hline 1890 & 26482 & 10414 & 19 & 0.02 & 82.24 & 0.075075 & 0.052052 & 13 & 186001107 & 13 & 186944653 & 13 & 186 \\
\hline 1769 & 26170 & 43367 & 23 & 0.02 & 82.26 & 0.096096 & 0.059059 & 13 & 65025003 & 13 & 65952730 & 13 & 65 \\
\hline 1056 & 34825 & 37890 & 15 & 0.02 & 82.28 & 0.076076 & 0.053053 & 7 & 19004434 & 7 & 19789994 & 7 & 19 \\
\hline 919 & 56822 & 18333 & 23 & 0.02 & 82.3 & 0.087087 & 0.053053 & 6 & 40053845 & 6 & 40937247 & 6 & 40 \\
\hline 2459 & 18441 & 55592 & 23 & 0.02 & 82.32 & 0.099099 & 0.058058 & 19 & 6003273 & 19 & 6798065 & 19 & 6 \\
\hline 1794 & 10050 & 26275 & 17 & 0.02 & 82.34 & 0.069069 & 0.037037 & 13 & 90039607 & 13 & 90998129 & 13 & 90 \\
\hline 1229 & 7043 & 24201 & 18 & 0.02 & 82.36 & 0.081081 & 0.047047 & 8 & 58000986 & 8 & 58901257 & 8 & 58 \\
\hline 1251 & 17128 & 59339 & 24 & 0.02 & 82.37 & 0.092092 & 0.068068 & 8 & 80038062 & 8 & 80973136 & 8 & 80 \\
\hline 2131 & 49580 & 44156 & 16 & 0.02 & 82.39 & 0.061061 & 0.031031 & 15 & 54046928 & 15 & 54991163 & 15 & 54 \\
\hline 1767 & 33639 & 9906 & 13 & 0.02 & 82.41 & 0.055055 & 0.041041 & 13 & 63250705 & 13 & 63994105 & 13 & 63 \\
\hline
\end{tabular}




\begin{tabular}{|c|c|c|c|c|c|c|c|c|c|c|c|c|}
\hline 707 & 4076 & 22145 & 19 & 0.02 & 82.43 & 0.081081 & 0.054054 & 4 & 84090680 & 4 & 84983220 & 4 \\
\hline 1564 & 25395 & 8680 & 24 & 0.02 & 82.45 & 0.084084 & 0.052052 & 11 & 12012805 & 11 & 12925634 & 11 \\
\hline 862 & 48866 & 41570 & 19 & 0.02 & 82.47 & 0.088088 & 0.049049 & 5 & 95007471 & 5 & 95971272 & 5 \\
\hline 925 & 14820 & 17282 & 12 & 0.02 & 82.49 & 0.052052 & 0.037037 & 6 & 46003088 & 6 & 46893592 & 6 \\
\hline 429 & 46459 & 37185 & 15 & 0.02 & 82.51 & 0.063063 & 0.037037 & 2 & 114047843 & 2 & 114975303 & 2 \\
\hline 943 & 45773 & 39692 & 19 & 0.02 & 82.53 & 0.074074 & 0.048048 & 6 & 64020985 & 6 & 64915744 & 6 \\
\hline 927 & 19027 & 33779 & 8 & 0.02 & 82.55 & 0.034034 & 0.026026 & 6 & 48031641 & 6 & 48792292 & 6 \\
\hline 361 & 2248 & 2251 & 19 & 0.02 & 82.57 & 0.08008 & 0.051051 & 2 & 45026774 & 2 & 45986058 & 2 \\
\hline 484 & 2800 & 54900 & 19 & 0.02 & 82.58 & 0.078078 & 0.046046 & 3 & 6011282 & 3 & 6591276 & 3 \\
\hline 95 & 724 & 19946 & 19 & 0.02 & 82.6 & 0.069069 & 0.038038 & 1 & 95010340 & 1 & 95995816 & 1 \\
\hline 2380 & 44678 & 28690 & 20 & 0.02 & 82.62 & 0.077077 & 0.048048 & 17 & 58007799 & 17 & 58990101 & 17 \\
\hline 1501 & 42788 & 54021 & 22 & 0.02 & 82.64 & 0.084084 & 0.054054 & 10 & 27028271 & 10 & 27940273 & 10 \\
\hline 2043 & 27336 & 11555 & 18 & 0.02 & 82.66 & 0.052052 & 0.033033 & 14 & 120063463 & 14 & 120986865 & 14 \\
\hline 71 & 32242 & 19839 & 21 & 0.02 & 82.68 & 0.076076 & 0.047047 & 1 & 71065994 & 1 & 71950726 & 1 \\
\hline 1442 & 52512 & 59241 & 22 & 0.02 & 82.7 & 0.111111 & 0.065065 & 9 & 122036577 & 9 & 122944920 & 9 \\
\hline 773 & 4608 & 22515 & 26 & 0.02 & 82.72 & 0.098098 & 0.06006 & 5 & 6072443 & 5 & 6980596 & 5 \\
\hline 941 & 31745 & 17047 & 13 & 0.02 & 82.74 & 0.058058 & 0.039039 & 6 & 62176946 & 6 & 62866629 & 6 \\
\hline 1959 & 47603 & 43714 & 22 & 0.02 & 82.75 & 0.104104 & 0.062062 & 14 & 36026991 & 14 & 36990529 & 14 \\
\hline 464 & 2743 & 32331 & 22 & 0.02 & 82.77 & 0.086086 & 0.047047 & 2 & 149106718 & 2 & 149987258 & 2 \\
\hline 641 & 37386 & 3558 & 21 & 0.02 & 82.79 & 0.087087 & 0.05005 & 4 & 18008464 & 4 & 18995385 & 4 \\
\hline 2281 & 39453 & 12934 & 14 & 0.02 & 82.81 & 0.058058 & 0.035035 & 16 & 46066010 & 16 & 46997060 & 16 \\
\hline 1163 & 42164 & 6718 & 26 & 0.02 & 82.83 & 0.089089 & 0.054054 & 7 & 127042709 & 7 & 127959806 & 7 \\
\hline 664 & 46595 & 3752 & 19 & 0.02 & 82.85 & 0.081081 & 0.052052 & 4 & 41008284 & 4 & 41937629 & 4 \\
\hline 1364 & 16627 & 7675 & 13 & 0.02 & 82.87 & 0.054054 & 0.038038 & 9 & 44432191 & 9 & 44949057 & 9 \\
\hline 1474 & 17129 & 8199 & 20 & 0.02 & 82.89 & 0.074074 & 0.054054 & 10 & 6354 & 10 & 933652 & 10 \\
\hline 345 & 32744 & 20816 & 21 & 0.02 & 82.91 & 0.076076 & 0.044044 & 2 & 29115937 & 2 & 29795889 & 2 \\
\hline 410 & 61803 & 53104 & 7 & 0.02 & 82.92 & 0.034034 & 0.025025 & 2 & 95014748 & 2 & 95957069 & 2 \\
\hline 1756 & 9830 & 9834 & 25 & 0.02 & 82.94 & 0.089089 & 0.064064 & 13 & 52016944 & 13 & 52962019 & 13 \\
\hline 2361 & 33221 & 13474 & 25 & 0.02 & 82.96 & 0.092092 & 0.059059 & 17 & 39094798 & 17 & 39980954 & 17 \\
\hline 333 & 60918 & 33459 & 16 & 0.02 & 82.98 & 0.064064 & 0.044044 & 2 & 17143182 & 2 & 17903452 & 2 \\
\hline 1274 & 18584 & 54793 & 19 & 0.02 & 83 & 0.081081 & 0.054054 & 8 & 103005406 & 8 & 103726346 & 8 \\
\hline 308 & 32581 & 14589 & 15 & 0.02 & 83.02 & 0.067067 & 0.041041 & 1 & 308111095 & 1 & 308984948 & 1 \\
\hline 114 & 53035 & 36756 & 18 & 0.02 & 83.04 & 0.074074 & 0.049049 & 1 & 114015245 & 1 & 114984686 & 1 \\
\hline 2163 & 27797 & 27803 & 24 & 0.02 & 83.06 & 0.096096 & 0.046046 & 15 & 86048980 & 15 & 86976928 & 15 \\
\hline 938 & 52125 & 56491 & 10 & 0.02 & 83.07 & 0.053053 & 0.036036 & 6 & 59213073 & 6 & 59962739 & 6 \\
\hline 1513 & 55805 & 38450 & 17 & 0.02 & 83.09 & 0.098098 & 0.056056 & 10 & 39007437 & 10 & 39667084 & 10 \\
\hline 1553 & 8601 & 25342 & 19 & 0.02 & 83.11 & 0.086086 & 0.052052 & 11 & 1000228 & 11 & 1873897 & 11 \\
\hline 1285 & 7280 & 51528 & 19 & 0.02 & 83.13 & 0.076076 & 0.047047 & 8 & 114008201 & 8 & 114746282 & 8 \\
\hline 2424 & 13862 & 51267 & 24 & 0.02 & 83.15 & 0.094094 & 0.056056 & 18 & 32091507 & 18 & 32986753 & 18 \\
\hline
\end{tabular}




\begin{tabular}{|c|c|c|c|c|c|c|c|c|c|c|c|c|c|}
\hline 1750 & 26109 & 9793 & 24 & 0.02 & 83.17 & 0.097097 & 0.063063 & 13 & 46015081 & 13 & 46975890 & 13 & 46 \\
\hline 2327 & 45104 & 13228 & 23 & 0.02 & 83.19 & 0.097097 & 0.065065 & 17 & 5041670 & 17 & 5999136 & 17 & 5 \\
\hline 1685 & 25858 & 16781 & 23 & 0.02 & 83.21 & 0.098098 & 0.048048 & 12 & 45034446 & 12 & 45985058 & 12 & 45 \\
\hline 852 & 50955 & 35158 & 16 & 0.02 & 83.22 & 0.069069 & 0.041041 & 5 & 85023135 & 5 & 85946546 & 5 & 85 \\
\hline 2124 & 12082 & 52048 & 6 & 0.02 & 83.24 & 0.04004 & 0.032032 & 15 & 47169627 & 15 & 47971398 & 15 & 47 \\
\hline 610 & 21628 & 21634 & 27 & 0.02 & 83.26 & 0.132132 & 0.076076 & 3 & 132154665 & 3 & 132947422 & 3 & 132 \\
\hline 441 & 2607 & 45560 & 20 & 0.02 & 83.28 & 0.098098 & 0.045045 & 2 & 126022431 & 2 & 126984010 & 2 & 126 \\
\hline 984 & 5478 & 23100 & 18 & 0.02 & 83.3 & 0.077077 & 0.051051 & 6 & 105043606 & 6 & 105987112 & 6 & 105 \\
\hline 102 & 39994 & 19971 & 17 & 0.02 & 83.32 & 0.076076 & 0.048048 & 1 & 102071378 & 1 & 102718610 & 1 & 102 \\
\hline 1962 & 26860 & 10910 & 24 & 0.02 & 83.34 & 0.084084 & 0.055055 & 14 & 39015629 & 14 & 39965589 & 14 & 39 \\
\hline 2336 & 28452 & 30353 & 16 & 0.02 & 83.35 & 0.066066 & 0.038038 & 17 & 14005506 & 17 & 14867578 & 17 & 14 \\
\hline 1239 & 29922 & 7089 & 14 & 0.02 & 83.37 & 0.063063 & 0.041041 & 8 & 68055002 & 8 & 68896436 & 8 & 68 \\
\hline 349 & 58230 & 40519 & 22 & 0.02 & 83.39 & 0.081081 & 0.053053 & 2 & 33011231 & 2 & 33982064 & 2 & 33 \\
\hline 2115 & 29826 & 50545 & 20 & 0.02 & 83.41 & 0.083083 & 0.051051 & 15 & 38003706 & 15 & 38894354 & 15 & 38 \\
\hline 1963 & 57119 & 10916 & 28 & 0.02 & 83.43 & 0.106106 & 0.067067 & 14 & 40008901 & 14 & 40922501 & 14 & 40 \\
\hline 2413 & 28840 & 28845 & 25 & 0.02 & 83.45 & 0.113113 & 0.056056 & 18 & 21017341 & 18 & 21942251 & 18 & 21 \\
\hline 1604 & 61681 & 25521 & 22 & 0.02 & 83.46 & 0.101101 & 0.06006 & 11 & 52010601 & 11 & 52969628 & 11 & 52 \\
\hline 2340 & 13302 & 13303 & 13 & 0.02 & 83.48 & 0.062062 & 0.035035 & 17 & 18057468 & 17 & 18983971 & 17 & 18 \\
\hline 417 & 40627 & 2448 & 15 & 0.02 & 83.5 & 0.069069 & 0.039039 & 2 & 102047786 & 2 & 102986466 & 2 & 102 \\
\hline 1043 & 5735 & 59074 & 23 & 0.02 & 83.52 & 0.111111 & 0.058058 & 7 & 6000797 & 7 & 6955465 & 7 & 6 \\
\hline 1820 & 54086 & 55132 & 15 & 0.02 & 83.54 & 0.065065 & 0.047047 & 13 & 116052233 & 13 & 116957243 & 13 & 116 \\
\hline 765 & 22474 & 4574 & 23 & 0.02 & 83.56 & 0.093093 & 0.067067 & 4 & 142008163 & 4 & 142982699 & 4 & 142 \\
\hline 174 & 40137 & 20211 & 11 & 0.02 & 83.58 & 0.047047 & 0.029029 & 1 & 174018173 & 1 & 174985817 & 1 & 174 \\
\hline 1612 & 47390 & 8973 & 18 & 0.02 & 83.59 & 0.07007 & 0.034034 & 11 & 60026383 & 11 & 60976002 & 11 & 60 \\
\hline 2360 & 28578 & 45482 & 21 & 0.02 & 83.61 & 0.094094 & 0.062062 & 17 & 38020355 & 17 & 38842836 & 17 & 38 \\
\hline 84 & 19900 & 19181 & 33 & 0.02 & 83.63 & 0.109109 & 0.064064 & 1 & 84007856 & 1 & 84960922 & 1 & 84 \\
\hline 739 & 4335 & 4345 & 25 & 0.02 & 83.65 & 0.097097 & 0.052052 & 4 & 116017042 & 4 & 116922748 & 4 & 116 \\
\hline 1611 & 47388 & 8964 & 15 & 0.02 & 83.67 & 0.069069 & 0.039039 & 11 & 59012574 & 11 & 59978485 & 11 & 59 \\
\hline 1378 & 24686 & 35799 & 12 & 0.02 & 83.69 & 0.043043 & 0.033033 & 9 & 58065674 & 9 & 58958297 & 9 & 58 \\
\hline 1465 & 8143 & 24963 & 30 & 0.02 & 83.7 & 0.097097 & 0.056056 & 9 & 145001798 & 9 & 145998807 & 9 & 145 \\
\hline 193 & 40177 & 1252 & 17 & 0.02 & 83.72 & 0.08008 & 0.046046 & 1 & 193062563 & 1 & 193998587 & 1 & 193 \\
\hline 624 & 4578 & 21669 & 24 & 0.02 & 83.74 & 0.096096 & 0.059059 & 4 & 1003897 & 4 & 1996854 & 4 & 1 \\
\hline 103 & 783 & 789 & 16 & 0.02 & 83.76 & 0.054054 & 0.035035 & 1 & 103024987 & 1 & 103980217 & 1 & 103 \\
\hline 767 & 51890 & 60972 & 19 & 0.02 & 83.78 & 0.072072 & 0.05005 & 5 & 144471 & 5 & 961240 & 5 & 0 \\
\hline 1372 & 29331 & 49183 & 22 & 0.02 & 83.79 & 0.099099 & 0.061061 & 9 & 52065210 & 9 & 52973032 & 9 & 52 \\
\hline 682 & 14600 & 3864 & 11 & 0.02 & 83.81 & 0.051051 & 0.028028 & 4 & 59063035 & 4 & 59967513 & 4 & 59 \\
\hline 2557 & 58250 & 45563 & 15 & 0.02 & 83.83 & 0.067067 & 0.042042 & 19 & 109003374 & 19 & 109562447 & 19 & 109 \\
\hline 569 & 31009 & 17174 & 12 & 0.02 & 83.85 & 0.064064 & 0.04004 & 3 & 91123979 & 3 & 91605127 & 3 & 91 \\
\hline 530 & 40810 & 2976 & 13 & 0.02 & 83.87 & 0.061061 & 0.042042 & 3 & 52025538 & 3 & 52990305 & 3 & 52 \\
\hline
\end{tabular}




\begin{tabular}{|c|c|c|c|c|c|c|c|c|c|c|c|c|c|}
\hline 1813 & 33181 & 56488 & 8 & 0.02 & 83.89 & 0.035035 & 0.022022 & 13 & 109045658 & 13 & 109986816 & 13 & 109 \\
\hline 1925 & 26610 & 10590 & 15 & 0.02 & 83.9 & 0.061061 & 0.042042 & 14 & 2036624 & 14 & 2993368 & 14 & 2 \\
\hline 1689 & 15974 & 43198 & 16 & 0.02 & 83.92 & 0.077077 & 0.041041 & 12 & 49085493 & 12 & 49990871 & 12 & 49 \\
\hline 1849 & 15373 & 59786 & 19 & 0.02 & 83.94 & 0.073073 & 0.045045 & 13 & 145009805 & 13 & 145772058 & 13 & 145 \\
\hline 2491 & 29091 & 14138 & 15 & 0.02 & 83.96 & 0.066066 & 0.043043 & 19 & 38023389 & 19 & 38708793 & 19 & 38 \\
\hline 1712 & 60854 & 38692 & 22 & 0.02 & 83.98 & 0.099099 & 0.048048 & 13 & 8050547 & 13 & 8963066 & 13 & 8 \\
\hline 1561 & 25378 & 47329 & 25 & 0.02 & 83.99 & 0.1001 & 0.06006 & 11 & 9066617 & 11 & 9980144 & 11 & 9 \\
\hline 242 & 1515 & 58127 & 26 & 0.02 & 84.01 & 0.122122 & 0.052052 & 1 & 242044327 & 1 & 242984908 & 1 & 242 \\
\hline 98 & 45980 & 752 & 17 & 0.02 & 84.03 & 0.072072 & 0.045045 & 1 & 98048248 & 1 & 98992614 & 1 & 98 \\
\hline 1336 & 42461 & 39693 & 22 & 0.02 & 84.05 & 0.076076 & 0.059059 & 9 & 16047080 & 9 & 16962945 & 9 & 16 \\
\hline 1304 & 38189 & 15169 & 19 & 0.02 & 84.06 & 0.078078 & 0.051051 & 8 & 133022001 & 8 & 133950496 & 8 & 133 \\
\hline 859 & 41563 & 53421 & 16 & 0.02 & 84.08 & 0.068068 & 0.043043 & 5 & 92073891 & 5 & 92957318 & 5 & 92 \\
\hline 1012 & 16379 & 23170 & 17 & 0.02 & 84.1 & 0.074074 & 0.046046 & 6 & 133030240 & 6 & 133929215 & 6 & 133 \\
\hline 2417 & 62026 & 28864 & 30 & 0.02 & 84.12 & 0.111111 & 0.068068 & 18 & 25027827 & 18 & 25981606 & 18 & 25 \\
\hline 1432 & 24841 & 42634 & 17 & 0.02 & 84.14 & 0.072072 & 0.046046 & 9 & 112012220 & 9 & 112995189 & 9 & 112 \\
\hline 502 & 33625 & 15928 & 25 & 0.02 & 84.15 & 0.078078 & 0.051051 & 3 & 24010664 & 3 & 24976420 & 3 & 24 \\
\hline 1270 & 24287 & 7209 & 14 & 0.02 & 84.17 & 0.055055 & 0.03003 & 8 & 99027479 & 8 & 99969028 & 8 & 99 \\
\hline 964 & 14784 & 16130 & 12 & 0.02 & 84.19 & 0.059059 & 0.036036 & 6 & 85010159 & 6 & 85994652 & 6 & 85 \\
\hline 1380 & 60744 & 7756 & 15 & 0.02 & 84.21 & 0.064064 & 0.047047 & 9 & 60085650 & 9 & 60985372 & 9 & 60 \\
\hline 1773 & 50726 & 9945 & 14 & 0.02 & 84.23 & 0.056056 & 0.035035 & 13 & 69080928 & 13 & 69987699 & 13 & 69 \\
\hline 1321 & 31799 & 42410 & 18 & 0.02 & 84.24 & 0.059059 & 0.045045 & 9 & 1120035 & 9 & 1707931 & 9 & 1 \\
\hline 2447 & 18489 & 19199 & 23 & 0.02 & 84.26 & 0.114114 & 0.07007 & 18 & 55026793 & 18 & 55963572 & 18 & 55 \\
\hline 2335 & 28428 & 28453 & 25 & 0.02 & 84.28 & 0.092092 & 0.055055 & 17 & 13015931 & 17 & 13983032 & 17 & 13 \\
\hline 794 & 15878 & 37554 & 15 & 0.02 & 84.3 & 0.067067 & 0.037037 & 5 & 27040193 & 5 & 27970414 & 5 & 27 \\
\hline 1287 & 30832 & 7292 & 16 & 0.02 & 84.31 & 0.057057 & 0.037037 & 8 & 116042287 & 8 & 116996260 & 8 & 116 \\
\hline 827 & 14795 & 4894 & 20 & 0.02 & 84.33 & 0.094094 & 0.057057 & 5 & 60196527 & 5 & 60978291 & 5 & 60 \\
\hline 68 & 19824 & 45924 & 14 & 0.02 & 84.35 & 0.075075 & 0.044044 & 1 & 68132380 & 1 & 68993279 & 1 & 68 \\
\hline 814 & 33661 & 4844 & 14 & 0.02 & 84.37 & 0.064064 & 0.039039 & 5 & 47023315 & 5 & 47940238 & 5 & 47 \\
\hline 2412 & 28837 & 60104 & 19 & 0.02 & 84.38 & 0.073073 & 0.037037 & 18 & 20017978 & 18 & 20991435 & 18 & 20 \\
\hline 2415 & 13788 & 33744 & 23 & 0.02 & 84.4 & 0.091091 & 0.051051 & 18 & 23005936 & 18 & 23969429 & 18 & 23 \\
\hline 387 & 15406 & 34389 & 8 & 0.02 & 84.42 & 0.039039 & 0.021021 & 2 & 72042065 & 2 & 72956647 & 2 & 72 \\
\hline 1199 & 24125 & 6914 & 17 & 0.02 & 84.44 & 0.069069 & 0.039039 & 8 & 28051243 & 8 & 28996107 & 8 & 28 \\
\hline 1853 & 57739 & 43486 & 15 & 0.02 & 84.45 & 0.07007 & 0.048048 & 13 & 149037360 & 13 & 149983411 & 13 & 149 \\
\hline 2134 & 51617 & 12148 & 22 & 0.02 & 84.47 & 0.083083 & 0.042042 & 15 & 57255533 & 15 & 57880292 & 15 & 57 \\
\hline 166 & 20193 & 17078 & 21 & 0.02 & 84.49 & 0.082082 & 0.048048 & 1 & 166046787 & 1 & 166934229 & 1 & 166 \\
\hline 2368 & 55597 & 54153 & 26 & 0.02 & 84.51 & 0.115115 & 0.049049 & 17 & 46016364 & 17 & 46995794 & 17 & 46 \\
\hline 1450 & 52285 & 50352 & 16 & 0.02 & 84.52 & 0.062062 & 0.04004 & 9 & 130383913 & 9 & 130992209 & 9 & 130 \\
\hline 2276 & 39440 & 12909 & 21 & 0.02 & 84.54 & 0.069069 & 0.036036 & 16 & 41035539 & 16 & 41985204 & 16 & 41 \\
\hline 1784 & 33755 & 43394 & 12 & 0.02 & 84.56 & 0.057057 & 0.037037 & 13 & 80063897 & 13 & 80987722 & 13 & 80 \\
\hline
\end{tabular}




\begin{tabular}{|c|c|c|c|c|c|c|c|c|c|c|c|c|c|}
\hline 1471 & 49212 & 18200 & 30 & 0.02 & 84.58 & 0.111111 & 0.062062 & 9 & 151005996 & 9 & 151986166 & 9 & 151 \\
\hline 231 & 34522 & 61459 & 27 & 0.02 & 84.59 & 0.117117 & 0.063063 & 1 & 231003149 & 1 & 231932843 & 1 & 231 \\
\hline 1715 & 59175 & 45146 & 25 & 0.02 & 84.61 & 0.092092 & 0.054054 & 13 & 11042615 & 13 & 11949152 & 13 & 11 \\
\hline 111 & 830 & 17200 & 11 & 0.02 & 84.63 & 0.054054 & 0.031031 & 1 & 111456164 & 1 & 111938688 & 1 & 111 \\
\hline 2154 & 12231 & 47897 & 8 & 0.02 & 84.65 & 0.037037 & 0.025025 & 15 & 77108630 & 15 & 77875540 & 15 & 77 \\
\hline 1213 & 6999 & 16788 & 13 & 0.02 & 84.66 & 0.05005 & 0.038038 & 8 & 42055005 & 8 & 42680025 & 8 & 42 \\
\hline 2590 & 31214 & 14353 & 18 & 0.02 & 84.68 & 0.076076 & 0.045045 & 19 & 142000976 & 19 & 142984588 & 19 & 142 \\
\hline 2136 & 55438 & 12162 & 18 & 0.02 & 84.7 & 0.071071 & 0.048048 & 15 & 59124311 & 15 & 59950764 & 15 & 59 \\
\hline 262 & 1631 & 20442 & 15 & 0.02 & 84.71 & 0.057057 & 0.039039 & 1 & 262039871 & 1 & 262967147 & 1 & 262 \\
\hline 588 & 21527 & 3257 & 30 & 0.02 & 84.73 & 0.111111 & 0.063063 & 3 & 110005181 & 3 & 110998569 & 3 & 110 \\
\hline 1826 & 10143 & 10147 & 13 & 0.02 & 84.75 & 0.068068 & 0.044044 & 13 & 122042291 & 13 & 122946774 & 13 & 122 \\
\hline 239 & 60667 & 51773 & 19 & 0.02 & 84.77 & 0.09009 & 0.046046 & 1 & 239045601 & 1 & 239987141 & 1 & 239 \\
\hline 1211 & 24173 & 61131 & 19 & 0.02 & 84.78 & 0.074074 & 0.058058 & 8 & 40020473 & 8 & 40919857 & 8 & 40 \\
\hline 113 & 34626 & 52920 & 20 & 0.02 & 84.8 & 0.086086 & 0.051051 & 1 & 113061808 & 1 & 113930519 & 1 & 113 \\
\hline 1679 & 16604 & 30363 & 25 & 0.02 & 84.82 & 0.094094 & 0.058058 & 12 & 39000847 & 12 & 39992894 & 12 & 39 \\
\hline 651 & 41035 & 3646 & 21 & 0.02 & 84.84 & 0.063063 & 0.043043 & 4 & 28013430 & 4 & 28935985 & 4 & 28 \\
\hline 209 & 1339 & 20317 & 17 & 0.02 & 84.85 & 0.073073 & 0.052052 & 1 & 209010763 & 1 & 209927137 & 1 & 209 \\
\hline 50 & 19725 & 53398 & 23 & 0.02 & 84.87 & 0.086086 & 0.057057 & 1 & 50015447 & 1 & 50999817 & 1 & 50 \\
\hline 940 & 29764 & 18999 & 15 & 0.02 & 84.89 & 0.067067 & 0.049049 & 6 & 61004887 & 6 & 61916523 & 6 & 61 \\
\hline 770 & 41351 & 41356 & 21 & 0.02 & 84.9 & 0.08008 & 0.047047 & 5 & 3060760 & 5 & 3844970 & 5 & 3 \\
\hline 1419 & 52081 & 17891 & 21 & 0.02 & 84.92 & 0.11011 & 0.061061 & 9 & 99173519 & 9 & 99988998 & 9 & 99 \\
\hline 316 & 29677 & 52837 & 13 & 0.02 & 84.94 & 0.075075 & 0.041041 & 2 & 16416 & 2 & 963130 & 2 & 0 \\
\hline 1079 & 57712 & 58557 & 23 & 0.02 & 84.95 & 0.095095 & 0.046046 & 7 & 42000072 & 7 & 42999450 & 7 & 42 \\
\hline 590 & 3263 & 21541 & 17 & 0.02 & 84.97 & 0.072072 & 0.045045 & 3 & 112022721 & 3 & 112989158 & 3 & 112 \\
\hline 303 & 20647 & 48493 & 28 & 0.02 & 84.99 & 0.136136 & 0.081081 & 1 & 303008877 & 1 & 303984121 & 1 & 303 \\
\hline 2588 & 14344 & 15258 & 13 & 0.02 & 85.01 & 0.071071 & 0.045045 & 19 & 140000914 & 19 & 140920145 & 19 & 140 \\
\hline 995 & 5511 & 5516 & 18 & 0.02 & 85.02 & 0.074074 & 0.045045 & 6 & 116010663 & 6 & 116969069 & 6 & 116 \\
\hline 1328 & 49162 & 24474 & 23 & 0.02 & 85.04 & 0.086086 & 0.052052 & 9 & 8047973 & 9 & 8994791 & 9 & 8 \\
\hline 2165 & 27812 & 12297 & 27 & 0.02 & 85.06 & 0.101101 & 0.049049 & 15 & 88036047 & 15 & 88943039 & 15 & 88 \\
\hline 1895 & 26501 & 33348 & 17 & 0.02 & 85.07 & 0.068068 & 0.039039 & 13 & 191101378 & 13 & 191971388 & 13 & 191 \\
\hline 430 & 37184 & 2534 & 12 & 0.02 & 85.09 & 0.044044 & 0.033033 & 2 & 115000618 & 2 & 115815947 & 2 & 115 \\
\hline 1893 & 15960 & 10432 & 20 & 0.02 & 85.11 & 0.074074 & 0.051051 & 13 & 189125669 & 13 & 189990288 & 13 & 189 \\
\hline 1790 & 26246 & 54261 & 18 & 0.02 & 85.12 & 0.077077 & 0.046046 & 13 & 86015154 & 13 & 86889866 & 13 & 86 \\
\hline 2430 & 32781 & 57380 & 17 & 0.02 & 85.14 & 0.081081 & 0.051051 & 18 & 38093304 & 18 & 38804587 & 18 & 38 \\
\hline 2132 & 27734 & 12132 & 19 & 0.02 & 85.16 & 0.082082 & 0.043043 & 15 & 55038222 & 15 & 55993215 & 15 & 55 \\
\hline 802 & 32054 & 22667 & 18 & 0.02 & 85.17 & 0.083083 & 0.048048 & 5 & 35168512 & 5 & 35945657 & 5 & 35 \\
\hline 534 & 53018 & 56376 & 23 & 0.02 & 85.19 & 0.089089 & 0.056056 & 3 & 56005947 & 3 & 56950047 & 3 & 56 \\
\hline 1807 & 10101 & 61186 & 20 & 0.02 & 85.21 & 0.081081 & 0.05005 & 13 & 103033108 & 13 & 103926061 & 13 & 103 \\
\hline 1622 & 25588 & 51088 & 20 & 0.02 & 85.22 & 0.077077 & 0.042042 & 11 & 70044668 & 11 & 70967934 & 11 & 70 \\
\hline
\end{tabular}




\begin{tabular}{|c|c|c|c|c|c|c|c|c|c|c|c|c|c|}
\hline 131 & 20062 & 48425 & 7 & 0.02 & 85.24 & 0.049049 & 0.033033 & 1 & 131011448 & 1 & 131973599 & 1 & 131 \\
\hline 227 & 20342 & 20345 & 16 & 0.02 & 85.26 & 0.065065 & 0.035035 & 1 & 227018020 & 1 & 227989037 & 1 & 227 \\
\hline 176 & 46150 & 20221 & 22 & 0.02 & 85.27 & 0.069069 & 0.043043 & 1 & 176015699 & 1 & 176979355 & 1 & 17 \\
\hline 1423 & 60183 & 24833 & 12 & 0.02 & 85.29 & 0.047047 & 0.026026 & 9 & 103035428 & 9 & 103976159 & 9 & \\
\hline 85 & 58030 & 667 & 21 & 0.02 & 85.31 & 0.082082 & 0.048048 & 1 & 85011343 & 1 & 85975562 & 1 & \\
\hline 915 & 14585 & 32592 & 17 & 0.02 & 85.32 & 0.063063 & 0.04004 & 6 & 36106926 & 6 & 36964748 & 6 & \\
\hline 1088 & 37933 & 41943 & 23 & 0.02 & 85.34 & 0.093093 & 0.056056 & 7 & 51027425 & 7 & 51991520 & 7 & \\
\hline 1001 & 35570 & 62070 & 17 & 0.02 & 85.36 & 0.069069 & 0.043043 & 6 & 122145146 & 6 & 122942504 & 6 & 12 \\
\hline 1028 & 5679 & 50895 & 15 & 0.02 & 85.37 & 0.076076 & 0.045045 & 6 & 149094962 & 6 & 149758688 & 6 & דו \\
\hline 592 & 59033 & 3281 & 21 & 0.02 & 85.39 & 0.077077 & 0.057057 & 3 & 114049876 & 3 & 114951968 & 3 & 11 \\
\hline 1370 & 60090 & 45237 & 20 & 0.02 & 85.41 & 0.076076 & 0.048048 & 9 & 50024764 & 9 & 50962419 & 9 & \\
\hline 931 & 59594 & 41639 & 12 & 0.02 & 85.42 & 0.05005 & 0.032032 & 6 & 52063034 & 6 & 52993696 & 6 & 5 \\
\hline 2589 & 56026 & 15779 & 10 & 0.02 & 85.44 & 0.056056 & 0.032032 & 19 & 141270018 & 19 & 141996499 & 19 & T \\
\hline 1101 & 49039 & 6227 & 21 & 0.02 & 85.45 & 0.09009 & 0.05005 & 7 & 64026660 & 7 & 64959971 & 7 & 6 \\
\hline 2585 & 58402 & 48256 & 6 & 0.02 & 85.47 & 0.035035 & 0.022022 & 19 & 137042297 & 19 & 137814306 & 19 & 10 \\
\hline 1783 & 31884 & 43393 & 14 & 0.02 & 85.49 & 0.06006 & 0.036036 & 13 & 79044236 & 13 & 79912229 & 13 & \\
\hline 2017 & 52375 & 27198 & 12 & 0.02 & 85.5 & 0.04004 & 0.03003 & 14 & 94036401 & 14 & 94994468 & 14 & 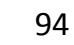 \\
\hline 1897 & 43543 & 45302 & 15 & 0.02 & 85.52 & 0.075075 & 0.031031 & 13 & 193019061 & 13 & 193793634 & 13 & 19 \\
\hline 921 & 40424 & 53713 & 10 & 0.02 & 85.54 & 0.059059 & 0.031031 & 6 & 42104347 & 6 & 42946643 & 6 & \\
\hline 517 & 56924 & 48619 & 8 & 0.02 & 85.55 & 0.035035 & 0.028028 & 3 & 39128984 & 3 & 39992614 & 3 & 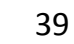 \\
\hline 1607 & 8927 & 38594 & 24 & 0.02 & 85.57 & 0.115115 & 0.059059 & 11 & 55001359 & 11 & 55975611 & 11 & 5 \\
\hline 255 & 36974 & 20417 & 21 & 0.02 & 85.59 & 0.079079 & 0.032032 & 1 & 255032767 & 1 & 255981511 & 1 & 255 \\
\hline 389 & 55233 & 60504 & 10 & 0.02 & 85.6 & 0.057057 & 0.033033 & 2 & 74063668 & 2 & 74917715 & 2 & 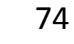 \\
\hline 1961 & 52781 & 34552 & 22 & 0.02 & 85.62 & 0.092092 & 0.058058 & 14 & 38017404 & 14 & 38996258 & 14 & 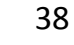 \\
\hline 463 & 52616 & 37243 & 13 & 0.02 & 85.63 & 0.05005 & 0.03003 & 2 & 148010312 & 2 & 148847293 & 2 & $14 \varepsilon$ \\
\hline 2552 & 52052 & 29148 & 14 & 0.02 & 85.65 & 0.065065 & 0.038038 & 19 & 104042870 & 19 & 104939296 & 19 & 10 \\
\hline 981 & 46921 & 23089 & 16 & 0.02 & 85.67 & 0.075075 & 0.043043 & 6 & 102098390 & 6 & 102973310 & 6 & 102 \\
\hline 1413 & 51718 & 24810 & 17 & 0.02 & 85.68 & 0.079079 & 0.047047 & 9 & 93035029 & 9 & 93976824 & 9 & 9 \\
\hline 1258 & 30390 & 32096 & 18 & 0.02 & 85.7 & 0.08008 & 0.042042 & 8 & 87000474 & 8 & 87954252 & 8 & 8 \\
\hline 2345 & 28486 & 48036 & 25 & 0.02 & 85.72 & 0.082082 & 0.044044 & 17 & 23024619 & 17 & 23961801 & 17 & 2 \\
\hline 2178 & 47925 & 16216 & 17 & 0.02 & 85.73 & 0.06006 & 0.036036 & 15 & 101102306 & 15 & 101921509 & 15 & 10 \\
\hline 1008 & 41710 & 29794 & 13 & 0.02 & 85.75 & 0.062062 & 0.039039 & 6 & 129010911 & 6 & 129902435 & 6 & 12 \\
\hline 257 & 36982 & 14478 & 28 & 0.02 & 85.76 & 0.113113 & 0.056056 & 1 & 257001258 & 1 & 257954281 & 1 & 25 \\
\hline 2026 & 27250 & 58170 & 21 & 0.02 & 85.78 & 0.092092 & 0.052052 & 14 & 103045309 & 14 & 103936269 & 14 & 10 \\
\hline 2171 & 12339 & 12343 & 17 & 0.02 & 85.8 & 0.085085 & 0.035035 & 15 & 94025320 & 15 & 94994150 & 15 & \\
\hline 39 & 55819 & 328 & 18 & 0.02 & 85.81 & 0.064064 & 0.036036 & 1 & 39019040 & 1 & 39987321 & 1 & \\
\hline 1774 & 43375 & 26185 & 15 & 0.02 & 85.83 & 0.066066 & 0.043043 & 13 & 70328466 & 13 & 70982729 & 13 & 7 \\
\hline 1104 & 6236 & 37962 & 15 & 0.02 & 85.84 & 0.065065 & 0.045045 & 7 & 67107544 & 7 & 67917223 & 7 & 6 \\
\hline 1835 & 10176 & 50151 & 13 & 0.02 & 85.86 & 0.076076 & 0.051051 & 13 & 131037563 & 13 & 131889566 & 13 & 13 \\
\hline
\end{tabular}




\begin{tabular}{|c|c|c|c|c|c|c|c|c|c|c|c|c|c|}
\hline 749 & 46769 & 60507 & 12 & 0.02 & 85.88 & 0.068068 & 0.054054 & 4 & 126080976 & 4 & 126984212 & 4 & 126 \\
\hline 72 & 19840 & 574 & 27 & 0.02 & 85.89 & 0.101101 & 0.047047 & 1 & 72028362 & 1 & 72982828 & 1 & 72 \\
\hline 2195 & 12434 & 12437 & 17 & 0.02 & 85.91 & 0.069069 & 0.05005 & 15 & 118002592 & 15 & 118920135 & 15 & 118 \\
\hline 2453 & 52415 & 17324 & 11 & 0.02 & 85.92 & 0.045045 & 0.033033 & 19 & 462585 & 19 & 930921 & 19 & 0 \\
\hline 44 & 61816 & 58745 & 18 & 0.02 & 85.94 & 0.078078 & 0.043043 & 1 & 44013987 & 1 & 44938878 & 1 & 44 \\
\hline 2075 & 51747 & 11812 & 20 & 0.02 & 85.96 & 0.078078 & 0.053053 & 14 & 152178755 & 14 & 152978092 & 14 & 152 \\
\hline 1656 & 61513 & 60787 & 23 & 0.02 & 85.97 & 0.102102 & 0.057057 & 12 & 16006733 & 12 & 16925639 & 12 & 16 \\
\hline 805 & 4801 & 4806 & 18 & 0.02 & 85.99 & 0.078078 & 0.044044 & 5 & 38006963 & 5 & 38988849 & 5 & 38 \\
\hline 890 & 15889 & 41604 & 18 & 0.02 & 86 & 0.069069 & 0.041041 & 6 & 11003757 & 6 & 11989602 & 6 & 11 \\
\hline 2137 & 44164 & 39270 & 18 & 0.02 & 86.02 & 0.074074 & 0.044044 & 15 & 60008146 & 15 & 60984129 & 15 & 60 \\
\hline 353 & 61746 & 2184 & 22 & 0.02 & 86.04 & 0.096096 & 0.055055 & 2 & 37273764 & 2 & 37988750 & 2 & 37 \\
\hline 204 & 51322 & 1309 & 22 & 0.02 & 86.05 & 0.089089 & 0.036036 & 1 & 204023854 & 1 & 204988249 & 1 & 204 \\
\hline 232 & 46276 & 20358 & 22 & 0.02 & 86.07 & 0.093093 & 0.044044 & 1 & 232040879 & 1 & 232978056 & 1 & 232 \\
\hline 1452 & 38385 & 18471 & 15 & 0.02 & 86.08 & 0.055055 & 0.031031 & 9 & 132023179 & 9 & 132827171 & 9 & 132 \\
\hline 1361 & 36017 & 24998 & 19 & 0.02 & 86.1 & 0.069069 & 0.043043 & 9 & 41043659 & 9 & 41978500 & 9 & 41 \\
\hline 2403 & 55403 & 28800 & 17 & 0.02 & 86.12 & 0.069069 & 0.036036 & 18 & 11037894 & 18 & 11928951 & 18 & 11 \\
\hline 945 & 30289 & 16795 & 18 & 0.02 & 86.13 & 0.055055 & 0.034034 & 6 & 66072075 & 6 & 66952267 & 6 & 66 \\
\hline 1303 & 55683 & 7363 & 8 & 0.02 & 86.15 & 0.043043 & 0.024024 & 8 & 132376091 & 8 & 132945670 & 8 & 132 \\
\hline 1782 & 19421 & 16161 & 14 & 0.02 & 86.16 & 0.064064 & 0.047047 & 13 & 78016930 & 13 & 78972578 & 13 & 78 \\
\hline 1281 & 36019 & 34079 & 19 & 0.02 & 86.18 & 0.067067 & 0.043043 & 8 & 110016042 & 8 & 110901720 & 8 & 110 \\
\hline 2326 & 28404 & 15858 & 19 & 0.02 & 86.19 & 0.082082 & 0.046046 & 17 & 4055697 & 17 & 4868955 & 17 & 4 \\
\hline 637 & 3516 & 57743 & 26 & 0.02 & 86.21 & 0.11011 & 0.054054 & 4 & 14003125 & 4 & 14995885 & 4 & 14 \\
\hline 276 & 46374 & 1727 & 17 & 0.02 & 86.23 & 0.065065 & 0.035035 & 1 & 276112956 & 1 & 276982150 & 1 & 276 \\
\hline 331 & 30756 & 40462 & 7 & 0.02 & 86.24 & 0.033033 & 0.028028 & 2 & 15670974 & 2 & 15981321 & 2 & 15 \\
\hline 982 & 23090 & 23093 & 17 & 0.02 & 86.26 & 0.051051 & 0.038038 & 6 & 103034132 & 6 & 103881022 & 6 & 103 \\
\hline 1847 & 43471 & 10225 & 10 & 0.02 & 86.27 & 0.042042 & 0.027027 & 13 & 143281651 & 13 & 143866440 & 13 & 143 \\
\hline 1565 & 25401 & 38511 & 18 & 0.02 & 86.29 & 0.085085 & 0.052052 & 11 & 13033569 & 11 & 13983161 & 11 & 13 \\
\hline 1384 & 56775 & 56245 & 24 & 0.02 & 86.3 & 0.085085 & 0.056056 & 9 & 64024041 & 9 & 64983107 & 9 & 64 \\
\hline 857 & 22834 & 57956 & 15 & 0.02 & 86.32 & 0.042042 & 0.036036 & 5 & 90128642 & 5 & 90976986 & 5 & 90 \\
\hline 205 & 40189 & 20308 & 27 & 0.02 & 86.34 & 0.102102 & 0.046046 & 1 & 205020361 & 1 & 205994796 & 1 & 205 \\
\hline 1838 & 43458 & 10203 & 20 & 0.02 & 86.35 & 0.091091 & 0.049049 & 13 & 134021456 & 13 & 134985031 & 13 & 134 \\
\hline 786 & 37546 & 22591 & 14 & 0.02 & 86.37 & 0.068068 & 0.044044 & 5 & 19053403 & 5 & 19631292 & 5 & 19 \\
\hline 2466 & 55739 & 30755 & 18 & 0.02 & 86.38 & 0.092092 & 0.039039 & 19 & 13061465 & 19 & 13993116 & 19 & 13 \\
\hline 59 & 45910 & 39917 & 20 & 0.02 & 86.4 & 0.08008 & 0.047047 & 1 & 59033524 & 1 & 59964653 & 1 & 59 \\
\hline 1047 & 48968 & 5775 & 20 & 0.02 & 86.41 & 0.078078 & 0.045045 & 7 & 10031634 & 7 & 10964520 & 7 & 10 \\
\hline 277 & 1730 & 56414 & 13 & 0.02 & 86.43 & 0.049049 & 0.034034 & 1 & 277105135 & 1 & 277975248 & 1 & 277 \\
\hline 1007 & 33730 & 5582 & 20 & 0.02 & 86.44 & 0.075075 & 0.048048 & 6 & 128062298 & 6 & 128989993 & 6 & 128 \\
\hline 2175 & 12362 & 16817 & 5 & 0.02 & 86.46 & 0.042042 & 0.023023 & 15 & 98011713 & 15 & 98451291 & 15 & 98 \\
\hline 743 & 41269 & 41272 & 22 & 0.02 & 86.48 & 0.091091 & 0.056056 & 4 & 120018660 & 4 & 120910957 & 4 & 120 \\
\hline
\end{tabular}




\begin{tabular}{|c|c|c|c|c|c|c|c|c|c|c|c|c|c|}
\hline 1122 & 53085 & 47114 & 20 & 0.02 & 86.49 & 0.079079 & 0.052052 & 7 & 86017385 & 7 & 86955735 & 7 & 86 \\
\hline 298 & 40378 & 40381 & 23 & 0.02 & 86.51 & 0.094094 & 0.042042 & 1 & 298042326 & 1 & 298972575 & 1 & 298 \\
\hline 1683 & 43188 & 58317 & 22 & 0.02 & 86.52 & 0.068068 & 0.039039 & 12 & 43068568 & 12 & 43987384 & 12 & 43 \\
\hline 70 & 554 & 19836 & 24 & 0.02 & 86.54 & 0.091091 & 0.048048 & 1 & 70002082 & 1 & 70950692 & 1 & 70 \\
\hline 1842 & 26367 & 26370 & 20 & 0.02 & 86.55 & 0.094094 & 0.053053 & 13 & 138039183 & 13 & 138941535 & 13 & 138 \\
\hline 2100 & 50128 & 32380 & 15 & 0.02 & 86.57 & 0.06006 & 0.032032 & 15 & 23067061 & 15 & 23987116 & 15 & 23 \\
\hline 2140 & 12176 & 12190 & 21 & 0.02 & 86.58 & 0.076076 & 0.048048 & 15 & 63029841 & 15 & 63998627 & 15 & 63 \\
\hline 1591 & 25488 & 8841 & 10 & 0.02 & 86.6 & 0.038038 & 0.029029 & 11 & 39122368 & 11 & 39964997 & 11 & 39 \\
\hline 1333 & 42453 & 7540 & 21 & 0.02 & 86.61 & 0.082082 & 0.048048 & 9 & 13001247 & 9 & 13977765 & 9 & 13 \\
\hline 2495 & 44924 & 14160 & 11 & 0.02 & 86.63 & 0.057057 & 0.034034 & 19 & 42112605 & 19 & 42984220 & 19 & 42 \\
\hline 1819 & 26321 & 31172 & 12 & 0.02 & 86.64 & 0.065065 & 0.045045 & 13 & 115027431 & 13 & 115846455 & 13 & 115 \\
\hline 106 & 3869 & 45988 & 19 & 0.02 & 86.66 & 0.067067 & 0.039039 & 1 & 106010779 & 1 & 106938537 & 1 & 106 \\
\hline 790 & 4720 & 22613 & 15 & 0.02 & 86.68 & 0.067067 & 0.032032 & 5 & 23002609 & 5 & 23994070 & 5 & 23 \\
\hline 1528 & 61080 & 15089 & 23 & 0.02 & 86.69 & 0.079079 & 0.05005 & 10 & 54096851 & 10 & 54960155 & 10 & 54 \\
\hline 1260 & 60446 & 7160 & 21 & 0.02 & 86.71 & 0.064064 & 0.041041 & 8 & 89162153 & 8 & 89981457 & 8 & 89 \\
\hline 2558 & 61325 & 39621 & 15 & 0.02 & 86.72 & 0.064064 & 0.046046 & 19 & 110165834 & 19 & 110841417 & 19 & 110 \\
\hline 930 & 19050 & 5356 & 11 & 0.02 & 86.74 & 0.072072 & 0.044044 & 6 & 51104922 & 6 & 51843873 & 6 & 51 \\
\hline 568 & 3144 & 31313 & 12 & 0.02 & 86.75 & 0.059059 & 0.035035 & 3 & 90020437 & 3 & 90913607 & 3 & 90 \\
\hline 533 & 2988 & 2994 & 18 & 0.02 & 86.77 & 0.074074 & 0.041041 & 3 & 55028381 & 3 & 55949229 & 3 & 55 \\
\hline 2168 & 44203 & 12325 & 21 & 0.02 & 86.78 & 0.109109 & 0.056056 & 15 & 91003938 & 15 & 91991367 & 15 & 91 \\
\hline 199 & 18328 & 36872 & 17 & 0.02 & 86.8 & 0.073073 & 0.043043 & 1 & 199041898 & 1 & 199973047 & 1 & 199 \\
\hline 1801 & 26289 & 26292 & 19 & 0.02 & 86.81 & 0.08008 & 0.047047 & 13 & 97001113 & 13 & 97966875 & 13 & 97 \\
\hline 2161 & 12256 & 12266 & 25 & 0.02 & 86.83 & 0.088088 & 0.038038 & 15 & 84109275 & 15 & 84990297 & 15 & 84 \\
\hline 1268 & 15253 & 7202 & 9 & 0.02 & 86.84 & 0.037037 & 0.028028 & 8 & 97285456 & 8 & 97956813 & 8 & 97 \\
\hline 499 & 2850 & 31100 & 26 & 0.02 & 86.86 & 0.113113 & 0.042042 & 3 & 21010393 & 3 & 21911837 & 3 & 21 \\
\hline 354 & 52281 & 40532 & 16 & 0.01 & 86.87 & 0.065065 & 0.043043 & 2 & 38144228 & 2 & 38997845 & 2 & 38 \\
\hline 1809 & 52849 & 34087 & 15 & 0.01 & 86.89 & 0.055055 & 0.04004 & 13 & 105053409 & 13 & 105786040 & 13 & 105 \\
\hline 1745 & 52335 & 9765 & 16 & 0.01 & 86.9 & 0.051051 & 0.033033 & 13 & 41087932 & 13 & 41882944 & 13 & 41 \\
\hline 1569 & 32219 & 52868 & 9 & 0.01 & 86.92 & 0.041041 & 0.03003 & 11 & 17133516 & 11 & 17982157 & 11 & 17 \\
\hline 1799 & 37420 & 10072 & 15 & 0.01 & 86.93 & 0.065065 & 0.046046 & 13 & 95181013 & 13 & 95969499 & 13 & 95 \\
\hline 812 & 39699 & 53034 & 9 & 0.01 & 86.95 & 0.037037 & 0.021021 & 5 & 45007479 & 5 & 45963766 & 5 & 45 \\
\hline 532 & 2983 & 56319 & 13 & 0.01 & 86.96 & 0.069069 & 0.036036 & 3 & 54006786 & 3 & 54995304 & 3 & 54 \\
\hline 2218 & 12581 & 12589 & 23 & 0.01 & 86.98 & 0.095095 & 0.055055 & 15 & 141026209 & 15 & 141938770 & 15 & 141 \\
\hline 2045 & 27346 & 58625 & 24 & 0.01 & 86.99 & 0.101101 & 0.042042 & 14 & 122043297 & 14 & 122966625 & 14 & 122 \\
\hline 1516 & 59400 & 54772 & 16 & 0.01 & 87.01 & 0.058058 & 0.041041 & 10 & 42034590 & 10 & 42921237 & 10 & 42 \\
\hline 500 & 30480 & 2858 & 14 & 0.01 & 87.02 & 0.06006 & 0.036036 & 3 & 22538991 & 3 & 22983641 & 3 & 22 \\
\hline 2025 & 11391 & 39118 & 16 & 0.01 & 87.04 & 0.084084 & 0.055055 & 14 & 102136878 & 14 & 102988071 & 14 & 102 \\
\hline 1752 & 9807 & 58918 & 17 & 0.01 & 87.05 & 0.091091 & 0.049049 & 13 & 48008516 & 13 & 48974796 & 13 & 48 \\
\hline 261 & 55745 & 40278 & 15 & 0.01 & 87.07 & 0.063063 & 0.04004 & 1 & 261028547 & 1 & 261972774 & 1 & 261 \\
\hline
\end{tabular}




\begin{tabular}{|c|c|c|c|c|c|c|c|c|c|c|c|c|c|}
\hline 2494 & 29114 & 44923 & 13 & 0.01 & 87.08 & 0.04004 & 0.029029 & 19 & 41097603 & 19 & 41984701 & 19 & 41 \\
\hline 587 & 3233 & 3241 & 22 & 0.01 & 87.1 & 0.073073 & 0.038038 & 3 & 109026074 & 3 & 109985693 & 3 & 109 \\
\hline 1583 & 8805 & 8808 & 16 & 0.01 & 87.11 & 0.073073 & 0.05005 & 11 & 31018065 & 11 & 31989556 & 11 & 31 \\
\hline 1167 & 6746 & 42182 & 10 & 0.01 & 87.12 & 0.051051 & 0.04004 & 7 & 131014179 & 7 & 131361899 & 7 & 131 \\
\hline 1290 & 54060 & 36044 & 11 & 0.01 & 87.14 & 0.046046 & 0.029029 & 8 & 119254143 & 8 & 119985447 & 8 & 119 \\
\hline 23 & 45415 & 183 & 21 & 0.01 & 87.15 & 0.086086 & 0.043043 & 1 & 23041590 & 1 & 23952116 & 1 & 23 \\
\hline 783 & 57492 & 50789 & 20 & 0.01 & 87.17 & 0.079079 & 0.048048 & 5 & 16001621 & 5 & 16920074 & 5 & 16 \\
\hline 988 & 57353 & 30835 & 13 & 0.01 & 87.18 & 0.067067 & 0.04004 & 6 & 109293981 & 6 & 109870440 & 6 & 109 \\
\hline 1404 & 38303 & 24791 & 20 & 0.01 & 87.2 & 0.071071 & 0.049049 & 9 & 84019420 & 9 & 84965987 & 9 & 84 \\
\hline 2144 & 47891 & 27772 & 10 & 0.01 & 87.21 & 0.032032 & 0.023023 & 15 & 67012516 & 15 & 67976297 & 15 & 67 \\
\hline 2243 & 52258 & 16934 & 8 & 0.01 & 87.23 & 0.042042 & 0.029029 & 16 & 8015400 & 16 & 8752527 & 16 & 8 \\
\hline 826 & 37656 & 4889 & 17 & 0.01 & 87.24 & 0.057057 & 0.037037 & 5 & 59023829 & 5 & 59906559 & 5 & 59 \\
\hline 586 & 40895 & 50683 & 18 & 0.01 & 87.26 & 0.076076 & 0.048048 & 3 & 108100697 & 3 & 108965502 & 3 & 108 \\
\hline 2431 & 28921 & 60700 & 12 & 0.01 & 87.27 & 0.06006 & 0.04004 & 18 & 39043858 & 18 & 39678645 & 18 & 39 \\
\hline 2554 & 44964 & 29153 & 15 & 0.01 & 87.29 & 0.061061 & 0.041041 & 19 & 106003938 & 19 & 106970823 & 19 & 106 \\
\hline 853 & 5070 & 37698 & 21 & 0.01 & 87.3 & 0.087087 & 0.046046 & 5 & 86094560 & 5 & 86906356 & 5 & 86 \\
\hline 2083 & 34640 & 11839 & 9 & 0.01 & 87.31 & 0.038038 & 0.026026 & 15 & 6040472 & 15 & 6978059 & 15 & 6 \\
\hline 1768 & 26168 & 38772 & 18 & 0.01 & 87.33 & 0.065065 & 0.037037 & 13 & 64033973 & 13 & 64999793 & 13 & 64 \\
\hline 2191 & 59858 & 52209 & 8 & 0.01 & 87.34 & 0.027027 & 0.02002 & 15 & 114055631 & 15 & 114987540 & 15 & 114 \\
\hline 2189 & 14991 & 12415 & 17 & 0.01 & 87.36 & 0.068068 & 0.034034 & 15 & 112258571 & 15 & 112984212 & 15 & 112 \\
\hline 1357 & 10587 & 38255 & 20 & 0.01 & 87.37 & 0.071071 & 0.045045 & 9 & 37031308 & 9 & 37972729 & 9 & 37 \\
\hline 2044 & 11556 & 43963 & 18 & 0.01 & 87.39 & 0.08008 & 0.033033 & 14 & 121000230 & 14 & 121970428 & 14 & 121 \\
\hline 572 & 3153 & 3161 & 13 & 0.01 & 87.4 & 0.045045 & 0.028028 & 3 & 94060695 & 3 & 94910712 & 3 & 94 \\
\hline 1414 & 53429 & 7913 & 15 & 0.01 & 87.42 & 0.064064 & 0.047047 & 9 & 94071916 & 9 & 94988727 & 9 & 94 \\
\hline 734 & 41236 & 59110 & 19 & 0.01 & 87.43 & 0.067067 & 0.046046 & 4 & 111222199 & 4 & 111993928 & 4 & 111 \\
\hline 2493 & 14146 & 61919 & 11 & 0.01 & 87.44 & 0.06006 & 0.037037 & 19 & 40075262 & 19 & 40954819 & 19 & 40 \\
\hline 555 & 39740 & 21445 & 19 & 0.01 & 87.46 & 0.084084 & 0.049049 & 3 & 77112515 & 3 & 77996061 & 3 & 77 \\
\hline 1816 & 26319 & 49415 & 13 & 0.01 & 87.47 & 0.058058 & 0.037037 & 13 & 112066136 & 13 & 112893275 & 13 & 112 \\
\hline 230 & 1448 & 46271 & 17 & 0.01 & 87.49 & 0.057057 & 0.037037 & 1 & 230021584 & 1 & 230928714 & 1 & 230 \\
\hline 454 & 2694 & 40699 & 23 & 0.01 & 87.5 & 0.072072 & 0.048048 & 2 & 139003438 & 2 & 139989204 & 2 & 139 \\
\hline 88 & 45956 & 33178 & 14 & 0.01 & 87.52 & 0.073073 & 0.048048 & 1 & 88055468 & 1 & 88863147 & 1 & 88 \\
\hline 1497 & 30190 & 49268 & 14 & 0.01 & 87.53 & 0.042042 & 0.031031 & 10 & 23465107 & 10 & 23970024 & 10 & 23 \\
\hline 213 & 40199 & 20327 & 21 & 0.01 & 87.54 & 0.076076 & 0.051051 & 1 & 213014666 & 1 & 213874782 & 1 & 213 \\
\hline 2151 & 32293 & 61304 & 16 & 0.01 & 87.56 & 0.048048 & 0.04004 & 15 & 74096437 & 15 & 74750835 & 15 & 74 \\
\hline 2095 & 48268 & 39231 & 19 & 0.01 & 87.57 & 0.094094 & 0.046046 & 15 & 18038664 & 15 & 18998615 & 15 & 18 \\
\hline 731 & 30743 & 62161 & 21 & 0.01 & 87.59 & 0.067067 & 0.045045 & 4 & 108032246 & 4 & 108995059 & 4 & 108 \\
\hline 18 & 45854 & 39807 & 23 & 0.01 & 87.6 & 0.093093 & 0.057057 & 1 & 18020929 & 1 & 18995163 & 1 & 18 \\
\hline 1351 & 15307 & 7628 & 22 & 0.01 & 87.62 & 0.083083 & 0.056056 & 9 & 31072493 & 9 & 31940771 & 9 & 31 \\
\hline 547 & 21419 & 3063 & 6 & 0.01 & 87.63 & 0.033033 & 0.028028 & 3 & 69657253 & 3 & 69935645 & 3 & 69 \\
\hline
\end{tabular}




\begin{tabular}{|c|c|c|c|c|c|c|c|c|c|c|c|c|c|}
\hline 1896 & 16270 & 36022 & 19 & 0.01 & 87.64 & 0.068068 & 0.04004 & 13 & 192020407 & 13 & 192997770 & 13 & 192 \\
\hline 1687 & 30484 & 43197 & 25 & 0.01 & 87.66 & 0.095095 & 0.049049 & 12 & 47002609 & 12 & 47948986 & 12 & 47 \\
\hline 1024 & 32024 & 30359 & 16 & 0.01 & 87.67 & 0.066066 & 0.038038 & 6 & 145065756 & 6 & 145998261 & 6 & 145 \\
\hline 212 & 31267 & 35110 & 14 & 0.01 & 87.69 & 0.048048 & 0.03003 & 1 & 212275513 & 1 & 212992088 & 1 & 212 \\
\hline 1447 & 24884 & 45761 & 12 & 0.01 & 87.7 & 0.058058 & 0.04004 & 9 & 127045581 & 9 & 127981472 & 9 & 127 \\
\hline 2149 & 45158 & 12222 & 13 & 0.01 & 87.71 & 0.05005 & 0.032032 & 15 & 72220380 & 15 & 72955165 & 15 & 72 \\
\hline 1185 & 31918 & 30985 & 17 & 0.01 & 87.73 & 0.08008 & 0.046046 & 8 & 14014229 & 8 & 14946506 & 8 & 14 \\
\hline 1798 & 26282 & 10066 & 13 & 0.01 & 87.74 & 0.051051 & 0.039039 & 13 & 94024615 & 13 & 94830010 & 13 & 94 \\
\hline 1902 & 15463 & 10477 & 14 & 0.01 & 87.76 & 0.058058 & 0.034034 & 13 & 198071598 & 13 & 198933332 & 13 & 198 \\
\hline 2252 & 56725 & 39399 & 16 & 0.01 & 87.77 & 0.064064 & 0.047047 & 16 & 17080961 & 16 & 17972248 & 16 & 17 \\
\hline 1293 & 15983 & 50491 & 17 & 0.01 & 87.78 & 0.069069 & 0.04004 & 8 & 122072309 & 8 & 122664524 & 8 & 122 \\
\hline 1145 & 42099 & 42101 & 19 & 0.01 & 87.8 & 0.086086 & 0.047047 & 7 & 109049333 & 7 & 109981964 & 7 & 109 \\
\hline 1802 & 55780 & 61319 & 14 & 0.01 & 87.81 & 0.05005 & 0.033033 & 13 & 98016719 & 13 & 98835795 & 13 & 98 \\
\hline 2408 & 13744 & 60027 & 25 & 0.01 & 87.83 & 0.134134 & 0.063063 & 18 & 16004972 & 18 & 16922697 & 18 & 16 \\
\hline 1473 & 24986 & 51569 & 14 & 0.01 & 87.84 & 0.055055 & 0.034034 & 9 & 153031450 & 9 & 153484548 & 9 & 153 \\
\hline 125 & 46032 & 903 & 17 & 0.01 & 87.85 & 0.061061 & 0.033033 & 1 & 125123092 & 1 & 125912707 & 1 & 125 \\
\hline 108 & 20000 & 821 & 17 & 0.01 & 87.87 & 0.064064 & 0.043043 & 1 & 108004707 & 1 & 108932925 & 1 & 108 \\
\hline 2071 & 44050 & 49536 & 18 & 0.01 & 87.88 & 0.076076 & 0.04004 & 14 & 148003749 & 14 & 148980215 & 14 & 148 \\
\hline 1235 & 2635 & 7065 & 13 & 0.01 & 87.89 & 0.051051 & 0.035035 & 8 & 64017753 & 8 & 64946356 & 8 & 64 \\
\hline 1758 & 56247 & 38751 & 20 & 0.01 & 87.91 & 0.078078 & 0.045045 & 13 & 54028991 & 13 & 54983756 & 13 & 54 \\
\hline 766 & 55176 & 61617 & 17 & 0.01 & 87.92 & 0.063063 & 0.038038 & 4 & 143021644 & 4 & 143462966 & 4 & 143 \\
\hline 1197 & 24112 & 47190 & 24 & 0.01 & 87.94 & 0.084084 & 0.046046 & 8 & 26012508 & 8 & 26961266 & 8 & 26 \\
\hline 1748 & 36142 & 26102 & 20 & 0.01 & 87.95 & 0.088088 & 0.048048 & 13 & 44014547 & 13 & 44990551 & 13 & 44 \\
\hline 1448 & 8038 & 56987 & 18 & 0.01 & 87.96 & 0.069069 & 0.04004 & 9 & 128025650 & 9 & 128986695 & 9 & 128 \\
\hline 2047 & 11569 & 27370 & 22 & 0.01 & 87.98 & 0.082082 & 0.041041 & 14 & 124020336 & 14 & 124905201 & 14 & 124 \\
\hline 1878 & 56155 & 10359 & 5 & 0.01 & 87.99 & 0.025025 & 0.02002 & 13 & 174247801 & 13 & 174758344 & 13 & 174 \\
\hline 1379 & 36229 & 29434 & 16 & 0.01 & 88 & 0.073073 & 0.043043 & 9 & 59032592 & 9 & 59952885 & 9 & 59 \\
\hline 2015 & 11305 & 43879 & 20 & 0.01 & 88.02 & 0.061061 & 0.041041 & 14 & 92014581 & 14 & 92993972 & 14 & 92 \\
\hline 1222 & 57485 & 57337 & 12 & 0.01 & 88.03 & 0.064064 & 0.036036 & 8 & 51018124 & 8 & 51942666 & 8 & 51 \\
\hline 1048 & 5776 & 41795 & 24 & 0.01 & 88.04 & 0.067067 & 0.045045 & 7 & 11038318 & 7 & 11979563 & 7 & 11 \\
\hline 2216 & 12566 & 57999 & 26 & 0.01 & 88.06 & 0.1001 & 0.046046 & 15 & 139070668 & 15 & 139947117 & 15 & 139 \\
\hline 1645 & 9197 & 61714 & 16 & 0.01 & 88.07 & 0.078078 & 0.053053 & 12 & 5026781 & 12 & 5900678 & 12 & 5 \\
\hline 173 & 1140 & 1146 & 14 & 0.01 & 88.08 & 0.052052 & 0.028028 & 1 & 173010521 & 1 & 173741598 & 1 & 173 \\
\hline 2577 & 14310 & 14315 & 15 & 0.01 & 88.1 & 0.039039 & 0.029029 & 19 & 129032125 & 19 & 129975535 & 19 & 129 \\
\hline 602 & 3327 & 21601 & 20 & 0.01 & 88.11 & 0.054054 & 0.039039 & 3 & 124002113 & 3 & 124901557 & 3 & 124 \\
\hline 2101 & 57738 & 45658 & 14 & 0.01 & 88.13 & 0.058058 & 0.039039 & 15 & 24019575 & 15 & 24972365 & 15 & 24 \\
\hline 675 & 59441 & 21991 & 8 & 0.01 & 88.14 & 0.043043 & 0.031031 & 4 & 52023825 & 4 & 52994578 & 4 & 52 \\
\hline 579 & 21489 & 31283 & 14 & 0.01 & 88.15 & 0.063063 & 0.037037 & 3 & 101222209 & 3 & 101813917 & 3 & 101 \\
\hline 1265 & 60260 & 7192 & 8 & 0.01 & 88.17 & 0.043043 & 0.029029 & 8 & 94303744 & 8 & 94994514 & 8 & 94 \\
\hline
\end{tabular}




\begin{tabular}{|c|c|c|c|c|c|c|c|c|c|c|c|c|c|}
\hline 1502 & 59887 & 30820 & 17 & 0.01 & 88.18 & 0.061061 & 0.039039 & 10 & 28265646 & 10 & 28963569 & 10 & 28 \\
\hline 79 & 626 & 33118 & 13 & 0.01 & 88.19 & 0.064064 & 0.04004 & 1 & 79048821 & 1 & 79931781 & 1 & 79 \\
\hline 1532 & 25254 & 32339 & 19 & 0.01 & 88.21 & 0.081081 & 0.041041 & 10 & 58040881 & 10 & 58966663 & 10 & 58 \\
\hline 1812 & 10110 & 10113 & 15 & 0.01 & 88.22 & 0.058058 & 0.038038 & 13 & 108017360 & 13 & 108900739 & 13 & 108 \\
\hline 2555 & 48223 & 52303 & 16 & 0.01 & 88.23 & 0.059059 & 0.037037 & 19 & 107000024 & 19 & 107876735 & 19 & 107 \\
\hline 2035 & 60680 & 39141 & 15 & 0.01 & 88.25 & 0.049049 & 0.034034 & 14 & 112008662 & 14 & 112992396 & 14 & 112 \\
\hline 899 & 34928 & 5286 & 13 & 0.01 & 88.26 & 0.052052 & 0.039039 & 6 & 20100569 & 6 & 20988489 & 6 & 20 \\
\hline 461 & 2734 & 18269 & 24 & 0.01 & 88.27 & 0.086086 & 0.05005 & 2 & 146082066 & 2 & 146937364 & 2 & 146 \\
\hline 1276 & 33314 & 61370 & 8 & 0.01 & 88.29 & 0.038038 & 0.027027 & 8 & 105078714 & 8 & 105991646 & 8 & 05 \\
\hline 709 & 55478 & 41167 & 17 & 0.01 & 88.3 & 0.065065 & 0.041041 & 4 & 86029839 & 4 & 86981509 & 4 & 86 \\
\hline 1278 & 7234 & 17005 & 18 & 0.01 & 88.31 & 0.076076 & 0.046046 & 8 & 107036658 & 8 & 107954353 & 8 & 107 \\
\hline 2562 & 49789 & 35551 & 21 & 0.01 & 88.33 & 0.079079 & 0.043043 & 19 & 114259111 & 19 & 114972696 & 19 & 114 \\
\hline 382 & 51768 & 51939 & 9 & 0.01 & 88.34 & 0.046046 & 0.03003 & 2 & 67308766 & 2 & 67981307 & 2 & 67 \\
\hline 167 & 60555 & 56555 & 13 & 0.01 & 88.35 & 0.057057 & 0.037037 & 1 & 167094891 & 1 & 167882120 & 1 & 167 \\
\hline 1554 & 42889 & 42892 & 19 & 0.01 & 88.37 & 0.074074 & 0.037037 & 11 & 2038539 & 11 & 2964267 & 11 & 2 \\
\hline 1759 & 56861 & 38754 & 23 & 0.01 & 88.38 & 0.077077 & 0.046046 & 13 & 55049361 & 13 & 55993218 & 13 & 55 \\
\hline 1175 & 53940 & 6769 & 14 & 0.01 & 88.39 & 0.055055 & 0.024024 & 8 & 4017057 & 8 & 4961037 & 8 & 4 \\
\hline 2441 & 13960 & 44819 & 20 & 0.01 & 88.41 & 0.081081 & 0.046046 & 18 & 49008785 & 18 & 49991095 & 18 & 49 \\
\hline 1547 & 31595 & 25327 & 20 & 0.01 & 88.42 & 0.073073 & 0.034034 & 10 & 73122633 & 10 & 73876295 & 10 & 73 \\
\hline 144 & 55775 & 20103 & 21 & 0.01 & 88.43 & 0.072072 & 0.049049 & 1 & 144001096 & 1 & 144941329 & 1 & 144 \\
\hline 1912 & 43572 & 60533 & 16 & 0.01 & 88.45 & 0.071071 & 0.034034 & 13 & 208012602 & 13 & 208919742 & 13 & 208 \\
\hline 214 & 20328 & 34752 & 23 & 0.01 & 88.46 & 0.074074 & 0.042042 & 1 & 214033032 & 1 & 214959896 & 1 & 214 \\
\hline 390 & 49816 & 15876 & 7 & 0.01 & 88.47 & 0.028028 & 0.022022 & 2 & 75198782 & 2 & 75900120 & 2 & 75 \\
\hline 2006 & 47680 & 51011 & 21 & 0.01 & 88.48 & 0.055055 & 0.033033 & 14 & 83128477 & 14 & 83999974 & 14 & 83 \\
\hline 2118 & 12060 & 30859 & 14 & 0.01 & 88.5 & 0.064064 & 0.042042 & 15 & 41012641 & 15 & 41967209 & 15 & 41 \\
\hline 840 & 4984 & 22787 & 11 & 0.01 & 88.51 & 0.055055 & 0.032032 & 5 & 73000608 & 5 & 73987917 & 5 & 73 \\
\hline 2425 & 28902 & 57284 & 9 & 0.01 & 88.52 & 0.046046 & 0.029029 & 18 & 33106672 & 18 & 33816366 & 18 & 33 \\
\hline 2231 & 18167 & 14535 & 7 & 0.01 & 88.54 & 0.043043 & 0.029029 & 15 & 154044342 & 15 & 154569347 & 15 & 154 \\
\hline 236 & 36931 & 46282 & 10 & 0.01 & 88.55 & 0.056056 & 0.028028 & 1 & 236058941 & 1 & 236949960 & 1 & 236 \\
\hline 2338 & 28456 & 51446 & 16 & 0.01 & 88.56 & 0.074074 & 0.034034 & 17 & 16023225 & 17 & 16985131 & 17 & 16 \\
\hline 828 & 22731 & 4902 & 20 & 0.01 & 88.58 & 0.073073 & 0.046046 & 5 & 61020546 & 5 & 61979581 & 5 & 61 \\
\hline 194 & 46184 & 36847 & 11 & 0.01 & 88.59 & 0.044044 & 0.026026 & 1 & 194020406 & 1 & 194987488 & 1 & 194 \\
\hline 868 & 5163 & 50582 & 10 & 0.01 & 88.6 & 0.044044 & 0.03003 & 5 & 101013243 & 5 & 101758816 & 5 & 101 \\
\hline 2194 & 27882 & 39307 & 15 & 0.01 & 88.62 & 0.07007 & 0.036036 & 15 & 117005136 & 15 & 117987234 & 15 & 117 \\
\hline 1403 & 57288 & 42610 & 16 & 0.01 & 88.63 & 0.062062 & 0.047047 & 9 & 83104537 & 9 & 83986835 & 9 & 83 \\
\hline 295 & 37052 & 1885 & 10 & 0.01 & 88.64 & 0.042042 & 0.023023 & 1 & 295017152 & 1 & 295976006 & 1 & 295 \\
\hline 663 & 46591 & 3745 & 14 & 0.01 & 88.65 & 0.055055 & 0.031031 & 4 & 40031986 & 4 & 40942240 & 4 & 40 \\
\hline 2569 & 30481 & 14437 & 15 & 0.01 & 88.67 & 0.057057 & 0.038038 & 19 & 121196405 & 19 & 121945530 & 19 & 121 \\
\hline 1259 & 16495 & 53477 & 20 & 0.01 & 88.68 & 0.065065 & 0.037037 & 8 & 88011897 & 8 & 88909379 & 8 & 88 \\
\hline
\end{tabular}




\begin{tabular}{|c|c|c|c|c|c|c|c|c|c|c|c|c|c|}
\hline 1011 & 50282 & 23165 & 22 & 0.01 & 88.69 & 0.096096 & 0.047047 & 6 & 132032339 & 6 & 132912255 & 6 & 132 \\
\hline 2234 & 12643 & 14963 & 11 & 0.01 & 88.71 & 0.045045 & 0.03003 & 15 & 157038929 & 15 & 157425891 & 15 & 157 \\
\hline 1588 & 42978 & 42980 & 11 & 0.01 & 88.72 & 0.052052 & 0.033033 & 11 & 36037488 & 11 & 36836674 & 11 & 36 \\
\hline 275 & 1718 & 54575 & 21 & 0.01 & 88.73 & 0.091091 & 0.051051 & 1 & 275078557 & 1 & 275995963 & 1 & 275 \\
\hline 1060 & 5875 & 5882 & 12 & 0.01 & 88.74 & 0.045045 & 0.031031 & 7 & 23022559 & 7 & 23977356 & 7 & 23 \\
\hline 2350 & 13389 & 61797 & 21 & 0.01 & 88.76 & 0.079079 & 0.039039 & 17 & 28091145 & 17 & 28931409 & 17 & 28 \\
\hline 1542 & 30117 & 42867 & 21 & 0.01 & 88.77 & 0.062062 & 0.041041 & 10 & 68013801 & 10 & 68989822 & 10 & 68 \\
\hline 2203 & 39339 & 39345 & 18 & 0.01 & 88.78 & 0.067067 & 0.03003 & 15 & 126029433 & 15 & 126862449 & 15 & 126 \\
\hline 259 & 1617 & 36988 & 16 & 0.01 & 88.79 & 0.07007 & 0.022022 & 1 & 259019570 & 1 & 259936367 & 1 & 259 \\
\hline 2564 & 14278 & 34635 & 10 & 0.01 & 88.81 & 0.043043 & 0.026026 & 19 & 116033785 & 19 & 116843785 & 19 & 116 \\
\hline 0 & 55418 & 36467 & 15 & 0.01 & 88.82 & 0.055055 & 0.034034 & 1 & 286933 & 1 & 985857 & 1 & 0 \\
\hline 2004 & 43846 & 11229 & 28 & 0.01 & 88.83 & 0.113113 & 0.042042 & 14 & 81000356 & 14 & 81990297 & 14 & 81 \\
\hline 2164 & 44194 & 61218 & 22 & 0.01 & 88.84 & 0.076076 & 0.04004 & 15 & 87027499 & 15 & 87942801 & 15 & 87 \\
\hline 1014 & 46941 & 5609 & 19 & 0.01 & 88.86 & 0.079079 & 0.043043 & 6 & 135074196 & 6 & 135878484 & 6 & 135 \\
\hline 2263 & 12803 & 18480 & 17 & 0.01 & 88.87 & 0.065065 & 0.034034 & 16 & 28006035 & 16 & 28850217 & 16 & 28 \\
\hline 195 & 35536 & 46188 & 13 & 0.01 & 88.88 & 0.05005 & 0.034034 & 1 & 195015099 & 1 & 195833378 & 1 & 195 \\
\hline 208 & 20313 & 36892 & 18 & 0.01 & 88.9 & 0.056056 & 0.034034 & 1 & 208051940 & 1 & 208999979 & 1 & 208 \\
\hline 566 & 37303 & 3136 & 10 & 0.01 & 88.91 & 0.046046 & 0.034034 & 3 & 88022523 & 3 & 88862483 & 3 & 88 \\
\hline 240 & 40237 & 1510 & 16 & 0.01 & 88.92 & 0.087087 & 0.046046 & 1 & 240011062 & 1 & 240994314 & 1 & 240 \\
\hline 1302 & 56387 & 31514 & 17 & 0.01 & 88.93 & 0.062062 & 0.044044 & 8 & 131014130 & 8 & 131837615 & 8 & 131 \\
\hline 1237 & 56113 & 38144 & 11 & 0.01 & 88.94 & 0.054054 & 0.034034 & 8 & 66027033 & 8 & 66847097 & 8 & 66 \\
\hline 811 & 60113 & 4831 & 16 & 0.01 & 88.96 & 0.047047 & 0.035035 & 5 & 44070464 & 5 & 44994712 & 5 & 44 \\
\hline 385 & 50904 & 37114 & 9 & 0.01 & 88.97 & 0.045045 & 0.021021 & 2 & 70061497 & 2 & 70869578 & 2 & 70 \\
\hline 1172 & 781 & 6763 & 14 & 0.01 & 88.98 & 0.057057 & 0.043043 & 8 & 1129887 & 8 & 1931528 & 8 & 1 \\
\hline 200 & 1272 & 20291 & 19 & 0.01 & 88.99 & 0.082082 & 0.038038 & 1 & 200027513 & 1 & 200973888 & 1 & 200 \\
\hline 1080 & 6030 & 23526 & 26 & 0.01 & 89.01 & 0.105105 & 0.047047 & 7 & 43037006 & 7 & 43924915 & 7 & 43 \\
\hline 115 & 40024 & 46008 & 14 & 0.01 & 89.02 & 0.057057 & 0.033033 & 1 & 115016840 & 1 & 115880586 & 1 & 115 \\
\hline 2580 & 54589 & 14326 & 8 & 0.01 & 89.03 & 0.041041 & 0.026026 & 19 & 132088343 & 19 & 132966392 & 19 & 132 \\
\hline 290 & 45109 & 40356 & 16 & 0.01 & 89.04 & 0.059059 & 0.03003 & 1 & 290260126 & 1 & 290972764 & 1 & 290 \\
\hline 711 & 46708 & 41171 & 20 & 0.01 & 89.06 & 0.07007 & 0.037037 & 4 & 88065653 & 4 & 88978451 & 4 & 88 \\
\hline 1326 & 32798 & 42426 & 19 & 0.01 & 89.07 & 0.073073 & 0.049049 & 9 & 6061399 & 9 & 6964469 & 9 & 6 \\
\hline 2357 & 33725 & 54545 & 12 & 0.01 & 89.08 & 0.043043 & 0.027027 & 17 & 35295082 & 17 & 35935548 & 17 & 35 \\
\hline 1704 & 53457 & 38679 & 22 & 0.01 & 89.09 & 0.077077 & 0.041041 & 13 & 88939 & 13 & 978869 & 13 & 0 \\
\hline 243 & 20376 & 29768 & 21 & 0.01 & 89.1 & 0.093093 & 0.037037 & 1 & 243011910 & 1 & 243989929 & 1 & 243 \\
\hline 2479 & 32427 & 54800 & 9 & 0.01 & 89.12 & 0.045045 & 0.028028 & 19 & 26000408 & 19 & 26951074 & 19 & 26 \\
\hline 1831 & 10165 & 59757 & 14 & 0.01 & 89.13 & 0.066066 & 0.044044 & 13 & 127026543 & 13 & 127992609 & 13 & 127 \\
\hline 518 & 53025 & 45565 & 6 & 0.01 & 89.14 & 0.038038 & 0.029029 & 3 & 40003536 & 3 & 40461817 & 3 & 40 \\
\hline 1796 & 15100 & 38818 & 13 & 0.01 & 89.15 & 0.056056 & 0.038038 & 13 & 92044292 & 13 & 92853911 & 13 & 92 \\
\hline 1266 & 56723 & 58266 & 13 & 0.01 & 89.16 & 0.057057 & 0.037037 & 8 & 95024815 & 8 & 95981359 & 8 & 95 \\
\hline
\end{tabular}




\begin{tabular}{|c|c|c|c|c|c|c|c|c|c|c|c|c|}
\hline 813 & 32984 & 37617 & 17 & 0.01 & 89.18 & 0.076076 & 0.041041 & 5 & 46029879 & 5 & 46748561 & 5 \\
\hline 1248 & 7118 & 33432 & 19 & 0.01 & 89.19 & 0.066066 & 0.043043 & 8 & 77021275 & 8 & 77966205 & 8 \\
\hline 2123 & 27711 & 39255 & 9 & 0.01 & 89.2 & 0.049049 & 0.033033 & 15 & 46072177 & 15 & 46554052 & 15 \\
\hline 1856 & 10262 & 18089 & 12 & 0.01 & 89.21 & 0.052052 & 0.037037 & 13 & 152053701 & 13 & 152969439 & 13 \\
\hline 841 & 22788 & 41528 & 18 & 0.01 & 89.22 & 0.074074 & 0.042042 & 5 & 74020663 & 5 & 74804208 & 5 \\
\hline 1118 & 6319 & 56737 & 22 & 0.01 & 89.24 & 0.079079 & 0.043043 & 7 & 81035442 & 7 & 81924254 & 7 \\
\hline 2196 & 44236 & 44238 & 17 & 0.01 & 89.25 & 0.061061 & 0.031031 & 15 & 119091942 & 15 & 119982356 & 15 \\
\hline 1746 & 16269 & 43323 & 13 & 0.01 & 89.26 & 0.053053 & 0.034034 & 13 & 42026635 & 13 & 42933805 & 13 \\
\hline 1282 & 53266 & 55824 & 13 & 0.01 & 89.27 & 0.053053 & 0.036036 & 8 & 111006793 & 8 & 111774978 & 8 \\
\hline 473 & 48589 & 2786 & 11 & 0.01 & 89.28 & 0.041041 & 0.029029 & 2 & 158014368 & 2 & 158680517 & 2 \\
\hline 621 & 17508 & 56944 & 14 & 0.01 & 89.3 & 0.053053 & 0.035035 & 3 & 143698872 & 3 & 143975041 & 3 \\
\hline 2042 & 27333 & 43955 & 14 & 0.01 & 89.31 & 0.057057 & 0.03003 & 14 & 119011148 & 14 & 119983210 & 14 \\
\hline 2275 & 12894 & 12902 & 18 & 0.01 & 89.32 & 0.06006 & 0.04004 & 16 & 40054179 & 16 & 40966989 & 16 \\
\hline 714 & 4116 & 46722 & 24 & 0.01 & 89.33 & 0.086086 & 0.049049 & 4 & 91007286 & 4 & 91938171 & 4 \\
\hline 202 & 20296 & 36884 & 24 & 0.01 & 89.34 & 0.027027 & 0.019019 & 1 & 202021384 & 1 & 202987973 & 1 \\
\hline 1446 & 8032 & 31662 & 11 & 0.01 & 89.35 & 0.054054 & 0.032032 & 9 & 126204363 & 9 & 126652137 & 9 \\
\hline 253 & 36959 & 36967 & 18 & 0.01 & 89.37 & 0.097097 & 0.03003 & 1 & 253029725 & 1 & 253914428 & 1 \\
\hline 1823 & 43439 & 56272 & 13 & 0.01 & 89.38 & 0.05005 & 0.027027 & 13 & 119023717 & 13 & 119979547 & 13 \\
\hline 187 & 20273 & 55985 & 21 & 0.01 & 89.39 & 0.069069 & 0.042042 & 1 & 187044656 & 1 & 187930689 & 1 \\
\hline 1410 & 47276 & 7892 & 13 & 0.01 & 89.4 & 0.048048 & 0.028028 & 9 & 90001442 & 9 & 90986895 & 9 \\
\hline 1868 & 62043 & 17042 & 6 & 0.01 & 89.41 & 0.031031 & 0.019019 & 13 & 164327059 & 13 & 164995422 & 13 \\
\hline 2135 & 27745 & 12154 & 17 & 0.01 & 89.42 & 0.074074 & 0.054054 & 15 & 58049980 & 15 & 58956076 & 15 \\
\hline 2468 & 31333 & 29063 & 24 & 0.01 & 89.43 & 0.085085 & 0.031031 & 19 & 15010224 & 19 & 15982273 & 19 \\
\hline 1010 & 18973 & 32353 & 13 & 0.01 & 89.45 & 0.055055 & 0.028028 & 6 & 131184737 & 6 & 131951039 & 6 \\
\hline 2169 & 44206 & 27833 & 24 & 0.01 & 89.46 & 0.105105 & 0.049049 & 15 & 92024785 & 15 & 92970795 & 15 \\
\hline 1401 & 24776 & 38297 & 11 & 0.01 & 89.47 & 0.051051 & 0.028028 & 9 & 81214756 & 9 & 81888480 & 9 \\
\hline 1562 & 47331 & 57928 & 17 & 0.01 & 89.48 & 0.072072 & 0.044044 & 11 & 10051760 & 11 & 10801527 & 11 \\
\hline 2116 & 12044 & 52725 & 15 & 0.01 & 89.49 & 0.069069 & 0.035035 & 15 & 39116703 & 15 & 39986267 & 15 \\
\hline 1596 & 8861 & 8865 & 9 & 0.01 & 89.5 & 0.046046 & 0.029029 & 11 & 44023659 & 11 & 44981824 & 11 \\
\hline 620 & 52313 & 45836 & 8 & 0.01 & 89.52 & 0.035035 & 0.025025 & 3 & 142329763 & 3 & 142766769 & 3 \\
\hline 2089 & 53026 & 27600 & 13 & 0.01 & 89.53 & 0.053053 & 0.036036 & 15 & 12155691 & 15 & 12984131 & 15 \\
\hline 67 & 36653 & 32143 & 24 & 0.01 & 89.54 & 0.076076 & 0.038038 & 1 & 67050038 & 1 & 67951385 & 1 \\
\hline 1851 & 15606 & 26395 & 14 & 0.01 & 89.55 & 0.039039 & 0.028028 & 13 & 147060010 & 13 & 147984539 & 13 \\
\hline 1480 & 38424 & 25033 & 14 & 0.01 & 89.56 & 0.059059 & 0.032032 & 10 & 6093540 & 10 & 6990318 & 10 \\
\hline 1422 & 59248 & 24831 & 15 & 0.01 & 89.57 & 0.054054 & 0.036036 & 9 & 102081959 & 9 & 102920120 & 9 \\
\hline 1818 & 18425 & 43436 & 8 & 0.01 & 89.58 & 0.044044 & 0.028028 & 13 & 114061850 & 13 & 114928166 & 13 \\
\hline 1032 & 5692 & 35463 & 17 & 0.01 & 89.6 & 0.064064 & 0.043043 & 6 & 153108429 & 6 & 153749532 & 6 \\
\hline 1817 & 14829 & 47482 & 5 & 0.01 & 89.61 & 0.025025 & 0.016016 & 13 & 113026364 & 13 & 113980170 & 13 \\
\hline 1026 & 48932 & 41734 & 15 & 0.01 & 89.62 & 0.058058 & 0.033033 & 6 & 147007251 & 6 & 147954934 & 6 \\
\hline
\end{tabular}




\begin{tabular}{|c|c|c|c|c|c|c|c|c|c|c|c|c|c|}
\hline 1806 & 45129 & 10100 & 12 & 0.01 & 89.63 & 0.056056 & 0.036036 & 13 & 102306232 & 13 & 102985566 & 13 & 102 \\
\hline 1585 & 38547 & 8820 & 12 & 0.01 & 89.64 & 0.042042 & 0.027027 & 11 & 33006254 & 11 & 33976329 & 11 & 33 \\
\hline 1678 & 9361 & 60754 & 13 & 0.01 & 89.65 & 0.043043 & 0.026026 & 12 & 38004200 & 12 & 38706000 & 12 & 38 \\
\hline 845 & 46861 & 5018 & 20 & 0.01 & 89.66 & 0.068068 & 0.034034 & 5 & 78067097 & 5 & 78990780 & 5 & 78 \\
\hline 274 & 46043 & 1717 & 15 & 0.01 & 89.67 & 0.054054 & 0.036036 & 1 & 274127937 & 1 & 274989579 & 1 & 274 \\
\hline 1240 & 15821 & 47211 & 19 & 0.01 & 89.69 & 0.069069 & 0.031031 & 8 & 69039573 & 8 & 69998730 & 8 & 69 \\
\hline 1848 & 10228 & 30828 & 18 & 0.01 & 89.7 & 0.059059 & 0.035035 & 13 & 144065180 & 13 & 144981309 & 13 & 144 \\
\hline 2472 & 14089 & 39592 & 12 & 0.01 & 89.71 & 0.064064 & 0.038038 & 19 & 19218547 & 19 & 19944045 & 19 & 19 \\
\hline 789 & 4719 & 22608 & 9 & 0.01 & 89.72 & 0.039039 & 0.027027 & 5 & 22536178 & 5 & 22969678 & 5 & 22 \\
\hline 2251 & 29802 & 52603 & 10 & 0.01 & 89.73 & 0.032032 & 0.025025 & 16 & 16006121 & 16 & 16893013 & 16 & 16 \\
\hline 1120 & 47101 & 49042 & 14 & 0.01 & 89.74 & 0.067067 & 0.034034 & 7 & 83057633 & 7 & 83944769 & 7 & 83 \\
\hline 1256 & 59544 & 50770 & 10 & 0.01 & 89.75 & 0.039039 & 0.021021 & 8 & 85070054 & 8 & 85901166 & 8 & 85 \\
\hline 186 & 20268 & 46172 & 18 & 0.01 & 89.76 & 0.067067 & 0.039039 & 1 & 186106230 & 1 & 186883993 & 1 & 186 \\
\hline 958 & 36436 & 14794 & 7 & 0.01 & 89.77 & 0.032032 & 0.023023 & 6 & 79026669 & 6 & 79924228 & 6 & 79 \\
\hline 1277 & 15787 & 17756 & 17 & 0.01 & 89.79 & 0.055055 & 0.039039 & 8 & 106188404 & 8 & 106955537 & 8 & 106 \\
\hline 224 & 51517 & 46256 & 14 & 0.01 & 89.8 & 0.06006 & 0.038038 & 1 & 224000157 & 1 & 224999683 & 1 & 224 \\
\hline 2296 & 35131 & 28284 & 19 & 0.01 & 89.81 & 0.088088 & 0.04004 & 16 & 61006434 & 16 & 61995301 & 16 & 61 \\
\hline 1273 & 7214 & 7222 & 20 & 0.01 & 89.82 & 0.05005 & 0.029029 & 8 & 102008930 & 8 & 102914965 & 8 & 102 \\
\hline 2553 & 14228 & 29149 & 10 & 0.01 & 89.83 & 0.056056 & 0.029029 & 19 & 105103691 & 19 & 105978406 & 19 & 105 \\
\hline 2193 & 12430 & 43603 & 6 & 0.01 & 89.84 & 0.029029 & 0.011011 & 15 & 116028994 & 15 & 116467644 & 15 & 116 \\
\hline 1638 & 15653 & 32721 & 7 & 0.01 & 89.85 & 0.04004 & 0.022022 & 11 & 86035052 & 11 & 86676975 & 11 & 86 \\
\hline 1828 & 26337 & 10153 & 20 & 0.01 & 89.86 & 0.065065 & 0.034034 & 13 & 124021372 & 13 & 124983364 & 13 & 124 \\
\hline 1515 & 55300 & 60314 & 9 & 0.01 & 89.87 & 0.05005 & 0.031031 & 10 & 41053714 & 10 & 41976069 & 10 & 41 \\
\hline 128 & 36769 & 20057 & 9 & 0.01 & 89.88 & 0.044044 & 0.027027 & 1 & 128248008 & 1 & 128958281 & 1 & 128 \\
\hline 2148 & 27777 & 12221 & 6 & 0.01 & 89.89 & 0.021021 & 0.013013 & 15 & 71357581 & 15 & 71846422 & 15 & 71 \\
\hline 1747 & 9770 & 50626 & 18 & 0.01 & 89.9 & 0.067067 & 0.036036 & 13 & 43007233 & 13 & 43923140 & 13 & 43 \\
\hline 2126 & 44145 & 12099 & 18 & 0.01 & 89.92 & 0.066066 & 0.027027 & 15 & 49103392 & 15 & 49896940 & 15 & 49 \\
\hline 1825 & 34940 & 10142 & 12 & 0.01 & 89.93 & 0.056056 & 0.035035 & 13 & 121174356 & 13 & 121983388 & 13 & 121 \\
\hline 1267 & 32646 & 7198 & 10 & 0.01 & 89.94 & 0.036036 & 0.02002 & 8 & 96051658 & 8 & 96992033 & 8 & 96 \\
\hline 1037 & 46949 & 46954 & 14 & 0.01 & 89.95 & 0.041041 & 0.023023 & 7 & 48748 & 7 & 992405 & 7 & 0 \\
\hline 2103 & 34907 & 44108 & 15 & 0.01 & 89.96 & 0.052052 & 0.029029 & 15 & 26156628 & 15 & 26975496 & 15 & 26 \\
\hline 1743 & 47438 & 26087 & 15 & 0.01 & 89.97 & 0.055055 & 0.029029 & 13 & 39012509 & 13 & 39992519 & 13 & 39 \\
\hline 1002 & 41701 & 16563 & 21 & 0.01 & 89.98 & 0.073073 & 0.047047 & 6 & 123075357 & 6 & 123855372 & 6 & 123 \\
\hline 815 & 46827 & 37623 & 13 & 0.01 & 89.99 & 0.058058 & 0.032032 & 5 & 48150113 & 5 & 48975686 & 5 & 48 \\
\hline 256 & 36979 & 20426 & 25 & 0.01 & 90 & 0.124124 & 0.037037 & 1 & 256001453 & 1 & 256989077 & 1 & 256 \\
\hline 816 & 37622 & 37627 & 20 & 0.01 & 90.01 & 0.074074 & 0.04004 & 5 & 49040715 & 5 & 49975876 & 5 & 49 \\
\hline 238 & 20361 & 20363 & 15 & 0.01 & 90.02 & 0.059059 & 0.03003 & 1 & 238102917 & 1 & 238827931 & 1 & 238 \\
\hline 215 & 1367 & 40204 & 16 & 0.01 & 90.03 & 0.043043 & 0.026026 & 1 & 215042412 & 1 & 215971588 & 1 & 215 \\
\hline 2579 & 49802 & 16578 & 8 & 0.01 & 90.04 & 0.032032 & 0.02002 & 19 & 131104850 & 19 & 131708696 & 19 & 131 \\
\hline
\end{tabular}




\begin{tabular}{|c|c|c|c|c|c|c|c|c|c|c|c|c|c|}
\hline 848 & 55686 & 5040 & 28 & 0.01 & 90.05 & 0.113113 & 0.044044 & 5 & 81043360 & 5 & 81968675 & 5 & 81 \\
\hline 1416 & 56662 & 57968 & 15 & 0.01 & 90.07 & 0.045045 & 0.029029 & 9 & 96199471 & 9 & 96887177 & 9 & 96 \\
\hline 1242 & 61053 & 16071 & 11 & 0.01 & 90.08 & 0.051051 & 0.031031 & 8 & 71042636 & 8 & 71847915 & 8 & 71 \\
\hline 1841 & 4230 & 38868 & 8 & 0.01 & 90.09 & 0.05005 & 0.025025 & 13 & 137076639 & 13 & 137988788 & 13 & 137 \\
\hline 2475 & 29069 & 48274 & 17 & 0.01 & 90.1 & 0.055055 & 0.039039 & 19 & 22009202 & 19 & 22855768 & 19 & 22 \\
\hline 601 & 21596 & 33223 & 11 & 0.01 & 90.11 & 0.05005 & 0.029029 & 3 & 123015205 & 3 & 123638149 & 3 & 123 \\
\hline 2274 & 59058 & 55382 & 19 & 0.01 & 90.12 & 0.058058 & 0.037037 & 16 & 39019226 & 16 & 39993955 & 16 & 39 \\
\hline 1703 & 9455 & 52184 & 13 & 0.01 & 90.13 & 0.059059 & 0.033033 & 12 & 63058856 & 12 & 63499825 & 12 & 63 \\
\hline 668 & 56820 & 21970 & 19 & 0.01 & 90.14 & 0.072072 & 0.038038 & 4 & 45031120 & 4 & 45986315 & 4 & 45 \\
\hline 2343 & 13329 & 13335 & 24 & 0.01 & 90.15 & 0.09009 & 0.045045 & 17 & 21010426 & 17 & 21985990 & 17 & 21 \\
\hline 1754 & 30265 & 38788 & 16 & 0.01 & 90.16 & 0.07007 & 0.04004 & 13 & 50015957 & 13 & 50853035 & 13 & 50 \\
\hline 2481 & 14115 & 58372 & 12 & 0.01 & 90.17 & 0.054054 & 0.027027 & 19 & 28014791 & 19 & 28988256 & 19 & 28 \\
\hline 2292 & 28260 & 25466 & 17 & 0.01 & 90.18 & 0.056056 & 0.036036 & 16 & 57003945 & 16 & 57990322 & 16 & 57 \\
\hline 2397 & 52494 & 32992 & 14 & 0.01 & 90.19 & 0.049049 & 0.033033 & 18 & 5064706 & 18 & 5999331 & 18 & 5 \\
\hline 2366 & 39525 & 28617 & 19 & 0.01 & 90.2 & 0.067067 & 0.039039 & 17 & 44237331 & 17 & 44986812 & 17 & 44 \\
\hline 1900 & 16228 & 38957 & 17 & 0.01 & 90.21 & 0.068068 & 0.037037 & 13 & 196079847 & 13 & 196931822 & 13 & 196 \\
\hline 2578 & 45007 & 29218 & 18 & 0.01 & 90.22 & 0.05005 & 0.03003 & 19 & 130002728 & 19 & 130728194 & 19 & 130 \\
\hline 1766 & 51886 & 57811 & 10 & 0.01 & 90.23 & 0.04004 & 0.022022 & 13 & 62006757 & 13 & 62924497 & 13 & 62 \\
\hline 594 & 53290 & 40917 & 15 & 0.01 & 90.24 & 0.064064 & 0.034034 & 3 & 116001962 & 3 & 116920902 & 3 & 116 \\
\hline 220 & 36899 & 36903 & 17 & 0.01 & 90.25 & 0.055055 & 0.03003 & 1 & 220102612 & 1 & 220815008 & 1 & 220 \\
\hline 221 & 36904 & 58253 & 8 & 0.01 & 90.26 & 0.042042 & 0.027027 & 1 & 221086679 & 1 & 221935450 & 1 & 221 \\
\hline 1038 & 46955 & 61799 & 20 & 0.01 & 90.27 & 0.072072 & 0.041041 & 7 & 1035119 & 7 & 1989754 & 7 & 1 \\
\hline 1250 & 16834 & 53947 & 16 & 0.01 & 90.28 & 0.053053 & 0.034034 & 8 & 79177836 & 8 & 79996042 & 8 & 79 \\
\hline 254 & 1579 & 60730 & 16 & 0.01 & 90.29 & 0.065065 & 0.016016 & 1 & 254016770 & 1 & 254948269 & 1 & 254 \\
\hline 924 & 51442 & 60367 & 12 & 0.01 & 90.3 & 0.049049 & 0.032032 & 6 & 45460886 & 6 & 45906794 & 6 & 45 \\
\hline 885 & 62032 & 22920 & 20 & 0.01 & 90.31 & 0.079079 & 0.05005 & 6 & 6052681 & 6 & 6921532 & 6 & 6 \\
\hline 2483 & 39608 & 14113 & 7 & 0.01 & 90.32 & 0.033033 & 0.019019 & 19 & 30064026 & 19 & 30775028 & 19 & 30 \\
\hline 2008 & 43858 & 35529 & 15 & 0.01 & 90.33 & 0.042042 & 0.026026 & 14 & 85028953 & 14 & 85991839 & 14 & 85 \\
\hline 313 & 1982 & 20697 & 6 & 0.01 & 90.34 & 0.016016 & 0.013013 & 1 & 313111618 & 1 & 313480723 & 1 & 313 \\
\hline 1800 & 47469 & 56585 & 7 & 0.01 & 90.35 & 0.035035 & 0.024024 & 13 & 96056270 & 13 & 96750659 & 13 & 96 \\
\hline 2398 & 16639 & 59864 & 10 & 0.01 & 90.36 & 0.043043 & 0.027027 & 18 & 6026724 & 18 & 6921239 & 18 & 6 \\
\hline 2321 & 54930 & 28387 & 13 & 0.01 & 90.37 & 0.056056 & 0.028028 & 16 & 86008309 & 16 & 86875355 & 16 & 86 \\
\hline 1353 & 61154 & 16463 & 6 & 0.01 & 90.38 & 0.03003 & 0.025025 & 9 & 33065256 & 9 & 33899905 & 9 & 33 \\
\hline 521 & 2941 & 21334 & 3 & 0.01 & 90.39 & 0.01001 & 0.008008 & 3 & 43240931 & 3 & 43336137 & 3 & 43 \\
\hline 2121 & 27710 & 52369 & 16 & 0.01 & 90.4 & 0.065065 & 0.019019 & 15 & 44059586 & 15 & 44862962 & 15 & 44 \\
\hline 1063 & 23419 & 35576 & 10 & 0.01 & 90.41 & 0.046046 & 0.031031 & 7 & 26157007 & 7 & 26954317 & 7 & 26 \\
\hline 2469 & 29064 & 14086 & 14 & 0.01 & 90.42 & 0.037037 & 0.017017 & 19 & 16023625 & 19 & 16853665 & 19 & 16 \\
\hline 182 & 20245 & 54836 & 18 & 0.01 & 90.43 & 0.076076 & 0.043043 & 1 & 182016926 & 1 & 182952918 & 1 & 182 \\
\hline 2488 & 48311 & 52236 & 15 & 0.01 & 90.44 & 0.056056 & 0.036036 & 19 & 35034444 & 19 & 35838914 & 19 & 35 \\
\hline
\end{tabular}




\begin{tabular}{|c|c|c|c|c|c|c|c|c|c|c|c|c|c|}
\hline 623 & 21658 & 30854 & 16 & 0.01 & 90.45 & 0.07007 & 0.034034 & 4 & 95368 & 4 & 779106 & 4 & 0 \\
\hline 127 & 50680 & 908 & 7 & 0.01 & 90.46 & 0.031031 & 0.019019 & 1 & 127172643 & 1 & 127733470 & 1 & 127 \\
\hline 2559 & 57530 & 14256 & 13 & 0.01 & 90.47 & 0.05005 & 0.028028 & 19 & 111015335 & 19 & 111965300 & 19 & 111 \\
\hline 1238 & 50172 & 7082 & 12 & 0.01 & 90.48 & 0.044044 & 0.029029 & 8 & 67026060 & 8 & 67911215 & 8 & b \\
\hline 296 & 1886 & 56783 & 13 & 0.01 & 90.49 & 0.059059 & 0.033033 & 1 & 296003591 & 1 & 296938083 & 1 & 296 \\
\hline 119 & 20031 & 20033 & 13 & 0.01 & 90.5 & 0.048048 & 0.023023 & 1 & 119004005 & 1 & 119835338 & 1 & 119 \\
\hline 2288 & 44400 & 44402 & 9 & 0.01 & 90.51 & 0.041041 & 0.02002 & 16 & 53033170 & 16 & 53927692 & 16 & \\
\hline 168 & 1120 & 46131 & 16 & 0.01 & 90.52 & 0.048048 & 0.034034 & 1 & 168136428 & 1 & 168987602 & 1 & 168 \\
\hline 2587 & 49805 & 29232 & 14 & 0.01 & 90.53 & 0.049049 & 0.022022 & 19 & 139042393 & 19 & 139986269 & 19 & 139 \\
\hline 1400 & 38294 & 38295 & 12 & 0.01 & 90.54 & 0.037037 & 0.028028 & 9 & 80094154 & 9 & 80677221 & 9 & \\
\hline 570 & 30508 & 57437 & 17 & 0.01 & 90.55 & 0.06006 & 0.038038 & 3 & 92012330 & 3 & 92847118 & 3 & \\
\hline 2190 & 47947 & 47949 & 11 & 0.01 & 90.56 & 0.032032 & 0.017017 & 15 & 113010695 & 15 & 113997526 & 15 & 113 \\
\hline 2150 & 59729 & 39282 & 10 & 0.01 & 90.57 & 0.047047 & 0.022022 & 15 & 73043848 & 15 & 73987697 & 15 & \\
\hline 1833 & 10172 & 53050 & 14 & 0.01 & 90.58 & 0.055055 & 0.033033 & 13 & 129131688 & 13 & 129811274 & 13 & 12 \\
\hline 1389 & 24736 & 50658 & 10 & 0.01 & 90.59 & 0.032032 & 0.017017 & 9 & 69016799 & 9 & 69976085 & 9 & 0 \\
\hline 2307 & 51174 & 58581 & 16 & 0.01 & 90.6 & 0.044044 & 0.021021 & 16 & 72137790 & 16 & 72974908 & 16 & \\
\hline 574 & 50485 & 3169 & 10 & 0.01 & 90.61 & 0.043043 & 0.028028 & 3 & 96012125 & 3 & 96641442 & 3 & \\
\hline 206 & 36887 & 36890 & 21 & 0.01 & 90.62 & 0.076076 & 0.034034 & 1 & 206003777 & 1 & 206994869 & 1 & 20 \\
\hline 165 & 46123 & 34521 & 12 & 0.01 & 90.63 & 0.049049 & 0.024024 & 1 & 165188787 & 1 & 165937460 & 1 & 165 \\
\hline 2156 & 50148 & 47900 & 7 & 0.01 & 90.64 & 0.035035 & 0.019019 & 15 & 79058727 & 15 & 79978032 & 15 & 7 \\
\hline 1226 & 7031 & 16568 & 9 & 0.01 & 90.65 & 0.05005 & 0.026026 & 8 & 55181102 & 8 & 55931429 & 8 & 5 \\
\hline 576 & 3173 & 50026 & 10 & 0.01 & 90.65 & 0.044044 & 0.03003 & 3 & 98100280 & 3 & 98991502 & 3 & 98 \\
\hline 2119 & 30162 & 17834 & 12 & 0.01 & 90.66 & 0.06006 & 0.031031 & 15 & 42105101 & 15 & 42956851 & 15 & 42 \\
\hline 2446 & 28996 & 45745 & 14 & 0.01 & 90.67 & 0.062062 & 0.031031 & 18 & 54010221 & 18 & 54902836 & 18 & 5 \\
\hline 133 & 50525 & 59418 & 12 & 0.01 & 90.68 & 0.024024 & 0.014014 & 1 & 133084921 & 1 & 133781212 & 1 & 133 \\
\hline 397 & 20925 & 18204 & 16 & 0.01 & 90.69 & 0.056056 & 0.032032 & 2 & 82002123 & 2 & 82969323 & 2 & 82 \\
\hline 246 & 20384 & 54725 & 14 & 0.01 & 90.7 & 0.047047 & 0.027027 & 1 & 246005498 & 1 & 246950678 & 1 & 24 \\
\hline 1829 & 26342 & 43449 & 17 & 0.01 & 90.71 & 0.057057 & 0.029029 & 13 & 125060509 & 13 & 125976825 & 13 & 12 \\
\hline 2584 & 53437 & 14337 & 12 & 0.01 & 90.72 & 0.043043 & 0.031031 & 19 & 136018066 & 19 & 136986704 & 19 & 13 \\
\hline 264 & 36114 & 40284 & 15 & 0.01 & 90.73 & 0.055055 & 0.033033 & 1 & 264047505 & 1 & 264938853 & 1 & 26 \\
\hline 258 & 59408 & 56575 & 18 & 0.01 & 90.74 & 0.071071 & 0.036036 & 1 & 258021815 & 1 & 258984538 & 1 & 25 \\
\hline 1412 & 24806 & 52927 & 8 & 0.01 & 90.75 & 0.032032 & 0.022022 & 9 & 92204161 & 9 & 92976421 & 9 & \\
\hline 1500 & 42785 & 54303 & 19 & 0.01 & 90.76 & 0.06006 & 0.038038 & 10 & 26120414 & 10 & 26980493 & 10 & \\
\hline 2465 & 48136 & 30608 & 22 & 0.01 & 90.77 & 0.094094 & 0.028028 & 19 & 12108725 & 19 & 12973924 & 19 & \\
\hline 2411 & 28833 & 48096 & 15 & 0.01 & 90.78 & 0.054054 & 0.03003 & 18 & 19003450 & 18 & 19972451 & 18 & \\
\hline 2496 & 29121 & 55243 & 16 & 0.01 & 90.79 & 0.046046 & 0.027027 & 19 & 43046630 & 19 & 43939946 & 19 & \\
\hline 2421 & 13843 & 56632 & 16 & 0.01 & 90.79 & 0.053053 & 0.029029 & 18 & 29008406 & 18 & 29980931 & 18 & \\
\hline 2233 & 55732 & 28058 & 11 & 0.01 & 90.8 & 0.046046 & 0.035035 & 15 & 156048507 & 15 & 156955591 & 15 & 15 \\
\hline 196 & 36852 & 20282 & 17 & 0.01 & 90.81 & 0.043043 & 0.024024 & 1 & 196015749 & 1 & 196836959 & 1 & 196 \\
\hline
\end{tabular}




\begin{tabular}{|c|c|c|c|c|c|c|c|c|c|c|c|c|c|}
\hline 524 & 16651 & 31263 & 8 & 0.01 & 90.82 & 0.033033 & 0.016016 & 3 & 46206833 & 3 & 46893143 & 3 & 46 \\
\hline 222 & 46252 & 1410 & 15 & 0.01 & 90.83 & 0.045045 & 0.026026 & 1 & 222007904 & 1 & 222962711 & 1 & 222 \\
\hline 2173 & 56079 & 52752 & 16 & 0.01 & 90.84 & 0.058058 & 0.027027 & 15 & 96071036 & 15 & 96930028 & 15 & 96 \\
\hline 1388 & 17134 & 7797 & 4 & 0.01 & 90.85 & 0.016016 & 0.011011 & 9 & 68169771 & 9 & 68936873 & 9 & 68 \\
\hline 1548 & 53347 & 51118 & 16 & 0.01 & 90.86 & 0.056056 & 0.034034 & 10 & 74004427 & 10 & 74943049 & 10 & 74 \\
\hline 1360 & 30358 & 8185 & 15 & 0.01 & 90.87 & 0.058058 & 0.032032 & 9 & 40024107 & 9 & 40991552 & 9 & 40 \\
\hline 2258 & 12768 & 12772 & 14 & 0.01 & 90.88 & 0.054054 & 0.033033 & 16 & 23020285 & 16 & 23986785 & 16 & 23 \\
\hline 355 & 17003 & 29455 & 12 & 0.01 & 90.88 & 0.053053 & 0.027027 & 2 & 39185102 & 2 & 39982124 & 2 & 39 \\
\hline 2104 & 27659 & 27662 & 15 & 0.01 & 90.89 & 0.074074 & 0.039039 & 15 & 27006998 & 15 & 27957695 & 15 & 27 \\
\hline 219 & 46246 & 1398 & 11 & 0.01 & 90.9 & 0.043043 & 0.023023 & 1 & 219151821 & 1 & 219966680 & 1 & 219 \\
\hline 2571 & 14724 & 48239 & 10 & 0.01 & 90.91 & 0.046046 & 0.024024 & 19 & 123376923 & 19 & 123800527 & 19 & 123 \\
\hline 1830 & 43450 & 56363 & 15 & 0.01 & 90.92 & 0.054054 & 0.03003 & 13 & 126026173 & 13 & 126943274 & 13 & 126 \\
\hline 1082 & 23533 & 47006 & 16 & 0.01 & 90.93 & 0.065065 & 0.04004 & 7 & 45004542 & 7 & 45911698 & 7 & 45 \\
\hline 1670 & 59231 & 9320 & 8 & 0.01 & 90.94 & 0.046046 & 0.028028 & 12 & 30149881 & 12 & 30998011 & 12 & 30 \\
\hline 272 & 57264 & 37006 & 13 & 0.01 & 90.95 & 0.041041 & 0.025025 & 1 & 272001095 & 1 & 272977191 & 1 & 272 \\
\hline 2484 & 39609 & 34918 & 4 & 0.01 & 90.96 & 0.024024 & 0.016016 & 19 & 31649425 & 19 & 31801282 & 19 & 31 \\
\hline 2155 & 54630 & 27785 & 10 & 0.01 & 90.97 & 0.031031 & 0.021021 & 15 & 78090341 & 15 & 78961640 & 15 & 78 \\
\hline 2290 & 35201 & 28257 & 15 & 0.01 & 90.97 & 0.047047 & 0.026026 & 16 & 55330045 & 16 & 55993053 & 16 & 55 \\
\hline 1917 & 32663 & 10556 & 13 & 0.01 & 90.98 & 0.051051 & 0.032032 & 13 & 213036330 & 13 & 213981854 & 13 & 213 \\
\hline 384 & 2300 & 32476 & 11 & 0.01 & 90.99 & 0.046046 & 0.028028 & 2 & 69103051 & 2 & 69823989 & 2 & 69 \\
\hline 346 & 2124 & 14705 & 16 & 0.01 & 91 & 0.052052 & 0.029029 & 2 & 30141776 & 2 & 30999341 & 2 & 30 \\
\hline 2502 & 29133 & 53472 & 7 & 0.01 & 91.01 & 0.023023 & 0.016016 & 19 & 49013199 & 19 & 49617748 & 19 & 49 \\
\hline 1840 & 26363 & 55569 & 15 & 0.01 & 91.02 & 0.057057 & 0.033033 & 13 & 136017764 & 13 & 136902376 & 13 & 136 \\
\hline 2426 & 13876 & 58370 & 12 & 0.01 & 91.03 & 0.042042 & 0.023023 & 18 & 34013193 & 18 & 34956874 & 18 & 34 \\
\hline 1171 & 45455 & 42191 & 7 & 0.01 & 91.04 & 0.032032 & 0.019019 & 8 & 424993 & 8 & 946879 & 8 & 0 \\
\hline 260 & 54409 & 46348 & 18 & 0.01 & 91.04 & 0.08008 & 0.029029 & 1 & 260134029 & 1 & 260985282 & 1 & 260 \\
\hline 207 & 46211 & 1332 & 16 & 0.01 & 91.05 & 0.05005 & 0.03003 & 1 & 207036700 & 1 & 207810204 & 1 & 207 \\
\hline 669 & 3787 & 41084 & 17 & 0.01 & 91.06 & 0.068068 & 0.037037 & 4 & 46040879 & 4 & 46986125 & 4 & 46 \\
\hline 2120 & 17308 & 27709 & 7 & 0.01 & 91.07 & 0.035035 & 0.013013 & 15 & 43053001 & 15 & 43972136 & 15 & 43 \\
\hline 976 & 35851 & 41680 & 13 & 0.01 & 91.08 & 0.047047 & 0.02002 & 6 & 97022833 & 6 & 97914488 & 6 & 97 \\
\hline 573 & 3162 & 3167 & 9 & 0.01 & 91.09 & 0.045045 & 0.023023 & 3 & 95002203 & 3 & 95944062 & 3 & 95 \\
\hline 37 & 39868 & 45877 & 20 & 0.01 & 91.1 & 0.086086 & 0.042042 & 1 & 37000384 & 1 & 37964303 & 1 & 37 \\
\hline 2109 & 59673 & 12012 & 18 & 0.01 & 91.1 & 0.069069 & 0.032032 & 15 & 32042636 & 15 & 32933373 & 15 & 32 \\
\hline 1770 & 58342 & 61624 & 13 & 0.01 & 91.11 & 0.05005 & 0.034034 & 13 & 66094535 & 13 & 66778422 & 13 & 66 \\
\hline 565 & 40856 & 21463 & 7 & 0.01 & 91.12 & 0.034034 & 0.023023 & 3 & 87200902 & 3 & 87941073 & 3 & 87 \\
\hline 241 & 40238 & 1514 & 12 & 0.01 & 91.13 & 0.042042 & 0.025025 & 1 & 241074745 & 1 & 241983957 & 1 & 241 \\
\hline 2470 & 15599 & 29065 & 11 & 0.01 & 91.14 & 0.05005 & 0.031031 & 19 & 17147091 & 19 & 17947980 & 19 & 17 \\
\hline 2482 & 34698 & 44903 & 7 & 0.01 & 91.15 & 0.043043 & 0.029029 & 19 & 29072100 & 19 & 29704103 & 19 & 29 \\
\hline 1751 & 9794 & 26113 & 20 & 0.01 & 91.16 & 0.071071 & 0.033033 & 13 & 47007358 & 13 & 47992156 & 13 & 47 \\
\hline
\end{tabular}




\begin{tabular}{|c|c|c|c|c|c|c|c|c|c|c|c|c|c|}
\hline 1601 & 57979 & 47361 & 18 & 0.01 & 91.16 & 0.079079 & 0.035035 & 11 & 49121567 & 11 & 49990774 & 11 & 49 \\
\hline 2170 & 44208 & 57869 & 27 & 0.01 & 91.17 & 0.11011 & 0.04004 & 15 & 93015426 & 15 & 93995497 & 15 & 93 \\
\hline 571 & 19398 & 3154 & 8 & 0.01 & 91.18 & 0.028028 & 0.017017 & 3 & 93074982 & 3 & 93929087 & 3 & 93 \\
\hline 1587 & 25483 & 50807 & 13 & 0.01 & 91.19 & 0.055055 & 0.022022 & 11 & 35006617 & 11 & 35896772 & 11 & 35 \\
\hline 1284 & 7274 & 61595 & 13 & 0.01 & 91.2 & 0.047047 & 0.03003 & 8 & 113050594 & 8 & 113825992 & 8 & 113 \\
\hline 712 & 4099 & 4107 & 21 & 0.01 & 91.21 & 0.097097 & 0.044044 & 4 & 89008992 & 4 & 89983155 & 4 & 89 \\
\hline 564 & 15618 & 52680 & 10 & 0.01 & 91.21 & 0.039039 & 0.029029 & 3 & 86085644 & 3 & 86960594 & 3 & 86 \\
\hline 705 & 4058 & 4068 & 23 & 0.01 & 91.22 & 0.068068 & 0.033033 & 4 & 82027009 & 4 & 82971117 & 4 & 82 \\
\hline 2473 & 39593 & 14096 & 8 & 0.01 & 91.23 & 0.04004 & 0.021021 & 19 & 20049731 & 19 & 20985182 & 19 & 20 \\
\hline 846 & 48854 & 5033 & 19 & 0.01 & 91.24 & 0.074074 & 0.034034 & 5 & 79010106 & 5 & 79988080 & 5 & 79 \\
\hline 1639 & 32281 & 49849 & 14 & 0.01 & 91.25 & 0.056056 & 0.025025 & 11 & 87115792 & 11 & 87660102 & 11 & 87 \\
\hline 918 & 32861 & 58371 & 6 & 0.01 & 91.25 & 0.032032 & 0.023023 & 6 & 39187023 & 6 & 39960478 & 6 & 39 \\
\hline 2301 & 13036 & 28297 & 13 & 0.01 & 91.26 & 0.049049 & 0.031031 & 16 & 66016093 & 16 & 66914976 & 16 & 66 \\
\hline 191 & 1240 & 17445 & 18 & 0.01 & 91.27 & 0.071071 & 0.024024 & 1 & 191055429 & 1 & 191937788 & 1 & 191 \\
\hline 198 & 46192 & 1268 & 15 & 0.01 & 91.28 & 0.061061 & 0.032032 & 1 & 198004282 & 1 & 198859209 & 1 & 198 \\
\hline 646 & 35912 & 3613 & 12 & 0.01 & 91.29 & 0.035035 & 0.018018 & 4 & 23215904 & 4 & 23861877 & 4 & 23 \\
\hline 1377 & 24684 & 54474 & 15 & 0.01 & 91.29 & 0.06006 & 0.035035 & 9 & 57027600 & 9 & 57905817 & 9 & 57 \\
\hline 1496 & 8347 & 56304 & 5 & 0.01 & 91.3 & 0.022022 & 0.017017 & 10 & 22010713 & 10 & 22730977 & 10 & 22 \\
\hline 877 & 5228 & 56131 & 9 & 0.01 & 91.31 & 0.035035 & 0.021021 & 5 & 110032368 & 5 & 110935968 & 5 & 110 \\
\hline 1103 & 42002 & 50423 & 8 & 0.01 & 91.32 & 0.046046 & 0.028028 & 7 & 66304912 & 7 & 66728436 & 7 & 66 \\
\hline 1236 & 42292 & 24203 & 11 & 0.01 & 91.33 & 0.042042 & 0.027027 & 8 & 65027400 & 8 & 65954370 & 8 & 65 \\
\hline 112 & 59732 & 838 & 15 & 0.01 & 91.33 & 0.06006 & 0.03003 & 1 & 112020122 & 1 & 112884757 & 1 & 112 \\
\hline 1815 & 17198 & 56349 & 3 & 0.01 & 91.34 & 0.012012 & 0.009009 & 13 & 111492781 & 13 & 111921377 & 13 & 111 \\
\hline 2377 & 28665 & 49688 & 16 & 0.01 & 91.35 & 0.065065 & 0.026026 & 17 & 55020501 & 17 & 55931769 & 17 & 55 \\
\hline 1234 & 7055 & 51212 & 12 & 0.01 & 91.36 & 0.04004 & 0.028028 & 8 & 63000451 & 8 & 63838720 & 8 & 63 \\
\hline 1662 & 53388 & 56697 & 6 & 0.01 & 91.37 & 0.023023 & 0.017017 & 12 & 22051275 & 12 & 22890196 & 12 & 22 \\
\hline 605 & 3342 & 61723 & 11 & 0.01 & 91.37 & 0.046046 & 0.019019 & 3 & 127121184 & 3 & 127967288 & 3 & 127 \\
\hline 1065 & 51247 & 41856 & 10 & 0.01 & 91.38 & 0.045045 & 0.032032 & 7 & 28014685 & 7 & 28889475 & 7 & 28 \\
\hline 1009 & 5585 & 14988 & 9 & 0.01 & 91.39 & 0.039039 & 0.021021 & 6 & 130083282 & 6 & 130959693 & 6 & 130 \\
\hline 1376 & 16622 & 19400 & 6 & 0.01 & 91.4 & 0.027027 & 0.019019 & 9 & 56048240 & 9 & 56975238 & 9 & 56 \\
\hline 791 & 36399 & 41425 & 9 & 0.01 & 91.4 & 0.041041 & 0.017017 & 5 & 24041995 & 5 & 24782967 & 5 & 24 \\
\hline 190 & 1235 & 36834 & 16 & 0.01 & 91.41 & 0.049049 & 0.027027 & 1 & 190244449 & 1 & 190921716 & 1 & 190 \\
\hline 154 & 40097 & 57614 & 13 & 0.01 & 91.42 & 0.039039 & 0.026026 & 1 & 154076660 & 1 & 154984965 & 1 & 154 \\
\hline 2576 & 48245 & 14309 & 13 & 0.01 & 91.43 & 0.028028 & 0.018018 & 19 & 128108120 & 19 & 128842928 & 19 & 128 \\
\hline 869 & 33288 & 5173 & 20 & 0.01 & 91.43 & 0.07007 & 0.027027 & 5 & 102247795 & 5 & 102993667 & 5 & 102 \\
\hline 1418 & 24821 & 15756 & 9 & 0.01 & 91.44 & 0.033033 & 0.022022 & 9 & 98059019 & 9 & 98984967 & 9 & 98 \\
\hline 1399 & 7842 & 31860 & 11 & 0.01 & 91.45 & 0.033033 & 0.024024 & 9 & 79010742 & 9 & 79894082 & 9 & 79 \\
\hline 1119 & 23711 & 61620 & 13 & 0.01 & 91.46 & 0.066066 & 0.033033 & 7 & 82003775 & 7 & 82993422 & 7 & 82 \\
\hline 2471 & 48144 & 16347 & 6 & 0.01 & 91.47 & 0.037037 & 0.025025 & 19 & 18005050 & 19 & 18720914 & 19 & 18 \\
\hline
\end{tabular}




\begin{tabular}{|c|c|c|c|c|c|c|c|c|c|c|c|c|c|}
\hline 2162 & 34679 & 27800 & 21 & 0.01 & 91.47 & 0.071071 & 0.028028 & 15 & 85023476 & 15 & 85874505 & 15 & 85 \\
\hline 1873 & 38894 & 34601 & 12 & 0.01 & 91.48 & 0.05005 & 0.031031 & 13 & 169000579 & 13 & 169988202 & 13 & 169 \\
\hline 2181 & 48328 & 53244 & 8 & 0.01 & 91.49 & 0.031031 & 0.016016 & 15 & 104065843 & 15 & 104904590 & 15 & 104 \\
\hline 1227 & 7034 & 7036 & 8 & 0.01 & 91.5 & 0.035035 & 0.022022 & 8 & 56064449 & 8 & 56805057 & 8 & 56 \\
\hline 2157 & 47903 & 54197 & 9 & 0.01 & 91.5 & 0.034034 & 0.022022 & 15 & 80299741 & 15 & 80987506 & 15 & 80 \\
\hline 2141 & 12191 & 12200 & 17 & 0.01 & 91.51 & 0.058058 & 0.032032 & 15 & 64019259 & 15 & 64980103 & 15 & 64 \\
\hline 237 & 61841 & 1490 & 10 & 0.01 & 91.52 & 0.038038 & 0.018018 & 1 & 237029410 & 1 & 237900874 & 1 & 237 \\
\hline 2575 & 14302 & 29208 & 12 & 0.01 & 91.52 & 0.032032 & 0.02002 & 19 & 127051537 & 19 & 127945580 & 19 & 127 \\
\hline 2574 & 14299 & 14303 & 9 & 0.01 & 91.53 & 0.038038 & 0.024024 & 19 & 126033395 & 19 & 126992902 & 19 & 126 \\
\hline 615 & 40958 & 51601 & 12 & 0.01 & 91.54 & 0.052052 & 0.024024 & 3 & 137058702 & 3 & 137907270 & 3 & 137 \\
\hline 810 & 18312 & 46823 & 7 & 0.01 & 91.55 & 0.032032 & 0.02002 & 5 & 43144872 & 5 & 43997881 & 5 & 43 \\
\hline 110 & 45994 & 58664 & 15 & 0.01 & 91.55 & 0.063063 & 0.027027 & 1 & 110118325 & 1 & 110903682 & 1 & 110 \\
\hline 676 & 46620 & 62092 & 11 & 0.01 & 91.56 & 0.027027 & 0.016016 & 4 & 53047867 & 4 & 53913712 & 4 & 53 \\
\hline 560 & 3116 & 58220 & 11 & 0.01 & 91.57 & 0.043043 & 0.024024 & 3 & 82013849 & 3 & 82697045 & 3 & 82 \\
\hline 2147 & 39277 & 12218 & 8 & 0.01 & 91.57 & 0.04004 & 0.023023 & 15 & 70035825 & 15 & 70797627 & 15 & 70 \\
\hline 2467 & 14076 & 18054 & 16 & 0.01 & 91.58 & 0.055055 & 0.026026 & 19 & 14154082 & 19 & 14724810 & 19 & 14 \\
\hline 1814 & 53063 & 57532 & 7 & 0.01 & 91.59 & 0.045045 & 0.026026 & 13 & 110176644 & 13 & 110802739 & 13 & 110 \\
\hline 1582 & 8802 & 61598 & 10 & 0.01 & 91.6 & 0.041041 & 0.018018 & 11 & 30218369 & 11 & 30982805 & 11 & 30 \\
\hline 1549 & 29461 & 57170 & 12 & 0.01 & 91.6 & 0.045045 & 0.021021 & 10 & 75064259 & 10 & 75940391 & 10 & 75 \\
\hline 1286 & 7308 & 56289 & 9 & 0.01 & 91.61 & 0.046046 & 0.025025 & 8 & 115099064 & 8 & 115989788 & 8 & 115 \\
\hline 1230 & 32950 & 7051 & 8 & 0.01 & 91.62 & 0.055055 & 0.028028 & 8 & 59002816 & 8 & 59957664 & 8 & 59 \\
\hline 2107 & 18787 & 11989 & 11 & 0.01 & 91.62 & 0.046046 & 0.024024 & 15 & 30007572 & 15 & 30928847 & 15 & 30 \\
\hline 2486 & 47198 & 29108 & 4 & 0.01 & 91.63 & 0.02002 & 0.014014 & 19 & 33571880 & 19 & 33819184 & 19 & 33 \\
\hline 1514 & 60653 & 34736 & 12 & 0.01 & 91.64 & 0.034034 & 0.013013 & 10 & 40186438 & 10 & 40950374 & 10 & 40 \\
\hline 181 & 50709 & 54926 & 18 & 0.01 & 91.64 & 0.049049 & 0.026026 & 1 & 181006837 & 1 & 181978498 & 1 & 181 \\
\hline 2186 & 12406 & 27866 & 8 & 0.01 & 91.65 & 0.034034 & 0.017017 & 15 & 109196356 & 15 & 109722503 & 15 & 109 \\
\hline 1804 & 38835 & 10090 & 14 & 0.01 & 91.66 & 0.048048 & 0.026026 & 13 & 100035462 & 13 & 100946188 & 13 & 100 \\
\hline 2153 & 16141 & 12230 & 4 & 0.01 & 91.67 & 0.017017 & 0.01001 & 15 & 76266102 & 15 & 76787826 & 15 & 76 \\
\hline 1589 & 8834 & 18833 & 9 & 0.01 & 91.67 & 0.046046 & 0.027027 & 11 & 37109307 & 11 & 37805603 & 11 & 37 \\
\hline 73 & 575 & 578 & 10 & 0.01 & 91.68 & 0.042042 & 0.025025 & 1 & 73100657 & 1 & 73922223 & 1 & 73 \\
\hline 935 & 18074 & 52580 & 10 & 0.01 & 91.69 & 0.043043 & 0.025025 & 6 & 56167422 & 6 & 56948678 & 6 & 56 \\
\hline 2583 & 14331 & 33021 & 7 & 0.01 & 91.69 & 0.027027 & 0.015015 & 19 & 135350565 & 19 & 135937088 & 19 & 135 \\
\hline 2187 & 27867 & 47941 & 10 & 0.01 & 91.7 & 0.055055 & 0.019019 & 15 & 110011380 & 15 & 110852498 & 15 & 110 \\
\hline 985 & 5487 & 23101 & 5 & 0.01 & 91.71 & 0.022022 & 0.015015 & 6 & 106010991 & 6 & 106324729 & 6 & 106 \\
\hline 149 & 36791 & 20124 & 9 & 0.01 & 91.71 & 0.043043 & 0.023023 & 1 & 149045152 & 1 & 149860127 & 1 & 149 \\
\hline 713 & 46714 & 4115 & 16 & 0.01 & 91.72 & 0.064064 & 0.031031 & 4 & 90010895 & 4 & 90944979 & 4 & 90 \\
\hline 817 & 37628 & 4853 & 10 & 0.01 & 91.73 & 0.05005 & 0.024024 & 5 & 50214568 & 5 & 50991368 & 5 & 50 \\
\hline 677 & 3836 & 53246 & 13 & 0.01 & 91.73 & 0.04004 & 0.022022 & 4 & 54122432 & 4 & 54937429 & 4 & 54 \\
\hline 150 & 56301 & 36794 & 13 & 0.01 & 91.74 & 0.039039 & 0.024024 & 1 & 150030731 & 1 & 150801717 & 1 & 150 \\
\hline
\end{tabular}




\begin{tabular}{|c|c|c|c|c|c|c|c|c|c|c|c|c|c|}
\hline 2551 & 44959 & 29147 & 8 & 0.01 & 91.75 & 0.04004 & 0.025025 & 19 & 103011287 & 19 & 103657659 & 19 & 103 \\
\hline 2291 & 49628 & 44413 & 20 & 0.01 & 91.75 & 0.079079 & 0.025025 & 16 & 56029991 & 16 & 56950872 & 16 & 56 \\
\hline 2420 & 13836 & 16284 & 14 & 0.01 & 91.76 & 0.044044 & 0.022022 & 18 & 28006207 & 18 & 28845095 & 18 & 28 \\
\hline 189 & 36824 & 16802 & 14 & 0.01 & 91.76 & 0.039039 & 0.019019 & 1 & 189025116 & 1 & 189978659 & 1 & 189 \\
\hline 561 & 39695 & 3121 & 16 & 0.01 & 91.77 & 0.049049 & 0.025025 & 3 & 83277793 & 3 & 83943514 & 3 & 83 \\
\hline 1428 & 47284 & 24839 & 11 & 0.01 & 91.78 & 0.037037 & 0.02002 & 9 & 108009662 & 9 & 108882963 & 9 & 108 \\
\hline 2560 & 33727 & 14261 & 7 & 0.01 & 91.78 & 0.03003 & 0.015015 & 19 & 112108617 & 19 & 112884986 & 19 & 112 \\
\hline 878 & 16810 & 33983 & 5 & 0.01 & 91.79 & 0.026026 & 0.021021 & 5 & 111137068 & 5 & 111371828 & 5 & 111 \\
\hline 153 & 40094 & 1037 & 11 & 0.01 & 91.8 & 0.03003 & 0.015015 & 1 & 153144281 & 1 & 153965399 & 1 & 153 \\
\hline 2436 & 13931 & 28955 & 11 & 0.01 & 91.8 & 0.059059 & 0.023023 & 18 & 44006428 & 18 & 44927369 & 18 & 44 \\
\hline 477 & 16695 & 30004 & 5 & 0.01 & 91.81 & 0.02002 & 0.016016 & 2 & 162084552 & 2 & 162298086 & 2 & 162 \\
\hline 161 & 20170 & 45524 & 8 & 0.01 & 91.81 & 0.024024 & 0.016016 & 1 & 161003628 & 1 & 161993748 & 1 & 161 \\
\hline 1356 & 38251 & 32207 & 14 & 0.01 & 91.82 & 0.05005 & 0.024024 & 9 & 36041037 & 9 & 36913567 & 9 & 36 \\
\hline 273 & 46366 & 1715 & 8 & 0.01 & 91.83 & 0.033033 & 0.017017 & 1 & 273002529 & 1 & 273825768 & 1 & 273 \\
\hline 1922 & 17126 & 17221 & 3 & 0.01 & 91.83 & 0.016016 & 0.014014 & 13 & 218194240 & 13 & 218594088 & 13 & 218 \\
\hline 1623 & 61017 & 25595 & 12 & 0.01 & 91.84 & 0.048048 & 0.024024 & 11 & 71130475 & 11 & 71993140 & 11 & 71 \\
\hline 178 & 1164 & 57414 & 13 & 0.01 & 91.85 & 0.042042 & 0.025025 & 1 & 178024855 & 1 & 178988459 & 1 & 178 \\
\hline 158 & 20157 & 53155 & 9 & 0.01 & 91.85 & 0.034034 & 0.013013 & 1 & 158219331 & 1 & 158936835 & 1 & 158 \\
\hline 934 & 29619 & 17874 & 8 & 0.01 & 91.86 & 0.025025 & 0.015015 & 6 & 55046176 & 6 & 55956683 & 6 & 55 \\
\hline 210 & 1344 & 35587 & 15 & 0.01 & 91.86 & 0.04004 & 0.023023 & 1 & 210025405 & 1 & 210956244 & 1 & 210 \\
\hline 928 & 30407 & 51539 & 6 & 0.01 & 91.87 & 0.031031 & 0.019019 & 6 & 49146524 & 6 & 49817264 & 6 & 49 \\
\hline 1241 & 24208 & 55886 & 9 & 0.01 & 91.88 & 0.026026 & 0.012012 & 8 & 70033358 & 8 & 70991752 & 8 & 70 \\
\hline 479 & 45521 & 15661 & 8 & 0.01 & 91.88 & 0.033033 & 0.019019 & 3 & 1456146 & 3 & 1997344 & 3 & 1 \\
\hline 2364 & 13489 & 44623 & 17 & 0.01 & 91.89 & 0.055055 & 0.032032 & 17 & 42024585 & 17 & 42998995 & 17 & 42 \\
\hline 2282 & 61888 & 12939 & 12 & 0.01 & 91.89 & 0.052052 & 0.023023 & 16 & 47011906 & 16 & 47944374 & 16 & 47 \\
\hline 2102 & 11975 & 11976 & 10 & 0.01 & 91.9 & 0.043043 & 0.023023 & 15 & 25026611 & 15 & 25947179 & 15 & 25 \\
\hline 1228 & 51731 & 7041 & 11 & 0.01 & 91.9 & 0.046046 & 0.029029 & 8 & 57262741 & 8 & 57960254 & 8 & 57 \\
\hline 1411 & 7893 & 7896 & 8 & 0.01 & 91.91 & 0.036036 & 0.019019 & 9 & 91008560 & 9 & 91927983 & 9 & 91 \\
\hline 320 & 1991 & 20705 & 11 & 0.01 & 91.92 & 0.041041 & 0.025025 & 2 & 4110608 & 2 & 4872634 & 2 & 4 \\
\hline 881 & 19394 & 50110 & 11 & 0.01 & 91.92 & 0.04004 & 0.025025 & 6 & 2042055 & 6 & 2952600 & 6 & 2 \\
\hline 234 & 1473 & 46281 & 10 & 0.01 & 91.93 & 0.042042 & 0.028028 & 1 & 234035171 & 1 & 234854917 & 1 & 234 \\
\hline 216 & 60848 & 1385 & 16 & 0.01 & 91.93 & 0.032032 & 0.014014 & 1 & 216019228 & 1 & 216980027 & 1 & 216 \\
\hline 2179 & 12379 & 50213 & 7 & 0.01 & 91.94 & 0.028028 & 0.01001 & 15 & 102338125 & 15 & 102673383 & 15 & 102 \\
\hline 912 & 59081 & 31946 & 8 & 0.01 & 91.94 & 0.039039 & 0.023023 & 6 & 33257346 & 6 & 33810961 & 6 & 33 \\
\hline 825 & 59570 & 33205 & 9 & 0.01 & 91.95 & 0.033033 & 0.018018 & 5 & 58028366 & 5 & 58957433 & 5 & 58 \\
\hline 880 & 30235 & 34251 & 8 & 0.01 & 91.95 & 0.039039 & 0.023023 & 6 & 1065220 & 6 & 1939451 & 6 & 1 \\
\hline 141 & 46069 & 985 & 9 & 0.01 & 91.96 & 0.037037 & 0.016016 & 1 & 141092891 & 1 & 141938208 & 1 & 141 \\
\hline 2455 & 14033 & 17778 & 12 & 0.01 & 91.97 & 0.047047 & 0.031031 & 19 & 2004788 & 19 & 2838767 & 19 & 2 \\
\hline 2145 & 47893 & 12213 & 6 & 0.01 & 91.97 & 0.018018 & 0.011011 & 15 & 68046201 & 15 & 68928476 & 15 & 68 \\
\hline
\end{tabular}




\begin{tabular}{|c|c|c|c|c|c|c|c|c|c|c|c|c|c|}
\hline 160 & 40115 & 53346 & 9 & 0.01 & 91.98 & 0.038038 & 0.024024 & 1 & 160024181 & 1 & 160991130 & 1 & 160 \\
\hline 978 & 58005 & 23083 & 6 & 0.01 & 91.98 & 0.014014 & 0.01001 & 6 & 99135401 & 6 & 99912189 & 6 & \\
\hline 2563 & 33535 & 29183 & 9 & 0.01 & 91.99 & 0.036036 & 0.014014 & 19 & 115004040 & 19 & 115895028 & 19 & 11 \\
\hline 297 & 40376 & 40377 & 12 & 0.01 & 91.99 & 0.051051 & 0.019019 & 1 & 297148442 & 1 & 297932234 & 1 & \\
\hline 1289 & 7307 & 59065 & 11 & 0.01 & 92 & 0.043043 & 0.031031 & 8 & 118016335 & 8 & 118874252 & 8 & \\
\hline 1253 & 56127 & 38156 & 8 & 0.01 & 92 & 0.022022 & 0.011011 & 8 & 82012032 & 8 & 82868297 & 8 & \\
\hline 1599 & 16106 & 19188 & 6 & 0.01 & 92.01 & 0.02002 & 0.011011 & 11 & 47014747 & 11 & 47907047 & 11 & \\
\hline 378 & 2313 & 57106 & 4 & 0.01 & 92.01 & 0.023023 & 0.01001 & 2 & 62015620 & 2 & 62521678 & 2 & \\
\hline 203 & 1293 & 33788 & 17 & 0.01 & 92.02 & 0.053053 & 0.022022 & 1 & 203020847 & 1 & 203809481 & 1 & 20 \\
\hline 197 & 1264 & 36858 & 12 & 0.01 & 92.02 & 0.029029 & 0.015015 & 1 & 197179651 & 1 & 197983246 & 1 & 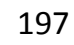 \\
\hline 225 & 34390 & 36921 & 9 & 0.01 & 92.03 & 0.03003 & 0.019019 & 1 & 225518654 & 1 & 225986595 & 1 & 22 \\
\hline 1408 & 17527 & 60222 & 10 & 0.01 & 92.03 & 0.034034 & 0.021021 & 9 & 88276225 & 9 & 88978634 & 9 & 8 \\
\hline 551 & 40842 & 18730 & 15 & 0.01 & 92.04 & 0.04004 & 0.021021 & 3 & 73025014 & 3 & 73996122 & 3 & \\
\hline 386 & 37115 & 29432 & 4 & 0.01 & 92.04 & 0.021021 & 0.012012 & 2 & 71036422 & 2 & 71990984 & 2 & \\
\hline 1421 & 32324 & 14801 & 8 & 0.01 & 92.05 & 0.037037 & 0.021021 & 9 & 101128149 & 9 & 101987835 & 9 & \\
\hline 1319 & 19319 & 53483 & 4 & 0.01 & 92.05 & 0.012012 & 0.011011 & 8 & 148060253 & 8 & 148158316 & 8 & 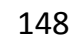 \\
\hline 2487 & 50326 & 50339 & 9 & 0 & 92.06 & 0.028028 & 0.016016 & 19 & 34450186 & 19 & 34925038 & 19 & \\
\hline 1451 & 16431 & 56850 & 14 & 0 & 92.06 & 0.041041 & 0.021021 & 9 & 131130851 & 9 & 131977434 & 9 & 13 \\
\hline 1852 & 54011 & 10247 & 12 & 0 & 92.07 & 0.028028 & 0.015015 & 13 & 148020127 & 13 & 148916021 & 13 & 14 \\
\hline 2501 & 35069 & 48166 & 7 & 0 & 92.07 & 0.021021 & 0.012012 & 19 & 48308192 & 19 & 48929906 & 19 & 4 \\
\hline 910 & 46907 & 41627 & 11 & 0 & 92.08 & 0.045045 & 0.025025 & 6 & 31009804 & 6 & 31994459 & 6 & 31 \\
\hline 2289 & 44403 & 51816 & 8 & 0 & 92.08 & 0.026026 & 0.017017 & 16 & 54031268 & 16 & 54979717 & 16 & 52 \\
\hline 2499 & 14168 & 48310 & 13 & 0 & 92.09 & 0.049049 & 0.021021 & 19 & 46048134 & 19 & 46763140 & 19 & 4 \\
\hline 478 & 52757 & 29919 & 3 & 0 & 92.09 & 0.011011 & 0.01001 & 3 & 72705 & 3 & 715273 & 3 & \\
\hline 170 & 46134 & 36805 & 12 & 0 & 92.1 & 0.043043 & 0.019019 & 1 & 170051398 & 1 & 170866800 & 1 & 17 \\
\hline 1427 & 17424 & 47283 & 11 & 0 & 92.1 & 0.036036 & 0.019019 & 9 & 107057752 & 9 & 107850450 & 9 & 10 \\
\hline 1218 & 53708 & 42271 & 6 & 0 & 92.11 & 0.031031 & 0.021021 & 8 & 47035822 & 8 & 47582919 & 8 & 4 \\
\hline 1232 & 7052 & 17227 & 7 & 0 & 92.11 & 0.033033 & 0.015015 & 8 & 61100526 & 8 & 61872918 & 8 & 6 \\
\hline 2474 & 58514 & 56432 & 6 & 0 & 92.11 & 0.025025 & 0.016016 & 19 & 21103213 & 19 & 21870366 & 19 & 2 \\
\hline 2477 & 39596 & 14109 & 7 & 0 & 92.12 & 0.029029 & 0.015015 & 19 & 24126684 & 19 & 24769857 & 19 & 2 \\
\hline 904 & 29840 & 17966 & 11 & 0 & 92.12 & 0.043043 & 0.017017 & 6 & 25114255 & 6 & 25995859 & 6 & 2 \\
\hline 1062 & 23413 & 41848 & 3 & 0 & 92.13 & 0.018018 & 0.014014 & 7 & 25105017 & 7 & 25215084 & 7 & 2 \\
\hline 369 & 55296 & 18057 & 7 & 0 & 92.13 & 0.032032 & 0.019019 & 2 & 53067453 & 2 & 53907735 & 2 & 5 \\
\hline 519 & 48621 & 48622 & 3 & 0 & 92.14 & 0.015015 & 0.009009 & 3 & 41738237 & 3 & 41843056 & 3 & 4 \\
\hline 933 & 5365 & 55604 & 4 & 0 & 92.14 & 0.026026 & 0.014014 & 6 & 54194696 & 6 & 54949995 & 6 & \\
\hline 211 & 1350 & 1355 & 11 & 0 & 92.15 & 0.039039 & 0.023023 & 1 & 211151578 & 1 & 211988436 & 1 & 21 \\
\hline 46 & 36592 & 33731 & 11 & 0 & 92.15 & 0.036036 & 0.018018 & 1 & 46028574 & 1 & 46907309 & 1 & \\
\hline 1855 & 10260 & 50836 & 9 & 0 & 92.15 & 0.027027 & 0.019019 & 13 & 151109312 & 13 & 151823774 & 13 & 15 \\
\hline 2572 & 52925 & 29195 & 9 & 0 & 92.16 & 0.04004 & 0.023023 & 19 & 124405844 & 19 & 124994817 & 19 & 12 \\
\hline
\end{tabular}




\begin{tabular}{|c|c|c|c|c|c|c|c|c|c|c|c|c|c|}
\hline 2177 & 47923 & 39299 & 10 & 0 & 92.16 & 0.036036 & 0.014014 & 15 & 100101961 & 15 & 100681987 & 15 & 100 \\
\hline 2565 & 48232 & 48234 & 5 & 0 & 92.17 & 0.019019 & 0.012012 & 19 & 117010968 & 19 & 117611622 & 19 & 117 \\
\hline 1216 & 18311 & 29762 & 7 & 0 & 92.17 & 0.032032 & 0.016016 & 8 & 45191096 & 8 & 45750461 & 8 & 45 \\
\hline 1225 & 53092 & 56296 & 5 & 0 & 92.17 & 0.012012 & 0.008008 & 8 & 54201619 & 8 & 54932026 & 8 & 54 \\
\hline 2567 & 44981 & 29185 & 6 & 0 & 92.18 & 0.023023 & 0.014014 & 19 & 119371829 & 19 & 119809654 & 19 & 119 \\
\hline 936 & 51555 & 55848 & 6 & 0 & 92.18 & 0.022022 & 0.012012 & 6 & 57107592 & 6 & 57971843 & 6 & 57 \\
\hline 937 & 50009 & 29369 & 6 & 0 & 92.19 & 0.016016 & 0.015015 & 6 & 58080576 & 6 & 58859012 & 6 & 58 \\
\hline 2524 & 14199 & 57355 & 2 & 0 & 92.19 & 0.009009 & 0.007007 & 19 & 73417360 & 19 & 73903037 & 19 & 73 \\
\hline 317 & 49927 & 35732 & 16 & 0 & 92.19 & 0.041041 & 0.017017 & 2 & 1021191 & 2 & 1881100 & 2 & 1 \\
\hline 2180 & 12383 & 12387 & 8 & 0 & 92.2 & 0.023023 & 0.009009 & 15 & 103012674 & 15 & 103608693 & 15 & 103 \\
\hline 2581 & 14327 & 29223 & 7 & 0 & 92.2 & 0.018018 & 0.008008 & 19 & 133074194 & 19 & 133995643 & 19 & 133 \\
\hline 1753 & 26115 & 17001 & 10 & 0 & 92.21 & 0.045045 & 0.021021 & 13 & 49008533 & 13 & 49979246 & 13 & 49 \\
\hline 2176 & 58951 & 54662 & 9 & 0 & 92.21 & 0.038038 & 0.014014 & 15 & 99006968 & 15 & 99784888 & 15 & 99 \\
\hline 2464 & 44876 & 44881 & 18 & 0 & 92.21 & 0.048048 & 0.01001 & 19 & 11030902 & 19 & 11783693 & 19 & 11 \\
\hline 1834 & 59047 & 52492 & 6 & 0 & 92.22 & 0.021021 & 0.017017 & 13 & 130011769 & 13 & 130982311 & 13 & 130 \\
\hline 2529 & 58310 & 19289 & 7 & 0 & 92.22 & 0.012012 & 0.009009 & 19 & 78000797 & 19 & 78893355 & 19 & 78 \\
\hline 1425 & 50828 & 7942 & 5 & 0 & 92.22 & 0.022022 & 0.016016 & 9 & 105545798 & 9 & 105742262 & 9 & 105 \\
\hline 2509 & 30426 & 51732 & 2 & 0 & 92.23 & 0.004004 & 0.004004 & 19 & 57019187 & 19 & 57672447 & 19 & 57 \\
\hline 192 & 46180 & 36841 & 9 & 0 & 92.23 & 0.037037 & 0.017017 & 1 & 192637720 & 1 & 192978414 & 1 & 192 \\
\hline 188 & 1226 & 36823 & 9 & 0 & 92.23 & 0.025025 & 0.01001 & 1 & 188025326 & 1 & 188990100 & 1 & 188 \\
\hline 1027 & 5674 & 17991 & 12 & 0 & 92.24 & 0.035035 & 0.015015 & 6 & 148004363 & 6 & 148742719 & 6 & 148 \\
\hline 2476 & 39594 & 29075 & 6 & 0 & 92.24 & 0.015015 & 0.011011 & 19 & 23047391 & 19 & 23980272 & 19 & 23 \\
\hline 1675 & 25819 & 9350 & 12 & 0 & 92.24 & 0.034034 & 0.015015 & 12 & 35017301 & 12 & 35990853 & 12 & 35 \\
\hline 218 & 20332 & 1394 & 12 & 0 & 92.25 & 0.039039 & 0.016016 & 1 & 218141657 & 1 & 218977436 & 1 & 218 \\
\hline 1168 & 17647 & 6747 & 10 & 0 & 92.25 & 0.033033 & 0.019019 & 7 & 132107366 & 7 & 132468484 & 7 & 132 \\
\hline 312 & 1979 & 34450 & 6 & 0 & 92.25 & 0.007007 & 0.005005 & 1 & 312364898 & 1 & 312829468 & 1 & 312 \\
\hline 2416 & 55471 & 48102 & 14 & 0 & 92.26 & 0.065065 & 0.023023 & 18 & 24076311 & 18 & 24990339 & 18 & 24 \\
\hline 1875 & 26464 & 32336 & 3 & 0 & 92.26 & 0.012012 & 0.005005 & 13 & 171148012 & 13 & 171444531 & 13 & 171 \\
\hline 1231 & 52585 & 47207 & 5 & 0 & 92.26 & 0.022022 & 0.016016 & 8 & 60101922 & 8 & 60981454 & 8 & 60 \\
\hline 1223 & 42277 & 59700 & 9 & 0 & 92.27 & 0.037037 & 0.013013 & 8 & 52134330 & 8 & 52765124 & 8 & 52 \\
\hline 315 & 53815 & 58893 & 2 & 0 & 92.27 & 0.014014 & 0.008008 & 1 & 315187351 & 1 & 315214500 & 1 & 315 \\
\hline 249 & 1546 & 1556 & 20 & 0 & 92.27 & 0.076076 & 0.017017 & 1 & 249012536 & 1 & 249998570 & 1 & 249 \\
\hline 1214 & 19274 & 56407 & 9 & 0 & 92.28 & 0.033033 & 0.014014 & 8 & 43068687 & 8 & 43955459 & 8 & 43 \\
\hline 171 & 46140 & 1132 & 10 & 0 & 92.28 & 0.043043 & 0.01001 & 1 & 171062342 & 1 & 171980469 & 1 & 171 \\
\hline 522 & 34570 & 395 & 7 & 0 & 92.28 & 0.025025 & 0.016016 & 3 & 44138379 & 3 & 44725638 & 3 & 44 \\
\hline 2517 & 18832 & 48182 & 6 & 0 & 92.28 & 0.01001 & 0.007007 & 19 & 66009586 & 19 & 66356168 & 19 & 66 \\
\hline 1169 & 24013 & 6753 & 7 & 0 & 92.29 & 0.024024 & 0.018018 & 7 & 133594228 & 7 & 133979586 & 7 & 133 \\
\hline 2527 & 14200 & 44951 & 7 & 0 & 92.29 & 0.017017 & 0.012012 & 19 & 76375371 & 19 & 76830183 & 19 & 76 \\
\hline 1233 & 7053 & 47208 & 3 & 0 & 92.29 & 0.016016 & 0.009009 & 8 & 62092621 & 8 & 62838673 & 8 & 62 \\
\hline
\end{tabular}




\begin{tabular}{|c|c|c|c|c|c|c|c|c|c|c|c|c|}
\hline 516 & 51371 & 15903 & 6 & 0 & 92.3 & 0.019019 & 0.006006 & 3 & 38091792 & 3 & 38949614 & 3 \\
\hline 879 & 16346 & 18574 & 9 & 0 & 92.3 & 0.025025 & 0.01001 & 6 & 79816 & 6 & 851457 & 6 \\
\hline 475 & 52115 & 18291 & 3 & 0 & 92.3 & 0.008008 & 0.006006 & 2 & 160085066 & 2 & 160434997 & 2 \\
\hline 2393 & 34828 & 15159 & 5 & 0 & 92.3 & 0.019019 & 0.01001 & 18 & 1454782 & 18 & 1943635 & 18 \\
\hline 2503 & 48168 & 54845 & 4 & 0 & 92.31 & 0.009009 & 0.007007 & 19 & 50662741 & 19 & 50904771 & 19 \\
\hline 562 & 3123 & 46501 & 3 & 0 & 92.31 & 0.007007 & 0.006006 & 3 & 84495609 & 3 & 84745984 & 3 \\
\hline 2534 & 48198 & 48200 & 7 & 0 & 92.31 & 0.007007 & 0.007007 & 19 & 85284931 & 19 & 85914898 & 19 \\
\hline 383 & 2301 & 35618 & 3 & 0 & 92.31 & 0.008008 & 0.006006 & 2 & 68894359 & 2 & 68965440 & 2 \\
\hline 2146 & 44177 & 27774 & 8 & 0 & 92.32 & 0.033033 & 0.013013 & 15 & 69276645 & 15 & 69714353 & 15 \\
\hline 2138 & 47889 & 29490 & 13 & 0 & 92.32 & 0.039039 & 0.012012 & 15 & 61018550 & 15 & 61994497 & 15 \\
\hline 1810 & 54050 & 33688 & 3 & 0 & 92.32 & 0.009009 & 0.007007 & 13 & 106051998 & 13 & 106808982 & 13 \\
\hline 151 & 50688 & 46093 & 9 & 0 & 92.33 & 0.022022 & 0.007007 & 1 & 151009336 & 1 & 151961290 & 1 \\
\hline 1430 & 7951 & 38347 & 7 & 0 & 92.33 & 0.032032 & 0.013013 & 9 & 110108860 & 9 & 110626460 & 9 \\
\hline 2512 & 14179 & 44942 & 2 & 0 & 92.33 & 0.006006 & 0.005005 & 19 & 60471958 & 19 & 60656889 & 19 \\
\hline 2530 & 32782 & 48196 & 4 & 0 & 92.33 & 0.001001 & 0.001001 & 19 & 79121304 & 19 & 79867005 & 19 \\
\hline 1827 & 38858 & 58008 & 7 & 0 & 92.33 & 0.025025 & 0.012012 & 13 & 123011640 & 13 & 123984079 & 13 \\
\hline 788 & 4712 & 4715 & 3 & 0 & 92.34 & 0.009009 & 0.008008 & 5 & 21017524 & 5 & 21547496 & 5 \\
\hline 681 & 3858 & 3861 & 6 & 0 & 92.34 & 0.008008 & 0.006006 & 4 & 58067211 & 4 & 58777597 & 4 \\
\hline 2337 & 59244 & 51284 & 13 & 0 & 92.34 & 0.046046 & 0.013013 & 17 & 15101576 & 17 & 15997261 & 17 \\
\hline 823 & 46842 & 33443 & 7 & 0 & 92.34 & 0.029029 & 0.01001 & 5 & 56054855 & 5 & 56865644 & 5 \\
\hline 2504 & 44936 & 48171 & 4 & 0 & 92.35 & 0.008008 & 0.006006 & 19 & 51624717 & 19 & 51916470 & 19 \\
\hline 2505 & 56773 & 56148 & 2 & 0 & 92.35 & 0.006006 & 0.004004 & 19 & 52288809 & 19 & 52526689 & 19 \\
\hline 2545 & 48206 & 35173 & 5 & 0 & 92.35 & 0.005005 & 0.004004 & 19 & 97152327 & 19 & 97832104 & 19 \\
\hline 1272 & 57677 & 24296 & 6 & 0 & 92.35 & 0.024024 & 0.012012 & 8 & 101158226 & 8 & 101976068 & 8 \\
\hline 2514 & 44944 & 14181 & 9 & 0 & 92.35 & 0.01001 & 0.004004 & 19 & 63109515 & 19 & 63891837 & 19 \\
\hline 370 & 2328 & 16864 & 4 & 0 & 92.36 & 0.012012 & 0.006006 & 2 & 54381571 & 2 & 54881273 & 2 \\
\hline 2152 & 12227 & 47896 & 6 & 0 & 92.36 & 0.017017 & 0.007007 & 15 & 75340693 & 15 & 75687558 & 15 \\
\hline 392 & 61052 & 14515 & 4 & 0 & 92.36 & 0.014014 & 0.006006 & 2 & 77011684 & 2 & 77635095 & 2 \\
\hline 2525 & 48190 & 55672 & 2 & 0 & 92.36 & 0.008008 & 0.006006 & 19 & 74064515 & 19 & 74704535 & 19 \\
\hline 1217 & 54877 & 58652 & 2 & 0 & 92.36 & 0.011011 & 0.006006 & 8 & 46447767 & 8 & 46706900 & 8 \\
\hline 152 & 20128 & 46098 & 11 & 0 & 92.36 & 0.034034 & 0.006006 & 1 & 152002404 & 1 & 152965216 & 1 \\
\hline 680 & 37447 & 3857 & 13 & 0 & 92.37 & 0.02002 & 0.009009 & 4 & 57099646 & 4 & 57935123 & 4 \\
\hline 372 & 60890 & 32196 & 3 & 0 & 92.37 & 0.021021 & 0.007007 & 2 & 56469735 & 2 & 56784798 & 2 \\
\hline 2592 & 33256 & 50059 & 3 & 0 & 92.37 & 0.008008 & 0.006006 & 19 & 144033871 & 19 & 144187103 & 19 \\
\hline 679 & 3849 & 46629 & 10 & 0 & 92.37 & 0.027027 & 0.005005 & 4 & 56000161 & 4 & 56894950 & 4 \\
\hline 678 & 3842 & 46626 & 16 & 0 & 92.37 & 0.035035 & 0.01001 & 4 & 55031488 & 4 & 55960525 & 4 \\
\hline 2523 & 14198 & 44949 & 4 & 0 & 92.37 & 0.007007 & 0.005005 & 19 & 72098978 & 19 & 72845131 & 19 \\
\hline 2544 & 60857 & 60857 & 1 & 0 & 92.38 & 0.009009 & 0.005005 & 19 & 96090467 & 19 & 96090467 & 19 \\
\hline 523 & 34532 & 46478 & 7 & 0 & 92.38 & 0.015015 & 0.011011 & 3 & 45453887 & 3 & 45994096 & 3 \\
\hline
\end{tabular}




\begin{tabular}{|c|c|c|c|c|c|c|c|c|c|c|c|c|c|}
\hline 2143 & 51748 & 50564 & 7 & 0 & 92.38 & 0.018018 & 0.011011 & 15 & 66122450 & 15 & 66907405 & 15 & 66 \\
\hline 2541 & 34567 & 58352 & 5 & 0 & 92.38 & 0.007007 & 0.004004 & 19 & 93230002 & 19 & 93517538 & 19 & 93 \\
\hline 180 & 61791 & 1180 & 13 & 0 & 92.38 & 0.03003 & 0.012012 & 1 & 180068581 & 1 & 180980648 & 1 & 180 \\
\hline 1220 & 47199 & 15356 & 4 & 0 & 92.38 & 0.012012 & 0.004004 & 8 & 49000633 & 8 & 49881116 & 8 & 49 \\
\hline 2582 & 14328 & 45012 & 8 & 0 & 92.39 & 0.011011 & 0.006006 & 19 & 134009220 & 19 & 134779719 & 19 & 134 \\
\hline 1424 & 24834 & 54969 & 3 & 0 & 92.39 & 0.013013 & 0.008008 & 9 & 104255261 & 9 & 104996366 & 9 & 104 \\
\hline 1224 & 58235 & 58859 & 2 & 0 & 92.39 & 0.007007 & 0.004004 & 8 & 53555323 & 8 & 53929233 & 8 & 53 \\
\hline 2516 & 48179 & 44946 & 4 & 0 & 92.39 & 0.002002 & 0.002002 & 19 & 65733803 & 19 & 65997012 & 19 & 65 \\
\hline 2500 & 14169 & 35127 & 7 & 0 & 92.39 & 0.013013 & 0.007007 & 19 & 47148219 & 19 & 47592822 & 19 & 47 \\
\hline 2538 & 51627 & 18556 & 5 & 0 & 92.39 & 0.009009 & 0.006006 & 19 & 90038588 & 19 & 90967583 & 19 & 90 \\
\hline 2521 & 14193 & 48186 & 3 & 0 & 92.39 & 0.005005 & 0.004004 & 19 & 70337525 & 19 & 70961131 & 19 & 70 \\
\hline 2391 & 17473 & 48084 & 5 & 0 & 92.39 & 0.017017 & 0.007007 & 17 & 69061472 & 17 & 69344035 & 17 & 69 \\
\hline 2513 & 29137 & 14187 & 8 & 0 & 92.39 & 0.009009 & 0.004004 & 19 & 62086511 & 19 & 62675407 & 19 & 62 \\
\hline 2550 & 59344 & 48219 & 6 & 0 & 92.4 & 0.022022 & 0.006006 & 19 & 102082896 & 19 & 102773567 & 19 & 102 \\
\hline 1590 & 47350 & 8837 & 3 & 0 & 92.4 & 0.01001 & 0.005005 & 11 & 38785701 & 11 & 38996126 & 11 & 38 \\
\hline 2543 & 18223 & 18223 & 1 & 0 & 92.4 & 0.006006 & 0.003003 & 19 & 95141795 & 19 & 95141795 & 19 & 95 \\
\hline 1215 & 7007 & 57562 & 2 & 0 & 92.4 & 0.009009 & 0.007007 & 8 & 44927836 & 8 & 44940909 & 8 & 44 \\
\hline 314 & 56225 & 60162 & 2 & 0 & 92.4 & 0.004004 & 0.004004 & 1 & 314046273 & 1 & 314869351 & 1 & 314 \\
\hline 1832 & 47501 & 55112 & 2 & 0 & 92.4 & 0.008008 & 0.004004 & 13 & 128100425 & 13 & 128280037 & 13 & 128 \\
\hline 2522 & 14195 & 14197 & 5 & 0 & 92.4 & 0.003003 & 0.002002 & 19 & 71022228 & 19 & 71708533 & 19 & 71 \\
\hline 2506 & 35161 & 39613 & 5 & 0 & 92.4 & 0.011011 & 0.005005 & 19 & 53047552 & 19 & 53798124 & 19 & 53 \\
\hline 2536 & 44954 & 14210 & 4 & 0 & 92.4 & 0.005005 & 0.003003 & 19 & 87347232 & 19 & 87901203 & 19 & 87 \\
\hline 2158 & 12241 & 12243 & 5 & 0 & 92.4 & 0.014014 & 0.003003 & 15 & 81428860 & 15 & 81992999 & 15 & 81 \\
\hline 2549 & 35143 & 14220 & 6 & 0 & 92.4 & 0.007007 & 0.002002 & 19 & 101068864 & 19 & 101736276 & 19 & 101 \\
\hline 2542 & 50082 & 50082 & 1 & 0 & 92.4 & 0.005005 & 0.003003 & 19 & 94468874 & 19 & 94468874 & 19 & 94 \\
\hline 2480 & 39602 & 29083 & 7 & 0 & 92.4 & 0.032032 & 0.001001 & 19 & 27034249 & 19 & 27962279 & 19 & 27 \\
\hline 2232 & 33451 & 51228 & 4 & 0 & 92.4 & 0.018018 & 0.003003 & 15 & 155667846 & 15 & 155782508 & 15 & 155 \\
\hline 476 & 55507 & 55507 & 1 & 0 & 92.41 & 0.009009 & 0.004004 & 2 & 161880071 & 2 & 161880071 & 2 & 161 \\
\hline 2568 & 14284 & 34641 & 3 & 0 & 92.41 & 0.006006 & 0.003003 & 19 & 120411731 & 19 & 120937826 & 19 & 120 \\
\hline 911 & 5337 & 32684 & 4 & 0 & 92.41 & 0.011011 & 0.001001 & 6 & 32034710 & 6 & 32835598 & 6 & 32 \\
\hline 2566 & 44980 & 48236 & 3 & 0 & 92.41 & 0.008008 & 0.003003 & 19 & 118208682 & 19 & 118515509 & 19 & 118 \\
\hline 1219 & 18326 & 53725 & 2 & 0 & 92.41 & 0.006006 & 0.002002 & 8 & 48642685 & 8 & 48649970 & 8 & 48 \\
\hline 2548 & 48213 & 14217 & 9 & 0 & 92.41 & 0.005005 & 0.001001 & 19 & 100022640 & 19 & 100911159 & 19 & 100 \\
\hline 2528 & 14201 & 48193 & 2 & 0 & 92.41 & 0.004004 & 0.001001 & 19 & 77046979 & 19 & 77333713 & 19 & 77 \\
\hline 2540 & 48205 & 14212 & 2 & 0 & 92.41 & 0.004004 & 0.003003 & 19 & 92108045 & 19 & 92882230 & 19 & 92 \\
\hline 520 & 15813 & 2940 & 2 & 0 & 92.41 & 0.005005 & 0.001001 & 3 & 42047929 & 3 & 42096113 & 3 & 42 \\
\hline 2546 & 44955 & 44955 & 1 & 0 & 92.41 & 0.005005 & 0.002002 & 19 & 98978944 & 19 & 98978944 & 19 & 98 \\
\hline 2485 & 14124 & 52161 & 3 & 0 & 92.41 & 0.004004 & 0.001001 & 19 & 32076524 & 19 & 32496364 & 19 & 32 \\
\hline 388 & 51705 & 53572 & 2 & 0 & 92.41 & 0.012012 & 0.001001 & 2 & 73076347 & 2 & 73385525 & 2 & 73 \\
\hline
\end{tabular}




\begin{tabular}{|c|c|c|c|c|c|c|c|c|c|c|c|c|c|}
\hline 1429 & 7948 & 47286 & 3 & 0 & 92.41 & 0.007007 & 0.002002 & 9 & 109096308 & 9 & 109683264 & 9 & 109 \\
\hline 2518 & 44948 & 29140 & 4 & 0 & 92.41 & 0.006006 & 0.001001 & 19 & 67110552 & 19 & 67818695 & 19 & 67 \\
\hline 2532 & 33245 & 33245 & 1 & 0 & 92.41 & 0.004004 & 0.001001 & 19 & 83670009 & 19 & 83670009 & 19 & 83 \\
\hline 2515 & 57187 & 29138 & 4 & 0 & 92.41 & 0.006006 & 0 & 19 & 64571859 & 19 & 64882657 & 19 & 64 \\
\hline 394 & 56802 & 58682 & 2 & 0 & 92.41 & 0.004004 & 0 & 2 & 79296283 & 2 & 79772013 & 2 & 79 \\
\hline 235 & 55965 & 20360 & 2 & 0 & 92.41 & 0.004004 & 0 & 1 & 235179193 & 1 & 235988757 & 1 & 235 \\
\hline 1121 & 47109 & 47109 & 1 & 0 & 92.41 & 0.005005 & 0 & 7 & 85445625 & 7 & 85445625 & 7 & 85 \\
\hline 2531 & 14204 & 49788 & 3 & 0 & 92.41 & 0.006006 & 0 & 19 & 80145695 & 19 & 80969428 & 19 & 80 \\
\hline 217 & 46241 & 54097 & 5 & 0 & 92.41 & 0.005005 & 0 & 1 & 217060222 & 1 & 217544827 & 1 & 217 \\
\hline 2539 & 14213 & 58883 & 2 & 0 & 92.41 & 0 & 0 & 19 & 91102005 & 19 & 91344424 & 19 & 91 \\
\hline 393 & 51163 & 51163 & 1 & 0 & 92.41 & 0 & 0 & 2 & 78174324 & 2 & 78174324 & 2 & 78 \\
\hline 2507 & 30650 & 30650 & 1 & 0 & 92.41 & 0 & 0 & 19 & 55073511 & 19 & 55073511 & 19 & 55 \\
\hline 2547 & 48208 & 48212 & 7 & 0 & 92.41 & 0 & 0 & 19 & 99091294 & 19 & 99836761 & 19 & 99 \\
\hline 2508 & 48172 & 44939 & 4 & 0 & 92.41 & 0 & 0 & 19 & 56659659 & 19 & 56808512 & 19 & 56 \\
\hline 2510 & 43884 & 44941 & 3 & 0 & 92.41 & 0 & 0 & 19 & 58094394 & 19 & 58911107 & 19 & 58 \\
\hline 2511 & 48173 & 14180 & 3 & 0 & 92.41 & 0 & 0 & 19 & 59339614 & 19 & 59411403 & 19 & 59 \\
\hline 2537 & 53069 & 58669 & 2 & 0 & 92.41 & 0 & 0 & 19 & 89259122 & 19 & 89918982 & 19 & 89 \\
\hline 2519 & 48185 & 61984 & 2 & 0 & 92.41 & 0 & 0 & 19 & 68685785 & 19 & 68863251 & 19 & 68 \\
\hline 2520 & 35034 & 37477 & 2 & 0 & 92.41 & 0 & 0 & 19 & 69322160 & 19 & 69589470 & 19 & 69 \\
\hline 2526 & 48298 & 34562 & 3 & 0 & 92.41 & 0 & 0 & 19 & 75081784 & 19 & 75703473 & 19 & 75 \\
\hline 2533 & 34565 & 48197 & 2 & 0 & 92.41 & 0 & 0 & 19 & 84602688 & 19 & 84914270 & 19 & 84 \\
\hline 2535 & 48201 & 34566 & 2 & 0 & 92.41 & 0 & 0 & 19 & 86201492 & 19 & 86254418 & 19 & 86 \\
\hline
\end{tabular}


Table S3: Regions with major clusters of SNPs associated with the largest effects on puberty onset.

\begin{tabular}{|c|c|c|c|c|}
\hline $\mathrm{SSC}$ & $5^{\prime}(b p)$ & 3' (bp) & $\begin{array}{c}\text { \# SNPs in top } 0.05 \% \\
(n=30)\end{array}$ & $\begin{array}{c}\text { \# SNPs in top } 0.5 \% \\
(n=300)\end{array}$ \\
\hline 1 & 25040176 & 25857749 & 0 & 4 \\
\hline 1 & 93750500 & 94228789 & 3 & 1 \\
\hline 1 & 285639839 & 288036346 & 0 & 12 \\
\hline 2 & 58607333 & 61161245 & 0 & 8 \\
\hline 2 & 87007147 & 87163628 & 2 & 3 \\
\hline 3 & 71708951 & 72344362 & 1 & 5 \\
\hline 4 & 104068028 & 104450564 & 0 & 4 \\
\hline 4 & 133429382 & 135045478 & 1 & 3 \\
\hline 5 & 30095581 & 31263199 & 0 & 6 \\
\hline 6 & 114372598 & 115802235 & 1 & 5 \\
\hline 6 & 143711252 & 144547947 & 2 & 2 \\
\hline 7 & 39512713 & 39709854 & 0 & 4 \\
\hline 8 & 35912308 & 37746259 & 2 & 8 \\
\hline 9 & 139207082 & 141784134 & 1 & 4 \\
\hline 12 & 940547 & 2621917 & 6 & 10 \\
\hline 13 & 117228983 & 118012606 & 2 & 1 \\
\hline 13 & 142153651 & 142431689 & 0 & 4 \\
\hline 14 & 66172017 & 68856362 & 1 & 9 \\
\hline
\end{tabular}


Table S4. Genetic variance of lifetime number of parities explained by $1 \mathrm{Mb}$ windows

\begin{tabular}{|c|c|c|c|c|c|c|c|c|c|c|c|c|c|c|c|c|}
\hline Window & start & end & \#SNPs & $\% \operatorname{Var}$ & Cum\%Var & $p>0$ & $p>$ Average & map & pos0 & map & posn & chr & $\mathrm{Mb}$ & var(ghat) & corr & regr \\
\hline 0 & 55418 & 36467 & 15 & 0.01 & 93.97 & 0.062062 & 0.044044 & 1 & 286933 & 1 & 985857 & 1 & 0 & 0.055 & 0.7085 & 4.7 \\
\hline 1 & 39749 & 17 & 23 & 0.04 & 57 & 0.108108 & 0.074074 & 1 & 1049272 & 1 & 1958514 & 1 & 1 & 0.031 & 0.6921 & 6.1 \\
\hline 2 & 19451 & 39756 & 29 & 0.06 & 38.93 & 0.136136 & 0.094094 & 1 & 2010349 & 1 & 2995480 & 1 & 2 & 0.021 & 0.6679 & 7.24 \\
\hline 3 & 50179 & 24 & 34 & 0.04 & 55.54 & 0.143143 & 0.101101 & 1 & 3008501 & 1 & 3977158 & 1 & 3 & 0.03 & 0.6919 & 6.18 \\
\hline 4 & 20 & 48373 & 30 & 0.04 & 64.93 & 0.118118 & 0.074074 & 1 & 4022389 & 1 & 4979417 & 1 & 4 & 0.036 & 0.6981 & 5.74 \\
\hline 5 & 62015 & 40 & 18 & 0.03 & 76.34 & 0.094094 & 0.058058 & 1 & 5008585 & 1 & 5732081 & 1 & 5 & 0.043 & 0.703 & 5.28 \\
\hline 6 & 41 & 15913 & 32 & 0.06 & 36.12 & 0.15015 & 0.099099 & 1 & 6010365 & 1 & 6986608 & 1 & 6 & 0.019 & 0.6595 & 7.4 \\
\hline 8 & 19474 & 19481 & 32 & 0.05 & 44.17 & 0.135135 & 0.08008 & 1 & 8004890 & 1 & 8988180 & 1 & 8 & 0.023 & 0.6785 & 6.9 \\
\hline 9 & 19480 & 75 & 31 & 0.04 & 61.13 & 0.141141 & 0.097097 & 1 & 9007321 & 1 & 9984842 & 1 & 9 & 0.034 & 0.6963 & 5.92 \\
\hline 10 & 58158 & 61852 & 32 & 0.04 & 54.63 & 0.143143 & 0.098098 & 1 & 10010333 & 1 & 10949247 & 1 & 10 & 0.03 & 0.6916 & 6.25 \\
\hline 11 & 45674 & 39779 & 30 & 0.05 & 46.84 & 0.145145 & 0.102102 & 1 & 11012998 & 1 & 11945736 & 1 & 11 & 0.025 & 0.682 & 6.69 \\
\hline 12 & 88 & 36478 & 29 & 0.04 & 59.28 & 0.141141 & 0.079079 & 1 & 12024877 & 1 & 12946299 & 1 & 12 & 0.033 & 0.6944 & 5.99 \\
\hline 13 & 39783 & 19509 & 32 & 0.05 & 44.91 & 0.117117 & 0.079079 & 1 & 13005744 & 1 & 13972300 & 1 & 13 & 0.024 & 0.6797 & 6.84 \\
\hline 14 & 51106 & 114 & 28 & 0.04 & 64.55 & 0.124124 & 0.084084 & 1 & 14038761 & 1 & 14874949 & 1 & 14 & 0.036 & 0.6977 & 5.75 \\
\hline 16 & 48394 & 18555 & 28 & 0.04 & 53.61 & 0.13013 & 0.081081 & 1 & 16038746 & 1 & 16870187 & 1 & 16 & 0.029 & 0.692 & 6.33 \\
\hline 17 & 53439 & 146 & 42 & 0.09 & 23.68 & 0.207207 & 0.151151 & 1 & 17004757 & 1 & 17991862 & 1 & 17 & 0.012 & 0.623 & 8.76 \\
\hline 18 & 45854 & 39807 & 23 & 0.03 & 75.78 & 0.095095 & 0.065065 & 1 & 18020929 & 1 & 18995163 & 1 & 18 & 0.043 & 0.7029 & 5.3 \\
\hline 19 & 48399 & 19566 & 28 & 0.04 & 50.88 & 0.14014 & 0.096096 & 1 & 19017181 & 1 & 19992800 & 1 & 19 & 0.027 & 0.6875 & 6.46 \\
\hline 20 & 162 & 39815 & 27 & 0.04 & 50.93 & 0.111111 & 0.071071 & 1 & 20009618 & 1 & 20996770 & 1 & 20 & 0.027 & 0.6877 & 6.46 \\
\hline 21 & 59958 & 19577 & 21 & 0.03 & 67.28 & 0.1001 & 0.067067 & 1 & 21017894 & 1 & 21899292 & 1 & 21 & 0.037 & 0.6993 & 5.64 \\
\hline 22 & 19578 & 19127 & 26 & 0.03 & 74.3 & 0.11011 & 0.07007 & 1 & 22042965 & 1 & 22993058 & 1 & 22 & 0.042 & 0.7019 & 5.34 \\
\hline 23 & 45415 & 183 & 21 & 0.02 & 87.69 & 0.104104 & 0.062062 & 1 & 23041590 & 1 & 23952116 & 1 & 23 & 0.051 & 0.7077 & 4.9 \\
\hline 24 & 50659 & 197 & 33 & 0.04 & 54.04 & 0.137137 & 0.085085 & 1 & 24006028 & 1 & 24953606 & 1 & 24 & 0.029 & 0.6914 & 6.29 \\
\hline 25 & 52428 & 36511 & 19 & 0.03 & 77.28 & 0.088088 & 0.058058 & 1 & 25010377 & 1 & 25927201 & 1 & 25 & 0.044 & 0.7035 & 5.24 \\
\hline 26 & 205 & 53569 & 24 & 0.07 & 27.33 & 0.132132 & 0.085085 & 1 & 26091832 & 1 & 26995287 & 1 & 26 & 0.014 & 0.6343 & 8.22 \\
\hline 27 & 36519 & 214 & 19 & 0.05 & 41.74 & 0.094094 & 0.055055 & 1 & 27020977 & 1 & 27899646 & 1 & 27 & 0.022 & 0.6756 & 7.1 \\
\hline 28 & 215 & 221 & 27 & 0.03 & 77.33 & 0.111111 & 0.056056 & 1 & 28050709 & 1 & 28899322 & 1 & 28 & 0.044 & 0.7035 & 5.24 \\
\hline 29 & 224 & 227 & 31 & 0.03 & 79.76 & 0.129129 & 0.077077 & 1 & 29026999 & 1 & 29949287 & 1 & 29 & 0.045 & 0.7048 & 5.16 \\
\hline 30 & 19613 & 31537 & 35 & 0.04 & 56.96 & 0.145145 & 0.112112 & 1 & 30017551 & 1 & 30978281 & 1 & 30 & 0.031 & 0.6918 & 6.09 \\
\hline 33 & 19636 & 36543 & 35 & 0.1 & 21.4 & 0.151151 & 0.106106 & 1 & 33008187 & 1 & 33957887 & 1 & 33 & 0.011 & 0.61 & 9.02 \\
\hline 34 & 275 & 39857 & 39 & 0.13 & 17.26 & 0.176176 & 0.118118 & 1 & 34001467 & 1 & 34993536 & 1 & 34 & 0.009 & 0.5872 & 9.68 \\
\hline 35 & 39858 & 54559 & 27 & 0.08 & 26.03 & 0.142142 & 0.106106 & 1 & 35046144 & 1 & 35987086 & 1 & 35 & 0.014 & 0.6295 & 8.35 \\
\hline 36 & 39862 & 308 & 38 & 0.07 & 28.72 & 0.184184 & 0.115115 & 1 & 36021536 & 1 & 36976294 & 1 & 36 & 0.015 & 0.6382 & 8.04 \\
\hline
\end{tabular}




\begin{tabular}{|c|c|c|c|c|c|c|c|c|c|c|c|c|c|c|c|c|}
\hline 37 & 39868 & 45877 & 20 & 0.02 & 87.03 & 0.085085 & 0.046046 & 1 & 37000384 & 1 & 37964303 & 1 & 37 & 0.05 & 0.7076 & 4.93 \\
\hline 38 & 36560 & 322 & 32 & 0.05 & 48.85 & 0.145145 & 0.101101 & 1 & 38044337 & 1 & 38953492 & 1 & 38 & 0.027 & 0.6838 & 6.54 \\
\hline 39 & 55819 & 328 & 18 & 0.05 & 46.89 & 0.079079 & 0.063063 & 1 & 39019040 & 1 & 39987321 & 1 & 39 & 0.025 & 0.6821 & 6.68 \\
\hline 40 & 39878 & 61731 & 23 & 0.05 & 47.64 & 0.111111 & 0.08008 & 1 & 40017655 & 1 & 40974578 & 1 & 40 & 0.026 & 0.6822 & 6.61 \\
\hline 41 & 334 & 36576 & 25 & 0.06 & 31.91 & 0.109109 & 0.067067 & 1 & 41017553 & 1 & 41894961 & 1 & 41 & 0.017 & 0.6474 & 7.75 \\
\hline 42 & 61252 & 348 & 20 & 0.03 & 75.98 & 0.083083 & 0.053053 & 1 & 42076935 & 1 & 42964863 & 1 & 42 & 0.043 & 0.7029 & 5.29 \\
\hline 43 & 349 & 357 & 26 & 0.06 & 34.06 & 0.111111 & 0.083083 & 1 & 43045248 & 1 & 43986463 & 1 & 43 & 0.018 & 0.6541 & 7.58 \\
\hline 44 & 61816 & 58745 & 18 & 0.02 & 87.51 & 0.072072 & 0.046046 & 1 & 44013987 & 1 & 44938878 & 1 & 44 & 0.05 & 0.7075 & 4.91 \\
\hline 45 & 19704 & 36591 & 29 & 0.05 & 46.41 & 0.124124 & 0.085085 & 1 & 45116274 & 1 & 45997932 & 1 & 45 & 0.025 & 0.6815 & 6.72 \\
\hline 46 & 36592 & 33731 & 11 & 0.03 & 68.15 & 0.057057 & 0.039039 & 1 & 46028574 & 1 & 46907309 & 1 & 46 & 0.038 & 0.6993 & 5.59 \\
\hline 47 & 18111 & 45887 & 21 & 0.04 & 62.36 & 0.098098 & 0.064064 & 1 & 47165930 & 1 & 47997868 & 1 & 47 & 0.034 & 0.6963 & 5.86 \\
\hline 48 & 374 & 19715 & 22 & 0.03 & 68.25 & 0.095095 & 0.06006 & 1 & 48013733 & 1 & 48925115 & 1 & 48 & 0.038 & 0.6991 & 5.59 \\
\hline 49 & 19716 & 392 & 31 & 0.05 & 42.2 & 0.124124 & 0.089089 & 1 & 49026306 & 1 & 49988544 & 1 & 49 & 0.022 & 0.6759 & 7.06 \\
\hline 50 & 19725 & 53398 & 23 & 0.03 & 72.38 & 0.078078 & 0.047047 & 1 & 50015447 & 1 & 50999817 & 1 & 50 & 0.041 & 0.701 & 5.42 \\
\hline 51 & 36604 & 36610 & 21 & 0.03 & 78.43 & 0.112112 & 0.068068 & 1 & 51037015 & 1 & 51998764 & 1 & 51 & 0.044 & 0.7041 & 5.21 \\
\hline 52 & 45898 & 19734 & 24 & 0.03 & 79.19 & 0.102102 & 0.065065 & 1 & 52062069 & 1 & 52999008 & 1 & 52 & 0.045 & 0.7046 & 5.19 \\
\hline 53 & 419 & 36621 & 25 & 0.04 & 60.72 & 0.111111 & 0.076076 & 1 & 53023914 & 1 & 53978051 & 1 & 53 & 0.033 & 0.6958 & 5.94 \\
\hline 54 & 432 & 45902 & 18 & 0.03 & 79.04 & 0.075075 & 0.049049 & 1 & 54001826 & 1 & 54991599 & 1 & 54 & 0.045 & 0.7045 & 5.19 \\
\hline 55 & 439 & 19744 & 28 & 0.04 & 55.13 & 0.128128 & 0.077077 & 1 & 55012053 & 1 & 55965347 & 1 & 55 & 0.03 & 0.6919 & 6.21 \\
\hline 56 & 60375 & 16290 & 28 & 0.04 & 53.95 & 0.128128 & 0.08008 & 1 & 56004411 & 1 & 56836525 & 1 & 56 & 0.029 & 0.6913 & 6.3 \\
\hline 57 & 53965 & 39908 & 29 & 0.07 & 30.12 & 0.151151 & 0.1001 & 1 & 57012775 & 1 & 57981015 & 1 & 57 & 0.016 & 0.6421 & 7.88 \\
\hline 58 & 36632 & 39914 & 34 & 0.1 & 20.71 & 0.172172 & 0.132132 & 1 & 58006061 & 1 & 58990256 & 1 & 58 & 0.011 & 0.6049 & 9.09 \\
\hline 59 & 45910 & 39917 & 20 & 0.08 & 25.64 & 0.106106 & 0.077077 & 1 & 59033524 & 1 & 59964653 & 1 & 59 & 0.013 & 0.6286 & 8.43 \\
\hline 60 & 36637 & 19773 & 28 & 0.06 & 34.67 & 0.119119 & 0.073073 & 1 & 60022622 & 1 & 60987629 & 1 & 60 & 0.018 & 0.6552 & 7.52 \\
\hline 61 & 39921 & 497 & 25 & 0.1 & 21.69 & 0.158158 & 0.121121 & 1 & 61002086 & 1 & 61949887 & 1 & 61 & 0.011 & 0.6111 & 8.97 \\
\hline 62 & 56204 & 508 & 29 & 0.05 & 42.61 & 0.137137 & 0.091091 & 1 & 62058511 & 1 & 62989866 & 1 & 62 & 0.023 & 0.6759 & 7.01 \\
\hline 63 & 19788 & 34371 & 29 & 0.05 & 40.41 & 0.13013 & 0.099099 & 1 & 63025137 & 1 & 63991546 & 1 & 63 & 0.021 & 0.6714 & 7.15 \\
\hline 64 & 19796 & 45919 & 24 & 0.03 & 78.14 & 0.111111 & 0.064064 & 1 & 64018444 & 1 & 64959341 & 1 & 64 & 0.044 & 0.7041 & 5.22 \\
\hline 65 & 50270 & 36650 & 33 & 0.03 & 69.37 & 0.138138 & 0.08008 & 1 & 65008123 & 1 & 65994430 & 1 & 65 & 0.039 & 0.6996 & 5.54 \\
\hline 66 & 19814 & 53423 & 26 & 0.04 & 63.62 & 0.106106 & 0.069069 & 1 & 66015914 & 1 & 66820206 & 1 & 66 & 0.035 & 0.6972 & 5.8 \\
\hline 67 & 36653 & 32143 & 24 & 0.03 & 69.21 & 0.109109 & 0.07007 & 1 & 67050038 & 1 & 67951385 & 1 & 67 & 0.039 & 0.6997 & 5.55 \\
\hline 68 & 19824 & 45924 & 14 & 0.02 & 88.99 & 0.067067 & 0.034034 & 1 & 68132380 & 1 & 68993279 & 1 & 68 & 0.051 & 0.7076 & 4.86 \\
\hline 69 & 549 & 553 & 22 & 0.03 & 73.5 & 0.105105 & 0.074074 & 1 & 69039001 & 1 & 69968051 & 1 & 69 & 0.041 & 0.7019 & 5.37 \\
\hline 70 & 554 & 19836 & 24 & 0.02 & 87.89 & 0.107107 & 0.054054 & 1 & 70002082 & 1 & 70950692 & 1 & 70 & 0.051 & 0.7078 & 4.9 \\
\hline 71 & 32242 & 19839 & 21 & 0.03 & 69.27 & 0.1001 & 0.074074 & 1 & 71065994 & 1 & 71950726 & 1 & 71 & 0.039 & 0.6996 & 5.55 \\
\hline 72 & 19840 & 574 & 27 & 0.02 & 84.71 & 0.119119 & 0.065065 & 1 & 72028362 & 1 & 72982828 & 1 & 72 & 0.049 & 0.7056 & 4.98 \\
\hline 73 & 575 & 578 & 10 & 0.01 & 92.85 & 0.049049 & 0.023023 & 1 & 73100657 & 1 & 73922223 & 1 & 73 & 0.054 & 0.7081 & 4.74 \\
\hline 74 & 36678 & 19858 & 25 & 0.04 & 64.4 & 0.101101 & 0.063063 & 1 & 74019662 & 1 & 74974844 & 1 & 74 & 0.036 & 0.6977 & 5.76 \\
\hline 75 & 591 & 19861 & 26 & 0.03 & 71.07 & 0.099099 & 0.07007 & 1 & 75002664 & 1 & 75994076 & 1 & 75 & 0.04 & 0.7005 & 5.47 \\
\hline
\end{tabular}




\begin{tabular}{|c|c|c|c|c|c|c|c|c|c|c|c|c|c|c|c|c|}
\hline 76 & 45935 & 36682 & 26 & 0.02 & 86.71 & 0.112112 & 0.07007 & 1 & 76030269 & 1 & 76975200 & 1 & 76 & 0.05 & 0.7073 & 4.93 \\
\hline 77 & 29511 & 36684 & 23 & 0.01 & 92.19 & 0.104104 & 0.052052 & 1 & 77011485 & 1 & 77999034 & 1 & 77 & 0.054 & 0.7081 & 4.76 \\
\hline 78 & 621 & 18214 & 20 & 0.02 & 87.63 & 0.068068 & 0.043043 & 1 & 78021030 & 1 & 78960119 & 1 & 78 & 0.051 & 0.7078 & 4.91 \\
\hline 79 & 626 & 33118 & 13 & 0.01 & 95.15 & 0.044044 & 0.025025 & 1 & 79048821 & 1 & 79931781 & 1 & 79 & 0.056 & 0.7087 & 4.67 \\
\hline 80 & 58855 & 19881 & 25 & 0.03 & 71.39 & 0.094094 & 0.047047 & 1 & 80057576 & 1 & 80997487 & 1 & 80 & 0.04 & 0.7008 & 5.46 \\
\hline 81 & 53680 & 52031 & 23 & 0.03 & 78.51 & 0.087087 & 0.059059 & 1 & 81036664 & 1 & 81998047 & 1 & 81 & 0.044 & 0.7041 & 5.21 \\
\hline 82 & 50124 & 652 & 23 & 0.02 & 81.4 & 0.095095 & 0.064064 & 1 & 82050327 & 1 & 82964894 & 1 & 82 & 0.046 & 0.7052 & 5.11 \\
\hline 83 & 53174 & 45952 & 23 & 0.04 & 58.66 & 0.095095 & 0.061061 & 1 & 83022518 & 1 & 83976962 & 1 & 83 & 0.032 & 0.6933 & 6.02 \\
\hline 84 & 19900 & 19181 & 33 & 0.06 & 34.55 & 0.155155 & 0.108108 & 1 & 84007856 & 1 & 84960922 & 1 & 84 & 0.018 & 0.6564 & 7.57 \\
\hline 85 & 58030 & 667 & 21 & 0.03 & 69.76 & 0.104104 & 0.065065 & 1 & 85011343 & 1 & 85975562 & 1 & 85 & 0.039 & 0.6999 & 5.53 \\
\hline 86 & 51445 & 19912 & 18 & 0.02 & 81.6 & 0.084084 & 0.056056 & 1 & 86014836 & 1 & 86991865 & 1 & 86 & 0.046 & 0.7052 & 5.1 \\
\hline 87 & 36699 & 39967 & 21 & 0.03 & 76.06 & 0.085085 & 0.058058 & 1 & 87012148 & 1 & 87852879 & 1 & 87 & 0.043 & 0.7028 & 5.29 \\
\hline 88 & 45956 & 33178 & 14 & 0.01 & 93.71 & 0.059059 & 0.031031 & 1 & 88055468 & 1 & 88863147 & 1 & 88 & 0.055 & 0.7082 & 4.71 \\
\hline 89 & 56625 & 51012 & 13 & 0.01 & 92.37 & 0.06006 & 0.028028 & 1 & 89210836 & 1 & 89896098 & 1 & 89 & 0.054 & 0.7082 & 4.76 \\
\hline 90 & 45959 & 697 & 19 & 0.04 & 64.12 & 0.085085 & 0.052052 & 1 & 90085632 & 1 & 90991697 & 1 & 90 & 0.035 & 0.6975 & 5.77 \\
\hline 91 & 19926 & 36128 & 28 & 0.08 & 27.18 & 0.139139 & 0.108108 & 1 & 91015459 & 1 & 91983106 & 1 & 91 & 0.014 & 0.633 & 8.21 \\
\hline 92 & 35188 & 710 & 24 & 0.05 & 42.82 & 0.118118 & 0.067067 & 1 & 92004937 & 1 & 92967007 & 1 & 92 & 0.023 & 0.6766 & 7.01 \\
\hline 93 & 50671 & 19939 & 23 & 0.03 & 66.32 & 0.1001 & 0.067067 & 1 & 93019315 & 1 & 93750500 & 1 & 93 & 0.037 & 0.6989 & 5.68 \\
\hline 94 & 45969 & 723 & 15 & 0.03 & 72.04 & 0.059059 & 0.041041 & 1 & 94180981 & 1 & 94935963 & 1 & 94 & 0.04 & 0.7011 & 5.44 \\
\hline 95 & 724 & 19946 & 19 & 0.03 & 76.4 & 0.085085 & 0.053053 & 1 & 95010340 & 1 & 95995816 & 1 & 95 & 0.043 & 0.7028 & 5.27 \\
\hline 96 & 729 & 54275 & 24 & 0.08 & 24.35 & 0.132132 & 0.099099 & 1 & 96015876 & 1 & 96971456 & 1 & 96 & 0.013 & 0.6253 & 8.65 \\
\hline 97 & 39983 & 39986 & 25 & 0.04 & 62.13 & 0.14014 & 0.086086 & 1 & 97039899 & 1 & 97978461 & 1 & 97 & 0.034 & 0.6962 & 5.87 \\
\hline 98 & 45980 & 752 & 17 & 0.02 & 82.69 & 0.075075 & 0.055055 & 1 & 98048248 & 1 & 98992614 & 1 & 98 & 0.047 & 0.7046 & 5.04 \\
\hline 99 & 45981 & 760 & 22 & 0.08 & 24.76 & 0.117117 & 0.079079 & 1 & 99051689 & 1 & 99989884 & 1 & 99 & 0.013 & 0.6263 & 8.57 \\
\hline 100 & 19961 & 766 & 23 & 0.14 & 15.5 & 0.139139 & 0.102102 & 1 & 100021690 & 1 & 100995739 & 1 & 100 & 0.008 & 0.5783 & 10.12 \\
\hline 101 & 767 & 45985 & 26 & 0.06 & 39.32 & 0.111111 & 0.08008 & 1 & 101018800 & 1 & 101977893 & 1 & 101 & 0.021 & 0.6682 & 7.2 \\
\hline 102 & 39994 & 19971 & 17 & 0.03 & 72.2 & 0.081081 & 0.058058 & 1 & 102071378 & 1 & 102718610 & 1 & 102 & 0.04 & 0.7011 & 5.43 \\
\hline 103 & 783 & 789 & 16 & 0.03 & 79.45 & 0.065065 & 0.044044 & 1 & 103024987 & 1 & 103980217 & 1 & 103 & 0.045 & 0.705 & 5.18 \\
\hline 104 & 54519 & 55902 & 22 & 0.08 & 24.1 & 0.101101 & 0.071071 & 1 & 104002361 & 1 & 104937362 & 1 & 104 & 0.013 & 0.6257 & 8.71 \\
\hline 105 & 50887 & 806 & 31 & 0.88 & 5.5 & 0.26026 & 0.221221 & 1 & 105018683 & 1 & 105985626 & 1 & 105 & 0.003 & 0.4766 & 12.63 \\
\hline 106 & 3869 & 45988 & 19 & 0.06 & 34.61 & 0.097097 & 0.074074 & 1 & 106010779 & 1 & 106938537 & 1 & 106 & 0.018 & 0.6562 & 7.56 \\
\hline 107 & 19994 & 19999 & 31 & 0.08 & 25.79 & 0.166166 & 0.118118 & 1 & 107013020 & 1 & 107964728 & 1 & 107 & 0.014 & 0.6287 & 8.38 \\
\hline 108 & 20000 & 821 & 17 & 0.03 & 65.07 & 0.078078 & 0.045045 & 1 & 108004707 & 1 & 108932925 & 1 & 108 & 0.036 & 0.6982 & 5.73 \\
\hline 109 & 54801 & 36750 & 17 & 0.03 & 78.54 & 0.083083 & 0.051051 & 1 & 109003582 & 1 & 109912929 & 1 & 109 & 0.044 & 0.7041 & 5.21 \\
\hline 110 & 45994 & 58664 & 15 & 0.02 & 91.53 & 0.067067 & 0.034034 & 1 & 110118325 & 1 & 110903682 & 1 & 110 & 0.053 & 0.7082 & 4.79 \\
\hline 111 & 830 & 17200 & 11 & 0.01 & 94.97 & 0.057057 & 0.037037 & 1 & 111456164 & 1 & 111938688 & 1 & 111 & 0.056 & 0.7087 & 4.68 \\
\hline 112 & 59732 & 838 & 15 & 0.02 & 90.15 & 0.059059 & 0.04004 & 1 & 112020122 & 1 & 112884757 & 1 & 112 & 0.052 & 0.7077 & 4.82 \\
\hline 113 & 34626 & 52920 & 20 & 0.03 & 68.98 & 0.09009 & 0.055055 & 1 & 113061808 & 1 & 113930519 & 1 & 113 & 0.038 & 0.7 & 5.57 \\
\hline 114 & 53035 & 36756 & 18 & 0.07 & 29 & 0.12012 & 0.087087 & 1 & 114015245 & 1 & 114984686 & 1 & 114 & 0.015 & 0.6391 & 8.01 \\
\hline
\end{tabular}




\begin{tabular}{|c|c|c|c|c|c|c|c|c|c|c|c|c|c|c|c|c|}
\hline 115 & 40024 & 46008 & 14 & 0.01 & 92.96 & 0.068068 & 0.042042 & 1 & 115016840 & 1 & 115880586 & 1 & 115 & 0.054 & 0.7082 & 4.74 \\
\hline 116 & 40026 & 34229 & 20 & 0.02 & 89.63 & 0.084084 & 0.049049 & 1 & 116031370 & 1 & 116992830 & 1 & 116 & 0.052 & 0.7077 & 4.84 \\
\hline 117 & 36762 & 48422 & 22 & 0.03 & 74.74 & 0.089089 & 0.055055 & 1 & 117018825 & 1 & 117930744 & 1 & 117 & 0.042 & 0.702 & 5.32 \\
\hline 118 & 866 & 40032 & 15 & 0.03 & 71.86 & 0.057057 & 0.026026 & 1 & 118042320 & 1 & 118992060 & 1 & 118 & 0.04 & 0.701 & 5.45 \\
\hline 119 & 20031 & 20033 & 13 & 0.02 & 91.43 & 0.047047 & 0.025025 & 1 & 119004005 & 1 & 119835338 & 1 & 119 & 0.053 & 0.7082 & 4.79 \\
\hline 120 & 20034 & 52208 & 25 & 0.04 & 53.74 & 0.103103 & 0.063063 & 1 & 120136077 & 1 & 120994029 & 1 & 120 & 0.029 & 0.6917 & 6.32 \\
\hline 121 & 46021 & 54553 & 15 & 0.01 & 95.38 & 0.049049 & 0.02002 & 1 & 121128221 & 1 & 121999146 & 1 & 121 & 0.056 & 0.7087 & 4.66 \\
\hline 122 & 52120 & 40042 & 20 & 0.03 & 77.6 & 0.089089 & 0.066066 & 1 & 122019338 & 1 & 122922363 & 1 & 122 & 0.044 & 0.7036 & 5.23 \\
\hline 123 & 46027 & 40044 & 13 & 0.02 & 88.12 & 0.056056 & 0.035035 & 1 & 123220315 & 1 & 123968294 & 1 & 123 & 0.051 & 0.7078 & 4.89 \\
\hline 124 & 895 & 36766 & 20 & 0.04 & 52.15 & 0.082082 & 0.059059 & 1 & 124014542 & 1 & 124987731 & 1 & 124 & 0.028 & 0.6897 & 6.41 \\
\hline 125 & 46032 & 903 & 17 & 0.02 & 80.66 & 0.055055 & 0.041041 & 1 & 125123092 & 1 & 125912707 & 1 & 125 & 0.046 & 0.7052 & 5.13 \\
\hline 126 & 46036 & 36768 & 17 & 0.02 & 90.61 & 0.083083 & 0.047047 & 1 & 126271024 & 1 & 126961319 & 1 & 126 & 0.053 & 0.7079 & 4.81 \\
\hline 127 & 50680 & 908 & 7 & 0.01 & 95.91 & 0.032032 & 0.022022 & 1 & 127172643 & 1 & 127733470 & 1 & 127 & 0.056 & 0.7088 & 4.65 \\
\hline 128 & 36769 & 20057 & 9 & 0.01 & 96.15 & 0.034034 & 0.022022 & 1 & 128248008 & 1 & 128958281 & 1 & 128 & 0.057 & 0.709 & 4.64 \\
\hline 129 & 34413 & 46044 & 15 & 0.02 & 82.31 & 0.066066 & 0.043043 & 1 & 129025180 & 1 & 129911267 & 1 & 129 & 0.047 & 0.7049 & 5.07 \\
\hline 130 & 46045 & 929 & 22 & 0.03 & 77.82 & 0.08008 & 0.05005 & 1 & 130035771 & 1 & 130848369 & 1 & 130 & 0.044 & 0.7037 & 5.23 \\
\hline 131 & 20062 & 48425 & 7 & 0.02 & 87.87 & 0.034034 & 0.024024 & 1 & 131011448 & 1 & 131973599 & 1 & 131 & 0.051 & 0.7078 & 4.9 \\
\hline 132 & 20064 & 40064 & 16 & 0.01 & 95.6 & 0.046046 & 0.024024 & 1 & 132029570 & 1 & 132999230 & 1 & 132 & 0.056 & 0.7088 & 4.66 \\
\hline 133 & 50525 & 59418 & 12 & 0.01 & 96.17 & 0.029029 & 0.013013 & 1 & 133084921 & 1 & 133781212 & 1 & 133 & 0.057 & 0.709 & 4.64 \\
\hline 134 & 20070 & 46053 & 12 & 0 & 96.46 & 0.036036 & 0.011011 & 1 & 134037038 & 1 & 134796719 & 1 & 134 & 0.057 & 0.7092 & 4.63 \\
\hline 135 & 55868 & 946 & 7 & 0.02 & 91.3 & 0.03003 & 0.02002 & 1 & 135320152 & 1 & 135930910 & 1 & 135 & 0.053 & 0.7081 & 4.79 \\
\hline 136 & 947 & 950 & 16 & 0.04 & 64.48 & 0.08008 & 0.05005 & 1 & 136015038 & 1 & 136891518 & 1 & 136 & 0.036 & 0.6977 & 5.76 \\
\hline 137 & 20075 & 51457 & 29 & 0.06 & 34.37 & 0.134134 & 0.078078 & 1 & 137035325 & 1 & 137998037 & 1 & 137 & 0.018 & 0.6557 & 7.57 \\
\hline 138 & 20078 & 40073 & 19 & 0.02 & 86.46 & 0.091091 & 0.061061 & 1 & 138050612 & 1 & 138927010 & 1 & 138 & 0.05 & 0.707 & 4.94 \\
\hline 139 & 46064 & 55827 & 23 & 0.04 & 61.32 & 0.102102 & 0.069069 & 1 & 139217790 & 1 & 139996752 & 1 & 139 & 0.034 & 0.6963 & 5.91 \\
\hline 140 & 977 & 20087 & 18 & 0.02 & 91 & 0.073073 & 0.04004 & 1 & 140022043 & 1 & 140842490 & 1 & 140 & 0.053 & 0.7081 & 4.8 \\
\hline 141 & 46069 & 985 & 9 & 0.01 & 95.7 & 0.029029 & 0.015015 & 1 & 141092891 & 1 & 141938208 & 1 & 141 & 0.056 & 0.7089 & 4.66 \\
\hline 142 & 46073 & 20096 & 20 & 0.02 & 80.96 & 0.107107 & 0.073073 & 1 & 142041071 & 1 & 142992212 & 1 & 142 & 0.046 & 0.7049 & 5.12 \\
\hline 143 & 54626 & 57690 & 16 & 0.03 & 80.04 & 0.068068 & 0.05005 & 1 & 143013233 & 1 & 143979942 & 1 & 143 & 0.045 & 0.7047 & 5.15 \\
\hline 144 & 55775 & 20103 & 21 & 0.03 & 77.58 & 0.087087 & 0.063063 & 1 & 144001096 & 1 & 144941329 & 1 & 144 & 0.044 & 0.7036 & 5.23 \\
\hline 145 & 61688 & 40086 & 21 & 0.03 & 75.69 & 0.084084 & 0.052052 & 1 & 145008895 & 1 & 145991718 & 1 & 145 & 0.043 & 0.7027 & 5.3 \\
\hline 146 & 999 & 1007 & 23 & 0.03 & 67.41 & 0.097097 & 0.064064 & 1 & 146032525 & 1 & 146988836 & 1 & 146 & 0.037 & 0.6992 & 5.63 \\
\hline 147 & 48429 & 40089 & 21 & 0.02 & 85.54 & 0.093093 & 0.062062 & 1 & 147019363 & 1 & 147989923 & 1 & 147 & 0.049 & 0.7062 & 4.96 \\
\hline 148 & 40088 & 1020 & 18 & 0.01 & 92.65 & 0.072072 & 0.036036 & 1 & 148011932 & 1 & 148999915 & 1 & 148 & 0.054 & 0.7081 & 4.74 \\
\hline 149 & 36791 & 20124 & 9 & 0.01 & 94.37 & 0.034034 & 0.021021 & 1 & 149045152 & 1 & 149860127 & 1 & 149 & 0.055 & 0.7083 & 4.69 \\
\hline 150 & 56301 & 36794 & 13 & 0.02 & 89.13 & 0.047047 & 0.03003 & 1 & 150030731 & 1 & 150801717 & 1 & 150 & 0.052 & 0.7076 & 4.85 \\
\hline 151 & 50688 & 46093 & 9 & 0 & 96.44 & 0.033033 & 0.009009 & 1 & 151009336 & 1 & 151961290 & 1 & 151 & 0.057 & 0.7091 & 4.63 \\
\hline 152 & 20128 & 46098 & 11 & 0 & 96.51 & 0.043043 & 0.006006 & 1 & 152002404 & 1 & 152965216 & 1 & 152 & 0.057 & 0.7092 & 4.63 \\
\hline 153 & 40094 & 1037 & 11 & 0.01 & 95.75 & 0.032032 & 0.013013 & 1 & 153144281 & 1 & 153965399 & 1 & 153 & 0.056 & 0.7089 & 4.66 \\
\hline
\end{tabular}




\begin{tabular}{|c|c|c|c|c|c|c|c|c|c|c|c|c|c|c|c|c|}
\hline 154 & 40097 & 57614 & 13 & 0.01 & 95.33 & 0.041041 & 0.021021 & 1 & 154076660 & 1 & 154984965 & 1 & 154 & 0.056 & 0.7086 & 4.66 \\
\hline 155 & 40101 & 1050 & 25 & 0.03 & 76.76 & 0.119119 & 0.06006 & 1 & 155011670 & 1 & 155984596 & 1 & 155 & 0.043 & 0.7032 & 5.26 \\
\hline 156 & 20141 & 46108 & 29 & 0.03 & 79.09 & 0.128128 & 0.079079 & 1 & 156019595 & 1 & 156990269 & 1 & 156 & 0.045 & 0.7045 & 5.19 \\
\hline 157 & 1061 & 61987 & 20 & 0.02 & 90 & 0.069069 & 0.037037 & 1 & 157003137 & 1 & 157966730 & 1 & 157 & 0.052 & 0.7077 & 4.83 \\
\hline 158 & 20157 & 53155 & 9 & 0.01 & 96.08 & 0.05005 & 0.022022 & 1 & 158219331 & 1 & 158936835 & 1 & 158 & 0.057 & 0.7091 & 4.64 \\
\hline 159 & 20159 & 40116 & 28 & 0.03 & 76.7 & 0.117117 & 0.068068 & 1 & 159034424 & 1 & 159990673 & 1 & 159 & 0.043 & 0.7031 & 5.26 \\
\hline 160 & 40115 & 53346 & 9 & 0.01 & 94.34 & 0.045045 & 0.028028 & 1 & 160024181 & 1 & 160991130 & 1 & 160 & 0.055 & 0.7083 & 4.69 \\
\hline 161 & 20170 & 45524 & 8 & 0.01 & 96.14 & 0.036036 & 0.018018 & 1 & 161003628 & 1 & 161993748 & 1 & 161 & 0.057 & 0.709 & 4.64 \\
\hline 162 & 45571 & 51307 & 28 & 0.03 & 67.11 & 0.106106 & 0.064064 & 1 & 162006116 & 1 & 162976215 & 1 & 162 & 0.037 & 0.6993 & 5.65 \\
\hline 163 & 20181 & 1098 & 24 & 0.04 & 54.33 & 0.102102 & 0.069069 & 1 & 163013923 & 1 & 163977023 & 1 & 163 & 0.029 & 0.6918 & 6.28 \\
\hline 164 & 46120 & 40123 & 26 & 0.02 & 90.84 & 0.078078 & 0.041041 & 1 & 164042900 & 1 & 164917958 & 1 & 164 & 0.053 & 0.708 & 4.8 \\
\hline 165 & 46123 & 34521 & 12 & 0.01 & 92.98 & 0.05005 & 0.029029 & 1 & 165188787 & 1 & 165937460 & 1 & 165 & 0.054 & 0.7082 & 4.74 \\
\hline 166 & 20193 & 17078 & 21 & 0.02 & 84.54 & 0.09009 & 0.049049 & 1 & 166046787 & 1 & 166934229 & 1 & 166 & 0.049 & 0.7054 & 4.98 \\
\hline 167 & 60555 & 56555 & 13 & 0.02 & 90.41 & 0.054054 & 0.032032 & 1 & 167094891 & 1 & 167882120 & 1 & 167 & 0.053 & 0.7077 & 4.81 \\
\hline 168 & 1120 & 46131 & 16 & 0.01 & 95.72 & 0.045045 & 0.022022 & 1 & 168136428 & 1 & 168987602 & 1 & 168 & 0.056 & 0.7089 & 4.66 \\
\hline 169 & 20203 & 60501 & 12 & 0.01 & 92.21 & 0.066066 & 0.029029 & 1 & 169007648 & 1 & 169984967 & 1 & 169 & 0.054 & 0.7081 & 4.76 \\
\hline 170 & 46134 & 36805 & 12 & 0.01 & 96.18 & 0.047047 & 0.021021 & 1 & 170051398 & 1 & 170866800 & 1 & 170 & 0.057 & 0.7091 & 4.64 \\
\hline 171 & 46140 & 1132 & 10 & 0 & 96.29 & 0.049049 & 0.011011 & 1 & 171062342 & 1 & 171980469 & 1 & 171 & 0.057 & 0.7091 & 4.64 \\
\hline 172 & 58719 & 17815 & 18 & 0.01 & 93.65 & 0.07007 & 0.038038 & 1 & 172013706 & 1 & 172960921 & 1 & 172 & 0.055 & 0.7081 & 4.71 \\
\hline 173 & 1140 & 1146 & 14 & 0.02 & 88.14 & 0.063063 & 0.04004 & 1 & 173010521 & 1 & 173741598 & 1 & 173 & 0.051 & 0.7077 & 4.89 \\
\hline 174 & 40137 & 20211 & 11 & 0.02 & 90.52 & 0.041041 & 0.025025 & 1 & 174018173 & 1 & 174985817 & 1 & 174 & 0.053 & 0.7078 & 4.81 \\
\hline 175 & 20212 & 1152 & 12 & 0.01 & 95.05 & 0.054054 & 0.032032 & 1 & 175226043 & 1 & 175979464 & 1 & 175 & 0.056 & 0.7087 & 4.67 \\
\hline 176 & 46150 & 20221 & 22 & 0.02 & 87.37 & 0.081081 & 0.05005 & 1 & 176015699 & 1 & 176979355 & 1 & 176 & 0.05 & 0.7077 & 4.92 \\
\hline 177 & 1158 & 46156 & 18 & 0.03 & 80.41 & 0.074074 & 0.051051 & 1 & 177006345 & 1 & 177966091 & 1 & 177 & 0.046 & 0.705 & 5.14 \\
\hline 178 & 1164 & 57414 & 13 & 0 & 96.26 & 0.039039 & 0.023023 & 1 & 178024855 & 1 & 178988459 & 1 & 178 & 0.057 & 0.7091 & 4.64 \\
\hline 179 & 1169 & 1177 & 24 & 0.02 & 87.92 & 0.075075 & 0.045045 & 1 & 179002367 & 1 & 179959509 & 1 & 179 & 0.051 & 0.7078 & 4.9 \\
\hline 180 & 61791 & 1180 & 13 & 0 & 96.48 & 0.043043 & 0.014014 & 1 & 180068581 & 1 & 180980648 & 1 & 180 & 0.057 & 0.7092 & 4.63 \\
\hline 181 & 50709 & 54926 & 18 & 0.01 & 93.94 & 0.061061 & 0.039039 & 1 & 181006837 & 1 & 181978498 & 1 & 181 & 0.055 & 0.7084 & 4.7 \\
\hline 182 & 20245 & 54836 & 18 & 0.03 & 76.67 & 0.079079 & 0.051051 & 1 & 182016926 & 1 & 182952918 & 1 & 182 & 0.043 & 0.703 & 5.26 \\
\hline 183 & 1189 & 1194 & 22 & 0.04 & 58.85 & 0.11011 & 0.074074 & 1 & 183094335 & 1 & 183971871 & 1 & 183 & 0.032 & 0.694 & 6.02 \\
\hline 184 & 20255 & 1200 & 22 & 0.03 & 65.42 & 0.097097 & 0.074074 & 1 & 184000929 & 1 & 184954399 & 1 & 184 & 0.036 & 0.6987 & 5.72 \\
\hline 185 & 1202 & 40165 & 32 & 0.06 & 38.2 & 0.131131 & 0.092092 & 1 & 185048329 & 1 & 185965822 & 1 & 185 & 0.02 & 0.6661 & 7.28 \\
\hline 186 & 20268 & 46172 & 18 & 0.02 & 91.46 & 0.081081 & 0.048048 & 1 & 186106230 & 1 & 186883993 & 1 & 186 & 0.053 & 0.7083 & 4.79 \\
\hline 187 & 20273 & 55985 & 21 & 0.02 & 86.13 & 0.094094 & 0.054054 & 1 & 187044656 & 1 & 187930689 & 1 & 187 & 0.049 & 0.7067 & 4.95 \\
\hline 188 & 1226 & 36823 & 9 & 0.01 & 95.92 & 0.039039 & 0.02002 & 1 & 188025326 & 1 & 188990100 & 1 & 188 & 0.056 & 0.7088 & 4.65 \\
\hline 189 & 36824 & 16802 & 14 & 0.01 & 95.48 & 0.048048 & 0.029029 & 1 & 189025116 & 1 & 189978659 & 1 & 189 & 0.056 & 0.7087 & 4.66 \\
\hline 190 & 1235 & 36834 & 16 & 0.01 & 94.57 & 0.077077 & 0.049049 & 1 & 190244449 & 1 & 190921716 & 1 & 190 & 0.055 & 0.7084 & 4.68 \\
\hline 191 & 1240 & 17445 & 18 & 0.01 & 92.78 & 0.07007 & 0.034034 & 1 & 191055429 & 1 & 191937788 & 1 & 191 & 0.054 & 0.7081 & 4.74 \\
\hline 192 & 46180 & 36841 & 9 & 0.01 & 95.21 & 0.044044 & 0.028028 & 1 & 192637720 & 1 & 192978414 & 1 & 192 & 0.056 & 0.7087 & 4.67 \\
\hline
\end{tabular}




\begin{tabular}{|c|c|c|c|c|c|c|c|c|c|c|c|c|c|c|c|c|}
\hline 193 & 40177 & 1252 & 17 & 0.02 & 85.3 & 0.085085 & 0.053053 & 1 & 193062563 & 1 & 193998587 & 1 & 193 & 0.049 & 0.7062 & 4.97 \\
\hline 194 & 46184 & 36847 & 11 & 0.01 & 94.59 & 0.047047 & 0.028028 & 1 & 194020406 & 1 & 194987488 & 1 & 194 & 0.056 & 0.7084 & 4.68 \\
\hline 195 & 35536 & 46188 & 13 & 0.02 & 91.5 & 0.06006 & 0.048048 & 1 & 195015099 & 1 & 195833378 & 1 & 195 & 0.053 & 0.7082 & 4.79 \\
\hline 196 & 36852 & 20282 & 17 & 0.03 & 78.78 & 0.061061 & 0.045045 & 1 & 196015749 & 1 & 196836959 & 1 & 196 & 0.045 & 0.7039 & 5.19 \\
\hline 197 & 1264 & 36858 & 12 & 0.01 & 93.22 & 0.037037 & 0.026026 & 1 & 197179651 & 1 & 197983246 & 1 & 197 & 0.054 & 0.7081 & 4.73 \\
\hline 198 & 46192 & 1268 & 15 & 0.01 & 95.18 & 0.068068 & 0.035035 & 1 & 198004282 & 1 & 198859209 & 1 & 198 & 0.056 & 0.7087 & 4.67 \\
\hline 199 & 18328 & 36872 & 17 & 0.02 & 84.36 & 0.098098 & 0.062062 & 1 & 199041898 & 1 & 199973047 & 1 & 199 & 0.049 & 0.7055 & 4.99 \\
\hline 200 & 1272 & 20291 & 19 & 0.03 & 67.07 & 0.096096 & 0.051051 & 1 & 200027513 & 1 & 200973888 & 1 & 200 & 0.037 & 0.6993 & 5.65 \\
\hline 201 & 36874 & 20295 & 31 & 0.02 & 80.91 & 0.102102 & 0.066066 & 1 & 201005516 & 1 & 201988814 & 1 & 201 & 0.046 & 0.7051 & 5.12 \\
\hline 202 & 20296 & 36884 & 24 & 0.01 & 93.51 & 0.061061 & 0.024024 & 1 & 202021384 & 1 & 202987973 & 1 & 202 & 0.055 & 0.7082 & 4.71 \\
\hline 203 & 1293 & 33788 & 17 & 0.01 & 92.81 & 0.058058 & 0.03003 & 1 & 203020847 & 1 & 203809481 & 1 & 203 & 0.054 & 0.7081 & 4.74 \\
\hline 204 & 51322 & 1309 & 22 & 0.02 & 88.18 & 0.087087 & 0.035035 & 1 & 204023854 & 1 & 204988249 & 1 & 204 & 0.051 & 0.7078 & 4.89 \\
\hline 205 & 40189 & 20308 & 27 & 0.01 & 95.29 & 0.1001 & 0.035035 & 1 & 205020361 & 1 & 205994796 & 1 & 205 & 0.056 & 0.7086 & 4.67 \\
\hline 206 & 36887 & 36890 & 21 & 0.01 & 94.93 & 0.072072 & 0.031031 & 1 & 206003777 & 1 & 206994869 & 1 & 206 & 0.056 & 0.7087 & 4.68 \\
\hline 207 & 46211 & 1332 & 16 & 0.01 & 95.03 & 0.059059 & 0.034034 & 1 & 207036700 & 1 & 207810204 & 1 & 207 & 0.056 & 0.7087 & 4.67 \\
\hline 208 & 20313 & 36892 & 18 & 0.02 & 91.4 & 0.061061 & 0.035035 & 1 & 208051940 & 1 & 208999979 & 1 & 208 & 0.053 & 0.7082 & 4.79 \\
\hline 209 & 1339 & 20317 & 17 & 0.01 & 93.04 & 0.076076 & 0.044044 & 1 & 209010763 & 1 & 209927137 & 1 & 209 & 0.054 & 0.7081 & 4.73 \\
\hline 210 & 1344 & 35587 & 15 & 0.01 & 93.69 & 0.038038 & 0.022022 & 1 & 210025405 & 1 & 210956244 & 1 & 210 & 0.055 & 0.7081 & 4.71 \\
\hline 211 & 1350 & 1355 & 11 & 0.01 & 95.89 & 0.046046 & 0.023023 & 1 & 211151578 & 1 & 211988436 & 1 & 211 & 0.056 & 0.7088 & 4.65 \\
\hline 212 & 31267 & 35110 & 14 & 0.01 & 92.4 & 0.064064 & 0.036036 & 1 & 212275513 & 1 & 212992088 & 1 & 212 & 0.054 & 0.7082 & 4.76 \\
\hline 213 & 40199 & 20327 & 21 & 0.03 & 77.39 & 0.091091 & 0.062062 & 1 & 213014666 & 1 & 213874782 & 1 & 213 & 0.044 & 0.7034 & 5.24 \\
\hline 214 & 20328 & 34752 & 23 & 0.02 & 87.19 & 0.075075 & 0.041041 & 1 & 214033032 & 1 & 214959896 & 1 & 214 & 0.05 & 0.7076 & 4.92 \\
\hline 215 & 1367 & 40204 & 16 & 0.01 & 94.56 & 0.05005 & 0.03003 & 1 & 215042412 & 1 & 215971588 & 1 & 215 & 0.055 & 0.7084 & 4.68 \\
\hline 216 & 60848 & 1385 & 16 & 0 & 96.54 & 0.028028 & 0.007007 & 1 & 216019228 & 1 & 216980027 & 1 & 216 & 0.057 & 0.7093 & 4.63 \\
\hline 217 & 46241 & 54097 & 5 & 0 & 96.56 & 0.003003 & 0 & 1 & 217060222 & 1 & 217544827 & 1 & 217 & 0.057 & 0.7093 & 4.63 \\
\hline 218 & 20332 & 1394 & 12 & 0.01 & 96.12 & 0.039039 & 0.014014 & 1 & 218141657 & 1 & 218977436 & 1 & 218 & 0.057 & 0.7091 & 4.64 \\
\hline 219 & 46246 & 1398 & 11 & 0.01 & 95.95 & 0.038038 & 0.025025 & 1 & 219151821 & 1 & 219966680 & 1 & 219 & 0.056 & 0.7088 & 4.65 \\
\hline 220 & 36899 & 36903 & 17 & 0.02 & 88.25 & 0.086086 & 0.05005 & 1 & 220102612 & 1 & 220815008 & 1 & 220 & 0.051 & 0.7076 & 4.88 \\
\hline 221 & 36904 & 58253 & 8 & 0.01 & 96.1 & 0.029029 & 0.019019 & 1 & 221086679 & 1 & 221935450 & 1 & 221 & 0.057 & 0.7091 & 4.64 \\
\hline 222 & 46252 & 1410 & 15 & 0.01 & 93.7 & 0.07007 & 0.038038 & 1 & 222007904 & 1 & 222962711 & 1 & 222 & 0.055 & 0.7081 & 4.71 \\
\hline 223 & 36910 & 53243 & 11 & 0.02 & 91.01 & 0.049049 & 0.033033 & 1 & 223043310 & 1 & 223992839 & 1 & 223 & 0.053 & 0.7081 & 4.8 \\
\hline 224 & 51517 & 46256 & 14 & 0.01 & 94.66 & 0.057057 & 0.036036 & 1 & 224000157 & 1 & 224999683 & 1 & 224 & 0.056 & 0.7085 & 4.68 \\
\hline 225 & 34390 & 36921 & 9 & 0 & 96.44 & 0.027027 & 0.012012 & 1 & 225518654 & 1 & 225986595 & 1 & 225 & 0.057 & 0.7092 & 4.63 \\
\hline 226 & 1418 & 36922 & 19 & 0.02 & 91.73 & 0.081081 & 0.042042 & 1 & 226149776 & 1 & 226989901 & 1 & 226 & 0.053 & 0.7081 & 4.78 \\
\hline 227 & 20342 & 20345 & 16 & 0.01 & 94.71 & 0.051051 & 0.03003 & 1 & 227018020 & 1 & 227989037 & 1 & 227 & 0.056 & 0.7085 & 4.68 \\
\hline 228 & 1433 & 1441 & 22 & 0.01 & 94.52 & 0.081081 & 0.047047 & 1 & 228038772 & 1 & 228976153 & 1 & 228 & 0.055 & 0.7084 & 4.68 \\
\hline 229 & 40225 & 40227 & 27 & 0.03 & 73.44 & 0.12012 & 0.075075 & 1 & 229000633 & 1 & 229999129 & 1 & 229 & 0.041 & 0.7018 & 5.38 \\
\hline 230 & 1448 & 46271 & 17 & 0.01 & 92.86 & 0.071071 & 0.043043 & 1 & 230021584 & 1 & 230928714 & 1 & 230 & 0.054 & 0.7081 & 4.74 \\
\hline 231 & 34522 & 61459 & 27 & 0.02 & 86.61 & 0.114114 & 0.059059 & 1 & 231003149 & 1 & 231932843 & 1 & 231 & 0.05 & 0.7071 & 4.94 \\
\hline
\end{tabular}




\begin{tabular}{|c|c|c|c|c|c|c|c|c|c|c|c|c|c|c|c|c|}
\hline 232 & 46276 & 20358 & 22 & 0.02 & 90.8 & 0.097097 & 0.045045 & 1 & 232040879 & 1 & 232978056 & 1 & 232 & 0.053 & 0.708 & 4.8 \\
\hline 233 & 34453 & 50432 & 19 & 0.01 & 93.88 & 0.072072 & 0.028028 & 1 & 233021161 & 1 & 233975946 & 1 & 233 & 0.055 & 0.7083 & 4.7 \\
\hline 234 & 1473 & 46281 & 10 & 0 & 96.38 & 0.033033 & 0.013013 & 1 & 234035171 & 1 & 234854917 & 1 & 234 & 0.057 & 0.7092 & 4.64 \\
\hline 235 & 55965 & 20360 & 2 & 0 & 96.56 & 0.005005 & 0.002002 & 1 & 235179193 & 1 & 235988757 & 1 & 235 & 0.057 & 0.7093 & 4.63 \\
\hline 236 & 36931 & 46282 & 10 & 0.01 & 96.03 & 0.036036 & 0.015015 & 1 & 236058941 & 1 & 236949960 & 1 & 236 & 0.057 & 0.709 & 4.65 \\
\hline 237 & 61841 & 1490 & 10 & 0.01 & 93.24 & 0.048048 & 0.018018 & 1 & 237029410 & 1 & 237900874 & 1 & 237 & 0.054 & 0.7081 & 4.73 \\
\hline 238 & 20361 & 20363 & 15 & 0.02 & 87.13 & 0.065065 & 0.032032 & 1 & 238102917 & 1 & 238827931 & 1 & 238 & 0.05 & 0.7076 & 4.92 \\
\hline 239 & 60667 & 51773 & 19 & 0.01 & 93.53 & 0.074074 & 0.04004 & 1 & 239045601 & 1 & 239987141 & 1 & 239 & 0.055 & 0.7082 & 4.71 \\
\hline 240 & 40237 & 1510 & 16 & 0.01 & 95.45 & 0.065065 & 0.032032 & 1 & 240011062 & 1 & 240994314 & 1 & 240 & 0.056 & 0.7087 & 4.66 \\
\hline 241 & 40238 & 1514 & 12 & 0.01 & 96.06 & 0.072072 & 0.029029 & 1 & 241074745 & 1 & 241983957 & 1 & 241 & 0.057 & 0.709 & 4.64 \\
\hline 242 & 1515 & 58127 & 26 & 0.01 & 95.64 & 0.13013 & 0.04004 & 1 & 242044327 & 1 & 242984908 & 1 & 242 & 0.056 & 0.7088 & 4.66 \\
\hline 243 & 20376 & 29768 & 21 & 0.02 & 88.4 & 0.089089 & 0.046046 & 1 & 243011910 & 1 & 243989929 & 1 & 243 & 0.051 & 0.7076 & 4.88 \\
\hline 244 & 1531 & 58547 & 12 & 0.02 & 89.24 & 0.051051 & 0.03003 & 1 & 244008725 & 1 & 244996464 & 1 & 244 & 0.052 & 0.7078 & 4.85 \\
\hline 245 & 36368 & 46306 & 24 & 0.01 & 94.74 & 0.073073 & 0.025025 & 1 & 245008421 & 1 & 245981247 & 1 & 245 & 0.056 & 0.7085 & 4.68 \\
\hline 246 & 20384 & 54725 & 14 & 0 & 96.39 & 0.045045 & 0.019019 & 1 & 246005498 & 1 & 246950678 & 1 & 246 & 0.057 & 0.7092 & 4.64 \\
\hline 247 & 45600 & 20396 & 29 & 0.02 & 84.45 & 0.108108 & 0.045045 & 1 & 247017119 & 1 & 247962534 & 1 & 247 & 0.049 & 0.7055 & 4.99 \\
\hline 248 & 36945 & 40255 & 31 & 0.05 & 42.56 & 0.152152 & 0.098098 & 1 & 248065286 & 1 & 248988370 & 1 & 248 & 0.022 & 0.6765 & 7.03 \\
\hline 249 & 1546 & 1556 & 20 & 0 & 96.28 & 0.071071 & 0.022022 & 1 & 249012536 & 1 & 249998570 & 1 & 249 & 0.057 & 0.7091 & 4.64 \\
\hline 250 & 40257 & 46323 & 28 & 0.01 & 95.84 & 0.121121 & 0.03003 & 1 & 250018705 & 1 & 250959302 & 1 & 250 & 0.056 & 0.7089 & 4.65 \\
\hline 251 & 60174 & 1566 & 21 & 0.02 & 82.83 & 0.076076 & 0.044044 & 1 & 251044409 & 1 & 251957006 & 1 & 251 & 0.047 & 0.7047 & 5.04 \\
\hline 252 & 40262 & 35883 & 26 & 0.04 & 53.19 & 0.118118 & 0.061061 & 1 & 252002865 & 1 & 252997864 & 1 & 252 & 0.029 & 0.6916 & 6.36 \\
\hline 253 & 36959 & 36967 & 18 & 0.01 & 95.36 & 0.087087 & 0.023023 & 1 & 253029725 & 1 & 253914428 & 1 & 253 & 0.056 & 0.7087 & 4.66 \\
\hline 254 & 1579 & 60730 & 16 & 0.01 & 95.71 & 0.061061 & 0.017017 & 1 & 254016770 & 1 & 254948269 & 1 & 254 & 0.056 & 0.7089 & 4.66 \\
\hline 255 & 36974 & 20417 & 21 & 0.02 & 82.5 & 0.107107 & 0.04004 & 1 & 255032767 & 1 & 255981511 & 1 & 255 & 0.047 & 0.7049 & 5.06 \\
\hline 256 & 36979 & 20426 & 25 & 0.04 & 50.48 & 0.129129 & 0.05005 & 1 & 256001453 & 1 & 256989077 & 1 & 256 & 0.027 & 0.6868 & 6.47 \\
\hline 257 & 36982 & 14478 & 28 & 0.03 & 75.32 & 0.122122 & 0.064064 & 1 & 257001258 & 1 & 257954281 & 1 & 257 & 0.042 & 0.7023 & 5.31 \\
\hline 258 & 59408 & 56575 & 18 & 0.02 & 88.61 & 0.068068 & 0.049049 & 1 & 258021815 & 1 & 258984538 & 1 & 258 & 0.051 & 0.7078 & 4.87 \\
\hline 259 & 1617 & 36988 & 16 & 0.02 & 88.31 & 0.071071 & 0.023023 & 1 & 259019570 & 1 & 259936367 & 1 & 259 & 0.051 & 0.7077 & 4.88 \\
\hline 260 & 54409 & 46348 & 18 & 0.02 & 91.6 & 0.095095 & 0.025025 & 1 & 260134029 & 1 & 260985282 & 1 & 260 & 0.053 & 0.7081 & 4.78 \\
\hline 261 & 55745 & 40278 & 15 & 0.01 & 94.6 & 0.06006 & 0.029029 & 1 & 261028547 & 1 & 261972774 & 1 & 261 & 0.056 & 0.7084 & 4.68 \\
\hline 262 & 1631 & 20442 & 15 & 0.01 & 93.46 & 0.051051 & 0.037037 & 1 & 262039871 & 1 & 262967147 & 1 & 262 & 0.055 & 0.7082 & 4.72 \\
\hline 263 & 53934 & 1646 & 17 & 0.01 & 94.11 & 0.065065 & 0.03003 & 1 & 263044178 & 1 & 263972707 & 1 & 263 & 0.055 & 0.7085 & 4.7 \\
\hline 264 & 36114 & 40284 & 15 & 0.02 & 89.89 & 0.057057 & 0.038038 & 1 & 264047505 & 1 & 264938853 & 1 & 264 & 0.052 & 0.7078 & 4.83 \\
\hline 265 & 51666 & 20455 & 22 & 0.04 & 62.06 & 0.087087 & 0.055055 & 1 & 265041087 & 1 & 265964227 & 1 & 265 & 0.034 & 0.6967 & 5.88 \\
\hline 266 & 1657 & 1670 & 30 & 0.04 & 60.69 & 0.114114 & 0.079079 & 1 & 266007391 & 1 & 266974660 & 1 & 266 & 0.033 & 0.6957 & 5.94 \\
\hline 267 & 1671 & 1683 & 34 & 0.06 & 32.23 & 0.158158 & 0.119119 & 1 & 267026000 & 1 & 267994055 & 1 & 267 & 0.017 & 0.6494 & 7.75 \\
\hline 268 & 60483 & 1693 & 31 & 0.05 & 41.89 & 0.15015 & 0.1001 & 1 & 268014122 & 1 & 268986440 & 1 & 268 & 0.022 & 0.675 & 7.07 \\
\hline 269 & 20483 & 46359 & 21 & 0.02 & 90.05 & 0.084084 & 0.051051 & 1 & 269008819 & 1 & 269972373 & 1 & 269 & 0.052 & 0.7078 & 4.82 \\
\hline 270 & 37003 & 59492 & 16 & 0.03 & 66.7 & 0.08008 & 0.055055 & 1 & 270102816 & 1 & 270881981 & 1 & 270 & 0.037 & 0.6992 & 5.66 \\
\hline
\end{tabular}




\begin{tabular}{|c|c|c|c|c|c|c|c|c|c|c|c|c|c|c|c|c|}
\hline 271 & 1703 & 20495 & 22 & 0.01 & 92.51 & 0.087087 & 0.05005 & 1 & 271028077 & 1 & 271984966 & 1 & 271 & 0.054 & 0.7081 & 4.75 \\
\hline 272 & 57264 & 37006 & 13 & 0.02 & 91.06 & 0.046046 & 0.027027 & 1 & 272001095 & 1 & 272977191 & 1 & 272 & 0.053 & 0.7081 & 4.8 \\
\hline 273 & 46366 & 1715 & 8 & 0.01 & 95.87 & 0.022022 & 0.015015 & 1 & 273002529 & 1 & 273825768 & 1 & 273 & 0.056 & 0.7088 & 4.65 \\
\hline 274 & 46043 & 1717 & 15 & 0.02 & 89.38 & 0.068068 & 0.047047 & 1 & 274127937 & 1 & 274989579 & 1 & 274 & 0.052 & 0.7076 & 4.84 \\
\hline 275 & 1718 & 54575 & 21 & 0.02 & 89.6 & 0.093093 & 0.05005 & 1 & 275078557 & 1 & 275995963 & 1 & 275 & 0.052 & 0.7077 & 4.84 \\
\hline 276 & 46374 & 1727 & 17 & 0.01 & 95.14 & 0.054054 & 0.022022 & 1 & 276112956 & 1 & 276982150 & 1 & 276 & 0.056 & 0.7087 & 4.67 \\
\hline 277 & 1730 & 56414 & 13 & 0.04 & 56.76 & 0.064064 & 0.044044 & 1 & 277105135 & 1 & 277975248 & 1 & 277 & 0.031 & 0.6917 & 6.1 \\
\hline 278 & 1735 & 46383 & 25 & 0.03 & 74.16 & 0.101101 & 0.073073 & 1 & 278256541 & 1 & 278989901 & 1 & 278 & 0.042 & 0.7021 & 5.35 \\
\hline 279 & 37018 & 58710 & 34 & 0.03 & 65.25 & 0.144144 & 0.075075 & 1 & 279014971 & 1 & 279972181 & 1 & 279 & 0.036 & 0.6984 & 5.73 \\
\hline 280 & 1752 & 40325 & 31 & 0.04 & 50.22 & 0.158158 & 0.104104 & 1 & 280005940 & 1 & 280937085 & 1 & 280 & 0.027 & 0.6863 & 6.49 \\
\hline 281 & 1765 & 30435 & 33 & 0.08 & 26.57 & 0.159159 & 0.112112 & 1 & 281095974 & 1 & 281969962 & 1 & 281 & 0.014 & 0.6304 & 8.26 \\
\hline 282 & 20526 & 52816 & 32 & 0.04 & 50.71 & 0.144144 & 0.092092 & 1 & 282001963 & 1 & 282998551 & 1 & 282 & 0.027 & 0.6869 & 6.46 \\
\hline 283 & 20534 & 40334 & 34 & 0.05 & 40.25 & 0.139139 & 0.081081 & 1 & 283010283 & 1 & 283959003 & 1 & 283 & 0.021 & 0.6718 & 7.17 \\
\hline 284 & 55247 & 19250 & 28 & 0.03 & 80.46 & 0.128128 & 0.069069 & 1 & 284045369 & 1 & 284998024 & 1 & 284 & 0.046 & 0.7051 & 5.14 \\
\hline 285 & 18644 & 40340 & 27 & 0.09 & 22.53 & 0.135135 & 0.092092 & 1 & 285026326 & 1 & 285985500 & 1 & 285 & 0.012 & 0.6164 & 8.88 \\
\hline 286 & 40341 & 20558 & 25 & 0.05 & 47.26 & 0.124124 & 0.085085 & 1 & 286007661 & 1 & 286998337 & 1 & 286 & 0.025 & 0.6825 & 6.66 \\
\hline 287 & 37036 & 40349 & 27 & 0.09 & 22.16 & 0.145145 & 0.113113 & 1 & 287061909 & 1 & 287999039 & 1 & 287 & 0.012 & 0.6141 & 8.91 \\
\hline 288 & 20567 & 20576 & 32 & 0.09 & 23.76 & 0.182182 & 0.132132 & 1 & 288024268 & 1 & 288885054 & 1 & 288 & 0.012 & 0.6234 & 8.74 \\
\hline 289 & 59996 & 40353 & 26 & 0.03 & 74.83 & 0.113113 & 0.075075 & 1 & 289048882 & 1 & 289983854 & 1 & 289 & 0.042 & 0.7021 & 5.32 \\
\hline 290 & 45109 & 40356 & 16 & 0.02 & 88.95 & 0.067067 & 0.039039 & 1 & 290260126 & 1 & 290972764 & 1 & 290 & 0.051 & 0.7077 & 4.86 \\
\hline 291 & 1845 & 1856 & 27 & 0.06 & 38.82 & 0.135135 & 0.084084 & 1 & 291021694 & 1 & 291903522 & 1 & 291 & 0.021 & 0.6679 & 7.25 \\
\hline 292 & 18920 & 37049 & 22 & 0.03 & 69.57 & 0.108108 & 0.074074 & 1 & 292036476 & 1 & 292998740 & 1 & 292 & 0.039 & 0.6997 & 5.54 \\
\hline 293 & 40360 & 20605 & 26 & 0.02 & 88.02 & 0.101101 & 0.055055 & 1 & 293020147 & 1 & 293991451 & 1 & 293 & 0.051 & 0.7078 & 4.89 \\
\hline 294 & 40365 & 19322 & 24 & 0.01 & 91.88 & 0.105105 & 0.056056 & 1 & 294012711 & 1 & 294890555 & 1 & 294 & 0.053 & 0.7081 & 4.77 \\
\hline 295 & 37052 & 1885 & 10 & 0.02 & 87.17 & 0.055055 & 0.03003 & 1 & 295017152 & 1 & 295976006 & 1 & 295 & 0.05 & 0.7076 & 4.92 \\
\hline 296 & 1886 & 56783 & 13 & 0.01 & 95.01 & 0.061061 & 0.031031 & 1 & 296003591 & 1 & 296938083 & 1 & 296 & 0.056 & 0.7087 & 4.68 \\
\hline 297 & 40376 & 40377 & 12 & 0 & 96.39 & 0.051051 & 0.018018 & 1 & 297148442 & 1 & 297932234 & 1 & 297 & 0.057 & 0.7092 & 4.64 \\
\hline 298 & 40378 & 40381 & 23 & 0.02 & 83.2 & 0.111111 & 0.062062 & 1 & 298042326 & 1 & 298972575 & 1 & 298 & 0.048 & 0.7045 & 5.02 \\
\hline 299 & 20625 & 16654 & 16 & 0.01 & 94.17 & 0.065065 & 0.027027 & 1 & 299033446 & 1 & 299937327 & 1 & 299 & 0.055 & 0.7084 & 4.69 \\
\hline 300 & 1904 & 40384 & 15 & 0.02 & 87.59 & 0.07007 & 0.041041 & 1 & 300316974 & 1 & 300929374 & 1 & 300 & 0.05 & 0.7078 & 4.91 \\
\hline 301 & 40386 & 40397 & 31 & 0.06 & 35.58 & 0.149149 & 0.097097 & 1 & 301003643 & 1 & 301983854 & 1 & 301 & 0.019 & 0.6571 & 7.42 \\
\hline 302 & 46407 & 1927 & 32 & 0.05 & 44.47 & 0.154154 & 0.095095 & 1 & 302009919 & 1 & 302980607 & 1 & 302 & 0.024 & 0.6795 & 6.89 \\
\hline 303 & 20647 & 48493 & 28 & 0.03 & 77.2 & 0.121121 & 0.068068 & 1 & 303008877 & 1 & 303984121 & 1 & 303 & 0.044 & 0.7034 & 5.25 \\
\hline 304 & 1935 & 54002 & 30 & 0.06 & 34.49 & 0.139139 & 0.101101 & 1 & 304042460 & 1 & 304994318 & 1 & 304 & 0.018 & 0.6564 & 7.57 \\
\hline 305 & 48495 & 48498 & 42 & 0.08 & 24.02 & 0.193193 & 0.126126 & 1 & 305110723 & 1 & 305987689 & 1 & 305 & 0.012 & 0.6259 & 8.74 \\
\hline 306 & 1952 & 48501 & 26 & 0.02 & 89.81 & 0.088088 & 0.042042 & 1 & 306010138 & 1 & 306907352 & 1 & 306 & 0.052 & 0.7077 & 4.83 \\
\hline 307 & 45528 & 60767 & 19 & 0.03 & 79.22 & 0.078078 & 0.05005 & 1 & 307006485 & 1 & 307934149 & 1 & 307 & 0.045 & 0.7046 & 5.19 \\
\hline 308 & 32581 & 14589 & 15 & 0.01 & 92.28 & 0.057057 & 0.034034 & 1 & 308111095 & 1 & 308984948 & 1 & 308 & 0.054 & 0.7081 & 4.76 \\
\hline 309 & 56395 & 1963 & 25 & 0.16 & 14.75 & 0.134134 & 0.094094 & 1 & 309037070 & 1 & 309932123 & 1 & 309 & 0.008 & 0.5727 & 10.28 \\
\hline
\end{tabular}




\begin{tabular}{|c|c|c|c|c|c|c|c|c|c|c|c|c|c|c|c|c|}
\hline 310 & 20687 & 1973 & 16 & 0.04 & 57.95 & 0.087087 & 0.057057 & 1 & 310005846 & 1 & 310999663 & 1 & 310 & 0.032 & 0.693 & 6.05 \\
\hline 311 & 20690 & 46418 & 18 & 0.01 & 94.75 & 0.071071 & 0.045045 & 1 & 311027658 & 1 & 311995820 & 1 & 311 & 0.056 & 0.7084 & 4.68 \\
\hline 312 & 1979 & 34450 & 6 & 0 & 96.43 & 0.018018 & 0.012012 & 1 & 312364898 & 1 & 312829468 & 1 & 312 & 0.057 & 0.7092 & 4.63 \\
\hline 313 & 1982 & 20697 & 6 & 0 & 96.45 & 0.009009 & 0.006006 & 1 & 313111618 & 1 & 313480723 & 1 & 313 & 0.057 & 0.7092 & 4.63 \\
\hline 314 & 56225 & 60162 & 2 & 0 & 96.56 & 0.001001 & 0.001001 & 1 & 314046273 & 1 & 314869351 & 1 & 314 & 0.057 & 0.7093 & 4.63 \\
\hline 315 & 53815 & 58893 & 2 & 0 & 96.4 & 0.013013 & 0.006006 & 1 & 315187351 & 1 & 315214500 & 1 & 315 & 0.057 & 0.7091 & 4.64 \\
\hline 316 & 29677 & 52837 & 13 & 0.02 & 83.39 & 0.073073 & 0.043043 & 2 & 16416 & 2 & 963130 & 2 & 0 & 0.048 & 0.7047 & 5.02 \\
\hline 317 & 49927 & 35732 & 16 & 0.01 & 95.08 & 0.06006 & 0.029029 & 2 & 1021191 & 2 & 1881100 & 2 & 1 & 0.056 & 0.7088 & 4.67 \\
\hline 318 & 45597 & 40436 & 23 & 0.03 & 67.31 & 0.111111 & 0.072072 & 2 & 2010989 & 2 & 2992926 & 2 & 2 & 0.037 & 0.6993 & 5.64 \\
\hline 319 & 48515 & 20702 & 16 & 0.03 & 70.44 & 0.08008 & 0.055055 & 2 & 3024804 & 2 & 3972464 & 2 & 3 & 0.039 & 0.7005 & 5.51 \\
\hline 320 & 1991 & 20705 & 11 & 0.01 & 95.43 & 0.04004 & 0.024024 & 2 & 4110608 & 2 & 4872634 & 2 & 4 & 0.056 & 0.7087 & 4.66 \\
\hline 321 & 20706 & 48521 & 30 & 0.02 & 80.84 & 0.115115 & 0.083083 & 2 & 5004637 & 2 & 5984348 & 2 & 5 & 0.046 & 0.7052 & 5.13 \\
\hline 322 & 20721 & 20726 & 37 & 0.04 & 50.97 & 0.138138 & 0.083083 & 2 & 6018125 & 2 & 6998911 & 2 & 6 & 0.028 & 0.6878 & 6.46 \\
\hline 323 & 54914 & 20737 & 20 & 0.01 & 91.85 & 0.071071 & 0.038038 & 2 & 7016156 & 2 & 7998883 & 2 & 7 & 0.053 & 0.7081 & 4.77 \\
\hline 324 & 20738 & 52444 & 27 & 0.13 & 17.13 & 0.162162 & 0.13013 & 2 & 8022443 & 2 & 8997005 & 2 & 8 & 0.009 & 0.5865 & 9.69 \\
\hline 325 & 2007 & 2015 & 31 & 0.04 & 50.04 & 0.126126 & 0.074074 & 2 & 9098547 & 2 & 9987663 & 2 & 9 & 0.027 & 0.6862 & 6.5 \\
\hline 326 & 2014 & 2017 & 30 & 0.03 & 70.24 & 0.132132 & 0.089089 & 2 & 10028746 & 2 & 10999714 & 2 & 10 & 0.039 & 0.7004 & 5.52 \\
\hline 327 & 33139 & 15348 & 19 & 0.02 & 84.8 & 0.086086 & 0.049049 & 2 & 11066709 & 2 & 11888058 & 2 & 11 & 0.049 & 0.7059 & 4.98 \\
\hline 328 & 2029 & 35259 & 38 & 0.07 & 28.14 & 0.147147 & 0.107107 & 2 & 12047540 & 2 & 12999985 & 2 & 12 & 0.015 & 0.636 & 8.09 \\
\hline 329 & 45775 & 40461 & 10 & 0.02 & 91.13 & 0.048048 & 0.03003 & 2 & 13005151 & 2 & 13902607 & 2 & 13 & 0.053 & 0.7081 & 4.8 \\
\hline 330 & 30120 & 30120 & 1 & 0 & 96.52 & 0.005005 & 0.004004 & 2 & 14035728 & 2 & 14035728 & 2 & 14 & 0.057 & 0.7092 & 4.63 \\
\hline 331 & 30756 & 40462 & 7 & 0.01 & 94.04 & 0.033033 & 0.026026 & 2 & 15670974 & 2 & 15981321 & 2 & 15 & 0.055 & 0.7084 & 4.7 \\
\hline 332 & 53436 & 46421 & 19 & 0.02 & 83.13 & 0.091091 & 0.051051 & 2 & 16030402 & 2 & 16770525 & 2 & 16 & 0.048 & 0.7045 & 5.02 \\
\hline 333 & 60918 & 33459 & 16 & 0.01 & 94.12 & 0.071071 & 0.04004 & 2 & 17143182 & 2 & 17903452 & 2 & 17 & 0.055 & 0.7085 & 4.7 \\
\hline 334 & 2047 & 40469 & 40 & 0.05 & 41.21 & 0.173173 & 0.117117 & 2 & 18091227 & 2 & 18926886 & 2 & 18 & 0.022 & 0.6731 & 7.1 \\
\hline 335 & 15309 & 51754 & 25 & 0.07 & 31.33 & 0.132132 & 0.099099 & 2 & 19099121 & 2 & 19991449 & 2 & 19 & 0.017 & 0.6464 & 7.8 \\
\hline 336 & 30990 & 2066 & 26 & 0.03 & 75.75 & 0.123123 & 0.077077 & 2 & 20008168 & 2 & 20975241 & 2 & 20 & 0.043 & 0.7028 & 5.3 \\
\hline 337 & 40478 & 61121 & 25 & 0.03 & 70.69 & 0.117117 & 0.074074 & 2 & 21003130 & 2 & 21970774 & 2 & 21 & 0.039 & 0.7004 & 5.49 \\
\hline 338 & 2077 & 40486 & 21 & 0.03 & 77.3 & 0.089089 & 0.05005 & 2 & 22230468 & 2 & 22996263 & 2 & 22 & 0.044 & 0.7035 & 5.24 \\
\hline 339 & 46425 & 40489 & 21 & 0.03 & 79.01 & 0.094094 & 0.065065 & 2 & 23018675 & 2 & 23936325 & 2 & 23 & 0.045 & 0.7045 & 5.19 \\
\hline 340 & 40490 & 2094 & 19 & 0.01 & 94.51 & 0.08008 & 0.045045 & 2 & 24003403 & 2 & 24998348 & 2 & 24 & 0.055 & 0.7084 & 4.68 \\
\hline 341 & 40493 & 2102 & 31 & 0.05 & 42.66 & 0.156156 & 0.109109 & 2 & 25056011 & 2 & 25977149 & 2 & 25 & 0.023 & 0.676 & 7.01 \\
\hline 342 & 2103 & 2108 & 29 & 0.04 & 52.2 & 0.139139 & 0.088088 & 2 & 26004268 & 2 & 26978443 & 2 & 26 & 0.028 & 0.6898 & 6.4 \\
\hline 343 & 54505 & 60363 & 39 & 0.05 & 39.98 & 0.169169 & 0.106106 & 2 & 27137409 & 2 & 27978087 & 2 & 27 & 0.021 & 0.6703 & 7.17 \\
\hline 344 & 19353 & 48275 & 39 & 0.05 & 41.11 & 0.168168 & 0.11011 & 2 & 28013337 & 2 & 28977392 & 2 & 28 & 0.022 & 0.6727 & 7.1 \\
\hline 345 & 32744 & 20816 & 21 & 0.04 & 61.8 & 0.106106 & 0.067067 & 2 & 29115937 & 2 & 29795889 & 2 & 29 & 0.034 & 0.6966 & 5.9 \\
\hline 346 & 2124 & 14705 & 16 & 0.02 & 87.85 & 0.06006 & 0.034034 & 2 & 30141776 & 2 & 30999341 & 2 & 30 & 0.051 & 0.7078 & 4.9 \\
\hline 347 & 56000 & 53156 & 25 & 0.03 & 79.24 & 0.104104 & 0.065065 & 2 & 31062461 & 2 & 31950516 & 2 & 31 & 0.045 & 0.7047 & 5.19 \\
\hline 348 & 20826 & 2148 & 31 & 0.05 & 44.22 & 0.139139 & 0.095095 & 2 & 32027800 & 2 & 32993535 & 2 & 32 & 0.023 & 0.6786 & 6.9 \\
\hline
\end{tabular}




\begin{tabular}{|c|c|c|c|c|c|c|c|c|c|c|c|c|c|c|c|c|}
\hline 349 & 58230 & 40519 & 22 & 0.02 & 88.42 & 0.105105 & 0.06006 & 2 & 33011231 & 2 & 33982064 & 2 & 33 & 0.051 & 0.7077 & 4.88 \\
\hline 350 & 2153 & 20840 & 18 & 0.02 & 90.74 & 0.072072 & 0.042042 & 2 & 34084545 & 2 & 34977540 & 2 & 34 & 0.053 & 0.7078 & 4.8 \\
\hline 351 & 57275 & 37093 & 25 & 0.07 & 27.77 & 0.13013 & 0.097097 & 2 & 35054445 & 2 & 35962897 & 2 & 35 & 0.015 & 0.6355 & 8.15 \\
\hline 352 & 60206 & 16792 & 22 & 0.03 & 69.08 & 0.099099 & 0.061061 & 2 & 36008750 & 2 & 36919501 & 2 & 36 & 0.038 & 0.6999 & 5.56 \\
\hline 353 & 61746 & 2184 & 22 & 0.08 & 26.64 & 0.12012 & 0.095095 & 2 & 37273764 & 2 & 37988750 & 2 & 37 & 0.014 & 0.631 & 8.27 \\
\hline 354 & 52281 & 40532 & 16 & 0.02 & 83.53 & 0.079079 & 0.047047 & 2 & 38144228 & 2 & 38997845 & 2 & 38 & 0.048 & 0.7051 & 5.02 \\
\hline 355 & 17003 & 29455 & 12 & 0.02 & 91.47 & 0.043043 & 0.026026 & 2 & 39185102 & 2 & 39982124 & 2 & 39 & 0.053 & 0.7083 & 4.79 \\
\hline 356 & 2192 & 48540 & 23 & 0.04 & 63.08 & 0.125125 & 0.087087 & 2 & 40072508 & 2 & 40993644 & 2 & 40 & 0.035 & 0.6965 & 5.82 \\
\hline 357 & 2202 & 2225 & 25 & 0.05 & 40.46 & 0.141141 & 0.097097 & 2 & 41011761 & 2 & 41998239 & 2 & 41 & 0.021 & 0.6715 & 7.14 \\
\hline 358 & 2224 & 2235 & 36 & 0.03 & 76 & 0.146146 & 0.084084 & 2 & 42026841 & 2 & 42989311 & 2 & 42 & 0.043 & 0.7029 & 5.29 \\
\hline 359 & 48542 & 2241 & 33 & 0.05 & 46.27 & 0.149149 & 0.097097 & 2 & 43009789 & 2 & 43985424 & 2 & 43 & 0.025 & 0.6815 & 6.73 \\
\hline 360 & 20868 & 2246 & 38 & 0.08 & 26.95 & 0.168168 & 0.12012 & 2 & 44033531 & 2 & 44984555 & 2 & 44 & 0.014 & 0.6322 & 8.24 \\
\hline 361 & 2248 & 2251 & 19 & 0.03 & 78.67 & 0.088088 & 0.059059 & 2 & 45026774 & 2 & 45986058 & 2 & 45 & 0.044 & 0.7038 & 5.2 \\
\hline 362 & 37107 & 20887 & 18 & 0.02 & 88.56 & 0.072072 & 0.048048 & 2 & 46053572 & 2 & 46987103 & 2 & 46 & 0.051 & 0.7077 & 4.87 \\
\hline 363 & 40549 & 2268 & 15 & 0.01 & 92.76 & 0.055055 & 0.04004 & 2 & 47055959 & 2 & 47984857 & 2 & 47 & 0.054 & 0.7081 & 4.74 \\
\hline 364 & 2269 & 53385 & 17 & 0.01 & 93.1 & 0.059059 & 0.034034 & 2 & 48125078 & 2 & 48927541 & 2 & 48 & 0.054 & 0.7081 & 4.73 \\
\hline 365 & 37111 & 30623 & 20 & 0.02 & 91.44 & 0.083083 & 0.047047 & 2 & 49185691 & 2 & 49903368 & 2 & 49 & 0.053 & 0.7083 & 4.79 \\
\hline 366 & 32305 & 31301 & 24 & 0.03 & 71.26 & 0.123123 & 0.081081 & 2 & 50036228 & 2 & 50999217 & 2 & 50 & 0.04 & 0.7007 & 5.47 \\
\hline 367 & 49893 & 46435 & 23 & 0.03 & 75.81 & 0.088088 & 0.053053 & 2 & 51003535 & 2 & 51995031 & 2 & 51 & 0.043 & 0.703 & 5.3 \\
\hline 368 & 2287 & 57780 & 10 & 0 & 96.28 & 0.043043 & 0.024024 & 2 & 52013286 & 2 & 52674728 & 2 & 52 & 0.057 & 0.7091 & 4.64 \\
\hline 369 & 55296 & 18057 & 7 & 0.01 & 95.1 & 0.031031 & 0.021021 & 2 & 53067453 & 2 & 53907735 & 2 & 53 & 0.056 & 0.7087 & 4.67 \\
\hline 370 & 2328 & 16864 & 4 & 0 & 96.55 & 0.013013 & 0.006006 & 2 & 54381571 & 2 & 54881273 & 2 & 54 & 0.057 & 0.7093 & 4.63 \\
\hline 371 & 16909 & 58969 & 7 & 0 & 96.34 & 0.021021 & 0.014014 & 2 & 55200111 & 2 & 55894230 & 2 & 55 & 0.057 & 0.7091 & 4.64 \\
\hline 372 & 60890 & 32196 & 3 & 0 & 96.41 & 0.014014 & 0.009009 & 2 & 56469735 & 2 & 56784798 & 2 & 56 & 0.057 & 0.7092 & 4.64 \\
\hline 373 & 59106 & 20917 & 11 & 0.01 & 95.54 & 0.046046 & 0.022022 & 2 & 57011989 & 2 & 57924527 & 2 & 57 & 0.056 & 0.7087 & 4.66 \\
\hline 374 & 57638 & 2321 & 9 & 0.01 & 93.34 & 0.032032 & 0.02002 & 2 & 58377827 & 2 & 58871412 & 2 & 58 & 0.055 & 0.7081 & 4.72 \\
\hline 375 & 53440 & 20913 & 10 & 0.01 & 95.77 & 0.051051 & 0.026026 & 2 & 59243470 & 2 & 59957369 & 2 & 59 & 0.056 & 0.7089 & 4.66 \\
\hline 376 & 20912 & 54049 & 17 & 0.01 & 94.32 & 0.045045 & 0.031031 & 2 & 60022633 & 2 & 60953615 & 2 & 60 & 0.055 & 0.7083 & 4.69 \\
\hline 377 & 36445 & 2314 & 5 & 0 & 96.47 & 0.017017 & 0.012012 & 2 & 61161245 & 2 & 61822605 & 2 & 61 & 0.057 & 0.7092 & 4.63 \\
\hline 378 & 2313 & 57106 & 4 & 0 & 96.56 & 0.016016 & 0.003003 & 2 & 62015620 & 2 & 62521678 & 2 & 62 & 0.057 & 0.7093 & 4.63 \\
\hline 379 & 14713 & 14713 & 1 & 0 & 96.52 & 0.003003 & 0.002002 & 2 & 64970665 & 2 & 64970665 & 2 & 64 & 0.057 & 0.7092 & 4.63 \\
\hline 380 & 32083 & 48550 & 13 & 0.01 & 94.06 & 0.051051 & 0.034034 & 2 & 65057680 & 2 & 65994755 & 2 & 65 & 0.055 & 0.7084 & 4.7 \\
\hline 381 & 2307 & 37117 & 15 & 0.01 & 93.98 & 0.05005 & 0.026026 & 2 & 66048253 & 2 & 66776495 & 2 & 66 & 0.055 & 0.7085 & 4.7 \\
\hline 382 & 51768 & 51939 & 9 & 0.02 & 91.49 & 0.042042 & 0.029029 & 2 & 67308766 & 2 & 67981307 & 2 & 67 & 0.053 & 0.7082 & 4.79 \\
\hline 383 & 2301 & 35618 & 3 & 0 & 96.25 & 0.017017 & 0.012012 & 2 & 68894359 & 2 & 68965440 & 2 & 68 & 0.057 & 0.7091 & 4.64 \\
\hline 384 & 2300 & 32476 & 11 & 0.01 & 95.84 & 0.047047 & 0.022022 & 2 & 69103051 & 2 & 69823989 & 2 & 69 & 0.056 & 0.7089 & 4.65 \\
\hline 385 & 50904 & 37114 & 9 & 0 & 96.51 & 0.032032 & 0.009009 & 2 & 70061497 & 2 & 70869578 & 2 & 70 & 0.057 & 0.7092 & 4.63 \\
\hline 386 & 37115 & 29432 & 4 & 0.01 & 96.14 & 0.014014 & 0.012012 & 2 & 71036422 & 2 & 71990984 & 2 & 71 & 0.057 & 0.709 & 4.64 \\
\hline 387 & 15406 & 34389 & 8 & 0 & 96.48 & 0.037037 & 0.014014 & 2 & 72042065 & 2 & 72956647 & 2 & 72 & 0.057 & 0.7092 & 4.63 \\
\hline
\end{tabular}




\begin{tabular}{|c|c|c|c|c|c|c|c|c|c|c|c|c|c|c|c|c|}
\hline 388 & 51705 & 53572 & 2 & 0 & 96.56 & 0.011011 & 0.002002 & 2 & 73076347 & 2 & 73385525 & 2 & 73 & 0.057 & 0.7093 & 4.63 \\
\hline 389 & 55233 & 60504 & 10 & 0.01 & 92.75 & 0.045045 & 0.023023 & 2 & 74063668 & 2 & 74917715 & 2 & 74 & 0.054 & 0.7081 & 4.74 \\
\hline 390 & 49816 & 15876 & 7 & 0.01 & 95.11 & 0.019019 & 0.013013 & 2 & 75198782 & 2 & 75900120 & 2 & 75 & 0.056 & 0.7087 & 4.67 \\
\hline 391 & 53479 & 54937 & 7 & 0.01 & 95.55 & 0.045045 & 0.02002 & 2 & 76037122 & 2 & 76667109 & 2 & 76 & 0.056 & 0.7088 & 4.66 \\
\hline 392 & 61052 & 14515 & 4 & 0 & 96.33 & 0.017017 & 0.01001 & 2 & 77011684 & 2 & 77635095 & 2 & 77 & 0.057 & 0.7091 & 4.64 \\
\hline 393 & 51163 & 51163 & 1 & 0 & 96.56 & 0 & 0 & 2 & 78174324 & 2 & 78174324 & 2 & 78 & 0.057 & 0.7093 & 4.63 \\
\hline 394 & 56802 & 58682 & 2 & 0 & 96.56 & 0.005005 & 0.002002 & 2 & 79296283 & 2 & 79772013 & 2 & 79 & 0.057 & 0.7093 & 4.63 \\
\hline 395 & 54741 & 29454 & 12 & 0.01 & 94.67 & 0.057057 & 0.032032 & 2 & 80129606 & 2 & 80974584 & 2 & 80 & 0.056 & 0.7086 & 4.68 \\
\hline 396 & 55142 & 40579 & 16 & 0.02 & 83.87 & 0.068068 & 0.043043 & 2 & 81034415 & 2 & 81845545 & 2 & 81 & 0.048 & 0.7053 & 5.01 \\
\hline 397 & 20925 & 18204 & 16 & 0.03 & 77.17 & 0.075075 & 0.052052 & 2 & 82002123 & 2 & 82969323 & 2 & 82 & 0.044 & 0.7034 & 5.25 \\
\hline 398 & 53826 & 37120 & 18 & 0.02 & 83.02 & 0.088088 & 0.05005 & 2 & 83080749 & 2 & 83985760 & 2 & 83 & 0.048 & 0.7045 & 5.03 \\
\hline 399 & 20929 & 37124 & 19 & 0.03 & 78.38 & 0.088088 & 0.06006 & 2 & 84026553 & 2 & 84987807 & 2 & 84 & 0.044 & 0.7041 & 5.21 \\
\hline 400 & 37125 & 40589 & 30 & 0.03 & 78.22 & 0.108108 & 0.063063 & 2 & 85036318 & 2 & 85956326 & 2 & 85 & 0.044 & 0.704 & 5.22 \\
\hline 401 & 2345 & 20942 & 20 & 0.02 & 89.35 & 0.083083 & 0.055055 & 2 & 86090869 & 2 & 86970329 & 2 & 86 & 0.052 & 0.7076 & 4.84 \\
\hline 402 & 48557 & 39685 & 32 & 0.04 & 57.12 & 0.131131 & 0.089089 & 2 & 87007147 & 2 & 87981729 & 2 & 87 & 0.031 & 0.692 & 6.09 \\
\hline 403 & 40599 & 2362 & 26 & 0.03 & 75.06 & 0.102102 & 0.068068 & 2 & 88090537 & 2 & 88987913 & 2 & 88 & 0.042 & 0.7021 & 5.31 \\
\hline 404 & 37134 & 2376 & 30 & 0.04 & 59.39 & 0.131131 & 0.084084 & 2 & 89007639 & 2 & 89995349 & 2 & 89 & 0.033 & 0.6943 & 5.99 \\
\hline 405 & 2374 & 40607 & 27 & 0.05 & 40.79 & 0.134134 & 0.098098 & 2 & 90053305 & 2 & 90971450 & 2 & 90 & 0.022 & 0.6719 & 7.12 \\
\hline 406 & 37135 & 40611 & 22 & 0.04 & 59.09 & 0.094094 & 0.063063 & 2 & 91003962 & 2 & 91992594 & 2 & 91 & 0.032 & 0.6941 & 6 \\
\hline 407 & 2388 & 32193 & 23 & 0.07 & 29.57 & 0.117117 & 0.089089 & 2 & 92017036 & 2 & 92965042 & 2 & 92 & 0.016 & 0.6412 & 7.96 \\
\hline 408 & 15455 & 37141 & 28 & 0.08 & 25.56 & 0.135135 & 0.095095 & 2 & 93152911 & 2 & 93992622 & 2 & 93 & 0.013 & 0.6292 & 8.47 \\
\hline 409 & 2403 & 46447 & 13 & 0.03 & 77.36 & 0.071071 & 0.043043 & 2 & 94085488 & 2 & 94631894 & 2 & 94 & 0.044 & 0.7035 & 5.24 \\
\hline 410 & 61803 & 53104 & 7 & 0.02 & 83.71 & 0.037037 & 0.022022 & 2 & 95014748 & 2 & 95957069 & 2 & 95 & 0.048 & 0.7053 & 5.01 \\
\hline 411 & 56707 & 20980 & 18 & 0.04 & 63.44 & 0.085085 & 0.063063 & 2 & 96053472 & 2 & 96992420 & 2 & 96 & 0.035 & 0.6971 & 5.8 \\
\hline 412 & 40618 & 2422 & 18 & 0.03 & 68.22 & 0.083083 & 0.052052 & 2 & 97005674 & 2 & 97884804 & 2 & 97 & 0.038 & 0.6992 & 5.59 \\
\hline 413 & 46449 & 2427 & 20 & 0.04 & 62.28 & 0.082082 & 0.059059 & 2 & 98002439 & 2 & 98997793 & 2 & 98 & 0.034 & 0.6963 & 5.86 \\
\hline 414 & 54043 & 58908 & 11 & 0.01 & 95.44 & 0.062062 & 0.033033 & 2 & 99022293 & 2 & 99964100 & 2 & 99 & 0.056 & 0.7087 & 4.66 \\
\hline 415 & 40621 & 40624 & 18 & 0.02 & 88.21 & 0.072072 & 0.053053 & 2 & 100248260 & 2 & 100951727 & 2 & 100 & 0.051 & 0.7077 & 4.89 \\
\hline 416 & 17209 & 58078 & 20 & 0.02 & 82.67 & 0.096096 & 0.062062 & 2 & 101065109 & 2 & 101995816 & 2 & 101 & 0.047 & 0.7047 & 5.05 \\
\hline 417 & 40627 & 2448 & 15 & 0.03 & 77.74 & 0.074074 & 0.046046 & 2 & 102047786 & 2 & 102986466 & 2 & 102 & 0.044 & 0.7037 & 5.23 \\
\hline 418 & 34648 & 46451 & 21 & 0.03 & 75.21 & 0.08008 & 0.052052 & 2 & 103008035 & 2 & 103803677 & 2 & 103 & 0.042 & 0.7022 & 5.31 \\
\hline 419 & 52106 & 36463 & 13 & 0.02 & 84.49 & 0.059059 & 0.038038 & 2 & 104256453 & 2 & 104830050 & 2 & 104 & 0.049 & 0.7054 & 4.98 \\
\hline 420 & 17253 & 61777 & 17 & 0.02 & 86.55 & 0.084084 & 0.06006 & 2 & 105029085 & 2 & 105980680 & 2 & 105 & 0.05 & 0.707 & 4.94 \\
\hline 421 & 17823 & 55280 & 18 & 0.02 & 90.49 & 0.054054 & 0.034034 & 2 & 106206301 & 2 & 106982661 & 2 & 106 & 0.053 & 0.7078 & 4.81 \\
\hline 422 & 31987 & 21011 & 26 & 0.04 & 57.56 & 0.119119 & 0.082082 & 2 & 107011848 & 2 & 107975061 & 2 & 107 & 0.032 & 0.6923 & 6.06 \\
\hline 423 & 40633 & 55037 & 20 & 0.02 & 87.71 & 0.079079 & 0.05005 & 2 & 108005632 & 2 & 108954634 & 2 & 108 & 0.051 & 0.7077 & 4.9 \\
\hline 424 & 35153 & 21019 & 21 & 0.03 & 66.18 & 0.088088 & 0.058058 & 2 & 109012528 & 2 & 109934488 & 2 & 109 & 0.037 & 0.6988 & 5.68 \\
\hline 425 & 37172 & 37174 & 22 & 0.03 & 75.26 & 0.085085 & 0.058058 & 2 & 110005387 & 2 & 110955851 & 2 & 110 & 0.042 & 0.7022 & 5.31 \\
\hline 426 & 60980 & 37178 & 27 & 0.02 & 86.87 & 0.103103 & 0.069069 & 2 & 111019898 & 2 & 111947482 & 2 & 111 & 0.05 & 0.7074 & 4.93 \\
\hline
\end{tabular}




\begin{tabular}{|c|c|c|c|c|c|c|c|c|c|c|c|c|c|c|c|c|}
\hline 427 & 2506 & 2509 & 16 & 0.02 & 82.76 & 0.078078 & 0.046046 & 2 & 112079833 & 2 & 112559732 & 2 & 112 & 0.047 & 0.7047 & 5.04 \\
\hline 428 & 40638 & 2524 & 22 & 0.03 & 76.12 & 0.092092 & 0.065065 & 2 & 113003444 & 2 & 113985753 & 2 & 113 & 0.043 & 0.7027 & 5.28 \\
\hline 429 & 46459 & 37185 & 15 & 0.01 & 94.95 & 0.051051 & 0.024024 & 2 & 114047843 & 2 & 114975303 & 2 & 114 & 0.056 & 0.7087 & 4.68 \\
\hline 430 & 37184 & 2534 & 12 & 0.02 & 90.93 & 0.06006 & 0.042042 & 2 & 115000618 & 2 & 115815947 & 2 & 115 & 0.053 & 0.7081 & 4.8 \\
\hline 431 & 2535 & 21047 & 19 & 0.02 & 86.34 & 0.075075 & 0.051051 & 2 & 116053045 & 2 & 116939471 & 2 & 116 & 0.05 & 0.707 & 4.95 \\
\hline 432 & 2541 & 2549 & 27 & 0.05 & 39.7 & 0.135135 & 0.099099 & 2 & 117038311 & 2 & 117962577 & 2 & 117 & 0.021 & 0.6692 & 7.17 \\
\hline 433 & 40646 & 2557 & 27 & 0.05 & 47.87 & 0.131131 & 0.089089 & 2 & 118010721 & 2 & 118934685 & 2 & 118 & 0.026 & 0.6819 & 6.59 \\
\hline 434 & 40657 & 2564 & 22 & 0.03 & 74.42 & 0.101101 & 0.068068 & 2 & 119044783 & 2 & 119970583 & 2 & 119 & 0.042 & 0.7018 & 5.33 \\
\hline 435 & 56294 & 2570 & 17 & 0.05 & 48.15 & 0.077077 & 0.054054 & 2 & 120009260 & 2 & 120974703 & 2 & 120 & 0.026 & 0.6823 & 6.57 \\
\hline 436 & 21064 & 21074 & 36 & 0.03 & 71.95 & 0.155155 & 0.082082 & 2 & 121032595 & 2 & 121991931 & 2 & 121 & 0.04 & 0.7012 & 5.44 \\
\hline 437 & 40665 & 2591 & 27 & 0.04 & 50.44 & 0.125125 & 0.087087 & 2 & 122010013 & 2 & 122886290 & 2 & 122 & 0.027 & 0.6866 & 6.48 \\
\hline 438 & 21082 & 32175 & 23 & 0.02 & 90.75 & 0.099099 & 0.053053 & 2 & 123010574 & 2 & 123982380 & 2 & 123 & 0.053 & 0.7078 & 4.8 \\
\hline 439 & 15493 & 54534 & 31 & 0.05 & 44.57 & 0.119119 & 0.086086 & 2 & 124021778 & 2 & 124984584 & 2 & 124 & 0.024 & 0.6795 & 6.88 \\
\hline 440 & 17984 & 2606 & 32 & 0.03 & 72.01 & 0.128128 & 0.088088 & 2 & 125009891 & 2 & 125989308 & 2 & 125 & 0.04 & 0.7011 & 5.44 \\
\hline 441 & 2607 & 45560 & 20 & 0.01 & 93.87 & 0.064064 & 0.036036 & 2 & 126022431 & 2 & 126984010 & 2 & 126 & 0.055 & 0.7083 & 4.7 \\
\hline 442 & 53661 & 18498 & 28 & 0.03 & 74.19 & 0.122122 & 0.078078 & 2 & 127056884 & 2 & 127964422 & 2 & 127 & 0.042 & 0.7021 & 5.35 \\
\hline 443 & 54670 & 46468 & 25 & 0.02 & 84.56 & 0.108108 & 0.057057 & 2 & 128229377 & 2 & 128982107 & 2 & 128 & 0.049 & 0.7055 & 4.98 \\
\hline 444 & 2621 & 17641 & 25 & 0.01 & 92.57 & 0.094094 & 0.049049 & 2 & 129003155 & 2 & 129999370 & 2 & 129 & 0.054 & 0.7081 & 4.75 \\
\hline 445 & 52718 & 51230 & 14 & 0.02 & 90.01 & 0.074074 & 0.052052 & 2 & 130043084 & 2 & 130977850 & 2 & 130 & 0.052 & 0.7077 & 4.83 \\
\hline 446 & 32191 & 2639 & 12 & 0.01 & 94.81 & 0.058058 & 0.036036 & 2 & 131005861 & 2 & 131999506 & 2 & 131 & 0.056 & 0.7085 & 4.68 \\
\hline 447 & 21116 & 2642 & 20 & 0.02 & 86.19 & 0.082082 & 0.061061 & 2 & 132012813 & 2 & 132997331 & 2 & 132 & 0.049 & 0.7071 & 4.95 \\
\hline 448 & 21120 & 16166 & 31 & 0.06 & 36.71 & 0.145145 & 0.097097 & 2 & 133019436 & 2 & 133937811 & 2 & 133 & 0.02 & 0.6618 & 7.37 \\
\hline 449 & 34248 & 31355 & 24 & 0.05 & 46.51 & 0.12012 & 0.072072 & 2 & 134056711 & 2 & 134992155 & 2 & 134 & 0.025 & 0.6813 & 6.71 \\
\hline 450 & 32857 & 29616 & 33 & 0.07 & 28.43 & 0.163163 & 0.11011 & 2 & 135007186 & 2 & 135935412 & 2 & 135 & 0.015 & 0.6373 & 8.07 \\
\hline 451 & 30625 & 59596 & 26 & 0.03 & 66.8 & 0.096096 & 0.062062 & 2 & 136043108 & 2 & 136947901 & 2 & 136 & 0.037 & 0.6992 & 5.66 \\
\hline 452 & 2669 & 21148 & 31 & 0.08 & 26.8 & 0.159159 & 0.109109 & 2 & 137046877 & 2 & 137986855 & 2 & 137 & 0.014 & 0.6318 & 8.25 \\
\hline 453 & 55412 & 46472 & 30 & 0.04 & 61.54 & 0.111111 & 0.073073 & 2 & 138000735 & 2 & 138911144 & 2 & 138 & 0.034 & 0.6962 & 5.9 \\
\hline 454 & 2694 & 40699 & 23 & 0.03 & 68.75 & 0.088088 & 0.056056 & 2 & 139003438 & 2 & 139989204 & 2 & 139 & 0.038 & 0.7001 & 5.58 \\
\hline 455 & 2702 & 21167 & 33 & 0.04 & 57.32 & 0.127127 & 0.085085 & 2 & 140002186 & 2 & 140996142 & 2 & 140 & 0.031 & 0.6923 & 6.08 \\
\hline 456 & 2711 & 31847 & 48 & 0.04 & 51.1 & 0.226226 & 0.117117 & 2 & 141030437 & 2 & 141983114 & 2 & 141 & 0.028 & 0.688 & 6.45 \\
\hline 457 & 17525 & 2723 & 27 & 0.02 & 80.64 & 0.12012 & 0.078078 & 2 & 142053892 & 2 & 142924314 & 2 & 142 & 0.046 & 0.7052 & 5.13 \\
\hline 458 & 14862 & 29926 & 33 & 0.04 & 64.76 & 0.127127 & 0.088088 & 2 & 143053245 & 2 & 143991472 & 2 & 143 & 0.036 & 0.698 & 5.75 \\
\hline 459 & 16843 & 31979 & 39 & 0.09 & 23.24 & 0.179179 & 0.128128 & 2 & 144000942 & 2 & 144958040 & 2 & 144 & 0.012 & 0.6214 & 8.84 \\
\hline 460 & 31325 & 35487 & 31 & 0.03 & 76.89 & 0.123123 & 0.067067 & 2 & 145012848 & 2 & 145963826 & 2 & 145 & 0.043 & 0.7034 & 5.26 \\
\hline 461 & 2734 & 18269 & 24 & 0.02 & 84.87 & 0.098098 & 0.063063 & 2 & 146082066 & 2 & 146937364 & 2 & 146 & 0.049 & 0.7061 & 4.98 \\
\hline 462 & 36434 & 36220 & 15 & 0.04 & 56.15 & 0.071071 & 0.046046 & 2 & 147103000 & 2 & 147952931 & 2 & 147 & 0.031 & 0.6919 & 6.15 \\
\hline 463 & 52616 & 37243 & 13 & 0.04 & 50.13 & 0.063063 & 0.044044 & 2 & 148010312 & 2 & 148847293 & 2 & 148 & 0.027 & 0.6863 & 6.49 \\
\hline 464 & 2743 & 32331 & 22 & 0.04 & 59.74 & 0.103103 & 0.067067 & 2 & 149106718 & 2 & 149987258 & 2 & 149 & 0.033 & 0.6953 & 5.98 \\
\hline 465 & 17098 & 31420 & 40 & 0.06 & 31.65 & 0.185185 & 0.13013 & 2 & 150000460 & 2 & 150931547 & 2 & 150 & 0.017 & 0.6469 & 7.77 \\
\hline
\end{tabular}




\begin{tabular}{|c|c|c|c|c|c|c|c|c|c|c|c|c|c|c|c|c|}
\hline 466 & 19201 & 59062 & 29 & 0.05 & 46.32 & 0.135135 & 0.083083 & 2 & 151012311 & 2 & 151909224 & 2 & 151 & 0.025 & 0.6815 & 6.73 \\
\hline 467 & 2756 & 2765 & 26 & 0.04 & 50.3 & 0.132132 & 0.084084 & 2 & 152016160 & 2 & 152972957 & 2 & 152 & 0.027 & 0.6863 & 6.48 \\
\hline 468 & 21211 & 33815 & 32 & 0.05 & 48.48 & 0.135135 & 0.081081 & 2 & 153002555 & 2 & 153940974 & 2 & 153 & 0.026 & 0.6829 & 6.55 \\
\hline 469 & 35662 & 33975 & 39 & 0.12 & 18.11 & 0.21021 & 0.146146 & 2 & 154040051 & 2 & 154980475 & 2 & 154 & 0.009 & 0.5923 & 9.51 \\
\hline 470 & 33029 & 32699 & 39 & 0.06 & 35.46 & 0.155155 & 0.108108 & 2 & 155011592 & 2 & 155939920 & 2 & 155 & 0.019 & 0.6566 & 7.43 \\
\hline 471 & 57006 & 61257 & 26 & 0.05 & 44.67 & 0.11011 & 0.079079 & 2 & 156071705 & 2 & 156982877 & 2 & 156 & 0.024 & 0.6798 & 6.87 \\
\hline 472 & 18404 & 2779 & 37 & 0.06 & 37.06 & 0.176176 & 0.124124 & 2 & 157004735 & 2 & 157957344 & 2 & 157 & 0.02 & 0.6628 & 7.34 \\
\hline 473 & 48589 & 2786 & 11 & 0.01 & 94.29 & 0.046046 & 0.036036 & 2 & 158014368 & 2 & 158680517 & 2 & 158 & 0.055 & 0.7083 & 4.69 \\
\hline 474 & 17340 & 17340 & 1 & 0 & 96.56 & 0.002002 & 0.001001 & 2 & 159250446 & 2 & 159250446 & 2 & 159 & 0.057 & 0.7093 & 4.63 \\
\hline 475 & 52115 & 18291 & 3 & 0 & 96.3 & 0.012012 & 0.008008 & 2 & 160085066 & 2 & 160434997 & 2 & 160 & 0.057 & 0.7091 & 4.64 \\
\hline 476 & 55507 & 55507 & 1 & 0 & 96.56 & 0.002002 & 0.001001 & 2 & 161880071 & 2 & 161880071 & 2 & 161 & 0.057 & 0.7093 & 4.63 \\
\hline 477 & 16695 & 30004 & 5 & 0.01 & 95.17 & 0.03003 & 0.019019 & 2 & 162084552 & 2 & 162298086 & 2 & 162 & 0.056 & 0.7086 & 4.67 \\
\hline 478 & 52757 & 29919 & 3 & 0 & 96.49 & 0.013013 & 0.006006 & 3 & 72705 & 3 & 715273 & 3 & 0 & 0.057 & 0.7092 & 4.63 \\
\hline 479 & 45521 & 15661 & 8 & 0 & 96.26 & 0.022022 & 0.014014 & 3 & 1456146 & 3 & 1997344 & 3 & 1 & 0.057 & 0.7091 & 4.64 \\
\hline 480 & 32077 & 61938 & 16 & 0.02 & 91.7 & 0.06006 & 0.039039 & 3 & 2013852 & 3 & 2992936 & 3 & 2 & 0.053 & 0.7081 & 4.78 \\
\hline 481 & 37250 & 33526 & 27 & 0.04 & 59.97 & 0.122122 & 0.068068 & 3 & 3029529 & 3 & 3936783 & 3 & 3 & 0.033 & 0.6955 & 5.97 \\
\hline 482 & 17466 & 16986 & 17 & 0.03 & 75.29 & 0.096096 & 0.068068 & 3 & 4032373 & 3 & 4980011 & 3 & 4 & 0.042 & 0.7022 & 5.31 \\
\hline 483 & 52620 & 48594 & 33 & 0.17 & 13.61 & 0.189189 & 0.138138 & 3 & 5004645 & 3 & 5897010 & 3 & 5 & 0.007 & 0.5659 & 10.66 \\
\hline 484 & 2800 & 54900 & 19 & 0.63 & 6.13 & 0.192192 & 0.17017 & 3 & 6011282 & 3 & 6591276 & 3 & 6 & 0.004 & 0.4895 & 12.6 \\
\hline 485 & 33489 & 35827 & 10 & 0.01 & 91.94 & 0.044044 & 0.027027 & 3 & 7099006 & 3 & 7846008 & 3 & 7 & 0.053 & 0.7081 & 4.77 \\
\hline 486 & 55141 & 60330 & 11 & 0.02 & 90.97 & 0.042042 & 0.022022 & 3 & 8021716 & 3 & 8941384 & 3 & 8 & 0.053 & 0.708 & 4.8 \\
\hline 487 & 59773 & 31966 & 23 & 0.04 & 51.98 & 0.101101 & 0.063063 & 3 & 9000666 & 3 & 9962466 & 3 & 9 & 0.028 & 0.689 & 6.41 \\
\hline 488 & 31616 & 31091 & 27 & 0.13 & 16.74 & 0.163163 & 0.132132 & 3 & 10004418 & 3 & 10973478 & 3 & 10 & 0.009 & 0.5836 & 9.76 \\
\hline 489 & 52392 & 2817 & 36 & 0.05 & 40.36 & 0.163163 & 0.111111 & 3 & 11054114 & 3 & 11960567 & 3 & 11 & 0.021 & 0.6715 & 7.15 \\
\hline 490 & 62076 & 51843 & 27 & 0.17 & 13.77 & 0.169169 & 0.129129 & 3 & 12001168 & 3 & 12944285 & 3 & 12 & 0.007 & 0.5675 & 10.63 \\
\hline 491 & 31278 & 2825 & 21 & 0.06 & 32.36 & 0.12012 & 0.073073 & 3 & 13040547 & 3 & 13922837 & 3 & 13 & 0.017 & 0.6502 & 7.74 \\
\hline 492 & 37254 & 55645 & 38 & 0.04 & 58.27 & 0.175175 & 0.103103 & 3 & 14007265 & 3 & 14998247 & 3 & 14 & 0.032 & 0.6932 & 6.03 \\
\hline 493 & 40755 & 18076 & 26 & 0.05 & 47.83 & 0.134134 & 0.09009 & 3 & 15010975 & 3 & 15925307 & 3 & 15 & 0.026 & 0.6821 & 6.59 \\
\hline 494 & 45465 & 19141 & 19 & 0.02 & 82.05 & 0.073073 & 0.04004 & 3 & 16029880 & 3 & 16920601 & 3 & 16 & 0.047 & 0.7049 & 5.08 \\
\hline 495 & 19363 & 40764 & 16 & 0.02 & 91.19 & 0.067067 & 0.043043 & 3 & 17091063 & 3 & 17859317 & 3 & 17 & 0.053 & 0.708 & 4.79 \\
\hline 496 & 31870 & 50533 & 20 & 0.03 & 76.65 & 0.092092 & 0.049049 & 3 & 18042146 & 3 & 18985624 & 3 & 18 & 0.043 & 0.703 & 5.26 \\
\hline 497 & 35761 & 2845 & 27 & 0.04 & 62.21 & 0.14014 & 0.09009 & 3 & 19077421 & 3 & 19951828 & 3 & 19 & 0.034 & 0.6962 & 5.87 \\
\hline 498 & 31622 & 21277 & 32 & 0.04 & 64.86 & 0.131131 & 0.075075 & 3 & 20058173 & 3 & 20983778 & 3 & 20 & 0.036 & 0.6979 & 5.74 \\
\hline 499 & 2850 & 31100 & 26 & 0.02 & 87.15 & 0.114114 & 0.062062 & 3 & 21010393 & 3 & 21911837 & 3 & 21 & 0.05 & 0.7076 & 4.92 \\
\hline 500 & 30480 & 2858 & 14 & 0.02 & 88.73 & 0.066066 & 0.045045 & 3 & 22538991 & 3 & 22983641 & 3 & 22 & 0.051 & 0.7077 & 4.87 \\
\hline 501 & 2859 & 31107 & 27 & 0.07 & 29.78 & 0.123123 & 0.084084 & 3 & 23015607 & 3 & 23987557 & 3 & 23 & 0.016 & 0.6415 & 7.93 \\
\hline 502 & 33625 & 15928 & 25 & 0.05 & 45.93 & 0.131131 & 0.093093 & 3 & 24010664 & 3 & 24976420 & 3 & 24 & 0.025 & 0.6813 & 6.77 \\
\hline 503 & 19654 & 31842 & 23 & 0.03 & 66.9 & 0.097097 & 0.054054 & 3 & 25018550 & 3 & 25990903 & 3 & 25 & 0.037 & 0.6992 & 5.65 \\
\hline 504 & 61170 & 18828 & 35 & 0.05 & 47.78 & 0.152152 & 0.102102 & 3 & 26002460 & 3 & 26978899 & 3 & 26 & 0.026 & 0.6821 & 6.6 \\
\hline
\end{tabular}




\begin{tabular}{|c|c|c|c|c|c|c|c|c|c|c|c|c|c|c|c|c|}
\hline 505 & 29633 & 21295 & 28 & 0.03 & 67.21 & 0.125125 & 0.074074 & 3 & 27041644 & 3 & 27988181 & 3 & 27 & 0.037 & 0.6991 & 5.64 \\
\hline 506 & 40772 & 2885 & 26 & 0.02 & 86.42 & 0.121121 & 0.061061 & 3 & 28053055 & 3 & 28995377 & 3 & 28 & 0.05 & 0.707 & 4.94 \\
\hline 507 & 15353 & 15494 & 29 & 0.03 & 79.5 & 0.125125 & 0.069069 & 3 & 29024154 & 3 & 29894909 & 3 & 29 & 0.045 & 0.705 & 5.18 \\
\hline 508 & 2890 & 30765 & 29 & 0.02 & 85.06 & 0.129129 & 0.071071 & 3 & 30021862 & 3 & 30988651 & 3 & 30 & 0.049 & 0.7062 & 4.98 \\
\hline 509 & 15333 & 51401 & 26 & 0.03 & 69.89 & 0.114114 & 0.066066 & 3 & 31012875 & 3 & 31931466 & 3 & 31 & 0.039 & 0.7 & 5.53 \\
\hline 510 & 34006 & 17010 & 21 & 0.03 & 73.53 & 0.081081 & 0.057057 & 3 & 32058308 & 3 & 32920176 & 3 & 32 & 0.041 & 0.7019 & 5.37 \\
\hline 511 & 30935 & 2918 & 18 & 0.02 & 86.32 & 0.082082 & 0.054054 & 3 & 33145490 & 3 & 33947247 & 3 & 33 & 0.05 & 0.707 & 4.95 \\
\hline 512 & 51158 & 48617 & 23 & 0.04 & 51.59 & 0.104104 & 0.077077 & 3 & 34016624 & 3 & 34989088 & 3 & 34 & 0.028 & 0.689 & 6.43 \\
\hline 513 & 2924 & 2927 & 22 & 0.05 & 49.76 & 0.103103 & 0.07007 & 3 & 35024144 & 3 & 35978570 & 3 & 35 & 0.027 & 0.6858 & 6.51 \\
\hline 514 & 40785 & 45456 & 24 & 0.06 & 32.55 & 0.118118 & 0.086086 & 3 & 36026450 & 3 & 36894931 & 3 & 36 & 0.017 & 0.65 & 7.71 \\
\hline 515 & 58503 & 55385 & 21 & 0.02 & 85.77 & 0.072072 & 0.048048 & 3 & 37023012 & 3 & 37919636 & 3 & 37 & 0.049 & 0.7064 & 4.95 \\
\hline 516 & 51371 & 15903 & 6 & 0 & 96.48 & 0.026026 & 0.009009 & 3 & 38091792 & 3 & 38949614 & 3 & 38 & 0.057 & 0.7092 & 4.63 \\
\hline 517 & 56924 & 48619 & 8 & 0.02 & 85.94 & 0.039039 & 0.033033 & 3 & 39128984 & 3 & 39992614 & 3 & 39 & 0.049 & 0.7066 & 4.95 \\
\hline 518 & 53025 & 45565 & 6 & 0.02 & 87.47 & 0.037037 & 0.028028 & 3 & 40003536 & 3 & 40461817 & 3 & 40 & 0.05 & 0.7076 & 4.91 \\
\hline 519 & 48621 & 48622 & 3 & 0 & 96.25 & 0.011011 & 0.008008 & 3 & 41738237 & 3 & 41843056 & 3 & 41 & 0.057 & 0.7091 & 4.64 \\
\hline 520 & 15813 & 2940 & 2 & 0 & 96.56 & 0.006006 & 0 & 3 & 42047929 & 3 & 42096113 & 3 & 42 & 0.057 & 0.7093 & 4.63 \\
\hline 521 & 2941 & 21334 & 3 & 0 & 96.55 & 0.008008 & 0.005005 & 3 & 43240931 & 3 & 43336137 & 3 & 43 & 0.057 & 0.7093 & 4.63 \\
\hline 522 & 34570 & 395 & 7 & 0.01 & 93.82 & 0.037037 & 0.023023 & 3 & 44138379 & 3 & 44725638 & 3 & 44 & 0.055 & 0.7082 & 4.71 \\
\hline 523 & 34532 & 46478 & 7 & 0 & 96.32 & 0.01001 & 0.005005 & 3 & 45453887 & 3 & 45994096 & 3 & 45 & 0.057 & 0.709 & 4.64 \\
\hline 524 & 16651 & 31263 & 8 & 0.01 & 95.9 & 0.029029 & 0.015015 & 3 & 46206833 & 3 & 46893143 & 3 & 46 & 0.056 & 0.7088 & 4.65 \\
\hline 525 & 59494 & 2950 & 16 & 0.02 & 87.43 & 0.065065 & 0.049049 & 3 & 47040078 & 3 & 47979489 & 3 & 47 & 0.05 & 0.7076 & 4.91 \\
\hline 526 & 40799 & 15457 & 19 & 0.02 & 89.56 & 0.067067 & 0.038038 & 3 & 48022855 & 3 & 48924957 & 3 & 48 & 0.052 & 0.7077 & 4.84 \\
\hline 527 & 21348 & 53475 & 32 & 0.03 & 69.53 & 0.133133 & 0.081081 & 3 & 49011623 & 3 & 49877554 & 3 & 49 & 0.039 & 0.6997 & 5.54 \\
\hline 528 & 55209 & 34822 & 21 & 0.03 & 70.28 & 0.118118 & 0.085085 & 3 & 50118060 & 3 & 50856818 & 3 & 50 & 0.039 & 0.7004 & 5.51 \\
\hline 529 & 17458 & 56912 & 26 & 0.04 & 58.35 & 0.116116 & 0.077077 & 3 & 51053503 & 3 & 51939345 & 3 & 51 & 0.032 & 0.693 & 6.02 \\
\hline 530 & 40810 & 2976 & 13 & 0.01 & 91.97 & 0.052052 & 0.036036 & 3 & 52025538 & 3 & 52990305 & 3 & 52 & 0.053 & 0.7082 & 4.77 \\
\hline 531 & 2975 & 53227 & 17 & 0.02 & 81.64 & 0.084084 & 0.055055 & 3 & 53003881 & 3 & 53810019 & 3 & 53 & 0.046 & 0.7052 & 5.1 \\
\hline 532 & 2983 & 56319 & 13 & 0.01 & 93.2 & 0.047047 & 0.03003 & 3 & 54006786 & 3 & 54995304 & 3 & 54 & 0.054 & 0.7082 & 4.73 \\
\hline 533 & 2988 & 2994 & 18 & 0.02 & 86.23 & 0.088088 & 0.058058 & 3 & 55028381 & 3 & 55949229 & 3 & 55 & 0.05 & 0.7071 & 4.95 \\
\hline 534 & 53018 & 56376 & 23 & 0.02 & 89.82 & 0.094094 & 0.061061 & 3 & 56005947 & 3 & 56950047 & 3 & 56 & 0.052 & 0.7077 & 4.83 \\
\hline 535 & 21371 & 46483 & 27 & 0.04 & 61.21 & 0.105105 & 0.076076 & 3 & 57006572 & 3 & 57996439 & 3 & 57 & 0.034 & 0.6963 & 5.92 \\
\hline 536 & 21376 & 21383 & 27 & 0.05 & 46.98 & 0.137137 & 0.095095 & 3 & 58013180 & 3 & 58999300 & 3 & 58 & 0.025 & 0.6821 & 6.67 \\
\hline 537 & 21384 & 21386 & 18 & 0.05 & 47.69 & 0.081081 & 0.051051 & 3 & 59012808 & 3 & 59995247 & 3 & 59 & 0.026 & 0.682 & 6.6 \\
\hline 538 & 40825 & 3023 & 22 & 0.06 & 36.59 & 0.098098 & 0.075075 & 3 & 60015652 & 3 & 60998171 & 3 & 60 & 0.02 & 0.6613 & 7.37 \\
\hline 539 & 36378 & 33523 & 21 & 0.03 & 73.33 & 0.099099 & 0.071071 & 3 & 61035420 & 3 & 61967071 & 3 & 61 & 0.041 & 0.7016 & 5.38 \\
\hline 540 & 29679 & 52501 & 28 & 0.03 & 78.11 & 0.098098 & 0.072072 & 3 & 62059336 & 3 & 62994493 & 3 & 62 & 0.044 & 0.7041 & 5.22 \\
\hline 541 & 35473 & 37273 & 27 & 0.02 & 84.03 & 0.106106 & 0.06006 & 3 & 63016890 & 3 & 63964964 & 3 & 63 & 0.048 & 0.7053 & 5 \\
\hline 542 & 3035 & 3039 & 14 & 0.01 & 91.9 & 0.061061 & 0.036036 & 3 & 64003964 & 3 & 64872620 & 3 & 64 & 0.053 & 0.7081 & 4.77 \\
\hline 543 & 55295 & 34223 & 14 & 0.02 & 84.07 & 0.05005 & 0.039039 & 3 & 65094505 & 3 & 65993379 & 3 & 65 & 0.048 & 0.7053 & 5 \\
\hline
\end{tabular}




\begin{tabular}{|c|c|c|c|c|c|c|c|c|c|c|c|c|c|c|c|c|}
\hline 544 & 30156 & 21408 & 28 & 0.08 & 24.84 & 0.144144 & 0.096096 & 3 & 66040600 & 3 & 66993596 & 3 & 66 & 0.013 & 0.6263 & 8.54 \\
\hline 545 & 54159 & 31836 & 26 & 0.08 & 26.26 & 0.128128 & 0.097097 & 3 & 67011470 & 3 & 67954066 & 3 & 67 & 0.014 & 0.6304 & 8.33 \\
\hline 546 & 3054 & 21418 & 21 & 0.04 & 57.28 & 0.096096 & 0.067067 & 3 & 68009516 & 3 & 68984089 & 3 & 68 & 0.031 & 0.6924 & 6.08 \\
\hline 547 & 21419 & 3063 & 6 & 0.01 & 95.44 & 0.03003 & 0.016016 & 3 & 69657253 & 3 & 69935645 & 3 & 69 & 0.056 & 0.7087 & 4.66 \\
\hline 549 & 18957 & 3074 & 24 & 0.03 & 76.59 & 0.088088 & 0.056056 & 3 & 71100014 & 3 & 71946686 & 3 & 71 & 0.043 & 0.703 & 5.26 \\
\hline 550 & 54572 & 15140 & 17 & 0.03 & 79.99 & 0.082082 & 0.06006 & 3 & 72009115 & 3 & 72838665 & 3 & 72 & 0.045 & 0.7046 & 5.15 \\
\hline 551 & 40842 & 18730 & 15 & 0.01 & 95.94 & 0.043043 & 0.025025 & 3 & 73025014 & 3 & 73996122 & 3 & 73 & 0.056 & 0.7088 & 4.65 \\
\hline 553 & 35325 & 61996 & 13 & 0.02 & 86.99 & 0.059059 & 0.038038 & 3 & 75020115 & 3 & 75980661 & 3 & 75 & 0.05 & 0.7076 & 4.93 \\
\hline 554 & 37294 & 15024 & 20 & 0.02 & 89.08 & 0.078078 & 0.038038 & 3 & 76044809 & 3 & 76994672 & 3 & 76 & 0.052 & 0.7074 & 4.85 \\
\hline 555 & 39740 & 21445 & 19 & 0.01 & 93.89 & 0.074074 & 0.043043 & 3 & 77112515 & 3 & 77996061 & 3 & 77 & 0.055 & 0.7084 & 4.7 \\
\hline 556 & 3098 & 30405 & 14 & 0.01 & 93.95 & 0.058058 & 0.042042 & 3 & 78029770 & 3 & 78944101 & 3 & 78 & 0.055 & 0.7084 & 4.7 \\
\hline 557 & 30375 & 40849 & 20 & 0.02 & 88 & 0.078078 & 0.059059 & 3 & 79053106 & 3 & 79981209 & 3 & 79 & 0.051 & 0.7078 & 4.89 \\
\hline 558 & 3105 & 21456 & 19 & 0.03 & 72.72 & 0.085085 & 0.064064 & 3 & 80053531 & 3 & 80978886 & 3 & 80 & 0.041 & 0.7012 & 5.41 \\
\hline 559 & 21457 & 36248 & 16 & 0.01 & 94.85 & 0.056056 & 0.028028 & 3 & 81005314 & 3 & 81986256 & 3 & 81 & 0.056 & 0.7085 & 4.68 \\
\hline 561 & 39695 & 3121 & 16 & 0.02 & 88.86 & 0.068068 & 0.04004 & 3 & 83277793 & 3 & 83943514 & 3 & 83 & 0.051 & 0.7076 & 4.86 \\
\hline 562 & 3123 & 46501 & 3 & 0.01 & 96.03 & 0.011011 & 0.006006 & 3 & 84495609 & 3 & 84745984 & 3 & 84 & 0.057 & 0.709 & 4.65 \\
\hline 563 & 54568 & 3128 & 19 & 0.01 & 94.69 & 0.073073 & 0.046046 & 3 & 85125505 & 3 & 85886007 & 3 & 85 & 0.056 & 0.7085 & 4.68 \\
\hline 564 & 15618 & 52680 & 10 & 0.01 & 94.98 & 0.04004 & 0.03003 & 3 & 86085644 & 3 & 86960594 & 3 & 86 & 0.056 & 0.7086 & 4.68 \\
\hline 565 & 40856 & 21463 & 7 & 0.02 & 90.66 & 0.037037 & 0.028028 & 3 & 87200902 & 3 & 87941073 & 3 & 87 & 0.053 & 0.7078 & 4.81 \\
\hline 566 & 37303 & 3136 & 10 & 0.01 & 96.09 & 0.033033 & 0.021021 & 3 & 88022523 & 3 & 88862483 & 3 & 88 & 0.057 & 0.7091 & 4.64 \\
\hline 567 & 60904 & 3143 & 22 & 0.03 & 65.46 & 0.101101 & 0.069069 & 3 & 89138287 & 3 & 89999192 & 3 & 89 & 0.036 & 0.6987 & 5.72 \\
\hline 568 & 3144 & 31313 & 12 & 0.01 & 93.67 & 0.058058 & 0.034034 & 3 & 90020437 & 3 & 90913607 & 3 & 90 & 0.055 & 0.7081 & 4.71 \\
\hline 569 & 31009 & 17174 & 12 & 0.03 & 65.7 & 0.06006 & 0.032032 & 3 & 91123979 & 3 & 91605127 & 3 & 91 & 0.036 & 0.6985 & 5.7 \\
\hline 570 & 30508 & 57437 & 17 & 0.02 & 83.69 & 0.07007 & 0.054054 & 3 & 92012330 & 3 & 92847118 & 3 & 92 & 0.048 & 0.7053 & 5.01 \\
\hline 571 & 19398 & 3154 & 8 & 0.02 & 90.17 & 0.038038 & 0.024024 & 3 & 93074982 & 3 & 93929087 & 3 & 93 & 0.052 & 0.7078 & 4.82 \\
\hline 572 & 3153 & 3161 & 13 & 0.02 & 82.2 & 0.055055 & 0.04004 & 3 & 94060695 & 3 & 94910712 & 3 & 94 & 0.047 & 0.705 & 5.07 \\
\hline 573 & 3162 & 3167 & 9 & 0.02 & 88.78 & 0.045045 & 0.027027 & 3 & 95002203 & 3 & 95944062 & 3 & 95 & 0.051 & 0.7077 & 4.87 \\
\hline 574 & 50485 & 3169 & 10 & 0.02 & 89.45 & 0.041041 & 0.029029 & 3 & 96012125 & 3 & 96641442 & 3 & 96 & 0.052 & 0.7077 & 4.84 \\
\hline 575 & 32369 & 21475 & 19 & 0.02 & 81.96 & 0.076076 & 0.049049 & 3 & 97223093 & 3 & 97974345 & 3 & 97 & 0.047 & 0.705 & 5.08 \\
\hline 578 & 21483 & 3187 & 26 & 0.04 & 60.2 & 0.108108 & 0.079079 & 3 & 100098050 & 3 & 100905327 & 3 & 100 & 0.033 & 0.6955 & 5.96 \\
\hline 579 & 21489 & 31283 & 14 & 0.01 & 94.41 & 0.059059 & 0.043043 & 3 & 101222209 & 3 & 101813917 & 3 & 101 & 0.055 & 0.7083 & 4.69 \\
\hline 580 & 46511 & 40883 & 25 & 0.04 & 63.91 & 0.118118 & 0.078078 & 3 & 102090846 & 3 & 102994163 & 3 & 102 & 0.035 & 0.6971 & 5.78 \\
\hline 581 & 21499 & 55369 & 17 & 0.03 & 68.78 & 0.085085 & 0.056056 & 3 & 103029683 & 3 & 103973714 & 3 & 103 & 0.038 & 0.7 & 5.58 \\
\hline 582 & 3203 & 3213 & 23 & 0.03 & 67.35 & 0.101101 & 0.065065 & 3 & 104064335 & 3 & 104974525 & 3 & 104 & 0.037 & 0.6993 & 5.63 \\
\hline
\end{tabular}




\begin{tabular}{|c|c|c|c|c|c|c|c|c|c|c|c|c|c|c|c|c|}
\hline 583 & 3214 & 21509 & 22 & 0.03 & 67.88 & 0.108108 & 0.076076 & 3 & 105002922 & 3 & 105933402 & 3 & 105 & 0.038 & 0.6996 & 5.62 \\
\hline 584 & 46516 & 37321 & 20 & 0.03 & 69.99 & 0.107107 & 0.071071 & 3 & 106010885 & 3 & 106987656 & 3 & 106 & 0.039 & 0.7001 & 5.52 \\
\hline 585 & 16076 & 31446 & 21 & 0.05 & 43.32 & 0.105105 & 0.075075 & 3 & 107073046 & 3 & 107985092 & 3 & 107 & 0.023 & 0.678 & 6.99 \\
\hline 586 & 40895 & 50683 & 18 & 0.02 & 88.33 & 0.088088 & 0.063063 & 3 & 108100697 & 3 & 108965502 & 3 & 108 & 0.051 & 0.7077 & 4.88 \\
\hline 588 & 21527 & 3257 & 30 & 0.03 & 66.49 & 0.103103 & 0.065065 & 3 & 110005181 & 3 & 110998569 & 3 & 110 & 0.037 & 0.6992 & 5.67 \\
\hline 589 & 37330 & 3262 & 21 & 0.04 & 51.85 & 0.111111 & 0.075075 & 3 & 111033114 & 3 & 111986113 & 3 & 111 & 0.028 & 0.689 & 6.42 \\
\hline 590 & 3263 & 21541 & 17 & 0.03 & 68.48 & 0.073073 & 0.051051 & 3 & 112022721 & 3 & 112989158 & 3 & 112 & 0.038 & 0.6994 & 5.58 \\
\hline 592 & 59033 & 3281 & 21 & 0.04 & 64.97 & 0.095095 & 0.065065 & 3 & 114049876 & 3 & 114951968 & 3 & 114 & 0.036 & 0.6981 & 5.74 \\
\hline 593 & 40910 & 57175 & 24 & 0.03 & 79.86 & 0.095095 & 0.062062 & 3 & 115009623 & 3 & 115996607 & 3 & 115 & 0.045 & 0.7048 & 5.16 \\
\hline 594 & 53290 & 40917 & 15 & 0.02 & 84.32 & 0.069069 & 0.049049 & 3 & 116001962 & 3 & 116920902 & 3 & 116 & 0.048 & 0.7055 & 4.99 \\
\hline 595 & 45806 & 3293 & 25 & 0.04 & 53.57 & 0.105105 & 0.071071 & 3 & 117022370 & 3 & 117988230 & 3 & 117 & 0.029 & 0.6919 & 6.34 \\
\hline 596 & 40918 & 35106 & 25 & 0.03 & 75.52 & 0.099099 & 0.068068 & 3 & 118132370 & 3 & 118993318 & 3 & 118 & 0.043 & 0.7024 & 5.3 \\
\hline 597 & 21576 & 3312 & 36 & 0.03 & 67.92 & 0.135135 & 0.085085 & 3 & 119006428 & 3 & 119979845 & 3 & 119 & 0.038 & 0.6996 & 5.62 \\
\hline 598 & 3310 & 33552 & 18 & 0.03 & 68.42 & 0.087087 & 0.058058 & 3 & 120040818 & 3 & 120863426 & 3 & 120 & 0.038 & 0.6994 & 5.58 \\
\hline 600 & 35543 & 3322 & 33 & 0.07 & 28.5 & 0.144144 & 0.085085 & 3 & 122042998 & 3 & 122976843 & 3 & 122 & 0.015 & 0.6377 & 8.07 \\
\hline 601 & 21596 & 33223 & 11 & 0.01 & 94.76 & 0.049049 & 0.022022 & 3 & 123015205 & 3 & 123638149 & 3 & 123 & 0.056 & 0.7084 & 4.68 \\
\hline 602 & 3327 & 21601 & 20 & 0.03 & 73.17 & 0.079079 & 0.059059 & 3 & 124002113 & 3 & 124901557 & 3 & 124 & 0.041 & 0.7016 & 5.39 \\
\hline 603 & 15834 & 40935 & 23 & 0.05 & 49.63 & 0.112112 & 0.064064 & 3 & 125018914 & 3 & 125979839 & 3 & 125 & 0.027 & 0.6857 & 6.52 \\
\hline 604 & 3336 & 3341 & 27 & 0.03 & 77.9 & 0.098098 & 0.062062 & 3 & 126010993 & 3 & 126989393 & 3 & 126 & 0.044 & 0.7039 & 5.23 \\
\hline 605 & 3342 & 61723 & 11 & 0.01 & 96.19 & 0.037037 & 0.019019 & 3 & 127121184 & 3 & 127967288 & 3 & 127 & 0.057 & 0.7091 & 4.64 \\
\hline 606 & 48654 & 21618 & 22 & 0.02 & 86.59 & 0.092092 & 0.064064 & 3 & 128135951 & 3 & 128988657 & 3 & 128 & 0.05 & 0.7071 & 4.94 \\
\hline 607 & 21619 & 40940 & 24 & 0.04 & 58.74 & 0.111111 & 0.068068 & 3 & 129005942 & 3 & 129847043 & 3 & 129 & 0.032 & 0.6936 & 6.01 \\
\hline 608 & 3358 & 21626 & 18 & 0.02 & 89.26 & 0.075075 & 0.046046 & 3 & 130036775 & 3 & 130877498 & 3 & 130 & 0.052 & 0.7078 & 4.85 \\
\hline 609 & 17394 & 55812 & 20 & 0.01 & 92.92 & 0.087087 & 0.036036 & 3 & 131057295 & 3 & 131906132 & 3 & 131 & 0.054 & 0.7082 & 4.74 \\
\hline 610 & 21628 & 21634 & 27 & 0.02 & 88.52 & 0.116116 & 0.061061 & 3 & 132154665 & 3 & 132947422 & 3 & 132 & 0.051 & 0.7078 & 4.88 \\
\hline 611 & 30377 & 50036 & 30 & 0.04 & 55.78 & 0.126126 & 0.083083 & 3 & 133094186 & 3 & 133992535 & 3 & 133 & 0.03 & 0.6922 & 6.18 \\
\hline 612 & 15104 & 21645 & 27 & 0.1 & 19.89 & 0.131131 & 0.087087 & 3 & 134008198 & 3 & 134990636 & 3 & 134 & 0.01 & 0.6024 & 9.28 \\
\hline 613 & 40955 & 30011 & 32 & 0.04 & 59.43 & 0.146146 & 0.082082 & 3 & 135011301 & 3 & 135940918 & 3 & 135 & 0.033 & 0.6944 & 5.99 \\
\hline 614 & 60112 & 58635 & 22 & 0.03 & 80.36 & 0.09009 & 0.056056 & 3 & 136029014 & 3 & 136995452 & 3 & 136 & 0.046 & 0.7049 & 5.14 \\
\hline 617 & 58200 & 9423 & 21 & 0.04 & 61.91 & 0.095095 & 0.061061 & 3 & 139055473 & 3 & 139956551 & 3 & 139 & 0.034 & 0.6969 & 5.9 \\
\hline 618 & 30981 & 61276 & 16 & 0.02 & 82.57 & 0.075075 & 0.05005 & 3 & 140070086 & 3 & 140798715 & 3 & 140 & 0.047 & 0.7049 & 5.06 \\
\hline 619 & 3396 & 57721 & 21 & 0.14 & 16.34 & 0.118118 & 0.093093 & 3 & 141006420 & 3 & 141885076 & 3 & 141 & 0.008 & 0.5824 & 9.88 \\
\hline 620 & 52313 & 45836 & 8 & 0.01 & 91.84 & 0.057057 & 0.038038 & 3 & 142329763 & 3 & 142766769 & 3 & 142 & 0.053 & 0.7081 & 4.77 \\
\hline 621 & 17508 & 56944 & 14 & 0.03 & 76.48 & 0.077077 & 0.047047 & 3 & 143698872 & 3 & 143975041 & 3 & 143 & 0.043 & 0.7031 & 5.27 \\
\hline
\end{tabular}




\begin{tabular}{|c|c|c|c|c|c|c|c|c|c|c|c|c|c|c|c|c|}
\hline 622 & 52046 & 52339 & 7 & 0.01 & 92.5 & 0.041041 & 0.026026 & 3 & 144009549 & 3 & 144353522 & 3 & 144 & 0.054 & 0.7081 & 4.75 \\
\hline 623 & 21658 & 30854 & 16 & 0.03 & 80.01 & 0.074074 & 0.044044 & 4 & 95368 & 4 & 779106 & 4 & 0 & 0.045 & 0.7047 & 5.15 \\
\hline 624 & 4578 & 21669 & 24 & 0.05 & 49.03 & 0.115115 & 0.08008 & 4 & 1003897 & 4 & 1996854 & 4 & 1 & 0.027 & 0.6844 & 6.54 \\
\hline 625 & 54334 & 21676 & 24 & 0.05 & 48.06 & 0.116116 & 0.077077 & 4 & 2035615 & 4 & 2990838 & 4 & 2 & 0.026 & 0.6819 & 6.56 \\
\hline 626 & 40968 & 59395 & 23 & 0.06 & 32.99 & 0.095095 & 0.061061 & 4 & 3004624 & 4 & 3984843 & 4 & 3 & 0.018 & 0.6503 & 7.65 \\
\hline 627 & 48680 & 3424 & 40 & 0.05 & 40.19 & 0.158158 & 0.115115 & 4 & 4001700 & 4 & 4957104 & 4 & 4 & 0.021 & 0.6717 & 7.17 \\
\hline 628 & 21699 & 21709 & 29 & 0.05 & 46.22 & 0.135135 & 0.087087 & 4 & 5006687 & 4 & 5985544 & 4 & 5 & 0.025 & 0.6815 & 6.74 \\
\hline 629 & 3436 & 21719 & 31 & 0.03 & 79.48 & 0.12012 & 0.086086 & 4 & 6007874 & 4 & 6977891 & 4 & 6 & 0.045 & 0.705 & 5.18 \\
\hline 630 & 21720 & 40992 & 34 & 0.05 & 40.73 & 0.148148 & 0.107107 & 4 & 7002305 & 4 & 7975375 & 4 & 7 & 0.022 & 0.6718 & 7.12 \\
\hline 631 & 48689 & 3465 & 27 & 0.04 & 57.08 & 0.125125 & 0.088088 & 4 & 8074964 & 4 & 8963064 & 4 & 8 & 0.031 & 0.6921 & 6.09 \\
\hline 632 & 21742 & 3476 & 27 & 0.08 & 25.87 & 0.133133 & 0.088088 & 4 & 9005118 & 4 & 9946393 & 4 & 9 & 0.014 & 0.629 & 8.37 \\
\hline 633 & 3477 & 37374 & 27 & 0.08 & 24.51 & 0.128128 & 0.08008 & 4 & 10016061 & 4 & 10835685 & 4 & 10 & 0.013 & 0.6267 & 8.64 \\
\hline 634 & 56887 & 52761 & 30 & 0.04 & 52.93 & 0.133133 & 0.084084 & 4 & 11002675 & 4 & 11982774 & 4 & 11 & 0.029 & 0.6913 & 6.38 \\
\hline 635 & 21769 & 3500 & 37 & 0.04 & 53.44 & 0.165165 & 0.104104 & 4 & 12003384 & 4 & 12980361 & 4 & 12 & 0.029 & 0.6917 & 6.35 \\
\hline 636 & 41007 & 3514 & 30 & 0.04 & 59.36 & 0.128128 & 0.088088 & 4 & 13008743 & 4 & 13903080 & 4 & 13 & 0.033 & 0.6944 & 5.99 \\
\hline 637 & 3516 & 57743 & 26 & 0.02 & 85.6 & 0.104104 & 0.059059 & 4 & 14003125 & 4 & 14995885 & 4 & 14 & 0.049 & 0.7062 & 4.96 \\
\hline 638 & 37381 & 3533 & 31 & 0.17 & 13.27 & 0.164164 & 0.095095 & 4 & 15016104 & 4 & 15977749 & 4 & 15 & 0.007 & 0.5654 & 10.82 \\
\hline 639 & 3531 & 16086 & 38 & 0.07 & 27.7 & 0.164164 & 0.11011 & 4 & 16001400 & 4 & 16995545 & 4 & 16 & 0.015 & 0.6352 & 8.15 \\
\hline 640 & 17151 & 34932 & 34 & 0.04 & 63.23 & 0.141141 & 0.095095 & 4 & 17033119 & 4 & 17975684 & 4 & 17 & 0.035 & 0.6967 & 5.81 \\
\hline 641 & 37386 & 3558 & 21 & 0.02 & 91.57 & 0.076076 & 0.046046 & 4 & 18008464 & 4 & 18995385 & 4 & 18 & 0.053 & 0.7082 & 4.79 \\
\hline 642 & 37392 & 51299 & 35 & 0.03 & 66.84 & 0.127127 & 0.087087 & 4 & 19015552 & 4 & 19958367 & 4 & 19 & 0.037 & 0.6992 & 5.66 \\
\hline 643 & 21835 & 3579 & 30 & 0.06 & 35.04 & 0.135135 & 0.087087 & 4 & 20010437 & 4 & 20985135 & 4 & 20 & 0.019 & 0.6561 & 7.48 \\
\hline 644 & 3583 & 37400 & 38 & 0.05 & 45.74 & 0.149149 & 0.102102 & 4 & 21086107 & 4 & 21990523 & 4 & 21 & 0.024 & 0.6815 & 6.79 \\
\hline 645 & 53331 & 3610 & 28 & 0.03 & 67.98 & 0.131131 & 0.088088 & 4 & 22012168 & 4 & 22978568 & 4 & 22 & 0.038 & 0.6995 & 5.61 \\
\hline 646 & 35912 & 3613 & 12 & 0.01 & 95.72 & 0.049049 & 0.024024 & 4 & 23215904 & 4 & 23861877 & 4 & 23 & 0.056 & 0.7089 & 4.66 \\
\hline 647 & 21856 & 52159 & 16 & 0.04 & 61.43 & 0.09009 & 0.064064 & 4 & 24167536 & 4 & 24884374 & 4 & 24 & 0.034 & 0.6962 & 5.9 \\
\hline 648 & 37405 & 21866 & 27 & 0.06 & 39.1 & 0.115115 & 0.077077 & 4 & 25019533 & 4 & 25984211 & 4 & 25 & 0.021 & 0.6683 & 7.23 \\
\hline 649 & 3626 & 61543 & 24 & 0.03 & 65.8 & 0.094094 & 0.066066 & 4 & 26012865 & 4 & 26994265 & 4 & 26 & 0.036 & 0.6985 & 5.7 \\
\hline 650 & 51018 & 37416 & 9 & 0.03 & 72.48 & 0.047047 & 0.034034 & 4 & 27041022 & 4 & 27846293 & 4 & 27 & 0.041 & 0.701 & 5.42 \\
\hline 651 & 41035 & 3646 & 21 & 0.03 & 75.66 & 0.086086 & 0.053053 & 4 & 28013430 & 4 & 28935985 & 4 & 28 & 0.043 & 0.7027 & 5.3 \\
\hline 652 & 37422 & 21881 & 21 & 0.07 & 27.99 & 0.093093 & 0.072072 & 4 & 29042837 & 4 & 29908735 & 4 & 29 & 0.015 & 0.6362 & 8.11 \\
\hline 653 & 3655 & 3663 & 24 & 0.07 & 31.2 & 0.143143 & 0.098098 & 4 & 30026623 & 4 & 30990527 & 4 & 30 & 0.017 & 0.6458 & 7.8 \\
\hline 654 & 21887 & 41040 & 19 & 0.01 & 91.82 & 0.082082 & 0.043043 & 4 & 31003651 & 4 & 31982461 & 4 & 31 & 0.053 & 0.7081 & 4.78 \\
\hline 655 & 3670 & 37429 & 19 & 0.04 & 53.53 & 0.092092 & 0.065065 & 4 & 32034432 & 4 & 32960684 & 4 & 32 & 0.029 & 0.6919 & 6.34 \\
\hline 656 & 52078 & 3692 & 31 & 0.03 & 66.63 & 0.118118 & 0.075075 & 4 & 33016884 & 4 & 33955625 & 4 & 33 & 0.037 & 0.6993 & 5.67 \\
\hline 657 & 21900 & 37432 & 31 & 0.06 & 33.12 & 0.138138 & 0.09009 & 4 & 34074856 & 4 & 34965838 & 4 & 34 & 0.018 & 0.6506 & 7.65 \\
\hline 658 & 21908 & 3716 & 29 & 0.04 & 59.89 & 0.144144 & 0.085085 & 4 & 35008828 & 4 & 35986572 & 4 & 35 & 0.033 & 0.6953 & 5.97 \\
\hline 659 & 3717 & 54368 & 24 & 0.03 & 74.95 & 0.116116 & 0.068068 & 4 & 36005051 & 4 & 36930500 & 4 & 36 & 0.042 & 0.7021 & 5.32 \\
\hline 660 & 52747 & 21926 & 25 & 0.03 & 75 & 0.107107 & 0.072072 & 4 & 37069150 & 4 & 37995302 & 4 & 37 & 0.042 & 0.7021 & 5.31 \\
\hline
\end{tabular}




\begin{tabular}{|c|c|c|c|c|c|c|c|c|c|c|c|c|c|c|c|c|}
\hline 661 & 3731 & 3735 & 20 & 0.02 & 90.71 & 0.081081 & 0.051051 & 4 & 38015849 & 4 & 38985579 & 4 & 38 & 0.053 & 0.7078 & 4.8 \\
\hline 662 & 21934 & 3741 & 23 & 0.03 & 73.02 & 0.096096 & 0.061061 & 4 & 39025906 & 4 & 39998067 & 4 & 39 & 0.041 & 0.7016 & 5.4 \\
\hline 663 & 46591 & 3745 & 14 & 0.02 & 86.85 & 0.057057 & 0.038038 & 4 & 40031986 & 4 & 40942240 & 4 & 40 & 0.05 & 0.7074 & 4.93 \\
\hline 664 & 46595 & 3752 & 19 & 0.04 & 62.87 & 0.101101 & 0.069069 & 4 & 41008284 & 4 & 41937629 & 4 & 41 & 0.035 & 0.6967 & 5.83 \\
\hline 665 & 21944 & 41073 & 24 & 0.04 & 52.24 & 0.115115 & 0.073073 & 4 & 42037215 & 4 & 42984787 & 4 & 42 & 0.028 & 0.6898 & 6.4 \\
\hline 666 & 21950 & 21956 & 26 & 0.03 & 74.39 & 0.103103 & 0.069069 & 4 & 43005049 & 4 & 43983124 & 4 & 43 & 0.042 & 0.7017 & 5.33 \\
\hline 667 & 61805 & 3782 & 27 & 0.05 & 45.4 & 0.135135 & 0.093093 & 4 & 44003320 & 4 & 44997961 & 4 & 44 & 0.024 & 0.6809 & 6.81 \\
\hline 668 & 56820 & 21970 & 19 & 0.21 & 11.49 & 0.149149 & 0.121121 & 4 & 45031120 & 4 & 45986315 & 4 & 45 & 0.006 & 0.5523 & 11.28 \\
\hline 669 & 3787 & 41084 & 17 & 0.02 & 84 & 0.069069 & 0.038038 & 4 & 46040879 & 4 & 46986125 & 4 & 46 & 0.048 & 0.7054 & 5 \\
\hline 670 & 37445 & 46612 & 24 & 0.05 & 43.92 & 0.126126 & 0.093093 & 4 & 47043904 & 4 & 47963187 & 4 & 47 & 0.023 & 0.6784 & 6.93 \\
\hline 671 & 3806 & 51466 & 16 & 0.02 & 86.73 & 0.072072 & 0.036036 & 4 & 48069819 & 4 & 48936417 & 4 & 48 & 0.05 & 0.7073 & 4.93 \\
\hline 672 & 17290 & 46616 & 9 & 0.02 & 90.95 & 0.049049 & 0.031031 & 4 & 49093079 & 4 & 49917991 & 4 & 49 & 0.053 & 0.708 & 4.8 \\
\hline 673 & 16349 & 3816 & 14 & 0.03 & 78.56 & 0.064064 & 0.036036 & 4 & 50110206 & 4 & 50997149 & 4 & 50 & 0.044 & 0.7041 & 5.21 \\
\hline 674 & 41090 & 3831 & 23 & 0.04 & 60.84 & 0.101101 & 0.069069 & 4 & 51036347 & 4 & 51937058 & 4 & 51 & 0.033 & 0.6958 & 5.93 \\
\hline 675 & 59441 & 21991 & 8 & 0.01 & 94.94 & 0.04004 & 0.02002 & 4 & 52023825 & 4 & 52994578 & 4 & 52 & 0.056 & 0.7087 & 4.68 \\
\hline 676 & 46620 & 62092 & 11 & 0.01 & 95.52 & 0.042042 & 0.016016 & 4 & 53047867 & 4 & 53913712 & 4 & 53 & 0.056 & 0.7087 & 4.66 \\
\hline 677 & 3836 & 53246 & 13 & 0.04 & 59.51 & 0.062062 & 0.045045 & 4 & 54122432 & 4 & 54937429 & 4 & 54 & 0.033 & 0.6943 & 5.98 \\
\hline 678 & 3842 & 46626 & 16 & 0.01 & 96.2 & 0.047047 & 0.012012 & 4 & 55031488 & 4 & 55960525 & 4 & 55 & 0.057 & 0.7091 & 4.64 \\
\hline 679 & 3849 & 46629 & 10 & 0.01 & 96.19 & 0.039039 & 0.014014 & 4 & 56000161 & 4 & 56894950 & 4 & 56 & 0.057 & 0.7091 & 4.64 \\
\hline 680 & 37447 & 3857 & 13 & 0 & 96.56 & 0.027027 & 0.004004 & 4 & 57099646 & 4 & 57935123 & 4 & 57 & 0.057 & 0.7093 & 4.63 \\
\hline 681 & 3858 & 3861 & 6 & 0 & 96.42 & 0.004004 & 0.003003 & 4 & 58067211 & 4 & 58777597 & 4 & 58 & 0.057 & 0.7092 & 4.64 \\
\hline 682 & 14600 & 3864 & 11 & 0.01 & 95.97 & 0.033033 & 0.023023 & 4 & 59063035 & 4 & 59967513 & 4 & 59 & 0.056 & 0.7089 & 4.65 \\
\hline 683 & 3866 & 3882 & 18 & 0.02 & 86 & 0.068068 & 0.039039 & 4 & 60030169 & 4 & 60899193 & 4 & 60 & 0.049 & 0.7066 & 4.95 \\
\hline 684 & 3886 & 35992 & 22 & 0.02 & 87.35 & 0.079079 & 0.046046 & 4 & 61038550 & 4 & 61946905 & 4 & 61 & 0.05 & 0.7076 & 4.92 \\
\hline 685 & 32042 & 35889 & 30 & 0.02 & 83.3 & 0.132132 & 0.088088 & 4 & 62027221 & 4 & 62973622 & 4 & 62 & 0.048 & 0.7045 & 5.02 \\
\hline 686 & 22021 & 55977 & 19 & 0.02 & 90.3 & 0.093093 & 0.056056 & 4 & 63054580 & 4 & 63988841 & 4 & 63 & 0.052 & 0.7077 & 4.81 \\
\hline 687 & 3910 & 3919 & 27 & 0.03 & 75.72 & 0.114114 & 0.075075 & 4 & 64137468 & 4 & 64983207 & 4 & 64 & 0.043 & 0.7027 & 5.29 \\
\hline 688 & 22029 & 41111 & 19 & 0.02 & 89.58 & 0.07007 & 0.048048 & 4 & 65016011 & 4 & 65976827 & 4 & 65 & 0.052 & 0.7077 & 4.84 \\
\hline 689 & 34682 & 46662 & 22 & 0.02 & 87.67 & 0.1001 & 0.053053 & 4 & 66002995 & 4 & 66988557 & 4 & 66 & 0.051 & 0.7077 & 4.9 \\
\hline 690 & 46663 & 22040 & 23 & 0.02 & 83.32 & 0.087087 & 0.054054 & 4 & 67015811 & 4 & 67992709 & 4 & 67 & 0.048 & 0.7045 & 5.02 \\
\hline 691 & 34624 & 52522 & 22 & 0.03 & 79.83 & 0.097097 & 0.07007 & 4 & 68024939 & 4 & 68766745 & 4 & 68 & 0.045 & 0.7048 & 5.16 \\
\hline 692 & 22049 & 41123 & 26 & 0.03 & 78.09 & 0.106106 & 0.064064 & 4 & 69018203 & 4 & 69896058 & 4 & 69 & 0.044 & 0.7042 & 5.23 \\
\hline 693 & 22058 & 45095 & 23 & 0.01 & 92.34 & 0.066066 & 0.031031 & 4 & 70092569 & 4 & 70979785 & 4 & 70 & 0.054 & 0.7082 & 4.76 \\
\hline 694 & 61238 & 46673 & 23 & 0.03 & 79.81 & 0.101101 & 0.062062 & 4 & 71081956 & 4 & 71941276 & 4 & 71 & 0.045 & 0.7048 & 5.16 \\
\hline 695 & 32940 & 3965 & 16 & 0.01 & 95.59 & 0.079079 & 0.028028 & 4 & 72005343 & 4 & 72955795 & 4 & 72 & 0.056 & 0.7088 & 4.66 \\
\hline 696 & 37458 & 46678 & 25 & 0.02 & 80.74 & 0.085085 & 0.056056 & 4 & 73017408 & 4 & 73907009 & 4 & 73 & 0.046 & 0.7052 & 5.13 \\
\hline 697 & 35695 & 46681 & 25 & 0.03 & 79.63 & 0.101101 & 0.063063 & 4 & 74095578 & 4 & 74999752 & 4 & 74 & 0.045 & 0.7048 & 5.17 \\
\hline 698 & 22082 & 48720 & 20 & 0.1 & 20.31 & 0.111111 & 0.086086 & 4 & 75016304 & 4 & 75991872 & 4 & 75 & 0.011 & 0.6016 & 9.12 \\
\hline 699 & 37460 & 46684 & 27 & 0.04 & 54.29 & 0.128128 & 0.079079 & 4 & 76004484 & 4 & 76985054 & 4 & 76 & 0.029 & 0.6917 & 6.28 \\
\hline
\end{tabular}




\begin{tabular}{|c|c|c|c|c|c|c|c|c|c|c|c|c|c|c|c|c|}
\hline 700 & 61827 & 22102 & 29 & 0.04 & 63.37 & 0.133133 & 0.077077 & 4 & 77034601 & 4 & 77999066 & 4 & 77 & 0.035 & 0.6971 & 5.81 \\
\hline 701 & 4016 & 22109 & 30 & 0.07 & 28.93 & 0.148148 & 0.105105 & 4 & 78027085 & 4 & 78946182 & 4 & 78 & 0.015 & 0.6393 & 8.03 \\
\hline 702 & 22110 & 46690 & 31 & 0.03 & 73.11 & 0.128128 & 0.086086 & 4 & 79008114 & 4 & 79794714 & 4 & 79 & 0.041 & 0.7015 & 5.39 \\
\hline 703 & 4041 & 4048 & 16 & 0.02 & 91.55 & 0.056056 & 0.035035 & 4 & 80111898 & 4 & 80963726 & 4 & 80 & 0.053 & 0.7081 & 4.79 \\
\hline 704 & 52553 & 41150 & 26 & 0.04 & 56.07 & 0.128128 & 0.081081 & 4 & 81026612 & 4 & 81984659 & 4 & 81 & 0.031 & 0.6917 & 6.15 \\
\hline 705 & 4058 & 4068 & 23 & 0.05 & 46.6 & 0.12012 & 0.076076 & 4 & 82027009 & 4 & 82971117 & 4 & 82 & 0.025 & 0.6811 & 6.68 \\
\hline 706 & 46700 & 22141 & 24 & 0.03 & 77 & 0.095095 & 0.058058 & 4 & 83025851 & 4 & 83846382 & 4 & 83 & 0.044 & 0.7033 & 5.25 \\
\hline 707 & 4076 & 22145 & 19 & 0.04 & 59.2 & 0.081081 & 0.062062 & 4 & 84090680 & 4 & 84983220 & 4 & 84 & 0.033 & 0.6943 & 6 \\
\hline 709 & 55478 & 41167 & 17 & 0.07 & 29.5 & 0.084084 & 0.063063 & 4 & 86029839 & 4 & 86981509 & 4 & 86 & 0.016 & 0.6412 & 7.97 \\
\hline 710 & 22147 & 22151 & 20 & 0.02 & 86.26 & 0.065065 & 0.045045 & 4 & 87007876 & 4 & 87889676 & 4 & 87 & 0.05 & 0.7071 & 4.95 \\
\hline 711 & 46708 & 41171 & 20 & 0.01 & 94.25 & 0.076076 & 0.051051 & 4 & 88065653 & 4 & 88978451 & 4 & 88 & 0.055 & 0.7083 & 4.69 \\
\hline 712 & 4099 & 4107 & 21 & 0.04 & 56.35 & 0.106106 & 0.061061 & 4 & 89008992 & 4 & 89983155 & 4 & 89 & 0.031 & 0.6917 & 6.13 \\
\hline 713 & 46714 & 4115 & 16 & 0.02 & 80.54 & 0.077077 & 0.045045 & 4 & 90010895 & 4 & 90944979 & 4 & 90 & 0.046 & 0.705 & 5.13 \\
\hline 714 & 4116 & 46722 & 24 & 0.02 & 81.21 & 0.089089 & 0.054054 & 4 & 91007286 & 4 & 91938171 & 4 & 91 & 0.046 & 0.705 & 5.11 \\
\hline 715 & 37473 & 22171 & 24 & 0.07 & 31 & 0.109109 & 0.071071 & 4 & 92004679 & 4 & 92991564 & 4 & 92 & 0.016 & 0.646 & 7.84 \\
\hline 717 & 4144 & 4156 & 28 & 0.04 & 61.25 & 0.117117 & 0.08008 & 4 & 94003968 & 4 & 94978683 & 4 & 94 & 0.034 & 0.6963 & 5.92 \\
\hline 718 & 46728 & 56180 & 34 & 0.09 & 22.62 & 0.161161 & 0.117117 & 4 & 95000157 & 4 & 95988078 & 4 & 95 & 0.012 & 0.6171 & 8.87 \\
\hline 719 & 22191 & 35230 & 26 & 0.05 & 48.62 & 0.119119 & 0.079079 & 4 & 96048342 & 4 & 96996047 & 4 & 96 & 0.026 & 0.6834 & 6.55 \\
\hline 720 & 22195 & 59950 & 29 & 0.06 & 35.4 & 0.151151 & 0.102102 & 4 & 97012116 & 4 & 97993218 & 4 & 97 & 0.019 & 0.6564 & 7.43 \\
\hline 721 & 46731 & 22211 & 29 & 0.03 & 77.14 & 0.126126 & 0.074074 & 4 & 98016950 & 4 & 98986789 & 4 & 98 & 0.044 & 0.7034 & 5.25 \\
\hline 722 & 22214 & 22217 & 22 & 0.05 & 49.67 & 0.089089 & 0.066066 & 4 & 99012920 & 4 & 99808905 & 4 & 99 & 0.027 & 0.6857 & 6.51 \\
\hline 723 & 22219 & 22229 & 26 & 0.05 & 43.17 & 0.109109 & 0.081081 & 4 & 100212108 & 4 & 100985004 & 4 & 100 & 0.023 & 0.6776 & 6.99 \\
\hline 724 & 41197 & 4211 & 22 & 0.04 & 61.4 & 0.102102 & 0.069069 & 4 & 101009461 & 4 & 101975122 & 4 & 101 & 0.034 & 0.6962 & 5.91 \\
\hline 725 & 29900 & 56117 & 27 & 0.28 & 9.6 & 0.193193 & 0.15015 & 4 & 102103941 & 4 & 102928877 & 4 & 102 & 0.005 & 0.5302 & 11.54 \\
\hline 726 & 46738 & 35076 & 28 & 0.12 & 17.75 & 0.158158 & 0.115115 & 4 & 103067357 & 4 & 103960092 & 4 & 103 & 0.009 & 0.5916 & 9.63 \\
\hline 727 & 41212 & 22251 & 27 & 0.05 & 43.67 & 0.108108 & 0.084084 & 4 & 104027961 & 4 & 104999962 & 4 & 104 & 0.023 & 0.6779 & 6.95 \\
\hline 728 & 4233 & 4240 & 26 & 0.04 & 58.31 & 0.117117 & 0.062062 & 4 & 105017514 & 4 & 105998663 & 4 & 105 & 0.032 & 0.6931 & 6.03 \\
\hline 729 & 22255 & 50937 & 29 & 0.03 & 65.63 & 0.115115 & 0.082082 & 4 & 106018138 & 4 & 106907287 & 4 & 106 & 0.036 & 0.6985 & 5.71 \\
\hline 730 & 52513 & 35537 & 31 & 0.06 & 33.18 & 0.139139 & 0.101101 & 4 & 107002176 & 4 & 107959349 & 4 & 107 & 0.018 & 0.6506 & 7.64 \\
\hline 731 & 30743 & 62161 & 21 & 0.03 & 73.65 & 0.096096 & 0.062062 & 4 & 108032246 & 4 & 108995059 & 4 & 108 & 0.041 & 0.702 & 5.37 \\
\hline 734 & 41236 & 59110 & 19 & 0.04 & 63.94 & 0.084084 & 0.059059 & 4 & 111222199 & 4 & 111993928 & 4 & 111 & 0.035 & 0.6972 & 5.78 \\
\hline 735 & 22288 & 53568 & 28 & 0.03 & 67.62 & 0.133133 & 0.078078 & 4 & 112014396 & 4 & 112988683 & 4 & 112 & 0.038 & 0.6991 & 5.62 \\
\hline 736 & 4291 & 4305 & 37 & 0.06 & 37.92 & 0.179179 & 0.114114 & 4 & 113016911 & 4 & 113982378 & 4 & 113 & 0.02 & 0.665 & 7.29 \\
\hline 737 & 4304 & 4323 & 33 & 0.03 & 67.95 & 0.148148 & 0.097097 & 4 & 114013243 & 4 & 114969990 & 4 & 114 & 0.038 & 0.6995 & 5.61 \\
\hline 738 & 4324 & 60684 & 25 & 0.04 & 62.5 & 0.105105 & 0.072072 & 4 & 115009742 & 4 & 115961449 & 4 & 115 & 0.034 & 0.6963 & 5.85 \\
\hline
\end{tabular}




\begin{tabular}{|c|c|c|c|c|c|c|c|c|c|c|c|c|c|c|c|c|}
\hline 739 & 4335 & 4345 & 25 & 0.04 & 62.65 & 0.119119 & 0.069069 & 4 & 116017042 & 4 & 116922748 & 4 & 116 & 0.034 & 0.6964 & 5.84 \\
\hline 740 & 46759 & 17226 & 31 & 0.05 & 41 & 0.131131 & 0.095095 & 4 & 117005641 & 4 & 117950885 & 4 & 117 & 0.022 & 0.6725 & 7.11 \\
\hline 741 & 22325 & 17379 & 28 & 0.03 & 66.6 & 0.121121 & 0.077077 & 4 & 118076498 & 4 & 118994554 & 4 & 118 & 0.037 & 0.6993 & 5.67 \\
\hline 742 & 4366 & 22338 & 27 & 0.04 & 60.8 & 0.122122 & 0.072072 & 4 & 119022315 & 4 & 119974157 & 4 & 119 & 0.033 & 0.6959 & 5.93 \\
\hline 743 & 41269 & 41272 & 22 & 0.02 & 82.15 & 0.096096 & 0.063063 & 4 & 120018660 & 4 & 120910957 & 4 & 120 & 0.047 & 0.705 & 5.07 \\
\hline 744 & 22346 & 41275 & 34 & 0.04 & 54.08 & 0.145145 & 0.097097 & 4 & 121010319 & 4 & 121989854 & 4 & 121 & 0.029 & 0.6916 & 6.29 \\
\hline 745 & 37490 & 22359 & 24 & 0.04 & 63.87 & 0.116116 & 0.084084 & 4 & 122078780 & 4 & 122988956 & 4 & 122 & 0.035 & 0.6972 & 5.78 \\
\hline 746 & 41276 & 4409 & 42 & 0.16 & 14.91 & 0.215215 & 0.149149 & 4 & 123001093 & 4 & 123983260 & 4 & 123 & 0.008 & 0.5731 & 10.22 \\
\hline 747 & 46767 & 4417 & 27 & 0.09 & 22.98 & 0.139139 & 0.103103 & 4 & 124043519 & 4 & 124982191 & 4 & 124 & 0.012 & 0.6193 & 8.84 \\
\hline 748 & 41286 & 22370 & 16 & 0.05 & 48.01 & 0.103103 & 0.074074 & 4 & 125054205 & 4 & 125948453 & 4 & 125 & 0.026 & 0.6822 & 6.58 \\
\hline 749 & 46769 & 60507 & 12 & 0.03 & 76.45 & 0.065065 & 0.044044 & 4 & 126080976 & 4 & 126984212 & 4 & 126 & 0.043 & 0.7029 & 5.27 \\
\hline 750 & 4430 & 14793 & 21 & 0.05 & 41.26 & 0.106106 & 0.076076 & 4 & 127072671 & 4 & 127895687 & 4 & 127 & 0.022 & 0.6736 & 7.1 \\
\hline 751 & 4435 & 62066 & 30 & 0.03 & 69.92 & 0.145145 & 0.087087 & 4 & 128001137 & 4 & 128996039 & 4 & 128 & 0.039 & 0.7001 & 5.52 \\
\hline 752 & 4447 & 4457 & 27 & 0.03 & 69.83 & 0.126126 & 0.077077 & 4 & 129016956 & 4 & 129991207 & 4 & 129 & 0.039 & 0.7001 & 5.53 \\
\hline 753 & 4458 & 4471 & 36 & 0.04 & 60.27 & 0.161161 & 0.099099 & 4 & 130003887 & 4 & 130976284 & 4 & 130 & 0.033 & 0.6956 & 5.96 \\
\hline 754 & 4472 & 4484 & 27 & 0.03 & 69.7 & 0.121121 & 0.079079 & 4 & 131001920 & 4 & 131918945 & 4 & 131 & 0.039 & 0.6998 & 5.53 \\
\hline 755 & 4485 & 17260 & 33 & 0.04 & 62.17 & 0.138138 & 0.089089 & 4 & 132039333 & 4 & 132970877 & 4 & 132 & 0.034 & 0.6962 & 5.87 \\
\hline 756 & 34031 & 35791 & 35 & 0.03 & 70.4 & 0.138138 & 0.088088 & 4 & 133019096 & 4 & 133997198 & 4 & 133 & 0.039 & 0.7006 & 5.51 \\
\hline 757 & 41307 & 58188 & 32 & 0.03 & 65 & 0.137137 & 0.096096 & 4 & 134029125 & 4 & 134976773 & 4 & 134 & 0.036 & 0.6981 & 5.74 \\
\hline 758 & 41310 & 34359 & 29 & 0.08 & 25.16 & 0.147147 & 0.111111 & 4 & 135010909 & 4 & 135982856 & 4 & 135 & 0.013 & 0.6268 & 8.49 \\
\hline 759 & 55823 & 22436 & 23 & 0.02 & 87.27 & 0.077077 & 0.045045 & 4 & 136004128 & 4 & 136979735 & 4 & 136 & 0.05 & 0.7075 & 4.92 \\
\hline 760 & 60100 & 4539 & 34 & 0.04 & 53.27 & 0.165165 & 0.104104 & 4 & 137017974 & 4 & 137950544 & 4 & 137 & 0.029 & 0.6917 & 6.36 \\
\hline 761 & 22445 & 22454 & 25 & 0.03 & 77.03 & 0.092092 & 0.061061 & 4 & 138123252 & 4 & 138987333 & 4 & 138 & 0.044 & 0.7034 & 5.25 \\
\hline 762 & 4547 & 22462 & 25 & 0.08 & 25.24 & 0.137137 & 0.104104 & 4 & 139015407 & 4 & 139989597 & 4 & 139 & 0.013 & 0.6273 & 8.47 \\
\hline 763 & 37531 & 22469 & 25 & 0.05 & 43.97 & 0.117117 & 0.082082 & 4 & 140011062 & 4 & 140899838 & 4 & 140 & 0.023 & 0.6785 & 6.92 \\
\hline 764 & 41334 & 4564 & 27 & 0.02 & 86.81 & 0.111111 & 0.064064 & 4 & 141117946 & 4 & 141966625 & 4 & 141 & 0.05 & 0.7074 & 4.93 \\
\hline 765 & 22474 & 4574 & 23 & 0.04 & 55.62 & 0.107107 & 0.075075 & 4 & 142008163 & 4 & 142982699 & 4 & 142 & 0.03 & 0.6919 & 6.18 \\
\hline 766 & 55176 & 61617 & 17 & 0.03 & 78.98 & 0.068068 & 0.047047 & 4 & 143021644 & 4 & 143462966 & 4 & 143 & 0.045 & 0.7045 & 5.19 \\
\hline 767 & 51890 & 60972 & 19 & 0.03 & 76.87 & 0.088088 & 0.065065 & 5 & 144471 & 5 & 961240 & 5 & 0 & 0.043 & 0.7033 & 5.26 \\
\hline 768 & 29255 & 41348 & 26 & 0.03 & 73.59 & 0.103103 & 0.073073 & 5 & 1007763 & 5 & 1981830 & 5 & 1 & 0.041 & 0.702 & 5.37 \\
\hline 769 & 41347 & 53366 & 29 & 0.06 & 37.57 & 0.133133 & 0.09009 & 5 & 2021416 & 5 & 2975876 & 5 & 2 & 0.02 & 0.6637 & 7.3 \\
\hline 770 & 41351 & 41356 & 21 & 0.04 & 52.11 & 0.087087 & 0.054054 & 5 & 3060760 & 5 & 3844970 & 5 & 3 & 0.028 & 0.6897 & 6.41 \\
\hline 771 & 48814 & 58654 & 24 & 0.06 & 31.72 & 0.123123 & 0.088088 & 5 & 4092291 & 5 & 4916323 & 5 & 4 & 0.017 & 0.647 & 7.76 \\
\hline 772 & 4597 & 22510 & 29 & 0.08 & 27.1 & 0.115115 & 0.078078 & 5 & 5011010 & 5 & 5995341 & 5 & 5 & 0.014 & 0.6325 & 8.2 \\
\hline 773 & 4608 & 22515 & 26 & 0.12 & 18.35 & 0.14014 & 0.101101 & 5 & 6072443 & 5 & 6980596 & 5 & 6 & 0.009 & 0.5939 & 9.51 \\
\hline 774 & 48820 & 4620 & 19 & 0.02 & 82.39 & 0.081081 & 0.053053 & 5 & 7013047 & 5 & 7992138 & 5 & 7 & 0.047 & 0.7049 & 5.07 \\
\hline 775 & 48823 & 4626 & 31 & 0.05 & 42.05 & 0.136136 & 0.088088 & 5 & 8031569 & 5 & 8937125 & 5 & 8 & 0.022 & 0.6754 & 7.06 \\
\hline 776 & 41383 & 22526 & 22 & 0.04 & 60.16 & 0.106106 & 0.068068 & 5 & 9096232 & 5 & 9942508 & 5 & 9 & 0.033 & 0.6955 & 5.96 \\
\hline 777 & 22527 & 22535 & 26 & 0.03 & 69.4 & 0.098098 & 0.065065 & 5 & 10080308 & 5 & 10947872 & 5 & 10 & 0.039 & 0.6996 & 5.54 \\
\hline
\end{tabular}




\begin{tabular}{|c|c|c|c|c|c|c|c|c|c|c|c|c|c|c|c|c|}
\hline 778 & 4642 & 46790 & 22 & 0.02 & 84.91 & 0.098098 & 0.061061 & 5 & 11029721 & 5 & 11981380 & 5 & 11 & 0.049 & 0.7061 & 4.98 \\
\hline 779 & 41393 & 22547 & 33 & 0.03 & 78.25 & 0.149149 & 0.075075 & 5 & 12002670 & 5 & 12992563 & 5 & 12 & 0.044 & 0.7041 & 5.22 \\
\hline 780 & 22548 & 55397 & 24 & 0.03 & 73.41 & 0.099099 & 0.061061 & 5 & 13000041 & 5 & 13990580 & 5 & 13 & 0.041 & 0.7017 & 5.38 \\
\hline 781 & 58093 & 4675 & 31 & 0.04 & 60.99 & 0.124124 & 0.085085 & 5 & 14008737 & 5 & 14972422 & 5 & 14 & 0.033 & 0.6962 & 5.93 \\
\hline 782 & 37544 & 56372 & 21 & 0.03 & 75.92 & 0.108108 & 0.065065 & 5 & 15052496 & 5 & 15908465 & 5 & 15 & 0.043 & 0.7029 & 5.29 \\
\hline 783 & 57492 & 50789 & 20 & 0.04 & 55.08 & 0.091091 & 0.063063 & 5 & 16001621 & 5 & 16920074 & 5 & 16 & 0.03 & 0.692 & 6.22 \\
\hline 784 & 56684 & 48832 & 30 & 0.04 & 60.91 & 0.127127 & 0.086086 & 5 & 17055970 & 5 & 17949392 & 5 & 17 & 0.033 & 0.6961 & 5.93 \\
\hline 785 & 51000 & 22588 & 33 & 0.04 & 58.23 & 0.155155 & 0.108108 & 5 & 18012619 & 5 & 18992892 & 5 & 18 & 0.032 & 0.6933 & 6.03 \\
\hline 786 & 37546 & 22591 & 14 & 0.01 & 93.28 & 0.064064 & 0.044044 & 5 & 19053403 & 5 & 19631292 & 5 & 19 & 0.055 & 0.708 & 4.72 \\
\hline 787 & 51925 & 4711 & 26 & 0.03 & 71.42 & 0.095095 & 0.062062 & 5 & 20035192 & 5 & 20954929 & 5 & 20 & 0.04 & 0.7008 & 5.46 \\
\hline 788 & 4712 & 4715 & 3 & 0 & 96.52 & 0.008008 & 0.006006 & 5 & 21017524 & 5 & 21547496 & 5 & 21 & 0.057 & 0.7092 & 4.63 \\
\hline 789 & 4719 & 22608 & 9 & 0.01 & 93.13 & 0.034034 & 0.027027 & 5 & 22536178 & 5 & 22969678 & 5 & 22 & 0.054 & 0.7081 & 4.73 \\
\hline 790 & 4720 & 22613 & 15 & 0.02 & 87.31 & 0.075075 & 0.049049 & 5 & 23002609 & 5 & 23994070 & 5 & 23 & 0.05 & 0.7077 & 4.92 \\
\hline 791 & 36399 & 41425 & 9 & 0.01 & 94.05 & 0.05005 & 0.028028 & 5 & 24041995 & 5 & 24782967 & 5 & 24 & 0.055 & 0.7084 & 4.7 \\
\hline 792 & 50436 & 4736 & 27 & 0.04 & 64.44 & 0.124124 & 0.072072 & 5 & 25027517 & 5 & 25995235 & 5 & 25 & 0.036 & 0.6978 & 5.76 \\
\hline 793 & 4741 & 57971 & 14 & 0.04 & 63.69 & 0.065065 & 0.05005 & 5 & 26081086 & 5 & 26929026 & 5 & 26 & 0.035 & 0.6972 & 5.79 \\
\hline 794 & 15878 & 37554 & 15 & 0.02 & 83.27 & 0.091091 & 0.058058 & 5 & 27040193 & 5 & 27970414 & 5 & 27 & 0.048 & 0.7045 & 5.02 \\
\hline 795 & 4753 & 4757 & 16 & 0.08 & 25.48 & 0.084084 & 0.063063 & 5 & 28124798 & 5 & 28898534 & 5 & 28 & 0.013 & 0.6288 & 8.47 \\
\hline 796 & 31262 & 22633 & 11 & 0.07 & 27.62 & 0.074074 & 0.06006 & 5 & 29078553 & 5 & 29952075 & 5 & 29 & 0.015 & 0.6352 & 8.16 \\
\hline 797 & 22634 & 22638 & 25 & 0.18 & 12.58 & 0.148148 & 0.11011 & 5 & 30056408 & 5 & 30913196 & 5 & 30 & 0.006 & 0.5584 & 10.91 \\
\hline 798 & 4764 & 41437 & 19 & 0.03 & 65.35 & 0.07007 & 0.044044 & 5 & 31161895 & 5 & 31980027 & 5 & 31 & 0.036 & 0.6985 & 5.72 \\
\hline 799 & 22640 & 4773 & 17 & 0.02 & 89.51 & 0.08008 & 0.047047 & 5 & 32015964 & 5 & 32986570 & 5 & 32 & 0.052 & 0.7078 & 4.84 \\
\hline 800 & 4774 & 50667 & 20 & 0.01 & 92.62 & 0.094094 & 0.049049 & 5 & 33007354 & 5 & 33957565 & 5 & 33 & 0.054 & 0.7081 & 4.75 \\
\hline 801 & 17044 & 4790 & 36 & 0.07 & 29.07 & 0.172172 & 0.113113 & 5 & 34011426 & 5 & 34945860 & 5 & 34 & 0.015 & 0.6392 & 8 \\
\hline 802 & 32054 & 22667 & 18 & 0.02 & 90.92 & 0.077077 & 0.043043 & 5 & 35168512 & 5 & 35945657 & 5 & 35 & 0.053 & 0.7081 & 4.8 \\
\hline 803 & 61241 & 22675 & 26 & 0.13 & 16.47 & 0.163163 & 0.13013 & 5 & 36115758 & 5 & 36973186 & 5 & 36 & 0.009 & 0.5832 & 9.85 \\
\hline 804 & 61199 & 46814 & 21 & 0.16 & 14.1 & 0.145145 & 0.109109 & 5 & 37025932 & 5 & 37974847 & 5 & 37 & 0.007 & 0.5703 & 10.54 \\
\hline 805 & 4801 & 4806 & 18 & 0.03 & 75.58 & 0.08008 & 0.039039 & 5 & 38006963 & 5 & 38988849 & 5 & 38 & 0.043 & 0.7024 & 5.3 \\
\hline 806 & 31940 & 46816 & 20 & 0.07 & 30.05 & 0.104104 & 0.069069 & 5 & 39028335 & 5 & 39916475 & 5 & 39 & 0.016 & 0.6422 & 7.9 \\
\hline 807 & 51608 & 37599 & 12 & 0.01 & 93.48 & 0.061061 & 0.038038 & 5 & 40106617 & 5 & 40915894 & 5 & 40 & 0.055 & 0.7082 & 4.72 \\
\hline 808 & 61891 & 37601 & 18 & 0.11 & 18.92 & 0.114114 & 0.091091 & 5 & 41151243 & 5 & 41831002 & 5 & 41 & 0.01 & 0.5964 & 9.39 \\
\hline 809 & 37602 & 41457 & 15 & 0.01 & 92.9 & 0.057057 & 0.04004 & 5 & 42081631 & 5 & 42945018 & 5 & 42 & 0.054 & 0.7081 & 4.74 \\
\hline 810 & 18312 & 46823 & 7 & 0.03 & 72.81 & 0.048048 & 0.026026 & 5 & 43144872 & 5 & 43997881 & 5 & 43 & 0.041 & 0.7014 & 5.41 \\
\hline 811 & 60113 & 4831 & 16 & 0.04 & 55.25 & 0.08008 & 0.056056 & 5 & 44070464 & 5 & 44994712 & 5 & 44 & 0.03 & 0.692 & 6.2 \\
\hline 812 & 39699 & 53034 & 9 & 0.03 & 76.31 & 0.036036 & 0.025025 & 5 & 45007479 & 5 & 45963766 & 5 & 45 & 0.043 & 0.7029 & 5.28 \\
\hline 813 & 32984 & 37617 & 17 & 0.03 & 71.04 & 0.087087 & 0.057057 & 5 & 46029879 & 5 & 46748561 & 5 & 46 & 0.04 & 0.7005 & 5.48 \\
\hline 814 & 33661 & 4844 & 14 & 0.01 & 93.75 & 0.048048 & 0.034034 & 5 & 47023315 & 5 & 47940238 & 5 & 47 & 0.055 & 0.7082 & 4.71 \\
\hline 815 & 46827 & 37623 & 13 & 0.01 & 95.8 & 0.065065 & 0.029029 & 5 & 48150113 & 5 & 48975686 & 5 & 48 & 0.056 & 0.7089 & 4.65 \\
\hline 816 & 37622 & 37627 & 20 & 0.03 & 78.49 & 0.083083 & 0.056056 & 5 & 49040715 & 5 & 49975876 & 5 & 49 & 0.044 & 0.7041 & 5.21 \\
\hline
\end{tabular}




\begin{tabular}{|c|c|c|c|c|c|c|c|c|c|c|c|c|c|c|c|c|}
\hline 817 & 37628 & 4853 & 10 & 0.01 & 96.22 & 0.039039 & 0.021021 & 5 & 50214568 & 5 & 50991368 & 5 & 50 & 0.057 & 0.7091 & 4.64 \\
\hline 818 & 46830 & 46832 & 15 & 0.03 & 80.06 & 0.058058 & 0.033033 & 5 & 51014577 & 5 & 51824431 & 5 & 51 & 0.045 & 0.7047 & 5.15 \\
\hline 819 & 29636 & 52487 & 19 & 0.03 & 66.22 & 0.085085 & 0.051051 & 5 & 52019205 & 5 & 52937921 & 5 & 52 & 0.037 & 0.6987 & 5.68 \\
\hline 820 & 4859 & 4862 & 13 & 0.02 & 85.13 & 0.058058 & 0.029029 & 5 & 53182979 & 5 & 53998123 & 5 & 53 & 0.049 & 0.7062 & 4.98 \\
\hline 821 & 18149 & 46840 & 11 & 0.02 & 87.65 & 0.042042 & 0.032032 & 5 & 54100199 & 5 & 54967272 & 5 & 54 & 0.051 & 0.7077 & 4.9 \\
\hline 822 & 37643 & 22718 & 20 & 0.03 & 66.87 & 0.087087 & 0.05005 & 5 & 55008930 & 5 & 55999452 & 5 & 55 & 0.037 & 0.6992 & 5.66 \\
\hline 823 & 46842 & 33443 & 7 & 0.01 & 95.64 & 0.03003 & 0.012012 & 5 & 56054855 & 5 & 56865644 & 5 & 56 & 0.056 & 0.7088 & 4.66 \\
\hline 824 & 52862 & 37653 & 21 & 0.02 & 82.08 & 0.094094 & 0.052052 & 5 & 57011829 & 5 & 57885800 & 5 & 57 & 0.047 & 0.7048 & 5.08 \\
\hline 825 & 59570 & 33205 & 9 & 0.01 & 94.96 & 0.032032 & 0.019019 & 5 & 58028366 & 5 & 58957433 & 5 & 58 & 0.056 & 0.7087 & 4.68 \\
\hline 826 & 37656 & 4889 & 17 & 0.03 & 76.62 & 0.057057 & 0.043043 & 5 & 59023829 & 5 & 59906559 & 5 & 59 & 0.043 & 0.703 & 5.26 \\
\hline 827 & 14795 & 4894 & 20 & 0.06 & 33.68 & 0.095095 & 0.074074 & 5 & 60196527 & 5 & 60978291 & 5 & 60 & 0.018 & 0.6517 & 7.6 \\
\hline 828 & 22731 & 4902 & 20 & 0.03 & 72.84 & 0.091091 & 0.058058 & 5 & 61020546 & 5 & 61979581 & 5 & 61 & 0.041 & 0.7014 & 5.4 \\
\hline 829 & 60143 & 33985 & 36 & 0.1 & 21.01 & 0.191191 & 0.142142 & 5 & 62000754 & 5 & 62972049 & 5 & 62 & 0.011 & 0.6059 & 9.02 \\
\hline 830 & 14362 & 4932 & 31 & 0.03 & 68.05 & 0.125125 & 0.077077 & 5 & 63039669 & 5 & 63996259 & 5 & 63 & 0.038 & 0.6995 & 5.6 \\
\hline 831 & 41491 & 37668 & 24 & 0.04 & 51.32 & 0.12012 & 0.09009 & 5 & 64000265 & 5 & 64971001 & 5 & 64 & 0.028 & 0.6887 & 6.44 \\
\hline 832 & 22748 & 17603 & 24 & 0.05 & 41.68 & 0.129129 & 0.086086 & 5 & 65003383 & 5 & 65950020 & 5 & 65 & 0.022 & 0.6753 & 7.1 \\
\hline 833 & 14412 & 48843 & 26 & 0.04 & 53.7 & 0.121121 & 0.085085 & 5 & 66015525 & 5 & 66918435 & 5 & 66 & 0.029 & 0.6918 & 6.32 \\
\hline 834 & 51848 & 4950 & 32 & 0.08 & 26.87 & 0.139139 & 0.1001 & 5 & 67063213 & 5 & 67994769 & 5 & 67 & 0.014 & 0.6321 & 8.25 \\
\hline 835 & 22760 & 48846 & 29 & 0.04 & 52.41 & 0.131131 & 0.083083 & 5 & 68011832 & 5 & 68998629 & 5 & 68 & 0.028 & 0.6906 & 6.4 \\
\hline 836 & 22766 & 16572 & 35 & 0.05 & 40.57 & 0.137137 & 0.097097 & 5 & 69012014 & 5 & 69897370 & 5 & 69 & 0.021 & 0.6719 & 7.14 \\
\hline 837 & 57141 & 22776 & 22 & 0.1 & 20.82 & 0.119119 & 0.089089 & 5 & 70001568 & 5 & 70984258 & 5 & 70 & 0.011 & 0.6054 & 9.08 \\
\hline 838 & 58110 & 41523 & 29 & 0.12 & 18.69 & 0.147147 & 0.111111 & 5 & 71056490 & 5 & 71996403 & 5 & 71 & 0.01 & 0.5947 & 9.42 \\
\hline 839 & 22778 & 4985 & 22 & 0.05 & 47.97 & 0.103103 & 0.075075 & 5 & 72009882 & 5 & 72971559 & 5 & 72 & 0.026 & 0.6824 & 6.59 \\
\hline 840 & 4984 & 22787 & 11 & 0.01 & 96.07 & 0.038038 & 0.016016 & 5 & 73000608 & 5 & 73987917 & 5 & 73 & 0.057 & 0.709 & 4.64 \\
\hline 841 & 22788 & 41528 & 18 & 0.04 & 61.88 & 0.082082 & 0.05005 & 5 & 74020663 & 5 & 74804208 & 5 & 74 & 0.034 & 0.6968 & 5.9 \\
\hline 842 & 54214 & 22797 & 25 & 0.05 & 42.87 & 0.114114 & 0.083083 & 5 & 75044596 & 5 & 75969214 & 5 & 75 & 0.023 & 0.6766 & 7.01 \\
\hline 843 & 22798 & 59361 & 15 & 0.03 & 76.78 & 0.08008 & 0.052052 & 5 & 76016635 & 5 & 76994567 & 5 & 76 & 0.043 & 0.7032 & 5.26 \\
\hline 844 & 61670 & 37683 & 23 & 0.03 & 74.45 & 0.091091 & 0.062062 & 5 & 77016025 & 5 & 77994370 & 5 & 77 & 0.042 & 0.7018 & 5.33 \\
\hline 845 & 46861 & 5018 & 20 & 0.01 & 93.93 & 0.078078 & 0.034034 & 5 & 78067097 & 5 & 78990780 & 5 & 78 & 0.055 & 0.7083 & 4.7 \\
\hline 846 & 48854 & 5033 & 19 & 0.01 & 95.25 & 0.071071 & 0.032032 & 5 & 79010106 & 5 & 79988080 & 5 & 79 & 0.056 & 0.7086 & 4.67 \\
\hline 847 & 53969 & 16989 & 28 & 0.04 & 57.2 & 0.14014 & 0.069069 & 5 & 80009101 & 5 & 80967561 & 5 & 80 & 0.031 & 0.6925 & 6.09 \\
\hline 848 & 55686 & 5040 & 28 & 0.01 & 94.89 & 0.114114 & 0.048048 & 5 & 81043360 & 5 & 81968675 & 5 & 81 & 0.056 & 0.7085 & 4.68 \\
\hline 849 & 5041 & 5049 & 21 & 0.01 & 93.39 & 0.093093 & 0.047047 & 5 & 82045018 & 5 & 82988313 & 5 & 82 & 0.055 & 0.7081 & 4.72 \\
\hline 850 & 38165 & 61626 & 22 & 0.02 & 84.89 & 0.107107 & 0.056056 & 5 & 83011120 & 5 & 83918631 & 5 & 83 & 0.049 & 0.7061 & 4.98 \\
\hline 851 & 5055 & 29541 & 36 & 0.05 & 43.82 & 0.164164 & 0.087087 & 5 & 84005513 & 5 & 84932150 & 5 & 84 & 0.023 & 0.678 & 6.94 \\
\hline 852 & 50955 & 35158 & 16 & 0.04 & 55.46 & 0.077077 & 0.054054 & 5 & 85023135 & 5 & 85946546 & 5 & 85 & 0.03 & 0.692 & 6.19 \\
\hline 853 & 5070 & 37698 & 21 & 0.02 & 91.29 & 0.089089 & 0.045045 & 5 & 86094560 & 5 & 86906356 & 5 & 86 & 0.053 & 0.7081 & 4.79 \\
\hline 854 & 34683 & 31894 & 22 & 0.06 & 36.53 & 0.11011 & 0.076076 & 5 & 87048659 & 5 & 87950997 & 5 & 87 & 0.02 & 0.6614 & 7.37 \\
\hline 855 & 45712 & 5079 & 15 & 0.01 & 94.23 & 0.069069 & 0.04004 & 5 & 88001856 & 5 & 88978805 & 5 & 88 & 0.055 & 0.7083 & 4.69 \\
\hline
\end{tabular}




\begin{tabular}{|c|c|c|c|c|c|c|c|c|c|c|c|c|c|c|c|c|}
\hline 856 & 5080 & 5085 & 18 & 0.02 & 84.12 & 0.085085 & 0.055055 & 5 & 89017490 & 5 & 89879433 & 5 & 89 & 0.048 & 0.7053 & 4.99 \\
\hline 857 & 22834 & 57956 & 15 & 0.01 & 93.64 & 0.044044 & 0.025025 & 5 & 90128642 & 5 & 90976986 & 5 & 90 & 0.055 & 0.7081 & 4.71 \\
\hline 858 & 46870 & 46875 & 32 & 0.03 & 77.49 & 0.126126 & 0.066066 & 5 & 91016023 & 5 & 91991652 & 5 & 91 & 0.044 & 0.7036 & 5.24 \\
\hline 859 & 41563 & 53421 & 16 & 0.01 & 94.36 & 0.068068 & 0.036036 & 5 & 92073891 & 5 & 92957318 & 5 & 92 & 0.055 & 0.7083 & 4.69 \\
\hline 860 & 19256 & 5107 & 26 & 0.03 & 69.31 & 0.101101 & 0.063063 & 5 & 93029076 & 5 & 93996098 & 5 & 93 & 0.039 & 0.6996 & 5.54 \\
\hline 861 & 5106 & 5116 & 29 & 0.04 & 59.7 & 0.146146 & 0.09009 & 5 & 94018164 & 5 & 94958858 & 5 & 94 & 0.033 & 0.6952 & 5.98 \\
\hline 862 & 48866 & 41570 & 19 & 0.04 & 54.21 & 0.099099 & 0.054054 & 5 & 95007471 & 5 & 95971272 & 5 & 95 & 0.029 & 0.6918 & 6.29 \\
\hline 863 & 58755 & 5129 & 18 & 0.18 & 12.76 & 0.121121 & 0.094094 & 5 & 96024130 & 5 & 96953817 & 5 & 96 & 0.006 & 0.561 & 10.92 \\
\hline 864 & 35055 & 33233 & 22 & 0.04 & 50.17 & 0.094094 & 0.056056 & 5 & 97022279 & 5 & 97980633 & 5 & 97 & 0.027 & 0.6863 & 6.49 \\
\hline 865 & 33611 & 22870 & 21 & 0.03 & 68.52 & 0.09009 & 0.051051 & 5 & 98025183 & 5 & 98986619 & 5 & 98 & 0.038 & 0.6995 & 5.58 \\
\hline 866 & 5160 & 5146 & 35 & 0.05 & 49.13 & 0.133133 & 0.075075 & 5 & 99010975 & 5 & 99972762 & 5 & 99 & 0.027 & 0.6846 & 6.54 \\
\hline 867 & 35078 & 52364 & 21 & 0.03 & 66.77 & 0.078078 & 0.058058 & 5 & 100037065 & 5 & 100881229 & 5 & 100 & 0.037 & 0.6992 & 5.66 \\
\hline 868 & 5163 & 50582 & 10 & 0.02 & 89.7 & 0.046046 & 0.03003 & 5 & 101013243 & 5 & 101758816 & 5 & 101 & 0.052 & 0.7077 & 4.83 \\
\hline 869 & 33288 & 5173 & 20 & 0.01 & 95.74 & 0.067067 & 0.032032 & 5 & 102247795 & 5 & 102993667 & 5 & 102 & 0.056 & 0.7089 & 4.66 \\
\hline 870 & 5174 & 14894 & 24 & 0.04 & 64.72 & 0.11011 & 0.073073 & 5 & 103013980 & 5 & 103936081 & 5 & 103 & 0.036 & 0.698 & 5.75 \\
\hline 871 & 15672 & 22889 & 26 & 0.02 & 83.37 & 0.11011 & 0.063063 & 5 & 104000831 & 5 & 104987144 & 5 & 104 & 0.048 & 0.7046 & 5.02 \\
\hline 872 & 41582 & 22893 & 32 & 0.05 & 41.42 & 0.129129 & 0.083083 & 5 & 105059227 & 5 & 105954521 & 5 & 105 & 0.022 & 0.6738 & 7.1 \\
\hline 873 & 22894 & 5207 & 22 & 0.03 & 72.35 & 0.104104 & 0.062062 & 5 & 106014977 & 5 & 106979109 & 5 & 106 & 0.041 & 0.7011 & 5.42 \\
\hline 874 & 5208 & 41588 & 29 & 0.05 & 48.39 & 0.112112 & 0.081081 & 5 & 107029477 & 5 & 107978337 & 5 & 107 & 0.026 & 0.6827 & 6.55 \\
\hline 875 & 5220 & 22906 & 22 & 0.02 & 81.91 & 0.09009 & 0.058058 & 5 & 108020226 & 5 & 108968821 & 5 & 108 & 0.047 & 0.7051 & 5.09 \\
\hline 876 & 5211 & 22917 & 20 & 0.02 & 89.75 & 0.079079 & 0.044044 & 5 & 109014722 & 5 & 109990527 & 5 & 109 & 0.052 & 0.7077 & 4.83 \\
\hline 877 & 5228 & 56131 & 9 & 0.01 & 94.72 & 0.035035 & 0.027027 & 5 & 110032368 & 5 & 110935968 & 5 & 110 & 0.056 & 0.7086 & 4.68 \\
\hline 878 & 16810 & 33983 & 5 & 0.01 & 96.07 & 0.027027 & 0.018018 & 5 & 111137068 & 5 & 111371828 & 5 & 111 & 0.057 & 0.7091 & 4.64 \\
\hline 879 & 16346 & 18574 & 9 & 0.01 & 95.61 & 0.037037 & 0.019019 & 6 & 79816 & 6 & 851457 & 6 & 0 & 0.056 & 0.7088 & 4.66 \\
\hline 880 & 30235 & 34251 & 8 & 0.01 & 92.05 & 0.046046 & 0.03003 & 6 & 1065220 & 6 & 1939451 & 6 & 1 & 0.053 & 0.7081 & 4.77 \\
\hline 881 & 19394 & 50110 & 11 & 0.01 & 94.3 & 0.04004 & 0.021021 & 6 & 2042055 & 6 & 2952600 & 6 & 2 & 0.055 & 0.7083 & 4.69 \\
\hline 882 & 59770 & 16401 & 24 & 0.03 & 80.44 & 0.095095 & 0.052052 & 6 & 3005254 & 6 & 3989070 & 6 & 3 & 0.046 & 0.705 & 5.14 \\
\hline 883 & 17685 & 33718 & 37 & 0.04 & 60.12 & 0.155155 & 0.09009 & 6 & 4060304 & 6 & 4871179 & 6 & 4 & 0.033 & 0.6955 & 5.97 \\
\hline 884 & 30000 & 37752 & 30 & 0.04 & 53.1 & 0.137137 & 0.1001 & 6 & 5041803 & 6 & 5951885 & 6 & 5 & 0.029 & 0.6916 & 6.37 \\
\hline 885 & 62032 & 22920 & 20 & 0.03 & 68.32 & 0.095095 & 0.056056 & 6 & 6052681 & 6 & 6921532 & 6 & 6 & 0.038 & 0.6994 & 5.59 \\
\hline 886 & 5242 & 5251 & 34 & 0.04 & 50.53 & 0.141141 & 0.1001 & 6 & 7000417 & 6 & 7943484 & 6 & 7 & 0.027 & 0.6869 & 6.47 \\
\hline 887 & 41597 & 52796 & 33 & 0.06 & 32.61 & 0.145145 & 0.103103 & 6 & 8019390 & 6 & 8966343 & 6 & 8 & 0.017 & 0.6502 & 7.71 \\
\hline 888 & 18733 & 29842 & 31 & 0.05 & 41.99 & 0.143143 & 0.084084 & 6 & 9149467 & 6 & 9957369 & 6 & 9 & 0.022 & 0.6754 & 7.06 \\
\hline 889 & 15933 & 57273 & 34 & 0.06 & 35.88 & 0.162162 & 0.099099 & 6 & 10012272 & 6 & 10950015 & 6 & 10 & 0.019 & 0.6584 & 7.41 \\
\hline 890 & 15889 & 41604 & 18 & 0.03 & 65.04 & 0.079079 & 0.053053 & 6 & 11003757 & 6 & 11989602 & 6 & 11 & 0.036 & 0.6982 & 5.74 \\
\hline 891 & 22929 & 52834 & 20 & 0.02 & 84.93 & 0.105105 & 0.061061 & 6 & 12005914 & 6 & 12980787 & 6 & 12 & 0.049 & 0.7062 & 4.98 \\
\hline 892 & 45319 & 18258 & 27 & 0.03 & 77.44 & 0.119119 & 0.07007 & 6 & 13006700 & 6 & 13977172 & 6 & 13 & 0.044 & 0.7036 & 5.24 \\
\hline 893 & 31738 & 5262 & 27 & 0.05 & 46.36 & 0.128128 & 0.087087 & 6 & 14016657 & 6 & 14947951 & 6 & 14 & 0.025 & 0.6817 & 6.73 \\
\hline 894 & 22936 & 53721 & 31 & 0.05 & 47.59 & 0.146146 & 0.09009 & 6 & 15000888 & 6 & 15985029 & 6 & 15 & 0.026 & 0.6822 & 6.62 \\
\hline
\end{tabular}




\begin{tabular}{|c|c|c|c|c|c|c|c|c|c|c|c|c|c|c|c|c|}
\hline 895 & 17873 & 15478 & 22 & 0.06 & 38.71 & 0.105105 & 0.079079 & 6 & 16013020 & 6 & 16859988 & 6 & 16 & 0.021 & 0.6671 & 7.24 \\
\hline 896 & 17827 & 36070 & 34 & 0.06 & 32.93 & 0.155155 & 0.103103 & 6 & 17189599 & 6 & 17980080 & 6 & 17 & 0.017 & 0.6503 & 7.67 \\
\hline 897 & 49937 & 5274 & 39 & 0.05 & 42.46 & 0.162162 & 0.103103 & 6 & 18019907 & 6 & 18982722 & 6 & 18 & 0.022 & 0.6765 & 7.05 \\
\hline 898 & 22948 & 5282 & 22 & 0.02 & 81.55 & 0.082082 & 0.054054 & 6 & 19002312 & 6 & 19952347 & 6 & 19 & 0.046 & 0.7053 & 5.1 \\
\hline 899 & 34928 & 5286 & 13 & 0.02 & 91.03 & 0.056056 & 0.034034 & 6 & 20100569 & 6 & 20988489 & 6 & 20 & 0.053 & 0.7081 & 4.8 \\
\hline 900 & 5287 & 46901 & 27 & 0.04 & 50.57 & 0.119119 & 0.07007 & 6 & 21014463 & 6 & 21988030 & 6 & 21 & 0.027 & 0.6869 & 6.47 \\
\hline 901 & 41616 & 34796 & 15 & 0.03 & 79.78 & 0.061061 & 0.042042 & 6 & 22021386 & 6 & 22868204 & 6 & 22 & 0.045 & 0.7048 & 5.16 \\
\hline 902 & 33237 & 41617 & 26 & 0.04 & 62.43 & 0.12012 & 0.088088 & 6 & 23005830 & 6 & 23918938 & 6 & 23 & 0.034 & 0.6964 & 5.86 \\
\hline 904 & 29840 & 17966 & 11 & 0 & 96.24 & 0.046046 & 0.017017 & 6 & 25114255 & 6 & 25995859 & 6 & 25 & 0.057 & 0.7091 & 4.64 \\
\hline 905 & 5311 & 19392 & 25 & 0.02 & 83.25 & 0.107107 & 0.066066 & 6 & 26110849 & 6 & 26948992 & 6 & 26 & 0.048 & 0.7045 & 5.02 \\
\hline 906 & 22965 & 29298 & 16 & 0.02 & 91.35 & 0.074074 & 0.039039 & 6 & 27402328 & 6 & 27995521 & 6 & 27 & 0.053 & 0.7082 & 4.79 \\
\hline 907 & 32847 & 60920 & 17 & 0.25 & 10.36 & 0.127127 & 0.092092 & 6 & 28054235 & 6 & 28694089 & 6 & 28 & 0.005 & 0.542 & 11.54 \\
\hline 908 & 61575 & 5328 & 27 & 0.04 & 64.37 & 0.134134 & 0.079079 & 6 & 29076106 & 6 & 29958963 & 6 & 29 & 0.036 & 0.6977 & 5.76 \\
\hline 909 & 5329 & 22985 & 27 & 0.05 & 48.71 & 0.11011 & 0.074074 & 6 & 30009402 & 6 & 30942669 & 6 & 30 & 0.026 & 0.6834 & 6.54 \\
\hline 910 & 46907 & 41627 & 11 & 0.01 & 95.79 & 0.038038 & 0.019019 & 6 & 31009804 & 6 & 31994459 & 6 & 31 & 0.056 & 0.7089 & 4.65 \\
\hline 912 & 59081 & 31946 & 8 & 0.02 & 81.93 & 0.03003 & 0.019019 & 6 & 33257346 & 6 & 33810961 & 6 & 33 & 0.047 & 0.705 & 5.08 \\
\hline 913 & 40575 & 14993 & 18 & 0.03 & 65.52 & 0.087087 & 0.052052 & 6 & 34031908 & 6 & 34960452 & 6 & 34 & 0.036 & 0.6984 & 5.71 \\
\hline 914 & 52509 & 29711 & 18 & 0.07 & 30.33 & 0.079079 & 0.062062 & 6 & 35011158 & 6 & 35967061 & 6 & 35 & 0.016 & 0.6427 & 7.86 \\
\hline 915 & 14585 & 32592 & 17 & 0.02 & 87.57 & 0.071071 & 0.045045 & 6 & 36106926 & 6 & 36964748 & 6 & 36 & 0.05 & 0.7078 & 4.91 \\
\hline 916 & 45445 & 18381 & 31 & 0.13 & 17.39 & 0.178178 & 0.142142 & 6 & 37040339 & 6 & 37983742 & 6 & 37 & 0.009 & 0.5901 & 9.74 \\
\hline 917 & 30003 & 55336 & 19 & 0.03 & 67.82 & 0.08008 & 0.061061 & 6 & 38026430 & 6 & 38961317 & 6 & 38 & 0.038 & 0.6995 & 5.62 \\
\hline 918 & 32861 & 58371 & 6 & 0.01 & 94.9 & 0.029029 & 0.023023 & 6 & 39187023 & 6 & 39960478 & 6 & 39 & 0.056 & 0.7085 & 4.68 \\
\hline 919 & 56822 & 18333 & 23 & 0.02 & 89.65 & 0.082082 & 0.052052 & 6 & 40053845 & 6 & 40937247 & 6 & 40 & 0.052 & 0.7076 & 4.83 \\
\hline 920 & 18166 & 50247 & 19 & 0.02 & 87.79 & 0.075075 & 0.054054 & 6 & 41004930 & 6 & 41983038 & 6 & 41 & 0.051 & 0.7078 & 4.9 \\
\hline 921 & 40424 & 53713 & 10 & 0.02 & 90.9 & 0.049049 & 0.027027 & 6 & 42104347 & 6 & 42946643 & 6 & 42 & 0.053 & 0.708 & 4.8 \\
\hline 922 & 48276 & 14624 & 18 & 0.03 & 79.94 & 0.088088 & 0.056056 & 6 & 43076940 & 6 & 43969345 & 6 & 43 & 0.045 & 0.7047 & 5.16 \\
\hline 923 & 51628 & 35328 & 11 & 0.01 & 93.42 & 0.049049 & 0.035035 & 6 & 44004031 & 6 & 44985575 & 6 & 44 & 0.055 & 0.7081 & 4.72 \\
\hline 924 & 51442 & 60367 & 12 & 0.02 & 82.95 & 0.058058 & 0.037037 & 6 & 45460886 & 6 & 45906794 & 6 & 45 & 0.048 & 0.7045 & 5.03 \\
\hline 925 & 14820 & 17282 & 12 & 0.01 & 92.52 & 0.053053 & 0.032032 & 6 & 46003088 & 6 & 46893592 & 6 & 46 & 0.054 & 0.7081 & 4.75 \\
\hline 926 & 17213 & 30893 & 11 & 0.01 & 95.4 & 0.041041 & 0.029029 & 6 & 47104925 & 6 & 47852634 & 6 & 47 & 0.056 & 0.7087 & 4.66 \\
\hline 929 & 45764 & 45793 & 21 & 0.02 & 85.81 & 0.097097 & 0.059059 & 6 & 50006716 & 6 & 50922233 & 6 & 50 & 0.049 & 0.7065 & 4.95 \\
\hline 930 & 19050 & 5356 & 11 & 0.01 & 96.02 & 0.05005 & 0.024024 & 6 & 51104922 & 6 & 51843873 & 6 & 51 & 0.057 & 0.709 & 4.65 \\
\hline 931 & 59594 & 41639 & 12 & 0.01 & 93.29 & 0.053053 & 0.034034 & 6 & 52063034 & 6 & 52993696 & 6 & 52 & 0.055 & 0.7081 & 4.72 \\
\hline 932 & 23002 & 5366 & 16 & 0.03 & 76.73 & 0.062062 & 0.04004 & 6 & 53001243 & 6 & 53966129 & 6 & 53 & 0.043 & 0.7032 & 5.26 \\
\hline 933 & 5365 & 55604 & 4 & 0.01 & 95.93 & 0.018018 & 0.011011 & 6 & 54194696 & 6 & 54949995 & 6 & 54 & 0.056 & 0.7088 & 4.65 \\
\hline
\end{tabular}




\begin{tabular}{|c|c|c|c|c|c|c|c|c|c|c|c|c|c|c|c|c|}
\hline 934 & 29619 & 17874 & 8 & 0.01 & 93.3 & 0.041041 & 0.028028 & 6 & 55046176 & 6 & 55956683 & 6 & 55 & 0.055 & 0.7081 & 4.72 \\
\hline 935 & 18074 & 52580 & 10 & 0.02 & 89.84 & 0.047047 & 0.03003 & 6 & 56167422 & 6 & 56948678 & 6 & 56 & 0.052 & 0.7078 & 4.83 \\
\hline 936 & 51555 & 55848 & 6 & 0.01 & 93.17 & 0.022022 & 0.019019 & 6 & 57107592 & 6 & 57971843 & 6 & 57 & 0.054 & 0.7082 & 4.73 \\
\hline 937 & 50009 & 29369 & 6 & 0.01 & 94.61 & 0.027027 & 0.021021 & 6 & 58080576 & 6 & 58859012 & 6 & 58 & 0.056 & 0.7085 & 4.68 \\
\hline 938 & 52125 & 56491 & 10 & 0.02 & 90.51 & 0.043043 & 0.03003 & 6 & 59213073 & 6 & 59962739 & 6 & 59 & 0.053 & 0.7078 & 4.81 \\
\hline 939 & 18379 & 14676 & 29 & 0.04 & 60.23 & 0.137137 & 0.091091 & 6 & 60051563 & 6 & 60993805 & 6 & 60 & 0.033 & 0.6956 & 5.96 \\
\hline 940 & 29764 & 18999 & 15 & 0.03 & 72.14 & 0.08008 & 0.045045 & 6 & 61004887 & 6 & 61916523 & 6 & 61 & 0.04 & 0.7013 & 5.44 \\
\hline 941 & 31745 & 17047 & 13 & 0.01 & 92.72 & 0.061061 & 0.035035 & 6 & 62176946 & 6 & 62866629 & 6 & 62 & 0.054 & 0.7081 & 4.74 \\
\hline 942 & 33525 & 53905 & 23 & 0.04 & 60.95 & 0.092092 & 0.067067 & 6 & 63053534 & 6 & 63986347 & 6 & 63 & 0.033 & 0.6962 & 5.93 \\
\hline 943 & 45773 & 39692 & 19 & 0.03 & 69.44 & 0.065065 & 0.049049 & 6 & 64020985 & 6 & 64915744 & 6 & 64 & 0.039 & 0.6995 & 5.54 \\
\hline 944 & 48911 & 5374 & 26 & 0.04 & 63.8 & 0.123123 & 0.086086 & 6 & 65081101 & 6 & 65940489 & 6 & 65 & 0.035 & 0.6973 & 5.79 \\
\hline 945 & 30289 & 16795 & 18 & 0.02 & 88.27 & 0.081081 & 0.059059 & 6 & 66072075 & 6 & 66952267 & 6 & 66 & 0.051 & 0.7076 & 4.88 \\
\hline 946 & 34221 & 45175 & 32 & 0.03 & 67.72 & 0.137137 & 0.087087 & 6 & 67024466 & 6 & 67975744 & 6 & 67 & 0.038 & 0.6991 & 5.62 \\
\hline 947 & 59771 & 23021 & 35 & 0.03 & 72.54 & 0.138138 & 0.08008 & 6 & 68066489 & 6 & 68993837 & 6 & 68 & 0.041 & 0.7011 & 5.42 \\
\hline 948 & 23020 & 45757 & 12 & 0.01 & 92.09 & 0.047047 & 0.03003 & 6 & 69006213 & 6 & 69930291 & 6 & 69 & 0.054 & 0.708 & 4.77 \\
\hline 949 & 18988 & 41654 & 34 & 0.08 & 24.18 & 0.155155 & 0.12012 & 6 & 70023840 & 6 & 70996798 & 6 & 70 & 0.013 & 0.6254 & 8.69 \\
\hline 950 & 14987 & 45195 & 35 & 0.07 & 27.4 & 0.168168 & 0.118118 & 6 & 71001575 & 6 & 71995060 & 6 & 71 & 0.015 & 0.6341 & 8.2 \\
\hline 951 & 60140 & 17969 & 24 & 0.04 & 55.95 & 0.105105 & 0.068068 & 6 & 72055444 & 6 & 72949935 & 6 & 72 & 0.031 & 0.6922 & 6.17 \\
\hline 952 & 29480 & 23030 & 23 & 0.04 & 63.59 & 0.116116 & 0.077077 & 6 & 73127988 & 6 & 73980831 & 6 & 73 & 0.035 & 0.6973 & 5.8 \\
\hline 953 & 23029 & 16694 & 41 & 0.04 & 51.72 & 0.171171 & 0.115115 & 6 & 74002918 & 6 & 74982947 & 6 & 74 & 0.028 & 0.6889 & 6.43 \\
\hline 954 & 33596 & 30815 & 31 & 0.02 & 88.48 & 0.092092 & 0.056056 & 6 & 75138271 & 6 & 75952956 & 6 & 75 & 0.051 & 0.7077 & 4.88 \\
\hline 955 & 17656 & 5390 & 27 & 0.03 & 77.95 & 0.096096 & 0.066066 & 6 & 76056354 & 6 & 76948482 & 6 & 76 & 0.044 & 0.704 & 5.23 \\
\hline 956 & 50125 & 29287 & 18 & 0.02 & 85.64 & 0.057057 & 0.04004 & 6 & 77035698 & 6 & 77847902 & 6 & 77 & 0.049 & 0.7062 & 4.96 \\
\hline 957 & 17263 & 56288 & 21 & 0.02 & 86.3 & 0.083083 & 0.051051 & 6 & 78048683 & 6 & 78938720 & 6 & 78 & 0.05 & 0.707 & 4.95 \\
\hline 958 & 36436 & 14794 & 7 & 0.01 & 94.31 & 0.038038 & 0.023023 & 6 & 79026669 & 6 & 79924228 & 6 & 79 & 0.055 & 0.7083 & 4.69 \\
\hline 959 & 34180 & 19311 & 31 & 0.04 & 53.78 & 0.139139 & 0.099099 & 6 & 80008558 & 6 & 80979535 & 6 & 80 & 0.029 & 0.6916 & 6.31 \\
\hline 960 & 45681 & 48918 & 31 & 0.06 & 34.92 & 0.149149 & 0.109109 & 6 & 81021081 & 6 & 81987568 & 6 & 81 & 0.019 & 0.6553 & 7.49 \\
\hline 961 & 5399 & 33393 & 25 & 0.03 & 69.34 & 0.088088 & 0.056056 & 6 & 82014983 & 6 & 82929466 & 6 & 82 & 0.039 & 0.6995 & 5.54 \\
\hline 962 & 53460 & 59865 & 19 & 0.03 & 66.01 & 0.1001 & 0.054054 & 6 & 83038514 & 6 & 83954643 & 6 & 83 & 0.037 & 0.6988 & 5.69 \\
\hline 963 & 29681 & 14395 & 11 & 0.01 & 92.67 & 0.044044 & 0.029029 & 6 & 84010187 & 6 & 84927021 & 6 & 84 & 0.054 & 0.7081 & 4.74 \\
\hline 964 & 14784 & 16130 & 12 & 0.01 & 92.42 & 0.051051 & 0.029029 & 6 & 85010159 & 6 & 85994652 & 6 & 85 & 0.054 & 0.7082 & 4.75 \\
\hline 965 & 32376 & 23056 & 23 & 0.03 & 77.09 & 0.105105 & 0.061061 & 6 & 86017603 & 6 & 86940350 & 6 & 86 & 0.044 & 0.7034 & 5.25 \\
\hline 966 & 61750 & 36045 & 16 & 0.03 & 80.39 & 0.07007 & 0.05005 & 6 & 87000361 & 6 & 87968836 & 6 & 87 & 0.046 & 0.7049 & 5.14 \\
\hline 967 & 23061 & 60617 & 28 & 0.05 & 45.98 & 0.138138 & 0.101101 & 6 & 88011983 & 6 & 88964845 & 6 & 88 & 0.025 & 0.6813 & 6.77 \\
\hline 968 & 35273 & 58618 & 20 & 0.04 & 55 & 0.094094 & 0.065065 & 6 & 89123167 & 6 & 89986075 & 6 & 89 & 0.03 & 0.6918 & 6.22 \\
\hline 969 & 49991 & 16685 & 17 & 0.02 & 89.86 & 0.09009 & 0.053053 & 6 & 90124294 & 6 & 90920481 & 6 & 90 & 0.052 & 0.7077 & 4.83 \\
\hline 970 & 5433 & 15678 & 29 & 0.06 & 33.81 & 0.126126 & 0.092092 & 6 & 91031831 & 6 & 91946493 & 6 & 91 & 0.018 & 0.6522 & 7.59 \\
\hline 971 & 33304 & 58628 & 16 & 0.04 & 61.69 & 0.09009 & 0.068068 & 6 & 92269778 & 6 & 92978013 & 6 & 92 & 0.034 & 0.6965 & 5.9 \\
\hline 972 & 58007 & 56126 & 29 & 0.03 & 73.2 & 0.126126 & 0.077077 & 6 & 93012456 & 6 & 93942039 & 6 & 93 & 0.041 & 0.7015 & 5.38 \\
\hline
\end{tabular}




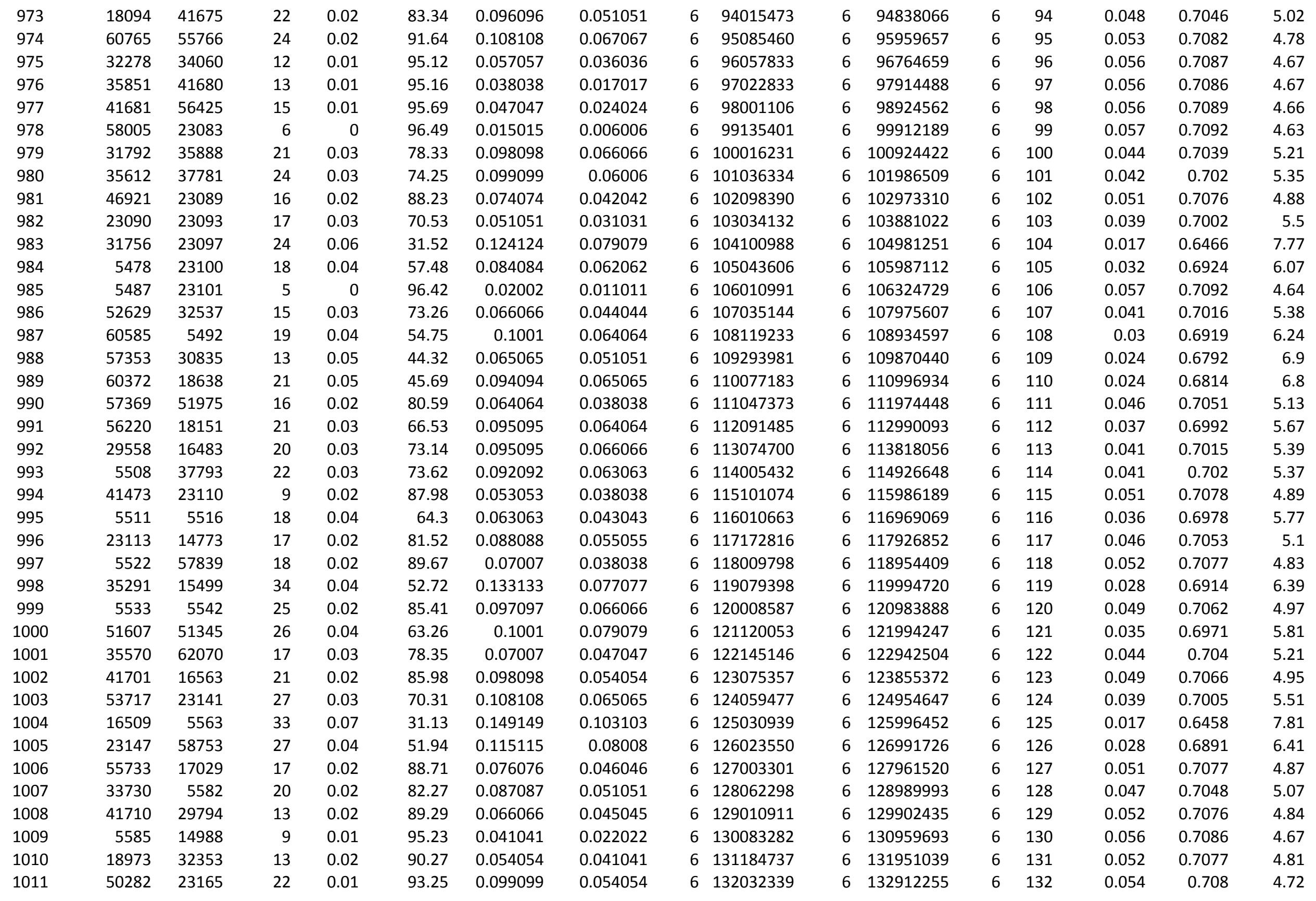




\begin{tabular}{|c|c|c|c|c|c|c|c|c|c|c|c|c|c|c|c|c|}
\hline 1012 & 16379 & 23170 & 17 & 0.01 & 92.02 & 0.076076 & 0.045045 & 6 & 133030240 & 6 & 133929215 & 6 & 133 & 0.053 & 0.7081 & 4.77 \\
\hline 1013 & 5599 & 45249 & 20 & 0.02 & 85.15 & 0.066066 & 0.045045 & 6 & 134051144 & 6 & 134996322 & 6 & 134 & 0.049 & 0.7062 & 4.98 \\
\hline 1014 & 46941 & 5609 & 19 & 0.02 & 84.63 & 0.06006 & 0.04004 & 6 & 135074196 & 6 & 135878484 & 6 & 135 & 0.049 & 0.7055 & 4.98 \\
\hline 1015 & 45289 & 31146 & 21 & 0.03 & 67.75 & 0.095095 & 0.071071 & 6 & 136045327 & 6 & 136990113 & 6 & 136 & 0.038 & 0.6992 & 5.61 \\
\hline 1016 & 30067 & 35315 & 26 & 0.07 & 27.47 & 0.118118 & 0.084084 & 6 & 137044171 & 6 & 137985648 & 6 & 137 & 0.015 & 0.6341 & 8.18 \\
\hline 1017 & 26600 & 46943 & 19 & 0.05 & 44.12 & 0.094094 & 0.063063 & 6 & 138009749 & 6 & 138921625 & 6 & 138 & 0.023 & 0.6785 & 6.9 \\
\hline 1018 & 59933 & 50769 & 24 & 0.06 & 36.36 & 0.135135 & 0.102102 & 6 & 139045500 & 6 & 139965340 & 6 & 139 & 0.019 & 0.6603 & 7.38 \\
\hline 1019 & 5627 & 5634 & 29 & 0.09 & 23.42 & 0.143143 & 0.105105 & 6 & 140009596 & 6 & 140829602 & 6 & 140 & 0.012 & 0.6225 & 8.81 \\
\hline 1020 & 5635 & 57523 & 32 & 0.05 & 46.12 & 0.152152 & 0.096096 & 6 & 141025042 & 6 & 141988774 & 6 & 141 & 0.025 & 0.6817 & 6.76 \\
\hline 1021 & 57735 & 5649 & 28 & 0.05 & 44.52 & 0.12012 & 0.087087 & 6 & 142036921 & 6 & 142989178 & 6 & 142 & 0.024 & 0.6796 & 6.88 \\
\hline 1022 & 41725 & 23200 & 24 & 0.02 & 81.03 & 0.107107 & 0.051051 & 6 & 143021550 & 6 & 143976085 & 6 & 143 & 0.046 & 0.705 & 5.12 \\
\hline 1023 & 17625 & 16282 & 23 & 0.04 & 62.72 & 0.112112 & 0.077077 & 6 & 144024419 & 6 & 144977414 & 6 & 144 & 0.035 & 0.6964 & 5.84 \\
\hline 1024 & 32024 & 30359 & 16 & 0.01 & 95.24 & 0.058058 & 0.037037 & 6 & 145065756 & 6 & 145998261 & 6 & 145 & 0.056 & 0.7086 & 4.67 \\
\hline 1025 & 34853 & 5672 & 21 & 0.03 & 71.89 & 0.088088 & 0.065065 & 6 & 146248545 & 6 & 146923286 & 6 & 146 & 0.04 & 0.7011 & 5.45 \\
\hline 1026 & 48932 & 41734 & 15 & 0.04 & 63.98 & 0.069069 & 0.045045 & 6 & 147007251 & 6 & 147954934 & 6 & 147 & 0.035 & 0.6972 & 5.77 \\
\hline 1027 & 5674 & 17991 & 12 & 0.01 & 95.32 & 0.055055 & 0.03003 & 6 & 148004363 & 6 & 148742719 & 6 & 148 & 0.056 & 0.7086 & 4.66 \\
\hline 1028 & 5679 & 50895 & 15 & 0.01 & 93.35 & 0.058058 & 0.04004 & 6 & 149094962 & 6 & 149758688 & 6 & 149 & 0.055 & 0.7081 & 4.72 \\
\hline 1029 & 15413 & 39742 & 24 & 0.03 & 66.73 & 0.105105 & 0.072072 & 6 & 150101096 & 6 & 150848334 & 6 & 150 & 0.037 & 0.6992 & 5.66 \\
\hline 1030 & 29748 & 58690 & 23 & 0.03 & 73.68 & 0.098098 & 0.06006 & 6 & 151053568 & 6 & 151865659 & 6 & 151 & 0.041 & 0.7021 & 5.37 \\
\hline 1031 & 34144 & 23224 & 28 & 0.03 & 66.94 & 0.123123 & 0.075075 & 6 & 152208520 & 6 & 152976959 & 6 & 152 & 0.037 & 0.6993 & 5.65 \\
\hline 1032 & 5692 & 35463 & 17 & 0.02 & 83.04 & 0.086086 & 0.047047 & 6 & 153108429 & 6 & 153749532 & 6 & 153 & 0.048 & 0.7045 & 5.03 \\
\hline 1033 & 23230 & 45268 & 28 & 0.06 & 34.12 & 0.13013 & 0.092092 & 6 & 154110204 & 6 & 154972467 & 6 & 154 & 0.018 & 0.6544 & 7.57 \\
\hline 1034 & 48936 & 30300 & 22 & 0.03 & 73.56 & 0.087087 & 0.053053 & 6 & 155139641 & 6 & 155985335 & 6 & 155 & 0.041 & 0.7019 & 5.37 \\
\hline 1035 & 61205 & 49847 & 27 & 0.07 & 28.86 & 0.121121 & 0.092092 & 6 & 156179469 & 6 & 156993286 & 6 & 156 & 0.015 & 0.6388 & 8.03 \\
\hline 1036 & 50006 & 51808 & 23 & 0.09 & 23.07 & 0.121121 & 0.097097 & 6 & 157003423 & 6 & 157734339 & 6 & 157 & 0.012 & 0.6196 & 8.83 \\
\hline 1037 & 46949 & 46954 & 14 & 0.04 & 53.02 & 0.06006 & 0.032032 & 7 & 48748 & 7 & 992405 & 7 & 0 & 0.029 & 0.6916 & 6.37 \\
\hline 1038 & 46955 & 61799 & 20 & 0.03 & 74.86 & 0.087087 & 0.054054 & 7 & 1035119 & 7 & 1989754 & 7 & 1 & 0.042 & 0.7021 & 5.32 \\
\hline 1039 & 52963 & 23259 & 27 & 0.05 & 45.06 & 0.133133 & 0.089089 & 7 & 2034057 & 7 & 2936612 & 7 & 2 & 0.024 & 0.6801 & 6.83 \\
\hline 1040 & 23258 & 48953 & 22 & 0.03 & 79.14 & 0.084084 & 0.054054 & 7 & 3056769 & 7 & 3993079 & 7 & 3 & 0.045 & 0.7046 & 5.19 \\
\hline 1041 & 5722 & 5729 & 29 & 0.06 & 34.79 & 0.127127 & 0.097097 & 7 & 4033259 & 7 & 4988931 & 7 & 4 & 0.018 & 0.6552 & 7.51 \\
\hline 1042 & 41768 & 5734 & 31 & 0.04 & 63.05 & 0.139139 & 0.097097 & 7 & 5020698 & 7 & 5975746 & 7 & 5 & 0.035 & 0.6965 & 5.82 \\
\hline 1043 & 5735 & 59074 & 23 & 0.04 & 57.72 & 0.11011 & 0.075075 & 7 & 6000797 & 7 & 6955465 & 7 & 6 & 0.032 & 0.6924 & 6.06 \\
\hline 1044 & 37848 & 23298 & 28 & 0.05 & 41.37 & 0.113113 & 0.078078 & 7 & 7001964 & 7 & 7981170 & 7 & 7 & 0.022 & 0.6736 & 7.1 \\
\hline 1045 & 23299 & 23308 & 25 & 0.07 & 30.19 & 0.129129 & 0.089089 & 7 & 8003501 & 7 & 8967851 & 7 & 8 & 0.016 & 0.6423 & 7.87 \\
\hline 1046 & 37854 & 5772 & 30 & 0.03 & 72.29 & 0.129129 & 0.073073 & 7 & 9003631 & 7 & 9976814 & 7 & 9 & 0.041 & 0.701 & 5.42 \\
\hline 1047 & 48968 & 5775 & 20 & 0.02 & 90.07 & 0.087087 & 0.044044 & 7 & 10031634 & 7 & 10964520 & 7 & 10 & 0.052 & 0.7078 & 4.82 \\
\hline 1048 & 5776 & 41795 & 24 & 0.06 & 36 & 0.103103 & 0.063063 & 7 & 11038318 & 7 & 11979563 & 7 & 11 & 0.019 & 0.6586 & 7.4 \\
\hline 1049 & 23328 & 23337 & 27 & 0.04 & 60.08 & 0.117117 & 0.078078 & 7 & 12019377 & 7 & 12984109 & 7 & 12 & 0.033 & 0.6955 & 5.97 \\
\hline 1050 & 23338 & 5803 & 25 & 0.02 & 88.46 & 0.082082 & 0.044044 & 7 & 13023831 & 7 & 13906473 & 7 & 13 & 0.051 & 0.7077 & 4.88 \\
\hline
\end{tabular}




\begin{tabular}{|c|c|c|c|c|c|c|c|c|c|c|c|c|c|c|c|c|}
\hline 1051 & 37863 & 5807 & 28 & 0.03 & 74.92 & 0.111111 & 0.071071 & 7 & 14059560 & 7 & 14901075 & 7 & 14 & 0.042 & 0.7021 & 5.32 \\
\hline 1052 & 37873 & 58620 & 27 & 0.04 & 61.84 & 0.104104 & 0.066066 & 7 & 15057420 & 7 & 15995030 & 7 & 15 & 0.034 & 0.6967 & 5.89 \\
\hline 1053 & 5817 & 37878 & 28 & 0.03 & 74.6 & 0.108108 & 0.073073 & 7 & 16062680 & 7 & 16974151 & 7 & 16 & 0.042 & 0.702 & 5.33 \\
\hline 1054 & 23365 & 37883 & 30 & 0.03 & 70.88 & 0.118118 & 0.078078 & 7 & 17027287 & 7 & 17994605 & 7 & 17 & 0.04 & 0.7004 & 5.48 \\
\hline 1055 & 5833 & 5843 & 33 & 0.04 & 61.06 & 0.129129 & 0.084084 & 7 & 18009952 & 7 & 18984509 & 7 & 18 & 0.033 & 0.6963 & 5.93 \\
\hline 1056 & 34825 & 37890 & 15 & 0.03 & 77.71 & 0.061061 & 0.043043 & 7 & 19004434 & 7 & 19789994 & 7 & 19 & 0.044 & 0.7036 & 5.23 \\
\hline 1057 & 5848 & 41835 & 37 & 0.05 & 42.15 & 0.143143 & 0.096096 & 7 & 20072507 & 7 & 20985785 & 7 & 20 & 0.022 & 0.6759 & 7.06 \\
\hline 1058 & 5855 & 58462 & 29 & 0.03 & 70.6 & 0.135135 & 0.08008 & 7 & 21006494 & 7 & 21875537 & 7 & 21 & 0.039 & 0.7003 & 5.49 \\
\hline 1059 & 41841 & 41843 & 20 & 0.03 & 73.8 & 0.092092 & 0.065065 & 7 & 22026124 & 7 & 22987329 & 7 & 22 & 0.042 & 0.7021 & 5.37 \\
\hline 1060 & 5875 & 5882 & 12 & 0.02 & 84.29 & 0.058058 & 0.047047 & 7 & 23022559 & 7 & 23977356 & 7 & 23 & 0.048 & 0.7056 & 4.99 \\
\hline 1061 & 23410 & 56644 & 21 & 0.03 & 68.91 & 0.081081 & 0.05005 & 7 & 24017747 & 7 & 24848207 & 7 & 24 & 0.038 & 0.7 & 5.57 \\
\hline 1062 & 23413 & 41848 & 3 & 0 & 96.54 & 0.012012 & 0.009009 & 7 & 25105017 & 7 & 25215084 & 7 & 25 & 0.057 & 0.7093 & 4.63 \\
\hline 1063 & 23419 & 35576 & 10 & 0.01 & 95.56 & 0.047047 & 0.023023 & 7 & 26157007 & 7 & 26954317 & 7 & 26 & 0.056 & 0.7088 & 4.66 \\
\hline 1064 & 23420 & 23424 & 29 & 0.05 & 49.4 & 0.109109 & 0.079079 & 7 & 27019705 & 7 & 27999311 & 7 & 27 & 0.027 & 0.6849 & 6.53 \\
\hline 1065 & 51247 & 41856 & 10 & 0.01 & 95.65 & 0.045045 & 0.025025 & 7 & 28014685 & 7 & 28889475 & 7 & 28 & 0.056 & 0.7089 & 4.66 \\
\hline 1066 & 35927 & 5911 & 26 & 0.03 & 73.23 & 0.11011 & 0.07007 & 7 & 29040724 & 7 & 29987393 & 7 & 29 & 0.041 & 0.7015 & 5.38 \\
\hline 1067 & 37906 & 5925 & 37 & 0.06 & 39.48 & 0.152152 & 0.103103 & 7 & 30036530 & 7 & 30978428 & 7 & 30 & 0.021 & 0.6691 & 7.19 \\
\hline 1068 & 5924 & 23446 & 36 & 0.04 & 51.5 & 0.156156 & 0.106106 & 7 & 31011932 & 7 & 31989509 & 7 & 31 & 0.028 & 0.6889 & 6.44 \\
\hline 1069 & 5937 & 41868 & 30 & 0.06 & 38.54 & 0.133133 & 0.092092 & 7 & 32016844 & 7 & 32997273 & 7 & 32 & 0.02 & 0.6672 & 7.27 \\
\hline 1070 & 23457 & 5953 & 35 & 0.06 & 37.4 & 0.171171 & 0.119119 & 7 & 33017627 & 7 & 33991469 & 7 & 33 & 0.02 & 0.6632 & 7.3 \\
\hline 1071 & 5954 & 46984 & 30 & 0.05 & 47.03 & 0.121121 & 0.079079 & 7 & 34026230 & 7 & 34978383 & 7 & 34 & 0.025 & 0.6823 & 6.67 \\
\hline 1072 & 5961 & 23481 & 21 & 0.03 & 76.14 & 0.102102 & 0.062062 & 7 & 35002839 & 7 & 35973567 & 7 & 35 & 0.043 & 0.7027 & 5.28 \\
\hline 1073 & 41880 & 5974 & 19 & 0.02 & 84.43 & 0.082082 & 0.056056 & 7 & 36004578 & 7 & 36991143 & 7 & 36 & 0.049 & 0.7055 & 4.99 \\
\hline 1074 & 23489 & 5987 & 36 & 0.04 & 57.64 & 0.135135 & 0.096096 & 7 & 37026669 & 7 & 37967748 & 7 & 37 & 0.032 & 0.6923 & 6.06 \\
\hline 1075 & 23496 & 55765 & 24 & 0.03 & 69.11 & 0.102102 & 0.066066 & 7 & 38006859 & 7 & 38984501 & 7 & 38 & 0.038 & 0.7 & 5.56 \\
\hline 1076 & 46993 & 23505 & 34 & 0.04 & 53.87 & 0.132132 & 0.083083 & 7 & 39051601 & 7 & 39985401 & 7 & 39 & 0.029 & 0.6914 & 6.3 \\
\hline 1077 & 62009 & 41903 & 33 & 0.07 & 30.39 & 0.151151 & 0.107107 & 7 & 40007647 & 7 & 40992238 & 7 & 40 & 0.016 & 0.6433 & 7.87 \\
\hline 1078 & 6014 & 6023 & 28 & 0.04 & 53.91 & 0.136136 & 0.094094 & 7 & 41004531 & 7 & 41984352 & 7 & 41 & 0.029 & 0.6915 & 6.3 \\
\hline 1079 & 57712 & 58557 & 23 & 0.01 & 93.12 & 0.084084 & 0.044044 & 7 & 42000072 & 7 & 42999450 & 7 & 42 & 0.054 & 0.7081 & 4.73 \\
\hline 1080 & 6030 & 23526 & 26 & 0.01 & 94.46 & 0.116116 & 0.047047 & 7 & 43037006 & 7 & 43924915 & 7 & 43 & 0.055 & 0.7083 & 4.69 \\
\hline 1081 & 23527 & 23532 & 23 & 0.02 & 83.43 & 0.104104 & 0.059059 & 7 & 44021990 & 7 & 44974706 & 7 & 44 & 0.048 & 0.7048 & 5.02 \\
\hline 1082 & 23533 & 47006 & 16 & 0.02 & 89.68 & 0.056056 & 0.03003 & 7 & 45004542 & 7 & 45911698 & 7 & 45 & 0.052 & 0.7077 & 4.83 \\
\hline 1083 & 47007 & 23541 & 18 & 0.03 & 80.49 & 0.09009 & 0.055055 & 7 & 46295528 & 7 & 46996404 & 7 & 46 & 0.046 & 0.7051 & 5.14 \\
\hline 1084 & 23542 & 23550 & 21 & 0.05 & 47.31 & 0.122122 & 0.087087 & 7 & 47037298 & 7 & 47934829 & 7 & 47 & 0.026 & 0.6826 & 6.66 \\
\hline 1085 & 6062 & 32577 & 27 & 0.08 & 25 & 0.149149 & 0.099099 & 7 & 48105743 & 7 & 48953568 & 7 & 48 & 0.013 & 0.6268 & 8.53 \\
\hline 1086 & 6073 & 49020 & 33 & 0.03 & 71.64 & 0.149149 & 0.078078 & 7 & 49013454 & 7 & 49982901 & 7 & 49 & 0.04 & 0.701 & 5.46 \\
\hline 1087 & 6091 & 53052 & 32 & 0.06 & 34.98 & 0.17017 & 0.102102 & 7 & 50028824 & 7 & 50991091 & 7 & 50 & 0.019 & 0.6559 & 7.49 \\
\hline 1088 & 37933 & 41943 & 23 & 0.02 & 87.05 & 0.089089 & 0.047047 & 7 & 51027425 & 7 & 51991520 & 7 & 51 & 0.05 & 0.7076 & 4.93 \\
\hline 1089 & 60256 & 36441 & 35 & 0.06 & 36.65 & 0.142142 & 0.096096 & 7 & 52038431 & 7 & 52978945 & 7 & 52 & 0.02 & 0.6615 & 7.37 \\
\hline
\end{tabular}




\begin{tabular}{|c|c|c|c|c|c|c|c|c|c|c|c|c|c|c|c|c|}
\hline 1090 & 37937 & 52186 & 35 & 0.06 & 36.3 & 0.18018 & 0.123123 & 7 & 53018942 & 7 & 53981796 & 7 & 53 & 0.019 & 0.6604 & 7.4 \\
\hline 1091 & 6132 & 45578 & 37 & 0.03 & 72.93 & 0.136136 & 0.083083 & 7 & 54010631 & 7 & 54997566 & 7 & 54 & 0.041 & 0.7015 & 5.4 \\
\hline 1092 & 56408 & 51854 & 36 & 0.05 & 43.42 & 0.156156 & 0.109109 & 7 & 55030156 & 7 & 55985056 & 7 & 55 & 0.023 & 0.678 & 6.98 \\
\hline 1093 & 60723 & 57604 & 39 & 0.06 & 37.46 & 0.154154 & 0.098098 & 7 & 56025148 & 7 & 56971231 & 7 & 56 & 0.02 & 0.6633 & 7.3 \\
\hline 1094 & 6171 & 53464 & 34 & 0.06 & 38.14 & 0.149149 & 0.099099 & 7 & 57012925 & 7 & 57971318 & 7 & 57 & 0.02 & 0.666 & 7.28 \\
\hline 1095 & 23626 & 23629 & 20 & 0.02 & 83.82 & 0.073073 & 0.046046 & 7 & 58003125 & 7 & 58927990 & 7 & 58 & 0.048 & 0.7053 & 5.01 \\
\hline 1096 & 6190 & 6195 & 23 & 0.02 & 91.75 & 0.078078 & 0.041041 & 7 & 59039109 & 7 & 59986169 & 7 & 59 & 0.053 & 0.7081 & 4.78 \\
\hline 1097 & 6196 & 35881 & 28 & 0.03 & 76.23 & 0.109109 & 0.065065 & 7 & 60010451 & 7 & 60969455 & 7 & 60 & 0.043 & 0.7028 & 5.28 \\
\hline 1098 & 47031 & 47040 & 14 & 0.01 & 93.57 & 0.066066 & 0.033033 & 7 & 61067623 & 7 & 61988185 & 7 & 61 & 0.055 & 0.7082 & 4.71 \\
\hline 1099 & 6208 & 41994 & 23 & 0.02 & 81.13 & 0.092092 & 0.049049 & 7 & 62010270 & 7 & 62954233 & 7 & 62 & 0.046 & 0.7051 & 5.12 \\
\hline 1100 & 47042 & 41997 & 21 & 0.02 & 81.11 & 0.11011 & 0.07007 & 7 & 63015572 & 7 & 63961157 & 7 & 63 & 0.046 & 0.7051 & 5.12 \\
\hline 1101 & 49039 & 6227 & 21 & 0.02 & 89.98 & 0.075075 & 0.042042 & 7 & 64026660 & 7 & 64959971 & 7 & 64 & 0.052 & 0.7077 & 4.83 \\
\hline 1102 & 23657 & 47057 & 24 & 0.03 & 79.71 & 0.096096 & 0.058058 & 7 & 65004224 & 7 & 65984458 & 7 & 65 & 0.045 & 0.7049 & 5.17 \\
\hline 1103 & 42002 & 50423 & 8 & 0 & 96.44 & 0.026026 & 0.011011 & 7 & 66304912 & 7 & 66728436 & 7 & 66 & 0.057 & 0.7091 & 4.63 \\
\hline 1104 & 6236 & 37962 & 15 & 0.01 & 94.18 & 0.062062 & 0.039039 & 7 & 67107544 & 7 & 67917223 & 7 & 67 & 0.055 & 0.7084 & 4.69 \\
\hline 1105 & 6242 & 37965 & 21 & 0.05 & 44.07 & 0.104104 & 0.076076 & 7 & 68047313 & 7 & 68854659 & 7 & 68 & 0.023 & 0.6785 & 6.92 \\
\hline 1106 & 42008 & 47066 & 24 & 0.02 & 82.6 & 0.102102 & 0.067067 & 7 & 69000948 & 7 & 69913988 & 7 & 69 & 0.047 & 0.7048 & 5.05 \\
\hline 1107 & 23672 & 6263 & 28 & 0.06 & 36.82 & 0.126126 & 0.081081 & 7 & 70000093 & 7 & 70975451 & 7 & 70 & 0.02 & 0.662 & 7.35 \\
\hline 1108 & 49040 & 6266 & 21 & 0.02 & 86.11 & 0.088088 & 0.051051 & 7 & 71050477 & 7 & 71974687 & 7 & 71 & 0.049 & 0.7067 & 4.95 \\
\hline 1109 & 37972 & 47075 & 16 & 0.02 & 88.59 & 0.067067 & 0.044044 & 7 & 72026564 & 7 & 72991035 & 7 & 72 & 0.051 & 0.7078 & 4.87 \\
\hline 1110 & 23689 & 47077 & 21 & 0.03 & 67.48 & 0.104104 & 0.062062 & 7 & 73011207 & 7 & 73938361 & 7 & 73 & 0.037 & 0.6992 & 5.63 \\
\hline 1111 & 37986 & 37989 & 17 & 0.02 & 84.69 & 0.076076 & 0.052052 & 7 & 74030057 & 7 & 74980796 & 7 & 74 & 0.049 & 0.7055 & 4.98 \\
\hline 1112 & 47078 & 47082 & 17 & 0.02 & 89.72 & 0.065065 & 0.036036 & 7 & 75033437 & 7 & 75893840 & 7 & 75 & 0.052 & 0.7078 & 4.83 \\
\hline 1113 & 6285 & 38001 & 9 & 0.02 & 88.06 & 0.047047 & 0.037037 & 7 & 76111756 & 7 & 76907606 & 7 & 76 & 0.051 & 0.7077 & 4.89 \\
\hline 1114 & 6289 & 38007 & 10 & 0.01 & 96.05 & 0.03003 & 0.021021 & 7 & 77009237 & 7 & 77973037 & 7 & 77 & 0.057 & 0.709 & 4.64 \\
\hline 1115 & 38008 & 38015 & 26 & 0.03 & 71.7 & 0.105105 & 0.072072 & 7 & 78031358 & 7 & 78971281 & 7 & 78 & 0.04 & 0.7009 & 5.45 \\
\hline 1116 & 6302 & 6311 & 21 & 0.03 & 76.81 & 0.089089 & 0.049049 & 7 & 79025097 & 7 & 79997832 & 7 & 79 & 0.043 & 0.7032 & 5.26 \\
\hline 1117 & 6312 & 6320 & 20 & 0.02 & 91.22 & 0.082082 & 0.048048 & 7 & 80027963 & 7 & 80985441 & 7 & 80 & 0.053 & 0.708 & 4.79 \\
\hline 1118 & 6319 & 56737 & 22 & 0.01 & 91.81 & 0.086086 & 0.046046 & 7 & 81035442 & 7 & 81924254 & 7 & 81 & 0.053 & 0.7081 & 4.78 \\
\hline 1119 & 23711 & 61620 & 13 & 0.01 & 95.28 & 0.065065 & 0.029029 & 7 & 82003775 & 7 & 82993422 & 7 & 82 & 0.056 & 0.7086 & 4.67 \\
\hline 1120 & 47101 & 49042 & 14 & 0.01 & 94.84 & 0.069069 & 0.035035 & 7 & 83057633 & 7 & 83944769 & 7 & 83 & 0.056 & 0.7085 & 4.68 \\
\hline 1121 & 47109 & 47109 & 1 & 0 & 96.56 & 0.004004 & 0 & 7 & 85445625 & 7 & 85445625 & 7 & 85 & 0.057 & 0.7093 & 4.63 \\
\hline 1122 & 53085 & 47114 & 20 & 0.01 & 93.37 & 0.062062 & 0.035035 & 7 & 86017385 & 7 & 86955735 & 7 & 86 & 0.055 & 0.7081 & 4.72 \\
\hline 1123 & 6350 & 52211 & 28 & 0.03 & 78.03 & 0.111111 & 0.081081 & 7 & 87029852 & 7 & 87977236 & 7 & 87 & 0.044 & 0.7039 & 5.22 \\
\hline 1124 & 23731 & 23742 & 27 & 0.03 & 78.96 & 0.101101 & 0.07007 & 7 & 88077718 & 7 & 88970583 & 7 & 88 & 0.045 & 0.7043 & 5.19 \\
\hline 1125 & 6369 & 47120 & 34 & 0.04 & 55.91 & 0.149149 & 0.1001 & 7 & 89014128 & 7 & 89999425 & 7 & 89 & 0.031 & 0.6922 & 6.17 \\
\hline 1126 & 47121 & 47126 & 26 & 0.06 & 37.52 & 0.123123 & 0.091091 & 7 & 90011443 & 7 & 90978100 & 7 & 90 & 0.02 & 0.6636 & 7.3 \\
\hline 1127 & 6394 & 38034 & 27 & 0.02 & 80.94 & 0.099099 & 0.054054 & 7 & 91019546 & 7 & 91996584 & 7 & 91 & 0.046 & 0.705 & 5.12 \\
\hline 1128 & 38033 & 23780 & 34 & 0.04 & 56.8 & 0.143143 & 0.097097 & 7 & 92008578 & 7 & 92964028 & 7 & 92 & 0.031 & 0.6916 & 6.1 \\
\hline
\end{tabular}




\begin{tabular}{|c|c|c|c|c|c|c|c|c|c|c|c|c|c|c|c|c|}
\hline 1129 & 6407 & 23771 & 28 & 0.02 & 87.94 & 0.112112 & 0.068068 & 7 & 93005460 & 7 & 93885374 & 7 & 93 & 0.051 & 0.7077 & 4.89 \\
\hline 1130 & 6399 & 47132 & 17 & 0.02 & 89.33 & 0.057057 & 0.039039 & 7 & 94045990 & 7 & 94980822 & 7 & 94 & 0.052 & 0.7076 & 4.84 \\
\hline 1131 & 42052 & 6439 & 33 & 0.03 & 70.12 & 0.136136 & 0.086086 & 7 & 95010323 & 7 & 95983910 & 7 & 95 & 0.039 & 0.7001 & 5.52 \\
\hline 1132 & 54761 & 6452 & 28 & 0.02 & 81.79 & 0.103103 & 0.059059 & 7 & 96000929 & 7 & 96970994 & 7 & 96 & 0.047 & 0.7051 & 5.09 \\
\hline 1133 & 23805 & 59484 & 27 & 0.03 & 67.51 & 0.115115 & 0.068068 & 7 & 97036575 & 7 & 97981809 & 7 & 97 & 0.037 & 0.699 & 5.62 \\
\hline 1134 & 6461 & 56328 & 36 & 0.05 & 46.55 & 0.145145 & 0.109109 & 7 & 98003978 & 7 & 98998276 & 7 & 98 & 0.025 & 0.6811 & 6.7 \\
\hline 1135 & 59132 & 42070 & 21 & 0.02 & 85.02 & 0.097097 & 0.057057 & 7 & 99021825 & 7 & 99992288 & 7 & 99 & 0.049 & 0.7062 & 4.98 \\
\hline 1136 & 6481 & 42072 & 21 & 0.03 & 70.85 & 0.086086 & 0.048048 & 7 & 100038150 & 7 & 100997092 & 7 & 100 & 0.04 & 0.7004 & 5.48 \\
\hline 1137 & 42073 & 6502 & 29 & 0.02 & 89.42 & 0.11011 & 0.059059 & 7 & 101022085 & 7 & 101965379 & 7 & 101 & 0.052 & 0.7077 & 4.84 \\
\hline 1138 & 23834 & 38044 & 23 & 0.03 & 65.49 & 0.097097 & 0.059059 & 7 & 102013846 & 7 & 102949951 & 7 & 102 & 0.036 & 0.6986 & 5.72 \\
\hline 1139 & 6512 & 49056 & 21 & 0.03 & 69.73 & 0.081081 & 0.059059 & 7 & 103001352 & 7 & 103999954 & 7 & 103 & 0.039 & 0.6999 & 5.53 \\
\hline 1140 & 23842 & 47156 & 21 & 0.02 & 81.18 & 0.095095 & 0.053053 & 7 & 104030356 & 7 & 104914125 & 7 & 104 & 0.046 & 0.7051 & 5.12 \\
\hline 1141 & 23847 & 23858 & 27 & 0.04 & 60.04 & 0.132132 & 0.073073 & 7 & 105022346 & 7 & 105980155 & 7 & 105 & 0.033 & 0.6955 & 5.97 \\
\hline 1142 & 6524 & 6538 & 29 & 0.05 & 47.45 & 0.124124 & 0.069069 & 7 & 106002380 & 7 & 106937807 & 7 & 106 & 0.026 & 0.6823 & 6.63 \\
\hline 1143 & 16438 & 18349 & 22 & 0.04 & 57.76 & 0.111111 & 0.072072 & 7 & 107177261 & 7 & 107995062 & 7 & 107 & 0.032 & 0.6924 & 6.05 \\
\hline 1144 & 47158 & 51503 & 19 & 0.02 & 89.93 & 0.085085 & 0.043043 & 7 & 108071051 & 7 & 108972110 & 7 & 108 & 0.052 & 0.7078 & 4.83 \\
\hline 1145 & 42099 & 42101 & 19 & 0.02 & 90.62 & 0.084084 & 0.041041 & 7 & 109049333 & 7 & 109981964 & 7 & 109 & 0.053 & 0.7079 & 4.81 \\
\hline 1146 & 23876 & 38050 & 22 & 0.01 & 92.79 & 0.078078 & 0.042042 & 7 & 110024659 & 7 & 110993314 & 7 & 110 & 0.054 & 0.7081 & 4.74 \\
\hline 1147 & 6569 & 47162 & 24 & 0.01 & 94.1 & 0.088088 & 0.037037 & 7 & 111019262 & 7 & 111972610 & 7 & 111 & 0.055 & 0.7085 & 4.7 \\
\hline 1148 & 6579 & 6588 & 21 & 0.03 & 76.28 & 0.084084 & 0.054054 & 7 & 112053041 & 7 & 112991344 & 7 & 112 & 0.043 & 0.7029 & 5.28 \\
\hline 1149 & 6589 & 57088 & 30 & 0.06 & 33.87 & 0.144144 & 0.095095 & 7 & 113020823 & 7 & 113908824 & 7 & 113 & 0.018 & 0.6528 & 7.59 \\
\hline 1150 & 6597 & 38063 & 23 & 0.03 & 66.04 & 0.1001 & 0.05005 & 7 & 114081936 & 7 & 114977348 & 7 & 114 & 0.037 & 0.6989 & 5.69 \\
\hline 1151 & 6606 & 6617 & 30 & 0.04 & 52.63 & 0.131131 & 0.098098 & 7 & 115014212 & 7 & 115998395 & 7 & 115 & 0.028 & 0.6911 & 6.39 \\
\hline 1152 & 51192 & 6632 & 34 & 0.04 & 55.7 & 0.156156 & 0.095095 & 7 & 116032422 & 7 & 116954912 & 7 & 116 & 0.03 & 0.692 & 6.18 \\
\hline 1153 & 6633 & 47173 & 30 & 0.04 & 53.23 & 0.127127 & 0.083083 & 7 & 117036639 & 7 & 117950905 & 7 & 117 & 0.029 & 0.6916 & 6.36 \\
\hline 1154 & 6642 & 42124 & 34 & 0.05 & 43.62 & 0.145145 & 0.095095 & 7 & 118001966 & 7 & 118979653 & 7 & 118 & 0.023 & 0.6778 & 6.95 \\
\hline 1155 & 51515 & 34475 & 27 & 0.05 & 46.7 & 0.119119 & 0.084084 & 7 & 119018157 & 7 & 119962301 & 7 & 119 & 0.025 & 0.6818 & 6.69 \\
\hline 1156 & 57055 & 6668 & 28 & 0.03 & 70.34 & 0.109109 & 0.075075 & 7 & 120016852 & 7 & 120936305 & 7 & 120 & 0.039 & 0.7004 & 5.51 \\
\hline 1157 & 23937 & 6673 & 18 & 0.02 & 83.62 & 0.089089 & 0.054054 & 7 & 121017867 & 7 & 121984372 & 7 & 121 & 0.048 & 0.7051 & 5.01 \\
\hline 1158 & 42143 & 49072 & 17 & 0.02 & 86.79 & 0.079079 & 0.046046 & 7 & 122018910 & 7 & 122910008 & 7 & 122 & 0.05 & 0.7073 & 4.93 \\
\hline 1159 & 23948 & 6684 & 23 & 0.03 & 77.85 & 0.103103 & 0.066066 & 7 & 123132345 & 7 & 123997457 & 7 & 123 & 0.044 & 0.7037 & 5.23 \\
\hline 1160 & 49073 & 14910 & 31 & 0.03 & 72.45 & 0.141141 & 0.08008 & 7 & 124018132 & 7 & 124941962 & 7 & 124 & 0.041 & 0.7011 & 5.42 \\
\hline 1161 & 49076 & 23969 & 31 & 0.04 & 55.21 & 0.149149 & 0.089089 & 7 & 125008742 & 7 & 125986792 & 7 & 125 & 0.03 & 0.6919 & 6.21 \\
\hline 1162 & 23970 & 49082 & 23 & 0.03 & 71.8 & 0.085085 & 0.053053 & 7 & 126014404 & 7 & 126852902 & 7 & 126 & 0.04 & 0.701 & 5.45 \\
\hline 1163 & 42164 & 6718 & 26 & 0.03 & 68.72 & 0.105105 & 0.071071 & 7 & 127042709 & 7 & 127959806 & 7 & 127 & 0.038 & 0.7001 & 5.58 \\
\hline 1164 & 23981 & 23992 & 30 & 0.06 & 38.43 & 0.132132 & 0.095095 & 7 & 128019403 & 7 & 128999444 & 7 & 128 & 0.02 & 0.6669 & 7.27 \\
\hline 1165 & 42173 & 6739 & 25 & 0.06 & 35.76 & 0.123123 & 0.099099 & 7 & 129019539 & 7 & 129994916 & 7 & 129 & 0.019 & 0.6575 & 7.42 \\
\hline 1166 & 23998 & 42180 & 25 & 0.04 & 63.51 & 0.112112 & 0.075075 & 7 & 130031038 & 7 & 130982643 & 7 & 130 & 0.035 & 0.6972 & 5.8 \\
\hline 1167 & 6746 & 42182 & 10 & 0.02 & 86.65 & 0.048048 & 0.033033 & 7 & 131014179 & 7 & 131361899 & 7 & 131 & 0.05 & 0.7073 & 4.93 \\
\hline
\end{tabular}




\begin{tabular}{|c|c|c|c|c|c|c|c|c|c|c|c|c|c|c|c|c|}
\hline 1168 & 17647 & 6747 & 10 & 0.01 & 91.91 & 0.052052 & 0.03003 & 7 & 132107366 & 7 & 132468484 & 7 & 132 & 0.053 & 0.7081 & 4.77 \\
\hline 1169 & 24013 & 6753 & 7 & 0.02 & 88.16 & 0.032032 & 0.021021 & 7 & 133594228 & 7 & 133979586 & 7 & 133 & 0.051 & 0.7078 & 4.89 \\
\hline 1170 & 24015 & 15870 & 27 & 0.03 & 75.41 & 0.129129 & 0.075075 & 7 & 134005327 & 7 & 134721771 & 7 & 134 & 0.043 & 0.7021 & 5.3 \\
\hline 1171 & 45455 & 42191 & 7 & 0.02 & 90.03 & 0.045045 & 0.031031 & 8 & 424993 & 8 & 946879 & 8 & 0 & 0.052 & 0.7077 & 4.83 \\
\hline 1172 & 781 & 6763 & 14 & 0.04 & 54.67 & 0.074074 & 0.063063 & 8 & 1129887 & 8 & 1931528 & 8 & 1 & 0.03 & 0.6918 & 6.25 \\
\hline 1173 & 6764 & 16676 & 23 & 0.06 & 32.87 & 0.095095 & 0.071071 & 8 & 2023121 & 8 & 2902485 & 8 & 2 & 0.017 & 0.6505 & 7.68 \\
\hline 1174 & 31570 & 24030 & 26 & 0.03 & 68.62 & 0.104104 & 0.072072 & 8 & 3027824 & 8 & 3982853 & 8 & 3 & 0.038 & 0.6997 & 5.58 \\
\hline 1175 & 53940 & 6769 & 14 & 0.02 & 89.15 & 0.067067 & 0.041041 & 8 & 4017057 & 8 & 4961037 & 8 & 4 & 0.052 & 0.7077 & 4.85 \\
\hline 1176 & 38085 & 33119 & 28 & 0.03 & 68.02 & 0.103103 & 0.062062 & 8 & 5043216 & 8 & 5984514 & 8 & 5 & 0.038 & 0.6995 & 5.61 \\
\hline 1177 & 34275 & 60870 & 26 & 0.06 & 33.93 & 0.121121 & 0.084084 & 8 & 6009251 & 8 & 6999002 & 8 & 6 & 0.018 & 0.6536 & 7.59 \\
\hline 1178 & 24041 & 17629 & 28 & 0.06 & 36.88 & 0.143143 & 0.109109 & 8 & 7050948 & 8 & 7964676 & 8 & 7 & 0.02 & 0.6626 & 7.36 \\
\hline 1179 & 14449 & 6790 & 16 & 0.02 & 84.76 & 0.085085 & 0.048048 & 8 & 8145247 & 8 & 8993808 & 8 & 8 & 0.049 & 0.7056 & 4.98 \\
\hline 1180 & 24046 & 42205 & 23 & 0.04 & 57.83 & 0.099099 & 0.07007 & 8 & 9026929 & 8 & 9891321 & 8 & 9 & 0.032 & 0.6926 & 6.05 \\
\hline 1181 & 14756 & 14648 & 26 & 0.05 & 46.08 & 0.114114 & 0.081081 & 8 & 10036134 & 8 & 10993731 & 8 & 10 & 0.025 & 0.6815 & 6.76 \\
\hline 1182 & 32605 & 6807 & 39 & 0.06 & 32.49 & 0.183183 & 0.129129 & 8 & 11003327 & 8 & 11977623 & 8 & 11 & 0.017 & 0.6502 & 7.72 \\
\hline 1183 & 14727 & 38092 & 24 & 0.04 & 51.81 & 0.102102 & 0.065065 & 8 & 12144490 & 8 & 12984077 & 8 & 12 & 0.028 & 0.6891 & 6.43 \\
\hline 1184 & 6812 & 38094 & 17 & 0.07 & 30.46 & 0.096096 & 0.074074 & 8 & 13111033 & 8 & 13951282 & 8 & 13 & 0.016 & 0.644 & 7.87 \\
\hline 1185 & 31918 & 30985 & 17 & 0.04 & 54.58 & 0.101101 & 0.07007 & 8 & 14014229 & 8 & 14946506 & 8 & 14 & 0.03 & 0.6919 & 6.26 \\
\hline 1186 & 56317 & 24076 & 32 & 0.08 & 26.41 & 0.174174 & 0.117117 & 8 & 15012662 & 8 & 15992070 & 8 & 15 & 0.014 & 0.63 & 8.28 \\
\hline 1187 & 38098 & 31881 & 29 & 0.08 & 27.25 & 0.147147 & 0.095095 & 8 & 16014162 & 8 & 16983112 & 8 & 16 & 0.014 & 0.6337 & 8.22 \\
\hline 1188 & 29688 & 15068 & 25 & 0.06 & 37.86 & 0.11011 & 0.07007 & 8 & 17019937 & 8 & 17992352 & 8 & 17 & 0.02 & 0.6647 & 7.29 \\
\hline 1189 & 24087 & 58423 & 33 & 0.04 & 51.67 & 0.151151 & 0.085085 & 8 & 18001094 & 8 & 18974551 & 8 & 18 & 0.028 & 0.6892 & 6.43 \\
\hline 1190 & 32735 & 24097 & 38 & 0.05 & 41.63 & 0.164164 & 0.116116 & 8 & 19014542 & 8 & 19969058 & 8 & 19 & 0.022 & 0.6747 & 7.1 \\
\hline 1191 & 6858 & 24098 & 26 & 0.05 & 47.22 & 0.124124 & 0.077077 & 8 & 20003228 & 8 & 20948717 & 8 & 20 & 0.025 & 0.6824 & 6.66 \\
\hline 1192 & 6861 & 34320 & 23 & 0.02 & 85.69 & 0.095095 & 0.059059 & 8 & 21000928 & 8 & 21987255 & 8 & 21 & 0.049 & 0.7062 & 4.96 \\
\hline 1193 & 18779 & 59134 & 19 & 0.03 & 72.9 & 0.079079 & 0.053053 & 8 & 22007040 & 8 & 22904022 & 8 & 22 & 0.041 & 0.7015 & 5.4 \\
\hline 1194 & 35004 & 18179 & 24 & 0.02 & 83.07 & 0.097097 & 0.053053 & 8 & 23033602 & 8 & 23935848 & 8 & 23 & 0.048 & 0.7045 & 5.03 \\
\hline 1195 & 29447 & 16102 & 18 & 0.03 & 72.51 & 0.073073 & 0.054054 & 8 & 24033377 & 8 & 24901498 & 8 & 24 & 0.041 & 0.7011 & 5.42 \\
\hline 1196 & 18440 & 24113 & 9 & 0.02 & 86.4 & 0.041041 & 0.027027 & 8 & 25020120 & 8 & 25948033 & 8 & 25 & 0.05 & 0.707 & 4.94 \\
\hline 1197 & 24112 & 47190 & 24 & 0.03 & 65.84 & 0.106106 & 0.07007 & 8 & 26012508 & 8 & 26961266 & 8 & 26 & 0.036 & 0.6986 & 5.7 \\
\hline 1198 & 47191 & 42240 & 28 & 0.04 & 53.4 & 0.105105 & 0.068068 & 8 & 27009610 & 8 & 27992239 & 8 & 27 & 0.029 & 0.6917 & 6.35 \\
\hline 1199 & 24125 & 6914 & 17 & 0.03 & 68.81 & 0.068068 & 0.047047 & 8 & 28051243 & 8 & 28996107 & 8 & 28 & 0.038 & 0.7 & 5.58 \\
\hline 1200 & 6915 & 57731 & 27 & 0.05 & 47.17 & 0.127127 & 0.09009 & 8 & 29026480 & 8 & 29990918 & 8 & 29 & 0.025 & 0.6822 & 6.66 \\
\hline 1201 & 24131 & 6930 & 32 & 0.03 & 71.29 & 0.125125 & 0.075075 & 8 & 30007914 & 8 & 30952610 & 8 & 30 & 0.04 & 0.7009 & 5.47 \\
\hline 1202 & 6931 & 37353 & 27 & 0.07 & 28.64 & 0.159159 & 0.118118 & 8 & 31034477 & 8 & 31972646 & 8 & 31 & 0.015 & 0.6383 & 8.05 \\
\hline 1203 & 32565 & 6945 & 25 & 0.04 & 54.12 & 0.122122 & 0.093093 & 8 & 32014830 & 8 & 32984498 & 8 & 32 & 0.029 & 0.6917 & 6.29 \\
\hline 1204 & 6944 & 6946 & 25 & 0.03 & 75.24 & 0.102102 & 0.063063 & 8 & 33033004 & 8 & 33985796 & 8 & 33 & 0.042 & 0.7022 & 5.31 \\
\hline 1205 & 60701 & 53391 & 29 & 0.05 & 39.76 & 0.142142 & 0.093093 & 8 & 34026040 & 8 & 34996041 & 8 & 34 & 0.021 & 0.6693 & 7.17 \\
\hline 1206 & 17252 & 33461 & 26 & 0.04 & 58.89 & 0.116116 & 0.07007 & 8 & 35031484 & 8 & 35944997 & 8 & 35 & 0.032 & 0.6938 & 6.01 \\
\hline
\end{tabular}




\begin{tabular}{|c|c|c|c|c|c|c|c|c|c|c|c|c|c|c|c|c|}
\hline 1207 & 30711 & 31235 & 19 & 0.09 & 23.51 & 0.099099 & 0.072072 & 8 & 36098692 & 8 & 36972953 & 8 & 36 & 0.012 & 0.623 & 8.8 \\
\hline 1208 & 32044 & 24165 & 19 & 0.04 & 58.03 & 0.085085 & 0.055055 & 8 & 37029609 & 8 & 37983458 & 8 & 37 & 0.032 & 0.693 & 6.04 \\
\hline 1209 & 38133 & 6984 & 19 & 0.06 & 38.03 & 0.09009 & 0.06006 & 8 & 38293338 & 8 & 38972975 & 8 & 38 & 0.02 & 0.6657 & 7.29 \\
\hline 1210 & 6985 & 6988 & 23 & 0.03 & 75.55 & 0.113113 & 0.068068 & 8 & 39014921 & 8 & 39851468 & 8 & 39 & 0.043 & 0.7025 & 5.3 \\
\hline 1211 & 24173 & 61131 & 19 & 0.06 & 33.99 & 0.11011 & 0.081081 & 8 & 40020473 & 8 & 40919857 & 8 & 40 & 0.018 & 0.6541 & 7.58 \\
\hline 1212 & 45666 & 6997 & 25 & 0.06 & 36.47 & 0.135135 & 0.087087 & 8 & 41047757 & 8 & 41970562 & 8 & 41 & 0.02 & 0.661 & 7.37 \\
\hline 1213 & 6999 & 16788 & 13 & 0.01 & 91.87 & 0.05005 & 0.04004 & 8 & 42055005 & 8 & 42680025 & 8 & 42 & 0.053 & 0.7081 & 4.77 \\
\hline 1214 & 19274 & 56407 & 9 & 0.01 & 95.75 & 0.039039 & 0.023023 & 8 & 43068687 & 8 & 43955459 & 8 & 43 & 0.056 & 0.7089 & 4.66 \\
\hline 1215 & 7007 & 57562 & 2 & 0 & 96.56 & 0.008008 & 0.003003 & 8 & 44927836 & 8 & 44940909 & 8 & 44 & 0.057 & 0.7093 & 4.63 \\
\hline 1216 & 18311 & 29762 & 7 & 0.01 & 95.89 & 0.025025 & 0.017017 & 8 & 45191096 & 8 & 45750461 & 8 & 45 & 0.056 & 0.7088 & 4.65 \\
\hline 1217 & 54877 & 58652 & 2 & 0 & 96.5 & 0.008008 & 0.007007 & 8 & 46447767 & 8 & 46706900 & 8 & 46 & 0.057 & 0.7092 & 4.63 \\
\hline 1218 & 53708 & 42271 & 6 & 0.01 & 95.58 & 0.027027 & 0.019019 & 8 & 47035822 & 8 & 47582919 & 8 & 47 & 0.056 & 0.7088 & 4.66 \\
\hline 1219 & 18326 & 53725 & 2 & 0 & 96.5 & 0.011011 & 0.007007 & 8 & 48642685 & 8 & 48649970 & 8 & 48 & 0.057 & 0.7092 & 4.63 \\
\hline 1220 & 47199 & 15356 & 4 & 0.01 & 96.04 & 0.02002 & 0.006006 & 8 & 49000633 & 8 & 49881116 & 8 & 49 & 0.057 & 0.709 & 4.64 \\
\hline 1221 & 24189 & 7023 & 18 & 0.06 & 35.82 & 0.1001 & 0.071071 & 8 & 50029364 & 8 & 50863927 & 8 & 50 & 0.019 & 0.6581 & 7.41 \\
\hline 1222 & 57485 & 57337 & 12 & 0.01 & 93.18 & 0.068068 & 0.037037 & 8 & 51018124 & 8 & 51942666 & 8 & 51 & 0.054 & 0.7082 & 4.73 \\
\hline 1223 & 42277 & 59700 & 9 & 0 & 96.55 & 0.042042 & 0.011011 & 8 & 52134330 & 8 & 52765124 & 8 & 52 & 0.057 & 0.7093 & 4.63 \\
\hline 1224 & 58235 & 58859 & 2 & 0.01 & 95.93 & 0.013013 & 0.012012 & 8 & 53555323 & 8 & 53929233 & 8 & 53 & 0.056 & 0.7088 & 4.65 \\
\hline 1225 & 53092 & 56296 & 5 & 0 & 96.51 & 0.017017 & 0.01001 & 8 & 54201619 & 8 & 54932026 & 8 & 54 & 0.057 & 0.7092 & 4.63 \\
\hline 1226 & 7031 & 16568 & 9 & 0.03 & 73.92 & 0.047047 & 0.034034 & 8 & 55181102 & 8 & 55931429 & 8 & 55 & 0.042 & 0.7022 & 5.36 \\
\hline 1227 & 7034 & 7036 & 8 & 0.02 & 88.44 & 0.049049 & 0.033033 & 8 & 56064449 & 8 & 56805057 & 8 & 56 & 0.051 & 0.7078 & 4.88 \\
\hline 1228 & 51731 & 7041 & 11 & 0.01 & 92.82 & 0.045045 & 0.029029 & 8 & 57262741 & 8 & 57960254 & 8 & 57 & 0.054 & 0.7081 & 4.74 \\
\hline 1229 & 7043 & 24201 & 18 & 0.02 & 86.17 & 0.063063 & 0.043043 & 8 & 58000986 & 8 & 58901257 & 8 & 58 & 0.049 & 0.7071 & 4.95 \\
\hline 1230 & 32950 & 7051 & 8 & 0.01 & 94.65 & 0.041041 & 0.029029 & 8 & 59002816 & 8 & 59957664 & 8 & 59 & 0.056 & 0.7085 & 4.68 \\
\hline 1231 & 52585 & 47207 & 5 & 0 & 96.41 & 0.026026 & 0.012012 & 8 & 60101922 & 8 & 60981454 & 8 & 60 & 0.057 & 0.7092 & 4.64 \\
\hline 1232 & 7052 & 17227 & 7 & 0 & 96.53 & 0.016016 & 0.01001 & 8 & 61100526 & 8 & 61872918 & 8 & 61 & 0.057 & 0.7092 & 4.63 \\
\hline 1233 & 7053 & 47208 & 3 & 0 & 96.55 & 0.011011 & 0.006006 & 8 & 62092621 & 8 & 62838673 & 8 & 62 & 0.057 & 0.7093 & 4.63 \\
\hline 1234 & 7055 & 51212 & 12 & 0.01 & 94.42 & 0.042042 & 0.023023 & 8 & 63000451 & 8 & 63838720 & 8 & 63 & 0.055 & 0.7083 & 4.69 \\
\hline 1235 & 2635 & 7065 & 13 & 0.02 & 89.02 & 0.056056 & 0.043043 & 8 & 64017753 & 8 & 64946356 & 8 & 64 & 0.052 & 0.7075 & 4.86 \\
\hline 1236 & 42292 & 24203 & 11 & 0.01 & 94.33 & 0.055055 & 0.03003 & 8 & 65027400 & 8 & 65954370 & 8 & 65 & 0.055 & 0.7083 & 4.69 \\
\hline 1237 & 56113 & 38144 & 11 & 0.01 & 94.45 & 0.038038 & 0.023023 & 8 & 66027033 & 8 & 66847097 & 8 & 66 & 0.055 & 0.7083 & 4.69 \\
\hline 1238 & 50172 & 7082 & 12 & 0.01 & 93.77 & 0.044044 & 0.027027 & 8 & 67026060 & 8 & 67911215 & 8 & 67 & 0.055 & 0.7082 & 4.71 \\
\hline 1239 & 29922 & 7089 & 14 & 0.01 & 93.5 & 0.056056 & 0.037037 & 8 & 68055002 & 8 & 68896436 & 8 & 68 & 0.055 & 0.7082 & 4.71 \\
\hline 1240 & 15821 & 47211 & 19 & 0.02 & 84.52 & 0.077077 & 0.043043 & 8 & 69039573 & 8 & 69998730 & 8 & 69 & 0.049 & 0.7054 & 4.98 \\
\hline 1241 & 24208 & 55886 & 9 & 0 & 96.54 & 0.02002 & 0.01001 & 8 & 70033358 & 8 & 70991752 & 8 & 70 & 0.057 & 0.7093 & 4.63 \\
\hline 1242 & 61053 & 16071 & 11 & 0.02 & 86.21 & 0.05005 & 0.036036 & 8 & 71042636 & 8 & 71847915 & 8 & 71 & 0.049 & 0.7071 & 4.95 \\
\hline 1243 & 57506 & 31821 & 14 & 0.02 & 87.07 & 0.052052 & 0.031031 & 8 & 72077897 & 8 & 72934282 & 8 & 72 & 0.05 & 0.7076 & 4.93 \\
\hline 1244 & 58287 & 7095 & 20 & 0.04 & 62.57 & 0.103103 & 0.055055 & 8 & 73036634 & 8 & 73926862 & 8 & 73 & 0.034 & 0.6963 & 5.84 \\
\hline 1245 & 7096 & 56258 & 23 & 0.16 & 14.59 & 0.135135 & 0.11011 & 8 & 74017045 & 8 & 74976900 & 8 & 74 & 0.007 & 0.5728 & 10.37 \\
\hline
\end{tabular}




\begin{tabular}{|c|c|c|c|c|c|c|c|c|c|c|c|c|c|c|c|c|}
\hline 1246 & 52937 & 7109 & 26 & 0.07 & 27.84 & 0.148148 & 0.104104 & 8 & 75063893 & 8 & 75981660 & 8 & 75 & 0.015 & 0.6357 & 8.14 \\
\hline 1247 & 50137 & 7117 & 32 & 0.25 & 10.11 & 0.186186 & 0.136136 & 8 & 76109453 & 8 & 76977941 & 8 & 76 & 0.005 & 0.5378 & 11.53 \\
\hline 1248 & 7118 & 33432 & 19 & 0.02 & 84.67 & 0.084084 & 0.044044 & 8 & 77021275 & 8 & 77966205 & 8 & 77 & 0.049 & 0.7055 & 4.98 \\
\hline 1249 & 56689 & 24235 & 23 & 0.01 & 93.06 & 0.085085 & 0.046046 & 8 & 78021944 & 8 & 78996665 & 8 & 78 & 0.054 & 0.7081 & 4.73 \\
\hline 1250 & 16834 & 53947 & 16 & 0.04 & 53.15 & 0.082082 & 0.051051 & 8 & 79177836 & 8 & 79996042 & 8 & 79 & 0.029 & 0.6916 & 6.37 \\
\hline 1251 & 17128 & 59339 & 24 & 0.04 & 60.5 & 0.106106 & 0.073073 & 8 & 80038062 & 8 & 80973136 & 8 & 80 & 0.033 & 0.6959 & 5.95 \\
\hline 1252 & 42309 & 16241 & 26 & 0.04 & 62.9 & 0.106106 & 0.077077 & 8 & 81017438 & 8 & 81998579 & 8 & 81 & 0.035 & 0.6965 & 5.83 \\
\hline 1253 & 56127 & 38156 & 8 & 0.01 & 95.34 & 0.027027 & 0.019019 & 8 & 82012032 & 8 & 82868297 & 8 & 82 & 0.056 & 0.7086 & 4.66 \\
\hline 1254 & 7134 & 7138 & 17 & 0.03 & 69.95 & 0.096096 & 0.064064 & 8 & 83018908 & 8 & 83968789 & 8 & 83 & 0.039 & 0.7001 & 5.52 \\
\hline 1255 & 33058 & 7148 & 22 & 0.04 & 55.41 & 0.104104 & 0.081081 & 8 & 84029728 & 8 & 84978671 & 8 & 84 & 0.03 & 0.692 & 6.19 \\
\hline 1256 & 59544 & 50770 & 10 & 0.01 & 92.31 & 0.051051 & 0.035035 & 8 & 85070054 & 8 & 85901166 & 8 & 85 & 0.054 & 0.7081 & 4.76 \\
\hline 1257 & 32418 & 35245 & 17 & 0.03 & 80.31 & 0.091091 & 0.053053 & 8 & 86016419 & 8 & 86928240 & 8 & 86 & 0.046 & 0.7048 & 5.15 \\
\hline 1258 & 30390 & 32096 & 18 & 0.02 & 84.96 & 0.075075 & 0.048048 & 8 & 87000474 & 8 & 87954252 & 8 & 87 & 0.049 & 0.7061 & 4.98 \\
\hline 1259 & 16495 & 53477 & 20 & 0.03 & 76.98 & 0.093093 & 0.058058 & 8 & 88011897 & 8 & 88909379 & 8 & 88 & 0.044 & 0.7034 & 5.25 \\
\hline 1260 & 60446 & 7160 & 21 & 0.02 & 89.88 & 0.075075 & 0.041041 & 8 & 89162153 & 8 & 89981457 & 8 & 89 & 0.052 & 0.7078 & 4.83 \\
\hline 1261 & 24263 & 7167 & 19 & 0.02 & 81.74 & 0.085085 & 0.05005 & 8 & 90096395 & 8 & 90995810 & 8 & 90 & 0.046 & 0.7052 & 5.09 \\
\hline 1262 & 38164 & 49136 & 20 & 0.04 & 56.19 & 0.093093 & 0.062062 & 8 & 91033467 & 8 & 91991327 & 8 & 91 & 0.031 & 0.6919 & 6.15 \\
\hline 1263 & 7178 & 42323 & 34 & 0.05 & 42.51 & 0.16016 & 0.103103 & 8 & 92067978 & 8 & 92963940 & 8 & 92 & 0.022 & 0.6767 & 7.04 \\
\hline 1264 & 33341 & 56846 & 36 & 0.04 & 61.02 & 0.139139 & 0.09009 & 8 & 93062852 & 8 & 93956344 & 8 & 93 & 0.033 & 0.6963 & 5.93 \\
\hline 1265 & 60260 & 7192 & 8 & 0.02 & 89.17 & 0.035035 & 0.025025 & 8 & 94303744 & 8 & 94994514 & 8 & 94 & 0.052 & 0.7078 & 4.85 \\
\hline 1266 & 56723 & 58266 & 13 & 0.03 & 68.28 & 0.07007 & 0.049049 & 8 & 95024815 & 8 & 95981359 & 8 & 95 & 0.038 & 0.6993 & 5.59 \\
\hline 1267 & 32646 & 7198 & 10 & 0.01 & 95.81 & 0.047047 & 0.026026 & 8 & 96051658 & 8 & 96992033 & 8 & 96 & 0.056 & 0.7089 & 4.65 \\
\hline 1268 & 15253 & 7202 & 9 & 0.01 & 91.96 & 0.046046 & 0.029029 & 8 & 97285456 & 8 & 97956813 & 8 & 97 & 0.053 & 0.7081 & 4.77 \\
\hline 1269 & 53884 & 24286 & 8 & 0.01 & 94.79 & 0.039039 & 0.03003 & 8 & 98547289 & 8 & 98985483 & 8 & 98 & 0.056 & 0.7085 & 4.68 \\
\hline 1270 & 24287 & 7209 & 14 & 0.04 & 63.3 & 0.068068 & 0.047047 & 8 & 99027479 & 8 & 99969028 & 8 & 99 & 0.035 & 0.6971 & 5.81 \\
\hline 1271 & 24291 & 24294 & 16 & 0.02 & 81.72 & 0.07007 & 0.036036 & 8 & 100219507 & 8 & 100921095 & 8 & 100 & 0.046 & 0.7052 & 5.09 \\
\hline 1272 & 57677 & 24296 & 6 & 0.02 & 90.67 & 0.02002 & 0.014014 & 8 & 101158226 & 8 & 101976068 & 8 & 101 & 0.053 & 0.7079 & 4.81 \\
\hline 1273 & 7214 & 7222 & 20 & 0.09 & 22.34 & 0.123123 & 0.088088 & 8 & 102008930 & 8 & 102914965 & 8 & 102 & 0.012 & 0.6151 & 8.91 \\
\hline 1274 & 18584 & 54793 & 19 & 0.03 & 79.66 & 0.078078 & 0.048048 & 8 & 103005406 & 8 & 103726346 & 8 & 103 & 0.045 & 0.7049 & 5.17 \\
\hline 1275 & 7228 & 14453 & 13 & 0.01 & 94.7 & 0.057057 & 0.031031 & 8 & 104001629 & 8 & 104961048 & 8 & 104 & 0.056 & 0.7085 & 4.68 \\
\hline 1276 & 33314 & 61370 & 8 & 0.01 & 95.78 & 0.039039 & 0.027027 & 8 & 105078714 & 8 & 105991646 & 8 & 105 & 0.056 & 0.7089 & 4.66 \\
\hline 1277 & 15787 & 17756 & 17 & 0.03 & 79.37 & 0.076076 & 0.051051 & 8 & 106188404 & 8 & 106955537 & 8 & 106 & 0.045 & 0.7048 & 5.18 \\
\hline 1278 & 7234 & 17005 & 18 & 0.03 & 70.37 & 0.081081 & 0.057057 & 8 & 107036658 & 8 & 107954353 & 8 & 107 & 0.039 & 0.7005 & 5.51 \\
\hline 1279 & 47235 & 34695 & 22 & 0.03 & 65.98 & 0.094094 & 0.066066 & 8 & 108068948 & 8 & 108982801 & 8 & 108 & 0.037 & 0.6988 & 5.69 \\
\hline 1280 & 42341 & 7256 & 25 & 0.04 & 64.09 & 0.106106 & 0.058058 & 8 & 109015913 & 8 & 109852339 & 8 & 109 & 0.035 & 0.6975 & 5.77 \\
\hline 1281 & 36019 & 34079 & 19 & 0.02 & 90.82 & 0.072072 & 0.047047 & 8 & 110016042 & 8 & 110901720 & 8 & 110 & 0.053 & 0.708 & 4.8 \\
\hline 1282 & 53266 & 55824 & 13 & 0.03 & 79.27 & 0.063063 & 0.04004 & 8 & 111006793 & 8 & 111774978 & 8 & 111 & 0.045 & 0.7047 & 5.18 \\
\hline 1283 & 18301 & 24327 & 24 & 0.04 & 55.58 & 0.119119 & 0.075075 & 8 & 112078084 & 8 & 112989184 & 8 & 112 & 0.03 & 0.6919 & 6.18 \\
\hline 1284 & 7274 & 61595 & 13 & 0.02 & 82.93 & 0.067067 & 0.056056 & 8 & 113050594 & 8 & 113825992 & 8 & 113 & 0.048 & 0.7045 & 5.03 \\
\hline
\end{tabular}




\begin{tabular}{|c|c|c|c|c|c|c|c|c|c|c|c|c|c|c|c|c|}
\hline 1285 & 7280 & 51528 & 19 & 0.03 & 70.72 & 0.082082 & 0.049049 & 8 & 114008201 & 8 & 114746282 & 8 & 114 & 0.04 & 0.7004 & 5.49 \\
\hline 1286 & 7308 & 56289 & 9 & 0.01 & 92.38 & 0.048048 & 0.03003 & 8 & 115099064 & 8 & 115989788 & 8 & 115 & 0.054 & 0.7082 & 4.76 \\
\hline 1287 & 30832 & 7292 & 16 & 0.02 & 88.65 & 0.073073 & 0.044044 & 8 & 116042287 & 8 & 116996260 & 8 & 116 & 0.051 & 0.7078 & 4.87 \\
\hline 1288 & 7293 & 7306 & 30 & 0.03 & 67.01 & 0.117117 & 0.075075 & 8 & 117017825 & 8 & 117994361 & 8 & 117 & 0.037 & 0.6993 & 5.65 \\
\hline 1289 & 7307 & 59065 & 11 & 0.01 & 95.2 & 0.047047 & 0.033033 & 8 & 118016335 & 8 & 118874252 & 8 & 118 & 0.056 & 0.7086 & 4.67 \\
\hline 1290 & 54060 & 36044 & 11 & 0.01 & 93.21 & 0.053053 & 0.026026 & 8 & 119254143 & 8 & 119985447 & 8 & 119 & 0.054 & 0.7081 & 4.73 \\
\hline 1291 & 53845 & 7318 & 25 & 0.12 & 18.46 & 0.14014 & 0.103103 & 8 & 120028517 & 8 & 120996107 & 8 & 120 & 0.01 & 0.5947 & 9.5 \\
\hline 1292 & 61470 & 16133 & 14 & 0.05 & 43.07 & 0.082082 & 0.062062 & 8 & 121026032 & 8 & 121909646 & 8 & 121 & 0.023 & 0.677 & 7 \\
\hline 1293 & 15983 & 50491 & 17 & 0.02 & 86.89 & 0.066066 & 0.043043 & 8 & 122072309 & 8 & 122664524 & 8 & 122 & 0.05 & 0.7074 & 4.93 \\
\hline 1294 & 34692 & 7327 & 12 & 0.02 & 83.73 & 0.059059 & 0.046046 & 8 & 123027874 & 8 & 123996880 & 8 & 123 & 0.048 & 0.7053 & 5.01 \\
\hline 1295 & 7326 & 7332 & 21 & 0.06 & 36.94 & 0.104104 & 0.074074 & 8 & 124059371 & 8 & 124932778 & 8 & 124 & 0.02 & 0.6628 & 7.36 \\
\hline 1296 & 7334 & 18435 & 16 & 0.03 & 77.79 & 0.081081 & 0.05005 & 8 & 125012854 & 8 & 125885539 & 8 & 125 & 0.044 & 0.7037 & 5.23 \\
\hline 1297 & 54817 & 7338 & 18 & 0.05 & 45.84 & 0.083083 & 0.057057 & 8 & 126024923 & 8 & 126952312 & 8 & 126 & 0.025 & 0.6813 & 6.78 \\
\hline 1298 & 58793 & 7341 & 17 & 0.04 & 62.54 & 0.084084 & 0.06006 & 8 & 127052406 & 8 & 127976364 & 8 & 127 & 0.034 & 0.6964 & 5.85 \\
\hline 1299 & 39715 & 33939 & 21 & 0.1 & 20.51 & 0.112112 & 0.085085 & 8 & 128039415 & 8 & 128966268 & 8 & 128 & 0.011 & 0.6042 & 9.14 \\
\hline 1300 & 15673 & 17137 & 23 & 0.03 & 71.73 & 0.105105 & 0.072072 & 8 & 129127706 & 8 & 129878095 & 8 & 129 & 0.04 & 0.701 & 5.45 \\
\hline 1301 & 49143 & 30100 & 27 & 0.02 & 82.24 & 0.118118 & 0.075075 & 8 & 130012979 & 8 & 130997013 & 8 & 130 & 0.047 & 0.7049 & 5.07 \\
\hline 1302 & 56387 & 31514 & 17 & 0.02 & 90.37 & 0.056056 & 0.033033 & 8 & 131014130 & 8 & 131837615 & 8 & 131 & 0.053 & 0.7077 & 4.81 \\
\hline 1303 & 55683 & 7363 & 8 & 0.01 & 95.82 & 0.041041 & 0.026026 & 8 & 132376091 & 8 & 132945670 & 8 & 132 & 0.056 & 0.7089 & 4.65 \\
\hline 1304 & 38189 & 15169 & 19 & 0.05 & 49.9 & 0.104104 & 0.068068 & 8 & 133022001 & 8 & 133950496 & 8 & 133 & 0.027 & 0.686 & 6.51 \\
\hline 1305 & 53922 & 33229 & 25 & 0.03 & 73.71 & 0.113113 & 0.074074 & 8 & 134024195 & 8 & 134960890 & 8 & 134 & 0.042 & 0.7022 & 5.37 \\
\hline 1306 & 42379 & 31043 & 23 & 0.04 & 64.69 & 0.1001 & 0.062062 & 8 & 135031919 & 8 & 135960757 & 8 & 135 & 0.036 & 0.6979 & 5.75 \\
\hline 1307 & 15992 & 38195 & 21 & 0.02 & 81.89 & 0.079079 & 0.045045 & 8 & 136113975 & 8 & 136985884 & 8 & 136 & 0.047 & 0.7051 & 5.09 \\
\hline 1308 & 30257 & 14621 & 17 & 0.04 & 56.11 & 0.083083 & 0.053053 & 8 & 137006114 & 8 & 137855568 & 8 & 137 & 0.031 & 0.6917 & 6.15 \\
\hline 1309 & 54482 & 7411 & 26 & 0.02 & 85.5 & 0.127127 & 0.069069 & 8 & 138036642 & 8 & 138999549 & 8 & 138 & 0.049 & 0.7063 & 4.96 \\
\hline 1310 & 38197 & 39652 & 36 & 0.04 & 60.01 & 0.131131 & 0.093093 & 8 & 139013496 & 8 & 139970751 & 8 & 139 & 0.033 & 0.6955 & 5.97 \\
\hline 1311 & 30826 & 56282 & 22 & 0.17 & 12.93 & 0.142142 & 0.115115 & 8 & 140246531 & 8 & 140986050 & 8 & 140 & 0.006 & 0.5641 & 10.95 \\
\hline 1312 & 24406 & 7430 & 32 & 0.12 & 18.23 & 0.166166 & 0.118118 & 8 & 141003882 & 8 & 141980614 & 8 & 141 & 0.009 & 0.5938 & 9.53 \\
\hline 1313 & 34735 & 50899 & 31 & 0.11 & 19.25 & 0.149149 & 0.115115 & 8 & 142003177 & 8 & 142950987 & 8 & 142 & 0.01 & 0.5989 & 9.37 \\
\hline 1314 & 7432 & 34330 & 34 & 0.11 & 19.36 & 0.184184 & 0.132132 & 8 & 143011607 & 8 & 143998135 & 8 & 143 & 0.01 & 0.5996 & 9.36 \\
\hline 1315 & 16475 & 16051 & 29 & 0.04 & 59.85 & 0.123123 & 0.076076 & 8 & 144034145 & 8 & 144999383 & 8 & 144 & 0.033 & 0.6954 & 5.98 \\
\hline 1316 & 29295 & 19170 & 47 & 0.06 & 37.11 & 0.209209 & 0.145145 & 8 & 145036187 & 8 & 145994240 & 8 & 145 & 0.02 & 0.6629 & 7.34 \\
\hline 1317 & 16299 & 15805 & 33 & 0.05 & 46.03 & 0.131131 & 0.088088 & 8 & 146042078 & 8 & 146958932 & 8 & 146 & 0.025 & 0.6814 & 6.76 \\
\hline 1318 & 15864 & 55548 & 25 & 0.04 & 53.06 & 0.104104 & 0.068068 & 8 & 147002408 & 8 & 147963935 & 8 & 147 & 0.029 & 0.6915 & 6.37 \\
\hline 1319 & 19319 & 53483 & 4 & 0.01 & 96.21 & 0.022022 & 0.015015 & 8 & 148060253 & 8 & 148158316 & 8 & 148 & 0.057 & 0.7091 & 4.64 \\
\hline 1320 & 51548 & 50018 & 36 & 0.06 & 38.26 & 0.17017 & 0.118118 & 9 & 21708 & 9 & 974310 & 9 & 0 & 0.02 & 0.6661 & 7.27 \\
\hline 1321 & 31799 & 42410 & 18 & 0.03 & 70.66 & 0.089089 & 0.064064 & 9 & 1120035 & 9 & 1707931 & 9 & 1 & 0.039 & 0.7004 & 5.49 \\
\hline 1322 & 16747 & 31404 & 28 & 0.04 & 57.8 & 0.114114 & 0.073073 & 9 & 2305353 & 9 & 2978499 & 9 & 2 & 0.032 & 0.6925 & 6.05 \\
\hline 1323 & 16236 & 62053 & 18 & 0.02 & 82.72 & 0.097097 & 0.067067 & 9 & 3016833 & 9 & 3993696 & 9 & 3 & 0.047 & 0.7046 & 5.04 \\
\hline
\end{tabular}




\begin{tabular}{|c|c|c|c|c|c|c|c|c|c|c|c|c|c|c|c|c|}
\hline 1324 & 24449 & 24456 & 27 & 0.03 & 76.51 & 0.113113 & 0.074074 & 9 & 4014490 & 9 & 4990218 & 9 & 4 & 0.043 & 0.7031 & 5.27 \\
\hline 1325 & 42421 & 38220 & 12 & 0.01 & 94.73 & 0.048048 & 0.026026 & 9 & 5226276 & 9 & 5988229 & 9 & 5 & 0.056 & 0.7085 & 4.68 \\
\hline 1326 & 32798 & 42426 & 19 & 0.04 & 61.47 & 0.109109 & 0.07007 & 9 & 6061399 & 9 & 6964469 & 9 & 6 & 0.034 & 0.6962 & 5.9 \\
\hline 1327 & 7496 & 57828 & 37 & 0.05 & 42.76 & 0.155155 & 0.104104 & 9 & 7010644 & 9 & 7987695 & 9 & 7 & 0.023 & 0.6765 & 7.01 \\
\hline 1328 & 49162 & 24474 & 23 & 0.07 & 30.6 & 0.12012 & 0.091091 & 9 & 8047973 & 9 & 8994791 & 9 & 8 & 0.016 & 0.6451 & 7.88 \\
\hline 1329 & 42430 & 24481 & 26 & 0.1 & 21.78 & 0.149149 & 0.103103 & 9 & 9019463 & 9 & 9995702 & 9 & 9 & 0.011 & 0.6129 & 9 \\
\hline 1330 & 57942 & 35967 & 35 & 0.06 & 38.48 & 0.138138 & 0.101101 & 9 & 10050483 & 9 & 10972428 & 9 & 10 & 0.02 & 0.667 & 7.27 \\
\hline 1331 & 7522 & 7528 & 32 & 0.04 & 61.1 & 0.131131 & 0.083083 & 9 & 11012156 & 9 & 11997728 & 9 & 11 & 0.033 & 0.6964 & 5.93 \\
\hline 1332 & 49169 & 35842 & 29 & 0.06 & 38.98 & 0.142142 & 0.098098 & 9 & 12012638 & 9 & 12980703 & 9 & 12 & 0.021 & 0.6681 & 7.24 \\
\hline 1333 & 42453 & 7540 & 21 & 0.01 & 93.73 & 0.065065 & 0.039039 & 9 & 13001247 & 9 & 13977765 & 9 & 13 & 0.055 & 0.7082 & 4.71 \\
\hline 1334 & 24505 & 16564 & 28 & 0.05 & 48.99 & 0.132132 & 0.095095 & 9 & 14007105 & 9 & 14991770 & 9 & 14 & 0.027 & 0.6843 & 6.54 \\
\hline 1335 & 19264 & 42460 & 25 & 0.04 & 60.42 & 0.103103 & 0.067067 & 9 & 15001911 & 9 & 15999539 & 9 & 15 & 0.033 & 0.6961 & 5.96 \\
\hline 1336 & 42461 & 39693 & 22 & 0.14 & 15.36 & 0.133133 & 0.101101 & 9 & 16047080 & 9 & 16962945 & 9 & 16 & 0.008 & 0.5784 & 10.18 \\
\hline 1337 & 15372 & 15154 & 29 & 0.04 & 59.93 & 0.128128 & 0.084084 & 9 & 17004309 & 9 & 17992016 & 9 & 17 & 0.033 & 0.6954 & 5.97 \\
\hline 1338 & 15737 & 36164 & 16 & 0.02 & 89.79 & 0.061061 & 0.038038 & 9 & 18002888 & 9 & 18971973 & 9 & 18 & 0.052 & 0.7077 & 4.83 \\
\hline 1339 & 33592 & 38230 & 26 & 0.03 & 70.08 & 0.104104 & 0.066066 & 9 & 19079143 & 9 & 19956110 & 9 & 19 & 0.039 & 0.7002 & 5.52 \\
\hline 1340 & 17589 & 42468 & 30 & 0.04 & 54.16 & 0.128128 & 0.078078 & 9 & 20002748 & 9 & 20979753 & 9 & 20 & 0.029 & 0.6917 & 6.29 \\
\hline 1341 & 24536 & 61555 & 28 & 0.05 & 49.31 & 0.125125 & 0.094094 & 9 & 21032490 & 9 & 21990942 & 9 & 21 & 0.027 & 0.6846 & 6.53 \\
\hline 1342 & 7582 & 18715 & 33 & 0.05 & 40.63 & 0.138138 & 0.103103 & 9 & 22008802 & 9 & 22985623 & 9 & 22 & 0.022 & 0.672 & 7.14 \\
\hline 1343 & 16035 & 61619 & 18 & 0.03 & 69.63 & 0.093093 & 0.069069 & 9 & 23018947 & 9 & 23888625 & 9 & 23 & 0.039 & 0.6998 & 5.53 \\
\hline 1344 & 24544 & 54125 & 25 & 0.08 & 25.4 & 0.138138 & 0.104104 & 9 & 24011516 & 9 & 24695732 & 9 & 24 & 0.013 & 0.6284 & 8.47 \\
\hline 1345 & 18378 & 18505 & 16 & 0.04 & 53.49 & 0.073073 & 0.051051 & 9 & 25082159 & 9 & 25971114 & 9 & 25 & 0.029 & 0.6921 & 6.35 \\
\hline 1346 & 52819 & 30658 & 32 & 0.06 & 38.09 & 0.137137 & 0.089089 & 9 & 26005437 & 9 & 26987809 & 9 & 26 & 0.02 & 0.6659 & 7.28 \\
\hline 1347 & 7606 & 33018 & 27 & 0.03 & 71.76 & 0.111111 & 0.073073 & 9 & 27001512 & 9 & 27995647 & 9 & 27 & 0.04 & 0.701 & 5.45 \\
\hline 1348 & 32410 & 59968 & 43 & 0.07 & 28.79 & 0.177177 & 0.118118 & 9 & 28035750 & 9 & 28998609 & 9 & 28 & 0.015 & 0.6382 & 8.04 \\
\hline 1349 & 16910 & 42483 & 22 & 0.03 & 68.58 & 0.093093 & 0.057057 & 9 & 29002522 & 9 & 29982654 & 9 & 29 & 0.038 & 0.6996 & 5.58 \\
\hline 1350 & 42484 & 54468 & 26 & 0.05 & 44.37 & 0.124124 & 0.085085 & 9 & 30061785 & 9 & 30795185 & 9 & 30 & 0.024 & 0.6794 & 6.9 \\
\hline 1351 & 15307 & 7628 & 22 & 0.02 & 82.88 & 0.09009 & 0.059059 & 9 & 31072493 & 9 & 31940771 & 9 & 31 & 0.047 & 0.7046 & 5.04 \\
\hline 1352 & 51795 & 33612 & 27 & 0.14 & 16.2 & 0.152152 & 0.103103 & 9 & 32073965 & 9 & 32928892 & 9 & 32 & 0.008 & 0.5817 & 9.9 \\
\hline 1353 & 61154 & 16463 & 6 & 0.03 & 70.5 & 0.044044 & 0.037037 & 9 & 33065256 & 9 & 33899905 & 9 & 33 & 0.039 & 0.7005 & 5.5 \\
\hline 1354 & 55573 & 24584 & 23 & 0.15 & 15.21 & 0.145145 & 0.115115 & 9 & 34056153 & 9 & 34993225 & 9 & 34 & 0.008 & 0.5763 & 10.17 \\
\hline 1355 & 24585 & 24591 & 22 & 0.05 & 44.77 & 0.091091 & 0.07007 & 9 & 35012606 & 9 & 35998370 & 9 & 35 & 0.024 & 0.6796 & 6.85 \\
\hline 1356 & 38251 & 32207 & 14 & 0.02 & 91.05 & 0.056056 & 0.044044 & 9 & 36041037 & 9 & 36913567 & 9 & 36 & 0.053 & 0.7081 & 4.8 \\
\hline 1357 & 10587 & 38255 & 20 & 0.07 & 29.64 & 0.119119 & 0.092092 & 9 & 37031308 & 9 & 37972729 & 9 & 37 & 0.016 & 0.6411 & 7.95 \\
\hline 1358 & 7645 & 7660 & 35 & 0.22 & 11.29 & 0.196196 & 0.156156 & 9 & 38006578 & 9 & 38993114 & 9 & 38 & 0.006 & 0.55 & 11.32 \\
\hline 1359 & 38257 & 14772 & 21 & 0.05 & 43.87 & 0.107107 & 0.069069 & 9 & 39034059 & 9 & 39882600 & 9 & 39 & 0.023 & 0.6784 & 6.94 \\
\hline 1360 & 30358 & 8185 & 15 & 0.03 & 75.03 & 0.069069 & 0.044044 & 9 & 40024107 & 9 & 40991552 & 9 & 40 & 0.042 & 0.7021 & 5.31 \\
\hline 1361 & 36017 & 24998 & 19 & 0.04 & 52.28 & 0.088088 & 0.066066 & 9 & 41043659 & 9 & 41978500 & 9 & 41 & 0.028 & 0.69 & 6.4 \\
\hline 1362 & 8190 & 61588 & 15 & 0.03 & 65.11 & 0.072072 & 0.049049 & 9 & 42014140 & 9 & 42938387 & 9 & 42 & 0.036 & 0.6983 & 5.73 \\
\hline
\end{tabular}




\begin{tabular}{|c|c|c|c|c|c|c|c|c|c|c|c|c|c|c|c|c|}
\hline 1363 & 15377 & 38261 & 34 & 0.06 & 32.42 & 0.15015 & 0.097097 & 9 & 43064592 & 9 & 43992201 & 9 & 43 & 0.017 & 0.6501 & 7.73 \\
\hline 1364 & 16627 & 7675 & 13 & 0.03 & 68.88 & 0.065065 & 0.046046 & 9 & 44432191 & 9 & 44949057 & 9 & 44 & 0.038 & 0.6999 & 5.57 \\
\hline 1365 & 42507 & 49821 & 34 & 0.06 & 32.8 & 0.147147 & 0.093093 & 9 & 45011963 & 9 & 45974573 & 9 & 45 & 0.017 & 0.6502 & 7.68 \\
\hline 1366 & 49178 & 50954 & 20 & 0.03 & 78.46 & 0.083083 & 0.054054 & 9 & 46041782 & 9 & 46991931 & 9 & 46 & 0.044 & 0.7041 & 5.21 \\
\hline 1367 & 19193 & 24642 & 29 & 0.06 & 33.37 & 0.151151 & 0.103103 & 9 & 47035782 & 9 & 47994172 & 9 & 47 & 0.018 & 0.6512 & 7.63 \\
\hline 1368 & 7693 & 7706 & 35 & 0.05 & 48.57 & 0.146146 & 0.107107 & 9 & 48020021 & 9 & 48972341 & 9 & 48 & 0.026 & 0.6832 & 6.55 \\
\hline 1369 & 24652 & 33877 & 29 & 0.04 & 51.06 & 0.122122 & 0.083083 & 9 & 49030981 & 9 & 49997890 & 9 & 49 & 0.028 & 0.6878 & 6.45 \\
\hline 1370 & 60090 & 45237 & 20 & 0.02 & 88.84 & 0.094094 & 0.054054 & 9 & 50024764 & 9 & 50962419 & 9 & 50 & 0.051 & 0.7077 & 4.86 \\
\hline 1371 & 56630 & 14511 & 25 & 0.03 & 68.85 & 0.1001 & 0.062062 & 9 & 51113384 & 9 & 51965451 & 9 & 51 & 0.038 & 0.7 & 5.58 \\
\hline 1372 & 29331 & 49183 & 22 & 0.02 & 84.18 & 0.104104 & 0.054054 & 9 & 52065210 & 9 & 52973032 & 9 & 52 & 0.048 & 0.7053 & 4.99 \\
\hline 1373 & 47265 & 42547 & 21 & 0.04 & 52.02 & 0.095095 & 0.057057 & 9 & 53008122 & 9 & 53975532 & 9 & 53 & 0.028 & 0.6893 & 6.41 \\
\hline 1374 & 7727 & 24675 & 21 & 0.03 & 77.22 & 0.1001 & 0.068068 & 9 & 54010624 & 9 & 54991166 & 9 & 54 & 0.044 & 0.7034 & 5.25 \\
\hline 1375 & 24676 & 24680 & 23 & 0.04 & 57.6 & 0.098098 & 0.064064 & 9 & 55009244 & 9 & 55977696 & 9 & 55 & 0.032 & 0.6923 & 6.06 \\
\hline 1376 & 16622 & 19400 & 6 & 0.01 & 94.27 & 0.028028 & 0.02002 & 9 & 56048240 & 9 & 56975238 & 9 & 56 & 0.055 & 0.7083 & 4.69 \\
\hline 1377 & 24684 & 54474 & 15 & 0.02 & 89.96 & 0.07007 & 0.047047 & 9 & 57027600 & 9 & 57905817 & 9 & 57 & 0.052 & 0.7078 & 4.83 \\
\hline 1378 & 24686 & 35799 & 12 & 0.01 & 95.67 & 0.039039 & 0.026026 & 9 & 58065674 & 9 & 58958297 & 9 & 58 & 0.056 & 0.7089 & 4.66 \\
\hline 1379 & 36229 & 29434 & 16 & 0.02 & 90.25 & 0.07007 & 0.05005 & 9 & 59032592 & 9 & 59952885 & 9 & 59 & 0.052 & 0.7077 & 4.81 \\
\hline 1380 & 60744 & 7756 & 15 & 0.02 & 90.64 & 0.061061 & 0.033033 & 9 & 60085650 & 9 & 60985372 & 9 & 60 & 0.053 & 0.7079 & 4.81 \\
\hline 1381 & 42570 & 24702 & 30 & 0.02 & 88.97 & 0.117117 & 0.058058 & 9 & 61003307 & 9 & 61986456 & 9 & 61 & 0.051 & 0.7076 & 4.86 \\
\hline 1382 & 38268 & 24708 & 27 & 0.03 & 77.11 & 0.114114 & 0.069069 & 9 & 62083176 & 9 & 62996659 & 9 & 62 & 0.044 & 0.7034 & 5.25 \\
\hline 1383 & 24709 & 58649 & 21 & 0.03 & 78.91 & 0.085085 & 0.054054 & 9 & 63029187 & 9 & 63978822 & 9 & 63 & 0.045 & 0.7042 & 5.19 \\
\hline 1384 & 56775 & 56245 & 24 & 0.02 & 81.5 & 0.083083 & 0.056056 & 9 & 64024041 & 9 & 64983107 & 9 & 64 & 0.046 & 0.7052 & 5.1 \\
\hline 1385 & 14587 & 24724 & 34 & 0.04 & 61.58 & 0.147147 & 0.098098 & 9 & 65022738 & 9 & 65959223 & 9 & 65 & 0.034 & 0.6963 & 5.9 \\
\hline 1386 & 24725 & 54763 & 34 & 0.03 & 77.55 & 0.135135 & 0.083083 & 9 & 66001271 & 9 & 66941538 & 9 & 66 & 0.044 & 0.7036 & 5.24 \\
\hline 1387 & 33254 & 51176 & 20 & 0.02 & 87.23 & 0.082082 & 0.063063 & 9 & 67063113 & 9 & 67814275 & 9 & 67 & 0.05 & 0.7075 & 4.92 \\
\hline 1388 & 17134 & 7797 & 4 & 0 & 96.5 & 0.013013 & 0.006006 & 9 & 68169771 & 9 & 68936873 & 9 & 68 & 0.057 & 0.7092 & 4.63 \\
\hline 1389 & 24736 & 50658 & 10 & 0.01 & 94.09 & 0.051051 & 0.028028 & 9 & 69016799 & 9 & 69976085 & 9 & 69 & 0.055 & 0.7084 & 4.7 \\
\hline 1390 & 51173 & 58953 & 25 & 0.03 & 72.11 & 0.118118 & 0.08008 & 9 & 70035504 & 9 & 70936501 & 9 & 70 & 0.04 & 0.7012 & 5.44 \\
\hline 1391 & 24746 & 45412 & 27 & 0.02 & 85.28 & 0.121121 & 0.074074 & 9 & 71170725 & 9 & 71989525 & 9 & 71 & 0.049 & 0.7061 & 4.97 \\
\hline 1392 & 60561 & 18765 & 22 & 0.02 & 89.31 & 0.084084 & 0.051051 & 9 & 72053046 & 9 & 72946896 & 9 & 72 & 0.052 & 0.7076 & 4.84 \\
\hline 1393 & 42732 & 42735 & 16 & 0.03 & 74.51 & 0.071071 & 0.051051 & 9 & 73040157 & 9 & 73942367 & 9 & 73 & 0.042 & 0.7022 & 5.34 \\
\hline 1394 & 25003 & 57148 & 19 & 0.02 & 83.41 & 0.071071 & 0.049049 & 9 & 74006034 & 9 & 74961114 & 9 & 74 & 0.048 & 0.7047 & 5.02 \\
\hline 1395 & 7807 & 7818 & 22 & 0.05 & 40.03 & 0.111111 & 0.087087 & 9 & 75067877 & 9 & 75973705 & 9 & 75 & 0.021 & 0.6707 & 7.17 \\
\hline 1396 & 24759 & 7829 & 19 & 0.03 & 69.86 & 0.093093 & 0.066066 & 9 & 76041058 & 9 & 76981023 & 9 & 76 & 0.039 & 0.7001 & 5.53 \\
\hline 1397 & 34397 & 7834 & 21 & 0.04 & 55.74 & 0.106106 & 0.077077 & 9 & 77019488 & 9 & 77970228 & 9 & 77 & 0.03 & 0.6921 & 6.18 \\
\hline 1398 & 15099 & 38289 & 23 & 0.06 & 34.3 & 0.102102 & 0.08008 & 9 & 78070603 & 9 & 78963458 & 9 & 78 & 0.018 & 0.6557 & 7.57 \\
\hline 1399 & 7842 & 31860 & 11 & 0.03 & 67.58 & 0.058058 & 0.042042 & 9 & 79010742 & 9 & 79894082 & 9 & 79 & 0.038 & 0.6991 & 5.62 \\
\hline 1400 & 38294 & 38295 & 12 & 0.02 & 87.45 & 0.052052 & 0.04004 & 9 & 80094154 & 9 & 80677221 & 9 & 80 & 0.05 & 0.7076 & 4.91 \\
\hline 1401 & 24776 & 38297 & 11 & 0.03 & 72.08 & 0.066066 & 0.051051 & 9 & 81214756 & 9 & 81888480 & 9 & 81 & 0.04 & 0.7012 & 5.44 \\
\hline
\end{tabular}




\begin{tabular}{|c|c|c|c|c|c|c|c|c|c|c|c|c|c|c|c|c|}
\hline 1402 & 7849 & 24787 & 21 & 0.02 & 83.78 & 0.093093 & 0.053053 & 9 & 82004163 & 9 & 82930808 & 9 & 82 & 0.048 & 0.7054 & 5.01 \\
\hline 1403 & 57288 & 42610 & 16 & 0.02 & 85.96 & 0.072072 & 0.05005 & 9 & 83104537 & 9 & 83986835 & 9 & 83 & 0.049 & 0.7066 & 4.95 \\
\hline 1404 & 38303 & 24791 & 20 & 0.02 & 82.29 & 0.096096 & 0.068068 & 9 & 84019420 & 9 & 84965987 & 9 & 84 & 0.047 & 0.7048 & 5.07 \\
\hline 1405 & 24792 & 24798 & 22 & 0.03 & 71.45 & 0.085085 & 0.055055 & 9 & 85001751 & 9 & 85988046 & 9 & 85 & 0.04 & 0.7008 & 5.46 \\
\hline 1406 & 24799 & 30566 & 15 & 0.03 & 74.57 & 0.071071 & 0.044044 & 9 & 86034248 & 9 & 86869637 & 9 & 86 & 0.042 & 0.702 & 5.33 \\
\hline 1407 & 33069 & 29544 & 12 & 0.02 & 82.74 & 0.072072 & 0.06006 & 9 & 87009539 & 9 & 87977671 & 9 & 87 & 0.047 & 0.7047 & 5.04 \\
\hline 1408 & 17527 & 60222 & 10 & 0.02 & 90.22 & 0.046046 & 0.027027 & 9 & 88276225 & 9 & 88978634 & 9 & 88 & 0.052 & 0.7076 & 4.81 \\
\hline 1409 & 33904 & 7887 & 6 & 0.01 & 95.81 & 0.037037 & 0.019019 & 9 & 89128614 & 9 & 89984248 & 9 & 89 & 0.056 & 0.7089 & 4.65 \\
\hline 1410 & 47276 & 7892 & 13 & 0.02 & 81.23 & 0.07007 & 0.043043 & 9 & 90001442 & 9 & 90986895 & 9 & 90 & 0.046 & 0.7052 & 5.11 \\
\hline 1411 & 7893 & 7896 & 8 & 0.02 & 83.55 & 0.049049 & 0.035035 & 9 & 91008560 & 9 & 91927983 & 9 & 91 & 0.048 & 0.7052 & 5.02 \\
\hline 1412 & 24806 & 52927 & 8 & 0.01 & 95.6 & 0.035035 & 0.023023 & 9 & 92204161 & 9 & 92976421 & 9 & 92 & 0.056 & 0.7088 & 4.66 \\
\hline 1413 & 51718 & 24810 & 17 & 0.05 & 46.65 & 0.088088 & 0.057057 & 9 & 93035029 & 9 & 93976824 & 9 & 93 & 0.025 & 0.6817 & 6.69 \\
\hline 1414 & 53429 & 7913 & 15 & 0.05 & 43.02 & 0.086086 & 0.064064 & 9 & 94071916 & 9 & 94988727 & 9 & 94 & 0.023 & 0.677 & 7 \\
\hline 1415 & 24811 & 53117 & 21 & 0.05 & 40.08 & 0.121121 & 0.086086 & 9 & 95008515 & 9 & 95973005 & 9 & 95 & 0.021 & 0.6713 & 7.18 \\
\hline 1416 & 56662 & 57968 & 15 & 0.02 & 85.04 & 0.061061 & 0.046046 & 9 & 96199471 & 9 & 96887177 & 9 & 96 & 0.049 & 0.7062 & 4.98 \\
\hline 1417 & 57174 & 38331 & 15 & 0.03 & 68.68 & 0.061061 & 0.044044 & 9 & 97051426 & 9 & 97912451 & 9 & 97 & 0.038 & 0.7 & 5.58 \\
\hline 1418 & 24821 & 15756 & 9 & 0.02 & 91.11 & 0.04004 & 0.023023 & 9 & 98059019 & 9 & 98984967 & 9 & 98 & 0.053 & 0.7081 & 4.8 \\
\hline 1419 & 52081 & 17891 & 21 & 0.04 & 64.9 & 0.087087 & 0.063063 & 9 & 99173519 & 9 & 99988998 & 9 & 99 & 0.036 & 0.698 & 5.74 \\
\hline 1420 & 45102 & 31415 & 34 & 0.07 & 29.43 & 0.156156 & 0.101101 & 9 & 100019532 & 9 & 100985297 & 9 & 100 & 0.016 & 0.641 & 7.98 \\
\hline 1421 & 32324 & 14801 & 8 & 0.01 & 95.62 & 0.032032 & 0.018018 & 9 & 101128149 & 9 & 101987835 & 9 & 101 & 0.056 & 0.7088 & 4.66 \\
\hline 1422 & 59248 & 24831 & 15 & 0.01 & 94.58 & 0.068068 & 0.036036 & 9 & 102081959 & 9 & 102920120 & 9 & 102 & 0.055 & 0.7084 & 4.68 \\
\hline 1423 & 60183 & 24833 & 12 & 0 & 96.33 & 0.036036 & 0.011011 & 9 & 103035428 & 9 & 103976159 & 9 & 103 & 0.057 & 0.7091 & 4.64 \\
\hline 1424 & 24834 & 54969 & 3 & 0.01 & 96.21 & 0.014014 & 0.01001 & 9 & 104255261 & 9 & 104996366 & 9 & 104 & 0.057 & 0.7091 & 4.64 \\
\hline 1425 & 50828 & 7942 & 5 & 0.01 & 93.99 & 0.029029 & 0.016016 & 9 & 105545798 & 9 & 105742262 & 9 & 105 & 0.055 & 0.7084 & 4.7 \\
\hline 1426 & 38339 & 30177 & 17 & 0.02 & 81.25 & 0.078078 & 0.052052 & 9 & 106274400 & 9 & 106920454 & 9 & 106 & 0.046 & 0.7051 & 5.11 \\
\hline 1427 & 17424 & 47283 & 11 & 0.01 & 93.52 & 0.048048 & 0.032032 & 9 & 107057752 & 9 & 107850450 & 9 & 107 & 0.055 & 0.7082 & 4.71 \\
\hline 1428 & 47284 & 24839 & 11 & 0.01 & 95.02 & 0.047047 & 0.025025 & 9 & 108009662 & 9 & 108882963 & 9 & 108 & 0.056 & 0.7087 & 4.67 \\
\hline 1429 & 7948 & 47286 & 3 & 0.01 & 96.13 & 0.018018 & 0.002002 & 9 & 109096308 & 9 & 109683264 & 9 & 109 & 0.057 & 0.709 & 4.64 \\
\hline 1430 & 7951 & 38347 & 7 & 0 & 96.51 & 0.033033 & 0.014014 & 9 & 110108860 & 9 & 110626460 & 9 & 110 & 0.057 & 0.7092 & 4.63 \\
\hline 1431 & 54155 & 7956 & 14 & 0.03 & 65.94 & 0.069069 & 0.049049 & 9 & 111000802 & 9 & 111952227 & 9 & 111 & 0.037 & 0.6986 & 5.69 \\
\hline 1432 & 24841 & 42634 & 17 & 0.06 & 35.1 & 0.11011 & 0.079079 & 9 & 112012220 & 9 & 112995189 & 9 & 112 & 0.019 & 0.656 & 7.46 \\
\hline 1433 & 24844 & 47287 & 13 & 0.02 & 91.63 & 0.046046 & 0.03003 & 9 & 113051200 & 9 & 113964106 & 9 & 113 & 0.053 & 0.7082 & 4.78 \\
\hline 1434 & 32716 & 17988 & 13 & 0.01 & 93.72 & 0.062062 & 0.039039 & 9 & 114021432 & 9 & 114916229 & 9 & 114 & 0.055 & 0.7082 & 4.71 \\
\hline 1435 & 39645 & 7974 & 16 & 0.02 & 90.69 & 0.062062 & 0.034034 & 9 & 115076491 & 9 & 115974419 & 9 & 115 & 0.053 & 0.7079 & 4.81 \\
\hline 1436 & 58155 & 34813 & 27 & 0.05 & 45.26 & 0.134134 & 0.077077 & 9 & 116099220 & 9 & 116927941 & 9 & 116 & 0.024 & 0.6805 & 6.82 \\
\hline 1437 & 7986 & 17888 & 14 & 0.04 & 60.57 & 0.078078 & 0.061061 & 9 & 117012701 & 9 & 117851340 & 9 & 117 & 0.033 & 0.6958 & 5.94 \\
\hline 1438 & 34121 & 7990 & 25 & 0.06 & 37.23 & 0.114114 & 0.077077 & 9 & 118021130 & 9 & 118989834 & 9 & 118 & 0.02 & 0.6627 & 7.31 \\
\hline 1439 & 55989 & 31056 & 15 & 0.02 & 86.28 & 0.072072 & 0.042042 & 9 & 119023630 & 9 & 119766208 & 9 & 119 & 0.05 & 0.7071 & 4.95 \\
\hline 1440 & 18433 & 7996 & 20 & 0.02 & 85.35 & 0.103103 & 0.064064 & 9 & 120039871 & 9 & 120972491 & 9 & 120 & 0.049 & 0.7062 & 4.97 \\
\hline
\end{tabular}




\begin{tabular}{|c|c|c|c|c|c|c|c|c|c|c|c|c|c|c|c|c|}
\hline 1441 & 7997 & 8010 & 31 & 0.04 & 63.12 & 0.131131 & 0.073073 & 9 & 121014874 & 9 & 121983147 & 9 & 121 & 0.035 & 0.6966 & 5.82 \\
\hline 1442 & 52512 & 59241 & 22 & 0.01 & 94.13 & 0.108108 & 0.044044 & 9 & 122036577 & 9 & 122944920 & 9 & 122 & 0.055 & 0.7085 & 4.7 \\
\hline 1443 & 18340 & 42650 & 17 & 0.01 & 93 & 0.077077 & 0.03003 & 9 & 123051900 & 9 & 123972496 & 9 & 123 & 0.054 & 0.7082 & 4.74 \\
\hline 1444 & 38377 & 47289 & 27 & 0.03 & 79.88 & 0.113113 & 0.06006 & 9 & 124079367 & 9 & 124959430 & 9 & 124 & 0.045 & 0.7048 & 5.16 \\
\hline 1446 & 8032 & 31662 & 11 & 0.01 & 94.19 & 0.062062 & 0.035035 & 9 & 126204363 & 9 & 126652137 & 9 & 126 & 0.055 & 0.7084 & 4.69 \\
\hline 1447 & 24884 & 45761 & 12 & 0.02 & 86.57 & 0.052052 & 0.039039 & 9 & 127045581 & 9 & 127981472 & 9 & 127 & 0.05 & 0.707 & 4.94 \\
\hline 1448 & 8038 & 56987 & 18 & 0.03 & 74.54 & 0.077077 & 0.057057 & 9 & 128025650 & 9 & 128986695 & 9 & 128 & 0.042 & 0.7021 & 5.34 \\
\hline 1450 & 52285 & 50352 & 16 & 0.02 & 90.57 & 0.057057 & 0.038038 & 9 & 130383913 & 9 & 130992209 & 9 & 130 & 0.053 & 0.7078 & 4.81 \\
\hline 1451 & 16431 & 56850 & 14 & 0.02 & 88.57 & 0.042042 & 0.021021 & 9 & 131130851 & 9 & 131977434 & 9 & 131 & 0.051 & 0.7078 & 4.87 \\
\hline 1452 & 38385 & 18471 & 15 & 0.02 & 86.52 & 0.062062 & 0.037037 & 9 & 132023179 & 9 & 132827171 & 9 & 132 & 0.05 & 0.707 & 4.94 \\
\hline 1453 & 51398 & 8072 & 22 & 0.06 & 37.29 & 0.097097 & 0.06006 & 9 & 133007769 & 9 & 133969269 & 9 & 133 & 0.02 & 0.6628 & 7.31 \\
\hline 1454 & 8073 & 42675 & 14 & 0.04 & 60.61 & 0.052052 & 0.044044 & 9 & 134004914 & 9 & 134966767 & 9 & 134 & 0.033 & 0.6957 & 5.94 \\
\hline 1455 & 24905 & 8084 & 30 & 0.05 & 45.64 & 0.148148 & 0.104104 & 9 & 135034104 & 9 & 135978071 & 9 & 135 & 0.024 & 0.6812 & 6.8 \\
\hline 1456 & 24911 & 8092 & 25 & 0.06 & 34.86 & 0.136136 & 0.102102 & 9 & 136006502 & 9 & 136977536 & 9 & 136 & 0.019 & 0.6553 & 7.5 \\
\hline 1458 & 8103 & 24932 & 34 & 0.08 & 24.27 & 0.169169 & 0.125125 & 9 & 138014373 & 9 & 138986678 & 9 & 138 & 0.013 & 0.6257 & 8.68 \\
\hline 1459 & 24933 & 36226 & 37 & 0.04 & 54.79 & 0.143143 & 0.085085 & 9 & 139005603 & 9 & 139976454 & 9 & 139 & 0.03 & 0.6917 & 6.23 \\
\hline 1460 & 38393 & 32548 & 24 & 0.03 & 77.06 & 0.095095 & 0.057057 & 9 & 140003794 & 9 & 140971189 & 9 & 140 & 0.044 & 0.7034 & 5.25 \\
\hline 1461 & 58102 & 59763 & 24 & 0.03 & 69.24 & 0.1001 & 0.071071 & 9 & 141246965 & 9 & 141992388 & 9 & 141 & 0.039 & 0.6997 & 5.55 \\
\hline 1462 & 45541 & 42701 & 28 & 0.07 & 29.98 & 0.142142 & 0.101101 & 9 & 142004595 & 9 & 142950929 & 9 & 142 & 0.016 & 0.6422 & 7.91 \\
\hline 1463 & 54512 & 17582 & 25 & 0.02 & 84.47 & 0.095095 & 0.064064 & 9 & 143029683 & 9 & 143890847 & 9 & 143 & 0.049 & 0.7054 & 4.98 \\
\hline 1464 & 29448 & 8146 & 33 & 0.03 & 78.41 & 0.138138 & 0.068068 & 9 & 144006070 & 9 & 144965956 & 9 & 144 & 0.044 & 0.7041 & 5.21 \\
\hline 1465 & 8143 & 24963 & 30 & 0.03 & 76.95 & 0.116116 & 0.073073 & 9 & 145001798 & 9 & 145998807 & 9 & 145 & 0.044 & 0.7034 & 5.25 \\
\hline 1466 & 8157 & 31453 & 27 & 0.03 & 78.27 & 0.109109 & 0.077077 & 9 & 146021041 & 9 & 146997147 & 9 & 146 & 0.044 & 0.704 & 5.22 \\
\hline 1467 & 56527 & 42719 & 35 & 0.06 & 36.18 & 0.137137 & 0.101101 & 9 & 147006399 & 9 & 147992536 & 9 & 147 & 0.019 & 0.6596 & 7.39 \\
\hline 1468 & 42721 & 34292 & 28 & 0.05 & 47.12 & 0.123123 & 0.085085 & 9 & 148001849 & 9 & 148997867 & 9 & 148 & 0.025 & 0.6821 & 6.66 \\
\hline 1469 & 31132 & 24979 & 20 & 0.02 & 83.23 & 0.099099 & 0.054054 & 9 & 149000274 & 9 & 149972040 & 9 & 149 & 0.048 & 0.7045 & 5.02 \\
\hline 1470 & 18814 & 24982 & 18 & 0.06 & 36.77 & 0.096096 & 0.068068 & 9 & 150097891 & 9 & 150980233 & 9 & 150 & 0.02 & 0.6618 & 7.36 \\
\hline 1471 & 49212 & 18200 & 30 & 0.05 & 48.43 & 0.118118 & 0.082082 & 9 & 151005996 & 9 & 151986166 & 9 & 151 & 0.026 & 0.6828 & 6.55 \\
\hline 1472 & 51756 & 8181 & 31 & 0.05 & 40.14 & 0.142142 & 0.094094 & 9 & 152109779 & 9 & 152989337 & 9 & 152 & 0.021 & 0.6714 & 7.18 \\
\hline 1475 & 25009 & 42737 & 19 & 0.03 & 80.14 & 0.076076 & 0.054054 & 10 & 1045768 & 10 & 1994574 & 10 & 1 & 0.045 & 0.7049 & 5.15 \\
\hline 1476 & 25011 & 8213 & 31 & 0.11 & 19.14 & 0.167167 & 0.132132 & 10 & 2015307 & 10 & 2870481 & 10 & 2 & 0.01 & 0.5979 & 9.37 \\
\hline 1477 & 15976 & 25019 & 21 & 0.03 & 71.2 & 0.092092 & 0.061061 & 10 & 3049187 & 10 & 3998062 & 10 & 3 & 0.04 & 0.7007 & 5.47 \\
\hline 1478 & 25018 & 25027 & 27 & 0.07 & 29.71 & 0.128128 & 0.094094 & 10 & 4010500 & 10 & 4939665 & 10 & 4 & 0.016 & 0.6416 & 7.94 \\
\hline 1479 & 51717 & 8238 & 23 & 0.03 & 73.47 & 0.101101 & 0.074074 & 10 & 5008012 & 10 & 5973172 & 10 & 5 & 0.041 & 0.7019 & 5.38 \\
\hline
\end{tabular}




\begin{tabular}{|c|c|c|c|c|c|c|c|c|c|c|c|c|c|c|c|c|}
\hline 1480 & 38424 & 25033 & 14 & 0.04 & 64.02 & 0.067067 & 0.05005 & 10 & 6093540 & 10 & 6990318 & 10 & 6 & 0.035 & 0.6973 & 5.77 \\
\hline 1481 & 25032 & 25038 & 24 & 0.06 & 37.74 & 0.119119 & 0.09009 & 10 & 7002238 & 10 & 7983066 & 10 & 7 & 0.02 & 0.6644 & 7.3 \\
\hline 1482 & 51292 & 25048 & 27 & 0.04 & 61.66 & 0.117117 & 0.071071 & 10 & 8003008 & 10 & 8965689 & 10 & 8 & 0.034 & 0.6964 & 5.9 \\
\hline 1483 & 25047 & 8270 & 27 & 0.04 & 58.5 & 0.138138 & 0.085085 & 10 & 9003663 & 10 & 9970331 & 10 & 9 & 0.032 & 0.6936 & 6.02 \\
\hline 1484 & 8271 & 42757 & 28 & 0.05 & 48.11 & 0.145145 & 0.085085 & 10 & 10002850 & 10 & 10995624 & 10 & 10 & 0.026 & 0.6821 & 6.57 \\
\hline 1485 & 50926 & 25069 & 31 & 0.04 & 50.84 & 0.129129 & 0.085085 & 10 & 11045137 & 10 & 11986190 & 10 & 11 & 0.027 & 0.6873 & 6.46 \\
\hline 1486 & 25070 & 25077 & 29 & 0.03 & 74.89 & 0.145145 & 0.08008 & 10 & 12003897 & 10 & 12991741 & 10 & 12 & 0.042 & 0.7021 & 5.32 \\
\hline 1487 & 8294 & 34771 & 37 & 0.05 & 41.05 & 0.186186 & 0.118118 & 10 & 13042978 & 10 & 13958065 & 10 & 13 & 0.022 & 0.6726 & 7.11 \\
\hline 1488 & 15962 & 31220 & 23 & 0.03 & 79.73 & 0.077077 & 0.049049 & 10 & 14031320 & 10 & 14993971 & 10 & 14 & 0.045 & 0.7048 & 5.16 \\
\hline 1489 & 36031 & 42771 & 26 & 0.06 & 37 & 0.124124 & 0.093093 & 10 & 15042704 & 10 & 15992149 & 10 & 15 & 0.02 & 0.6626 & 7.34 \\
\hline 1490 & 8313 & 8318 & 26 & 0.07 & 30.26 & 0.15015 & 0.101101 & 10 & 16004340 & 10 & 16993029 & 10 & 16 & 0.016 & 0.6422 & 7.86 \\
\hline 1491 & 8319 & 25102 & 24 & 0.04 & 50.62 & 0.105105 & 0.072072 & 10 & 17018794 & 10 & 17948352 & 10 & 17 & 0.027 & 0.6868 & 6.47 \\
\hline 1492 & 25101 & 57818 & 28 & 0.03 & 65.77 & 0.13013 & 0.092092 & 10 & 18001886 & 10 & 18973140 & 10 & 18 & 0.036 & 0.6986 & 5.7 \\
\hline 1493 & 30638 & 45259 & 31 & 0.03 & 66.25 & 0.131131 & 0.088088 & 10 & 19081508 & 10 & 19975279 & 10 & 19 & 0.037 & 0.6988 & 5.68 \\
\hline 1494 & 8338 & 50400 & 24 & 0.04 & 56.84 & 0.111111 & 0.069069 & 10 & 20017052 & 10 & 20952466 & 10 & 20 & 0.031 & 0.6917 & 6.1 \\
\hline 1495 & 17792 & 49237 & 14 & 0.07 & 28.28 & 0.086086 & 0.067067 & 10 & 21021888 & 10 & 21954770 & 10 & 21 & 0.015 & 0.6368 & 8.08 \\
\hline 1496 & 8347 & 56304 & 5 & 0.01 & 94.16 & 0.029029 & 0.018018 & 10 & 22010713 & 10 & 22730977 & 10 & 22 & 0.055 & 0.7084 & 4.7 \\
\hline 1497 & 30190 & 49268 & 14 & 0.02 & 83.66 & 0.067067 & 0.037037 & 10 & 23465107 & 10 & 23970024 & 10 & 23 & 0.048 & 0.7053 & 5.01 \\
\hline 1498 & 8595 & 25116 & 21 & 0.07 & 29.21 & 0.103103 & 0.074074 & 10 & 24011021 & 10 & 24984526 & 10 & 24 & 0.016 & 0.6399 & 7.99 \\
\hline 1499 & 15273 & 25118 & 21 & 0.04 & 60.76 & 0.1001 & 0.066066 & 10 & 25028197 & 10 & 25912932 & 10 & 25 & 0.033 & 0.6958 & 5.93 \\
\hline 1500 & 42785 & 54303 & 19 & 0.02 & 84.05 & 0.085085 & 0.05005 & 10 & 26120414 & 10 & 26980493 & 10 & 26 & 0.048 & 0.7053 & 5 \\
\hline 1501 & 42788 & 54021 & 22 & 0.05 & 49.72 & 0.106106 & 0.077077 & 10 & 27028271 & 10 & 27940273 & 10 & 27 & 0.027 & 0.6858 & 6.51 \\
\hline 1502 & 59887 & 30820 & 17 & 0.03 & 72.69 & 0.081081 & 0.05005 & 10 & 28265646 & 10 & 28963569 & 10 & 28 & 0.041 & 0.7012 & 5.41 \\
\hline 1503 & 25131 & 8374 & 17 & 0.03 & 70.05 & 0.095095 & 0.063063 & 10 & 29045919 & 10 & 29991683 & 10 & 29 & 0.039 & 0.7002 & 5.52 \\
\hline 1504 & 25138 & 17677 & 25 & 0.04 & 58.46 & 0.123123 & 0.081081 & 10 & 30012240 & 10 & 30978174 & 10 & 30 & 0.032 & 0.6934 & 6.03 \\
\hline 1505 & 51971 & 42795 & 21 & 0.02 & 85.79 & 0.089089 & 0.055055 & 10 & 31124017 & 10 & 31983675 & 10 & 31 & 0.049 & 0.7064 & 4.95 \\
\hline 1506 & 8380 & 42798 & 26 & 0.03 & 75.89 & 0.116116 & 0.073073 & 10 & 32008178 & 10 & 32971037 & 10 & 32 & 0.043 & 0.7029 & 5.29 \\
\hline 1507 & 38445 & 25162 & 18 & 0.02 & 91.25 & 0.068068 & 0.039039 & 10 & 33060083 & 10 & 33996942 & 10 & 33 & 0.053 & 0.708 & 4.79 \\
\hline 1508 & 8391 & 25168 & 20 & 0.04 & 63.66 & 0.085085 & 0.063063 & 10 & 34168331 & 10 & 34945662 & 10 & 34 & 0.035 & 0.6971 & 5.79 \\
\hline 1509 & 17080 & 54705 & 14 & 0.02 & 91.79 & 0.056056 & 0.037037 & 10 & 35068814 & 10 & 35912867 & 10 & 35 & 0.053 & 0.7082 & 4.78 \\
\hline 1510 & 45136 & 8399 & 22 & 0.02 & 89.06 & 0.084084 & 0.054054 & 10 & 36057791 & 10 & 36991693 & 10 & 36 & 0.052 & 0.7075 & 4.85 \\
\hline 1511 & 51024 & 59520 & 16 & 0.02 & 91.21 & 0.067067 & 0.046046 & 10 & 37039997 & 10 & 37984810 & 10 & 37 & 0.053 & 0.708 & 4.79 \\
\hline 1512 & 25179 & 8412 & 19 & 0.04 & 56.27 & 0.094094 & 0.054054 & 10 & 38009455 & 10 & 38986300 & 10 & 38 & 0.031 & 0.6918 & 6.14 \\
\hline 1513 & 55805 & 38450 & 17 & 0.09 & 22.06 & 0.112112 & 0.087087 & 10 & 39007437 & 10 & 39667084 & 10 & 39 & 0.011 & 0.6133 & 8.91 \\
\hline 1514 & 60653 & 34736 & 12 & 0.01 & 92.22 & 0.046046 & 0.027027 & 10 & 40186438 & 10 & 40950374 & 10 & 40 & 0.054 & 0.7081 & 4.76 \\
\hline 1515 & 55300 & 60314 & 9 & 0.01 & 93.05 & 0.045045 & 0.024024 & 10 & 41053714 & 10 & 41976069 & 10 & 41 & 0.054 & 0.7081 & 4.73 \\
\hline 1516 & 59400 & 54772 & 16 & 0.03 & 78.59 & 0.061061 & 0.041041 & 10 & 42034590 & 10 & 42921237 & 10 & 42 & 0.044 & 0.704 & 5.2 \\
\hline 1517 & 54523 & 57266 & 22 & 0.06 & 34.18 & 0.1001 & 0.079079 & 10 & 43015513 & 10 & 43858335 & 10 & 43 & 0.018 & 0.6546 & 7.57 \\
\hline 1518 & 25193 & 31354 & 20 & 0.04 & 57.36 & 0.093093 & 0.06006 & 10 & 44144334 & 10 & 44971089 & 10 & 44 & 0.031 & 0.6924 & 6.08 \\
\hline
\end{tabular}




\begin{tabular}{|c|c|c|c|c|c|c|c|c|c|c|c|c|c|c|c|c|}
\hline 1519 & 29913 & 38459 & 16 & 0.02 & 88.2 & 0.078078 & 0.047047 & 10 & 45101309 & 10 & 45903094 & 10 & 45 & 0.051 & 0.7078 & 4.89 \\
\hline 1520 & 25201 & 8447 & 17 & 0.01 & 93.01 & 0.062062 & 0.044044 & 10 & 46084707 & 10 & 46983347 & 10 & 46 & 0.054 & 0.7082 & 4.74 \\
\hline 1521 & 25204 & 25210 & 23 & 0.02 & 85.47 & 0.09009 & 0.056056 & 10 & 47025504 & 10 & 47958278 & 10 & 47 & 0.049 & 0.7063 & 4.96 \\
\hline 1522 & 25211 & 49249 & 33 & 0.04 & 50.08 & 0.138138 & 0.095095 & 10 & 48014763 & 10 & 48960017 & 10 & 48 & 0.027 & 0.6863 & 6.5 \\
\hline 1523 & 38463 & 25228 & 26 & 0.03 & 67.65 & 0.133133 & 0.088088 & 10 & 49042878 & 10 & 49993854 & 10 & 49 & 0.038 & 0.6991 & 5.62 \\
\hline 1524 & 8467 & 42833 & 23 & 0.04 & 58.54 & 0.123123 & 0.08008 & 10 & 50018944 & 10 & 50993659 & 10 & 50 & 0.032 & 0.6935 & 6.02 \\
\hline 1525 & 42834 & 8477 & 26 & 0.05 & 43.47 & 0.131131 & 0.083083 & 10 & 51028164 & 10 & 51975023 & 10 & 51 & 0.023 & 0.678 & 6.97 \\
\hline 1526 & 25241 & 29360 & 29 & 0.06 & 34.73 & 0.156156 & 0.111111 & 10 & 52025204 & 10 & 52991516 & 10 & 52 & 0.018 & 0.6552 & 7.51 \\
\hline 1527 & 29405 & 34983 & 15 & 0.02 & 81.06 & 0.07007 & 0.046046 & 10 & 53037792 & 10 & 53939074 & 10 & 53 & 0.046 & 0.705 & 5.12 \\
\hline 1528 & 61080 & 15089 & 23 & 0.03 & 74.98 & 0.104104 & 0.062062 & 10 & 54096851 & 10 & 54960155 & 10 & 54 & 0.042 & 0.7021 & 5.32 \\
\hline 1529 & 31097 & 25246 & 17 & 0.01 & 93.02 & 0.082082 & 0.047047 & 10 & 55063135 & 10 & 55999312 & 10 & 55 & 0.054 & 0.7081 & 4.73 \\
\hline 1530 & 25247 & 42843 & 31 & 0.04 & 55.17 & 0.155155 & 0.103103 & 10 & 56024865 & 10 & 56998330 & 10 & 56 & 0.03 & 0.6918 & 6.21 \\
\hline 1531 & 8506 & 8509 & 23 & 0.02 & 84.23 & 0.11011 & 0.057057 & 10 & 57044576 & 10 & 57997759 & 10 & 57 & 0.048 & 0.7055 & 4.99 \\
\hline 1532 & 25254 & 32339 & 19 & 0.01 & 93.8 & 0.084084 & 0.038038 & 10 & 58040881 & 10 & 58966663 & 10 & 58 & 0.055 & 0.7082 & 4.71 \\
\hline 1533 & 60045 & 32115 & 30 & 0.06 & 33.75 & 0.139139 & 0.104104 & 10 & 59082877 & 10 & 59954227 & 10 & 59 & 0.018 & 0.6522 & 7.6 \\
\hline 1534 & 53777 & 38465 & 20 & 0.07 & 29.28 & 0.095095 & 0.077077 & 10 & 60034333 & 10 & 60965150 & 10 & 60 & 0.016 & 0.6406 & 7.99 \\
\hline 1535 & 8530 & 58456 & 37 & 0.06 & 33.56 & 0.159159 & 0.109109 & 10 & 61014593 & 10 & 61965891 & 10 & 61 & 0.018 & 0.6514 & 7.61 \\
\hline 1536 & 59203 & 29373 & 31 & 0.04 & 62.46 & 0.143143 & 0.089089 & 10 & 62022807 & 10 & 62985219 & 10 & 62 & 0.034 & 0.6963 & 5.85 \\
\hline 1537 & 56537 & 18536 & 27 & 0.03 & 74.27 & 0.108108 & 0.073073 & 10 & 63088598 & 10 & 63934312 & 10 & 63 & 0.042 & 0.7018 & 5.34 \\
\hline 1538 & 8547 & 42859 & 33 & 0.05 & 45.3 & 0.138138 & 0.087087 & 10 & 64020297 & 10 & 64996997 & 10 & 64 & 0.024 & 0.6811 & 6.83 \\
\hline 1539 & 19076 & 17601 & 29 & 0.04 & 59.62 & 0.12012 & 0.083083 & 10 & 65022553 & 10 & 65996645 & 10 & 65 & 0.033 & 0.695 & 5.98 \\
\hline 1540 & 14859 & 25303 & 26 & 0.13 & 16.87 & 0.146146 & 0.102102 & 10 & 66020622 & 10 & 66971701 & 10 & 66 & 0.009 & 0.5852 & 9.76 \\
\hline 1541 & 8566 & 51447 & 20 & 0.02 & 88.8 & 0.082082 & 0.053053 & 10 & 67023689 & 10 & 67993259 & 10 & 67 & 0.051 & 0.7077 & 4.87 \\
\hline 1542 & 30117 & 42867 & 21 & 0.03 & 79.6 & 0.08008 & 0.047047 & 10 & 68013801 & 10 & 68989822 & 10 & 68 & 0.045 & 0.7048 & 5.17 \\
\hline 1543 & 25307 & 49263 & 44 & 0.05 & 43.27 & 0.16016 & 0.088088 & 10 & 69008968 & 10 & 69985989 & 10 & 69 & 0.023 & 0.678 & 6.99 \\
\hline 1544 & 8576 & 53828 & 33 & 0.06 & 34.24 & 0.158158 & 0.105105 & 10 & 70020888 & 10 & 70904101 & 10 & 70 & 0.018 & 0.655 & 7.57 \\
\hline 1545 & 18323 & 60676 & 13 & 0.03 & 68.65 & 0.058058 & 0.037037 & 10 & 71078073 & 10 & 71713257 & 10 & 71 & 0.038 & 0.6999 & 5.58 \\
\hline 1546 & 39669 & 45537 & 21 & 0.03 & 75.64 & 0.109109 & 0.064064 & 10 & 72248720 & 10 & 72941077 & 10 & 72 & 0.043 & 0.7028 & 5.3 \\
\hline 1547 & 31595 & 25327 & 20 & 0.03 & 72.42 & 0.107107 & 0.065065 & 10 & 73122633 & 10 & 73876295 & 10 & 73 & 0.041 & 0.701 & 5.42 \\
\hline 1548 & 53347 & 51118 & 16 & 0.01 & 94.54 & 0.063063 & 0.042042 & 10 & 74004427 & 10 & 74943049 & 10 & 74 & 0.055 & 0.7084 & 4.68 \\
\hline 1549 & 29461 & 57170 & 12 & 0.01 & 95.68 & 0.053053 & 0.026026 & 10 & 75064259 & 10 & 75940391 & 10 & 75 & 0.056 & 0.7089 & 4.66 \\
\hline 1550 & 58359 & 16921 & 13 & 0.02 & 82.12 & 0.071071 & 0.036036 & 10 & 76156036 & 10 & 76994582 & 10 & 76 & 0.047 & 0.7049 & 5.08 \\
\hline 1551 & 29275 & 31887 & 17 & 0.02 & 81.62 & 0.075075 & 0.054054 & 10 & 77119998 & 10 & 77903646 & 10 & 77 & 0.046 & 0.7052 & 5.1 \\
\hline 1552 & 42880 & 56735 & 23 & 0.03 & 73.98 & 0.085085 & 0.06006 & 11 & 22488 & 11 & 939424 & 11 & 0 & 0.042 & 0.7021 & 5.36 \\
\hline 1553 & 8601 & 25342 & 19 & 0.02 & 86.91 & 0.093093 & 0.048048 & 11 & 1000228 & 11 & 1873897 & 11 & 1 & 0.05 & 0.7074 & 4.93 \\
\hline 1554 & 42889 & 42892 & 19 & 0.02 & 85.52 & 0.089089 & 0.051051 & 11 & 2038539 & 11 & 2964267 & 11 & 2 & 0.049 & 0.7062 & 4.96 \\
\hline 1555 & 61046 & 25349 & 25 & 0.1 & 20.1 & 0.116116 & 0.08008 & 11 & 3251630 & 11 & 3991836 & 11 & 3 & 0.01 & 0.6013 & 9.17 \\
\hline 1556 & 54532 & 8624 & 25 & 3.16 & 3.16 & 0.366366 & 0.34034 & 11 & 4003595 & 11 & 4989840 & 11 & 4 & 0.003 & 0.4577 & 13.97 \\
\hline 1557 & 25354 & 38484 & 24 & 0.04 & 54.71 & 0.107107 & 0.063063 & 11 & 5016353 & 11 & 5987234 & 11 & 5 & 0.03 & 0.6916 & 6.24 \\
\hline
\end{tabular}




\begin{tabular}{|c|c|c|c|c|c|c|c|c|c|c|c|c|c|c|c|c|}
\hline 1558 & 8633 & 57537 & 24 & 0.04 & 62.32 & 0.112112 & 0.071071 & 11 & 6056322 & 11 & 6988731 & 11 & 6 & 0.034 & 0.6964 & 5.86 \\
\hline 1559 & 38486 & 42909 & 25 & 0.16 & 14.27 & 0.133133 & 0.093093 & 11 & 7000946 & 11 & 7976689 & 11 & 7 & 0.007 & 0.5728 & 10.53 \\
\hline 1560 & 47324 & 25377 & 28 & 0.04 & 64.19 & 0.115115 & 0.082082 & 11 & 8012239 & 11 & 8927330 & 11 & 8 & 0.035 & 0.6977 & 5.77 \\
\hline 1561 & 25378 & 47329 & 25 & 0.03 & 70.79 & 0.111111 & 0.049049 & 11 & 9066617 & 11 & 9980144 & 11 & 9 & 0.04 & 0.7004 & 5.48 \\
\hline 1562 & 47331 & 57928 & 17 & 0.02 & 81.01 & 0.083083 & 0.057057 & 11 & 10051760 & 11 & 10801527 & 11 & 10 & 0.046 & 0.705 & 5.12 \\
\hline 1563 & 59349 & 25394 & 25 & 0.02 & 85.11 & 0.1001 & 0.055055 & 11 & 11007618 & 11 & 11987246 & 11 & 11 & 0.049 & 0.7062 & 4.98 \\
\hline 1564 & 25395 & 8680 & 24 & 0.04 & 51.76 & 0.108108 & 0.075075 & 11 & 12012805 & 11 & 12925634 & 11 & 12 & 0.028 & 0.689 & 6.43 \\
\hline 1565 & 25401 & 38511 & 18 & 0.02 & 89.53 & 0.092092 & 0.055055 & 11 & 13033569 & 11 & 13983161 & 11 & 13 & 0.052 & 0.7077 & 4.84 \\
\hline 1566 & 58185 & 8686 & 21 & 0.04 & 53.36 & 0.093093 & 0.066066 & 11 & 14013915 & 11 & 14978831 & 11 & 14 & 0.029 & 0.6916 & 6.35 \\
\hline 1567 & 61641 & 8693 & 21 & 0.02 & 84.74 & 0.1001 & 0.063063 & 11 & 15065868 & 11 & 15982103 & 11 & 15 & 0.049 & 0.7056 & 4.98 \\
\hline 1568 & 25413 & 53591 & 29 & 0.05 & 46.79 & 0.128128 & 0.077077 & 11 & 16046333 & 11 & 16996424 & 11 & 16 & 0.025 & 0.6821 & 6.69 \\
\hline 1569 & 32219 & 52868 & 9 & 0.01 & 95.42 & 0.021021 & 0.013013 & 11 & 17133516 & 11 & 17982157 & 11 & 17 & 0.056 & 0.7087 & 4.66 \\
\hline 1570 & 8708 & 47337 & 17 & 0.02 & 86.5 & 0.067067 & 0.035035 & 11 & 18175690 & 11 & 18868868 & 11 & 18 & 0.05 & 0.7069 & 4.94 \\
\hline 1571 & 32103 & 31549 & 18 & 0.03 & 78.01 & 0.089089 & 0.055055 & 11 & 19306043 & 11 & 19998464 & 11 & 19 & 0.044 & 0.7039 & 5.22 \\
\hline 1572 & 35913 & 25432 & 34 & 0.05 & 47.41 & 0.125125 & 0.083083 & 11 & 20016772 & 11 & 20989421 & 11 & 20 & 0.026 & 0.6823 & 6.64 \\
\hline 1573 & 35946 & 57717 & 23 & 0.03 & 66.35 & 0.084084 & 0.065065 & 11 & 21011462 & 11 & 21968682 & 11 & 21 & 0.037 & 0.699 & 5.68 \\
\hline 1574 & 8741 & 8745 & 22 & 0.02 & 84.85 & 0.102102 & 0.054054 & 11 & 22013261 & 11 & 22990483 & 11 & 22 & 0.049 & 0.7059 & 4.98 \\
\hline 1575 & 47339 & 25448 & 30 & 0.05 & 40.52 & 0.13013 & 0.101101 & 11 & 23031513 & 11 & 23991998 & 11 & 23 & 0.021 & 0.6716 & 7.14 \\
\hline 1576 & 8755 & 8764 & 30 & 0.05 & 49.17 & 0.127127 & 0.088088 & 11 & 24007939 & 11 & 24974538 & 11 & 24 & 0.027 & 0.6846 & 6.54 \\
\hline 1577 & 8768 & 34020 & 35 & 0.08 & 25.95 & 0.167167 & 0.131131 & 11 & 25001589 & 11 & 25970861 & 11 & 25 & 0.014 & 0.6295 & 8.36 \\
\hline 1578 & 19431 & 42965 & 27 & 0.05 & 44.86 & 0.124124 & 0.082082 & 11 & 26022055 & 11 & 26971685 & 11 & 26 & 0.024 & 0.6797 & 6.84 \\
\hline 1579 & 42964 & 54130 & 25 & 0.04 & 52.59 & 0.113113 & 0.076076 & 11 & 27007918 & 11 & 27954931 & 11 & 27 & 0.028 & 0.6909 & 6.39 \\
\hline 1580 & 42962 & 8784 & 22 & 0.03 & 80.51 & 0.084084 & 0.053053 & 11 & 28019183 & 11 & 28992353 & 11 & 28 & 0.046 & 0.7051 & 5.14 \\
\hline 1581 & 8783 & 25476 & 14 & 0.01 & 95.3 & 0.052052 & 0.026026 & 11 & 29014105 & 11 & 29994790 & 11 & 29 & 0.056 & 0.7086 & 4.67 \\
\hline 1582 & 8802 & 61598 & 10 & 0.01 & 94.88 & 0.042042 & 0.018018 & 11 & 30218369 & 11 & 30982805 & 11 & 30 & 0.056 & 0.7085 & 4.68 \\
\hline 1583 & 8805 & 8808 & 16 & 0.01 & 92.87 & 0.063063 & 0.039039 & 11 & 31018065 & 11 & 31989556 & 11 & 31 & 0.054 & 0.7081 & 4.74 \\
\hline 1584 & 38540 & 47347 & 19 & 0.01 & 92.15 & 0.092092 & 0.056056 & 11 & 32013457 & 11 & 32913253 & 11 & 32 & 0.054 & 0.7081 & 4.77 \\
\hline 1585 & 38547 & 8820 & 12 & 0.01 & 95.41 & 0.049049 & 0.024024 & 11 & 33006254 & 11 & 33976329 & 11 & 33 & 0.056 & 0.7087 & 4.66 \\
\hline 1586 & 8822 & 47348 & 14 & 0.01 & 93.55 & 0.057057 & 0.034034 & 11 & 34013189 & 11 & 34890371 & 11 & 34 & 0.055 & 0.7082 & 4.71 \\
\hline 1587 & 25483 & 50807 & 13 & 0 & 96.24 & 0.048048 & 0.021021 & 11 & 35006617 & 11 & 35896772 & 11 & 35 & 0.057 & 0.7091 & 4.64 \\
\hline 1588 & 42978 & 42980 & 11 & 0.01 & 93.92 & 0.051051 & 0.026026 & 11 & 36037488 & 11 & 36836674 & 11 & 36 & 0.055 & 0.7083 & 4.7 \\
\hline 1589 & 8834 & 18833 & 9 & 0.01 & 95.88 & 0.031031 & 0.025025 & 11 & 37109307 & 11 & 37805603 & 11 & 37 & 0.056 & 0.7088 & 4.65 \\
\hline 1590 & 47350 & 8837 & 3 & 0 & 96.36 & 0.019019 & 0.008008 & 11 & 38785701 & 11 & 38996126 & 11 & 38 & 0.057 & 0.7091 & 4.64 \\
\hline 1591 & 25488 & 8841 & 10 & 0.02 & 86.95 & 0.046046 & 0.031031 & 11 & 39122368 & 11 & 39964997 & 11 & 39 & 0.05 & 0.7074 & 4.93 \\
\hline 1592 & 59345 & 8847 & 13 & 0.02 & 89.04 & 0.057057 & 0.042042 & 11 & 40013884 & 11 & 40982195 & 11 & 40 & 0.052 & 0.7075 & 4.85 \\
\hline 1593 & 38556 & 25494 & 17 & 0.02 & 81.43 & 0.065065 & 0.042042 & 11 & 41062108 & 11 & 41970836 & 11 & 41 & 0.046 & 0.7052 & 5.1 \\
\hline 1594 & 14540 & 30172 & 13 & 0.01 & 92.94 & 0.059059 & 0.039039 & 11 & 42043634 & 11 & 42953643 & 11 & 42 & 0.054 & 0.7081 & 4.74 \\
\hline 1595 & 8852 & 54236 & 22 & 0.04 & 64.33 & 0.091091 & 0.068068 & 11 & 43085921 & 11 & 43951738 & 11 & 43 & 0.036 & 0.6977 & 5.76 \\
\hline 1596 & 8861 & 8865 & 9 & 0.01 & 95.46 & 0.037037 & 0.024024 & 11 & 44023659 & 11 & 44981824 & 11 & 44 & 0.056 & 0.7087 & 4.66 \\
\hline
\end{tabular}




\begin{tabular}{|c|c|c|c|c|c|c|c|c|c|c|c|c|c|c|c|c|}
\hline 1597 & 25501 & 38578 & 15 & 0.02 & 82.22 & 0.085085 & 0.052052 & 11 & 45049289 & 11 & 45992991 & 11 & 45 & 0.047 & 0.7049 & 5.07 \\
\hline 1598 & 8870 & 15264 & 22 & 0.01 & 93.6 & 0.086086 & 0.038038 & 11 & 46050226 & 11 & 46997995 & 11 & 46 & 0.055 & 0.7081 & 4.71 \\
\hline 1599 & 16106 & 19188 & 6 & 0.01 & 95.19 & 0.029029 & 0.018018 & 11 & 47014747 & 11 & 47907047 & 11 & 47 & 0.056 & 0.7086 & 4.67 \\
\hline 1600 & 8877 & 59105 & 26 & 0.02 & 84.16 & 0.115115 & 0.068068 & 11 & 48014416 & 11 & 48937000 & 11 & 48 & 0.048 & 0.7053 & 4.99 \\
\hline 1601 & 57979 & 47361 & 18 & 0.01 & 95.51 & 0.072072 & 0.036036 & 11 & 49121567 & 11 & 49990774 & 11 & 49 & 0.056 & 0.7087 & 4.66 \\
\hline 1602 & 25511 & 8897 & 20 & 0.05 & 45.5 & 0.103103 & 0.076076 & 11 & 50012027 & 11 & 50973313 & 11 & 50 & 0.024 & 0.6812 & 6.82 \\
\hline 1603 & 8898 & 58640 & 30 & 0.04 & 59.82 & 0.138138 & 0.074074 & 11 & 51024403 & 11 & 51986201 & 11 & 51 & 0.033 & 0.6953 & 5.98 \\
\hline 1604 & 61681 & 25521 & 22 & 0.06 & 31.97 & 0.099099 & 0.068068 & 11 & 52010601 & 11 & 52969628 & 11 & 52 & 0.017 & 0.6481 & 7.75 \\
\hline 1605 & 8909 & 47371 & 26 & 0.02 & 85.37 & 0.099099 & 0.052052 & 11 & 53000238 & 11 & 53920963 & 11 & 53 & 0.049 & 0.7062 & 4.97 \\
\hline 1606 & 47372 & 38593 & 22 & 0.02 & 84.38 & 0.088088 & 0.045045 & 11 & 54006230 & 11 & 54954986 & 11 & 54 & 0.049 & 0.7055 & 4.99 \\
\hline 1607 & 8927 & 38594 & 24 & 0.02 & 84.78 & 0.098098 & 0.053053 & 11 & 55001359 & 11 & 55975611 & 11 & 55 & 0.049 & 0.7057 & 4.98 \\
\hline 1608 & 25529 & 8944 & 27 & 0.03 & 69.5 & 0.117117 & 0.075075 & 11 & 56034371 & 11 & 56992066 & 11 & 56 & 0.039 & 0.6997 & 5.54 \\
\hline 1609 & 43009 & 38598 & 16 & 0.02 & 81.33 & 0.074074 & 0.053053 & 11 & 57041894 & 11 & 57992782 & 11 & 57 & 0.046 & 0.7053 & 5.11 \\
\hline 1610 & 47386 & 8956 & 11 & 0.02 & 85.58 & 0.058058 & 0.044044 & 11 & 58007243 & 11 & 58988824 & 11 & 58 & 0.049 & 0.7062 & 4.96 \\
\hline 1611 & 47388 & 8964 & 15 & 0.02 & 86.97 & 0.067067 & 0.035035 & 11 & 59012574 & 11 & 59978485 & 11 & 59 & 0.05 & 0.7076 & 4.93 \\
\hline 1612 & 47390 & 8973 & 18 & 0.03 & 74.48 & 0.086086 & 0.047047 & 11 & 60026383 & 11 & 60976002 & 11 & 60 & 0.042 & 0.7021 & 5.34 \\
\hline 1613 & 8974 & 8978 & 11 & 0.01 & 94.91 & 0.046046 & 0.029029 & 11 & 61033874 & 11 & 61996760 & 11 & 61 & 0.056 & 0.7087 & 4.68 \\
\hline 1614 & 49307 & 53735 & 16 & 0.03 & 78.06 & 0.08008 & 0.045045 & 11 & 62019748 & 11 & 62976117 & 11 & 62 & 0.044 & 0.7041 & 5.23 \\
\hline 1615 & 38602 & 47398 & 13 & 0.01 & 94.87 & 0.055055 & 0.03003 & 11 & 63178157 & 11 & 63978325 & 11 & 63 & 0.056 & 0.7085 & 4.68 \\
\hline 1616 & 8997 & 25559 & 17 & 0.06 & 38.76 & 0.071071 & 0.057057 & 11 & 64018740 & 11 & 64995107 & 11 & 64 & 0.021 & 0.6675 & 7.25 \\
\hline 1617 & 9002 & 25562 & 18 & 0.02 & 83 & 0.082082 & 0.045045 & 11 & 65022245 & 11 & 65973607 & 11 & 65 & 0.048 & 0.7045 & 5.03 \\
\hline 1618 & 9013 & 9025 & 32 & 0.06 & 37.63 & 0.166166 & 0.099099 & 11 & 66014418 & 11 & 66991408 & 11 & 66 & 0.02 & 0.664 & 7.3 \\
\hline 1619 & 25566 & 9037 & 28 & 0.04 & 59.59 & 0.133133 & 0.087087 & 11 & 67039090 & 11 & 67975596 & 11 & 67 & 0.033 & 0.6946 & 5.98 \\
\hline 1620 & 9035 & 25582 & 34 & 0.23 & 10.83 & 0.206206 & 0.159159 & 11 & 68089906 & 11 & 68982930 & 11 & 68 & 0.006 & 0.5442 & 11.34 \\
\hline 1621 & 25581 & 9054 & 22 & 0.02 & 83.89 & 0.075075 & 0.052052 & 11 & 69005136 & 11 & 69986366 & 11 & 69 & 0.048 & 0.7053 & 5.01 \\
\hline 1622 & 25588 & 51088 & 20 & 0.02 & 83.48 & 0.092092 & 0.055055 & 11 & 70044668 & 11 & 70967934 & 11 & 70 & 0.048 & 0.705 & 5.02 \\
\hline 1623 & 61017 & 25595 & 12 & 0.02 & 87.39 & 0.066066 & 0.039039 & 11 & 71130475 & 11 & 71993140 & 11 & 71 & 0.05 & 0.7077 & 4.92 \\
\hline 1624 & 43040 & 9077 & 26 & 0.04 & 52.89 & 0.129129 & 0.087087 & 11 & 72031850 & 11 & 72966366 & 11 & 72 & 0.028 & 0.6914 & 6.38 \\
\hline 1625 & 14740 & 43042 & 38 & 0.11 & 19.68 & 0.191191 & 0.132132 & 11 & 73002067 & 11 & 73978500 & 11 & 73 & 0.01 & 0.601 & 9.29 \\
\hline 1626 & 43043 & 43047 & 33 & 0.04 & 54.46 & 0.131131 & 0.089089 & 11 & 74019999 & 11 & 74978970 & 11 & 74 & 0.03 & 0.6917 & 6.26 \\
\hline 1627 & 25614 & 36383 & 32 & 0.03 & 70.95 & 0.156156 & 0.097097 & 11 & 75009361 & 11 & 75996081 & 11 & 75 & 0.04 & 0.7004 & 5.48 \\
\hline 1628 & 9093 & 43057 & 29 & 0.05 & 40.89 & 0.136136 & 0.093093 & 11 & 76044951 & 11 & 76999888 & 11 & 76 & 0.022 & 0.6722 & 7.11 \\
\hline 1629 & 25626 & 25633 & 36 & 0.03 & 72.63 & 0.138138 & 0.075075 & 11 & 77015017 & 11 & 77964900 & 11 & 77 & 0.041 & 0.701 & 5.41 \\
\hline 1630 & 25634 & 47412 & 29 & 0.03 & 70.02 & 0.131131 & 0.092092 & 11 & 78000180 & 11 & 78952414 & 11 & 78 & 0.039 & 0.7001 & 5.52 \\
\hline 1631 & 9121 & 59190 & 26 & 0.02 & 85.22 & 0.115115 & 0.078078 & 11 & 79016735 & 11 & 79920806 & 11 & 79 & 0.049 & 0.7061 & 4.97 \\
\hline 1632 & 25641 & 9142 & 31 & 0.04 & 51.02 & 0.136136 & 0.093093 & 11 & 80007010 & 11 & 80996526 & 11 & 80 & 0.028 & 0.6878 & 6.46 \\
\hline 1633 & 38630 & 25654 & 34 & 0.06 & 39.54 & 0.164164 & 0.113113 & 11 & 81016745 & 11 & 81997858 & 11 & 81 & 0.021 & 0.6692 & 7.19 \\
\hline 1634 & 34637 & 9166 & 28 & 0.04 & 51.28 & 0.131131 & 0.099099 & 11 & 82019810 & 11 & 82972974 & 11 & 82 & 0.028 & 0.6887 & 6.45 \\
\hline 1635 & 25663 & 60061 & 26 & 0.09 & 21.97 & 0.118118 & 0.075075 & 11 & 83011472 & 11 & 83976808 & 11 & 83 & 0.011 & 0.6129 & 8.94 \\
\hline
\end{tabular}




\begin{tabular}{|c|c|c|c|c|c|c|c|c|c|c|c|c|c|c|c|c|}
\hline 1636 & 49330 & 25681 & 24 & 0.12 & 17.63 & 0.16016 & 0.134134 & 11 & 84167373 & 11 & 84977439 & 11 & 84 & 0.009 & 0.5915 & 9.68 \\
\hline 1637 & 25682 & 9186 & 21 & 0.04 & 59.01 & 0.096096 & 0.066066 & 11 & 85016603 & 11 & 85932032 & 11 & 85 & 0.032 & 0.6941 & 6.01 \\
\hline 1638 & 15653 & 32721 & 7 & 0.01 & 94.92 & 0.039039 & 0.025025 & 11 & 86035052 & 11 & 86676975 & 11 & 86 & 0.056 & 0.7087 & 4.68 \\
\hline 1639 & 32281 & 49849 & 14 & 0.03 & 71.01 & 0.072072 & 0.046046 & 11 & 87115792 & 11 & 87660102 & 11 & 87 & 0.04 & 0.7005 & 5.48 \\
\hline 1640 & 45525 & 50520 & 21 & 0.14 & 15.65 & 0.137137 & 0.112112 & 12 & 79502 & 12 & 980500 & 12 & 0 & 0.008 & 0.5775 & 10.02 \\
\hline 1641 & 29706 & 49340 & 16 & 0.16 & 14.43 & 0.131131 & 0.105105 & 12 & 1169606 & 12 & 1998701 & 12 & 1 & 0.007 & 0.5725 & 10.44 \\
\hline 1642 & 43103 & 30214 & 25 & 0.14 & 16.06 & 0.139139 & 0.107107 & 12 & 2065310 & 12 & 2979580 & 12 & 2 & 0.008 & 0.5809 & 9.95 \\
\hline 1643 & 18062 & 31826 & 17 & 0.04 & 51.19 & 0.084084 & 0.06006 & 12 & 3097741 & 12 & 3928785 & 12 & 3 & 0.028 & 0.6884 & 6.45 \\
\hline 1644 & 43107 & 9196 & 20 & 0.03 & 74.71 & 0.081081 & 0.052052 & 12 & 4010569 & 12 & 4992763 & 12 & 4 & 0.042 & 0.702 & 5.33 \\
\hline 1645 & 9197 & 61714 & 16 & 0.01 & 93.56 & 0.063063 & 0.032032 & 12 & 5026781 & 12 & 5900678 & 12 & 5 & 0.055 & 0.7083 & 4.71 \\
\hline 1646 & 48323 & 29730 & 39 & 0.07 & 30.66 & 0.185185 & 0.136136 & 12 & 6120038 & 12 & 6998897 & 12 & 6 & 0.016 & 0.6453 & 7.87 \\
\hline 1647 & 58634 & 33422 & 30 & 0.02 & 82.01 & 0.105105 & 0.068068 & 12 & 7009507 & 12 & 7976640 & 12 & 7 & 0.047 & 0.7051 & 5.08 \\
\hline 1648 & 25713 & 25719 & 40 & 0.04 & 50.35 & 0.144144 & 0.093093 & 12 & 8013844 & 12 & 8987734 & 12 & 8 & 0.027 & 0.6865 & 6.48 \\
\hline 1649 & 9211 & 53205 & 28 & 0.06 & 35.34 & 0.126126 & 0.1001 & 12 & 9004984 & 12 & 9992535 & 12 & 9 & 0.019 & 0.6561 & 7.43 \\
\hline 1650 & 25726 & 53234 & 23 & 0.03 & 73.08 & 0.093093 & 0.06006 & 12 & 10017988 & 12 & 10938363 & 12 & 10 & 0.041 & 0.7016 & 5.39 \\
\hline 1651 & 43131 & 56868 & 22 & 0.03 & 69.6 & 0.094094 & 0.057057 & 12 & 11015651 & 12 & 11918052 & 12 & 11 & 0.039 & 0.6997 & 5.53 \\
\hline 1652 & 25739 & 38637 & 25 & 0.05 & 49.58 & 0.116116 & 0.083083 & 12 & 12040820 & 12 & 12957003 & 12 & 12 & 0.027 & 0.6854 & 6.52 \\
\hline 1653 & 25745 & 45545 & 33 & 0.04 & 56.64 & 0.138138 & 0.097097 & 12 & 13007194 & 12 & 13901807 & 12 & 13 & 0.031 & 0.6916 & 6.11 \\
\hline 1654 & 61557 & 49358 & 30 & 0.06 & 35.94 & 0.132132 & 0.098098 & 12 & 14013189 & 12 & 14984702 & 12 & 14 & 0.019 & 0.6586 & 7.41 \\
\hline 1655 & 29638 & 9253 & 32 & 0.04 & 62.25 & 0.138138 & 0.1001 & 12 & 15000817 & 12 & 15974791 & 12 & 15 & 0.034 & 0.6963 & 5.86 \\
\hline 1656 & 61513 & 60787 & 23 & 0.05 & 43.37 & 0.111111 & 0.063063 & 12 & 16006733 & 12 & 16925639 & 12 & 16 & 0.023 & 0.6781 & 6.98 \\
\hline 1657 & 9262 & 17231 & 24 & 0.1 & 21.21 & 0.138138 & 0.102102 & 12 & 17081939 & 12 & 17946048 & 12 & 17 & 0.011 & 0.6081 & 9.03 \\
\hline 1658 & 50249 & 54233 & 30 & 0.06 & 35.16 & 0.131131 & 0.092092 & 12 & 18147178 & 12 & 18890652 & 12 & 18 & 0.019 & 0.656 & 7.45 \\
\hline 1659 & 25772 & 53295 & 25 & 0.06 & 35.64 & 0.126126 & 0.089089 & 12 & 19195079 & 12 & 19884388 & 12 & 19 & 0.019 & 0.657 & 7.41 \\
\hline 1660 & 18588 & 43151 & 15 & 0.02 & 86.38 & 0.059059 & 0.041041 & 12 & 20135020 & 12 & 20987983 & 12 & 20 & 0.05 & 0.707 & 4.94 \\
\hline 1661 & 38645 & 38646 & 21 & 0.03 & 73.77 & 0.104104 & 0.065065 & 12 & 21017764 & 12 & 21955485 & 12 & 21 & 0.042 & 0.7021 & 5.37 \\
\hline 1662 & 53388 & 56697 & 6 & 0 & 96.27 & 0.021021 & 0.014014 & 12 & 22051275 & 12 & 22890196 & 12 & 22 & 0.057 & 0.7091 & 4.64 \\
\hline 1663 & 52557 & 55255 & 23 & 0.03 & 78.17 & 0.079079 & 0.052052 & 12 & 23012589 & 12 & 23998144 & 12 & 23 & 0.044 & 0.704 & 5.22 \\
\hline 1664 & 25781 & 45651 & 32 & 0.03 & 67.18 & 0.124124 & 0.088088 & 12 & 24025660 & 12 & 24979525 & 12 & 24 & 0.037 & 0.6992 & 5.64 \\
\hline 1665 & 29997 & 9297 & 24 & 0.06 & 39.15 & 0.118118 & 0.079079 & 12 & 25006232 & 12 & 25983929 & 12 & 25 & 0.021 & 0.6686 & 7.23 \\
\hline 1666 & 55925 & 43162 & 27 & 0.05 & 49.45 & 0.123123 & 0.082082 & 12 & 26022727 & 12 & 26969612 & 12 & 26 & 0.027 & 0.6848 & 6.52 \\
\hline 1667 & 9300 & 25792 & 32 & 0.05 & 47.5 & 0.131131 & 0.089089 & 12 & 27013251 & 12 & 27972588 & 12 & 27 & 0.026 & 0.6822 & 6.63 \\
\hline 1668 & 43163 & 14943 & 19 & 0.03 & 76.42 & 0.088088 & 0.065065 & 12 & 28071542 & 12 & 28779167 & 12 & 28 & 0.043 & 0.7027 & 5.27 \\
\hline 1669 & 34952 & 17691 & 9 & 0.01 & 92 & 0.039039 & 0.026026 & 12 & 29022063 & 12 & 29700187 & 12 & 29 & 0.053 & 0.7082 & 4.77 \\
\hline 1670 & 59231 & 9320 & 8 & 0.01 & 95.99 & 0.031031 & 0.021021 & 12 & 30149881 & 12 & 30998011 & 12 & 30 & 0.056 & 0.709 & 4.65 \\
\hline 1671 & 55472 & 51029 & 17 & 0.03 & 74.77 & 0.06006 & 0.041041 & 12 & 31037865 & 12 & 31975933 & 12 & 31 & 0.042 & 0.7019 & 5.32 \\
\hline 1672 & 35905 & 25804 & 30 & 0.03 & 77.63 & 0.129129 & 0.066066 & 12 & 32009788 & 12 & 32777362 & 12 & 32 & 0.044 & 0.7036 & 5.23 \\
\hline 1673 & 9330 & 33165 & 22 & 0.02 & 84.09 & 0.084084 & 0.051051 & 12 & 33064079 & 12 & 33982636 & 12 & 33 & 0.048 & 0.7053 & 4.99 \\
\hline 1674 & 32709 & 43177 & 41 & 0.1 & 21.11 & 0.198198 & 0.128128 & 12 & 34002369 & 12 & 34995000 & 12 & 34 & 0.011 & 0.6069 & 9.03 \\
\hline
\end{tabular}




\begin{tabular}{|c|c|c|c|c|c|c|c|c|c|c|c|c|c|c|c|c|}
\hline 1675 & 25819 & 9350 & 12 & 0.05 & 48.29 & 0.054054 & 0.042042 & 12 & 35017301 & 12 & 35990853 & 12 & 35 & 0.026 & 0.6825 & 6.55 \\
\hline 1676 & 25826 & 31535 & 27 & 0.04 & 53.83 & 0.119119 & 0.081081 & 12 & 36015100 & 12 & 36829763 & 12 & 36 & 0.029 & 0.6913 & 6.3 \\
\hline 1677 & 52789 & 25830 & 18 & 0.02 & 88.37 & 0.083083 & 0.046046 & 12 & 37005248 & 12 & 37778827 & 12 & 37 & 0.051 & 0.7076 & 4.88 \\
\hline 1678 & 9361 & 60754 & 13 & 0.01 & 92.08 & 0.059059 & 0.032032 & 12 & 38004200 & 12 & 38706000 & 12 & 38 & 0.054 & 0.708 & 4.77 \\
\hline 1679 & 16604 & 30363 & 25 & 0.23 & 11.06 & 0.158158 & 0.121121 & 12 & 39000847 & 12 & 39992894 & 12 & 39 & 0.006 & 0.5477 & 11.31 \\
\hline 1680 & 54822 & 25837 & 16 & 0.1 & 21.59 & 0.1001 & 0.072072 & 12 & 40019047 & 12 & 40544362 & 12 & 40 & 0.011 & 0.6116 & 9.01 \\
\hline 1681 & 51978 & 18544 & 27 & 0.05 & 42.71 & 0.112112 & 0.076076 & 12 & 41032283 & 12 & 41951866 & 12 & 41 & 0.023 & 0.6763 & 7.01 \\
\hline 1682 & 16358 & 50592 & 45 & 0.09 & 23.59 & 0.188188 & 0.135135 & 12 & 42035618 & 12 & 42984312 & 12 & 42 & 0.012 & 0.6228 & 8.77 \\
\hline 1683 & 43188 & 58317 & 22 & 0.05 & 44.72 & 0.111111 & 0.086086 & 12 & 43068568 & 12 & 43987384 & 12 & 43 & 0.024 & 0.6797 & 6.86 \\
\hline 1684 & 43190 & 9384 & 21 & 0.04 & 61.77 & 0.094094 & 0.061061 & 12 & 44080140 & 12 & 44971304 & 12 & 44 & 0.034 & 0.6966 & 5.9 \\
\hline 1685 & 25858 & 16781 & 23 & 0.04 & 58.11 & 0.111111 & 0.069069 & 12 & 45034446 & 12 & 45985058 & 12 & 45 & 0.032 & 0.6932 & 6.04 \\
\hline 1686 & 14877 & 54189 & 25 & 0.08 & 25.08 & 0.131131 & 0.097097 & 12 & 46023473 & 12 & 46979274 & 12 & 46 & 0.013 & 0.6269 & 8.51 \\
\hline 1687 & 30484 & 43197 & 25 & 0.06 & 38.31 & 0.108108 & 0.075075 & 12 & 47002609 & 12 & 47948986 & 12 & 47 & 0.02 & 0.6664 & 7.27 \\
\hline 1688 & 38667 & 33231 & 16 & 0.03 & 79.32 & 0.052052 & 0.031031 & 12 & 48001470 & 12 & 48863354 & 12 & 48 & 0.045 & 0.7047 & 5.18 \\
\hline 1689 & 15974 & 43198 & 16 & 0.02 & 87.91 & 0.088088 & 0.058058 & 12 & 49085493 & 12 & 49990871 & 12 & 49 & 0.051 & 0.7078 & 4.9 \\
\hline 1690 & 9402 & 25876 & 23 & 0.02 & 86.03 & 0.083083 & 0.051051 & 12 & 50043147 & 12 & 50907615 & 12 & 50 & 0.049 & 0.7066 & 4.95 \\
\hline 1691 & 9413 & 9414 & 17 & 0.03 & 71.98 & 0.077077 & 0.053053 & 12 & 51190108 & 12 & 51984256 & 12 & 51 & 0.04 & 0.7012 & 5.44 \\
\hline 1692 & 9416 & 43212 & 30 & 0.06 & 32.68 & 0.16016 & 0.107107 & 12 & 52006043 & 12 & 52989009 & 12 & 52 & 0.017 & 0.6502 & 7.71 \\
\hline 1693 & 9419 & 32021 & 25 & 0.03 & 69.04 & 0.102102 & 0.075075 & 12 & 53007660 & 12 & 53999623 & 12 & 53 & 0.038 & 0.7 & 5.56 \\
\hline 1694 & 53957 & 9421 & 13 & 0.02 & 88.87 & 0.058058 & 0.041041 & 12 & 54003633 & 12 & 54837708 & 12 & 54 & 0.051 & 0.7076 & 4.86 \\
\hline 1695 & 54085 & 35831 & 20 & 0.01 & 93.85 & 0.08008 & 0.053053 & 12 & 55002053 & 12 & 55962023 & 12 & 55 & 0.055 & 0.7083 & 4.7 \\
\hline 1696 & 15978 & 49389 & 23 & 0.02 & 89.09 & 0.096096 & 0.056056 & 12 & 56000017 & 12 & 56868845 & 12 & 56 & 0.052 & 0.7076 & 4.85 \\
\hline 1697 & 14997 & 9441 & 32 & 0.04 & 54.5 & 0.131131 & 0.089089 & 12 & 57002739 & 12 & 57950908 & 12 & 57 & 0.03 & 0.6917 & 6.26 \\
\hline 1698 & 43220 & 33169 & 26 & 0.03 & 65.66 & 0.102102 & 0.068068 & 12 & 58042164 & 12 & 58992046 & 12 & 58 & 0.036 & 0.6985 & 5.7 \\
\hline 1699 & 52072 & 14562 & 30 & 0.05 & 49.08 & 0.139139 & 0.096096 & 12 & 59032217 & 12 & 59982065 & 12 & 59 & 0.027 & 0.6845 & 6.54 \\
\hline 1700 & 14464 & 14590 & 48 & 0.09 & 22.89 & 0.204204 & 0.14014 & 12 & 60002492 & 12 & 60982548 & 12 & 60 & 0.012 & 0.6183 & 8.84 \\
\hline 1701 & 31005 & 45198 & 31 & 0.02 & 81.77 & 0.117117 & 0.074074 & 12 & 61042519 & 12 & 61949547 & 12 & 61 & 0.047 & 0.7052 & 5.09 \\
\hline 1702 & 55843 & 33509 & 34 & 0.11 & 19.79 & 0.189189 & 0.135135 & 12 & 62026594 & 12 & 62962634 & 12 & 62 & 0.01 & 0.6012 & 9.27 \\
\hline 1703 & 9455 & 52184 & 13 & 0.02 & 87.73 & 0.071071 & 0.044044 & 12 & 63058856 & 12 & 63499825 & 12 & 63 & 0.051 & 0.7077 & 4.9 \\
\hline 1704 & 53457 & 38679 & 22 & 0.02 & 85.9 & 0.105105 & 0.06006 & 13 & 88939 & 13 & 978869 & 13 & 0 & 0.049 & 0.7066 & 4.95 \\
\hline 1705 & 43234 & 25916 & 23 & 0.02 & 88.74 & 0.089089 & 0.047047 & 13 & 1022508 & 13 & 1982864 & 13 & 1 & 0.051 & 0.7077 & 4.87 \\
\hline 1706 & 9470 & 43237 & 15 & 0.01 & 94.55 & 0.042042 & 0.023023 & 13 & 2049440 & 13 & 2947636 & 13 & 2 & 0.055 & 0.7084 & 4.68 \\
\hline 1707 & 49399 & 16338 & 31 & 0.04 & 64.05 & 0.133133 & 0.092092 & 13 & 3072331 & 13 & 3982402 & 13 & 3 & 0.035 & 0.6974 & 5.77 \\
\hline 1708 & 33588 & 25928 & 20 & 0.03 & 75.46 & 0.082082 & 0.058058 & 13 & 4023767 & 13 & 4922900 & 13 & 4 & 0.043 & 0.7022 & 5.3 \\
\hline 1709 & 47431 & 25934 & 18 & 0.05 & 41.32 & 0.099099 & 0.066066 & 13 & 5013989 & 13 & 5963268 & 13 & 5 & 0.022 & 0.6737 & 7.1 \\
\hline 1710 & 9490 & 9504 & 28 & 0.04 & 57.16 & 0.121121 & 0.076076 & 13 & 6000732 & 13 & 6978113 & 13 & 6 & 0.031 & 0.6924 & 6.09 \\
\hline 1711 & 9505 & 50269 & 29 & 0.06 & 34.43 & 0.134134 & 0.088088 & 13 & 7091760 & 13 & 7981207 & 13 & 7 & 0.018 & 0.6561 & 7.57 \\
\hline 1712 & 60854 & 38692 & 22 & 0.04 & 57.91 & 0.092092 & 0.059059 & 13 & 8050547 & 13 & 8963066 & 13 & 8 & 0.032 & 0.6928 & 6.05 \\
\hline 1713 & 51119 & 25952 & 26 & 0.04 & 63.15 & 0.11011 & 0.067067 & 13 & 9056247 & 13 & 9889880 & 13 & 9 & 0.035 & 0.6966 & 5.81 \\
\hline
\end{tabular}




\begin{tabular}{|c|c|c|c|c|c|c|c|c|c|c|c|c|c|c|c|c|}
\hline 1714 & 43249 & 17565 & 16 & 0.01 & 95.07 & 0.066066 & 0.033033 & 13 & 10009490 & 13 & 10819124 & 13 & 10 & 0.056 & 0.7087 & 4.67 \\
\hline 1715 & 59175 & 45146 & 25 & 0.02 & 83.57 & 0.108108 & 0.063063 & 13 & 11042615 & 13 & 11949152 & 13 & 11 & 0.048 & 0.7051 & 5.02 \\
\hline 1716 & 45087 & 9550 & 38 & 0.04 & 56.6 & 0.159159 & 0.108108 & 13 & 12004442 & 13 & 12986957 & 13 & 12 & 0.031 & 0.6916 & 6.11 \\
\hline 1717 & 52035 & 17912 & 28 & 0.05 & 48.94 & 0.132132 & 0.086086 & 13 & 13026095 & 13 & 13971542 & 13 & 13 & 0.027 & 0.684 & 6.54 \\
\hline 1718 & 14782 & 9567 & 38 & 0.05 & 41.47 & 0.154154 & 0.105105 & 13 & 14054487 & 13 & 14975251 & 13 & 14 & 0.022 & 0.6741 & 7.1 \\
\hline 1719 & 43256 & 25981 & 26 & 0.04 & 57.44 & 0.124124 & 0.092092 & 13 & 15058867 & 13 & 15976193 & 13 & 15 & 0.032 & 0.6925 & 6.07 \\
\hline 1720 & 58329 & 9593 & 22 & 0.03 & 77.68 & 0.103103 & 0.068068 & 13 & 16082398 & 13 & 16946257 & 13 & 16 & 0.044 & 0.7036 & 5.23 \\
\hline 1721 & 25983 & 9603 & 32 & 0.04 & 64.62 & 0.14014 & 0.087087 & 13 & 17000021 & 13 & 17956613 & 13 & 17 & 0.036 & 0.6978 & 5.75 \\
\hline 1722 & 59215 & 61918 & 25 & 0.03 & 76.56 & 0.114114 & 0.066066 & 13 & 18076357 & 13 & 18960253 & 13 & 18 & 0.043 & 0.7032 & 5.27 \\
\hline 1723 & 25991 & 34226 & 29 & 0.05 & 48.67 & 0.129129 & 0.083083 & 13 & 19066947 & 13 & 19932514 & 13 & 19 & 0.026 & 0.6835 & 6.55 \\
\hline 1724 & 32249 & 59457 & 27 & 0.03 & 72.66 & 0.118118 & 0.069069 & 13 & 20004436 & 13 & 20991970 & 13 & 20 & 0.041 & 0.7011 & 5.41 \\
\hline 1725 & 52499 & 9627 & 26 & 0.04 & 58.15 & 0.111111 & 0.074074 & 13 & 21009181 & 13 & 21999054 & 13 & 21 & 0.032 & 0.6931 & 6.03 \\
\hline 1726 & 9628 & 9638 & 26 & 0.03 & 74.04 & 0.117117 & 0.064064 & 13 & 22030419 & 13 & 22892852 & 13 & 22 & 0.042 & 0.7022 & 5.36 \\
\hline 1727 & 9639 & 29949 & 21 & 0.01 & 93.38 & 0.095095 & 0.039039 & 13 & 23032326 & 13 & 23944165 & 13 & 23 & 0.055 & 0.7081 & 4.72 \\
\hline 1728 & 9640 & 31794 & 33 & 0.04 & 63.55 & 0.142142 & 0.087087 & 13 & 24026709 & 13 & 24969288 & 13 & 24 & 0.035 & 0.6973 & 5.8 \\
\hline 1729 & 16980 & 32836 & 32 & 0.03 & 69.01 & 0.128128 & 0.075075 & 13 & 25073183 & 13 & 25929578 & 13 & 25 & 0.038 & 0.7 & 5.57 \\
\hline 1730 & 43283 & 54454 & 34 & 0.03 & 75.12 & 0.126126 & 0.073073 & 13 & 26001164 & 13 & 26988390 & 13 & 26 & 0.042 & 0.7022 & 5.31 \\
\hline 1731 & 36190 & 26033 & 35 & 0.07 & 28.07 & 0.176176 & 0.131131 & 13 & 27003930 & 13 & 27982732 & 13 & 27 & 0.015 & 0.636 & 8.1 \\
\hline 1732 & 38724 & 9683 & 27 & 0.08 & 24.68 & 0.144144 & 0.11011 & 13 & 28078702 & 13 & 28974982 & 13 & 28 & 0.013 & 0.6266 & 8.6 \\
\hline 1733 & 9684 & 9690 & 33 & 0.13 & 17 & 0.177177 & 0.127127 & 13 & 29002222 & 13 & 29980015 & 13 & 29 & 0.009 & 0.5845 & 9.67 \\
\hline 1734 & 9691 & 43291 & 23 & 0.12 & 17.87 & 0.139139 & 0.099099 & 13 & 30023501 & 13 & 30990361 & 13 & 30 & 0.009 & 0.5902 & 9.53 \\
\hline 1735 & 38727 & 45082 & 24 & 0.43 & 7.65 & 0.208208 & 0.178178 & 13 & 31070091 & 13 & 31969896 & 13 & 31 & 0.004 & 0.515 & 12.5 \\
\hline 1736 & 9705 & 26055 & 30 & 0.26 & 9.85 & 0.212212 & 0.174174 & 13 & 32068194 & 13 & 32997777 & 13 & 32 & 0.005 & 0.5316 & 11.45 \\
\hline 1737 & 48353 & 43296 & 21 & 0.03 & 69.47 & 0.088088 & 0.06006 & 13 & 33023006 & 13 & 33967886 & 13 & 33 & 0.039 & 0.6996 & 5.54 \\
\hline 1738 & 18534 & 26069 & 26 & 0.05 & 45.88 & 0.115115 & 0.086086 & 13 & 34112306 & 13 & 34993798 & 13 & 34 & 0.025 & 0.6813 & 6.78 \\
\hline 1739 & 26070 & 62127 & 27 & 0.06 & 31.46 & 0.116116 & 0.081081 & 13 & 35018578 & 13 & 35985818 & 13 & 35 & 0.017 & 0.6469 & 7.79 \\
\hline 1740 & 43305 & 43310 & 28 & 0.04 & 59.32 & 0.105105 & 0.072072 & 13 & 36016140 & 13 & 36964009 & 13 & 36 & 0.033 & 0.6944 & 5.99 \\
\hline 1741 & 61426 & 43314 & 22 & 0.01 & 93.16 & 0.084084 & 0.051051 & 13 & 37122998 & 13 & 37987928 & 13 & 37 & 0.054 & 0.7081 & 4.73 \\
\hline 1742 & 38732 & 9749 & 24 & 0.04 & 62.94 & 0.1001 & 0.068068 & 13 & 38085096 & 13 & 38948602 & 13 & 38 & 0.035 & 0.6965 & 5.82 \\
\hline 1743 & 47438 & 26087 & 15 & 0.02 & 89.4 & 0.072072 & 0.042042 & 13 & 39012509 & 13 & 39992519 & 13 & 39 & 0.052 & 0.7076 & 4.84 \\
\hline 1744 & 9757 & 30598 & 20 & 0.03 & 68.08 & 0.096096 & 0.064064 & 13 & 40050308 & 13 & 40905892 & 13 & 40 & 0.038 & 0.6995 & 5.6 \\
\hline 1745 & 52335 & 9765 & 16 & 0.09 & 22.25 & 0.093093 & 0.063063 & 13 & 41087932 & 13 & 41882944 & 13 & 41 & 0.012 & 0.6146 & 8.91 \\
\hline 1746 & 16269 & 43323 & 13 & 0.04 & 64.16 & 0.072072 & 0.052052 & 13 & 42026635 & 13 & 42933805 & 13 & 42 & 0.035 & 0.6976 & 5.77 \\
\hline 1747 & 9770 & 50626 & 18 & 0.02 & 81.57 & 0.077077 & 0.04004 & 13 & 43007233 & 13 & 43923140 & 13 & 43 & 0.046 & 0.7053 & 5.1 \\
\hline 1748 & 36142 & 26102 & 20 & 0.04 & 61.73 & 0.086086 & 0.059059 & 13 & 44014547 & 13 & 44990551 & 13 & 44 & 0.034 & 0.6965 & 5.9 \\
\hline 1749 & 38737 & 9783 & 27 & 0.04 & 52.33 & 0.128128 & 0.081081 & 13 & 45053798 & 13 & 45985901 & 13 & 45 & 0.028 & 0.6901 & 6.39 \\
\hline 1750 & 26109 & 9793 & 24 & 0.02 & 80.69 & 0.102102 & 0.055055 & 13 & 46015081 & 13 & 46975890 & 13 & 46 & 0.046 & 0.7052 & 5.13 \\
\hline 1751 & 9794 & 26113 & 20 & 0.01 & 94.5 & 0.079079 & 0.033033 & 13 & 47007358 & 13 & 47992156 & 13 & 47 & 0.055 & 0.7084 & 4.69 \\
\hline 1752 & 9807 & 58918 & 17 & 0.02 & 82.64 & 0.082082 & 0.046046 & 13 & 48008516 & 13 & 48974796 & 13 & 48 & 0.047 & 0.7047 & 5.05 \\
\hline
\end{tabular}




\begin{tabular}{|c|c|c|c|c|c|c|c|c|c|c|c|c|c|c|c|c|}
\hline 1753 & 26115 & 17001 & 10 & 0.09 & 22.44 & 0.072072 & 0.049049 & 13 & 49008533 & 13 & 49979246 & 13 & 49 & 0.012 & 0.6156 & 8.89 \\
\hline 1754 & 30265 & 38788 & 16 & 0.04 & 51.89 & 0.098098 & 0.07007 & 13 & 50015957 & 13 & 50853035 & 13 & 50 & 0.028 & 0.6891 & 6.42 \\
\hline 1755 & 9824 & 53467 & 20 & 0.05 & 43.12 & 0.092092 & 0.064064 & 13 & 51115180 & 13 & 51982923 & 13 & 51 & 0.023 & 0.6775 & 7 \\
\hline 1756 & 9830 & 9834 & 25 & 0.04 & 60.46 & 0.105105 & 0.085085 & 13 & 52016944 & 13 & 52962019 & 13 & 52 & 0.033 & 0.696 & 5.96 \\
\hline 1757 & 9835 & 59902 & 23 & 0.03 & 79.55 & 0.099099 & 0.07007 & 13 & 53002465 & 13 & 53914708 & 13 & 53 & 0.045 & 0.7048 & 5.17 \\
\hline 1758 & 56247 & 38751 & 20 & 0.02 & 89.28 & 0.086086 & 0.052052 & 13 & 54028991 & 13 & 54983756 & 13 & 54 & 0.052 & 0.7077 & 4.85 \\
\hline 1759 & 56861 & 38754 & 23 & 0.03 & 79.58 & 0.09009 & 0.046046 & 13 & 55049361 & 13 & 55993218 & 13 & 55 & 0.045 & 0.7047 & 5.17 \\
\hline 1760 & 47450 & 9861 & 26 & 0.05 & 42.97 & 0.119119 & 0.081081 & 13 & 56018886 & 13 & 56933573 & 13 & 56 & 0.023 & 0.6765 & 6.99 \\
\hline 1761 & 38756 & 34634 & 16 & 0.04 & 62.02 & 0.079079 & 0.055055 & 13 & 57191330 & 13 & 57921952 & 13 & 57 & 0.034 & 0.6967 & 5.88 \\
\hline 1762 & 9869 & 9874 & 28 & 0.07 & 30.73 & 0.134134 & 0.088088 & 13 & 58006732 & 13 & 58977959 & 13 & 58 & 0.016 & 0.6453 & 7.87 \\
\hline 1763 & 17218 & 26152 & 39 & 0.07 & 30.87 & 0.157157 & 0.122122 & 13 & 59015422 & 13 & 59941517 & 13 & 59 & 0.016 & 0.6456 & 7.85 \\
\hline 1764 & 9882 & 38767 & 30 & 0.04 & 51.41 & 0.135135 & 0.087087 & 13 & 60024211 & 13 & 60980258 & 13 & 60 & 0.028 & 0.6889 & 6.44 \\
\hline 1765 & 9894 & 30145 & 23 & 0.06 & 35.22 & 0.101101 & 0.077077 & 13 & 61014675 & 13 & 61982576 & 13 & 61 & 0.019 & 0.656 & 7.44 \\
\hline 1766 & 51886 & 57811 & 10 & 0.01 & 92.47 & 0.034034 & 0.022022 & 13 & 62006757 & 13 & 62924497 & 13 & 62 & 0.054 & 0.7081 & 4.75 \\
\hline 1767 & 33639 & 9906 & 13 & 0.03 & 65.87 & 0.075075 & 0.055055 & 13 & 63250705 & 13 & 63994105 & 13 & 63 & 0.037 & 0.6986 & 5.69 \\
\hline 1768 & 26168 & 38772 & 18 & 0.03 & 71.51 & 0.075075 & 0.05005 & 13 & 64033973 & 13 & 64999793 & 13 & 64 & 0.04 & 0.7008 & 5.46 \\
\hline 1769 & 26170 & 43367 & 23 & 0.05 & 49.35 & 0.123123 & 0.087087 & 13 & 65025003 & 13 & 65952730 & 13 & 65 & 0.027 & 0.6847 & 6.53 \\
\hline 1770 & 58342 & 61624 & 13 & 0.01 & 93.47 & 0.054054 & 0.04004 & 13 & 66094535 & 13 & 66778422 & 13 & 66 & 0.055 & 0.7081 & 4.72 \\
\hline 1771 & 17550 & 43371 & 17 & 0.03 & 65.18 & 0.092092 & 0.066066 & 13 & 67066590 & 13 & 67934836 & 13 & 67 & 0.036 & 0.6983 & 5.73 \\
\hline 1772 & 52171 & 9941 & 18 & 0.04 & 56.43 & 0.091091 & 0.054054 & 13 & 68100531 & 13 & 68968779 & 13 & 68 & 0.031 & 0.6914 & 6.12 \\
\hline 1773 & 50726 & 9945 & 14 & 0.04 & 64.79 & 0.076076 & 0.057057 & 13 & 69080928 & 13 & 69987699 & 13 & 69 & 0.036 & 0.6979 & 5.74 \\
\hline 1774 & 43375 & 26185 & 15 & 0.07 & 27.92 & 0.079079 & 0.053053 & 13 & 70328466 & 13 & 70982729 & 13 & 70 & 0.015 & 0.6359 & 8.13 \\
\hline 1775 & 38786 & 26194 & 22 & 0.06 & 37.17 & 0.121121 & 0.083083 & 13 & 71007063 & 13 & 71998014 & 13 & 71 & 0.02 & 0.663 & 7.33 \\
\hline 1776 & 56340 & 9962 & 21 & 0.03 & 75.44 & 0.083083 & 0.056056 & 13 & 72033973 & 13 & 72975884 & 13 & 72 & 0.043 & 0.7021 & 5.3 \\
\hline 1777 & 9963 & 56515 & 29 & 0.09 & 22.71 & 0.144144 & 0.098098 & 13 & 73023057 & 13 & 73966542 & 13 & 73 & 0.012 & 0.6176 & 8.85 \\
\hline 1778 & 16584 & 32736 & 22 & 0.04 & 61.36 & 0.094094 & 0.065065 & 13 & 74047780 & 13 & 74874236 & 13 & 74 & 0.034 & 0.6963 & 5.91 \\
\hline 1779 & 26203 & 51448 & 24 & 0.04 & 63.77 & 0.097097 & 0.07007 & 13 & 75066887 & 13 & 75849184 & 13 & 75 & 0.035 & 0.6972 & 5.79 \\
\hline 1780 & 29911 & 60459 & 18 & 0.02 & 83.6 & 0.085085 & 0.052052 & 13 & 76026446 & 13 & 76870123 & 13 & 76 & 0.048 & 0.705 & 5.01 \\
\hline 1781 & 32349 & 26217 & 15 & 0.06 & 36.06 & 0.098098 & 0.068068 & 13 & 77087193 & 13 & 77894260 & 13 & 77 & 0.019 & 0.6587 & 7.39 \\
\hline 1782 & 19421 & 16161 & 14 & 0.03 & 65.39 & 0.066066 & 0.043043 & 13 & 78016930 & 13 & 78972578 & 13 & 78 & 0.036 & 0.6984 & 5.72 \\
\hline 1783 & 31884 & 43393 & 14 & 0.02 & 87.49 & 0.057057 & 0.045045 & 13 & 79044236 & 13 & 79912229 & 13 & 79 & 0.05 & 0.7075 & 4.91 \\
\hline 1784 & 33755 & 43394 & 12 & 0.02 & 90.34 & 0.056056 & 0.042042 & 13 & 80063897 & 13 & 80987722 & 13 & 80 & 0.052 & 0.7076 & 4.81 \\
\hline 1785 & 43395 & 55189 & 20 & 0.03 & 67.85 & 0.088088 & 0.06006 & 13 & 81015331 & 13 & 81902962 & 13 & 81 & 0.038 & 0.6994 & 5.62 \\
\hline 1786 & 9996 & 43399 & 29 & 0.04 & 58.62 & 0.104104 & 0.076076 & 13 & 82003709 & 13 & 82988910 & 13 & 82 & 0.032 & 0.6933 & 6.02 \\
\hline 1787 & 53061 & 31248 & 28 & 0.05 & 46.46 & 0.113113 & 0.082082 & 13 & 83003977 & 13 & 83965641 & 13 & 83 & 0.025 & 0.6812 & 6.71 \\
\hline 1788 & 61695 & 10019 & 15 & 0.03 & 78.8 & 0.055055 & 0.036036 & 13 & 84062043 & 13 & 84751325 & 13 & 84 & 0.045 & 0.7038 & 5.19 \\
\hline 1789 & 10020 & 47465 & 12 & 0.01 & 92.44 & 0.06006 & 0.035035 & 13 & 85011406 & 13 & 85970514 & 13 & 85 & 0.054 & 0.7081 & 4.75 \\
\hline 1790 & 26246 & 54261 & 18 & 0.03 & 75.83 & 0.084084 & 0.058058 & 13 & 86015154 & 13 & 86889866 & 13 & 86 & 0.043 & 0.7029 & 5.3 \\
\hline 1791 & 36302 & 36334 & 18 & 0.02 & 86.44 & 0.074074 & 0.046046 & 13 & 87089543 & 13 & 87988310 & 13 & 87 & 0.05 & 0.707 & 4.94 \\
\hline
\end{tabular}




\begin{tabular}{|c|c|c|c|c|c|c|c|c|c|c|c|c|c|c|c|c|}
\hline 1792 & 10033 & 26259 & 31 & 0.07 & 29.14 & 0.168168 & 0.12012 & 13 & 88018134 & 13 & 88989523 & 13 & 88 & 0.016 & 0.6394 & 7.99 \\
\hline 1793 & 26260 & 38806 & 27 & 0.06 & 36.24 & 0.127127 & 0.081081 & 13 & 89014753 & 13 & 89982824 & 13 & 89 & 0.019 & 0.6604 & 7.4 \\
\hline 1794 & 10050 & 26275 & 17 & 0.04 & 61.62 & 0.084084 & 0.068068 & 13 & 90039607 & 13 & 90998129 & 13 & 90 & 0.034 & 0.6964 & 5.9 \\
\hline 1795 & 35327 & 38815 & 28 & 0.07 & 30.93 & 0.134134 & 0.096096 & 13 & 91031788 & 13 & 91993044 & 13 & 91 & 0.016 & 0.6458 & 7.85 \\
\hline 1796 & 15100 & 38818 & 13 & 0.02 & 91.17 & 0.057057 & 0.041041 & 13 & 92044292 & 13 & 92853911 & 13 & 92 & 0.053 & 0.7081 & 4.79 \\
\hline 1797 & 30209 & 38825 & 15 & 0.05 & 48.76 & 0.085085 & 0.062062 & 13 & 93191917 & 13 & 93956431 & 13 & 93 & 0.026 & 0.6835 & 6.54 \\
\hline 1798 & 26282 & 10066 & 13 & 0.04 & 61.95 & 0.072072 & 0.052052 & 13 & 94024615 & 13 & 94830010 & 13 & 94 & 0.034 & 0.6969 & 5.89 \\
\hline 1799 & 37420 & 10072 & 15 & 0.03 & 71.67 & 0.081081 & 0.056056 & 13 & 95181013 & 13 & 95969499 & 13 & 95 & 0.04 & 0.7009 & 5.45 \\
\hline 1800 & 47469 & 56585 & 7 & 0.02 & 82.9 & 0.053053 & 0.033033 & 13 & 96056270 & 13 & 96750659 & 13 & 96 & 0.048 & 0.7045 & 5.03 \\
\hline 1801 & 26289 & 26292 & 19 & 0.04 & 62.79 & 0.084084 & 0.052052 & 13 & 97001113 & 13 & 97966875 & 13 & 97 & 0.035 & 0.6965 & 5.84 \\
\hline 1802 & 55780 & 61319 & 14 & 0.01 & 94.2 & 0.045045 & 0.032032 & 13 & 98016719 & 13 & 98835795 & 13 & 98 & 0.055 & 0.7084 & 4.69 \\
\hline 1803 & 30291 & 10087 & 22 & 0.04 & 63.19 & 0.108108 & 0.073073 & 13 & 99016394 & 13 & 99980492 & 13 & 99 & 0.035 & 0.6967 & 5.81 \\
\hline 1804 & 38835 & 10090 & 14 & 0.03 & 71.23 & 0.062062 & 0.045045 & 13 & 100035462 & 13 & 100946188 & 13 & 100 & 0.04 & 0.7007 & 5.47 \\
\hline 1805 & 52500 & 10098 & 17 & 0.03 & 76.03 & 0.081081 & 0.054054 & 13 & 101029096 & 13 & 101993885 & 13 & 101 & 0.043 & 0.7029 & 5.29 \\
\hline 1806 & 45129 & 10100 & 12 & 0.02 & 88.5 & 0.06006 & 0.041041 & 13 & 102306232 & 13 & 102985566 & 13 & 102 & 0.051 & 0.7078 & 4.88 \\
\hline 1807 & 10101 & 61186 & 20 & 0.02 & 83.18 & 0.094094 & 0.052052 & 13 & 103033108 & 13 & 103926061 & 13 & 103 & 0.048 & 0.7045 & 5.02 \\
\hline 1808 & 34971 & 31213 & 12 & 0.01 & 95.04 & 0.054054 & 0.036036 & 13 & 104031218 & 13 & 104807793 & 13 & 104 & 0.056 & 0.7087 & 4.67 \\
\hline 1809 & 52849 & 34087 & 15 & 0.02 & 87.75 & 0.052052 & 0.039039 & 13 & 105053409 & 13 & 105786040 & 13 & 105 & 0.051 & 0.7078 & 4.9 \\
\hline 1810 & 54050 & 33688 & 3 & 0 & 96.34 & 0.007007 & 0.004004 & 13 & 106051998 & 13 & 106808982 & 13 & 106 & 0.057 & 0.7091 & 4.64 \\
\hline 1811 & 53091 & 52772 & 22 & 0.02 & 86.93 & 0.08008 & 0.058058 & 13 & 107023094 & 13 & 107980182 & 13 & 107 & 0.05 & 0.7074 & 4.93 \\
\hline 1812 & 10110 & 10113 & 15 & 0.01 & 93.78 & 0.055055 & 0.033033 & 13 & 108017360 & 13 & 108900739 & 13 & 108 & 0.055 & 0.7083 & 4.71 \\
\hline 1813 & 33181 & 56488 & 8 & 0.01 & 96.22 & 0.033033 & 0.02002 & 13 & 109045658 & 13 & 109986816 & 13 & 109 & 0.057 & 0.7091 & 4.64 \\
\hline 1814 & 53063 & 57532 & 7 & 0.01 & 95.98 & 0.022022 & 0.012012 & 13 & 110176644 & 13 & 110802739 & 13 & 110 & 0.056 & 0.7089 & 4.65 \\
\hline 1815 & 17198 & 56349 & 3 & 0 & 96.42 & 0.011011 & 0.007007 & 13 & 111492781 & 13 & 111921377 & 13 & 111 & 0.057 & 0.7092 & 4.64 \\
\hline 1816 & 26319 & 49415 & 13 & 0.01 & 93.09 & 0.059059 & 0.04004 & 13 & 112066136 & 13 & 112893275 & 13 & 112 & 0.054 & 0.708 & 4.73 \\
\hline 1817 & 14829 & 47482 & 5 & 0 & 96.31 & 0.022022 & 0.012012 & 13 & 113026364 & 13 & 113980170 & 13 & 113 & 0.057 & 0.709 & 4.64 \\
\hline 1818 & 18425 & 43436 & 8 & 0.01 & 95.66 & 0.042042 & 0.029029 & 13 & 114061850 & 13 & 114928166 & 13 & 114 & 0.056 & 0.7089 & 4.66 \\
\hline 1819 & 26321 & 31172 & 12 & 0.01 & 92.74 & 0.058058 & 0.042042 & 13 & 115027431 & 13 & 115846455 & 13 & 115 & 0.054 & 0.7081 & 4.74 \\
\hline 1820 & 54086 & 55132 & 15 & 0.02 & 86.69 & 0.064064 & 0.042042 & 13 & 116052233 & 13 & 116957243 & 13 & 116 & 0.05 & 0.7074 & 4.93 \\
\hline 1821 & 51646 & 47490 & 11 & 0.01 & 94.03 & 0.047047 & 0.033033 & 13 & 117014839 & 13 & 117860412 & 13 & 117 & 0.055 & 0.7084 & 4.7 \\
\hline 1822 & 30134 & 26325 & 15 & 0.02 & 87.29 & 0.063063 & 0.048048 & 13 & 118012221 & 13 & 118998431 & 13 & 118 & 0.05 & 0.7076 & 4.92 \\
\hline 1823 & 43439 & 56272 & 13 & 0.03 & 77.25 & 0.062062 & 0.046046 & 13 & 119023717 & 13 & 119979547 & 13 & 119 & 0.044 & 0.7035 & 5.25 \\
\hline 1824 & 26327 & 38849 & 13 & 0.02 & 91.69 & 0.067067 & 0.04004 & 13 & 120017132 & 13 & 120825463 & 13 & 120 & 0.053 & 0.7081 & 4.78 \\
\hline 1825 & 34940 & 10142 & 12 & 0.02 & 91.16 & 0.048048 & 0.029029 & 13 & 121174356 & 13 & 121983388 & 13 & 121 & 0.053 & 0.7081 & 4.79 \\
\hline 1826 & 10143 & 10147 & 13 & 0.02 & 90.24 & 0.052052 & 0.036036 & 13 & 122042291 & 13 & 122946774 & 13 & 122 & 0.052 & 0.7077 & 4.81 \\
\hline 1827 & 38858 & 58008 & 7 & 0.02 & 91.33 & 0.04004 & 0.026026 & 13 & 123011640 & 13 & 123984079 & 13 & 123 & 0.053 & 0.7082 & 4.79 \\
\hline 1828 & 26337 & 10153 & 20 & 0.02 & 80.81 & 0.081081 & 0.048048 & 13 & 124021372 & 13 & 124983364 & 13 & 124 & 0.046 & 0.7052 & 5.13 \\
\hline 1829 & 26342 & 43449 & 17 & 0.02 & 91.41 & 0.06006 & 0.04004 & 13 & 125060509 & 13 & 125976825 & 13 & 125 & 0.053 & 0.7082 & 4.79 \\
\hline 1830 & 43450 & 56363 & 15 & 0.02 & 82.17 & 0.069069 & 0.047047 & 13 & 126026173 & 13 & 126943274 & 13 & 126 & 0.047 & 0.705 & 5.07 \\
\hline
\end{tabular}




\begin{tabular}{|c|c|c|c|c|c|c|c|c|c|c|c|c|c|c|c|c|}
\hline 1831 & 10165 & 59757 & 14 & 0.02 & 85.86 & 0.059059 & 0.039039 & 13 & 127026543 & 13 & 127992609 & 13 & 127 & 0.049 & 0.7066 & 4.95 \\
\hline 1832 & 47501 & 55112 & 2 & 0 & 96.56 & 0.008008 & 0.001001 & 13 & 128100425 & 13 & 128280037 & 13 & 128 & 0.057 & 0.7093 & 4.63 \\
\hline 1833 & 10172 & 53050 & 14 & 0.01 & 94.8 & 0.034034 & 0.021021 & 13 & 129131688 & 13 & 129811274 & 13 & 129 & 0.056 & 0.7085 & 4.68 \\
\hline 1834 & 59047 & 52492 & 6 & 0 & 96.3 & 0.015015 & 0.006006 & 13 & 130011769 & 13 & 130982311 & 13 & 130 & 0.057 & 0.7091 & 4.64 \\
\hline 1835 & 10176 & 50151 & 13 & 0.02 & 89.54 & 0.055055 & 0.035035 & 13 & 131037563 & 13 & 131889566 & 13 & 131 & 0.052 & 0.7077 & 4.84 \\
\hline 1836 & 51019 & 10186 & 24 & 0.02 & 82.86 & 0.094094 & 0.056056 & 13 & 132017643 & 13 & 132985654 & 13 & 132 & 0.047 & 0.7047 & 5.04 \\
\hline 1837 & 10187 & 34408 & 23 & 0.02 & 81.35 & 0.083083 & 0.047047 & 13 & 133032413 & 13 & 133979002 & 13 & 133 & 0.046 & 0.7053 & 5.11 \\
\hline 1838 & 43458 & 10203 & 20 & 0.05 & 48.8 & 0.084084 & 0.056056 & 13 & 134021456 & 13 & 134985031 & 13 & 134 & 0.026 & 0.6836 & 6.54 \\
\hline 1839 & 10202 & 10205 & 14 & 0.02 & 90.85 & 0.045045 & 0.028028 & 13 & 135034976 & 13 & 135995420 & 13 & 135 & 0.053 & 0.708 & 4.8 \\
\hline 1840 & 26363 & 55569 & 15 & 0.01 & 94.86 & 0.069069 & 0.033033 & 13 & 136017764 & 13 & 136902376 & 13 & 136 & 0.056 & 0.7085 & 4.68 \\
\hline 1841 & 4230 & 38868 & 8 & 0.01 & 95.79 & 0.035035 & 0.022022 & 13 & 137076639 & 13 & 137988788 & 13 & 137 & 0.056 & 0.7089 & 4.65 \\
\hline 1842 & 26367 & 26370 & 20 & 0.05 & 49.95 & 0.082082 & 0.059059 & 13 & 138039183 & 13 & 138941535 & 13 & 138 & 0.027 & 0.6861 & 6.51 \\
\hline 1843 & 26371 & 54558 & 16 & 0.02 & 87.77 & 0.04004 & 0.03003 & 13 & 139142736 & 13 & 139998680 & 13 & 139 & 0.051 & 0.7078 & 4.9 \\
\hline 1844 & 57629 & 52216 & 16 & 0.02 & 84.82 & 0.069069 & 0.042042 & 13 & 140032848 & 13 & 140851043 & 13 & 140 & 0.049 & 0.7059 & 4.98 \\
\hline 1845 & 43467 & 51393 & 21 & 0.05 & 48.34 & 0.089089 & 0.059059 & 13 & 141032836 & 13 & 141902985 & 13 & 141 & 0.026 & 0.6827 & 6.55 \\
\hline 1846 & 57493 & 60468 & 16 & 0.03 & 66.42 & 0.08008 & 0.061061 & 13 & 142131038 & 13 & 142902305 & 13 & 142 & 0.037 & 0.699 & 5.67 \\
\hline 1847 & 43471 & 10225 & 10 & 0.03 & 79.53 & 0.037037 & 0.028028 & 13 & 143281651 & 13 & 143866440 & 13 & 143 & 0.045 & 0.705 & 5.18 \\
\hline 1848 & 10228 & 30828 & 18 & 0.03 & 78.72 & 0.066066 & 0.048048 & 13 & 144065180 & 13 & 144981309 & 13 & 144 & 0.045 & 0.7038 & 5.19 \\
\hline 1849 & 15373 & 59786 & 19 & 0.04 & 60.87 & 0.081081 & 0.055055 & 13 & 145009805 & 13 & 145772058 & 13 & 145 & 0.033 & 0.696 & 5.93 \\
\hline 1850 & 15050 & 43483 & 21 & 0.02 & 80.56 & 0.081081 & 0.058058 & 13 & 146120125 & 13 & 146920356 & 13 & 146 & 0.046 & 0.705 & 5.13 \\
\hline 1851 & 15606 & 26395 & 14 & 0.02 & 83.91 & 0.053053 & 0.037037 & 13 & 147060010 & 13 & 147984539 & 13 & 147 & 0.048 & 0.7054 & 5.01 \\
\hline 1852 & 54011 & 10247 & 12 & 0.01 & 92.32 & 0.048048 & 0.024024 & 13 & 148020127 & 13 & 148916021 & 13 & 148 & 0.054 & 0.7082 & 4.76 \\
\hline 1853 & 57739 & 43486 & 15 & 0.02 & 87.61 & 0.063063 & 0.043043 & 13 & 149037360 & 13 & 149983411 & 13 & 149 & 0.05 & 0.7078 & 4.91 \\
\hline 1854 & 10254 & 54605 & 14 & 0.02 & 84.25 & 0.068068 & 0.042042 & 13 & 150022400 & 13 & 150958697 & 13 & 150 & 0.048 & 0.7055 & 4.99 \\
\hline 1855 & 10260 & 50836 & 9 & 0.01 & 95.35 & 0.035035 & 0.014014 & 13 & 151109312 & 13 & 151823774 & 13 & 151 & 0.056 & 0.7086 & 4.66 \\
\hline 1856 & 10262 & 18089 & 12 & 0.01 & 93.81 & 0.042042 & 0.018018 & 13 & 152053701 & 13 & 152969439 & 13 & 152 & 0.055 & 0.7083 & 4.71 \\
\hline 1857 & 15094 & 51557 & 16 & 0.01 & 93.66 & 0.073073 & 0.039039 & 13 & 153245989 & 13 & 153940892 & 13 & 153 & 0.055 & 0.7081 & 4.71 \\
\hline 1858 & 52073 & 58834 & 13 & 0.02 & 88.69 & 0.067067 & 0.031031 & 13 & 154021170 & 13 & 154899831 & 13 & 154 & 0.051 & 0.7077 & 4.87 \\
\hline 1859 & 18749 & 10284 & 20 & 0.02 & 88.39 & 0.078078 & 0.052052 & 13 & 155052948 & 13 & 155870725 & 13 & 155 & 0.051 & 0.7076 & 4.88 \\
\hline 1860 & 32613 & 26418 & 16 & 0.05 & 41.79 & 0.081081 & 0.051051 & 13 & 156202355 & 13 & 156983040 & 13 & 156 & 0.022 & 0.6748 & 7.07 \\
\hline 1861 & 10288 & 26421 & 17 & 0.08 & 26.34 & 0.088088 & 0.061061 & 13 & 157024403 & 13 & 157993717 & 13 & 157 & 0.014 & 0.6292 & 8.28 \\
\hline 1862 & 58565 & 10297 & 21 & 0.33 & 8.73 & 0.15015 & 0.127127 & 13 & 158099578 & 13 & 158992883 & 13 & 158 & 0.005 & 0.5171 & 11.68 \\
\hline 1863 & 52197 & 26432 & 20 & 0.57 & 6.7 & 0.17017 & 0.143143 & 13 & 159004143 & 13 & 159985503 & 13 & 159 & 0.004 & 0.4976 & 12.53 \\
\hline 1864 & 26433 & 26436 & 20 & 0.24 & 10.6 & 0.116116 & 0.087087 & 13 & 160014224 & 13 & 160894746 & 13 & 160 & 0.006 & 0.5396 & 11.29 \\
\hline 1865 & 26437 & 43503 & 24 & 0.07 & 31.06 & 0.134134 & 0.099099 & 13 & 161008278 & 13 & 161962013 & 13 & 161 & 0.017 & 0.6457 & 7.82 \\
\hline 1866 & 43502 & 58439 & 13 & 0.03 & 80.34 & 0.077077 & 0.049049 & 13 & 162011185 & 13 & 162889030 & 13 & 162 & 0.046 & 0.7049 & 5.14 \\
\hline 1867 & 15397 & 57476 & 16 & 0.08 & 24.43 & 0.097097 & 0.074074 & 13 & 163002035 & 13 & 163963155 & 13 & 163 & 0.013 & 0.626 & 8.64 \\
\hline 1868 & 62043 & 17042 & 6 & 0.1 & 20.61 & 0.064064 & 0.05005 & 13 & 164327059 & 13 & 164995422 & 13 & 164 & 0.011 & 0.6049 & 9.12 \\
\hline 1869 & 33372 & 10325 & 13 & 0.04 & 54.96 & 0.067067 & 0.051051 & 13 & 165058078 & 13 & 165948194 & 13 & 165 & 0.03 & 0.6918 & 6.22 \\
\hline
\end{tabular}




\begin{tabular}{|c|c|c|c|c|c|c|c|c|c|c|c|c|c|c|c|c|}
\hline 1870 & 26449 & 26455 & 12 & 0.02 & 85.84 & 0.062062 & 0.038038 & 13 & 166070864 & 13 & 166984099 & 13 & 166 & 0.049 & 0.7065 & 4.95 \\
\hline 1871 & 26454 & 16773 & 18 & 0.04 & 56.88 & 0.09009 & 0.068068 & 13 & 167022760 & 13 & 167997645 & 13 & 167 & 0.031 & 0.6918 & 6.1 \\
\hline 1872 & 10335 & 43510 & 10 & 0.02 & 90.56 & 0.055055 & 0.038038 & 13 & 168097806 & 13 & 168856970 & 13 & 168 & 0.053 & 0.7078 & 4.81 \\
\hline 1873 & 38894 & 34601 & 12 & 0.01 & 92.45 & 0.055055 & 0.043043 & 13 & 169000579 & 13 & 169988202 & 13 & 169 & 0.054 & 0.7081 & 4.75 \\
\hline 1874 & 26461 & 19317 & 15 & 0.01 & 95.09 & 0.043043 & 0.025025 & 13 & 170055214 & 13 & 170572438 & 13 & 170 & 0.056 & 0.7087 & 4.67 \\
\hline 1875 & 26464 & 32336 & 3 & 0 & 96.55 & 0.015015 & 0.007007 & 13 & 171148012 & 13 & 171444531 & 13 & 171 & 0.057 & 0.7093 & 4.63 \\
\hline 1876 & 38897 & 59204 & 8 & 0.01 & 94.39 & 0.036036 & 0.021021 & 13 & 172047752 & 13 & 172744897 & 13 & 172 & 0.055 & 0.7083 & 4.69 \\
\hline 1877 & 43517 & 43519 & 14 & 0.02 & 82.97 & 0.07007 & 0.04004 & 13 & 173078781 & 13 & 173838695 & 13 & 173 & 0.048 & 0.7045 & 5.03 \\
\hline 1878 & 56155 & 10359 & 5 & 0.01 & 95.49 & 0.021021 & 0.014014 & 13 & 174247801 & 13 & 174758344 & 13 & 174 & 0.056 & 0.7087 & 4.66 \\
\hline 1879 & 26465 & 43522 & 11 & 0.01 & 94.68 & 0.05005 & 0.032032 & 13 & 175005291 & 13 & 175985883 & 13 & 175 & 0.056 & 0.7086 & 4.68 \\
\hline 1880 & 43523 & 57865 & 15 & 0.01 & 93.76 & 0.056056 & 0.033033 & 13 & 176029424 & 13 & 176997245 & 13 & 176 & 0.055 & 0.7082 & 4.71 \\
\hline 1881 & 34925 & 60213 & 16 & 0.02 & 90.47 & 0.066066 & 0.033033 & 13 & 177033643 & 13 & 177999254 & 13 & 177 & 0.053 & 0.7078 & 4.81 \\
\hline 1882 & 51545 & 39670 & 15 & 0.02 & 91.08 & 0.067067 & 0.046046 & 13 & 178024707 & 13 & 178860766 & 13 & 178 & 0.053 & 0.7081 & 4.8 \\
\hline 1883 & 10378 & 56500 & 14 & 0.02 & 88.89 & 0.062062 & 0.033033 & 13 & 179045118 & 13 & 179990010 & 13 & 179 & 0.051 & 0.7076 & 4.86 \\
\hline 1884 & 10380 & 10384 & 13 & 0.01 & 94.38 & 0.053053 & 0.034034 & 13 & 180057818 & 13 & 180923495 & 13 & 180 & 0.055 & 0.7083 & 4.69 \\
\hline 1885 & 58248 & 52122 & 6 & 0.01 & 94.15 & 0.021021 & 0.014014 & 13 & 181014066 & 13 & 181995091 & 13 & 181 & 0.055 & 0.7085 & 4.7 \\
\hline 1886 & 60429 & 10389 & 6 & 0.01 & 95.5 & 0.026026 & 0.018018 & 13 & 182315832 & 13 & 182817467 & 13 & 182 & 0.056 & 0.7087 & 4.66 \\
\hline 1887 & 38920 & 10393 & 13 & 0.01 & 92.29 & 0.054054 & 0.035035 & 13 & 183007199 & 13 & 183927662 & 13 & 183 & 0.054 & 0.7081 & 4.76 \\
\hline 1888 & 34357 & 60318 & 16 & 0.03 & 77.76 & 0.072072 & 0.05005 & 13 & 184018627 & 13 & 184921505 & 13 & 184 & 0.044 & 0.7036 & 5.23 \\
\hline 1889 & 10399 & 43531 & 26 & 0.03 & 71.36 & 0.126126 & 0.085085 & 13 & 185067136 & 13 & 185935406 & 13 & 185 & 0.04 & 0.7008 & 5.47 \\
\hline 1890 & 26482 & 10414 & 19 & 0.02 & 81.69 & 0.082082 & 0.054054 & 13 & 186001107 & 13 & 186944653 & 13 & 186 & 0.046 & 0.7052 & 5.09 \\
\hline 1891 & 38928 & 10419 & 23 & 0.02 & 80.89 & 0.103103 & 0.07007 & 13 & 187024787 & 13 & 187968272 & 13 & 187 & 0.046 & 0.7051 & 5.12 \\
\hline 1892 & 33188 & 38939 & 26 & 0.02 & 89.61 & 0.092092 & 0.053053 & 13 & 188095940 & 13 & 188953021 & 13 & 188 & 0.052 & 0.7077 & 4.84 \\
\hline 1893 & 15960 & 10432 & 20 & 0.02 & 84.21 & 0.091091 & 0.048048 & 13 & 189125669 & 13 & 189990288 & 13 & 189 & 0.048 & 0.7054 & 4.99 \\
\hline 1894 & 38943 & 57167 & 24 & 0.03 & 74.63 & 0.078078 & 0.048048 & 13 & 190029235 & 13 & 190969594 & 13 & 190 & 0.042 & 0.7021 & 5.33 \\
\hline 1895 & 26501 & 33348 & 17 & 0.03 & 70.76 & 0.072072 & 0.048048 & 13 & 191101378 & 13 & 191971388 & 13 & 191 & 0.04 & 0.7004 & 5.49 \\
\hline 1896 & 16270 & 36022 & 19 & 0.03 & 72.17 & 0.091091 & 0.052052 & 13 & 192020407 & 13 & 192997770 & 13 & 192 & 0.04 & 0.7012 & 5.44 \\
\hline 1897 & 43543 & 45302 & 15 & 0.04 & 52.76 & 0.108108 & 0.067067 & 13 & 193019061 & 13 & 193793634 & 13 & 193 & 0.028 & 0.6914 & 6.39 \\
\hline 1898 & 15621 & 38950 & 20 & 0.03 & 76.37 & 0.076076 & 0.043043 & 13 & 194099336 & 13 & 194986995 & 13 & 194 & 0.043 & 0.7029 & 5.28 \\
\hline 1899 & 38951 & 30784 & 11 & 0.01 & 92.83 & 0.052052 & 0.029029 & 13 & 195007274 & 13 & 195871973 & 13 & 195 & 0.054 & 0.7081 & 4.74 \\
\hline 1900 & 16228 & 38957 & 17 & 0.01 & 93.91 & 0.086086 & 0.033033 & 13 & 196079847 & 13 & 196931822 & 13 & 196 & 0.055 & 0.7084 & 4.7 \\
\hline 1901 & 30701 & 51285 & 15 & 0.02 & 81.45 & 0.075075 & 0.052052 & 13 & 197079707 & 13 & 197960155 & 13 & 197 & 0.046 & 0.7052 & 5.1 \\
\hline 1902 & 15463 & 10477 & 14 & 0.03 & 80.29 & 0.072072 & 0.046046 & 13 & 198071598 & 13 & 198933332 & 13 & 198 & 0.046 & 0.7049 & 5.15 \\
\hline 1903 & 36152 & 10488 & 27 & 0.05 & 44.02 & 0.124124 & 0.082082 & 13 & 199017905 & 13 & 199927172 & 13 & 199 & 0.023 & 0.6786 & 6.92 \\
\hline 1904 & 29324 & 14927 & 17 & 0.02 & 90.79 & 0.058058 & 0.032032 & 13 & 200005691 & 13 & 200940291 & 13 & 200 & 0.053 & 0.7079 & 4.8 \\
\hline 1905 & 57209 & 10495 & 11 & 0.08 & 23.93 & 0.082082 & 0.057057 & 13 & 201035915 & 13 & 201929107 & 13 & 201 & 0.012 & 0.6252 & 8.74 \\
\hline 1906 & 32040 & 47542 & 22 & 0.05 & 48.9 & 0.098098 & 0.071071 & 13 & 202031500 & 13 & 202882988 & 13 & 202 & 0.027 & 0.684 & 6.54 \\
\hline 1907 & 26535 & 26540 & 28 & 0.05 & 40.95 & 0.128128 & 0.083083 & 13 & 203080799 & 13 & 203989627 & 13 & 203 & 0.022 & 0.6725 & 7.11 \\
\hline 1908 & 26541 & 16206 & 11 & 0.02 & 81.47 & 0.061061 & 0.036036 & 13 & 204016747 & 13 & 204969242 & 13 & 204 & 0.046 & 0.7052 & 5.1 \\
\hline
\end{tabular}




\begin{tabular}{|c|c|c|c|c|c|c|c|c|c|c|c|c|c|c|c|c|}
\hline 1909 & 16239 & 45642 & 22 & 0.18 & 11.86 & 0.143143 & 0.116116 & 13 & 205022285 & 13 & 205872598 & 13 & 205 & 0.006 & 0.5516 & 11.07 \\
\hline 1910 & 17196 & 15151 & 23 & 0.08 & 25.72 & 0.122122 & 0.09009 & 13 & 206171736 & 13 & 206956346 & 13 & 206 & 0.014 & 0.629 & 8.42 \\
\hline 1911 & 33808 & 49428 & 23 & 0.03 & 72.96 & 0.106106 & 0.072072 & 13 & 207108562 & 13 & 207969771 & 13 & 207 & 0.041 & 0.7015 & 5.4 \\
\hline 1912 & 43572 & 60533 & 16 & 0.03 & 67.68 & 0.078078 & 0.053053 & 13 & 208012602 & 13 & 208919742 & 13 & 208 & 0.038 & 0.6991 & 5.62 \\
\hline 1913 & 54867 & 26560 & 15 & 0.02 & 91.61 & 0.052052 & 0.038038 & 13 & 209095174 & 13 & 209954223 & 13 & 209 & 0.053 & 0.7082 & 4.78 \\
\hline 1914 & 47545 & 10531 & 26 & 0.03 & 78.3 & 0.111111 & 0.067067 & 13 & 210026312 & 13 & 210866603 & 13 & 210 & 0.044 & 0.704 & 5.22 \\
\hline 1915 & 60573 & 10541 & 30 & 0.03 & 68.18 & 0.108108 & 0.069069 & 13 & 211000701 & 13 & 211989807 & 13 & 211 & 0.038 & 0.6992 & 5.59 \\
\hline 1916 & 26576 & 57961 & 30 & 0.05 & 43.57 & 0.135135 & 0.093093 & 13 & 212011618 & 13 & 212967360 & 13 & 212 & 0.023 & 0.6779 & 6.96 \\
\hline 1917 & 32663 & 10556 & 13 & 0.02 & 90.98 & 0.059059 & 0.037037 & 13 & 213036330 & 13 & 213981854 & 13 & 213 & 0.053 & 0.7081 & 4.8 \\
\hline 1918 & 26587 & 26593 & 23 & 0.03 & 76.92 & 0.105105 & 0.071071 & 13 & 214005140 & 13 & 214973846 & 13 & 214 & 0.044 & 0.7034 & 5.25 \\
\hline 1919 & 55230 & 30948 & 27 & 0.03 & 68.55 & 0.136136 & 0.084084 & 13 & 215012282 & 13 & 215936425 & 13 & 215 & 0.038 & 0.6995 & 5.58 \\
\hline 1920 & 17160 & 29629 & 15 & 0.01 & 92.64 & 0.059059 & 0.035035 & 13 & 216046101 & 13 & 216721489 & 13 & 216 & 0.054 & 0.7082 & 4.75 \\
\hline 1921 & 45363 & 43595 & 11 & 0.02 & 90.77 & 0.043043 & 0.024024 & 13 & 217027218 & 13 & 217743121 & 13 & 217 & 0.053 & 0.7078 & 4.8 \\
\hline 1922 & 17126 & 17221 & 3 & 0.01 & 96.11 & 0.024024 & 0.013013 & 13 & 218194240 & 13 & 218594088 & 13 & 218 & 0.057 & 0.709 & 4.64 \\
\hline 1923 & 43597 & 49442 & 19 & 0.06 & 33.43 & 0.1001 & 0.06006 & 14 & 167845 & 14 & 957393 & 14 & 0 & 0.018 & 0.6513 & 7.62 \\
\hline 1924 & 43599 & 55048 & 23 & 0.03 & 73.39 & 0.122122 & 0.076076 & 14 & 1000181 & 14 & 1929126 & 14 & 1 & 0.041 & 0.7017 & 5.38 \\
\hline 1925 & 26610 & 10590 & 15 & 0.04 & 62.39 & 0.057057 & 0.041041 & 14 & 2036624 & 14 & 2993368 & 14 & 2 & 0.034 & 0.6963 & 5.86 \\
\hline 1926 & 26615 & 10597 & 19 & 0.03 & 76.84 & 0.094094 & 0.065065 & 14 & 3034421 & 14 & 3966701 & 14 & 3 & 0.043 & 0.7032 & 5.26 \\
\hline 1927 & 10598 & 43606 & 21 & 0.04 & 57.68 & 0.104104 & 0.072072 & 14 & 4117715 & 14 & 4999819 & 14 & 4 & 0.032 & 0.6924 & 6.06 \\
\hline 1928 & 47556 & 26639 & 25 & 0.08 & 23.85 & 0.14014 & 0.093093 & 14 & 5009281 & 14 & 5995892 & 14 & 5 & 0.012 & 0.6241 & 8.74 \\
\hline 1929 & 26641 & 61516 & 22 & 0.08 & 24.6 & 0.122122 & 0.099099 & 14 & 6036903 & 14 & 6992243 & 14 & 6 & 0.013 & 0.6272 & 8.64 \\
\hline 1930 & 10620 & 36194 & 25 & 0.02 & 85.24 & 0.098098 & 0.054054 & 14 & 7028801 & 14 & 7976149 & 14 & 7 & 0.049 & 0.7061 & 4.97 \\
\hline 1931 & 43619 & 10635 & 24 & 0.03 & 67.45 & 0.102102 & 0.069069 & 14 & 8228429 & 14 & 8993832 & 14 & 8 & 0.037 & 0.6993 & 5.63 \\
\hline 1932 & 43625 & 10642 & 22 & 0.03 & 70.56 & 0.095095 & 0.063063 & 14 & 9010230 & 14 & 9915945 & 14 & 9 & 0.039 & 0.7003 & 5.5 \\
\hline 1933 & 10647 & 10663 & 30 & 0.05 & 43.77 & 0.132132 & 0.094094 & 14 & 10012490 & 14 & 10994010 & 14 & 10 & 0.023 & 0.6779 & 6.94 \\
\hline 1934 & 26673 & 10675 & 37 & 0.06 & 38.87 & 0.146146 & 0.103103 & 14 & 11022529 & 14 & 11989764 & 14 & 11 & 0.021 & 0.6678 & 7.24 \\
\hline 1935 & 26683 & 10686 & 32 & 0.07 & 30.53 & 0.163163 & 0.115115 & 14 & 12010383 & 14 & 12997847 & 14 & 12 & 0.016 & 0.6442 & 7.87 \\
\hline 1936 & 10687 & 38982 & 27 & 0.04 & 58.58 & 0.112112 & 0.073073 & 14 & 13030843 & 14 & 13982953 & 14 & 13 & 0.032 & 0.6935 & 6.02 \\
\hline 1937 & 57122 & 26708 & 29 & 0.04 & 50.26 & 0.123123 & 0.089089 & 14 & 14005194 & 14 & 14998570 & 14 & 14 & 0.027 & 0.6863 & 6.48 \\
\hline 1938 & 26709 & 61669 & 30 & 0.03 & 72.57 & 0.121121 & 0.077077 & 14 & 15020070 & 14 & 15993135 & 14 & 15 & 0.041 & 0.7011 & 5.41 \\
\hline 1939 & 31367 & 26720 & 31 & 0.03 & 71.55 & 0.134134 & 0.083083 & 14 & 16009830 & 14 & 16993535 & 14 & 16 & 0.04 & 0.7008 & 5.46 \\
\hline 1940 & 38987 & 43657 & 25 & 0.03 & 78.75 & 0.094094 & 0.065065 & 14 & 17079159 & 14 & 17981100 & 14 & 17 & 0.045 & 0.7038 & 5.19 \\
\hline 1941 & 10745 & 10730 & 25 & 0.03 & 65.91 & 0.105105 & 0.071071 & 14 & 18052581 & 14 & 18990098 & 14 & 18 & 0.037 & 0.6986 & 5.69 \\
\hline 1942 & 10729 & 10757 & 27 & 0.03 & 69.66 & 0.122122 & 0.079079 & 14 & 19012273 & 14 & 19994178 & 14 & 19 & 0.039 & 0.6998 & 5.53 \\
\hline 1943 & 10758 & 26750 & 27 & 0.03 & 79.06 & 0.095095 & 0.057057 & 14 & 20034189 & 14 & 20912572 & 14 & 20 & 0.045 & 0.7045 & 5.19 \\
\hline 1944 & 10762 & 26755 & 18 & 0.02 & 87.81 & 0.079079 & 0.045045 & 14 & 21017604 & 14 & 21963563 & 14 & 21 & 0.051 & 0.7078 & 4.9 \\
\hline 1945 & 54961 & 26760 & 24 & 0.03 & 73.86 & 0.095095 & 0.056056 & 14 & 22000499 & 14 & 22998419 & 14 & 22 & 0.042 & 0.7022 & 5.36 \\
\hline 1946 & 43663 & 43668 & 25 & 0.03 & 73.3 & 0.106106 & 0.074074 & 14 & 23027457 & 14 & 23992439 & 14 & 23 & 0.041 & 0.7016 & 5.38 \\
\hline 1947 & 10785 & 58020 & 23 & 0.02 & 87.11 & 0.098098 & 0.062062 & 14 & 24039632 & 14 & 24906177 & 14 & 24 & 0.05 & 0.7076 & 4.92 \\
\hline
\end{tabular}




\begin{tabular}{|c|c|c|c|c|c|c|c|c|c|c|c|c|c|c|c|c|}
\hline 1948 & 26773 & 26784 & 24 & 0.03 & 76.26 & 0.113113 & 0.073073 & 14 & 25077228 & 14 & 25976982 & 14 & 25 & 0.043 & 0.7028 & 5.28 \\
\hline 1949 & 26783 & 10812 & 22 & 0.02 & 87.53 & 0.094094 & 0.05005 & 14 & 26020243 & 14 & 26951421 & 14 & 26 & 0.05 & 0.7075 & 4.91 \\
\hline 1950 & 47583 & 57648 & 19 & 0.02 & 87.09 & 0.074074 & 0.051051 & 14 & 27119203 & 14 & 27942025 & 14 & 27 & 0.05 & 0.7076 & 4.92 \\
\hline 1951 & 10819 & 49467 & 37 & 0.06 & 37.69 & 0.146146 & 0.104104 & 14 & 28021727 & 14 & 28972892 & 14 & 28 & 0.02 & 0.6641 & 7.3 \\
\hline 1952 & 52661 & 43688 & 32 & 0.05 & 44.82 & 0.142142 & 0.093093 & 14 & 29004529 & 14 & 29985434 & 14 & 29 & 0.024 & 0.6795 & 6.84 \\
\hline 1953 & 10843 & 10849 & 26 & 0.03 & 66.66 & 0.112112 & 0.069069 & 14 & 30116923 & 14 & 30970606 & 14 & 30 & 0.037 & 0.6993 & 5.67 \\
\hline 1954 & 47592 & 10856 & 31 & 0.03 & 75.15 & 0.139139 & 0.1001 & 14 & 31036695 & 14 & 31983579 & 14 & 31 & 0.042 & 0.7022 & 5.31 \\
\hline 1955 & 51375 & 43700 & 17 & 0.02 & 85.45 & 0.064064 & 0.046046 & 14 & 32000259 & 14 & 32974206 & 14 & 32 & 0.049 & 0.7063 & 4.97 \\
\hline 1956 & 57718 & 26827 & 23 & 0.04 & 58.78 & 0.104104 & 0.074074 & 14 & 33040198 & 14 & 33959799 & 14 & 33 & 0.032 & 0.6936 & 6.01 \\
\hline 1957 & 47600 & 26837 & 26 & 0.04 & 60.35 & 0.122122 & 0.093093 & 14 & 34028866 & 14 & 34991538 & 14 & 34 & 0.033 & 0.6959 & 5.96 \\
\hline 1958 & 59895 & 10883 & 27 & 0.05 & 45.35 & 0.14014 & 0.098098 & 14 & 35036848 & 14 & 35973121 & 14 & 35 & 0.024 & 0.6809 & 6.82 \\
\hline 1959 & 47603 & 43714 & 22 & 0.02 & 80.71 & 0.104104 & 0.06006 & 14 & 36026991 & 14 & 36990529 & 14 & 36 & 0.046 & 0.7051 & 5.13 \\
\hline 1960 & 43715 & 43721 & 32 & 0.04 & 55.83 & 0.145145 & 0.09009 & 14 & 37100789 & 14 & 37968250 & 14 & 37 & 0.03 & 0.6922 & 6.17 \\
\hline 1961 & 52781 & 34552 & 22 & 0.02 & 88.91 & 0.083083 & 0.045045 & 14 & 38017404 & 14 & 38996258 & 14 & 38 & 0.051 & 0.7077 & 4.86 \\
\hline 1962 & 26860 & 10910 & 24 & 0.05 & 42.1 & 0.113113 & 0.092092 & 14 & 39015629 & 14 & 39965589 & 14 & 39 & 0.022 & 0.6758 & 7.06 \\
\hline 1963 & 57119 & 10916 & 28 & 0.3 & 9.32 & 0.229229 & 0.189189 & 14 & 40008901 & 14 & 40922501 & 14 & 40 & 0.005 & 0.5287 & 11.73 \\
\hline 1964 & 26879 & 26888 & 26 & 0.04 & 53.66 & 0.121121 & 0.09009 & 14 & 41000376 & 14 & 41993819 & 14 & 41 & 0.029 & 0.6919 & 6.33 \\
\hline 1965 & 26887 & 47615 & 19 & 0.04 & 56.39 & 0.075075 & 0.046046 & 14 & 42013177 & 14 & 42997445 & 14 & 42 & 0.031 & 0.6914 & 6.12 \\
\hline 1966 & 26893 & 26901 & 26 & 0.05 & 42.25 & 0.123123 & 0.083083 & 14 & 43015985 & 14 & 43990421 & 14 & 43 & 0.022 & 0.6758 & 7.05 \\
\hline 1967 & 47616 & 26906 & 27 & 0.04 & 60.65 & 0.133133 & 0.08008 & 14 & 44011983 & 14 & 44977107 & 14 & 44 & 0.033 & 0.6957 & 5.94 \\
\hline 1968 & 10944 & 26913 & 20 & 0.04 & 55.04 & 0.105105 & 0.075075 & 14 & 45068764 & 14 & 45950397 & 14 & 45 & 0.03 & 0.692 & 6.22 \\
\hline 1969 & 36069 & 54351 & 33 & 0.05 & 47.08 & 0.137137 & 0.106106 & 14 & 46043439 & 14 & 46983442 & 14 & 46 & 0.025 & 0.6821 & 6.66 \\
\hline 1970 & 47623 & 57316 & 31 & 0.03 & 68.12 & 0.135135 & 0.086086 & 14 & 47010896 & 14 & 47976511 & 14 & 47 & 0.038 & 0.6994 & 5.6 \\
\hline 1971 & 26937 & 43754 & 28 & 0.03 & 65.32 & 0.113113 & 0.074074 & 14 & 48046775 & 14 & 48973279 & 14 & 48 & 0.036 & 0.6983 & 5.72 \\
\hline 1972 & 47627 & 47631 & 22 & 0.03 & 77.47 & 0.087087 & 0.062062 & 14 & 49115640 & 14 & 49990314 & 14 & 49 & 0.044 & 0.7035 & 5.24 \\
\hline 1973 & 55273 & 43758 & 31 & 0.05 & 44.96 & 0.143143 & 0.108108 & 14 & 50012997 & 14 & 50992818 & 14 & 50 & 0.024 & 0.6799 & 6.84 \\
\hline 1974 & 10991 & 10997 & 21 & 0.06 & 38.65 & 0.117117 & 0.087087 & 14 & 51006694 & 14 & 51937785 & 14 & 51 & 0.021 & 0.6672 & 7.25 \\
\hline 1975 & 47635 & 36432 & 20 & 0.03 & 66.46 & 0.092092 & 0.07007 & 14 & 52003103 & 14 & 52994112 & 14 & 52 & 0.037 & 0.6993 & 5.68 \\
\hline 1976 & 11004 & 26978 & 22 & 0.02 & 85.73 & 0.092092 & 0.062062 & 14 & 53021844 & 14 & 53902642 & 14 & 53 & 0.049 & 0.7063 & 4.96 \\
\hline 1977 & 55282 & 57093 & 15 & 0.01 & 92.1 & 0.077077 & 0.05005 & 14 & 54006581 & 14 & 54867498 & 14 & 54 & 0.054 & 0.7081 & 4.77 \\
\hline 1978 & 26984 & 17883 & 15 & 0.02 & 85.62 & 0.058058 & 0.038038 & 14 & 55003669 & 14 & 55935436 & 14 & 55 & 0.049 & 0.7063 & 4.96 \\
\hline 1979 & 39017 & 57764 & 14 & 0.02 & 90.44 & 0.068068 & 0.038038 & 14 & 56002473 & 14 & 56894114 & 14 & 56 & 0.053 & 0.7077 & 4.81 \\
\hline 1980 & 43776 & 11033 & 21 & 0.04 & 51.24 & 0.112112 & 0.085085 & 14 & 57113686 & 14 & 57982523 & 14 & 57 & 0.028 & 0.6886 & 6.45 \\
\hline 1981 & 51624 & 47641 & 30 & 0.04 & 50.79 & 0.132132 & 0.083083 & 14 & 58002810 & 14 & 58984594 & 14 & 58 & 0.027 & 0.6871 & 6.46 \\
\hline 1982 & 43779 & 51270 & 23 & 0.02 & 83.09 & 0.088088 & 0.05005 & 14 & 59004595 & 14 & 59986607 & 14 & 59 & 0.048 & 0.7045 & 5.02 \\
\hline 1983 & 11044 & 11054 & 29 & 0.03 & 72.26 & 0.105105 & 0.069069 & 14 & 60106278 & 14 & 60939220 & 14 & 60 & 0.04 & 0.7011 & 5.43 \\
\hline 1984 & 27028 & 11061 & 27 & 0.03 & 67.04 & 0.105105 & 0.075075 & 14 & 61003207 & 14 & 61937913 & 14 & 61 & 0.037 & 0.6993 & 5.65 \\
\hline 1985 & 11064 & 43795 & 20 & 0.02 & 91.24 & 0.083083 & 0.052052 & 14 & 62002777 & 14 & 62975744 & 14 & 62 & 0.053 & 0.708 & 4.79 \\
\hline 1986 & 39030 & 11073 & 20 & 0.02 & 87.83 & 0.076076 & 0.042042 & 14 & 63006966 & 14 & 63962796 & 14 & 63 & 0.051 & 0.7078 & 4.9 \\
\hline
\end{tabular}




\begin{tabular}{|c|c|c|c|c|c|c|c|c|c|c|c|c|c|c|c|c|}
\hline 1987 & 11074 & 27051 & 30 & 0.03 & 75.49 & 0.117117 & 0.068068 & 14 & 64014394 & 14 & 64974040 & 14 & 64 & 0.043 & 0.7023 & 5.3 \\
\hline 1988 & 27052 & 43804 & 21 & 0.04 & 60.38 & 0.105105 & 0.069069 & 14 & 65015603 & 14 & 65969593 & 14 & 65 & 0.033 & 0.696 & 5.96 \\
\hline 1989 & 11090 & 11108 & 30 & 0.05 & 49.81 & 0.139139 & 0.099099 & 14 & 66049762 & 14 & 66992067 & 14 & 66 & 0.027 & 0.6859 & 6.51 \\
\hline 1990 & 47650 & 11116 & 22 & 0.03 & 71.48 & 0.085085 & 0.055055 & 14 & 67012779 & 14 & 67973978 & 14 & 67 & 0.04 & 0.7008 & 5.46 \\
\hline 1991 & 39038 & 59753 & 32 & 0.06 & 37.8 & 0.13013 & 0.082082 & 14 & 68005023 & 14 & 68982424 & 14 & 68 & 0.02 & 0.6644 & 7.29 \\
\hline 1992 & 27075 & 39049 & 28 & 0.06 & 31.84 & 0.128128 & 0.094094 & 14 & 69015482 & 14 & 69980983 & 14 & 69 & 0.017 & 0.6472 & 7.75 \\
\hline 1993 & 11130 & 39052 & 25 & 0.03 & 66.08 & 0.116116 & 0.068068 & 14 & 70019958 & 14 & 70993366 & 14 & 70 & 0.037 & 0.6988 & 5.69 \\
\hline 1994 & 27085 & 47659 & 22 & 0.03 & 73.95 & 0.086086 & 0.062062 & 14 & 71155797 & 14 & 71975247 & 14 & 71 & 0.042 & 0.7021 & 5.36 \\
\hline 1995 & 27092 & 43822 & 29 & 0.04 & 54.37 & 0.104104 & 0.063063 & 14 & 72010321 & 14 & 72950668 & 14 & 72 & 0.03 & 0.6917 & 6.27 \\
\hline 1996 & 11156 & 39059 & 23 & 0.03 & 77.66 & 0.114114 & 0.066066 & 14 & 73021315 & 14 & 73984104 & 14 & 73 & 0.044 & 0.7036 & 5.23 \\
\hline 1997 & 39060 & 39065 & 16 & 0.02 & 89.94 & 0.082082 & 0.051051 & 14 & 74155370 & 14 & 74990114 & 14 & 74 & 0.052 & 0.7078 & 4.83 \\
\hline 1998 & 27107 & 11179 & 30 & 0.03 & 67.38 & 0.117117 & 0.073073 & 14 & 75048108 & 14 & 75991444 & 14 & 75 & 0.037 & 0.6993 & 5.63 \\
\hline 1999 & 11180 & 39077 & 30 & 0.01 & 92.68 & 0.094094 & 0.044044 & 14 & 76005264 & 14 & 76979840 & 14 & 76 & 0.054 & 0.7081 & 4.74 \\
\hline 2000 & 43829 & 11331 & 17 & 0.02 & 81.38 & 0.065065 & 0.049049 & 14 & 77013888 & 14 & 77974347 & 14 & 77 & 0.046 & 0.7053 & 5.11 \\
\hline 2001 & 11332 & 27119 & 30 & 0.04 & 51.46 & 0.123123 & 0.084084 & 14 & 78011535 & 14 & 78995471 & 14 & 78 & 0.028 & 0.6888 & 6.44 \\
\hline 2002 & 55239 & 55906 & 29 & 0.02 & 91.58 & 0.096096 & 0.047047 & 14 & 79023018 & 14 & 79985244 & 14 & 79 & 0.053 & 0.7082 & 4.78 \\
\hline 2003 & 27127 & 11220 & 31 & 0.04 & 62.1 & 0.121121 & 0.062062 & 14 & 80010535 & 14 & 80984032 & 14 & 80 & 0.034 & 0.6966 & 5.88 \\
\hline 2004 & 43846 & 11229 & 28 & 0.02 & 84.41 & 0.101101 & 0.042042 & 14 & 81000356 & 14 & 81990297 & 14 & 81 & 0.049 & 0.7055 & 4.99 \\
\hline 2005 & 36169 & 47679 & 29 & 0.02 & 89.2 & 0.107107 & 0.037037 & 14 & 82017521 & 14 & 82965316 & 14 & 82 & 0.052 & 0.7078 & 4.85 \\
\hline 2006 & 47680 & 51011 & 21 & 0.01 & 92.58 & 0.058058 & 0.025025 & 14 & 83128477 & 14 & 83999974 & 14 & 83 & 0.054 & 0.7081 & 4.75 \\
\hline 2007 & 11245 & 43857 & 24 & 0.02 & 90.29 & 0.077077 & 0.04004 & 14 & 84042439 & 14 & 84917246 & 14 & 84 & 0.052 & 0.7077 & 4.81 \\
\hline 2008 & 43858 & 35529 & 15 & 0.03 & 80.21 & 0.064064 & 0.036036 & 14 & 85028953 & 14 & 85991839 & 14 & 85 & 0.045 & 0.705 & 5.15 \\
\hline 2009 & 11258 & 35673 & 22 & 0.04 & 59.78 & 0.091091 & 0.055055 & 14 & 86073571 & 14 & 86979241 & 14 & 86 & 0.033 & 0.6952 & 5.98 \\
\hline 2010 & 43864 & 61591 & 29 & 0.04 & 52.54 & 0.113113 & 0.076076 & 14 & 87034503 & 14 & 87975446 & 14 & 87 & 0.028 & 0.6908 & 6.39 \\
\hline 2011 & 47696 & 56621 & 27 & 0.04 & 54.88 & 0.111111 & 0.078078 & 14 & 88077735 & 14 & 88987258 & 14 & 88 & 0.03 & 0.6917 & 6.23 \\
\hline 2012 & 11281 & 11289 & 20 & 0.02 & 91.36 & 0.076076 & 0.047047 & 14 & 89013097 & 14 & 89670530 & 14 & 89 & 0.053 & 0.7082 & 4.79 \\
\hline 2013 & 27176 & 11298 & 27 & 0.03 & 75.38 & 0.105105 & 0.068068 & 14 & 90035986 & 14 & 90968956 & 14 & 90 & 0.043 & 0.7019 & 5.3 \\
\hline 2014 & 43877 & 43878 & 20 & 0.04 & 54.25 & 0.111111 & 0.074074 & 14 & 91013066 & 14 & 91762659 & 14 & 91 & 0.029 & 0.6917 & 6.28 \\
\hline 2015 & 11305 & 43879 & 20 & 0.02 & 87.41 & 0.073073 & 0.055055 & 14 & 92014581 & 14 & 92993972 & 14 & 92 & 0.05 & 0.7076 & 4.91 \\
\hline 2016 & 43880 & 51099 & 29 & 0.04 & 54.54 & 0.14014 & 0.089089 & 14 & 93096651 & 14 & 93968851 & 14 & 93 & 0.03 & 0.6916 & 6.26 \\
\hline 2017 & 52375 & 27198 & 12 & 0.06 & 38.59 & 0.064064 & 0.043043 & 14 & 94036401 & 14 & 94994468 & 14 & 94 & 0.021 & 0.6669 & 7.25 \\
\hline 2018 & 11327 & 51323 & 19 & 0.09 & 23.33 & 0.109109 & 0.079079 & 14 & 95195496 & 14 & 95961878 & 14 & 95 & 0.012 & 0.622 & 8.82 \\
\hline 2019 & 47711 & 11349 & 24 & 0.04 & 52.5 & 0.121121 & 0.086086 & 14 & 96103451 & 14 & 96986147 & 14 & 96 & 0.028 & 0.6909 & 6.39 \\
\hline 2020 & 27213 & 50842 & 32 & 0.05 & 41.16 & 0.122122 & 0.081081 & 14 & 97018128 & 14 & 97994997 & 14 & 97 & 0.022 & 0.6729 & 7.1 \\
\hline 2021 & 11363 & 43897 & 26 & 0.04 & 62.97 & 0.103103 & 0.071071 & 14 & 98020117 & 14 & 98941715 & 14 & 98 & 0.035 & 0.6965 & 5.82 \\
\hline 2022 & 43899 & 11376 & 18 & 0.05 & 46.93 & 0.097097 & 0.065065 & 14 & 99111537 & 14 & 99978645 & 14 & 99 & 0.025 & 0.6822 & 6.68 \\
\hline 2023 & 59709 & 31236 & 24 & 0.02 & 90.12 & 0.089089 & 0.056056 & 14 & 100004352 & 14 & 100971708 & 14 & 100 & 0.052 & 0.7078 & 4.82 \\
\hline 2024 & 39109 & 47718 & 23 & 0.04 & 59.66 & 0.099099 & 0.06006 & 14 & 101124119 & 14 & 101995631 & 14 & 101 & 0.033 & 0.695 & 5.98 \\
\hline 2025 & 11391 & 39118 & 16 & 0.03 & 76.53 & 0.077077 & 0.055055 & 14 & 102136878 & 14 & 102988071 & 14 & 102 & 0.043 & 0.7031 & 5.27 \\
\hline
\end{tabular}




\begin{tabular}{|c|c|c|c|c|c|c|c|c|c|c|c|c|c|c|c|c|}
\hline 2026 & 27250 & 58170 & 21 & 0.02 & 85.88 & 0.085085 & 0.053053 & 14 & 103045309 & 14 & 103936269 & 14 & 103 & 0.049 & 0.7066 & 4.95 \\
\hline 2027 & 27255 & 11416 & 30 & 0.04 & 52.8 & 0.132132 & 0.086086 & 14 & 104029253 & 14 & 104954378 & 14 & 104 & 0.028 & 0.6914 & 6.39 \\
\hline 2028 & 39126 & 34756 & 23 & 0.02 & 91.78 & 0.103103 & 0.065065 & 14 & 105006631 & 14 & 105966472 & 14 & 105 & 0.053 & 0.7081 & 4.78 \\
\hline 2029 & 39130 & 27265 & 20 & 0.01 & 92.54 & 0.09009 & 0.051051 & 14 & 106074877 & 14 & 106992825 & 14 & 106 & 0.054 & 0.7081 & 4.75 \\
\hline 2030 & 11431 & 11442 & 28 & 0.03 & 69.14 & 0.118118 & 0.083083 & 14 & 107033485 & 14 & 107993512 & 14 & 107 & 0.038 & 0.6999 & 5.56 \\
\hline 2031 & 27273 & 11451 & 21 & 0.03 & 72.32 & 0.093093 & 0.054054 & 14 & 108016508 & 14 & 108992984 & 14 & 108 & 0.041 & 0.701 & 5.42 \\
\hline 2032 & 49505 & 11462 & 30 & 0.05 & 45.55 & 0.136136 & 0.085085 & 14 & 109016085 & 14 & 109988613 & 14 & 109 & 0.024 & 0.6814 & 6.81 \\
\hline 2033 & 43922 & 11470 & 23 & 0.05 & 40.84 & 0.082082 & 0.059059 & 14 & 110103413 & 14 & 110983342 & 14 & 110 & 0.022 & 0.672 & 7.11 \\
\hline 2034 & 43926 & 27299 & 30 & 0.04 & 61.99 & 0.118118 & 0.087087 & 14 & 111089602 & 14 & 111970799 & 14 & 111 & 0.034 & 0.6969 & 5.89 \\
\hline 2035 & 60680 & 39141 & 15 & 0.01 & 93.86 & 0.048048 & 0.029029 & 14 & 112008662 & 14 & 112992396 & 14 & 112 & 0.055 & 0.7083 & 4.7 \\
\hline 2036 & 11487 & 11494 & 27 & 0.03 & 77.98 & 0.096096 & 0.062062 & 14 & 113018677 & 14 & 113973779 & 14 & 113 & 0.044 & 0.704 & 5.23 \\
\hline 2037 & 34461 & 27313 & 23 & 0.05 & 48.2 & 0.098098 & 0.07007 & 14 & 114007859 & 14 & 114987900 & 14 & 114 & 0.026 & 0.6825 & 6.57 \\
\hline 2038 & 11499 & 18041 & 18 & 0.05 & 45.16 & 0.091091 & 0.064064 & 14 & 115013554 & 14 & 115960354 & 14 & 115 & 0.024 & 0.6805 & 6.83 \\
\hline 2039 & 39144 & 52595 & 23 & 0.05 & 46.74 & 0.103103 & 0.08008 & 14 & 116309545 & 14 & 116970199 & 14 & 116 & 0.025 & 0.682 & 6.69 \\
\hline 2040 & 11517 & 43949 & 27 & 0.06 & 35.52 & 0.132132 & 0.102102 & 14 & 117011153 & 14 & 117886869 & 14 & 117 & 0.019 & 0.6569 & 7.43 \\
\hline 2041 & 27326 & 11539 & 26 & 0.03 & 71.14 & 0.098098 & 0.063063 & 14 & 118017826 & 14 & 118988272 & 14 & 118 & 0.04 & 0.7005 & 5.47 \\
\hline 2042 & 27333 & 43955 & 14 & 0.02 & 85.43 & 0.08008 & 0.046046 & 14 & 119011148 & 14 & 119983210 & 14 & 119 & 0.049 & 0.7062 & 4.96 \\
\hline 2043 & 27336 & 11555 & 18 & 0.02 & 90.59 & 0.058058 & 0.029029 & 14 & 120063463 & 14 & 120986865 & 14 & 120 & 0.053 & 0.7078 & 4.81 \\
\hline 2044 & 11556 & 43963 & 18 & 0.02 & 90.1 & 0.087087 & 0.033033 & 14 & 121000230 & 14 & 121970428 & 14 & 121 & 0.052 & 0.7078 & 4.82 \\
\hline 2045 & 27346 & 58625 & 24 & 0.04 & 59.16 & 0.126126 & 0.058058 & 14 & 122043297 & 14 & 122966625 & 14 & 122 & 0.032 & 0.6943 & 6 \\
\hline 2046 & 53060 & 39149 & 23 & 0.02 & 82.43 & 0.096096 & 0.053053 & 14 & 123006309 & 14 & 123957615 & 14 & 123 & 0.047 & 0.7049 & 5.06 \\
\hline 2047 & 11569 & 27370 & 22 & 0.03 & 77.93 & 0.102102 & 0.056056 & 14 & 124020336 & 14 & 124905201 & 14 & 124 & 0.044 & 0.7039 & 5.23 \\
\hline 2048 & 39150 & 11581 & 19 & 0.01 & 95.37 & 0.07007 & 0.038038 & 14 & 125016745 & 14 & 125978735 & 14 & 125 & 0.056 & 0.7087 & 4.66 \\
\hline 2049 & 11582 & 11590 & 20 & 0.01 & 93.14 & 0.079079 & 0.044044 & 14 & 126018137 & 14 & 126980313 & 14 & 126 & 0.054 & 0.7081 & 4.73 \\
\hline 2050 & 27380 & 47783 & 19 & 0.06 & 32.29 & 0.076076 & 0.055055 & 14 & 127001828 & 14 & 127981685 & 14 & 127 & 0.017 & 0.6499 & 7.75 \\
\hline 2051 & 47784 & 47788 & 15 & 0.01 & 92.18 & 0.053053 & 0.03003 & 14 & 128001688 & 14 & 128987436 & 14 & 128 & 0.054 & 0.7081 & 4.76 \\
\hline 2052 & 11598 & 43981 & 19 & 0.01 & 92.03 & 0.076076 & 0.043043 & 14 & 129055323 & 14 & 129966461 & 14 & 129 & 0.053 & 0.7081 & 4.77 \\
\hline 2053 & 61369 & 27393 & 24 & 0.03 & 79.3 & 0.092092 & 0.058058 & 14 & 130079124 & 14 & 130962219 & 14 & 130 & 0.045 & 0.7047 & 5.18 \\
\hline 2054 & 11610 & 27398 & 29 & 0.04 & 57.24 & 0.119119 & 0.066066 & 14 & 131033758 & 14 & 131991212 & 14 & 131 & 0.031 & 0.6925 & 6.09 \\
\hline 2055 & 27399 & 27405 & 36 & 0.06 & 35.7 & 0.149149 & 0.09009 & 14 & 132022319 & 14 & 132972206 & 14 & 132 & 0.019 & 0.6572 & 7.41 \\
\hline 2056 & 43991 & 11641 & 21 & 0.03 & 74.01 & 0.096096 & 0.055055 & 14 & 133005108 & 14 & 133783308 & 14 & 133 & 0.042 & 0.7021 & 5.36 \\
\hline 2057 & 59279 & 11648 & 27 & 0.03 & 74.68 & 0.096096 & 0.058058 & 14 & 134026947 & 14 & 134988252 & 14 & 134 & 0.042 & 0.702 & 5.33 \\
\hline 2058 & 47805 & 11667 & 37 & 0.03 & 80.24 & 0.137137 & 0.077077 & 14 & 135016019 & 14 & 135991043 & 14 & 135 & 0.045 & 0.7049 & 5.15 \\
\hline 2059 & 27422 & 11676 & 30 & 0.03 & 73.74 & 0.128128 & 0.082082 & 14 & 136018609 & 14 & 136939847 & 14 & 136 & 0.042 & 0.7021 & 5.37 \\
\hline 2060 & 44006 & 11688 & 26 & 0.02 & 88.08 & 0.128128 & 0.056056 & 14 & 137063119 & 14 & 137979007 & 14 & 137 & 0.051 & 0.7078 & 4.89 \\
\hline 2061 & 34382 & 27445 & 33 & 0.3 & 9.02 & 0.238238 & 0.2002 & 14 & 138031007 & 14 & 138971702 & 14 & 138 & 0.005 & 0.5238 & 11.76 \\
\hline 2062 & 27447 & 11707 & 28 & 0.08 & 27.02 & 0.146146 & 0.107107 & 14 & 139040157 & 14 & 139971668 & 14 & 139 & 0.014 & 0.6322 & 8.21 \\
\hline 2063 & 44017 & 47824 & 40 & 0.1 & 20 & 0.179179 & 0.131131 & 14 & 140004840 & 14 & 140944805 & 14 & 140 & 0.01 & 0.603 & 9.27 \\
\hline 2064 & 39191 & 27474 & 38 & 0.08 & 26.18 & 0.19019 & 0.139139 & 14 & 141004641 & 14 & 141996996 & 14 & 141 & 0.014 & 0.6305 & 8.35 \\
\hline
\end{tabular}




\begin{tabular}{|c|c|c|c|c|c|c|c|c|c|c|c|c|c|c|c|c|}
\hline 2065 & 11741 & 11750 & 25 & 0.11 & 19.47 & 0.121121 & 0.076076 & 14 & 142014167 & 14 & 142951787 & 14 & 142 & 0.01 & 0.6003 & 9.35 \\
\hline 2066 & 33925 & 47829 & 26 & 0.14 & 15.93 & 0.158158 & 0.124124 & 14 & 143000381 & 14 & 143979213 & 14 & 143 & 0.008 & 0.5799 & 9.99 \\
\hline 2067 & 54405 & 52164 & 30 & 0.07 & 29.84 & 0.159159 & 0.11011 & 14 & 144001271 & 14 & 144927544 & 14 & 144 & 0.016 & 0.6412 & 7.91 \\
\hline 2068 & 58897 & 44040 & 27 & 0.04 & 56.03 & 0.115115 & 0.085085 & 14 & 145001826 & 14 & 145945750 & 14 & 145 & 0.031 & 0.6918 & 6.16 \\
\hline 2069 & 44041 & 62004 & 35 & 0.04 & 63.33 & 0.132132 & 0.08008 & 14 & 146002787 & 14 & 146982251 & 14 & 146 & 0.035 & 0.6971 & 5.81 \\
\hline 2070 & 56792 & 11783 & 29 & 0.04 & 56.92 & 0.113113 & 0.079079 & 14 & 147003080 & 14 & 147903117 & 14 & 147 & 0.031 & 0.6918 & 6.1 \\
\hline 2071 & 44050 & 49536 & 18 & 0.03 & 74.36 & 0.09009 & 0.053053 & 14 & 148003749 & 14 & 148980215 & 14 & 148 & 0.042 & 0.7018 & 5.34 \\
\hline 2072 & 11794 & 27527 & 32 & 0.03 & 72.87 & 0.138138 & 0.079079 & 14 & 149002010 & 14 & 149990362 & 14 & 149 & 0.041 & 0.7015 & 5.4 \\
\hline 2073 & 49544 & 53633 & 25 & 0.03 & 80.11 & 0.113113 & 0.065065 & 14 & 150010047 & 14 & 150984209 & 14 & 150 & 0.045 & 0.7049 & 5.15 \\
\hline 2074 & 49549 & 56774 & 36 & 0.04 & 58.81 & 0.155155 & 0.09009 & 14 & 151015261 & 14 & 151993696 & 14 & 151 & 0.032 & 0.6937 & 6.01 \\
\hline 2075 & 51747 & 11812 & 20 & 0.02 & 87.55 & 0.076076 & 0.042042 & 14 & 152178755 & 14 & 152978092 & 14 & 152 & 0.05 & 0.7077 & 4.91 \\
\hline 2076 & 47846 & 44070 & 13 & 0.02 & 86.15 & 0.063063 & 0.049049 & 14 & 153114220 & 14 & 153786761 & 14 & 153 & 0.049 & 0.7069 & 4.95 \\
\hline 2077 & 31605 & 11886 & 23 & 0.04 & 62.68 & 0.104104 & 0.077077 & 15 & 4535 & 15 & 993283 & 15 & 0 & 0.034 & 0.6964 & 5.84 \\
\hline 2078 & 27597 & 27585 & 24 & 0.02 & 85.26 & 0.091091 & 0.057057 & 15 & 1015164 & 15 & 1975870 & 15 & 1 & 0.049 & 0.7061 & 4.97 \\
\hline 2079 & 11871 & 53733 & 32 & 0.04 & 51.63 & 0.135135 & 0.085085 & 15 & 2009887 & 15 & 2990508 & 15 & 2 & 0.028 & 0.689 & 6.43 \\
\hline 2080 & 14524 & 27558 & 27 & 0.04 & 56.31 & 0.126126 & 0.078078 & 15 & 3017242 & 15 & 3985728 & 15 & 3 & 0.031 & 0.6918 & 6.14 \\
\hline 2081 & 11823 & 44077 & 30 & 0.04 & 51.15 & 0.134134 & 0.09009 & 15 & 4061992 & 15 & 4920014 & 15 & 4 & 0.028 & 0.6884 & 6.46 \\
\hline 2082 & 27562 & 11838 & 13 & 0.01 & 94.07 & 0.056056 & 0.038038 & 15 & 5034616 & 15 & 5936184 & 15 & 5 & 0.055 & 0.7084 & 4.7 \\
\hline 2083 & 34640 & 11839 & 9 & 0.01 & 93.43 & 0.049049 & 0.036036 & 15 & 6040472 & 15 & 6978059 & 15 & 6 & 0.055 & 0.7081 & 4.72 \\
\hline 2084 & 11840 & 19362 & 27 & 0.04 & 52.67 & 0.113113 & 0.079079 & 15 & 7009701 & 15 & 7998827 & 15 & 7 & 0.028 & 0.6911 & 6.39 \\
\hline 2085 & 15676 & 27573 & 21 & 0.02 & 89.74 & 0.084084 & 0.058058 & 15 & 8023920 & 15 & 8907355 & 15 & 8 & 0.052 & 0.7078 & 4.83 \\
\hline 2086 & 27574 & 11858 & 23 & 0.03 & 74.8 & 0.094094 & 0.064064 & 15 & 9026506 & 15 & 9997960 & 15 & 9 & 0.042 & 0.7019 & 5.32 \\
\hline 2087 & 39210 & 27582 & 23 & 0.03 & 66.15 & 0.113113 & 0.072072 & 15 & 10038766 & 15 & 10975125 & 15 & 10 & 0.037 & 0.6987 & 5.68 \\
\hline 2088 & 39213 & 60898 & 14 & 0.02 & 91.66 & 0.063063 & 0.035035 & 15 & 11001978 & 15 & 11904651 & 15 & 11 & 0.053 & 0.7081 & 4.78 \\
\hline 2089 & 53026 & 27600 & 13 & 0.02 & 82.62 & 0.057057 & 0.043043 & 15 & 12155691 & 15 & 12984131 & 15 & 12 & 0.047 & 0.7048 & 5.05 \\
\hline 2090 & 27601 & 27609 & 20 & 0.03 & 79.4 & 0.08008 & 0.057057 & 15 & 13000324 & 15 & 13990176 & 15 & 13 & 0.045 & 0.7049 & 5.18 \\
\hline 2091 & 27608 & 11902 & 17 & 0.04 & 58.42 & 0.081081 & 0.059059 & 15 & 14019896 & 15 & 14940640 & 15 & 14 & 0.032 & 0.6933 & 6.02 \\
\hline 2092 & 54749 & 50716 & 23 & 0.02 & 83.46 & 0.098098 & 0.048048 & 15 & 15047103 & 15 & 15904702 & 15 & 15 & 0.048 & 0.705 & 5.02 \\
\hline 2093 & 36390 & 45309 & 25 & 0.06 & 32.04 & 0.133133 & 0.099099 & 15 & 16070249 & 15 & 16953219 & 15 & 16 & 0.017 & 0.6487 & 7.76 \\
\hline 2094 & 27624 & 56383 & 27 & 0.05 & 41.58 & 0.117117 & 0.08008 & 15 & 17030766 & 15 & 17976658 & 15 & 17 & 0.022 & 0.6747 & 7.1 \\
\hline 2095 & 48268 & 39231 & 19 & 0.02 & 84.98 & 0.104104 & 0.051051 & 15 & 18038664 & 15 & 18998615 & 15 & 18 & 0.049 & 0.7061 & 4.98 \\
\hline 2096 & 11933 & 11944 & 29 & 0.04 & 49.99 & 0.138138 & 0.094094 & 15 & 19076645 & 15 & 19989529 & 15 & 19 & 0.027 & 0.6863 & 6.51 \\
\hline 2097 & 60291 & 11949 & 20 & 0.04 & 54.83 & 0.103103 & 0.072072 & 15 & 20011263 & 15 & 20934106 & 15 & 20 & 0.03 & 0.6919 & 6.23 \\
\hline 2098 & 44097 & 11960 & 27 & 0.05 & 49.49 & 0.131131 & 0.096096 & 15 & 21094100 & 15 & 21993474 & 15 & 21 & 0.027 & 0.6851 & 6.52 \\
\hline 2099 & 27644 & 11969 & 28 & 0.08 & 26.49 & 0.126126 & 0.079079 & 15 & 22028463 & 15 & 22961387 & 15 & 22 & 0.014 & 0.6302 & 8.27 \\
\hline 2100 & 50128 & 32380 & 15 & 0.03 & 73.05 & 0.08008 & 0.047047 & 15 & 23067061 & 15 & 23987116 & 15 & 23 & 0.041 & 0.7016 & 5.4 \\
\hline 2101 & 57738 & 45658 & 14 & 0.04 & 64.26 & 0.074074 & 0.05005 & 15 & 24019575 & 15 & 24972365 & 15 & 24 & 0.036 & 0.6977 & 5.77 \\
\hline 2102 & 11975 & 11976 & 10 & 0.07 & 30.8 & 0.077077 & 0.051051 & 15 & 25026611 & 15 & 25947179 & 15 & 25 & 0.016 & 0.6455 & 7.86 \\
\hline 2103 & 34907 & 44108 & 15 & 0.01 & 92.55 & 0.048048 & 0.031031 & 15 & 26156628 & 15 & 26975496 & 15 & 26 & 0.054 & 0.7082 & 4.75 \\
\hline
\end{tabular}




\begin{tabular}{|c|c|c|c|c|c|c|c|c|c|c|c|c|c|c|c|c|}
\hline 2104 & 27659 & 27662 & 15 & 0.03 & 66.39 & 0.073073 & 0.04004 & 15 & 27006998 & 15 & 27957695 & 15 & 27 & 0.037 & 0.699 & 5.68 \\
\hline 2105 & 27663 & 59905 & 21 & 0.04 & 56.48 & 0.087087 & 0.056056 & 15 & 28020404 & 15 & 28991507 & 15 & 28 & 0.031 & 0.6915 & 6.11 \\
\hline 2106 & 45643 & 39240 & 29 & 0.05 & 49.54 & 0.123123 & 0.067067 & 15 & 29029368 & 15 & 29947470 & 15 & 29 & 0.027 & 0.6855 & 6.53 \\
\hline 2107 & 18787 & 11989 & 11 & 0.01 & 94.99 & 0.038038 & 0.025025 & 15 & 30007572 & 15 & 30928847 & 15 & 30 & 0.056 & 0.7087 & 4.68 \\
\hline 2108 & 34926 & 19281 & 31 & 0.03 & 67.14 & 0.137137 & 0.095095 & 15 & 31033635 & 15 & 31995248 & 15 & 31 & 0.037 & 0.6994 & 5.65 \\
\hline 2109 & 59673 & 12012 & 18 & 0.02 & 90.72 & 0.075075 & 0.049049 & 15 & 32042636 & 15 & 32933373 & 15 & 32 & 0.053 & 0.7078 & 4.8 \\
\hline 2110 & 12013 & 44120 & 23 & 0.04 & 64.58 & 0.106106 & 0.06006 & 15 & 33013541 & 15 & 33883655 & 15 & 33 & 0.036 & 0.6978 & 5.75 \\
\hline 2111 & 27681 & 12024 & 23 & 0.03 & 70.21 & 0.109109 & 0.066066 & 15 & 34128573 & 15 & 34973841 & 15 & 34 & 0.039 & 0.7004 & 5.51 \\
\hline 2112 & 12025 & 27687 & 27 & 0.03 & 72.23 & 0.124124 & 0.082082 & 15 & 35010372 & 15 & 35973145 & 15 & 35 & 0.04 & 0.7011 & 5.43 \\
\hline 2113 & 44127 & 59289 & 19 & 0.02 & 82.1 & 0.096096 & 0.064064 & 15 & 36011179 & 15 & 36905893 & 15 & 36 & 0.047 & 0.7048 & 5.08 \\
\hline 2114 & 12032 & 45481 & 23 & 0.04 & 54.42 & 0.115115 & 0.075075 & 15 & 37073268 & 15 & 37957138 & 15 & 37 & 0.03 & 0.6916 & 6.27 \\
\hline 2115 & 29826 & 50545 & 20 & 0.03 & 73.83 & 0.087087 & 0.058058 & 15 & 38003706 & 15 & 38894354 & 15 & 38 & 0.042 & 0.7022 & 5.36 \\
\hline 2116 & 12044 & 52725 & 15 & 0.02 & 88.82 & 0.061061 & 0.041041 & 15 & 39116703 & 15 & 39986267 & 15 & 39 & 0.051 & 0.7077 & 4.87 \\
\hline 2117 & 14888 & 47863 & 26 & 0.09 & 22.8 & 0.132132 & 0.098098 & 15 & 40012527 & 15 & 40974908 & 15 & 40 & 0.012 & 0.6178 & 8.84 \\
\hline 2118 & 12060 & 30859 & 14 & 0.12 & 17.99 & 0.086086 & 0.068068 & 15 & 41012641 & 15 & 41967209 & 15 & 41 & 0.009 & 0.5908 & 9.51 \\
\hline 2119 & 30162 & 17834 & 12 & 0.02 & 90.13 & 0.06006 & 0.038038 & 15 & 42105101 & 15 & 42956851 & 15 & 42 & 0.052 & 0.7078 & 4.82 \\
\hline 2120 & 17308 & 27709 & 7 & 0 & 96.45 & 0.028028 & 0.011011 & 15 & 43053001 & 15 & 43972136 & 15 & 43 & 0.057 & 0.7091 & 4.63 \\
\hline 2121 & 27710 & 52369 & 16 & 0 & 96.45 & 0.069069 & 0.018018 & 15 & 44059586 & 15 & 44862962 & 15 & 44 & 0.057 & 0.7092 & 4.63 \\
\hline 2122 & 12071 & 12075 & 16 & 0.06 & 39.37 & 0.061061 & 0.033033 & 15 & 45022930 & 15 & 45981536 & 15 & 45 & 0.021 & 0.6685 & 7.2 \\
\hline 2123 & 27711 & 39255 & 9 & 0 & 96.41 & 0.029029 & 0.012012 & 15 & 46072177 & 15 & 46554052 & 15 & 46 & 0.057 & 0.7092 & 4.64 \\
\hline 2124 & 12082 & 52048 & 6 & 0.01 & 96.17 & 0.029029 & 0.02002 & 15 & 47169627 & 15 & 47971398 & 15 & 47 & 0.057 & 0.7091 & 4.64 \\
\hline 2125 & 33919 & 47869 & 11 & 0.03 & 77.41 & 0.049049 & 0.038038 & 15 & 48336880 & 15 & 48970076 & 15 & 48 & 0.044 & 0.7036 & 5.24 \\
\hline 2126 & 44145 & 12099 & 18 & 0.03 & 80.09 & 0.075075 & 0.046046 & 15 & 49103392 & 15 & 49896940 & 15 & 49 & 0.045 & 0.7049 & 5.15 \\
\hline 2127 & 32696 & 27721 & 19 & 0.04 & 56.56 & 0.086086 & 0.064064 & 15 & 50037970 & 15 & 50967710 & 15 & 50 & 0.031 & 0.6917 & 6.11 \\
\hline 2128 & 60799 & 12113 & 22 & 0.1 & 21.5 & 0.123123 & 0.09009 & 15 & 51028020 & 15 & 51979193 & 15 & 51 & 0.011 & 0.6109 & 9.02 \\
\hline 2129 & 12115 & 12118 & 26 & 0.07 & 29.91 & 0.121121 & 0.096096 & 15 & 52006633 & 15 & 52955798 & 15 & 52 & 0.016 & 0.6419 & 7.91 \\
\hline 2130 & 52901 & 47879 & 24 & 0.04 & 64.65 & 0.09009 & 0.054054 & 15 & 53067599 & 15 & 53869001 & 15 & 53 & 0.036 & 0.6978 & 5.75 \\
\hline 2131 & 49580 & 44156 & 16 & 0.02 & 90.39 & 0.071071 & 0.032032 & 15 & 54046928 & 15 & 54991163 & 15 & 54 & 0.053 & 0.7077 & 4.81 \\
\hline 2132 & 27734 & 12132 & 19 & 0.01 & 92.61 & 0.065065 & 0.032032 & 15 & 55038222 & 15 & 55993215 & 15 & 55 & 0.054 & 0.7082 & 4.75 \\
\hline 2133 & 44158 & 47882 & 14 & 0.01 & 95.5 & 0.06006 & 0.031031 & 15 & 56060543 & 15 & 56937444 & 15 & 56 & 0.056 & 0.7087 & 4.66 \\
\hline 2134 & 51617 & 12148 & 22 & 0.04 & 54.92 & 0.101101 & 0.068068 & 15 & 57255533 & 15 & 57880292 & 15 & 57 & 0.03 & 0.6917 & 6.22 \\
\hline 2135 & 27745 & 12154 & 17 & 0.01 & 95.63 & 0.068068 & 0.038038 & 15 & 58049980 & 15 & 58956076 & 15 & 58 & 0.056 & 0.7088 & 4.66 \\
\hline 2136 & 55438 & 12162 & 18 & 0.02 & 87.33 & 0.082082 & 0.052052 & 15 & 59124311 & 15 & 59950764 & 15 & 59 & 0.05 & 0.7076 & 4.92 \\
\hline 2137 & 44164 & 39270 & 18 & 0.02 & 88.35 & 0.074074 & 0.045045 & 15 & 60008146 & 15 & 60984129 & 15 & 60 & 0.051 & 0.7077 & 4.88 \\
\hline 2138 & 47889 & 29490 & 13 & 0.01 & 94.47 & 0.047047 & 0.016016 & 15 & 61018550 & 15 & 61994497 & 15 & 61 & 0.055 & 0.7083 & 4.69 \\
\hline 2139 & 30906 & 12175 & 26 & 0.02 & 90.32 & 0.112112 & 0.046046 & 15 & 62033445 & 15 & 62990144 & 15 & 62 & 0.052 & 0.7077 & 4.81 \\
\hline 2140 & 12176 & 12190 & 21 & 0.04 & 53.32 & 0.088088 & 0.064064 & 15 & 63029841 & 15 & 63998627 & 15 & 63 & 0.029 & 0.6917 & 6.36 \\
\hline 2141 & 12191 & 12200 & 17 & 0.03 & 78.62 & 0.079079 & 0.038038 & 15 & 64019259 & 15 & 64980103 & 15 & 64 & 0.044 & 0.704 & 5.2 \\
\hline 2142 & 56423 & 12204 & 24 & 0.04 & 58.39 & 0.095095 & 0.066066 & 15 & 65014590 & 15 & 65866223 & 15 & 65 & 0.032 & 0.693 & 6.02 \\
\hline
\end{tabular}




\begin{tabular}{|c|c|c|c|c|c|c|c|c|c|c|c|c|c|c|c|c|}
\hline 2143 & 51748 & 50564 & 7 & 0.01 & 96.11 & 0.031031 & 0.015015 & 15 & 66122450 & 15 & 66907405 & 15 & 66 & 0.057 & 0.709 & 4.64 \\
\hline 2144 & 47891 & 27772 & 10 & 0.01 & 92.25 & 0.044044 & 0.027027 & 15 & 67012516 & 15 & 67976297 & 15 & 67 & 0.054 & 0.7081 & 4.76 \\
\hline 2145 & 47893 & 12213 & 6 & 0 & 96.56 & 0.017017 & 0.006006 & 15 & 68046201 & 15 & 68928476 & 15 & 68 & 0.057 & 0.7093 & 4.63 \\
\hline 2146 & 44177 & 27774 & 8 & 0.01 & 95.26 & 0.03003 & 0.019019 & 15 & 69276645 & 15 & 69714353 & 15 & 69 & 0.056 & 0.7086 & 4.67 \\
\hline 2147 & 39277 & 12218 & 8 & 0.01 & 95.97 & 0.039039 & 0.02002 & 15 & 70035825 & 15 & 70797627 & 15 & 70 & 0.056 & 0.7089 & 4.65 \\
\hline 2148 & 27777 & 12221 & 6 & 0.02 & 91.67 & 0.028028 & 0.017017 & 15 & 71357581 & 15 & 71846422 & 15 & 71 & 0.053 & 0.7081 & 4.78 \\
\hline 2149 & 45158 & 12222 & 13 & 0.02 & 82.46 & 0.077077 & 0.051051 & 15 & 72220380 & 15 & 72955165 & 15 & 72 & 0.047 & 0.705 & 5.06 \\
\hline 2150 & 59729 & 39282 & 10 & 0.01 & 96.01 & 0.039039 & 0.017017 & 15 & 73043848 & 15 & 73987697 & 15 & 73 & 0.057 & 0.709 & 4.65 \\
\hline 2151 & 32293 & 61304 & 16 & 0.02 & 81.98 & 0.071071 & 0.046046 & 15 & 74096437 & 15 & 74750835 & 15 & 74 & 0.047 & 0.705 & 5.08 \\
\hline 2152 & 12227 & 47896 & 6 & 0.01 & 96.04 & 0.025025 & 0.01001 & 15 & 75340693 & 15 & 75687558 & 15 & 75 & 0.057 & 0.709 & 4.64 \\
\hline 2153 & 16141 & 12230 & 4 & 0.01 & 96.16 & 0.013013 & 0.012012 & 15 & 76266102 & 15 & 76787826 & 15 & 76 & 0.057 & 0.709 & 4.64 \\
\hline 2154 & 12231 & 47897 & 8 & 0.01 & 92.48 & 0.032032 & 0.018018 & 15 & 77108630 & 15 & 77875540 & 15 & 77 & 0.054 & 0.7082 & 4.75 \\
\hline 2155 & 54630 & 27785 & 10 & 0.01 & 96.06 & 0.028028 & 0.013013 & 15 & 78090341 & 15 & 78961640 & 15 & 78 & 0.057 & 0.709 & 4.64 \\
\hline 2156 & 50148 & 47900 & 7 & 0.01 & 95.91 & 0.037037 & 0.019019 & 15 & 79058727 & 15 & 79978032 & 15 & 79 & 0.056 & 0.7088 & 4.65 \\
\hline 2157 & 47903 & 54197 & 9 & 0.01 & 96.1 & 0.035035 & 0.02002 & 15 & 80299741 & 15 & 80987506 & 15 & 80 & 0.057 & 0.7091 & 4.64 \\
\hline 2158 & 12241 & 12243 & 5 & 0 & 96.39 & 0.01001 & 0.007007 & 15 & 81428860 & 15 & 81992999 & 15 & 81 & 0.057 & 0.7092 & 4.64 \\
\hline 2159 & 12245 & 12246 & 7 & 0.01 & 96.23 & 0.029029 & 0.019019 & 15 & 82310352 & 15 & 82835779 & 15 & 82 & 0.057 & 0.7091 & 4.64 \\
\hline 2160 & 54612 & 27790 & 21 & 0.03 & 71.92 & 0.099099 & 0.066066 & 15 & 83086792 & 15 & 83881219 & 15 & 83 & 0.04 & 0.7012 & 5.44 \\
\hline 2161 & 12256 & 12266 & 25 & 0.01 & 92.16 & 0.106106 & 0.042042 & 15 & 84109275 & 15 & 84990297 & 15 & 84 & 0.054 & 0.7081 & 4.76 \\
\hline 2162 & 34679 & 27800 & 21 & 0.01 & 93.45 & 0.081081 & 0.032032 & 15 & 85023476 & 15 & 85874505 & 15 & 85 & 0.055 & 0.7082 & 4.72 \\
\hline 2163 & 27797 & 27803 & 24 & 0.02 & 88.29 & 0.075075 & 0.044044 & 15 & 86048980 & 15 & 86976928 & 15 & 86 & 0.051 & 0.7077 & 4.88 \\
\hline 2164 & 44194 & 61218 & 22 & 0.02 & 91.38 & 0.083083 & 0.049049 & 15 & 87027499 & 15 & 87942801 & 15 & 87 & 0.053 & 0.7082 & 4.79 \\
\hline 2165 & 27812 & 12297 & 27 & 0.02 & 91.27 & 0.127127 & 0.063063 & 15 & 88036047 & 15 & 88943039 & 15 & 88 & 0.053 & 0.708 & 4.79 \\
\hline 2166 & 12298 & 39291 & 26 & 0.02 & 82.48 & 0.113113 & 0.059059 & 15 & 89097103 & 15 & 89953222 & 15 & 89 & 0.047 & 0.7049 & 5.06 \\
\hline 2167 & 27823 & 12318 & 30 & 0.01 & 92.24 & 0.113113 & 0.047047 & 15 & 90020714 & 15 & 90992066 & 15 & 90 & 0.054 & 0.7081 & 4.76 \\
\hline 2168 & 44203 & 12325 & 21 & 0.02 & 85.75 & 0.113113 & 0.048048 & 15 & 91003938 & 15 & 91991367 & 15 & 91 & 0.049 & 0.7064 & 4.96 \\
\hline 2169 & 44206 & 27833 & 24 & 0.02 & 83.64 & 0.112112 & 0.058058 & 15 & 92024785 & 15 & 92970795 & 15 & 92 & 0.048 & 0.7053 & 5.01 \\
\hline 2170 & 44208 & 57869 & 27 & 0.02 & 91.32 & 0.121121 & 0.057057 & 15 & 93015426 & 15 & 93995497 & 15 & 93 & 0.053 & 0.7082 & 4.79 \\
\hline 2171 & 12339 & 12343 & 17 & 0.01 & 95.55 & 0.072072 & 0.02002 & 15 & 94025320 & 15 & 94994150 & 15 & 94 & 0.056 & 0.7088 & 4.66 \\
\hline 2172 & 14575 & 48272 & 19 & 0.02 & 83.94 & 0.088088 & 0.047047 & 15 & 95042255 & 15 & 95919710 & 15 & 95 & 0.048 & 0.7054 & 5 \\
\hline 2173 & 56079 & 52752 & 16 & 0.01 & 94.63 & 0.061061 & 0.031031 & 15 & 96071036 & 15 & 96930028 & 15 & 96 & 0.056 & 0.7085 & 4.68 \\
\hline 2174 & 12357 & 47922 & 11 & 0.02 & 83.75 & 0.06006 & 0.035035 & 15 & 97254108 & 15 & 97940788 & 15 & 97 & 0.048 & 0.7053 & 5.01 \\
\hline 2175 & 12362 & 16817 & 5 & 0.04 & 51.37 & 0.043043 & 0.03003 & 15 & 98011713 & 15 & 98451291 & 15 & 98 & 0.028 & 0.6889 & 6.44 \\
\hline 2176 & 58951 & 54662 & 9 & 0.01 & 95.96 & 0.031031 & 0.016016 & 15 & 99006968 & 15 & 99784888 & 15 & 99 & 0.056 & 0.7089 & 4.65 \\
\hline 2177 & 47923 & 39299 & 10 & 0 & 96.38 & 0.039039 & 0.019019 & 15 & 100101961 & 15 & 100681987 & 15 & 100 & 0.057 & 0.7092 & 4.64 \\
\hline 2178 & 47925 & 16216 & 17 & 0.02 & 90.36 & 0.069069 & 0.044044 & 15 & 101102306 & 15 & 101921509 & 15 & 101 & 0.052 & 0.7077 & 4.81 \\
\hline 2179 & 12379 & 50213 & 7 & 0 & 96.43 & 0.027027 & 0.008008 & 15 & 102338125 & 15 & 102673383 & 15 & 102 & 0.057 & 0.7092 & 4.64 \\
\hline 2180 & 12383 & 12387 & 8 & 0.01 & 92.97 & 0.044044 & 0.024024 & 15 & 103012674 & 15 & 103608693 & 15 & 103 & 0.054 & 0.7082 & 4.74 \\
\hline 2181 & 48328 & 53244 & 8 & 0.01 & 94.24 & 0.03003 & 0.015015 & 15 & 104065843 & 15 & 104904590 & 15 & 104 & 0.055 & 0.7084 & 4.69 \\
\hline
\end{tabular}




\begin{tabular}{|c|c|c|c|c|c|c|c|c|c|c|c|c|c|c|c|c|}
\hline 2182 & 12389 & 31116 & 10 & 0.01 & 94.4 & 0.043043 & 0.029029 & 15 & 105198145 & 15 & 105993430 & 15 & 105 & 0.055 & 0.7083 & 4.69 \\
\hline 2183 & 61219 & 27857 & 17 & 0.02 & 83.85 & 0.074074 & 0.048048 & 15 & 106045290 & 15 & 106977147 & 15 & 106 & 0.048 & 0.7053 & 5.01 \\
\hline 2184 & 59912 & 12400 & 14 & 0.02 & 85.71 & 0.061061 & 0.034034 & 15 & 107048443 & 15 & 107945927 & 15 & 107 & 0.049 & 0.7062 & 4.95 \\
\hline 2185 & 57606 & 34669 & 14 & 0.01 & 95.47 & 0.072072 & 0.031031 & 15 & 108002667 & 15 & 108889109 & 15 & 108 & 0.056 & 0.7087 & 4.66 \\
\hline 2186 & 12406 & 27866 & 8 & 0.01 & 95.39 & 0.046046 & 0.03003 & 15 & 109196356 & 15 & 109722503 & 15 & 109 & 0.056 & 0.7087 & 4.66 \\
\hline 2187 & 27867 & 47941 & 10 & 0.01 & 95.95 & 0.046046 & 0.01001 & 15 & 110011380 & 15 & 110852498 & 15 & 110 & 0.056 & 0.7088 & 4.65 \\
\hline 2188 & 12409 & 35070 & 20 & 0.03 & 78.83 & 0.097097 & 0.063063 & 15 & 111088143 & 15 & 111917903 & 15 & 111 & 0.045 & 0.7038 & 5.19 \\
\hline 2189 & 14991 & 12415 & 17 & 0.02 & 89.91 & 0.066066 & 0.034034 & 15 & 112258571 & 15 & 112984212 & 15 & 112 & 0.052 & 0.7078 & 4.83 \\
\hline 2190 & 47947 & 47949 & 11 & 0.01 & 95.68 & 0.037037 & 0.022022 & 15 & 113010695 & 15 & 113997526 & 15 & 113 & 0.056 & 0.7089 & 4.66 \\
\hline 2191 & 59858 & 52209 & 8 & 0.02 & 86.75 & 0.039039 & 0.024024 & 15 & 114055631 & 15 & 114987540 & 15 & 114 & 0.05 & 0.7073 & 4.93 \\
\hline 2192 & 12422 & 44235 & 15 & 0.02 & 89.77 & 0.067067 & 0.036036 & 15 & 115026418 & 15 & 115836301 & 15 & 115 & 0.052 & 0.7077 & 4.83 \\
\hline 2193 & 12430 & 43603 & 6 & 0 & 96.55 & 0.024024 & 0.004004 & 15 & 116028994 & 15 & 116467644 & 15 & 116 & 0.057 & 0.7093 & 4.63 \\
\hline 2194 & 27882 & 39307 & 15 & 0.02 & 90.89 & 0.062062 & 0.041041 & 15 & 117005136 & 15 & 117987234 & 15 & 117 & 0.053 & 0.708 & 4.8 \\
\hline 2195 & 12434 & 12437 & 17 & 0.02 & 88.54 & 0.068068 & 0.044044 & 15 & 118002592 & 15 & 118920135 & 15 & 118 & 0.051 & 0.7078 & 4.88 \\
\hline 2196 & 44236 & 44238 & 17 & 0.02 & 85 & 0.081081 & 0.051051 & 15 & 119091942 & 15 & 119982356 & 15 & 119 & 0.049 & 0.7061 & 4.98 \\
\hline 2197 & 39316 & 12447 & 24 & 0.03 & 74.07 & 0.099099 & 0.067067 & 15 & 120015248 & 15 & 120942551 & 15 & 120 & 0.042 & 0.7022 & 5.36 \\
\hline 2198 & 44240 & 47953 & 22 & 0.03 & 80.16 & 0.109109 & 0.072072 & 15 & 121000357 & 15 & 121778781 & 15 & 121 & 0.045 & 0.705 & 5.15 \\
\hline 2199 & 12455 & 45428 & 13 & 0.01 & 92.06 & 0.074074 & 0.042042 & 15 & 122166717 & 15 & 122998623 & 15 & 122 & 0.054 & 0.7081 & 4.77 \\
\hline 2200 & 33559 & 12466 & 15 & 0.01 & 95.22 & 0.042042 & 0.029029 & 15 & 123052463 & 15 & 123902773 & 15 & 123 & 0.056 & 0.7087 & 4.67 \\
\hline 2201 & 27903 & 34783 & 14 & 0.02 & 90.46 & 0.066066 & 0.028028 & 15 & 124030230 & 15 & 124792285 & 15 & 124 & 0.053 & 0.7077 & 4.81 \\
\hline 2202 & 12471 & 12477 & 28 & 0.04 & 58.93 & 0.114114 & 0.071071 & 15 & 125026754 & 15 & 125988978 & 15 & 125 & 0.032 & 0.694 & 6.01 \\
\hline 2203 & 39339 & 39345 & 18 & 0.02 & 87.21 & 0.082082 & 0.042042 & 15 & 126029433 & 15 & 126862449 & 15 & 126 & 0.05 & 0.7076 & 4.92 \\
\hline 2204 & 39346 & 27915 & 21 & 0.05 & 42.3 & 0.096096 & 0.069069 & 15 & 127201004 & 15 & 127982302 & 15 & 127 & 0.022 & 0.676 & 7.05 \\
\hline 2205 & 39348 & 12494 & 20 & 0.06 & 31.39 & 0.118118 & 0.076076 & 15 & 128002384 & 15 & 128894663 & 15 & 128 & 0.017 & 0.6469 & 7.8 \\
\hline 2206 & 14907 & 61833 & 19 & 0.06 & 39.59 & 0.093093 & 0.066066 & 15 & 129031744 & 15 & 129798767 & 15 & 129 & 0.021 & 0.6696 & 7.2 \\
\hline 2207 & 12503 & 14405 & 24 & 0.06 & 31.78 & 0.132132 & 0.093093 & 15 & 130001470 & 15 & 130968782 & 15 & 130 & 0.017 & 0.6474 & 7.76 \\
\hline 2208 & 16001 & 58144 & 35 & 0.04 & 58.7 & 0.131131 & 0.087087 & 15 & 131030369 & 15 & 131996123 & 15 & 131 & 0.032 & 0.6934 & 6.02 \\
\hline 2209 & 27936 & 56680 & 26 & 0.03 & 74.1 & 0.102102 & 0.073073 & 15 & 132116033 & 15 & 132949028 & 15 & 132 & 0.042 & 0.7022 & 5.36 \\
\hline 2210 & 27944 & 27951 & 29 & 0.05 & 45.21 & 0.14014 & 0.1001 & 15 & 133052815 & 15 & 133970166 & 15 & 133 & 0.024 & 0.6806 & 6.83 \\
\hline 2211 & 36231 & 12532 & 22 & 0.03 & 78.19 & 0.105105 & 0.065065 & 15 & 134006803 & 15 & 134994861 & 15 & 134 & 0.044 & 0.7041 & 5.22 \\
\hline 2212 & 27983 & 12553 & 34 & 0.16 & 15.07 & 0.197197 & 0.158158 & 15 & 135059821 & 15 & 135999617 & 15 & 135 & 0.008 & 0.5752 & 10.21 \\
\hline 2213 & 12552 & 44258 & 29 & 0.05 & 49.86 & 0.133133 & 0.098098 & 15 & 136021602 & 15 & 136981095 & 15 & 136 & 0.027 & 0.6859 & 6.51 \\
\hline 2214 & 12540 & 44255 & 23 & 0.04 & 63.48 & 0.101101 & 0.069069 & 15 & 137038624 & 15 & 137973386 & 15 & 137 & 0.035 & 0.6971 & 5.8 \\
\hline 2215 & 27964 & 60269 & 21 & 0.04 & 55.66 & 0.096096 & 0.062062 & 15 & 138046832 & 15 & 138987291 & 15 & 138 & 0.03 & 0.6919 & 6.18 \\
\hline 2216 & 12566 & 57999 & 26 & 0.03 & 80.26 & 0.105105 & 0.059059 & 15 & 139070668 & 15 & 139947117 & 15 & 139 & 0.045 & 0.7049 & 5.15 \\
\hline 2217 & 49597 & 12580 & 33 & 0.03 & 66.56 & 0.134134 & 0.078078 & 15 & 140007776 & 15 & 140944699 & 15 & 140 & 0.037 & 0.6993 & 5.67 \\
\hline 2218 & 12581 & 12589 & 23 & 0.03 & 78.85 & 0.119119 & 0.079079 & 15 & 141026209 & 15 & 141938770 & 15 & 141 & 0.045 & 0.7042 & 5.19 \\
\hline 2219 & 15908 & 12594 & 30 & 0.04 & 56.52 & 0.112112 & 0.073073 & 15 & 142018466 & 15 & 142992475 & 15 & 142 & 0.031 & 0.6916 & 6.11 \\
\hline 2220 & 55924 & 50445 & 24 & 0.02 & 89.44 & 0.094094 & 0.054054 & 15 & 143021121 & 15 & 143987209 & 15 & 143 & 0.052 & 0.7076 & 4.84 \\
\hline
\end{tabular}




\begin{tabular}{|c|c|c|c|c|c|c|c|c|c|c|c|c|c|c|c|c|}
\hline 2221 & 50825 & 39364 & 35 & 0.04 & 55.29 & 0.16016 & 0.098098 & 15 & 144002309 & 15 & 144989420 & 15 & 144 & 0.03 & 0.6921 & 6.2 \\
\hline 2222 & 12608 & 44292 & 27 & 0.02 & 86.67 & 0.103103 & 0.067067 & 15 & 145007171 & 15 & 145990809 & 15 & 145 & 0.05 & 0.7074 & 4.93 \\
\hline 2223 & 49601 & 44295 & 26 & 0.04 & 64.51 & 0.127127 & 0.08008 & 15 & 146016611 & 15 & 146927575 & 15 & 146 & 0.036 & 0.6977 & 5.76 \\
\hline 2224 & 28039 & 12623 & 23 & 0.06 & 39.26 & 0.116116 & 0.071071 & 15 & 147013651 & 15 & 147944719 & 15 & 147 & 0.021 & 0.6687 & 7.22 \\
\hline 2225 & 12624 & 50103 & 16 & 0.01 & 92.35 & 0.046046 & 0.03003 & 15 & 148072735 & 15 & 148972866 & 15 & 148 & 0.054 & 0.7082 & 4.76 \\
\hline 2226 & 12631 & 16591 & 25 & 0.02 & 80.79 & 0.109109 & 0.067067 & 15 & 149140529 & 15 & 149953164 & 15 & 149 & 0.046 & 0.7052 & 5.13 \\
\hline 2227 & 31461 & 31910 & 39 & 0.05 & 47.73 & 0.161161 & 0.11011 & 15 & 150020921 & 15 & 150963456 & 15 & 150 & 0.026 & 0.6821 & 6.6 \\
\hline 2228 & 49606 & 28051 & 34 & 0.05 & 39.87 & 0.148148 & 0.098098 & 15 & 151027575 & 15 & 151968517 & 15 & 151 & 0.021 & 0.6701 & 7.17 \\
\hline 2229 & 55291 & 30146 & 18 & 0.02 & 82.34 & 0.083083 & 0.052052 & 15 & 152015494 & 15 & 152938240 & 15 & 152 & 0.047 & 0.7049 & 5.07 \\
\hline 2230 & 53489 & 32220 & 11 & 0.02 & 89.19 & 0.061061 & 0.042042 & 15 & 153014360 & 15 & 153829568 & 15 & 153 & 0.052 & 0.7078 & 4.85 \\
\hline 2231 & 18167 & 14535 & 7 & 0.01 & 96.15 & 0.035035 & 0.015015 & 15 & 154044342 & 15 & 154569347 & 15 & 154 & 0.057 & 0.709 & 4.64 \\
\hline 2232 & 33451 & 51228 & 4 & 0 & 96.56 & 0.018018 & 0.006006 & 15 & 155667846 & 15 & 155782508 & 15 & 155 & 0.057 & 0.7093 & 4.63 \\
\hline 2233 & 55732 & 28058 & 11 & 0.02 & 90.87 & 0.051051 & 0.03003 & 15 & 156048507 & 15 & 156955591 & 15 & 156 & 0.053 & 0.708 & 4.8 \\
\hline 2234 & 12643 & 14963 & 11 & 0.01 & 94.26 & 0.036036 & 0.027027 & 15 & 157038929 & 15 & 157425891 & 15 & 157 & 0.055 & 0.7083 & 4.69 \\
\hline 2235 & 44393 & 12650 & 20 & 0.17 & 13.94 & 0.126126 & 0.098098 & 16 & 15148 & 16 & 973962 & 16 & 0 & 0.007 & 0.5689 & 10.58 \\
\hline 2236 & 28064 & 16889 & 21 & 0.05 & 42.92 & 0.094094 & 0.073073 & 16 & 1016806 & 16 & 1883120 & 16 & 1 & 0.023 & 0.6766 & 7 \\
\hline 2237 & 55021 & 44306 & 16 & 0.12 & 18.58 & 0.093093 & 0.071071 & 16 & 2142400 & 16 & 2996993 & 16 & 2 & 0.01 & 0.5938 & 9.43 \\
\hline 2238 & 28072 & 28078 & 30 & 1.46 & 4.61 & 0.308308 & 0.278278 & 16 & 3010789 & 16 & 3964902 & 16 & 3 & 0.003 & 0.4668 & 13.05 \\
\hline 2239 & 12664 & 12674 & 26 & 0.04 & 61.51 & 0.11011 & 0.073073 & 16 & 4016044 & 16 & 4964893 & 16 & 4 & 0.034 & 0.6962 & 5.9 \\
\hline 2240 & 44315 & 14603 & 27 & 0.05 & 48.25 & 0.139139 & 0.094094 & 16 & 5008766 & 16 & 5923147 & 16 & 5 & 0.026 & 0.6826 & 6.57 \\
\hline 2241 & 59592 & 28090 & 36 & 0.09 & 23.16 & 0.173173 & 0.117117 & 16 & 6013914 & 16 & 6989080 & 16 & 6 & 0.012 & 0.62 & 8.82 \\
\hline 2242 & 49614 & 39381 & 11 & 0.02 & 85.92 & 0.062062 & 0.038038 & 16 & 7013478 & 16 & 7634155 & 16 & 7 & 0.049 & 0.7066 & 4.95 \\
\hline 2243 & 52258 & 16934 & 8 & 0 & 96.3 & 0.028028 & 0.022022 & 16 & 8015400 & 16 & 8752527 & 16 & 8 & 0.057 & 0.7091 & 4.64 \\
\hline 2244 & 28095 & 44324 & 19 & 0.03 & 79.68 & 0.081081 & 0.054054 & 16 & 9060044 & 16 & 9999843 & 16 & 9 & 0.045 & 0.7049 & 5.17 \\
\hline 2245 & 44325 & 28100 & 10 & 0.01 & 94 & 0.036036 & 0.019019 & 16 & 10023136 & 16 & 10985374 & 16 & 10 & 0.055 & 0.7084 & 4.7 \\
\hline 2246 & 44326 & 52133 & 21 & 0.03 & 79.35 & 0.087087 & 0.057057 & 16 & 11013570 & 16 & 11999198 & 16 & 11 & 0.045 & 0.7047 & 5.18 \\
\hline 2247 & 12709 & 28104 & 19 & 0.02 & 85.32 & 0.066066 & 0.047047 & 16 & 12073651 & 16 & 12976031 & 16 & 12 & 0.049 & 0.7061 & 4.97 \\
\hline 2248 & 12717 & 39394 & 13 & 0.03 & 70.91 & 0.063063 & 0.051051 & 16 & 13007287 & 16 & 13970080 & 16 & 13 & 0.04 & 0.7004 & 5.48 \\
\hline 2249 & 12721 & 30312 & 18 & 0.04 & 61.28 & 0.089089 & 0.075075 & 16 & 14013912 & 16 & 14823848 & 16 & 14 & 0.034 & 0.6962 & 5.91 \\
\hline 2250 & 33334 & 45602 & 19 & 0.02 & 82.41 & 0.085085 & 0.056056 & 16 & 15022443 & 16 & 15992155 & 16 & 15 & 0.047 & 0.7049 & 5.06 \\
\hline 2251 & 29802 & 52603 & 10 & 0.01 & 96 & 0.033033 & 0.02002 & 16 & 16006121 & 16 & 16893013 & 16 & 16 & 0.056 & 0.709 & 4.65 \\
\hline 2252 & 56725 & 39399 & 16 & 0.02 & 88.1 & 0.069069 & 0.041041 & 16 & 17080961 & 16 & 17972248 & 16 & 17 & 0.051 & 0.7078 & 4.89 \\
\hline 2253 & 39400 & 28125 & 35 & 0.05 & 41.94 & 0.153153 & 0.101101 & 16 & 18027175 & 16 & 18998881 & 16 & 18 & 0.022 & 0.6751 & 7.06 \\
\hline 2254 & 28126 & 12747 & 21 & 0.17 & 13.44 & 0.151151 & 0.119119 & 16 & 19022068 & 16 & 19997593 & 16 & 19 & 0.007 & 0.5645 & 10.7 \\
\hline 2255 & 44339 & 32998 & 34 & 0.35 & 8.4 & 0.252252 & 0.204204 & 16 & 20018423 & 16 & 20985329 & 16 & 20 & 0.005 & 0.5182 & 11.97 \\
\hline 2256 & 60138 & 14578 & 28 & 0.13 & 16.61 & 0.156156 & 0.114114 & 16 & 21011283 & 16 & 21993561 & 16 & 21 & 0.009 & 0.584 & 9.84 \\
\hline 2257 & 12758 & 12770 & 24 & 0.07 & 29.35 & 0.124124 & 0.083083 & 16 & 22095735 & 16 & 22991610 & 16 & 22 & 0.016 & 0.6405 & 7.97 \\
\hline 2258 & 12768 & 12772 & 14 & 0.03 & 75.35 & 0.069069 & 0.049049 & 16 & 23020285 & 16 & 23986785 & 16 & 23 & 0.043 & 0.7021 & 5.3 \\
\hline 2259 & 30054 & 44360 & 28 & 0.06 & 35.28 & 0.138138 & 0.098098 & 16 & 24096772 & 16 & 24940106 & 16 & 24 & 0.019 & 0.6559 & 7.43 \\
\hline
\end{tabular}




\begin{tabular}{|c|c|c|c|c|c|c|c|c|c|c|c|c|c|c|c|c|}
\hline 2260 & 18827 & 12787 & 23 & 0.02 & 80.86 & 0.095095 & 0.055055 & 16 & 25038049 & 16 & 25999742 & 16 & 25 & 0.046 & 0.7052 & 5.13 \\
\hline 2261 & 51282 & 53898 & 30 & 0.03 & 65.73 & 0.122122 & 0.084084 & 16 & 26019525 & 16 & 26898740 & 16 & 26 & 0.036 & 0.6985 & 5.7 \\
\hline 2262 & 39411 & 49620 & 25 & 0.04 & 55.37 & 0.103103 & 0.075075 & 16 & 27017719 & 16 & 27972337 & 16 & 27 & 0.03 & 0.692 & 6.19 \\
\hline 2263 & 12803 & 18480 & 17 & 0.08 & 26.72 & 0.086086 & 0.062062 & 16 & 28006035 & 16 & 28850217 & 16 & 28 & 0.014 & 0.6312 & 8.26 \\
\hline 2264 & 45160 & 44369 & 22 & 0.05 & 43.22 & 0.12012 & 0.085085 & 16 & 29044133 & 16 & 29952113 & 16 & 29 & 0.023 & 0.6778 & 6.99 \\
\hline 2265 & 12813 & 16826 & 23 & 0.06 & 39.04 & 0.111111 & 0.085085 & 16 & 30081210 & 16 & 30976457 & 16 & 30 & 0.021 & 0.6683 & 7.24 \\
\hline 2266 & 12822 & 12829 & 23 & 0.05 & 44.62 & 0.096096 & 0.072072 & 16 & 31016837 & 16 & 31983325 & 16 & 31 & 0.024 & 0.6797 & 6.88 \\
\hline 2267 & 39421 & 12836 & 32 & 0.05 & 44.27 & 0.141141 & 0.093093 & 16 & 32024005 & 16 & 32979912 & 16 & 32 & 0.023 & 0.6787 & 6.9 \\
\hline 2268 & 28176 & 35962 & 28 & 0.04 & 64.23 & 0.12012 & 0.081081 & 16 & 33002382 & 16 & 33954882 & 16 & 33 & 0.035 & 0.6977 & 5.77 \\
\hline 2269 & 12847 & 36370 & 22 & 0.04 & 57.4 & 0.098098 & 0.069069 & 16 & 34003949 & 16 & 34854206 & 16 & 34 & 0.032 & 0.6924 & 6.08 \\
\hline 2270 & 31841 & 28189 & 31 & 0.05 & 49.26 & 0.129129 & 0.086086 & 16 & 35014537 & 16 & 35997155 & 16 & 35 & 0.027 & 0.6847 & 6.53 \\
\hline 2271 & 28190 & 53481 & 28 & 0.03 & 68.95 & 0.114114 & 0.083083 & 16 & 36026675 & 16 & 36933143 & 16 & 36 & 0.038 & 0.7001 & 5.57 \\
\hline 2272 & 61969 & 28200 & 25 & 0.06 & 33.25 & 0.106106 & 0.075075 & 16 & 37039866 & 16 & 37966104 & 16 & 37 & 0.018 & 0.6506 & 7.64 \\
\hline 2273 & 12883 & 12890 & 27 & 0.12 & 17.51 & 0.155155 & 0.108108 & 16 & 38004706 & 16 & 38989787 & 16 & 38 & 0.009 & 0.5915 & 9.74 \\
\hline 2274 & 59058 & 55382 & 19 & 0.09 & 21.88 & 0.111111 & 0.076076 & 16 & 39019226 & 16 & 39993955 & 16 & 39 & 0.011 & 0.6126 & 8.96 \\
\hline 2275 & 12894 & 12902 & 18 & 0.11 & 18.81 & 0.097097 & 0.071071 & 16 & 40054179 & 16 & 40966989 & 16 & 40 & 0.01 & 0.5953 & 9.39 \\
\hline 2276 & 39440 & 12909 & 21 & 0.1 & 20.91 & 0.133133 & 0.097097 & 16 & 41035539 & 16 & 41985204 & 16 & 41 & 0.011 & 0.6053 & 9.03 \\
\hline 2277 & 12910 & 53804 & 15 & 0.04 & 57.04 & 0.07007 & 0.044044 & 16 & 42082484 & 16 & 42978682 & 16 & 42 & 0.031 & 0.6921 & 6.1 \\
\hline 2278 & 12918 & 53338 & 8 & 0.02 & 89.37 & 0.038038 & 0.024024 & 16 & 43093614 & 16 & 43762162 & 16 & 43 & 0.052 & 0.7076 & 4.84 \\
\hline 2279 & 14366 & 28225 & 16 & 0.03 & 78.64 & 0.075075 & 0.053053 & 16 & 44062106 & 16 & 44919236 & 16 & 44 & 0.044 & 0.704 & 5.2 \\
\hline 2280 & 39448 & 28227 & 16 & 0.03 & 72.99 & 0.08008 & 0.054054 & 16 & 45027412 & 16 & 45829750 & 16 & 45 & 0.041 & 0.7015 & 5.4 \\
\hline 2281 & 39453 & 12934 & 14 & 0.02 & 84.34 & 0.073073 & 0.041041 & 16 & 46066010 & 16 & 46997060 & 16 & 46 & 0.049 & 0.7055 & 4.99 \\
\hline 2282 & 61888 & 12939 & 12 & 0.02 & 91.72 & 0.051051 & 0.029029 & 16 & 47011906 & 16 & 47944374 & 16 & 47 & 0.053 & 0.7081 & 4.78 \\
\hline 2283 & 12940 & 28241 & 25 & 0.03 & 68.45 & 0.103103 & 0.065065 & 16 & 48020257 & 16 & 48994238 & 16 & 48 & 0.038 & 0.6995 & 5.58 \\
\hline 2284 & 28242 & 28245 & 20 & 0.02 & 89.47 & 0.095095 & 0.056056 & 16 & 49050041 & 16 & 49868072 & 16 & 49 & 0.052 & 0.7077 & 4.84 \\
\hline 2285 & 56469 & 53505 & 14 & 0.02 & 81.81 & 0.075075 & 0.051051 & 16 & 50384871 & 16 & 50977092 & 16 & 50 & 0.047 & 0.7051 & 5.09 \\
\hline 2286 & 30153 & 48339 & 11 & 0.01 & 91.93 & 0.053053 & 0.034034 & 16 & 51117642 & 16 & 51990539 & 16 & 51 & 0.053 & 0.7081 & 4.77 \\
\hline 2287 & 49627 & 28253 & 15 & 0.03 & 67.55 & 0.071071 & 0.05005 & 16 & 52012266 & 16 & 52903897 & 16 & 52 & 0.038 & 0.6991 & 5.62 \\
\hline 2288 & 44400 & 44402 & 9 & 0.01 & 96.18 & 0.039039 & 0.02002 & 16 & 53033170 & 16 & 53927692 & 16 & 53 & 0.057 & 0.7091 & 4.64 \\
\hline 2289 & 44403 & 51816 & 8 & 0.01 & 95.99 & 0.025025 & 0.015015 & 16 & 54031268 & 16 & 54979717 & 16 & 54 & 0.056 & 0.709 & 4.65 \\
\hline 2290 & 35201 & 28257 & 15 & 0.03 & 70.98 & 0.074074 & 0.048048 & 16 & 55330045 & 16 & 55993053 & 16 & 55 & 0.04 & 0.7004 & 5.48 \\
\hline 2291 & 49628 & 44413 & 20 & 0.02 & 89 & 0.084084 & 0.039039 & 16 & 56029991 & 16 & 56950872 & 16 & 56 & 0.052 & 0.7075 & 4.86 \\
\hline 2292 & 28260 & 25466 & 17 & 0.02 & 83.98 & 0.07007 & 0.045045 & 16 & 57003945 & 16 & 57990322 & 16 & 57 & 0.048 & 0.7054 & 5 \\
\hline 2293 & 28264 & 28269 & 13 & 0.01 & 93.33 & 0.057057 & 0.027027 & 16 & 58031082 & 16 & 58593026 & 16 & 58 & 0.055 & 0.7081 & 4.72 \\
\hline 2294 & 44419 & 44425 & 26 & 0.03 & 74.22 & 0.124124 & 0.067067 & 16 & 59138735 & 16 & 59988426 & 16 & 59 & 0.042 & 0.7021 & 5.35 \\
\hline 2295 & 28275 & 56116 & 27 & 0.04 & 64.83 & 0.119119 & 0.079079 & 16 & 60082082 & 16 & 60988882 & 16 & 60 & 0.036 & 0.6979 & 5.74 \\
\hline 2296 & 35131 & 28284 & 19 & 0.03 & 76.17 & 0.088088 & 0.057057 & 16 & 61006434 & 16 & 61995301 & 16 & 61 & 0.043 & 0.7027 & 5.28 \\
\hline 2297 & 44430 & 13014 & 16 & 0.02 & 84.6 & 0.058058 & 0.034034 & 16 & 62091147 & 16 & 62939945 & 16 & 62 & 0.049 & 0.7054 & 4.98 \\
\hline 2298 & 13017 & 34982 & 15 & 0.02 & 88.76 & 0.062062 & 0.039039 & 16 & 63072207 & 16 & 63944017 & 16 & 63 & 0.051 & 0.7077 & 4.87 \\
\hline
\end{tabular}




\begin{tabular}{|c|c|c|c|c|c|c|c|c|c|c|c|c|c|c|c|c|}
\hline 2299 & 13022 & 44435 & 24 & 0.03 & 75.18 & 0.11011 & 0.073073 & 16 & 64085517 & 16 & 64990189 & 16 & 64 & 0.042 & 0.7022 & 5.31 \\
\hline 2300 & 44436 & 13031 & 17 & 0.03 & 74.33 & 0.09009 & 0.052052 & 16 & 65033887 & 16 & 65908026 & 16 & 65 & 0.042 & 0.7017 & 5.34 \\
\hline 2301 & 13036 & 28297 & 13 & 0.01 & 95.85 & 0.044044 & 0.021021 & 16 & 66016093 & 16 & 66914976 & 16 & 66 & 0.056 & 0.7089 & 4.65 \\
\hline 2302 & 59624 & 61393 & 13 & 0.02 & 89.22 & 0.07007 & 0.042042 & 16 & 67092208 & 16 & 67897442 & 16 & 67 & 0.052 & 0.7078 & 4.85 \\
\hline 2303 & 45432 & 28301 & 22 & 0.02 & 81.16 & 0.089089 & 0.064064 & 16 & 68067101 & 16 & 68845016 & 16 & 68 & 0.046 & 0.7051 & 5.12 \\
\hline 2304 & 49638 & 28309 & 16 & 0.02 & 81.67 & 0.084084 & 0.051051 & 16 & 69042784 & 16 & 69938536 & 16 & 69 & 0.046 & 0.7053 & 5.1 \\
\hline 2305 & 44442 & 13063 & 27 & 0.05 & 46.17 & 0.136136 & 0.086086 & 16 & 70031425 & 16 & 70989867 & 16 & 70 & 0.025 & 0.6818 & 6.76 \\
\hline 2306 & 28315 & 31773 & 25 & 0.03 & 65.14 & 0.118118 & 0.061061 & 16 & 71043012 & 16 & 71984043 & 16 & 71 & 0.036 & 0.6984 & 5.73 \\
\hline 2307 & 51174 & 58581 & 16 & 0.02 & 90.42 & 0.059059 & 0.028028 & 16 & 72137790 & 16 & 72974908 & 16 & 72 & 0.053 & 0.7077 & 4.81 \\
\hline 2308 & 28320 & 49641 & 27 & 0.06 & 33.06 & 0.134134 & 0.094094 & 16 & 73039198 & 16 & 73996806 & 16 & 73 & 0.018 & 0.6505 & 7.65 \\
\hline 2309 & 13096 & 13106 & 32 & 0.04 & 63.84 & 0.137137 & 0.08008 & 16 & 74053569 & 16 & 74982852 & 16 & 74 & 0.035 & 0.6972 & 5.78 \\
\hline 2310 & 28331 & 54335 & 37 & 0.11 & 19.03 & 0.197197 & 0.138138 & 16 & 75024639 & 16 & 75983297 & 16 & 75 & 0.01 & 0.5969 & 9.37 \\
\hline 2311 & 28336 & 57447 & 25 & 0.04 & 59.47 & 0.103103 & 0.072072 & 16 & 76026438 & 16 & 76990076 & 16 & 76 & 0.033 & 0.6943 & 5.98 \\
\hline 2312 & 56411 & 56478 & 29 & 0.05 & 40.68 & 0.137137 & 0.086086 & 16 & 77046740 & 16 & 77939638 & 16 & 77 & 0.022 & 0.672 & 7.13 \\
\hline 2313 & 44466 & 44470 & 28 & 0.02 & 82.55 & 0.11011 & 0.064064 & 16 & 78001888 & 16 & 78984075 & 16 & 78 & 0.047 & 0.7048 & 5.06 \\
\hline 2314 & 39478 & 28358 & 27 & 0.03 & 77.87 & 0.116116 & 0.072072 & 16 & 79012137 & 16 & 79991804 & 16 & 79 & 0.044 & 0.7038 & 5.23 \\
\hline 2315 & 44471 & 57102 & 29 & 0.03 & 72.78 & 0.109109 & 0.071071 & 16 & 80020682 & 16 & 80946630 & 16 & 80 & 0.041 & 0.7014 & 5.41 \\
\hline 2316 & 17367 & 13154 & 29 & 0.02 & 81.86 & 0.112112 & 0.072072 & 16 & 81058129 & 16 & 81997191 & 16 & 81 & 0.047 & 0.7051 & 5.09 \\
\hline 2317 & 44476 & 28376 & 39 & 0.05 & 41.53 & 0.175175 & 0.115115 & 16 & 82017160 & 16 & 82981442 & 16 & 82 & 0.022 & 0.6741 & 7.1 \\
\hline 2318 & 52112 & 45828 & 32 & 0.04 & 60.54 & 0.124124 & 0.079079 & 16 & 83023456 & 16 & 83958725 & 16 & 83 & 0.033 & 0.696 & 5.95 \\
\hline 2319 & 32088 & 45124 & 21 & 0.03 & 76.2 & 0.093093 & 0.059059 & 16 & 84005681 & 16 & 84890972 & 16 & 84 & 0.043 & 0.7029 & 5.28 \\
\hline 2320 & 32464 & 49655 & 20 & 0.06 & 32.17 & 0.11011 & 0.073073 & 16 & 85011017 & 16 & 85843098 & 16 & 85 & 0.017 & 0.6492 & 7.75 \\
\hline 2321 & 54930 & 28387 & 13 & 0.01 & 94.01 & 0.049049 & 0.025025 & 16 & 86008309 & 16 & 86875355 & 16 & 86 & 0.055 & 0.7084 & 4.7 \\
\hline 2322 & 28388 & 13180 & 20 & 0.02 & 83.96 & 0.079079 & 0.05005 & 17 & 15175 & 17 & 927919 & 17 & 0 & 0.048 & 0.7054 & 5 \\
\hline 2323 & 57301 & 39484 & 23 & 0.04 & 62.83 & 0.098098 & 0.068068 & 17 & 1018025 & 17 & 1963091 & 17 & 1 & 0.035 & 0.6967 & 5.84 \\
\hline 2324 & 13194 & 13200 & 22 & 0.04 & 52.07 & 0.101101 & 0.07007 & 17 & 2011617 & 17 & 2991834 & 17 & 2 & 0.028 & 0.6896 & 6.41 \\
\hline 2325 & 13203 & 13209 & 18 & 0.05 & 42.36 & 0.093093 & 0.065065 & 17 & 3129112 & 17 & 3989769 & 17 & 3 & 0.022 & 0.6764 & 7.05 \\
\hline 2326 & 28404 & 15858 & 19 & 0.03 & 79.43 & 0.085085 & 0.06006 & 17 & 4055697 & 17 & 4868955 & 17 & 4 & 0.045 & 0.705 & 5.18 \\
\hline 2327 & 45104 & 13228 & 23 & 0.03 & 71.33 & 0.107107 & 0.071071 & 17 & 5041670 & 17 & 5999136 & 17 & 5 & 0.04 & 0.7009 & 5.47 \\
\hline 2328 & 13229 & 35077 & 17 & 0.02 & 89.49 & 0.068068 & 0.036036 & 17 & 6024213 & 17 & 6989362 & 17 & 6 & 0.052 & 0.7077 & 4.84 \\
\hline 2329 & 58631 & 18713 & 20 & 0.07 & 28.21 & 0.098098 & 0.073073 & 17 & 7027710 & 17 & 7923768 & 17 & 7 & 0.015 & 0.6367 & 8.1 \\
\hline 2330 & 44499 & 28423 & 27 & 0.05 & 45.01 & 0.14014 & 0.09009 & 17 & 8048013 & 17 & 8987674 & 17 & 8 & 0.024 & 0.68 & 6.84 \\
\hline 2331 & 32511 & 58474 & 25 & 0.04 & 57.87 & 0.111111 & 0.084084 & 17 & 9014186 & 17 & 9992606 & 17 & 9 & 0.032 & 0.6928 & 6.05 \\
\hline 2332 & 28445 & 49658 & 21 & 0.03 & 73.36 & 0.093093 & 0.062062 & 17 & 10039697 & 17 & 10939739 & 17 & 10 & 0.041 & 0.7017 & 5.38 \\
\hline 2333 & 13268 & 28437 & 31 & 0.03 & 66.97 & 0.115115 & 0.074074 & 17 & 11026407 & 17 & 11952380 & 17 & 11 & 0.037 & 0.6993 & 5.65 \\
\hline 2334 & 48019 & 52200 & 23 & 0.03 & 75.61 & 0.088088 & 0.055055 & 17 & 12059087 & 17 & 12967074 & 17 & 12 & 0.043 & 0.7026 & 5.3 \\
\hline 2335 & 28428 & 28453 & 25 & 0.03 & 78.93 & 0.119119 & 0.07007 & 17 & 13015931 & 17 & 13983032 & 17 & 13 & 0.045 & 0.7043 & 5.19 \\
\hline 2336 & 28452 & 30353 & 16 & 0.02 & 86.07 & 0.045045 & 0.034034 & 17 & 14005506 & 17 & 14867578 & 17 & 14 & 0.049 & 0.7066 & 4.95 \\
\hline 2337 & 59244 & 51284 & 13 & 0 & 96.41 & 0.04004 & 0.014014 & 17 & 15101576 & 17 & 15997261 & 17 & 15 & 0.057 & 0.7092 & 4.64 \\
\hline
\end{tabular}




\begin{tabular}{|c|c|c|c|c|c|c|c|c|c|c|c|c|c|c|c|c|}
\hline 2338 & 28456 & 51446 & 16 & 0.05 & 45.11 & 0.097097 & 0.053053 & 17 & 16023225 & 17 & 16985131 & 17 & 16 & 0.024 & 0.6804 & 6.83 \\
\hline 2339 & 60615 & 48027 & 17 & 0.01 & 92.41 & 0.063063 & 0.04004 & 17 & 17004115 & 17 & 17972781 & 17 & 17 & 0.054 & 0.7082 & 4.75 \\
\hline 2340 & 13302 & 13303 & 13 & 0.01 & 95.31 & 0.057057 & 0.033033 & 17 & 18057468 & 17 & 18983971 & 17 & 18 & 0.056 & 0.7086 & 4.67 \\
\hline 2341 & 13304 & 44529 & 30 & 0.04 & 62.61 & 0.129129 & 0.08008 & 17 & 19005250 & 17 & 19978814 & 17 & 19 & 0.034 & 0.6963 & 5.84 \\
\hline 2342 & 13318 & 28472 & 25 & 0.03 & 72.6 & 0.121121 & 0.074074 & 17 & 20006406 & 17 & 20971854 & 17 & 20 & 0.041 & 0.701 & 5.41 \\
\hline 2343 & 13329 & 13335 & 24 & 0.03 & 65.28 & 0.12012 & 0.068068 & 17 & 21010426 & 17 & 21985990 & 17 & 21 & 0.036 & 0.6984 & 5.72 \\
\hline 2344 & 13336 & 54404 & 27 & 0.04 & 52.84 & 0.124124 & 0.085085 & 17 & 22009494 & 17 & 22937340 & 17 & 22 & 0.028 & 0.6915 & 6.39 \\
\hline 2345 & 28486 & 48036 & 25 & 0.03 & 69.79 & 0.116116 & 0.061061 & 17 & 23024619 & 17 & 23961801 & 17 & 23 & 0.039 & 0.7 & 5.53 \\
\hline 2346 & 13351 & 28499 & 24 & 0.06 & 33.5 & 0.133133 & 0.084084 & 17 & 24013333 & 17 & 24856383 & 17 & 24 & 0.018 & 0.6514 & 7.62 \\
\hline 2347 & 13361 & 61623 & 26 & 0.18 & 12.41 & 0.161161 & 0.123123 & 17 & 25073964 & 17 & 25894376 & 17 & 25 & 0.006 & 0.5558 & 10.89 \\
\hline 2348 & 28504 & 56461 & 19 & 0.05 & 39.65 & 0.099099 & 0.063063 & 17 & 26152267 & 17 & 26991897 & 17 & 26 & 0.021 & 0.6695 & 7.19 \\
\hline 2349 & 13377 & 13386 & 21 & 0.04 & 50.66 & 0.091091 & 0.069069 & 17 & 27035671 & 17 & 27855668 & 17 & 27 & 0.027 & 0.6868 & 6.46 \\
\hline 2350 & 13389 & 61797 & 21 & 0.03 & 65.59 & 0.102102 & 0.061061 & 17 & 28091145 & 17 & 28931409 & 17 & 28 & 0.036 & 0.6986 & 5.71 \\
\hline 2351 & 13393 & 39515 & 26 & 0.08 & 25.32 & 0.145145 & 0.111111 & 17 & 29081895 & 17 & 29964161 & 17 & 29 & 0.013 & 0.6276 & 8.47 \\
\hline 2352 & 61285 & 50865 & 27 & 0.04 & 59.12 & 0.147147 & 0.09009 & 17 & 30009989 & 17 & 30994140 & 17 & 30 & 0.032 & 0.6942 & 6 \\
\hline 2353 & 13407 & 13417 & 31 & 0.05 & 47.55 & 0.141141 & 0.1001 & 17 & 31034493 & 17 & 31972942 & 17 & 31 & 0.026 & 0.6821 & 6.62 \\
\hline 2354 & 60959 & 44566 & 25 & 0.02 & 84.27 & 0.098098 & 0.061061 & 17 & 32009043 & 17 & 32986542 & 17 & 32 & 0.048 & 0.7055 & 4.99 \\
\hline 2355 & 13423 & 49666 & 31 & 0.03 & 73.89 & 0.131131 & 0.082082 & 17 & 33009145 & 17 & 33993354 & 17 & 33 & 0.042 & 0.7022 & 5.36 \\
\hline 2356 & 13429 & 44578 & 27 & 0.06 & 39.43 & 0.122122 & 0.081081 & 17 & 34032332 & 17 & 34975107 & 17 & 34 & 0.021 & 0.6689 & 7.19 \\
\hline 2357 & 33725 & 54545 & 12 & 0.02 & 83.8 & 0.052052 & 0.037037 & 17 & 35295082 & 17 & 35935548 & 17 & 35 & 0.048 & 0.7054 & 5.01 \\
\hline 2358 & 28563 & 44588 & 30 & 0.06 & 31.59 & 0.158158 & 0.124124 & 17 & 36007878 & 17 & 36999204 & 17 & 36 & 0.017 & 0.6468 & 7.77 \\
\hline 2359 & 44589 & 44602 & 28 & 0.1 & 20.2 & 0.155155 & 0.114114 & 17 & 37018486 & 17 & 37982038 & 17 & 37 & 0.011 & 0.6011 & 9.13 \\
\hline 2360 & 28578 & 45482 & 21 & 0.11 & 19.57 & 0.119119 & 0.094094 & 17 & 38020355 & 17 & 38842836 & 17 & 38 & 0.01 & 0.6006 & 9.31 \\
\hline 2361 & 33221 & 13474 & 25 & 0.18 & 12.23 & 0.159159 & 0.131131 & 17 & 39094798 & 17 & 39980954 & 17 & 39 & 0.006 & 0.5563 & 11.02 \\
\hline 2362 & 13473 & 44612 & 20 & 0.14 & 15.79 & 0.141141 & 0.104104 & 17 & 40021954 & 17 & 40996438 & 17 & 40 & 0.008 & 0.5782 & 9.99 \\
\hline 2363 & 13478 & 13488 & 35 & 0.06 & 33.31 & 0.175175 & 0.117117 & 17 & 41034762 & 17 & 41957670 & 17 & 41 & 0.018 & 0.6508 & 7.63 \\
\hline 2364 & 13489 & 44623 & 17 & 0.02 & 83.5 & 0.08008 & 0.049049 & 17 & 42024585 & 17 & 42998995 & 17 & 42 & 0.048 & 0.7051 & 5.02 \\
\hline 2365 & 44622 & 55572 & 21 & 0.04 & 62.76 & 0.104104 & 0.055055 & 17 & 43013416 & 17 & 43931913 & 17 & 43 & 0.035 & 0.6965 & 5.84 \\
\hline 2366 & 39525 & 28617 & 19 & 0.03 & 71.17 & 0.097097 & 0.064064 & 17 & 44237331 & 17 & 44986812 & 17 & 44 & 0.04 & 0.7006 & 5.47 \\
\hline 2367 & 44625 & 44633 & 27 & 0.03 & 72.75 & 0.112112 & 0.075075 & 17 & 45002413 & 17 & 45985972 & 17 & 45 & 0.041 & 0.7014 & 5.41 \\
\hline 2368 & 55597 & 54153 & 26 & 0.02 & 80.61 & 0.101101 & 0.047047 & 17 & 46016364 & 17 & 46995794 & 17 & 46 & 0.046 & 0.7051 & 5.13 \\
\hline 2369 & 13525 & 56669 & 22 & 0.06 & 38.37 & 0.105105 & 0.07007 & 17 & 47086750 & 17 & 47981889 & 17 & 47 & 0.02 & 0.6665 & 7.27 \\
\hline 2370 & 53621 & 28634 & 19 & 0.01 & 95.06 & 0.08008 & 0.043043 & 17 & 48108999 & 17 & 48985762 & 17 & 48 & 0.056 & 0.7087 & 4.67 \\
\hline 2371 & 28637 & 28639 & 17 & 0.19 & 11.68 & 0.107107 & 0.072072 & 17 & 49014076 & 17 & 49985893 & 17 & 49 & 0.006 & 0.55 & 11.09 \\
\hline 2372 & 13537 & 28644 & 29 & 0.02 & 84.65 & 0.104104 & 0.064064 & 17 & 50025811 & 17 & 50988229 & 17 & 50 & 0.049 & 0.7055 & 4.98 \\
\hline 2373 & 16275 & 32952 & 32 & 0.04 & 51.54 & 0.13013 & 0.091091 & 17 & 51005799 & 17 & 51974554 & 17 & 51 & 0.028 & 0.689 & 6.44 \\
\hline 2374 & 33960 & 28654 & 24 & 0.02 & 80.98 & 0.103103 & 0.069069 & 17 & 52023322 & 17 & 52970123 & 17 & 52 & 0.046 & 0.705 & 5.12 \\
\hline 2375 & 57872 & 13567 & 26 & 0.03 & 75.09 & 0.098098 & 0.06006 & 17 & 53166870 & 17 & 53996435 & 17 & 53 & 0.042 & 0.7022 & 5.31 \\
\hline 2376 & 13568 & 28664 & 20 & 0.02 & 88.63 & 0.098098 & 0.048048 & 17 & 54025507 & 17 & 54986042 & 17 & 54 & 0.051 & 0.7078 & 4.87 \\
\hline
\end{tabular}




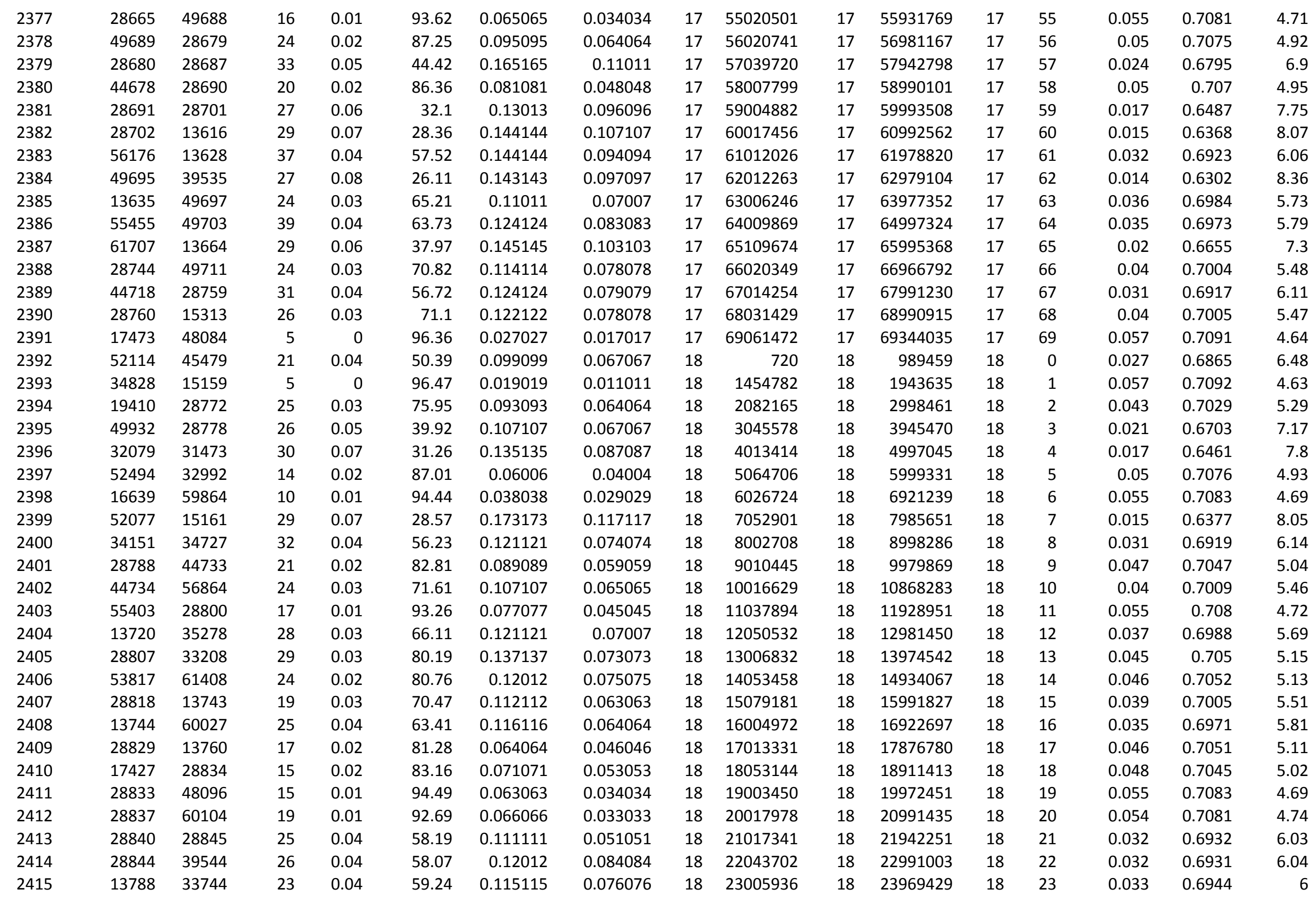




\begin{tabular}{|c|c|c|c|c|c|c|c|c|c|c|c|c|c|c|c|c|}
\hline 2416 & 55471 & 48102 & 14 & 0.01 & 92.89 & 0.066066 & 0.035035 & 18 & 24076311 & 18 & 24990339 & 18 & 24 & 0.054 & 0.7081 & 4.74 \\
\hline 2417 & 62026 & 28864 & 30 & 0.03 & 68.35 & 0.105105 & 0.065065 & 18 & 25027827 & 18 & 25981606 & 18 & 25 & 0.038 & 0.6995 & 5.59 \\
\hline 2418 & 13810 & 28871 & 29 & 0.05 & 47.92 & 0.14014 & 0.085085 & 18 & 26004852 & 18 & 26977792 & 18 & 26 & 0.026 & 0.6822 & 6.59 \\
\hline 2419 & 13823 & 13835 & 31 & 0.05 & 43.52 & 0.145145 & 0.091091 & 18 & 27001689 & 18 & 27981092 & 18 & 27 & 0.023 & 0.6781 & 6.97 \\
\hline 2420 & 13836 & 16284 & 14 & 0.02 & 84.14 & 0.078078 & 0.048048 & 18 & 28006207 & 18 & 28845095 & 18 & 28 & 0.048 & 0.7053 & 4.99 \\
\hline 2421 & 13843 & 56632 & 16 & 0.03 & 79.91 & 0.077077 & 0.044044 & 18 & 29008406 & 18 & 29980931 & 18 & 29 & 0.045 & 0.7048 & 5.16 \\
\hline 2422 & 55319 & 13856 & 27 & 0.03 & 69.18 & 0.11011 & 0.069069 & 18 & 30091996 & 18 & 30963795 & 18 & 30 & 0.039 & 0.6998 & 5.56 \\
\hline 2423 & 50754 & 61143 & 26 & 0.04 & 56.68 & 0.118118 & 0.08008 & 18 & 31024660 & 18 & 31995431 & 18 & 31 & 0.031 & 0.6917 & 6.11 \\
\hline 2424 & 13862 & 51267 & 24 & 0.04 & 61.17 & 0.091091 & 0.062062 & 18 & 32091507 & 18 & 32986753 & 18 & 32 & 0.034 & 0.6963 & 5.92 \\
\hline 2425 & 28902 & 57284 & 9 & 0.02 & 90.08 & 0.052052 & 0.034034 & 18 & 33106672 & 18 & 33816366 & 18 & 33 & 0.052 & 0.7078 & 4.82 \\
\hline 2426 & 13876 & 58370 & 12 & 0.01 & 95.13 & 0.049049 & 0.029029 & 18 & 34013193 & 18 & 34956874 & 18 & 34 & 0.056 & 0.7087 & 4.67 \\
\hline 2427 & 30201 & 30921 & 18 & 0.02 & 89.11 & 0.067067 & 0.046046 & 18 & 35243439 & 18 & 35993025 & 18 & 35 & 0.052 & 0.7076 & 4.85 \\
\hline 2428 & 36233 & 55359 & 21 & 0.02 & 88.67 & 0.087087 & 0.053053 & 18 & 36145263 & 18 & 36938024 & 18 & 36 & 0.051 & 0.7078 & 4.87 \\
\hline 2429 & 31656 & 13898 & 24 & 0.02 & 81.3 & 0.084084 & 0.053053 & 18 & 37000204 & 18 & 37926205 & 18 & 37 & 0.046 & 0.7052 & 5.11 \\
\hline 2430 & 32781 & 57380 & 17 & 0.03 & 71.83 & 0.076076 & 0.052052 & 18 & 38093304 & 18 & 38804587 & 18 & 38 & 0.04 & 0.7011 & 5.45 \\
\hline 2431 & 28921 & 60700 & 12 & 0.02 & 81.08 & 0.052052 & 0.037037 & 18 & 39043858 & 18 & 39678645 & 18 & 39 & 0.046 & 0.705 & 5.12 \\
\hline 2432 & 31687 & 44795 & 31 & 0.04 & 52.37 & 0.126126 & 0.082082 & 18 & 40067536 & 18 & 40992858 & 18 & 40 & 0.028 & 0.6905 & 6.4 \\
\hline 2433 & 28934 & 36288 & 23 & 0.05 & 45.45 & 0.122122 & 0.084084 & 18 & 41063892 & 18 & 41968802 & 18 & 41 & 0.024 & 0.6811 & 6.82 \\
\hline 2434 & 28937 & 48119 & 22 & 0.03 & 77.52 & 0.083083 & 0.051051 & 18 & 42030856 & 18 & 42938796 & 18 & 42 & 0.044 & 0.7036 & 5.24 \\
\hline 2435 & 28947 & 48120 & 24 & 0.03 & 67.78 & 0.11011 & 0.073073 & 18 & 43012691 & 18 & 43976955 & 18 & 43 & 0.038 & 0.6994 & 5.62 \\
\hline 2436 & 13931 & 28955 & 11 & 0.01 & 95.36 & 0.034034 & 0.02002 & 18 & 44006428 & 18 & 44927369 & 18 & 44 & 0.056 & 0.7087 & 4.66 \\
\hline 2437 & 54052 & 18288 & 22 & 0.05 & 49.22 & 0.109109 & 0.078078 & 18 & 45142069 & 18 & 45949396 & 18 & 45 & 0.027 & 0.6847 & 6.53 \\
\hline 2438 & 55793 & 13941 & 24 & 0.04 & 58.97 & 0.095095 & 0.067067 & 18 & 46190367 & 18 & 46983072 & 18 & 46 & 0.032 & 0.6941 & 6.01 \\
\hline 2439 & 15130 & 30144 & 37 & 0.05 & 39.81 & 0.173173 & 0.094094 & 18 & 47041773 & 18 & 47988831 & 18 & 47 & 0.021 & 0.6697 & 7.17 \\
\hline 2440 & 13951 & 28968 & 29 & 0.02 & 86.09 & 0.105105 & 0.065065 & 18 & 48005643 & 18 & 48998765 & 18 & 48 & 0.049 & 0.7067 & 4.95 \\
\hline 2441 & 13960 & 44819 & 20 & 0.02 & 85.56 & 0.071071 & 0.04004 & 18 & 49008785 & 18 & 49991095 & 18 & 49 & 0.049 & 0.7062 & 4.96 \\
\hline 2442 & 44820 & 35514 & 27 & 0.04 & 60.31 & 0.122122 & 0.082082 & 18 & 50016394 & 18 & 50984974 & 18 & 50 & 0.033 & 0.6959 & 5.96 \\
\hline 2443 & 33039 & 13976 & 31 & 0.07 & 27.55 & 0.134134 & 0.101101 & 18 & 51012467 & 18 & 51975074 & 18 & 51 & 0.015 & 0.6347 & 8.18 \\
\hline 2444 & 28984 & 28988 & 28 & 0.1 & 20.41 & 0.149149 & 0.111111 & 18 & 52001599 & 18 & 52977587 & 18 & 52 & 0.011 & 0.6028 & 9.13 \\
\hline 2445 & 49749 & 44830 & 25 & 0.04 & 52.97 & 0.121121 & 0.079079 & 18 & 53009145 & 18 & 53889747 & 18 & 53 & 0.029 & 0.6915 & 6.38 \\
\hline 2446 & 28996 & 45745 & 14 & 0.01 & 92.12 & 0.054054 & 0.031031 & 18 & 54010221 & 18 & 54902836 & 18 & 54 & 0.054 & 0.7081 & 4.77 \\
\hline 2447 & 18489 & 19199 & 23 & 0.04 & 54 & 0.113113 & 0.078078 & 18 & 55026793 & 18 & 55963572 & 18 & 55 & 0.029 & 0.6914 & 6.29 \\
\hline 2448 & 28999 & 29008 & 24 & 0.05 & 45.79 & 0.13013 & 0.09009 & 18 & 56020136 & 18 & 56988923 & 18 & 56 & 0.024 & 0.6815 & 6.79 \\
\hline 2449 & 29007 & 14015 & 21 & 0.03 & 71.58 & 0.082082 & 0.056056 & 18 & 57033078 & 18 & 57957917 & 18 & 57 & 0.04 & 0.7009 & 5.46 \\
\hline 2450 & 55697 & 29015 & 19 & 0.06 & 36.42 & 0.095095 & 0.06006 & 18 & 58006718 & 18 & 58958866 & 18 & 58 & 0.019 & 0.6606 & 7.37 \\
\hline 2451 & 56337 & 54740 & 15 & 0.53 & 7.22 & 0.194194 & 0.176176 & 18 & 59086075 & 18 & 59971337 & 18 & 59 & 0.004 & 0.507 & 12.46 \\
\hline 2452 & 44846 & 14947 & 12 & 0.18 & 12.04 & 0.102102 & 0.079079 & 18 & 60063061 & 18 & 60911033 & 18 & 60 & 0.006 & 0.5531 & 11.01 \\
\hline 2453 & 52415 & 17324 & 11 & 0.02 & 87.96 & 0.047047 & 0.031031 & 19 & 462585 & 19 & 930921 & 19 & 0 & 0.051 & 0.7078 & 4.89 \\
\hline 2454 & 29637 & 44851 & 26 & 0.02 & 83.11 & 0.11011 & 0.075075 & 19 & 1141455 & 19 & 1968265 & 19 & 1 & 0.048 & 0.7045 & 5.02 \\
\hline
\end{tabular}




\begin{tabular}{|c|c|c|c|c|c|c|c|c|c|c|c|c|c|c|c|c|}
\hline 2455 & 14033 & 17778 & 12 & 0.02 & 86.48 & 0.056056 & 0.042042 & 19 & 2004788 & 19 & 2838767 & 19 & 2 & 0.05 & 0.7069 & 4.94 \\
\hline 2456 & 14641 & 32232 & 31 & 0.06 & 33.62 & 0.141141 & 0.099099 & 19 & 3024537 & 19 & 3979293 & 19 & 3 & 0.018 & 0.6517 & 7.61 \\
\hline 2457 & 54441 & 44855 & 26 & 0.05 & 48.53 & 0.103103 & 0.073073 & 19 & 4006006 & 19 & 4977485 & 19 & 4 & 0.026 & 0.683 & 6.55 \\
\hline 2458 & 14037 & 59417 & 36 & 0.06 & 32.74 & 0.166166 & 0.114114 & 19 & 5061093 & 19 & 5997290 & 19 & 5 & 0.017 & 0.6499 & 7.68 \\
\hline 2459 & 18441 & 55592 & 23 & 0.03 & 78.7 & 0.094094 & 0.058058 & 19 & 6003273 & 19 & 6798065 & 19 & 6 & 0.045 & 0.7037 & 5.19 \\
\hline 2460 & 14047 & 29036 & 20 & 0.03 & 79.11 & 0.089089 & 0.06006 & 19 & 7185187 & 19 & 7979887 & 19 & 7 & 0.045 & 0.7046 & 5.19 \\
\hline 2461 & 14051 & 59871 & 18 & 0.02 & 90.2 & 0.074074 & 0.05005 & 19 & 8044093 & 19 & 8938817 & 19 & 8 & 0.052 & 0.7076 & 4.81 \\
\hline 2462 & 29042 & 14061 & 19 & 0.02 & 88.93 & 0.071071 & 0.054054 & 19 & 9077280 & 19 & 9970257 & 19 & 9 & 0.051 & 0.7076 & 4.86 \\
\hline 2463 & 55031 & 60055 & 30 & 0.05 & 45.59 & 0.138138 & 0.089089 & 19 & 10005053 & 19 & 10911377 & 19 & 10 & 0.024 & 0.6814 & 6.81 \\
\hline 2464 & 44876 & 44881 & 18 & 0.01 & 95.57 & 0.064064 & 0.018018 & 19 & 11030902 & 19 & 11783693 & 19 & 11 & 0.056 & 0.7088 & 4.66 \\
\hline 2465 & 48136 & 30608 & 22 & 0.01 & 94.62 & 0.108108 & 0.034034 & 19 & 12108725 & 19 & 12973924 & 19 & 12 & 0.056 & 0.7085 & 4.68 \\
\hline 2466 & 55739 & 30755 & 18 & 0.02 & 82.03 & 0.077077 & 0.042042 & 19 & 13061465 & 19 & 13993116 & 19 & 13 & 0.047 & 0.7049 & 5.08 \\
\hline 2467 & 14076 & 18054 & 16 & 0.05 & 40.3 & 0.063063 & 0.04004 & 19 & 14154082 & 19 & 14724810 & 19 & 14 & 0.021 & 0.6715 & 7.16 \\
\hline 2468 & 31333 & 29063 & 24 & 0.02 & 90.19 & 0.12012 & 0.039039 & 19 & 15010224 & 19 & 15982273 & 19 & 15 & 0.052 & 0.7076 & 4.82 \\
\hline 2469 & 29064 & 14086 & 14 & 0.02 & 82.79 & 0.046046 & 0.03003 & 19 & 16023625 & 19 & 16853665 & 19 & 16 & 0.047 & 0.7047 & 5.04 \\
\hline 2470 & 15599 & 29065 & 11 & 0.01 & 93.32 & 0.052052 & 0.031031 & 19 & 17147091 & 19 & 17947980 & 19 & 17 & 0.055 & 0.7081 & 4.72 \\
\hline 2471 & 48144 & 16347 & 6 & 0.03 & 65.56 & 0.045045 & 0.03003 & 19 & 18005050 & 19 & 18720914 & 19 & 18 & 0.036 & 0.6986 & 5.71 \\
\hline 2472 & 14089 & 39592 & 12 & 0.01 & 95.76 & 0.044044 & 0.026026 & 19 & 19218547 & 19 & 19944045 & 19 & 19 & 0.056 & 0.7089 & 4.66 \\
\hline 2473 & 39593 & 14096 & 8 & 0.01 & 95.83 & 0.043043 & 0.02002 & 19 & 20049731 & 19 & 20985182 & 19 & 20 & 0.056 & 0.7089 & 4.65 \\
\hline 2474 & 58514 & 56432 & 6 & 0 & 96.35 & 0.028028 & 0.016016 & 19 & 21103213 & 19 & 21870366 & 19 & 21 & 0.057 & 0.7091 & 4.64 \\
\hline 2475 & 29069 & 48274 & 17 & 0.02 & 86.77 & 0.063063 & 0.045045 & 19 & 22009202 & 19 & 22855768 & 19 & 22 & 0.05 & 0.7074 & 4.93 \\
\hline 2476 & 39594 & 29075 & 6 & 0 & 96.49 & 0.017017 & 0.008008 & 19 & 23047391 & 19 & 23980272 & 19 & 23 & 0.057 & 0.7092 & 4.63 \\
\hline 2477 & 39596 & 14109 & 7 & 0.01 & 95.53 & 0.036036 & 0.02002 & 19 & 24126684 & 19 & 24769857 & 19 & 24 & 0.056 & 0.7087 & 4.66 \\
\hline 2478 & 55085 & 59759 & 13 & 0.04 & 59.55 & 0.088088 & 0.066066 & 19 & 25015600 & 19 & 25930877 & 19 & 25 & 0.033 & 0.6945 & 5.98 \\
\hline 2479 & 32427 & 54800 & 9 & 0.02 & 91.76 & 0.046046 & 0.034034 & 19 & 26000408 & 19 & 26951074 & 19 & 26 & 0.053 & 0.7081 & 4.78 \\
\hline 2480 & 39602 & 29083 & 7 & 0 & 96.56 & 0.022022 & 0.001001 & 19 & 27034249 & 19 & 27962279 & 19 & 27 & 0.057 & 0.7093 & 4.63 \\
\hline 2481 & 14115 & 58372 & 12 & 0.03 & 68.38 & 0.073073 & 0.048048 & 19 & 28014791 & 19 & 28988256 & 19 & 28 & 0.038 & 0.6995 & 5.59 \\
\hline 2482 & 34698 & 44903 & 7 & 0.02 & 88.04 & 0.039039 & 0.031031 & 19 & 29072100 & 19 & 29704103 & 19 & 29 & 0.051 & 0.7077 & 4.89 \\
\hline 2483 & 39608 & 14113 & 7 & 0 & 96.31 & 0.035035 & 0.02002 & 19 & 30064026 & 19 & 30775028 & 19 & 30 & 0.057 & 0.709 & 4.64 \\
\hline 2484 & 39609 & 34918 & 4 & 0.01 & 92.6 & 0.03003 & 0.023023 & 19 & 31649425 & 19 & 31801282 & 19 & 31 & 0.054 & 0.7081 & 4.75 \\
\hline 2485 & 14124 & 52161 & 3 & 0 & 96.49 & 0.008008 & 0.004004 & 19 & 32076524 & 19 & 32496364 & 19 & 32 & 0.057 & 0.7092 & 4.63 \\
\hline 2486 & 47198 & 29108 & 4 & 0 & 96.29 & 0.024024 & 0.015015 & 19 & 33571880 & 19 & 33819184 & 19 & 33 & 0.057 & 0.7091 & 4.64 \\
\hline 2487 & 50326 & 50339 & 9 & 0.02 & 86.63 & 0.064064 & 0.047047 & 19 & 34450186 & 19 & 34925038 & 19 & 34 & 0.05 & 0.7072 & 4.93 \\
\hline 2488 & 48311 & 52236 & 15 & 0.03 & 70.63 & 0.067067 & 0.041041 & 19 & 35034444 & 19 & 35838914 & 19 & 35 & 0.039 & 0.7004 & 5.49 \\
\hline 2489 & 48295 & 30457 & 9 & 0.02 & 91.52 & 0.044044 & 0.025025 & 19 & 36313690 & 19 & 36933196 & 19 & 36 & 0.053 & 0.7082 & 4.79 \\
\hline 2490 & 53138 & 29090 & 16 & 0.04 & 63.01 & 0.085085 & 0.056056 & 19 & 37029211 & 19 & 37990102 & 19 & 37 & 0.035 & 0.6965 & 5.82 \\
\hline 2491 & 29091 & 14138 & 15 & 0.04 & 55.33 & 0.081081 & 0.053053 & 19 & 38023389 & 19 & 38708793 & 19 & 38 & 0.03 & 0.6919 & 6.19 \\
\hline 2492 & 48158 & 49782 & 30 & 0.05 & 43.72 & 0.145145 & 0.092092 & 19 & 39062455 & 19 & 39994318 & 19 & 39 & 0.023 & 0.6779 & 6.94 \\
\hline 2493 & 14146 & 61919 & 11 & 0.02 & 82.53 & 0.061061 & 0.042042 & 19 & 40075262 & 19 & 40954819 & 19 & 40 & 0.047 & 0.7049 & 5.06 \\
\hline
\end{tabular}




\begin{tabular}{|c|c|c|c|c|c|c|c|c|c|c|c|c|c|c|c|c|}
\hline 2494 & 29114 & 44923 & 13 & 0.01 & 95.73 & 0.034034 & 0.023023 & 19 & 41097603 & 19 & 41984701 & 19 & 41 & 0.056 & 0.7088 & 4.65 \\
\hline 2495 & 44924 & 14160 & 11 & 0.01 & 92.13 & 0.04004 & 0.029029 & 19 & 42112605 & 19 & 42984220 & 19 & 42 & 0.054 & 0.7081 & 4.77 \\
\hline 2496 & 29121 & 55243 & 16 & 0.02 & 81.84 & 0.058058 & 0.026026 & 19 & 43046630 & 19 & 43939946 & 19 & 43 & 0.047 & 0.7051 & 5.09 \\
\hline 2497 & 44927 & 30069 & 14 & 0.02 & 85.09 & 0.067067 & 0.047047 & 19 & 44092699 & 19 & 44988606 & 19 & 44 & 0.049 & 0.7062 & 4.98 \\
\hline 2498 & 29543 & 44930 & 25 & 0.02 & 85.39 & 0.104104 & 0.061061 & 19 & 45004996 & 19 & 45972443 & 19 & 45 & 0.049 & 0.7062 & 4.96 \\
\hline 2499 & 14168 & 48310 & 13 & 0.02 & 85.19 & 0.064064 & 0.041041 & 19 & 46048134 & 19 & 46763140 & 19 & 46 & 0.049 & 0.706 & 4.97 \\
\hline 2500 & 14169 & 35127 & 7 & 0 & 96.36 & 0.014014 & 0.009009 & 19 & 47148219 & 19 & 47592822 & 19 & 47 & 0.057 & 0.7091 & 4.64 \\
\hline 2501 & 35069 & 48166 & 7 & 0 & 96.48 & 0.02002 & 0.009009 & 19 & 48308192 & 19 & 48929906 & 19 & 48 & 0.057 & 0.7092 & 4.63 \\
\hline 2502 & 29133 & 53472 & 7 & 0 & 96.4 & 0.019019 & 0.01001 & 19 & 49013199 & 19 & 49617748 & 19 & 49 & 0.057 & 0.7091 & 4.64 \\
\hline 2503 & 48168 & 54845 & 4 & 0 & 96.52 & 0.01001 & 0.003003 & 19 & 50662741 & 19 & 50904771 & 19 & 50 & 0.057 & 0.7092 & 4.63 \\
\hline 2504 & 44936 & 48171 & 4 & 0 & 96.47 & 0.009009 & 0.003003 & 19 & 51624717 & 19 & 51916470 & 19 & 51 & 0.057 & 0.7092 & 4.63 \\
\hline 2505 & 56773 & 56148 & 2 & 0 & 96.53 & 0.012012 & 0.004004 & 19 & 52288809 & 19 & 52526689 & 19 & 52 & 0.057 & 0.7092 & 4.63 \\
\hline 2506 & 35161 & 39613 & 5 & 0 & 96.55 & 0.018018 & 0.007007 & 19 & 53047552 & 19 & 53798124 & 19 & 53 & 0.057 & 0.7093 & 4.63 \\
\hline 2507 & 30650 & 30650 & 1 & 0 & 96.56 & 0.002002 & 0 & 19 & 55073511 & 19 & 55073511 & 19 & 55 & 0.057 & 0.7093 & 4.63 \\
\hline 2508 & 48172 & 44939 & 4 & 0 & 96.56 & 0.007007 & 0 & 19 & 56659659 & 19 & 56808512 & 19 & 56 & 0.057 & 0.7093 & 4.63 \\
\hline 2509 & 30426 & 51732 & 2 & 0 & 96.56 & 0.004004 & 0.001001 & 19 & 57019187 & 19 & 57672447 & 19 & 57 & 0.057 & 0.7093 & 4.63 \\
\hline 2510 & 43884 & 44941 & 3 & 0 & 96.56 & 0.001001 & 0 & 19 & 58094394 & 19 & 58911107 & 19 & 58 & 0.057 & 0.7093 & 4.63 \\
\hline 2511 & 48173 & 14180 & 3 & 0 & 96.56 & 0 & 0 & 19 & 59339614 & 19 & 59411403 & 19 & 59 & 0.057 & 0.7093 & 4.63 \\
\hline 2512 & 14179 & 44942 & 2 & 0 & 96.35 & 0.005005 & 0.004004 & 19 & 60471958 & 19 & 60656889 & 19 & 60 & 0.057 & 0.7091 & 4.64 \\
\hline 2513 & 29137 & 14187 & 8 & 0.01 & 95.87 & 0.017017 & 0.01001 & 19 & 62086511 & 19 & 62675407 & 19 & 62 & 0.056 & 0.7088 & 4.65 \\
\hline 2514 & 44944 & 14181 & 9 & 0 & 96.47 & 0.026026 & 0.01001 & 19 & 63109515 & 19 & 63891837 & 19 & 63 & 0.057 & 0.7092 & 4.63 \\
\hline 2515 & 57187 & 29138 & 4 & 0 & 96.56 & 0.007007 & 0.001001 & 19 & 64571859 & 19 & 64882657 & 19 & 64 & 0.057 & 0.7093 & 4.63 \\
\hline 2516 & 48179 & 44946 & 4 & 0 & 96.5 & 0.01001 & 0.003003 & 19 & 65733803 & 19 & 65997012 & 19 & 65 & 0.057 & 0.7092 & 4.63 \\
\hline 2517 & 18832 & 48182 & 6 & 0 & 96.46 & 0.019019 & 0.006006 & 19 & 66009586 & 19 & 66356168 & 19 & 66 & 0.057 & 0.7091 & 4.63 \\
\hline 2518 & 44948 & 29140 & 4 & 0 & 96.54 & 0.009009 & 0.004004 & 19 & 67110552 & 19 & 67818695 & 19 & 67 & 0.057 & 0.7092 & 4.63 \\
\hline 2519 & 48185 & 61984 & 2 & 0 & 96.56 & 0.005005 & 0 & 19 & 68685785 & 19 & 68863251 & 19 & 68 & 0.057 & 0.7093 & 4.63 \\
\hline 2520 & 35034 & 37477 & 2 & 0 & 96.56 & 0 & 0 & 19 & 69322160 & 19 & 69589470 & 19 & 69 & 0.057 & 0.7093 & 4.63 \\
\hline 2521 & 14193 & 48186 & 3 & 0 & 96.53 & 0.004004 & 0.004004 & 19 & 70337525 & 19 & 70961131 & 19 & 70 & 0.057 & 0.7092 & 4.63 \\
\hline 2522 & 14195 & 14197 & 5 & 0 & 96.56 & 0.006006 & 0.004004 & 19 & 71022228 & 19 & 71708533 & 19 & 71 & 0.057 & 0.7093 & 4.63 \\
\hline 2523 & 14198 & 44949 & 4 & 0 & 96.46 & 0.01001 & 0.005005 & 19 & 72098978 & 19 & 72845131 & 19 & 72 & 0.057 & 0.7092 & 4.63 \\
\hline 2524 & 14199 & 57355 & 2 & 0 & 96.33 & 0.01001 & 0.008008 & 19 & 73417360 & 19 & 73903037 & 19 & 73 & 0.057 & 0.709 & 4.64 \\
\hline 2525 & 48190 & 55672 & 2 & 0 & 96.56 & 0.002002 & 0.002002 & 19 & 74064515 & 19 & 74704535 & 19 & 74 & 0.057 & 0.7093 & 4.63 \\
\hline 2526 & 48298 & 34562 & 3 & 0 & 96.56 & 0.006006 & 0 & 19 & 75081784 & 19 & 75703473 & 19 & 75 & 0.057 & 0.7093 & 4.63 \\
\hline 2527 & 14200 & 44951 & 7 & 0 & 96.32 & 0.022022 & 0.01001 & 19 & 76375371 & 19 & 76830183 & 19 & 76 & 0.057 & 0.709 & 4.64 \\
\hline 2528 & 14201 & 48193 & 2 & 0 & 96.37 & 0.01001 & 0.005005 & 19 & 77046979 & 19 & 77333713 & 19 & 77 & 0.057 & 0.7091 & 4.64 \\
\hline 2529 & 58310 & 19289 & 7 & 0 & 96.37 & 0.013013 & 0.011011 & 19 & 78000797 & 19 & 78893355 & 19 & 78 & 0.057 & 0.7091 & 4.64 \\
\hline 2530 & 32782 & 48196 & 4 & 0 & 96.56 & 0.004004 & 0.002002 & 19 & 79121304 & 19 & 79867005 & 19 & 79 & 0.057 & 0.7093 & 4.63 \\
\hline 2531 & 14204 & 49788 & 3 & 0 & 96.56 & 0.009009 & 0 & 19 & 80145695 & 19 & 80969428 & 19 & 80 & 0.057 & 0.7093 & 4.63 \\
\hline 2532 & 33245 & 33245 & 1 & 0 & 96.56 & 0.003003 & 0.001001 & 19 & 83670009 & 19 & 83670009 & 19 & 83 & 0.057 & 0.7093 & 4.63 \\
\hline
\end{tabular}




\begin{tabular}{|c|c|c|c|c|c|c|c|c|c|c|c|c|c|c|c|c|}
\hline 2533 & 34565 & 48197 & 2 & 0 & 96.56 & 0.001001 & 0 & 19 & 84602688 & 19 & 84914270 & 19 & 84 & 0.057 & 0.7093 & 4.63 \\
\hline 2534 & 48198 & 48200 & 7 & 0 & 96.53 & 0.012012 & 0.007007 & 19 & 85284931 & 19 & 85914898 & 19 & 85 & 0.057 & 0.7092 & 4.63 \\
\hline 2535 & 48201 & 34566 & 2 & 0 & 96.56 & 0.003003 & 0 & 19 & 86201492 & 19 & 86254418 & 19 & 86 & 0.057 & 0.7093 & 4.63 \\
\hline 2536 & 44954 & 14210 & 4 & 0 & 96.52 & 0.01001 & 0.008008 & 19 & 87347232 & 19 & 87901203 & 19 & 87 & 0.057 & 0.7092 & 4.63 \\
\hline 2537 & 53069 & 58669 & 2 & 0 & 96.56 & 0.004004 & 0 & 19 & 89259122 & 19 & 89918982 & 19 & 89 & 0.057 & 0.7093 & 4.63 \\
\hline 2538 & 51627 & 18556 & 5 & 0 & 96.37 & 0.008008 & 0.006006 & 19 & 90038588 & 19 & 90967583 & 19 & 90 & 0.057 & 0.7091 & 4.64 \\
\hline 2539 & 14213 & 58883 & 2 & 0 & 96.56 & 0.003003 & 0 & 19 & 91102005 & 19 & 91344424 & 19 & 91 & 0.057 & 0.7093 & 4.63 \\
\hline 2540 & 48205 & 14212 & 2 & 0 & 96.44 & 0.005005 & 0.003003 & 19 & 92108045 & 19 & 92882230 & 19 & 92 & 0.057 & 0.7092 & 4.63 \\
\hline 2541 & 34567 & 58352 & 5 & 0 & 96.56 & 0.008008 & 0.003003 & 19 & 93230002 & 19 & 93517538 & 19 & 93 & 0.057 & 0.7093 & 4.63 \\
\hline 2542 & 50082 & 50082 & 1 & 0 & 96.56 & 0.002002 & 0.001001 & 19 & 94468874 & 19 & 94468874 & 19 & 94 & 0.057 & 0.7093 & 4.63 \\
\hline 2543 & 18223 & 18223 & 1 & 0 & 96.54 & 0.011011 & 0.006006 & 19 & 95141795 & 19 & 95141795 & 19 & 95 & 0.057 & 0.7093 & 4.63 \\
\hline 2544 & 60857 & 60857 & 1 & 0 & 96.56 & 0.007007 & 0.004004 & 19 & 96090467 & 19 & 96090467 & 19 & 96 & 0.057 & 0.7093 & 4.63 \\
\hline 2545 & 48206 & 35173 & 5 & 0 & 96.56 & 0.011011 & 0.002002 & 19 & 97152327 & 19 & 97832104 & 19 & 97 & 0.057 & 0.7093 & 4.63 \\
\hline 2546 & 44955 & 44955 & 1 & 0 & 96.56 & 0.004004 & 0.003003 & 19 & 98978944 & 19 & 98978944 & 19 & 98 & 0.057 & 0.7093 & 4.63 \\
\hline 2547 & 48208 & 48212 & 7 & 0 & 96.56 & 0.006006 & 0 & 19 & 99091294 & 19 & 99836761 & 19 & 99 & 0.057 & 0.7093 & 4.63 \\
\hline 2548 & 48213 & 14217 & 9 & 0 & 96.46 & 0.01001 & 0.003003 & 19 & 100022640 & 19 & 100911159 & 19 & 100 & 0.057 & 0.7092 & 4.63 \\
\hline 2549 & 35143 & 14220 & 6 & 0 & 96.55 & 0.018018 & 0.01001 & 19 & 101068864 & 19 & 101736276 & 19 & 101 & 0.057 & 0.7093 & 4.63 \\
\hline 2550 & 59344 & 48219 & 6 & 0 & 96.53 & 0.014014 & 0.005005 & 19 & 102082896 & 19 & 102773567 & 19 & 102 & 0.057 & 0.7092 & 4.63 \\
\hline 2551 & 44959 & 29147 & 8 & 0.01 & 93.08 & 0.035035 & 0.022022 & 19 & 103011287 & 19 & 103657659 & 19 & 103 & 0.054 & 0.7081 & 4.73 \\
\hline 2552 & 52052 & 29148 & 14 & 0.01 & 92.93 & 0.054054 & 0.028028 & 19 & 104042870 & 19 & 104939296 & 19 & 104 & 0.054 & 0.7082 & 4.74 \\
\hline 2553 & 14228 & 29149 & 10 & 0.01 & 96.16 & 0.037037 & 0.02002 & 19 & 105103691 & 19 & 105978406 & 19 & 105 & 0.057 & 0.709 & 4.64 \\
\hline 2554 & 44964 & 29153 & 15 & 0.02 & 82.36 & 0.069069 & 0.05005 & 19 & 106003938 & 19 & 106970823 & 19 & 106 & 0.047 & 0.7049 & 5.07 \\
\hline 2555 & 48223 & 52303 & 16 & 0.04 & 55.5 & 0.097097 & 0.068068 & 19 & 107000024 & 19 & 107876735 & 19 & 107 & 0.03 & 0.6917 & 6.18 \\
\hline 2556 & 45258 & 14243 & 14 & 0.01 & 94.22 & 0.054054 & 0.038038 & 19 & 108206131 & 19 & 108915748 & 19 & 108 & 0.055 & 0.7083 & 4.69 \\
\hline 2557 & 58250 & 45563 & 15 & 0.03 & 75.86 & 0.053053 & 0.04004 & 19 & 109003374 & 19 & 109562447 & 19 & 109 & 0.043 & 0.7028 & 5.29 \\
\hline 2558 & 61325 & 39621 & 15 & 0.03 & 67.24 & 0.083083 & 0.06006 & 19 & 110165834 & 19 & 110841417 & 19 & 110 & 0.037 & 0.6991 & 5.64 \\
\hline 2559 & 57530 & 14256 & 13 & 0.05 & 47.36 & 0.073073 & 0.043043 & 19 & 111015335 & 19 & 111965300 & 19 & 111 & 0.026 & 0.6824 & 6.65 \\
\hline 2560 & 33727 & 14261 & 7 & 0.17 & 13.1 & 0.081081 & 0.066066 & 19 & 112108617 & 19 & 112884986 & 19 & 112 & 0.007 & 0.564 & 10.86 \\
\hline 2561 & 14262 & 34117 & 20 & 0.39 & 8.04 & 0.161161 & 0.139139 & 19 & 113078996 & 19 & 113962590 & 19 & 113 & 0.004 & 0.5182 & 12.36 \\
\hline 2562 & 49789 & 35551 & 21 & 0.04 & 59.05 & 0.1001 & 0.06006 & 19 & 114259111 & 19 & 114972696 & 19 & 114 & 0.032 & 0.6942 & 6.01 \\
\hline 2563 & 33535 & 29183 & 9 & 0.01 & 92.27 & 0.044044 & 0.026026 & 19 & 115004040 & 19 & 115895028 & 19 & 115 & 0.054 & 0.7081 & 4.76 \\
\hline 2564 & 14278 & 34635 & 10 & 0.02 & 84.58 & 0.041041 & 0.032032 & 19 & 116033785 & 19 & 116843785 & 19 & 116 & 0.049 & 0.7054 & 4.98 \\
\hline 2565 & 48232 & 48234 & 5 & 0 & 96.43 & 0.014014 & 0.01001 & 19 & 117010968 & 19 & 117611622 & 19 & 117 & 0.057 & 0.7092 & 4.64 \\
\hline 2566 & 44980 & 48236 & 3 & 0 & 96.54 & 0.013013 & 0.008008 & 19 & 118208682 & 19 & 118515509 & 19 & 118 & 0.057 & 0.7093 & 4.63 \\
\hline 2567 & 44981 & 29185 & 6 & 0 & 96.4 & 0.018018 & 0.011011 & 19 & 119371829 & 19 & 119809654 & 19 & 119 & 0.057 & 0.7091 & 4.64 \\
\hline 2568 & 14284 & 34641 & 3 & 0 & 96.52 & 0.009009 & 0.006006 & 19 & 120411731 & 19 & 120937826 & 19 & 120 & 0.057 & 0.7092 & 4.63 \\
\hline 2569 & 30481 & 14437 & 15 & 0.02 & 91.14 & 0.054054 & 0.042042 & 19 & 121196405 & 19 & 121945530 & 19 & 121 & 0.053 & 0.708 & 4.79 \\
\hline 2570 & 29188 & 31667 & 13 & 0.02 & 91.09 & 0.056056 & 0.031031 & 19 & 122081895 & 19 & 122863117 & 19 & 122 & 0.053 & 0.708 & 4.8 \\
\hline 2571 & 14724 & 48239 & 10 & 0.01 & 94.48 & 0.055055 & 0.032032 & 19 & 123376923 & 19 & 123800527 & 19 & 123 & 0.055 & 0.7083 & 4.69 \\
\hline
\end{tabular}




\begin{tabular}{|c|c|c|c|c|c|c|c|c|c|c|c|c|c|c|c|c|}
\hline 2572 & 52925 & 29195 & 9 & 0.01 & 95.27 & 0.05005 & 0.032032 & 19 & 124405844 & 19 & 124994817 & 19 & 124 & 0.056 & 0.7086 & 4.67 \\
\hline 2573 & 29194 & 29203 & 31 & 0.05 & 42.41 & 0.156156 & 0.092092 & 19 & 125006304 & 19 & 125972086 & 19 & 125 & 0.022 & 0.6766 & 7.05 \\
\hline 2574 & 14299 & 14303 & 9 & 0.01 & 94.77 & 0.04004 & 0.033033 & 19 & 126033395 & 19 & 126992902 & 19 & 126 & 0.056 & 0.7084 & 4.68 \\
\hline 2575 & 14302 & 29208 & 12 & 0.01 & 93.61 & 0.043043 & 0.02002 & 19 & 127051537 & 19 & 127945580 & 19 & 127 & 0.055 & 0.7081 & 4.71 \\
\hline 2576 & 48245 & 14309 & 13 & 0 & 96.5 & 0.031031 & 0.01001 & 19 & 128108120 & 19 & 128842928 & 19 & 128 & 0.057 & 0.7092 & 4.63 \\
\hline 2577 & 14310 & 14315 & 15 & 0.03 & 70.15 & 0.062062 & 0.04004 & 19 & 129032125 & 19 & 129975535 & 19 & 129 & 0.039 & 0.7002 & 5.52 \\
\hline 2578 & 45007 & 29218 & 18 & 0.01 & 93.83 & 0.065065 & 0.039039 & 19 & 130002728 & 19 & 130728194 & 19 & 130 & 0.055 & 0.7083 & 4.7 \\
\hline 2579 & 49802 & 16578 & 8 & 0.01 & 96.2 & 0.026026 & 0.015015 & 19 & 131104850 & 19 & 131708696 & 19 & 131 & 0.057 & 0.7091 & 4.64 \\
\hline 2580 & 54589 & 14326 & 8 & 0.01 & 95.26 & 0.025025 & 0.016016 & 19 & 132088343 & 19 & 132966392 & 19 & 132 & 0.056 & 0.7086 & 4.67 \\
\hline 2581 & 14327 & 29223 & 7 & 0.01 & 96.09 & 0.023023 & 0.013013 & 19 & 133074194 & 19 & 133995643 & 19 & 133 & 0.057 & 0.7091 & 4.64 \\
\hline 2582 & 14328 & 45012 & 8 & 0.01 & 93.59 & 0.017017 & 0.01001 & 19 & 134009220 & 19 & 134779719 & 19 & 134 & 0.055 & 0.7082 & 4.71 \\
\hline 2583 & 14331 & 33021 & 7 & 0 & 96.23 & 0.025025 & 0.013013 & 19 & 135350565 & 19 & 135937088 & 19 & 135 & 0.057 & 0.7091 & 4.64 \\
\hline 2584 & 53437 & 14337 & 12 & 0.01 & 95 & 0.053053 & 0.028028 & 19 & 136018066 & 19 & 136986704 & 19 & 136 & 0.056 & 0.7087 & 4.68 \\
\hline 2585 & 58402 & 48256 & 6 & 0.01 & 96.12 & 0.016016 & 0.008008 & 19 & 137042297 & 19 & 137814306 & 19 & 137 & 0.057 & 0.709 & 4.64 \\
\hline 2586 & 45015 & 14343 & 20 & 0.03 & 78.88 & 0.085085 & 0.062062 & 19 & 138175988 & 19 & 138988497 & 19 & 138 & 0.045 & 0.7042 & 5.19 \\
\hline 2587 & 49805 & 29232 & 14 & 0.01 & 96.01 & 0.056056 & 0.024024 & 19 & 139042393 & 19 & 139986269 & 19 & 139 & 0.057 & 0.709 & 4.65 \\
\hline 2588 & 14344 & 15258 & 13 & 0.01 & 95.86 & 0.048048 & 0.029029 & 19 & 140000914 & 19 & 140920145 & 19 & 140 & 0.056 & 0.7088 & 4.65 \\
\hline 2589 & 56026 & 15779 & 10 & 0.01 & 92.71 & 0.061061 & 0.034034 & 19 & 141270018 & 19 & 141996499 & 19 & 141 & 0.054 & 0.7081 & 4.74 \\
\hline 2590 & 31214 & 14353 & 18 & 0.04 & 55.99 & 0.082082 & 0.047047 & 19 & 142000976 & 19 & 142984588 & 19 & 142 & 0.031 & 0.6919 & 6.16 \\
\hline 2591 & 49812 & 19344 & 14 & 0.02 & 86.83 & 0.059059 & 0.042042 & 19 & 143013614 & 19 & 143838682 & 19 & 143 & 0.05 & 0.7074 & 4.93 \\
\hline 2592 & 33256 & 50059 & 3 & 0 & 96.53 & 0.01001 & 0.006006 & 19 & 144033871 & 19 & 144187103 & 19 & 144 & 0.057 & 0.7092 & 4.63 \\
\hline 2593 & 30809 & 14634 & 4799 & 9.76 & 0 & 1 & 1 & 21 & 0 & 21 & 0 & 21 & 0 & 0.001 & 0.5572 & 28.97 \\
\hline
\end{tabular}

